\title{
The $r^{p}$-Weighted Energy Method of Dafermos and Rodnianski in General Asymptotically Flat Spacetimes and Applications
}

\author{
Georgios Moschidis ${ }^{1}$
}

Received: 16 December 2015 / Accepted: 25 March 2016 / Published online: 2 May 2016

(C) Springer International Publishing AG 2016

\begin{abstract}
In [11], Dafermos and Rodnianski presented a novel approach to establish uniform decay rates for solutions $\varphi$ to the scalar wave equation $\square_{g} \varphi=0$ on Minkowski, Schwarzschild and other asymptotically flat backgrounds. This paper generalises the methods and results of [11] to a broad class of asymptotically flat spacetimes $(\mathcal{M}, g)$, including Kerr spacetimes in the full subextremal range $|a|<M$, but also radiating spacetimes with no exact symmetries in general dimension $d+1$, $d \geq 3$. As a soft corollary, it is shown that the Friedlander radiation field for $\varphi$ is well defined on future null infinity. Moreover, polynomial decay rates are established for $\varphi$, provided that an integrated local energy decay statement (possibly with a finite loss of derivatives) holds and the near region of $(\mathcal{M}, g)$ satisfies some mild geometric conditions. The latter conditions allow for $(\mathcal{M}, g)$ to be the exterior of a black hole spacetime with a non-degenerate event horizon (having possibly complicated topology) or the exterior of a compact moving obstacle in an ambient globally hyperbolic spacetime satisfying suitable geometric conditions.
\end{abstract}

Keywords Asymptotically flat $\cdot$ Improved polynomial decay $\cdot$ Friedlander radiation field

\section{Contents}

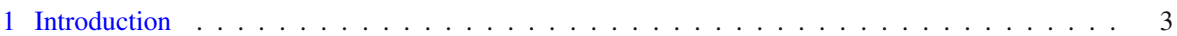

1.1 The Klainerman Vector Field Method . . . . . . . . . . . . . . . . . . . . . . . . . . . . . . . .

1.2 The Dafermos-Rodnianski Method . . . . . . . . . . . . . . . . . . . . . 5

1.3 Non-technical Statements of the Main Results and Applications . . . . . . . . . . . . . . 7

1.4 Comparison of the Two Approaches . . . . . . . . . . . . . . . . . . . . . 11

$凶$ Georgios Moschidis gm6@math.princeton.edu

1 Department of Mathematics, Princeton University, Fine Hall, Washington Road, Princeton, NJ 08544, USA 
1.5 Statement of the Main Results . . . . . . . . . . . . . . . . . . . . . . . . . . . 14

1.6 Applications of the $r^{p}$-Weighted Energy Method . . . . . . . . . . . . . . . . . 21

1.7 Outline of the Paper and Technical Comments . . . . . . . . . . . . . . . . . . 28

2 Notational Conventions . . . . . . . . . . . . . . . . . . . . . . 30

2.1 Conventions on Constants and Inequality Symbols . . . . . . . . . . . . . . . . . . . 30

2.2 Convention on Connections and Volume form Notations . . . . . . . . . . . . . . . . . . . 31

2.3 Conventions on Notations for Derivatives on $\mathbb{S}^{d-1} \ldots \ldots \ldots \ldots \ldots$

2.4 Convention on the $O(\cdot)$ Notation . . . . . . . . . . . . . . . . . . 33

2.5 Conventions on Integration . . . . . . . . . . . . . . . . . . . . . . . . . . . . . . . . . . . . . . . 34

2.6 Notations for Vector Field Multipliers and Currents . . . . . . . . . . . . . . . . . . 34

3 Geometry of the Asymptotically Flat Regions $\left(\mathcal{N}_{a f}, g\right) \ldots \ldots \ldots \ldots$

3.1 Spacelike Hyperboloidal Hypersurfaces Terminating at $\mathcal{I}^{+} \ldots \ldots$. . . . . . . . . . . . 36

3.2 Expression of the Wave Equation in the Coordinate Chart $(u, v, \sigma) \ldots \ldots$. . . . . . . 38

4 Some $\partial_{r}$-Morawetz Type and Energy Boundedness Estimates . . . . . . . . . . . . . . . . . 38

4.1 A First $\partial_{r}$-Morawetz Type Estimate . . . . . . . . . . . . . . . . . . . . . . . . . 39

4.2 An Improved $\partial_{r}$-Morawetz Type Estimate . . . . . . . . . . . . . . . . . . . . . . . . . . . . . . . . . . . . . . . . .

4.3 Estimates for the $J^{T}$-Energy $\ldots \ldots \ldots \ldots \ldots \ldots \ldots \ldots$

5 The Extension of the $r^{p}$-Weighted Energy Hierarchy . . . . . . . . . . . . . . . . . . 54

5.1 Statement of the $r^{p}$-Weighted Energy Hierarchy . . . . . . . . . . . . . . . . . . 54

5.2 Proof of Theorem $5.1 \ldots \ldots \ldots \ldots \ldots \ldots$

6 The Improved $r^{p}$-Weighted Energy Hierarchy for Higher Order Derivatives . . . . . . . . . . . 69

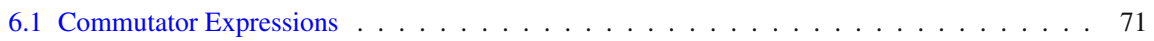

6.2 Proof of Theorem $6.1 \ldots \ldots \ldots \ldots \ldots \ldots \ldots \ldots$

7 Friedlander Radiation Field on $\mathcal{I}^{+} \ldots \ldots \ldots \ldots \ldots \ldots$. . . . . . . . . . . . . . . 88

7.1 Assumptions on the Spacetimes Under Consideration . . . . . . . . . . . . . . . . . . 88

7.2 Existence of the Friedlander Radiation Field on Future Null Infinity . . . . . . . . . . . . . 89

7.3 Estimates for $\Phi_{\mathcal{I}^{+}}$Provided by Lemma $5.1 \ldots \ldots \ldots$. . . . . . . . . . . . . 93

8 Polynomial Decay $\bar{t}^{-1}$ for Solutions to $\square_{g} \varphi=0 \ldots \ldots \ldots \ldots \ldots \ldots$. . . . . . . . 94

8.1 Assumptions on the Class of Spacetimes $(\mathcal{M}, g)$ Under Consideration . . . . . . . . . . . 94

8.2 A First Polynomial Decay Result . . . . . . . . . . . . . . . . . . . . . . . . . . . 101

8.3 Energy Boundedness with Loss of Derivatives . . . . . . . . . . . . . . . . . . . 105

8.4 Proof of Theorem $8.1 \ldots \ldots \ldots \ldots$. . . . . . . . . . . . . . . 108

9 Improved Polynomial Decay $\bar{t}^{-\frac{d}{2}}$ for Solutions to $\square_{g} \varphi=0$ in Dimensions $d \geq 3 \ldots \ldots$. . . . . . 112

9.1 Further Assumptions and Geometric Constructions on $(\mathcal{M}, g) \ldots \ldots$. . . . . . . . 113

9.2 Shorthand Notation for Energy Norms . . . . . . . . . . . . . . . . . . . . . . . 120

9.3 Statement of the Results on Improved Polynomial Decay . . . . . . . . . . . . . . . . . . . . . . . . . . . . . . . . . .

9.4 Sketch of the Proof of Theorem 9.1 and Corollary $9.2 \ldots$. . . . . . . . . . . . . . . . . . 125

9.5 Commutation with $T, K_{R_{C}}$ and Control of the Error Terms . . . . . . . . . . . . 127

9.6 Integrated Local Energy Decay After Commuting with $T, K_{R_{C}} \ldots \ldots$. . . . . . . . . . 129

9.7 Proof of Theorem 9.1 on Improved Polynomial Decay . . . . . . . . . . . . . . . . . . . 136

9.8 Gagliardo-Nirenberg Type Inequalities on the Hyperboloids $\{\bar{t}=$ const $\}$. . . . . . . . . . 145

9.9 Proof of Corollary $9.2 \ldots \ldots \ldots \ldots$. . . . . . . . . . . . . . . . . . 153

Appendix A: Construction of the Natural Riemannian Metrics $h_{\tau, N}$ and $h_{\tau, K, \Phi} \ldots \ldots \ldots 5$

A.1 Construction of $h_{\tau, N} \ldots \ldots \ldots \ldots \ldots \ldots \ldots \ldots$

A.2 Construction of $h_{\tau, K, \Phi} \ldots \ldots \ldots \ldots \ldots \ldots \ldots \ldots$. . . . . . . . . . . . . 157

Appendix B: Elliptic Estimates on Asymptotically Euclidean Riemannian Manifolds with Boundary 158

B.1 Geometric Constructions on $(\mathcal{S}, h) \ldots \ldots \ldots \ldots \ldots \ldots$

B.2 Elliptic Estimates on $(\mathcal{S}, h) \ldots \ldots \ldots \ldots \ldots \ldots \ldots$

B.3 Lemma on Harmonic Functions on $(\mathcal{S}, h) \ldots \ldots \ldots \ldots$. . . . . . . . . . . . . 182

Appendix C: Hardy Type Inequalities . . . . . . . . . . . . . . . . . . . . . . . . . . . . . . . 190

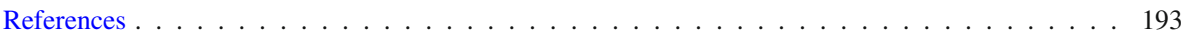




\section{Introduction}

The covariant wave equation

$$
\square_{g} \varphi=\frac{1}{\sqrt{-g}} \partial_{\mu}\left(g^{\mu \nu} \sqrt{-g} \partial_{\nu} \varphi\right)=0,
$$

where $g$ is the Lorentzian metric of a background manifold $\mathcal{M}$, arises in various areas of mathematical physics, including fluid mechanics, where $g$ is the so called acoustical metric of a fluid in motion, as well as general relativity, in which case $g$ corresponds to the spacetime metric of a $3+1$ dimensional model of our universe.

Of fundamental importance in most settings where equation (1.1) appears is the case where the background $(\mathcal{M}, g)$ is flat or almost flat, that is, when $\mathcal{M}=\mathbb{R}^{d+1}$ and $g$ is the Minkowski metric $\eta$

$$
\eta=-d t^{2}+\left(d x^{1}\right)^{2}+\ldots+\left(d x^{d}\right)^{2}
$$

(in the usual $\left(t, x^{1}, \ldots, x^{d}\right)$ coordinates of $\left.\mathbb{R}^{d+1}\right)$ or small perturbations of it, respectively. These are the simplest settings for which the stability properties (i.e. uniform boundedness and decay properties) of solutions to (1.1) have been studied extensively. Of particular interest for applications is also the study of the stability properties of equation (1.1) on backgrounds $(\mathcal{M}, g)$ which are far from Minkowski, but which are asymptotically flat, i.e. asymptotically approach (as one moves to "infinity" along any null direction) the geometry of $\left(\mathbb{R}^{d+1}, \eta\right)$. Such backgrounds include, for instance, various black hole spacetimes appearing in general relativity (see [13]).

In this paper, we will develop a general approach for establishing decay estimates for equation (1.1) on a general class of asymptotically flat backgrounds $(\mathcal{M}, g)$, generalising the methods of [11]. In order to better clarify the motivation behind our approach and our assumptions on the backgrounds $(\mathcal{M}, g)$, we will first briefly highlight the main techniques that have been developed so far for obtaining stability estimates for equation (1.1), and state a non-technical summary of our results. We will then revisit and compare the main techniques that already exist in the literature, before, finally, presenting our results in detail.

\subsection{The Klainerman Vector Field Method}

One of the most successful approaches for obtaining decay estimates for solutions $\varphi$ to (1.1) on flat or almost flat backgounds has been the so called vector field method (see e.g. [35]), which utilises the vector fields generating the conformal isometries of Minkowski spacetime in two ways:

1. As multipliers: For any conformally Killing vector field $X$ of $\left(\mathbb{R}^{d+1}, \eta\right)$, one can multiply equation (1.1) with $X(\varphi)+w \varphi$ (where $w$ is a smooth function on $\mathbb{R}^{d+1}$ depending on the choice of $X$ ) and then integrate the resulting expression over a domain $\Omega$ of $\mathbb{R}^{d+1}$ bounded by two achronal hypersurfaces $\mathcal{S}_{1}, \mathcal{S}_{2}$, with $\mathcal{S}_{2}$ being 
in the future of $\mathcal{S}_{1}$ (e.g. $\Omega$ can be of the form $\{0 \leq t \leq T\}$ ). For the right choices of $X, w$, performing an integration by parts yields an identity of the form

$$
\int_{\mathcal{S}_{2}} \mathcal{E}_{X, w}[\varphi]=\int_{\mathcal{S}_{1}} \mathcal{E}_{X, w}[\varphi],
$$

where $\mathcal{E}_{X, w}[\varphi]$ is a positive definite weighted quadratic expression in $\varphi$ and its first derivatives. Notice that the identity (1.3) only contains terms on the boundary of $\Omega$ and can be interpreted as an estimate of the "final" energy norm $\int_{\mathcal{S}_{2}} \mathcal{E}_{X, w}[\varphi]$ in terms of the "initial" energy norm $\int_{\mathcal{S}_{1}} \mathcal{E}_{X, w}[\varphi]$. This approach can be traced back to Morawetz (see [28]).

2. As commutation vector fields: For certain elements $X$ of the algebra of conformally Killing vector fields of $\mathbb{R}^{d+1}$, the commutator $\left[\square_{g}, X\right]$ is is either 0 or a multiple of $\square_{g}$. Thus, equation (1.1) is also satisfied by $X \varphi$ (or even higher derivatives of $\varphi$ ), and this fact allows the establishment of $L^{2}$ estimates for higher order derivatives of $\varphi$, which in turn yield pointwise decay estimates for $\varphi$ itself through suitable global Sobolev inequalities. This approach was initiated and developed by Klainerman (see e.g. [21,22]).

The vector field method has turned out to be especially fruitful in the study of non linear variants of (1.1), culminating in the proof of the non linear stability of Minkowski spacetime in [7].

Preceding the use of conformally Killing vector fields $X$ as multipliers for equation (1.1), Morawetz [27] utilised more general first order operators generating "positive bulk terms" in $\varphi$, i.e. estimates for the $L^{2}$ norm of $\varphi$ integrated over spacetime. In particular, studying the decay properties of solutions $\varphi$ to equation (1.1) on the exterior of a compact star-shaped obstacle $\mathcal{O}$ in $\mathbb{R}^{d}$ with reflecting boundary conditions on $\partial \mathcal{O}$, Morawetz derived an integrated local energy decay statement for $\varphi$, that is an estimate of the form

$$
\begin{aligned}
& \int_{0}^{\infty} \int_{\{t=\tau\} \cap\{r \leq R\}}\left(|\partial \varphi|^{2}+|\varphi|^{2}\right) d x d \tau+\int_{0}^{\infty} \int_{\{t=\tau\} \cap \partial \mathcal{O}}|\partial \varphi|^{2} d \sigma d \tau \\
& \quad \lesssim \int_{\{t=0\}}|\partial \varphi|^{2} d x .
\end{aligned}
$$

This estimate was obtained in [27] by using the (not conformally Killing) radial vector field $\partial_{r}$ as a multiplier for (1.1).

The exterior of a compact obstacle $\mathcal{O}$ in $\mathbb{R}^{d}$ (where suitable boundary conditions for solutions $\varphi$ to (1.1) are imposed on the boundary $\partial \mathcal{O}$ of $\mathcal{O}$ ) is already an example of a background for equation (1.1) which is not a globally small perturbation of Minkowski spacetime. More complicated examples far from Minkowski include spacetimes $\left(\mathcal{M}^{d+1}, g\right), d \geq 3$, which contain black hole regions, like Schwarzschild or Kerr (see [13]). Such backgrounds are of particular interest to general relativity. One common feature that the exterior of a compact obstacle $\mathcal{O}$ in flat space and the exterior of a black hole spacetime share is the fact that they are naturally separated into two regions where different geometric mechanisms contribute to the long time behaviour of solutions to (1.1) on them: 
- In the "near" region of these backgrounds, the long time behaviour of solutions to (1.1) is strongly affected by the characteristics of the null geodesic flow, such as the existence of trapped null geodesics which are reflected on the obstacle or orbit around the black hole. In the black hole case, the existence of such geodesics is unavoidable. A further geometric aspect of a black hole spacetime $(\mathcal{M}, g)$ which is absent in the obstacle case is the so called event horizon $\mathcal{H}$. In most interesting examples, the geometric structure of $\mathcal{H}$ leads to the celebrated red-shift effect, which forces "wave packets" travelling along the null generators of $\mathcal{H}$ to decay fast. For this reason, the null geodesics spanning $\mathcal{H}$ are not considered trapped in this case. ${ }^{1}$

- In the "far away" region of these backgrounds, there exists a coordinate chart $\left(t, x^{1}, \ldots, x^{d}\right)$ in which the metric $g$ is pointwise close to the Minkowski metric $\eta$ (1.2) and tends to it along all outgoing null directions (of course, in the exterior of a compact obstacle in flat space, $g$ is identically equal to the Minkowski metric $\eta$ in this region). Thus, setting $r=\sqrt{\left(x^{1}\right)^{2}+\ldots+\left(x^{d}\right)^{2}}$, the area of the $\{r, t=$ const $\}$ surfaces increases to infinity along the outgoing null directions, and this fact serves as a decay mechanism for solutions to (1.1). In particular, the quantity $r^{\frac{d-1}{2}} \varphi$ is expected to have a finite limit on future null infinity $\mathcal{I}^{+}$, provided that $\varphi$ arises from suitably decaying initial data (see [17] and Section 7). Notice that on a general asymptotically flat spacetime $(\mathcal{M}, g)$, with its asymptotically flat region foliated by a set of outgoing null hypersurfaces $\left\{\mathcal{S}_{\tau}\right\}_{\tau \in \mathbb{R}}, \mathcal{I}^{+}$can be abstractly defined and is parametrised by the "points at infinity" of the null geodesics generating $\left\{\mathcal{S}_{\tau}\right\}_{\tau \in \mathbb{R}}$.

The issue of matching the estimates obtained for solutions to (1.1) in different regions of a black hole spacetime implicitly appeared in $[1,4,5,8,9,12,13,37,38]$, where definitive boundedness and decay estimates were established for solutions to (1.1) on Schwarzschild and very slowly rotating Kerr exterior spacetimes (i.e. for Kerr spacetimes with angular momentum $a$ and mass $M$ satisfying the relation $|a| \ll M$ ). This was achieved by the use of a Morawetz-type integrated local energy decay statement, in conjunction with an adaptation of techniques previously applied on flat spacetime.

\subsection{The Dafermos-Rodnianski Method}

In [11], Dafermos and Rodnianski suggested a more flexible strategy for proving polynomial decay estimates for solutions to (1.1), which is explicitly tied to the aforementioned partition of a general asymptotically flat spacetime. This approach makes use of first order multipliers producing both positive boundary terms (like in (1.3)) and positive bulk terms (like in (1.4)), and each term contains weights which grow towards $\mathcal{I}^{+}$but are time-translation invariant. For the sake of simplicity of our exposition, we will discuss here the approach of [11] restricted to the case of Schwarzschild spacetime.

\footnotetext{
1 They are considered trapped, however, in the case when $\mathcal{H}$ is degenerate and the red-shift effect is absent, which happens in extremal black hole spacetimes. See [3].
} 
On the exterior of Schwarzschild spacetime $\left(\mathcal{M}_{S c h}, g_{M}\right)$ of mass $M$, fix the $(u, v, \sigma)$ double null coordinate system (where $u=\frac{1}{2}\left(t-r^{*}\right)$ and $v=\frac{1}{2}\left(t+r^{*}\right)$, see [13]) and let $\left\{\mathcal{S}_{\tau}\right\}_{\tau \in \mathbb{R}}$ be a foliation of $\mathcal{M}_{S c h}$ by spacelike hypersurfaces terminating at $\mathcal{I}^{+}$(see Section 3 for the relevant definition), such that $\mathcal{S}_{\tau_{2}}$ is in the future domain of dependence of $\mathcal{S}_{\tau_{1}}$ when $\tau_{2}>\tau_{1}$. Let also $T$ denote the stationary Killing field of $\left(\mathcal{M}_{S c h}, g_{M}\right)$, and let $N$ be a globally timelike vector field on $\mathcal{M}_{S c h}$ such that $[T, N]=0$ and $T \equiv N$ in the far away region $\{r \gg 1\}$. Then the following estimates hold for solutions $\varphi$ to (1.1) (See Section 2 for the notations on vector field currents):

Non degenerate energy boundedness: For any $\tau_{1}<\tau_{2}$ :

$$
\int_{\mathcal{S}_{\tau_{2}}} J_{\mu}^{N}(\varphi) n_{\mathcal{S}_{\tau_{2}}}^{\mu} \leq C \cdot \int_{\mathcal{S}_{\tau_{1}}} J_{\mu}^{N}(\varphi) n_{\mathcal{S}_{\tau_{1}}}^{\mu}
$$

where $n_{\mathcal{S}_{\tau}}$ is the future directed unit normal on the leaves of the foliation $\left\{\mathcal{S}_{\tau}\right\}$, and the constant $C$ in (1.5) depends only on the precise choice of the foliation $\left\{\mathcal{S}_{\tau}\right\}_{\tau \in \mathbb{R}}$ and the vectro field $N$. See [9] for a proof of (1.5).

Integrated local energy decay in the near region: There exists an $m>0$, such that for any $R>0$ and $\tau \in \mathbb{R}$ :

$$
\int_{\mathcal{D}^{+}\left(\mathcal{S}_{\tau}\right) \cap\{r \leq R\}}\left(|\partial \varphi|^{2}+|\varphi|^{2}\right) d g_{\mathcal{M}} \leq C(R) \cdot \sum_{j=0}^{m} \int_{\mathcal{S}_{\tau}} J_{\mu}^{N}\left(T^{j} \varphi\right) n_{\mathcal{S}_{\tau}}^{\mu},
$$

where $d g_{\mathcal{M}}$ is the spacetime volume form, $n_{\mathcal{S}_{\tau}}$ is the future directed unit normal on $\mathcal{S}_{\tau}$ and the constant $C(R)$ depends only on $R$ and the precise choice of the foliation $\left\{\mathcal{S}_{\tau}\right\}_{\tau \in \mathbb{R}}$. This was established in $[4,8,9]$.

Remark Notice that (1.6) is actually valid for $m=1$. However, due to the existence of trapped null geodesics on $\left(\mathcal{M}_{S c h}, g_{M}\right)$, the requirement that $m>0$ is necessary in this case. Notice also that it is the red shift effect that allows the integrand in the left hand side of (1.6) to be non-degenerate up to the event horizon $\mathcal{H}$ of $\left(\mathcal{M}_{S c h}, g_{M}\right)$ (see [13]).

Using as ingredients the estimates (1.5) and (1.6), the novel approach of [11] for establishing polynomial decay rates for solutions $\varphi$ to (1.1) lies in the proof of a hierarchy of $r^{p}$-weighted energy estimates for $\varphi$ in a neighborhood of $\mathcal{I}^{+}$and the repeated use of the pigeonhole principle on the resulting set of estimates in order to obtain polynomial decay rates for various weighted energies of $\varphi$. In particular, the following result was established in [11]:

Theorem (Dafermos-Rodnianski [11], specialised here to Schwarzschild) On Schwarzschild exterior spacetime $\left(\mathcal{M}_{S c h}, g_{M}\right)$, the following statements hold for any solution $\varphi$ to the wave equation (1.1): 
1. An $r^{p}$-weighted energy hierarchy of the form

$$
\begin{aligned}
& \int_{\{r \geq R\} \cap\left\{u=\tau_{2}\right\}} r^{p}\left|\partial_{v}(r \varphi)\right|^{2} d v d \sigma+\int_{\{r \geq R\} \cap \mathcal{D}_{\tau_{1}}^{\tau_{2}}} r^{p-1}\left(p\left|\partial_{v}(r \varphi)\right|^{2}\right. \\
& \left.\quad+(2-p)\left|r^{-1} \partial_{\sigma}(r \varphi)\right|^{2}\right) d u d v d \sigma \\
& \lesssim \int_{\{r \geq R\} \cap\left\{u=\tau_{1}\right\}} r^{p}\left|\partial_{v}(r \varphi)\right|^{2} d v d \sigma+\int_{\{r \sim R\} \cap \mathcal{D}_{\tau_{1}}^{\tau_{2}}}\left(|\partial \varphi|^{2}+|\varphi|^{2}\right),
\end{aligned}
$$

for $p \in[0,2]$ holds, where $\mathcal{D}_{\tau_{1}}^{\tau_{2}}=\left\{\tau_{1} \leq u \leq \tau_{2}\right\}$ for $\tau_{1}<\tau_{2}$, and the derivatives are considered with respect to the double null coordinate system $(u, v, \sigma)$ on $\mathcal{M}_{\text {Sch }}$. The hierarchy (1.7) is stable under suitable perturbations of the background metric.

2. Let $\bar{t}$ be a time function on $\mathcal{M}_{S c h}$ with spacelike level sets intersecting the future event horizon $\mathcal{H}^{+}$and terminating at null infinity $\mathcal{I}^{+}$, such that $T(\bar{t})=1$. In view of (1.6), (1.5) and (1.7), $\bar{t}^{-1}$ polynomial decay estimates hold for $\varphi$, provided its initial data on $\mathcal{S}_{0}$ (or on the hypersurface $\{t=0\}$, where $t$ is the usual Schwarzschild exterior time coordinate) are sufficiently smooth and decaying.

3. (Schlue [34]) In the near region of $\left(\mathcal{M}_{S c h}, g_{M}\right), \bar{t}^{-\frac{3}{2}+\delta}$ polynomial decay rates for $\varphi$ hold, provided its initial data on $\mathcal{S}_{0}$ (or on the hypersurface $\{t=0\}$ ) are sufficiently smooth and decaying.

See [11] for a more detailed description of the above result and an explanation of how the proof immediately carries over to a certain wider class of spacetimes.

\subsection{Non-technical Statements of the Main Results and Applications}

The goal of the present paper is to introduce a broad class of asymptotically flat Lorentzian manifolds $\left(\mathcal{M}^{d+1}, g\right), d \geq 3$, on which the methods of [11,34] (suitably adapted) can be generalised. In particular, this class (described in Section 3) is broad enough to include spacetimes which radiate Bondi mass through future null infinity $\mathcal{I}^{+}$and are allowed to have a timelike boundary $\partial_{\text {tim }} \mathcal{M}$ with compact spacelike crosssections (modeling the boundary of a compact, possibly moving, obstacle in an ambient globally hyperbolic spacetime). An increasing hierarchy of geometric conditions will be imposed on this class of spacetimes, with each additional set of conditions leading to additional decay estimates for solutions $\varphi$ to the wave equation $(1.1)$ on $(\mathcal{M}, g)$. These conditions are partly motivated by the geometric structure of Kerr spacetime (and perturbations of it).

In particular, we will establish the following three results, each following from the previous under additional assumptions on the structure of $(\mathcal{M}, g)$ :

Theorem Let $\left(\mathcal{M}^{d+1}, g\right), d \geq 3$, be a Lorentzian manifold with the asymptotics (1.14), possibly with non-empty timelike boundary $\partial_{\text {tim }} \mathcal{M}$ with compact spacelike cross-sections. Then the following statements hold for any solution $\varphi$ to the wave equation (1.1) on $(\mathcal{M}, g)$ :

1. Weighted energy hierarchy. An $r^{p}$-weighted energy hierarchy holds, similar to (1.7). See Theorems 5.1 and 6.1. 
2. Slow polynomial decay. Assume that an integrated local energy decay statement of the form (1.6) holds for solutions $\varphi$ to (1.1) on $(\mathcal{M}, g)$ (satisfying suitable boundary conditions on $\partial_{\text {tim }} \mathcal{M}$, if non empty). Then $\bar{t}^{-1}$ polynomial decay estimates hold for $\varphi$, provided its initial data are sufficiently smooth and decaying, where $\bar{t}$ is a suitably defined time function on M. See Theorem 8.1.

3. Improved polynomial decay. Assume, in addition to the previous integrated local energy decay assumption, that $(\mathcal{M}, g$ ) possesses two vector fields $\{T, K\}$ (not necessarily distinct) with timelike span and with slowly decaying in time deformation tensor. Then provided the initial data for $\varphi$ are sufficiently smooth and decaying (and that suitable boundary conditions have been imposed on $\partial_{\text {tim }} \mathcal{M}$ ):

- In case $d$ is odd, a $\bar{t}^{-\frac{d}{2}}$ decay rate for $\varphi$ and a $\bar{t}^{-\frac{d+1}{2}}$ decay rate for the derivatives of $\varphi$ hold.

- In case d is even, a $\bar{t}^{-\frac{d}{2}+\delta}$ decay rate for $\varphi$ and its derivatives holds. See Theorem 9.1.

See also Sections 1.5.1, 1.5.2 and 1.5.3 for a more detailed statement of Parts 1, 2 and 3 of the above theorem.

Remark We should note that in fact, the integrated local energy estimate assumed in Parts 2 and 3 of the above theorem is weaker than (1.6), as we allow for an additional $\int_{\mathcal{D}^{+}\left(\mathcal{S}_{\tau}\right)} r^{-1} J_{\mu}^{N}\left(T^{j} \varphi\right) n_{\mathcal{S}}^{\mu}$ summand on the right hand side. On general spacetimes $(\mathcal{M}, g$ ) with $g$ having radiating asymptotics (without satisfying any special monotonicity condition), this additional "error" term appears necessary for (1.6) to hold (see Sections 4 and 8). Furthermore, in Part 3 above we can relax the condition that the deformation tensors of $T, K$ decay in time, replacing this with the statement that they are merely uniformly $\in$-small, provided there is no loss of derivatives in the assumed integrated local energy decay estimate. In this case, however, there is an extra $O(\varepsilon)$ loss in the exponents of $\bar{t}$ in the related decay estimates. See also the remark in Section 1.5.3.

As an application of Part 1 of the above theorem, we will establish that solutions to (1.1) on general asymptotically flat spacetimes (without any assumptions posed on the structure of their near region) have a well defined radiation field on future null infinity $\mathcal{I}^{+}$:

Theorem (Existence of radiation field at $\mathcal{I}^{+}$) Let $\left(\mathcal{M}^{d+1}, g\right), d \geq 3$, be a Lorentzian manifold with the asymptotics (1.14). Then for any smooth solution $\varphi$ to (1.1) with suitably decaying intial data on a spacelike hypersurface $\Sigma$ of $\mathcal{M}$ which is asymptotically of the form $\{t=$ const $\}$, the Friedlander radiation field $\Phi_{\mathcal{I}^{+}}$of $\varphi$ on future null infinity:

$$
\Phi_{\mathcal{I}^{+}}(u, \sigma)=\lim _{r \rightarrow+\infty}(\Omega \cdot \varphi(u, r, \sigma))
$$

where $\Omega=r^{\frac{d-1}{2}}\left(1+O\left(r^{-1}\right)\right)$, exists and is a smooth function of $(u, \sigma)$. See Theorem 7.1.

The assumption of an integrated local energy decay estimate for solutions $\varphi$ to (1.1), stated in Part 2 of the above theorem, does not hold on general spacetimes $(\mathcal{M}, g)$ 
without restricting the structure of their trapped set. In particular, in the case when $(\mathcal{M}, g)$ contains a stably trapped null geodesic, the local energy of $\varphi$ will not decay faster than logarithmically, see e.g. [32]. Hence, in that case, no ILED statement with finite loss of derivatives (i.e. of the form (1.6)) can hold on $(\mathcal{M}, g)$.

Even in the case where no ILED statement holds, however, the $r^{p}$-weighted energy hierarchy (1.7) can still yield decay estimates for $\varphi$ provided some decay estimate for the local energy of $\varphi$ can be established. In [29], it is shown that on a general class of stationary and asymptotically flat spacetimes $(\mathcal{M}, g)$, the local energy of solutions $\varphi$ to (1.1) decays logarithmically in time. Combining Part 1 of the above theorem with the logarithmic local energy decay estimate established in [29], we will thus be able to infer that the energy of $\varphi$ through a hyperboloidal foliation of $\mathcal{M}$ decays logarithmically in time:

Theorem (Logarithmic decay of the energy flux through a hyperboloidal foliation, [29] ) Let $\left(\mathcal{M}^{d+1}, g\right), d \geq 3$, be a globally hyperbolic spacetime with a Cauchy hypersurface $\Sigma$.

Assume that $(\mathcal{M}, g)$ is stationary, with stationary Killing field $T$, and asymptotically flat. If $\mathcal{M}$ contains a black hole region bounded by an event horizon $\mathcal{H}$, assume that $\mathcal{H}$ has positive surface gravity and that the ergoregion (i.e. the set where $g(T, T)>0$ ) is "small" (see [29] for the precise statement of these assumptions). Finally, assume that an energy boundedness statement of the form (1.5) holds for solutions to $\square \varphi=0$ on the domain of outer communications $\mathcal{D}$ of $\mathcal{M}$.

It then follows that the energy flux through a T-translated hyperboloidal foliation of $\mathcal{M}$ terminating at $\mathcal{I}^{+}$of any smooth solution $\varphi$ to $(1.1)$ on $(\mathcal{M}, g)$ with suitably decaying initial data on a Cauchy hypersurface $\Sigma$ of $\mathcal{M}$ decays at least logarithmically in time. See [29].

We will now give some examples of spacetimes $(\mathcal{M}, g)$ satisfying the assumptions of the above theorem. On these spacetimes, polynomial decay rates for solutions to (1.1) will be inferred as a result of Parts 2 and 3 of the above theorem.

Our first example will be the exterior region of a subextremal Kerr spacetime (with parameters $a, M$ in the full subextremal range $|a|<M$ ). This satisfies all the geometric assumptions of Parts 1,2 and 3 of the above theorem. We should remark that, in fact, our assumption on the properties of the vector fields $T, K$ of Part 3 of the above theorem was motivated by the geometric properties of the subextremal Kerr family. In view of the integrated local energy decay statement and the energy boundedness estimate established in [16], we will be able to infer Corollary 3.1 of [16]:

Theorem (Polynomial decay on subextremal Kerr exterior for $|a|<M$, [16]) Corollary 3.1 of [16] holds, that is to say, a $\bar{t}^{-\frac{3}{2}}$ pointwise decay rate for $\varphi$ and $\bar{t}^{-2}$ decay rate for the derivatives of $\varphi$ hold for solutions $\varphi$ to the wave equation (1.1) on subextremal Kerr spacetimes in the full parameter range $|a|<M$.

See Section 1.6.3 for a precise statement of this result.

Notice also that, in view of the integrated local energy decay estimate established in [23], the results of the present paper also imply a $\bar{t}^{-2+\delta}$ decay estimate for solutions $\varphi$ to (1.1) on very slowly rotating $4+1$ dimensional Myers-Perry spacetimes. 
For our second example, we will first need to introduce a definition: A metric $g$ on $\mathbb{R}^{d+1}$ will be called a radiating uniformly small perturbation of Minkowski spacetime $\left(\mathbb{R}^{d+1}, \eta\right)$ if it has the asymptotics (1.14), and moreover there exists a small $\varepsilon_{0}>0$ such that $r \cdot(g-\eta)$ and all its derivatives are $\varepsilon_{0}$-globally small, with each differentiation of this tensor with respect to $\partial_{t}$ except for the first one yielding additional decay in terms of $|u|$ (see (1.42) and (1.43) for a more precise definition). For such spacetimes, the geometric assumptions of Parts 1, 2 and 3 are satisfied and an integrated loacal energy decay estimate of the form (1.6) without loss of derivatives holds (in view of the stability to small perturbations of the estimates provided by the $\partial_{r}$-Morawetz current, combined with the estimates of Section 4 of the present paper). Examples of such spacetimes include the vacuum dynamical perturbations of Minkowski spacetime considered in [7].

We will infer the following result:

Theorem (Improved polynomial decay on radiating uniformly small perturbations of Minkowski) If $\left(\mathbb{R}^{d+1}, g\right)$ is a radiating uniformly small perturbation of Minkowski spacetime and $\varepsilon_{0}$ is small enough, then any solution $\varphi$ to $\square_{g} \varphi=0$ on $\left(\mathbb{R}^{d+1}, g\right)$ with suitably decaying initial data on $\{t=0\}$ will satisfy a $\bar{t}^{-\frac{d}{2}+O\left(\varepsilon_{0}\right)}$ decay estimate. If, in addition, the deformation tensor of the vector field $\partial_{t}$ is $O\left(\bar{t}^{-\delta_{0}}\right)$ decaying for some $\delta_{0}$, then $\varphi$ will satisfy a $\bar{t}^{-\frac{d}{2}}$ decay rate.

See Section 1.6.4 for a precise statement of this result. Let us remark that this theorem extends a recent result of Oliver [31].

Our final example will concern the class of radiating black hole exterior spacetimes $(\mathcal{M}, g)$ dynamically settling down to the exterior region of a subextremal Kerr spacetime. In order to present our example in the most simple form that can be deduced without computation from previous results, we will retrict ourselves to spacetimes $(\mathcal{M}, g)$ settling down to Schwarzschild exterior at a sufficiently fast polynomial rate. This class includes the dynamical vacuum spacetimes constructed in [14] (which actually approach Schwarzschild at an exponential rate).

The energy current yielding the integrated local energy decay statement for Schwarzschild exterior constructed in [8], combined with the estimates of Section 4 of the present paper and the fast rate at which $g$ approaches the Schwarzschild metric $g_{M}$, immediately imply that an integrated local energy decay statement of the form (1.6) also holds on $(\mathcal{M}, g)$. Furthermore, it is straightforward to check that $(\mathcal{M}, g)$ satisfies the assumptions of Parts 1, 2 and 3 of the above Theorem (in view of the fast approach to the Schwarzschild exterior metric, which satisfies these assumptions). Thus, on these spacetimes we will be able to infer the following result:

Theorem (Improved polynomial decay on dynamical, radiating black hole spacetimes) If $\left(\mathcal{M}^{3+1}, g\right)$ is a radiating black hole spacetime settling down to a Schwarzschild exterior at a sufficiently fast polynomial decay rate (such us the ones constructed in [14]), then any solution $\varphi$ to $\square_{g} \varphi=0$ on $(\mathcal{M}, g)$ with suitably decaying initial data on a Cauchy hypersurface will satisfy a $\bar{t}^{-\frac{3}{2}}$ decay estimate.

We will discuss in more detail the results of this paper and their applications in the next sections of the introduction. But first, we will review in more detail the "old" 
approach of using the conformal isometries of Minkowski spacetime for establishing decay rates for solutions to (1.1) on asymptotically flat spacetimes, and compare it to the method of [11].

\subsection{Comparison of the Two Approaches}

\subsubsection{The "Old" Approach}

The use of first order operators as multipliers and commutators for (1.1) has been implemented extensively during the last 50 years to deal with linear and non linear wave equations on small perturbations of Minkowski spacetime $\left(\mathbb{R}^{d+1}, \eta\right)$.

Following Morawetz (see e.g. [28]), one way to obtain decay for the local energy of solutions $\varphi$ to (1.1) is to apply the conformal Killing field $Z$ of $\left(\mathbb{R}^{d+1}, \eta\right)$

$$
Z=\left(t^{2}+r^{2}\right) \partial_{t}+2 t r \partial_{r}
$$

as multiplier for (1.1). On Minkowski spacetime itself for $d \geq 3$, the vector field $Z$ gives rise to a conserved positive definite energy norm $E_{Z}[\varphi](t)$ with weights growing in $t$. In particular, one can bound:

$$
E_{Z}[\varphi](\tau) \gtrsim \int_{\{t=\tau\}}\left(v^{2}\left|\partial_{v} \varphi\right|^{2}+u^{2}\left|\partial_{u} \varphi\right|^{2}+\left|\partial_{\sigma} \varphi\right|^{2}+|\varphi|^{2}\right) d x
$$

where $(t, r, \sigma)$ is the usual polar coordinate system on Minkowski space $\mathbb{R}^{3+1}, v=$ $t+r, u=t-r$ and $d x$ denotes the usual integration measure on $\{t=$ const $\}$ slices of $\mathbb{R}^{d+1}$ (see also Section 2 for the $\sigma$ notation). Thus, the preservation of $E_{Z}[\varphi](t)$ and the growth in time of the weights in the expression (1.10) can be used to establish polynomial decay in time estimates for the $L^{2}$ norm of certain derivatives of $\varphi$.

The above approach has been also implemented in the treatment of the wave equation (1.1) on the complement of a compact obstacle $\mathcal{O}$ in flat space, with suitable boundary conditions imposed on the boundary of $\mathcal{O}$. In [27,28], for instance, pointwise polynomial decay rates were established for solutions $\varphi$ to (1.1) on the complement of a star shaped obstacle with Dirichlet boundary conditions, and this was achieved with the use of the conformally Killing vector field $Z$ and the radial vector field $\partial_{r}$ as multipliers for equation (1.1). Moreover, the use of $\partial_{r}$ as a multiplier for (1.1) yielded the integrated local energy decay statement (1.4).

Another method for obtaining refined pointwise decay rates for solutions $\varphi$ to (1.1) on flat spacetime is the commutation vector field method, introduced by Klainerman: By commuting equation (1.1) with the generators of the isometries of $\left(\mathbb{R}^{d+1}, \eta\right)$ plus the dilation vector fieldand the dilation vector field $S$ :

$$
S=t \partial_{t}+r \partial_{r}
$$

$((t, r)$ being the usual time and radius coordinates on Minkowski space), and using the conservation of the $E_{Z}$ energy norm $(1.10)$ on $\left(\mathbb{R}^{d+1}, \eta\right)$ together with a modified 
version of the Sobolev embedding theorem due to Klainerman (see [21,22]), one can attain a pointwise decay estimate for $\varphi(d \geq 3)$ :

$$
\left|\partial_{u}^{l_{1}} \partial_{v}^{l_{2}}\left(r^{-1} \partial_{\sigma}\right)^{l_{3}} \varphi\right| \lesssim(1+|t-r|)^{-\frac{1}{2}-l_{1}}(1+t+r)^{-\frac{d-1}{2}-l_{2}-l_{3}} \sqrt{\mathcal{E}_{l_{1}, l_{2}, l_{3}}},
$$

where $l_{1}, l_{2}, l_{3} \geq 0$ are integers and $\mathcal{E}_{l_{1}, l_{2}, l_{3}}$ is a weighted higher order energy norm of the initial data for $\varphi$ on $\{t=0\}$. See [35] for more details on the commutation vector field approach.

Notice that the $t^{-\frac{d}{2}}$ decay rate for $|\varphi|$ in the region $\{r \lesssim 1\}$ provided by (1.12) guarantees that $|\varphi(t, x)|$ is integrable in $t$ in dimensions $d \geq 3$, and this fact is of fundamental importance in the treatment of non linear variants of the wave equation (1.1).

The aforementioned techniques have been also extended to the exterior of black hole spacetimes, such as the Schwarzschild and very slowly rotating (i.e with $|a| \ll M$ ) Kerr exterior spacetimes, see $[1,5,8,9]$. In these works, a variant of the conformally Killing vector field $Z$ of Minkowski spacetime was constructed and used, but this construction came at a cost: Since $Z$ is not a conformally Killing vector field on these black hole spacetimes, decay estimates obtained in this way for solutions to (1.1) were coupled with error terms in the near region of the spacetimes under consideration, and these error terms carried weights growing in time.

In view also of the unavoidable presence of trapping in the near region of a black hole spacetime, the error terms associated to the use of the modified $Z$ vector field as a multiplier for (1.1) required additional effort in order to be controlled. An essential step towards controlling these error terms was the establishment of an integrated local energy decay statement of the form (1.6), with the use of carefully chosen first order multipliers for (1.1) capturing the red-shift effect near the horizon $\mathcal{H}$ and the structure of the trapped set in the near region $\{r \lesssim 1\}$ (these multipliers being equal to $\partial_{r}$ plus a lower order correction in the far away region $\left.\{r \gg 1\}\right)$. See $[1,4,8-$ $10,13,38]$.

The above approach of using an adaptation of the Morawetz $Z$ vector field and an integrated local energy decay statement yielded $t^{-1}$ decay estimates for solutions $\varphi$ to (1.1) on Schwarzschild exterior spacetimes and $t^{-1+\delta(a)}$ decay estimates on slowly rotating Kerr exterior spacetimes, with $\delta(a) \rightarrow 0$ as $a \rightarrow 0$ (see [13]). In [24,25], Luk was able to obtain improved $t^{-\frac{3}{2}+\delta}$ decay estimates for $\varphi$ in the near region of these backgrounds by commuting the wave equation (1.1) with an analogue of the dilation vector field $S$ (1.11) of Minkowski spacetime.

Let us note at this point that the vector field approach has been effectively applied in the case of non linear wave equations on a radiating spacetime which is globally close to $\left(\mathbb{R}^{3+1}, \eta\right)$ : This can be viewed as a corollary of the monumental proof of the non linear stability of Minkowski spacetime in the context of the Einstein equations, by Christodoulou and Klainerman (see [7]). These techniques have also been applied in the study of non linear wave equations on black hole spacetimes (see the work of Luk [26]). See also [31] for the treatment of the linear wave equation $(1.1)$ on radiating spacetimes which are globally close to $\left(\mathbb{R}^{3+1}, \eta\right)$ 
(where, among other decay results, a $t^{-\frac{3}{2}}$ decay rate in the near region is established).

The difficulties in extending the "old" approach of establishing decay estimates for solutions to (1.1) on more general black hole spacetimes led the authors of [11] to suggest a more flexible approach that does not involve multipliers and commutators with weights growing in time. This is the approach that we will now discuss.

\subsubsection{The ${ }^{p}$-Weighted Energy Method}

The crux of the new method of obtaining decay estimates for solutions to (1.1) introduced in [11] lies in the establishment of a hierarchy of estimates for $r^{p}$-weighted energies, $0 \leq p \leq 2$, using as a multiplier an $r^{p}$-weighted outgoing null vector field. On Minkowski spacetime, this hierarchy of estimates takes the following form for any solution $\varphi$ to the wave equation (1.1) and any $\tau_{1} \leq \tau_{2}, R>0$ :

$$
\begin{aligned}
& \int_{\left\{u=\tau_{2}\right\} \cap\{r \geq R\}} r^{p} \cdot\left|\partial_{v}(r \varphi)\right|^{2} d v d \sigma \\
& \quad+\int_{\left\{\tau_{1} \leq u \leq \tau_{2}\right\} \cap\{r \geq R\}} r^{p-1}\left(p\left|\partial_{v}(r \varphi)\right|^{2}+(2-p)\left|r^{-1} \partial_{\sigma}(r \varphi)\right|^{2}\right) d u d v d \sigma \\
& \leq \int_{\left\{u=\tau_{2}\right\} \cap\{r \geq R\}} r^{p} \cdot\left|\partial_{v}(r \varphi)\right|^{2} d v d \sigma \\
& \quad+\int_{\left\{\tau_{1} \leq u \leq \tau_{2}\right\} \cap\{r=R\}} r^{p} \cdot\left(\left|r^{-1} \partial_{\sigma}(r \varphi)\right|^{2}-\left|\partial_{v}(r \varphi)\right|^{2}\right) d u d \sigma .
\end{aligned}
$$

In the above, $u=t-r, v=t+r$. For the $\sigma$ notation on the angular variables, see Section 2. Moreover, the right hand side of (1.13) also controls the angular derivatives of the radiation field of $\varphi$ on future null infinity $\mathcal{I}^{+}$, but we have dropped these terms for simplicity. A similar expression is also valid on Schwarzschild spacetimes and suitable perturbations, see [11].

The importance of the hierarchy (1.13) lies in the fact that the left hand side of (1.13) contains a positive definite bulk term, while the "error" term in the near region (namely the last term of the right hand side) does not carry weights growing in $t$. Thus, combining (1.13) with the integrated local energy decay statement (1.6) and the energy boundedness estimate (1.5), the authors of [11] were able to obtain uniform polynomial decay rates for $r^{\frac{1}{2}} \varphi$ and $r \varphi$ in terms of $u$.

A noteable aspect of this novel approach of [11] is that decay rates for $\varphi$ are obtained by repeatedly applying the pigeonhole principle on the positive definite bulk term (i.e. the second term of the left hand side) controlled in (1.13). This is in contrast to the older approach (described in the previous section), which yielded decay rates for $\varphi$ by establishing uniform bounds for $t$-weighted energy norms of $\varphi$ on suitable hypersurfaces.

Moreover, the new method of [11] allows one to obtain the result of Luk ([25]), namely to establish improved (i.e. $t^{-\frac{3}{2}+\delta}$ ) polynomial decay estimates for $\varphi$ in the 
near region of Schwarzschild exterior: This was achieved by Schlue in [34], where it was established that commuting (1.1) with the outgoing null vector field $\partial_{v}$ (as well as the generators of the isometries of Schwarzschild) leads to an improvement of the $p$-hierarchy (1.13). ${ }^{2}$ In particular, it was established that higher order $\partial_{v}$ and $r^{-1} \partial_{\sigma}$ derivatives of $\varphi$ satisfy (1.13) for larger values of $p$. This better decay rate in $r$ was then translated into a better decay rate in $u$ by using the expression of the wave equation (1.1) as well as the pigeonhole principle argument of [11].

This novel approach has also been implemented in the case of non-linear wave equations: In $[39,40]$, Yang established a small data global existence result for non-linear (in fact, quasi-linear) wave equations on a certain class of asymptotically flat backgrounds, using the techniques of [11] (see also [41]). In [2], Angelopoulos obtained a small data global existence result for spherically symmetric solutions to a class of semi-linear wave equations on extremal Reissner-Nordström backgrounds. A variant of the $r^{p}$-weighted energy method has also been effectively used in the case of the Einstein equations themselves: In [14,20], the authors established an $r^{p}$-weighted energy hierarchy, a proper tensorial analogue of (1.13), for radiating solutions to the Einstein equations $\operatorname{Ric}(g)=0$ that approach the Schwarzschild exterior in the future. Notice also that [14] utilised the $r^{p}$-weighted energy method for the Einstein equations in the scattering setting.

\subsection{Statement of the Main Results}

The present paper introduces a broad class of asymptotically flat spacetimes $\left(\mathcal{M}^{d+1}, g\right), d \geq 3$, on which the techniques of [11] and [34] can be generalised. See Section 3 for a more detailed discussion of the class of spacetimes under consideration. Notice that this class of metrics includes spacetimes with non-constant Bondi mass at null infinity, see e.g. [6,33], such as the dynamical vacuum perturbations of Minkowski spacetime (see [7]). Moreover, spacetimes in this class are allowed to have a timelike boundary $\partial_{\text {tim }} \mathcal{M}$ with compact spacelike cross-sections.

We will now proceed to briefly review the results established in the following sections of the paper.

\subsubsection{The $r^{p}$-Weighted Energy Hierarchy in Dimensions $d \geq 3$}

In this Section, all results will be stated on an asymptotic region $\mathcal{N}_{\text {af }} \subset \mathcal{M}$ of a general radiating asymptotically flat ${ }^{3}$ spacetime $(\mathcal{M}, g)$. In particular, let $\left(\mathcal{N}_{a f}^{d+1}, g\right)$, $d \geq 3$, be a Lorentzian manifold diffeomorphic to $\mathbb{R} \times\left[R_{0},+\infty\right) \times \mathbb{S}^{d-1}$ for some $R_{0}>0$, on which a single $(u, r, \sigma)$ coordinate chart has been fixed. Assume that in this chart $g$ takes the form

\footnotetext{
2 Note also that [34] also deals with the case of higher dimensional Schwarzschild spacetimes.

3 Let us also remark that the results of this paper also hold on spacetimes which are asymptotically conic instead of asymptotically flat. However, we will not pursue this issue further in this paper.
} 


$$
\begin{aligned}
g= & -4\left(1-\frac{2 M(u, \sigma)}{r}+O\left(r^{-1-a}\right)\right) d u^{2}-\left(4+O\left(r^{-1-a}\right)\right) d u d r \\
& +r^{2} \cdot\left(g_{\mathbb{S}^{d-1}}+O\left(r^{-1}\right)\right)+O(1) d u d \sigma \\
& +O\left(r^{-a}\right) d r d \sigma+O\left(r^{-2-a}\right) d r^{2},
\end{aligned}
$$

where $M(u, \sigma)$ is a bounded and sufficiently regular function of $u, \sigma$. Notice that $\left(\mathcal{N}_{a f}, g\right)$ is not in general globally hyperbolic.

We will extend the hierarchy $(1.13)$ to $\left(\mathcal{N}_{a f}, g\right)$ as follows (see the remark below for explanation of the notation):

Theorem 1.1 Let $\mathcal{S}_{1}, \mathcal{S}_{2}$ be two spacelike hyperboloidal hypersurfaces of $\left(\mathcal{N}_{a f}^{d+1}, g\right)$, $d \geq 3$, terminating at $\mathcal{I}^{+}$, such that $\mathcal{S}_{2} \subset J^{+}\left(\mathcal{S}_{1}\right)$. Then for any $0<p \leq 2$, any given $0<\eta<a, 0<\delta<1$ and $R>0$ large, the following inequality is true for any smooth function $\varphi: \mathcal{N}_{a f} \rightarrow \mathbb{C}$ (setting also $\Phi \doteq \Omega \cdot \varphi$, where $\left.\Omega=(-\operatorname{det}(g))^{\frac{1}{4}}\right)$ :

$$
\begin{aligned}
& \int_{\mathcal{S}_{2} \cap\{r \gtrsim R\}} r^{p}\left|\partial_{r} \Phi\right|^{2} d v d \sigma \\
& +\int_{\mathcal{S}_{2} \cap\{r \gtrsim R\}}\left(r^{p}\left|r^{-1} \partial_{\sigma} \Phi\right|^{2}+\max \left\{(d-3), r^{-\delta}\right\} \cdot r^{p-2}|\Phi|^{2}\right) d u d \sigma \\
& +\int_{J^{+}\left(\mathcal{S}_{1}\right) \cap J^{-}\left(\mathcal{S}_{2}\right) \cap\{r \gtrsim R\}} \chi_{R} \cdot\left(p r^{p-1}\left|\partial_{r} \Phi\right|^{2}+(2-p) r^{p-1}\left|r^{-1} \partial_{\sigma} \Phi\right|^{2}\right. \\
& \left.+\max \left\{(2-p)(d-3), r^{-\delta}\right\} \cdot r^{p-3}|\Phi|^{2}\right) d u d v d \sigma \lesssim_{p, \eta, \delta} \\
& \lesssim_{p, \eta, \delta} \int_{\mathcal{S}_{1} \cap\{r \gtrsim R\}} r^{p}\left|\partial_{r} \Phi\right|^{2} d v d \sigma+\int_{\mathcal{S}_{2} \cap\{r \gtrsim R\}}\left(r^{p}\left|r^{-1} \partial_{\sigma} \Phi\right|^{2}\right. \\
& \left.+\max \left\{(d-3), r^{-\delta}\right\} \cdot r^{p-2}|\Phi|^{2}\right) d u d \sigma \\
& +\int_{J^{+}\left(\mathcal{S}_{1}\right) \cap J^{-}\left(\mathcal{S}_{2}\right) \cap\{r \sim R\}}\left(r^{p}|\partial \Phi|^{2}+r^{p-2}|\Phi|^{2}\right) d u d v d \sigma+\int_{\mathcal{S}_{1} \cap\{r \gtrsim R\}} J_{\mu}^{\partial_{u}}(\varphi) n_{\mathcal{S}}^{\mu} \\
& +\int_{J^{+}\left(\mathcal{S}_{1}\right) \cap J^{-}\left(\mathcal{S}_{2}\right) \cap\{r \gtrsim R\}} \chi_{R} \cdot\left(r^{p+1}+r^{1+\eta}\right) \cdot\left|\Omega \cdot \square_{g} \varphi\right|^{2} d u d v d \sigma .
\end{aligned}
$$

In the above, the constants implicit in the $\lesssim p, \eta, \delta$ notation depend only on $p, \eta, \delta$ and on the geometry of $\left(\mathcal{N}_{a f}, g\right)$. The partial derivatives $\partial_{r}, \partial_{\sigma}$ are considered with respect to the cooordinate chart $(u, r, \sigma)$ and the notation $\partial_{\sigma}$ is explained in Section 2.

Remark Notice that the $d u d \sigma$ volume form on the hyperboloidal hypersurfaces $\mathcal{S}_{i}$ degenerates as $r \rightarrow+\infty$ when compared to $d v d \sigma$. The notion of a spacelike hyperboloidal hypersurface terminating at $\mathcal{I}^{+}$is given in Section 3.1. For the notations on vector field currents see Section 2.

For a more detailed statement of the above result, see Theorems 5.1 and 5.3 in Section 5.

We will also establish the following improved $r^{p}$-weighted hierarchy for higher derivatives of $\varphi$ : 
Theorem 1.2 With the notations as in Theorem 1.1, for any $k \in \mathbb{N}$, any $2 k-2<p \leq$ $2 k$, any given $0<\eta<a$ and $R>0$ large, the following inequality is true for any smooth function $\varphi: \mathcal{N}_{a f} \rightarrow \mathbb{C}$ (setting also $\Phi \doteq \Omega \cdot \varphi$ ):

$$
\begin{aligned}
& \int_{\mathcal{S}_{2} \cap\{r \gtrsim R\}} r^{p}\left|\partial_{r} \nabla_{\mathcal{S}}^{k-1} \Phi\right|^{2} d v d \sigma+\int_{\mathcal{S}_{2} \cap\{r \gtrsim R\}} r^{p}\left|r^{-1} \partial_{\sigma} \nabla_{\mathcal{S}}^{k-1} \Phi\right|^{2} d u d \sigma \\
& +\int_{J^{+}\left(\mathcal{S}_{1}\right) \cap J^{-}\left(\mathcal{S}_{2}\right) \cap\{r \gtrsim R\}} \chi_{R} \cdot\left(r^{p-1}\left|\partial_{r} \nabla_{\mathcal{S}}^{k-1} \Phi\right|^{2}\right. \\
& \left.\quad+(2 k-p) r^{p-1}\left|r^{-1} \partial_{\sigma} \nabla_{\mathcal{S}}^{k-1} \Phi\right|^{2}\right) d u d v d \sigma \lesssim_{p, \eta} \\
& \lesssim_{p, \eta} \sum_{j=1}^{k} \int_{\mathcal{S}_{1} \cap\{r \gtrsim R\}} r^{p-2(k-j)}\left|\partial_{r} \nabla_{\mathcal{S}}^{j-1} \Phi\right|^{2} d v d \sigma \\
& \quad+\sum_{j=1}^{k} \int_{\mathcal{S}_{1} \cap\{r \gtrsim R\}}\left(r^{p-2(k-j)}\left|r^{-1} \partial_{\sigma} \nabla_{\mathcal{S}}^{j-1} \Phi\right|^{2}+r^{p-2 k}|\Phi|^{2}\right) d u d \sigma \\
& \quad+\sum_{j=0}^{k} \int_{J^{+}\left(\mathcal{S}_{1}\right) \cap J^{-}\left(\mathcal{S}_{2}\right) \cap\{r \sim R\}} r^{p-2(k-j)}\left|\partial^{j} \Phi\right|^{2} d u d v d \sigma \\
& \quad+\sum_{j=1}^{k} \int_{\mathcal{S}_{1} \cap\{r \gtrsim R\}} J_{\mu}^{\partial_{u}}\left(\nabla_{\mathcal{S}}^{j-1} \varphi\right) n_{\mathcal{S}}^{\mu}+\sum_{j=1}^{k} \int_{J^{+}\left(\mathcal{S}_{1}\right) \cap J-\left(\mathcal{S}_{2}\right) \cap\{r \gtrsim R\}} \chi_{R} \\
& \quad \cdot\left(r^{p+1-2(k-j)}+r^{1+\eta}\right) \cdot\left|\nabla_{\mathcal{S}}^{j-1}\left(\Omega \square_{g} \varphi\right)\right|^{2} d u d v d \sigma .
\end{aligned}
$$

In the above,

$$
\left|\nabla_{S}^{j} \psi\right|^{2} \doteq \sum_{j_{1}+j_{2}+j_{3}=j}\left|r^{-j_{2}-j_{3}} \partial_{r}^{j_{1}} \partial_{\sigma}^{j_{2}} \partial_{u}^{j_{3}} \psi\right|^{2}
$$

and the constants implicit in the $\lesssim_{p, \eta}$ notation depend only on $p, \eta$ and on the geometry of $\left(\mathcal{N}_{a f}, g\right)$.

See Section 6 for a more detailed statement of the above result.

\subsection{2 $A \bar{t}^{-1}$ Polynomial Decay Estimate for Solutions to the Wave Equation $\square \varphi=0$}

In this section, we will be concerned with obtaining results for (1.1) on the whole spacetime $(\mathcal{M}, g)$ (and not merely the asymptotic region $\mathcal{N}_{a f}$ ). Provided that an integrated local energy decay statement (possibly with loss of derivatives) holds for solutions to $\square_{g} \varphi=F$ on a spacetime $\left(\mathcal{M}^{d+1}, g\right), d \geq 3$, with $g$ asymptotically of the form (1.14), we will establish polynomial decay rates for $\varphi$ with respect to a hyperboloidal foliation of $\mathcal{M}$. 
In particular, let $\left(\mathcal{M}^{d+1}, g\right), d \geq 3$, be a Lorentzian manifold with possibly nonempty boundary $\partial \mathcal{M}$, which can be split as

$$
\partial \mathcal{M}=\partial_{\text {tim }} \mathcal{M} \oplus \partial_{\text {hor }} \mathcal{M}
$$

where $\partial_{\text {tim }} \mathcal{M}$ is smooth and timelike and $\partial_{\text {hor }} \mathcal{M}$ is piecewise smooth and null. Assume also that $(\mathcal{M}, g)$ is globally hyperbolic as a manifold with timelike boundary, which means that the double $\left(\tilde{\mathcal{M}}_{\text {tim }}, g\right)$ of $(\mathcal{M}, g)$ across $\partial_{\text {tim }} \mathcal{M}$ is globally hyperbolic.

Suppose that $(\mathcal{M}, g)$ satisfies the following geometric assumptions:

(GM1) Asymptotic flatness: $(\mathcal{M}, g)$ is asymptotically flat in the sense that there exists an open subset $\mathcal{N}_{a f} \subset \mathcal{M}$ such that each connected component of $\mathcal{N}_{a f}$ is mapped diffeomorphically on $\mathbb{R} \times\left(R_{0},+\infty\right) \times \mathbb{S}^{d-1}$ through a coordinate chart $(u, r, \sigma)$, and in this coordinate chart $g$ has the form (1.14).

(GM2) Existence of a well behaved time function: There exists a function $\bar{t}: \mathcal{M} \rightarrow \mathbb{R}$ with level sets which are spacelike hyperboloids terminating at future null infinity, such that on each component of $\mathcal{N}_{a f}$ the difference $|\bar{t}-u|$ is bounded, and the foliation $\{\bar{t}=$ const $\}$ in the region $\mathcal{M} \backslash \mathcal{N}_{a f}$ is sufficiently "regular" (see Section 8 for the precise relevant assumptions on $\bar{t}$ ).

In fact, the precise description of Assumptions (GM1)-(GM2)is more complicated, and requires the splitting of these assumptions into a larger number of statements: see Assumptions (G1)-(G13) of Sections 7.1 and 8.1.1.

For convenience, we define the globally timelike vector field $N$ so that $N \equiv \operatorname{grad}(\bar{t})$ on $\mathcal{M} \backslash \mathcal{N}_{a f}$ and $N=\partial_{u}$ on each connected component of the region $\mathcal{N}_{a f} \cap\{r \gg 1\}$.

Suppose also that on $(\mathcal{M}, g)$ the following integrated local energy decay estimate holds:

(ILED1) Integrated local energy decay with polynomial loss of derivatives: There exists an integer $k \geq 0$, such that for any solution $\varphi$ to $\square \varphi=F$ with suitable boundary conditions on $\partial_{\text {tim }} \mathcal{M}$, any $m \in \mathbb{N}, 0 \leq \tau_{1} \leq \tau_{2}, \eta>0$ and $R>0$ :

$$
\begin{aligned}
& \sum_{j=0}^{m} \int_{\left\{\tau_{1} \leq \bar{t} \leq \tau_{2}\right\} \cap\{r \leq R\}}\left|\nabla^{j} \varphi\right|^{2}+\sum_{j=1}^{m} \int_{\left\{\tau_{1} \leq \bar{t} \leq \tau_{2}\right\} \cap \partial_{t i m} \mathcal{M}}\left|\nabla^{j} \varphi\right|^{2} \\
& \leq C_{m, \eta}(R) \sum_{j=0}^{m+k-1} \int_{\left\{\bar{t}=\tau_{1}\right\}} J_{\mu}^{N}\left(N^{j} \varphi\right) \bar{n}^{\mu} \\
& +C_{m, \eta} \sum_{j=0}^{m-1} \int_{\left\{\tau_{1} \leq \bar{t} \leq \tau_{2}\right\} \cap\{r \geq R\}} r^{-1} J_{\mu}^{N}\left(N^{j} \varphi\right) \bar{n}^{\mu} \\
& +C_{m, \eta}(R) \sum_{j=0}^{m+k-1} \int_{\left\{\tau_{1} \leq \bar{t} \leq \tau_{2}\right\}} r^{1+\eta}\left|\nabla^{j} F\right|^{2},
\end{aligned}
$$

where $C_{m, \eta}(R)$ depends only on $m, \eta, R$ and the geometry of $(\mathcal{M}, g)$. 
See Section 8.1.2 for a more detailed description of Assumption (ILED1). For an alternative to the Assumption (ILED1), see the remarks in Section 8.1.2 (and in particular (8.21) and (8.22)).

On any spacetime $(\mathcal{M}, g)$ satisfying the above assumptions we will establish the following decay statement for solutions to (1.1):

Theorem 1.3 Let $\left(\mathcal{M}^{d+1}, g\right)$ satisfy the geometric assumptions (GM1) and (GM2), and the integrated local energy decay assumption (ILEDI), and let $\bar{t}$ and $N$ be as above. Then the following decay estimates hold for any $0<\delta<1$, any $\tau \geq 0$ and any solution $\varphi$ to the inhomogeneous wave equation $\square_{g} \varphi=F$ with suitably decaying inital data on $\{\bar{t}=0\}$ (and satisfying suitable boundary conditions on $\partial_{\text {tim }} \mathcal{M}$ ):

$$
\begin{aligned}
& \int_{\{\bar{t}=\tau\}} J_{\mu}^{N}(\varphi) \bar{n}^{\mu} \leq \frac{C_{\delta}}{\tau^{2-\delta}} \mathcal{E}^{2, k}[\varphi](0)+\mathcal{F}^{2, k, d, \delta}[F](\tau), \\
& \sup _{\{\bar{t}=\tau\}} r^{d-2} \cdot|\varphi|^{2} \leq \frac{C_{\delta}}{\tau^{2-\delta}} \mathcal{E}^{2, k, d}[\varphi](0)+\mathcal{F}^{2, k, d, \delta}[F](\tau)
\end{aligned}
$$

and

$$
\sup _{\{\bar{t}=\tau\}} r^{d-1} \cdot|\varphi|^{2} \leq \frac{C}{\tau} \mathcal{E}^{2, k, d}[\varphi](0)+\mathcal{F}^{1, k, d}[F](\tau) .
$$

Furthermore, in case the vector field $T=\partial_{u}$ in the coordinate chart $(u, r, \sigma)$ in the asymptotically flat region $\{r \gg 1\}$ of $\mathcal{M}$ satisfies for some (small) $\delta_{0}>0$ and any $k \in \mathbb{N}$ :

$$
\begin{aligned}
\mathcal{L}_{T}^{k} g= & O\left(|u|^{-\delta 0}\right)\left\{O\left(r^{-1-a}\right) d v d u+O(r) d \sigma d \sigma+O(1) d u d \sigma\right. \\
& \left.+O\left(r^{-a}\right) d v d \sigma+O\left(r^{-1}\right) d u^{2}+O\left(r^{-2-a}\right) d v^{2}\right\}
\end{aligned}
$$

and the second term of the right hand side of the integrated local energy decay estimate (1.19) is replaced by

$$
C_{m, \eta} \sum_{j=0}^{m-1} \int_{\left\{\tau_{1} \leq \bar{t} \leq \tau_{2}\right\} \cap\{r \geq R\}}|u|^{-\delta 0} r^{-1} J_{\mu}^{N}\left(N^{j} \varphi\right) \bar{n}^{\mu},
$$

then the $\delta$-loss in the decay estimates (1.20) and (1.21) can be removed:

$$
\int_{\{\bar{t}=\tau\}} J_{\mu}^{N}(\varphi) \bar{n}^{\mu} \leq \frac{C}{\tau^{2}} \mathcal{E}^{2, k}[\varphi](0)+\mathcal{F}^{2, k, d}[F](\tau)
$$

and

$$
\sup _{\{\bar{t}=\tau\}} r^{d-2} \cdot|\varphi|^{2} \leq \frac{C}{\tau^{2}} \mathcal{E}^{2, k, d}[\varphi](0)+\mathcal{F}^{2, k, d}[F](\tau) .
$$

See Theorem 8.1 (and the remark below it) in Section 8 for a more detailed statement of the above result and the definition of the weighted energy norms of $\varphi$ and $F$ in the right hand side of (1.20), (1.21) and (1.22). 
Remark Let us remark that the initial weighted energy norm on the hyperboloid $\{\bar{t}=0\}$ in the right hand sides of (1.20)-(1.26) can be readily replaced by a similar weighted norm on a hypersurface $\Sigma$ terminating at spacelike infinity (e.g. a hypersurface which in the asymptotically flat region is of the form $\{t=$ const $\}$ ). In that case, the source spacetime energy norms $\mathcal{F}[F]$ in (1.20)-(1.26) are replaced by similar weighted spacetime norms of $F$ over the region $J^{+}(\Sigma) \cap\{\bar{t} \leq \tau\}$.

\subsubsection{An Improved $\bar{t}^{-\frac{d}{2}}$ Polynomial Decay Estimate for Solutions to the Wave Equation $\square \varphi=0$ in Dimensions $d \geq 3$}

Finally, we will also be able to establish improved polynomial decay rates for $\varphi$, under some additional restrictions on the spacetimes $(\mathcal{M}, g)$. Let $(\mathcal{M}, g)$, satisfy the geometric assumptions (GM1) and (GM2) of the previous section, as well as the integrated local energy decay assumption (ILED1). Let also $\bar{t}$ and $N$ be as in the statement of Theorem 1.3.

Assume furthermore that $(\mathcal{M}, g)$ satisfies the following two geometric conditions:

(GM3) There exist two smooth vector fields $T, K$ (not necessarily distinct) on $(\mathcal{M}, g)$ such that:

1. $d \bar{t}(T)=d \bar{t}(K)=1$

2. The span of $\{T, K\}$ is everywhere timelike on $\mathcal{M} \backslash \mathcal{H}^{+}$(where $\mathcal{H}^{+}$is the future event horizon of $(\mathcal{M}, g)$, which is required to be a subset of $\left.\partial \mathcal{M}_{\text {hor }}\right)$.

3 . In the coordinate chart $(u, r, \sigma)$ on each connected component of the region $r \gg 1$, $T=\partial_{u}$ and $K=T+\Phi$ (where $\Phi$ is the generator of a rotation of $\mathbb{S}^{d-1}$, allowed to be identically 0 ).

4. The vector fields $T$ and $K$ are almost Killing in the sense that there exists a small $\delta_{0}>0$ such that their deformation tensor satisfies the $O\left(\bar{t}^{-\delta_{0}}\right)$ decay estimates (9.5) and (9.6).

(GM4) The span of $\{T, K\}$ is tangential to the future event horizon $\mathcal{H}^{+}$of $(\mathcal{M}, g)$ (if non-empty). Moreover, $\mathcal{H}^{+}$is non-degenerate with respect to $K$, in the sense that $K$ satisfies $g(K, K)=0$ and $d(g(K, K)) \neq 0$ on $\mathcal{H}^{+} .4$

(GM5) The constants in the elliptic, Sobolev and Gagliardo-Nirenberg type estimates on the leaves of the foliation $\{\bar{t}=\tau\}$ stated in Section 9.1 can be chosen to be independent of $\tau \geq 0$.

Remark Assumption (GM5) holds automatically on spacetimes $(\mathcal{M}, g)$ which are near stationary or time periodic.

Again, the precise description of Assumptions (GM3)-(GM5) is actually more complicated, and will require the splitting of these assumptions into a larger number of statements: see Assumptions (EG1)-(EG8) in Section 9.1.

We will also assume that the following stronger form of Assumption (ILED1) holds:

(ILED2) Integrated local energy decay with polynomial loss of derivatives: There exists an integer $k \geq 0$, such that for any solution $\varphi$ to $\square \varphi=F$ with

\footnotetext{
${ }^{4}$ Hence, $K$ should be viewed as the analogue of the Hawking vector field of the Kerr spacetime.
} 
suitable boundary conditions on $\partial_{\text {tim }} \mathcal{M}$, any $m \in \mathbb{N}, 0 \leq \tau_{1} \leq \tau_{2}, \eta>0$, $R>0$ and any integers $i_{1}, i_{2} \geq 0$ we can bound:

$$
\begin{aligned}
& \sum_{j=0}^{m} \int_{\left\{\tau_{1} \leq \bar{t} \leq \tau_{2}\right\} \cap\{r \leq R\}}\left|\nabla^{j}\left(T^{i_{1}} K^{i_{2}} \varphi\right)\right|^{2}+\sum_{j=1}^{m} \int_{\left\{\tau_{1} \leq \bar{t} \leq \tau_{2}\right\} \cap \partial_{t i m} \mathcal{M}}\left|\nabla^{j}\left(T^{i_{1}} K^{i_{2}} \varphi\right)\right|^{2} \\
& \leq C_{m, \eta, i_{1}, i_{2}}(R) \sum_{j=0}^{m+k-1} \int_{\left\{\bar{t}=\tau_{1}\right\}} J_{\mu}^{N}\left(N^{j} T^{i_{1}} K^{i_{2}} \varphi\right) \bar{n}^{\mu} \\
& \quad+C_{m, \eta, i_{1}, i_{2}} \sum_{j=0}^{m-1} \int_{\left\{\tau_{1} \leq \bar{t} \leq \tau_{2}\right\} \cap\{r \geq R\}}|\bar{t}|^{-\delta 0} r^{-1} J_{\mu}^{N}\left(N^{j} T^{i_{1}} K^{i_{2}} \varphi\right) \bar{n}^{\mu} \\
& \quad+C_{m, \eta, i_{1}, i_{2}}(R) \sum_{j=0}^{m+k-1} \int_{\left\{\tau_{1} \leq \bar{t} \leq \tau_{2}\right\}} r^{1+\eta}\left|\nabla^{j} \square\left(T^{i_{1}} K^{i_{2}} \varphi\right)\right|^{2} .
\end{aligned}
$$

See Section 9.1 for a more detailed description of Assumption (ILED2)

For spacetimes $(\mathcal{M}, g)$ as above, we will infer the following improved decay result:

Theorem 1.4 Let $\left(\mathcal{M}^{d+1}, g\right)$ satisfy the geometric assumptions (GM1), (GM2), (GM3), (GM4) and (GM5) and the integrated local energy decay assumption (ILED2), and let $\bar{t}$ and $N$ be as above. Then for any integer $1 \leq q \leq\left\lfloor\frac{d+1}{2}\right\rfloor$, any $0<\varepsilon \ll \delta_{0}$, any $\tau \geq 0$ and any solution $\varphi$ to the inhomogeneous wave equation $\square_{g} \varphi=F$ with suitably decaying intial data on $\{\bar{t}=0\}$ (and satisfying suitable boundary conditions on $\left.\partial_{\text {tim }} \mathcal{M}\right)$ the following estimates hold:

$$
\begin{aligned}
& \mathcal{E}_{\text {en }}^{(0, q)}[\varphi](\tau)+\int_{\tau}^{+\infty} \mathcal{E}_{\text {en }}^{(-1+\varepsilon, q)}[\varphi](s) d s \\
& \quad \lesssim m, \varepsilon \tau^{-2 q+C \varepsilon} \mathcal{E}_{i n}^{\left(2 q, k, \delta_{0}\right)}[\varphi](0)+\mathcal{F}_{\varepsilon}^{\left(q, k, m, \delta_{0}\right)}[F](\tau)
\end{aligned}
$$

and

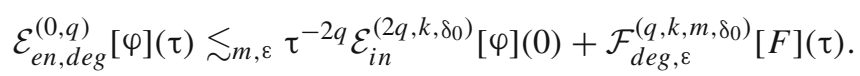

In the above, $\mathcal{E}_{\text {en }}^{(a, q)}[\varphi](\tau)$ is the non degenerate $L^{2}$ norm on $\{\bar{t}=\tau\}$ of all derivatives of $\varphi$ of order $q$, with $r^{a}$ weights near infinity, and $\mathcal{E}_{\text {en,deg }}^{(a, q)}[\varphi](\tau)$ is similar to $\mathcal{E}_{\text {en }}^{(a, q)}[\varphi](\tau)$ but with a degeneracy on $\mathcal{H}^{+}$. See Section 9 for a more precise definition of these norms.

Moreover, the following pointwise decay rates for $\varphi$ are established:

1. In case the dimension $d$ is odd, we can bound:

$$
\sup _{\{\bar{t}=\tau\}}|\varphi| \lesssim m, \varepsilon \tau^{-\frac{d}{2}} \sqrt{\mathcal{E}_{0, d}[\varphi](0)}+\mathcal{F}_{p w, \varepsilon}^{\left(q, k, 0, \delta_{0}\right)}[F](\tau),
$$


and for any integer $m \geq 1$ :

$$
\sup _{\{\bar{t}=\tau\}}\left|\nabla^{m} \varphi\right|_{h} \lesssim m, \varepsilon \tau^{-\frac{d+1}{2}} \sqrt{\mathcal{E}_{m+2, d}[\varphi](0)}+\mathcal{F}_{p w, \varepsilon}^{\left(q, k, m+2, \delta_{0}\right)}[F](\tau) .
$$

2. In case the dimension $d$ is even, for any integer $m \geq 0$ we can bound:

$$
\sup _{\{\bar{t}=\tau\}}\left|\nabla^{m} \varphi\right|_{h} \lesssim m, \varepsilon \tau^{-\frac{d}{2}+C \varepsilon} \sqrt{\mathcal{E}_{m, d}[\varphi](0)}+\mathcal{F}_{p w, \varepsilon}^{(q, k, \mu, \delta 0)}[F](\tau) .
$$

For the definition of the weighted energy norms of $\varphi$ and $F$ appearing in the right hand sides of the inequalities above, see Section 9.

See Theorem 9.1 and Corollary 9.2 in Section 9 for more details.

Remark Let us remark at this point that in the case the integrated local energy decay statement in Assumption (ILED1) does not lose derivatives (i.e. $k=0$ ), we can relax the assumption that the deformation tensors of $T$ and $K$ decay like $\bar{t}^{-\delta_{0}}$ (i.e. (9.5) and (9.6)) by replacing it with a uniform $\varepsilon_{0}$-smallness assumption (i.e . (9.42)- (9.44)) for some $\varepsilon_{0}>0$. In this case, we can still obtain (1.28), (1.32), (1.29) and (1.30), at a cost of an $O\left(\varepsilon_{0}\right)$ loss in the exponent of $\tau$ in all these inequalities. Thus, in the absence of trapping, the $r^{p}$-weighted energy method of [11] is robust enough to yield the full "improved" polynomial hierarchy on spacetimes that do not settle down to a stationary background. See also the remark below Theorem 9.1.

We should also notice that in the case when the vector fields $T$ and $K$ are exactly Killing, the proof of Theorem 9.1 yields that for any solution $\varphi$ to $\square \varphi=0$ with compactly supported initial data and any integer $k \geq 0$ :

$$
\left|T^{k} \varphi\right| \lesssim k \bar{t}^{-1-k}
$$

Therefore, using the frequency cut-off techniques of [12] or [29], from (1.33) (and the corresponding statement for decay of the energy of $T^{k} \varphi$ on the foliation $\{\bar{t}=\tau\}$ ) we can deduce that for any $\omega_{0}>0, \varphi_{\geq \omega_{0}}$ decays superpolynomially in $\bar{t}$ (where $\varphi_{\geq \omega_{0}}$ is the part of $\varphi$ supported in the frequency range $|\omega|>\omega_{0}$ with respect to the $\bar{t}$ variable in a coordinate chart where $T=\partial_{\bar{t}}$ ).

Finally, as before, we should note that the initial weighted energy norm on the hyperboloid $\{\bar{t}=0\}$ in the right hand sides of (1.28)-(1.32) can be readily replaced by a similar weighted norm on a hypersurface $\Sigma$ terminating at spacelike infinity. In that case, the source spacetime energy norms $\mathcal{F}[F]$ in (1.28)-(1.32) are replaced by similar weighted spacetime norms of $F$ over the region $J^{+}(\Sigma) \cap\{\bar{t} \leq \tau\}$.

\subsection{Applications of the $r^{p}$-Weighted Energy Method}

We will now discuss some applications of Theorems 1.1-1.4. 


\subsubsection{The Friedlander Radiation Field for Solutions to the Wave Equation $\square \varphi=0$}

On any product Lorentzian manifold of the form $\left(\mathcal{M}^{d+1}, g\right)=\left(\mathbb{R} \times \mathcal{S}^{d},-d t^{2}+\right.$ $\bar{g}),{ }^{5}$ where $\left(\mathcal{S}^{d}, \bar{g}\right)$ is an asymptotically Euclidean Riemannian manifold for $d \geq 2$, Friedlander [17] has established that for any smooth solution $\varphi$ to the wave equation $\square_{g} \varphi=0$ on $(\mathcal{M}, g)$ with compactly supported initial data on $\{t=0\}, r^{\frac{d-1}{2}} \cdot \varphi$ has a well defined and smooth limit on future null infinity. This limit is called the future radiation field of $\varphi$. In order to deduce this result, Friedlander utilised the Penrose compactification method.

As a soft corollary of the hierarchy of $r^{p}$-weighted estimates (1.15), we will extend the result of Friedlander to more general asymptotically flat spacetimes $\left(\mathcal{M}^{d+1}, g\right)$, not necessarily of product type, with $d \geq 3$ :

Theorem 1.5 Let $\left(\mathcal{M}^{d+1}, g\right), d \geq 3$, be a Lorentzian manifold with with the asymptotics (1.14), in the sense that each connected component of an open subset $\mathcal{N}_{a f, \mathcal{M}}$ of $\mathcal{M}$ is mapped diffeomorphically on $\mathbb{R} \times\left(R_{0},+\infty\right) \times \mathbb{S}^{d-1}$ through a $(u, r, \sigma)$ coordinate chart, in which $g$ has the form (1.14). Then for any smooth solution $\varphi$ to the inhomogeneous wave equation $\square_{g} \varphi=F$ on $(\mathcal{M}, g)$ with $\left.(\varphi, \partial \varphi)\right|_{\{t=0\}}$ and $F$ suitably decaying in $r$, the limit

$$
\left(\lim _{r \rightarrow+\infty} \Omega \cdot \varphi\right)(u, r, \sigma) \doteq \Phi_{\mathcal{I}^{+}}(u, \sigma),
$$

where $\Omega=r^{\frac{d-1}{2}}\left(1+O\left(r^{-1}\right)\right)$, exists on all connected components of $\mathcal{N}_{\text {af, } \mathcal{M}}$ and defines a smooth function on $\mathbb{R} \times \mathbb{S}^{d-1}$. Moreover, the following limit exists and is finite for all integers $j_{1}, j_{2}, j_{3} \geq 0$

$$
\lim _{r \rightarrow+\infty}\left(r^{j_{1}} \partial_{r}^{j_{1}} \partial_{\sigma}^{j_{2}} \partial_{u}^{j_{3}}(\Omega \varphi)\right)<+\infty,
$$

where the coordinate derivatives $\partial_{r}, \partial_{\sigma}$ and $\partial_{u}$ are considered with respect to the $(u, r, \sigma)$ coordinate system in the region $\{r \gg 1\}$.

This result will be established in Section 7. For the required decay rates for the initial data of $\varphi$ and the source term $F$, see the statement of Theorem 7.1.

Remark Notice that Theorem 1.5 applies also on spacetimes $(\mathcal{M}, g)$ where the decay rate of $g$ near the future null infinity does not allow for a smooth conformal compactification of the spacetime. Let us also notice that we actually expect the limit (1.35) to be identically 0 when $j_{1} \geq 1$ and $\varphi$ solves $\square \varphi=0$ with compactly supported initial data, but we do not establish this fact here. ${ }^{6}$

The above result will be established in Section 7. For the required decay rates for the initial data of $\varphi$ and the source terms $F$, see the statement of Theorem 7.1.

\footnotetext{
5 As usual for product Lorentzian manifolds, $t$ will denote the projection onto the first factor of $\mathbb{R} \times \mathcal{S}$.

${ }^{6}$ In case $(\mathcal{M}, g)$ admits a conformal compactification near $\mathcal{I}^{+}$, the stronger statement $\lim _{r \rightarrow+\infty}\left(r^{2 j_{1}} \partial_{r}^{j_{1}} \partial_{\sigma}^{j_{2}} \partial_{u}^{j_{3}}(\Omega \varphi)\right)<+\infty$ is known to hold for solutions $\varphi$ to $\square \varphi=0$ with compactly supported initial data.
} 
1.6.2 Logarithmic Hyperboloidal Energy Decay for Solutions to the Wave Equation $\square \varphi=0$ on a General Class of Stationary Asymptotically Flat Spacetimes

As described before, the results of the present paper have been used in our [29] to establish the following result:

Theorem (Corollary 2.2 of [29]). Let $\left(\mathcal{M}^{d+1}, g\right), d \geq 3$, be a globally hyperbolic spacetime with a Cauchy hypersurface $\Sigma$.

Assume that $(\mathcal{M}, g)$ is stationary, with stationary Killing field $T$, and asymptotically flat. If $\mathcal{M}$ contains a black hole region bounded by an event horizon $\mathcal{H}$, assume that $\mathcal{H}$ has positive surface gravity and that the ergoregion (i.e. the set where $g(T, T)>0$ ) is "small" (see [29] for the precise statement of these assumptions). Finally, assume that an energy boundedness statement is true for solutions to $\square \varphi=0$ on the domain of outer communications $\mathcal{D}$ of $\mathcal{M}$.

It then follows that any smooth solution $\varphi$ to $\square_{g} \varphi=0$ on $\mathcal{M}$ with suitably decaying initial data on a Cauchy hypersurface $\Sigma$ of $\mathcal{M}$ satisfies on $\mathcal{D}$ for any integer $m>0$ :

$$
E_{\text {hyp }}(\bar{t}) \leq \frac{C_{m}}{\{\log (2+\bar{t})\}^{2 m}}\left(E_{\text {hyp }}^{(m)}(0)+E_{w, h y p}(0)\right) .
$$

In the above, $\bar{t} \geq 0$ is a suitable time function on $J^{+}(\Sigma) \cap \mathcal{D}$ with hyperboloidal level sets, satisfying $T(\bar{t})=1$, and $E_{\text {hyp }}(\bar{t})$ is the energy flux of $\varphi$ with respect to the level sets of the time functiont $\bar{t} E_{\text {hyp }}^{(m)}(0)$ is the energy of the first $m$ derivatives of $\varphi$ at $\{\bar{t}=0\}$, while $E_{w}(0)$ is a suitable weighted energy of $\varphi$ at $\{\bar{t}=0\}$. The constant $C$ on the right hand side depends on the geometry of $(\mathcal{D}, g)$ and the precise choice of the function $\bar{t}$, while in addition to that, $C_{m}$ also depends on the number $m$ of derivatives of $\varphi$ in $E^{(m)}(0)$.

For a more detailed statement of the above result, see [29].

\subsubsection{Polynomial Decay for Solutions to the Wave Equation $\square \varphi=0$ on the Exterior of Subextremal Kerr Spacetimes for $|a|<M$}

In the next three sections, we will introduce some examples of spacetimes $(\mathcal{M}, g)$ which satisfy the assumptions of Theorems 1.1-1.4.

Our first such example will be the exterior of a subextremal Kerr spacetime $\left(\mathcal{M}_{a, M}, g_{a, M}\right)$ with parameters lying in the full subextremal range $|a|<M$. Notice that this spacetime satisfies all the geometric assumptions of Theorems 1.1-1.4. In fact, the form of the assumptions of Theorem 1.4 was motivated by the geometry of the subextremal Kerr family. In [10], the authors have established an energy boundedness and integrated local energy decay statement for solutions to (1.1) on $\left(\mathcal{M}_{a, M}, g_{a, M}\right)$. As already noted in [10], by applying Theorems 8.1 and 9.1 one can thus readily upgrade these results to polynomial decay estimates for solutions to (1.1), and therefore establish Corollary 3.1 of [10], which we state here with the notation of [10]:

Corollary (Corollary 3.1 of [16]) Let $\left(\mathcal{M}_{a, M}, g_{a, M}\right)$ be the exterior of a Kerr black hole spacetime of mass $M$ and angular momentum $a$, such that $|a|<M$. Let $\tilde{\Sigma}_{0}$ be a 
smooth spacelike hypersurface of $\left(\mathcal{M}_{a, M}, g_{a, M}\right)$ intersecting transversally the future event horizon $\mathcal{H}^{+}$and terminating at fututre null infinity $\mathcal{I}^{+}$. Let also $\tilde{\Sigma}_{\tau}$ denote the image of $\tilde{\Sigma}_{0}$ under the flow of the stationary Killing field $T$ of $\left(\mathcal{M}_{a, M}, g_{a, M}\right)$ (see [16]), and let $N$ be a globally timelike, future directed and T-invariant vector field on $\mathcal{M}_{a, M}$ coinciding with $T$ in the region $\{r \gg 1\}$.

Then, for any $\delta>0$, there exists a constant $C=C\left(a, M, \tilde{\Sigma}_{0}, \delta\right)>0$ such that for any smooth solution $\varphi$ to the wave equation (1.1) on $J^{+}\left(\tilde{\Sigma}_{0}\right) \subset\left(\mathcal{M}_{a, M}, g_{a, M}\right)$ with suitably decaying initial data on $\tilde{\Sigma}_{0}$ the following energy decay estimates hold:

$$
\begin{aligned}
& \int_{\tilde{\Sigma}_{\tau}} J_{\mu}^{N}(\varphi) n_{\tilde{\Sigma}}^{\mu} \leq C \cdot E \tau^{-2}, \\
& \int_{\tilde{\Sigma}_{\tau}} J_{\mu}^{N}(N \varphi) n_{\tilde{\Sigma}}^{\mu} \leq C \cdot E \tau^{-4+\delta} .
\end{aligned}
$$

Moreover, the following pointwise decay estimates hold:

$$
\begin{aligned}
& \sup _{\tilde{\Sigma}_{\tau}} r|\varphi| \leq C \sqrt{E} \cdot \tau^{-\frac{1}{2}}, \\
& \sup _{\tilde{\Sigma}_{\tau}}|\varphi| \leq C \sqrt{E} \cdot \tau^{-\frac{3}{2}}
\end{aligned}
$$

and

$$
\sup _{\tilde{\Sigma}_{\tau}}\left(|N \varphi|+\left|\nabla_{\tilde{\Sigma}} \varphi\right|\right) \leq C \sqrt{E} \cdot \tau^{-2} .
$$

In the above, $E$ denotes a suitable higher order weighted energy norm of the intial data of $\varphi$ on $\tilde{\Sigma}_{0}$, and is not necessarily the same quantity in all of the above estimates.

See [16] for more details.

Remark Notice that the slowly rotating $4+1$ dimensional Myers-Perry spacetimes satisfy all the geometric assumptions of Theorem 1.4. Therefore, in view of the integrated local energy decay estimate established in [23], Theorem 1.4 implies that any solution $\varphi$ to (1.1) on a slowly rotating $4+1$ dimensional Myers-Perry spacetime with suitably decaying initial data satisfies a $\bar{t}^{-2+\delta}$ pointwise decay estimate.

\subsubsection{Improved Polynomial Decay on Radiating Uniformly Small Perturbations of Minkowski Spacetime}

For our second example of a spacetime satisfying the assumptions of Theorems 1.11.4 , we will need to introduce a definition: We will define a metric $g$ on $\mathbb{R}^{d+1}, d \geq 3$, to be a radiating uniformly small perturbation of Minkowski spacetime if there exists a (small) $\varepsilon_{0}>0$ and an $R>0$ such that, in the $(u, r, \sigma)$ coordinate system on $\mathbb{R}^{d+1}$ in the region $\{r \geq R\}, g$ is of the form (3.3) for some $0<a \leq 1$, and moreover:

- For any integers $m_{1}, m_{2} \geq 0$ we have the global bound:

$$
\sup _{\mathbb{R}^{d+1}}\left|\mathcal{L}_{T}^{m_{1}} \nabla_{e}^{m_{2}}(g-\eta)\right|_{e} \lesssim m_{1}, m_{2} \varepsilon_{0}(1+r)^{-1} \min \left\{1,|u|^{1-m_{1}}\right\}
$$


- In the region $\{r \geq R\}$ we can estimate for any $m \geq 1$ in the $(u, r, \sigma)$ coordinate system:

$$
\begin{aligned}
\mathcal{L}_{T}^{m} g= & O_{m}\left(\varepsilon_{0} \min \left\{1,|u|^{1-m}\right\}\right)\left\{O\left(r^{-1-a}\right) d r d u+O(r) d \sigma d \sigma+O(1) d u d \sigma\right. \\
& \left.+O\left(r^{-a}\right) d r d \sigma+O\left(r^{-1}\right) d u^{2}+O\left(r^{-2-a}\right) d r^{2}\right\}
\end{aligned}
$$

In the above, $T$ is the vector field $\partial_{t}$ in the Cartesian coordinate system $\left(t, x^{1}, \ldots, x^{d}\right)$ on $\mathbb{R}^{d+1}, e$ is the usual Euclidean metric on $\mathbb{R}^{d+1}$ and $\nabla_{e}$ is the flat connection on $\mathbb{R}^{d+1}$. Notice that if $\varepsilon_{0}$ is smaller than an absolute constant, $T$ is everywhere timelike and furthermore $\left(\mathbb{R}^{d+1}, g\right)$ can not contain any trapped geodesics. In fact, if $\varepsilon_{0}$ is small enough, the $\partial_{r}$-Morawetz current of Minkowski spacetime (combined with the estimates of Section 4 of the present paper) yields an integrated local energy decay estimate of the form (1.19) without loss of derivatives. Furthermore, the rest of the geometric Assumptions of Theorems 1.1-1.4 are satisfied, except for the assumption on the $\bar{t}^{-\delta_{0}}$ decay of deformation tensors of $T$ and $K$ which is replaced by a uniform $\varepsilon_{0}$-smallness assumption (see the remark below Theorem 1.4).

For such a spacetime $\left(\mathbb{R}^{d+1}, g\right)$, we will fix $\mathcal{S} \subset \mathbb{R}^{d+1}$ to be a smooth spacelike hypersurface of $\left(\mathbb{R}^{d+1}, g\right)$ which terminates at $\mathcal{I}^{+}$, and let $\bar{t}: \mathbb{R}^{d+1} \rightarrow \mathbb{R}$ be defined by the condition $T(\bar{t})=1$ and $\left.\bar{t}\right|_{\mathcal{S}}=0$.

One can deduce from [7] that dynamical solutions of the vacuum Einstein equations arising from initial data which are close to the ones for Minkowski spacetime are included in this class.

The following pointwise decay estimate for solutions to the wave equation on radiating uniformly small perturbations of Minkowski spacetime is a straightforward application of Theorems 1.3 and 1.4:

Corollary Let $\left(\mathbb{R}^{d+1}, g\right), d \geq 3$, be a uniformly small perturbation of Minkowski spacetime, in the sense that for some $\varepsilon_{0}>0$ and $R>0, g$ is of the form (3.3) in the region $\{r \geq R\}$ and (1.42) and (1.43) hold. Let also $\bar{t}:\left(\mathbb{R}^{d+1}, g\right) \rightarrow \mathbb{R}$ be constructed as above. Then, provided $\varepsilon_{0}$ is smaller than an absolute constant, for any solution $\varphi$ to the wave equation $\square_{g} \varphi=0$ on $\left(\mathbb{R}^{d+1}, g\right)$ and any $\tau \geq 0$ we can bound

$$
\sup _{\{\bar{t}=\tau\}}|\varphi| \lesssim \tau^{-\frac{d}{2}+O\left(\varepsilon_{0}\right)} \sqrt{\mathcal{E}_{0, d}[\varphi](0)}
$$

and, for any integer $m \geq 1$ :

$$
\sup _{\{\bar{t}=\tau\}}\left|\nabla^{m} \varphi\right|_{e} \lesssim \tau^{-\frac{d+1}{2}+O\left(\varepsilon_{0}\right)} \sqrt{\mathcal{E}_{m+2, d}[\varphi](0)}
$$

In case the following stronger assumptions on the deformation tensor of $T$ hold for some $\delta_{0}>0$ and any $m_{1} \geq 1, m_{2} \geq 0$ in place of (1.42) and (1.43):

$$
\sup _{\mathbb{R}^{d+1}}\left|r \mathcal{L}_{T}^{m_{1}} \nabla_{e}^{m_{2}}(g-\eta)\right|_{e} \lesssim m_{1}, m_{2}|u|^{1-m_{1}-\delta_{0}}
$$


and in the region $\{r \geq R\}$ for any $m \geq 1$ :

$$
\begin{aligned}
\mathcal{L}_{T}^{m} g= & O_{m}\left(|u|^{1-m-\delta_{0}}\right)\left\{O\left(r^{-1-a}\right) d r d u+O(r) d \sigma d \sigma+O(1) d u d \sigma\right. \\
& \left.+O\left(r^{-a}\right) d r d \sigma+O\left(r^{-1}\right) d u^{2}+O\left(r^{-2-a}\right) d r^{2}\right\}
\end{aligned}
$$

then (1.44) and (1.45) can be upgraded to

$$
\sup _{\{\bar{t}=\tau\}}|\varphi| \lesssim \tau^{-\frac{d}{2}} \sqrt{\mathcal{E}_{0, d}[\varphi](0)}
$$

and, for any integer $m \geq 1$ :

$$
\sup _{\{\bar{t}=\tau\}}\left|\nabla^{m} \varphi\right|_{e} \lesssim \tau^{-\frac{d+1}{2}} \sqrt{\mathcal{E}_{m+2, d}[\varphi](0)} .
$$

For the definition of the initial energy norms $\mathcal{E}_{0, d}[\varphi](0)$ and $\mathcal{E}_{m+2, d}[\varphi](0)$ on the hypersurfaces $\{\bar{t}=0\}$ (which can also be replaced by norms on $\{t=0\}$ ), see Section 9.2.

Remark Notice that the above corollary extends a recent result of Oliver [31].

\subsubsection{Improved Polynomial Decay on Dynamical, Radiating Black Hole Spacetimes}

A final example of a class of spacetimes satisfying the assumptions of Theorems 1.1-1.4 will concern the exterior region of black hole spacetimes dynamically settling down to a subextremal Kerr spacetime. Here, we will restrict ourselves only to spacetimes $\left(\mathcal{M}_{S c h}, g\right)$ (where $\mathcal{M}_{S c h}$ has the differentiable structure of the Schwarzschild exterior) settling down to the Schwarzschild exterior spacetime $\left(\mathcal{M}_{S c h}, g_{M}\right)$ for some $M>0$ at a sufficiently fast polynomial rate. In particular, we will assume that we can fix a double null foliation on $\left(\mathcal{M}_{S c h}, g\right)$ such that the components of $g$ with respect to this foliation approach the components of the Schwarzschild metric $g_{M}$ at a sufficiently fast polynomial rate towards "timelike infinity". This class of spacetimes includes, in particular, the radiating spacetimes constructed in [14], which approach the Schwarschild metric at an exponential rate. We will not provide more details of this setup here, but instead we will refer the reader to [14]. The reason for this restriction is that it is straightforward to check (essentially without calculation) that these spacetimes $\left(\mathcal{M}_{S c h}, g\right)$ satisfy the assumptions of Theorems 1.1-1.4 (we will omit the details).

On spacetimes $\left(\mathcal{M}_{S c h}, g\right)$ as above, the energy current yielding the integrated local energy decay statement for the Schwarzschild exterior $\left(\mathcal{M}_{S c h}, g_{M}\right)$ constructed in [8], combined with the estimates of Section 4 of the present paper and the fast rate at which $g$ approaches the Schwarzschild metric $g_{M}$, imply that an integrated local energy decay statement of the form (1.27) also holds on $\left(\mathcal{M}_{S c h}, g\right)$. Furthermore, $\left(\mathcal{M}_{S c h}, g\right)$ also satisfies the rest of the geometric assumptions of Theorem 1.4. Therefore, as an application of Theorem 1.4, we obtain the following result: 
Corollary Let $\left(\mathcal{M}_{S c h}, g\right)$ be a radiating spacetime approaching $\left(\mathcal{M}_{S c h}, g_{M}\right)$ in the future at a sufficiently fast polynomial rate (in the sense described above). Let also $\bar{t}: \mathcal{M}_{S c h} \rightarrow \mathbb{R}$ be a function with spacelike level sets intersecting $\mathcal{H}^{+}$transversally and terminating at $\mathcal{I}^{+}$, such that $T(\bar{t})=1$ (where $T$ is the Schwarzschild stationary Killing field). Then for any solution $\varphi$ to $\square_{g} \varphi=0$ on $\left(\mathcal{M}_{S c h}, g\right)$ with suitably decaying initial data on a Cauchy hypersurface, the following pointwise decay estimates hold:

$$
\sup _{\{\bar{t}=\tau\}}|\varphi| \lesssim_{m, \varepsilon} \tau^{-\frac{3}{2}} \sqrt{\mathcal{E}_{0}[\varphi](0)}
$$

and for any integer $m \geq 1$ :

$$
\sup _{\{\bar{t}=\tau\}}\left|\nabla^{m} \varphi\right| \lesssim m \tau^{-2} \sqrt{\mathcal{E}_{m+2}[\varphi](0)}
$$

For the definition of the initial energy norms $\mathcal{E}_{0}[\varphi](0)$ and $\mathcal{E}_{m+2}[\varphi](0)$ on the hypersurfaces $\{\bar{t}=0\}$ (which can also be replaced by norms on a Cauchy hypersurface), see Section 9.2

Remark We should notice that the spacetimes constructed in [14] are only $C^{l}$ on the future event horizon $\mathcal{H}^{+}$for some sufficiently large $l$, but not $C^{\infty}$. However, Theorem 1.4 still applies in this case, and the above Corollary holds provided the integer $m$ in (1.51) is restricted to lie below some constant $C(l)$ depending on the order of differentiability of $g$ on $\mathcal{H}^{+}$.

\subsubsection{Expected Applications in the Case of Non-linear Wave Equations and the Einstein Equations}

As we mentioned in Section 1.4.2, the $r^{p}$-weighted energy method of [11] has already been applied to the study of solutions to non-linear wave equations on backgrounds close to Minkowski spacetime $\left(\mathbb{R}^{3+1}, \eta\right)$. In particular, in [40], Yang established the global existence of small data solutions to quasilinear wave equations satisfying the null condition on non-stationary backgrounds $(\mathcal{M}, g)$ which are $C^{1}$-close to $\left(\mathbb{R}^{3+1}, \eta\right)$ and approach $\left(\mathbb{R}^{3+1}, \eta\right)$ towards $\mathcal{I}^{+}$.

We expect that the estimates established in Sections 5-9 are robust enough to be generalised to the case of small data solutions to systems of quasilinear wave equations satisfying the null condition on general radiating asymptotically flat spacetimes $(\mathcal{M}, g)$ satisfying Assumptions (GM1), (GM2), (GM3), (GM4), (GM5) and the integrated local energy decay assumption (ILED2). This would serve as an extension of the results of [40] on this much broader class of spacetimes which are not necessarily globally close to $\left(\mathbb{R}^{3+1}, \eta\right.$ ) (and equipped with a metric $g$ decaying to $\eta$ at a weaker rate in a neighborhood of $\mathcal{I}^{+}$), and moreover only satisfy an integrated local energy decay statement with loss of derivatives.

In [20] and [14], the techniques of [11] have been extended to the case of the vacuum Einstein equations

$$
\operatorname{Ric}(g)=0
$$


establishing an $r^{p}$-weighted hierarchy of estimates for the curvature components of dynamical solutions to (1.52) approaching the Schwarzschild exterior in the future. In view of the properties of the Kerr exterior spacetime, the asymptotic dynamical behaviour of solutions to (1.52) arising as small perturbations of Minkowski spacetime, established in [7], and the decay estimates established for the linearised vacuum perturbations of Schwarzschild exterior in [15], it would be reasonable to expect that a proof of the well-known subextremal Kerr exterior stability conjecture (see [15]) ${ }^{7}$ would also establish that all vacuum small perturbations of the subextremal Kerr exterior spacetime satisfy Assumptions (GM1)-(GM5) and (ILED2). For this reason, we expect the results of Sections 5-9, suitably adapted according to [14,20], to be relevant to the ongoing research aimed at establishing the Kerr exterior stability conjecture.

\subsection{Outline of the Paper and Technical Comments}

In this section, we will describe briefly how the current paper is organised and we will sketch the difficulties arising in the proof of the main statements. In particular, we will point out the new difficulties that appear in comparison to [11,34]. The reader might find it helpful to return to this section after viewing the detailed setup of the Theorems in Sections 4-9.

The geometry of the asymptotically fat region $\mathcal{N}_{a f}$ of the spacetimes $(\mathcal{M}, g)$ under consideration is introduced in Section 3. In this region, a function $\bar{t}$ with hyperboloidal level sets is constructed. It is also shown that in $\mathcal{N}_{a f}$, the wave operator takes the form:

$$
\begin{aligned}
\Omega \cdot \square \varphi= & -\left(1+O\left(r^{-1-a}\right)\right) \cdot \partial_{u} \partial_{v}(\Omega \varphi)+r^{-2} \Delta_{g_{\mathbb{S} d-1}+O\left(r^{-1}\right)}(\Omega \varphi) \\
& -\frac{(d-1)(d-3)}{4} r^{-2} \cdot(\Omega \varphi)+\operatorname{Err}(\Omega \varphi)
\end{aligned}
$$

where $\Omega=r^{\frac{d-1}{2}}\left(1+O\left(r^{-1}\right)\right)$ and the "error" terms $\operatorname{Err}(\Omega \varphi)$ have the form (3.15). Notice that the particular choice of the factor $\Omega$ serves to eliminate some terms in the expression for $\operatorname{Err}(\Omega \varphi)$ which would be "problematic" in the derivation of the $r^{p}$ weighted energy estimates (1.15) and (1.16) (such terms would appear, for instance, if one substituted $\Omega$ by $r^{\frac{d-1}{2}}$ in the case when $\partial_{u} M \neq 0$ in (1.14))

In Section 4, we establish $\partial_{r}$-Morawetz and $J^{T}$-energy boundedness estimates of the form

$$
\begin{aligned}
& \int_{\left\{\tau_{1} \leq \bar{t} \leq \tau_{2}\right\} \cap\{r \geq R\}} r^{-1-\eta}\left(|\partial \varphi|^{2}+r^{-2}|\varphi|^{2}\right) \lesssim \eta \int_{\left\{\bar{t}=\tau_{1}\right\} \cap\{r \geq R\}} J_{\mu}^{T}(\varphi) \bar{n}^{\mu} \\
& \quad+\int_{\left\{\tau_{1} \leq \bar{t} \leq \tau_{2}\right\} \cap\{r \sim R\}}\left(|\partial \varphi|^{2}+r^{-2}|\varphi|^{2}\right)+\int_{\left\{\tau_{1} \leq \bar{t} \leq \tau_{2}\right\} \cap\{r \geq R\}} r^{1+\eta}|\square \varphi|^{2} \\
& +\int_{\left\{\tau_{1} \leq \bar{t} \leq \tau_{2}\right\} \cap\{r \geq R\}} r^{-1}\left(\left|\partial_{v} \varphi\right|^{2}+\left|r^{-1} \partial_{\sigma} \varphi\right|^{2}+r^{-2}|\varphi|^{2}\right)
\end{aligned}
$$

\footnotetext{
7 Provided, of course, that the conjecture is true!
} 
and

$$
\begin{aligned}
& \int_{\left\{\bar{t}=\tau_{1}\right\} \cap\{r \geq R\}} J_{\mu}^{T}(\varphi) \bar{n}^{\mu} \lesssim \eta \int_{\left\{\bar{t}=\tau_{1}\right\} \cap\{r \geq R\}} J_{\mu}^{T}(\varphi) \bar{n}^{\mu} \\
& \quad+\int_{\left\{\tau_{1} \leq \bar{t} \leq \tau_{2}\right\} \cap\{r \sim R\}}\left(|\partial \varphi|^{2}+r^{-2}|\varphi|^{2}\right) \\
& \quad+\int_{\left\{\tau_{1} \leq \bar{t} \leq \tau_{2}\right\} \cap\{r \geq R\}} r^{1+\eta}|\square \varphi|^{2} \\
& \quad+\int_{\left\{\tau_{1} \leq \bar{t} \leq \tau_{2}\right\} \cap\{r \geq R\}} r^{-1}\left(\left|\partial_{v} \varphi\right|^{2}+\left|r^{-1} \partial_{\sigma} \varphi\right|^{2}+r^{-2}|\varphi|^{2}\right),
\end{aligned}
$$

respectively. We notice that the last terms of the right hand sides of (1.54) and (1.55) appear due to the radiating asymptotics of (1.14), and can be completely dropped in the case the spacetime is non radiating or when the radiating components of (1.14) satisfy some special monotonicity conditions (which are satisfied in the case when $\partial_{u} M \leq 0$ in (1.14) and the spacetime is spherically symmetric).

In Section 5, the $r^{p}$-weighted energy hierarchy (1.15) is established. This is achieved by multiplying the expression (1.53) by $r^{p} \partial_{v}(\Omega \varphi)$ and then integrating by parts (in the top order terms) over a region of the form $\left\{\tau_{1} \leq \bar{t} \leq \tau_{2}\right\}$ (athough regions of different "shape" are also treated). In this integration by parts procedure, the error terms occuring from the $\operatorname{Err}(\Omega \varphi)$ summands are controlled with the help of the already positive definite terms in the resulting expression, after adding to it the estimates (1.54) and (1.55), using also a Hardy-type inequality for the zeroth order terms. It is in this procedure that the elimination of the "worst" terms in $\operatorname{Err}(\Omega \varphi)$, resulting from the precise choice of the factor $\Omega$ in (1.53), is important.

In Section 6, the higher order $r^{p}$-weighted energy hierarchy (1.16) is established. This is achieved by commuting equation (1.53) with $\partial_{v}, r^{-1} \partial_{\sigma}$ and $r^{-1} \partial_{u}$, and repeating the proof leading to (1.15), after noticing that the first two commutation vector fields lead to the appearence of some new bulk terms with favorable sign. Notice that in this procedure, multiple integrations by parts are performed also on lower order terms, in order to guarantee that (1.16) is valid even at the (upper) endpoint $p=2 k$.

In Section 7, Theorem 1.5 concerning the existence of the Friedlander radiation field is formulated and established with the use of the boundary terms controlled by the $r^{p}$-weighted energy hierarchy (1.16) (for $2 k-1<p \leq 2$ ), combined simply with the fundamental theorem of calculus. As a corollary of Theorem 1.5, it is shown the $L^{2}$ norm of certain derivatives of the radiation field of $\varphi$ on $\mathcal{I}^{+}$are also controlled by the right hand side of (1.15) and (1.16).

In Section 8, the geometric conditions and the integrated local energy decay assumption (consistent with the right hand side error term in (1.54)) on the spacetimes $(\mathcal{M}, g)$, on which Theorem 1.3 (concerning the $\bar{t}^{-1}$ decay estimates for $\varphi$ ) applies, are introduced. The proof of Theorem 1.3 then follows by applying the pigeonhole principle on the positive bulk terms of the hierarchy (1.15), as was first done in [11]. Notice that, since no energy boundedness statement is a priori assumed, in order to 
obtain the final decay estimate in this procedure, an energy boundedness estimate with loss of derivatives (and right hand side error terms consistent with (1.55)) is established. ${ }^{8}$

Finally, in Section 9, the extra geometric conditions on $(\mathcal{M}, g)$, required for Theorem 1.4 (concerning the $\bar{t}^{-\frac{d}{2}}$ decay estimates for $\varphi$ ) to hold, are formulated. The proof of Theorem 1.4 then follows by repeated applying the pigeonhole principle argument on the higher order hierarchy (1.16) for higher $T$ and $\chi \cdot K$ derivatives of $\varphi$ (where $\chi$ is a suitable compactly supported cut-off function). In each step in this procedure, the wave equation is used to substitute derivatives of $\varphi$ tangential to the hyperboloids $\{\bar{t}=$ const $\}$ with $T$ and $\chi \cdot K$ derivatives of $\varphi$, in a fashion similar to [34]. In the end, however, the wave equation is used again to transform decay estimates of $T$ and $\chi \cdot K$ derivatives of $\varphi$ into decay estimates of certain elliptic operators on $\{\bar{t}=$ const $\}$ applied on $\varphi$, and then the elliptic estimates of Section B of the Appendix yield decay estimates for the energy of all higher order derivatives of $\varphi$. Pointwise decay estimates for $\varphi$ then follow by applying the Gagliardo-Nirenberg type estimates of Section 9.8. Thus, our method for extracting $\bar{t}^{-\frac{d}{2}}$ decay estimates differs substantially from the method implemented in [34] (which yielded $\bar{t}^{-\frac{3}{2}+\delta}$ decay estimates). For a more detailed sketch of the proof of Theorem 1.4, see Section 9.4. Let us remark that the elliptic estimates of Section B are also used to control error terms arising from the commutations with the truncated vector field $\chi \cdot K$.

\section{Notational Conventions}

\subsection{Conventions on Constants and Inequality Symbols}

We will use capital letters (e.g. $C$ ) to denote "large" constants, namely constants that appear on the right hand side of inequalities, and hence can be replaced by larger ones without affecting the validity of the inequality. Lower case letters (e.g. $c$ ) will be used to denote small constants (which can similarly freely be replaced by smaller ones). Moreover, the same character might be used to denote different constants even in adjacent lines or formulas.

We will not keep track of the dependence of constants on the specific geometric aspects of our spacetime, except for some very specific cases. However, we will always keep track of the dependence of all constants on each parameter that has not been fixed. Once a parameter is fixed (which will be clearly stated in the text), we will feel free to drop the dependence of constants on it.

The notation $f_{1} \lesssim f_{2}$ for two real functions $f_{1}, f_{2}$ will as usual mean that there exists some $C>0$, such that $f_{1} \leq C \cdot f_{2}$. Of course, it should be stated clearly in each case whether this constant $C$ depends on any free parameters. If nothing is stated regarding the dependence of this constant on parameters, it should be assumed that it only depends on the geometry of the background under consideration.

\footnotetext{
8 Notice, however, that this energy estimate can yield a "true" energy boundedness estimate only in the case where the spacetime $(\mathcal{M}, g)$ is non-radiating or when the radiating components of (1.14) satisfy some special monotonicity conditions.
} 
We will also write $f_{1} \sim f_{2}$ if $f_{1} \lesssim f_{2}$ and $f_{2} \lesssim f_{1}$. Moreover, $f_{1} \ll f_{2}$ will mean that the quotient $\frac{\left|f_{1}\right|}{\left|f_{2}\right|}$ can be bounded from above by some sufficiently small positive constant, the magnitude and the dependence of which on variable parameters will be clear in each case from the context. Furthermore, for any function $f: \mathcal{M} \rightarrow[0,+\infty)$ defined on some set $\mathcal{M}$, we will denote with $\{f \gg 1\}$ the subset $\{f \geq R\}$ of $\mathcal{M}$ for some constant $R \gg 1$.

For functions $f_{1}, f_{2}$ of some variable $x$ taking values in a semi-infinite interval $[a,+\infty)$, writing $f_{1}=o\left(f_{2}\right)$ will imply that $\frac{f_{1}}{f_{2}}$ can be bounded by some continuous function $h:[a,+\infty) \rightarrow \mathbb{R}_{+}$such that $h(x) \rightarrow 0$ as $x \rightarrow+\infty$. Again, the dependence of this bound $h$ on any free parameter will be clear from the context.

\subsection{Convention on Connections and Volume form Notations}

We will frequently denote the natural connection of a pseudo-Riemannian manifold $\left(\mathcal{N}, h_{\mathcal{N}}\right)$ as $\nabla^{h_{\mathcal{N}}}$ or $\nabla_{h_{\mathcal{N}}}$, and the associated volume form as $d h_{\mathcal{N}}$. If $h_{\mathcal{N}}$ is Riemannian, we will denote the associated norm on $\oplus_{n, m \in \mathbb{N}}\left(\otimes^{n} T \mathcal{N} \otimes^{m} T^{*} \mathcal{N}\right)$ with $|\cdot|_{h_{\mathcal{N}}}$.

For any integer $j \geq 0,\left(\nabla^{h_{\mathcal{N}}}\right)^{j}$ or $\nabla_{h_{\mathcal{N}}}^{j}$ will as usual denote the higher order operator

$$
\underbrace{\nabla_{h_{\mathcal{N}}} \cdots \nabla_{h_{\mathcal{N}}}}_{j \text { times }} .
$$

Notice that the above product is not symmetrised. We will always use Latin characters to denote such powers of covariant derivative operators, while Greek characters will be used for the indices of a tensor in an abstract index notation.

Example Under these conventions, for a $(n, m)$-tensor $k$ and a function $u$ on a pseudoRiemannian manifold $\left(\mathcal{N}, h_{\mathcal{N}}\right)$, the quantity

$$
k_{\beta_{1} \ldots \beta_{m}}^{\alpha_{1} \ldots \alpha_{n}} \cdot\left(\nabla_{h_{\mathcal{N}}}^{n+m}\right)_{\alpha_{1} \ldots \alpha_{n}}^{\beta_{1} \ldots \beta_{m}} u
$$

denotes a contraction of $k$ with the higher order derivative $\nabla_{h_{\mathcal{N}}}^{n+m} u$ of $u$, where the metric $h_{\mathcal{N}}$ was used to raise the first $m$ indices of $\nabla_{h_{\mathcal{N}}}^{n+m} u$. Notice that the abstract index notation used above is independent of the choice of an underlying coordinate chart for the indices.

\subsection{Conventions on Notations for Derivatives on $\mathbb{S}^{d-1}$}

In this paper we will frequently work in polar coordinates, and hence it will prove convenient to introduce some shorthand notation regarding iterated derivatives on the unit sphere $\mathbb{S}^{d-1}, d \geq 2$.

We will denote with $g_{\mathbb{S}^{d-1}}$ the usual round metric on the sphere $\mathbb{S}^{d-1}$, which is the induced metric on the unit sphere of $\mathbb{R}^{d}$. The metric $g_{\mathbb{S}^{d-1}}$ extends naturally to an inner product on the tensor bundle $\oplus_{n, m \in \mathbb{N}}\left(\otimes^{n} T \mathbb{S}^{d-1} \otimes^{m} T^{*} \mathbb{S}^{d-1}\right)$ over $\mathbb{S}^{d-1}$; we will still 
denote this inner product as $g_{\mathbb{S}^{d-1}}$. For any tensor field $\mathfrak{m}$ on $\mathbb{S}^{d-1},|\mathfrak{m}|_{\mathbb{S}^{d-1}}$ will as usual denote the norm of $\mathfrak{m}$ with respect to $g_{\mathbb{S}^{d-1}}$. We will also denote with $\nabla^{\mathbb{S}^{d-1}}$ or $\nabla_{\mathbb{S}^{d-1}}$ the covariant derivative associated with $g_{\mathbb{S}^{d-1}}$. We will denote with $\Delta_{g_{\mathbb{S}} d-1}$ the Laplace-Beltrami operator on $\left(\mathbb{S}^{d-1}, g_{\mathbb{S}^{d-1}}\right)$. For any smooth $\left(k_{1}, k_{2}\right)$-tensor field $\mathfrak{m}$ on $\mathbb{S}^{d-1},\left(\nabla^{\mathbb{S}^{d-1}}\right)^{k} \mathfrak{m}\left(\right.$ or $\left.\nabla_{\mathbb{S}^{d-1}}^{k} \mathfrak{m}\right)$ will denote the $\left(k_{1}, k_{2}+k\right)$-tensor field on $\mathbb{S}^{d-1}$ obtained after applying the operator $\nabla^{\mathbb{S}^{d-1}}$ on $\mathfrak{m} k$ times.

We will frequently work on regions $\mathcal{U}$ of a spacetime $\mathcal{M}^{d+1}$ where there exists a coordinate "chart" mapping $\mathcal{U}$ diffeomorphically onto $\mathbb{R}_{+} \times \mathbb{R}_{+} \times \mathbb{S}^{d-1}$. In any such a coordinate "chart", $\sigma$ will denote the projection $\sigma: \mathcal{U} \rightarrow \mathbb{S}^{d-1}$. Notice that with this notation, for any $x \in \mathcal{M}, \sigma(x)$ is a point on $\mathbb{S}^{d-1}$ and not just the coordinates of this point in a coordinate chart on $\mathbb{S}^{d-1}$. We will not need to fix a coordinate atlas on $\mathbb{S}^{d-1}$. We will use the same $\sigma$ notation also for the spherical variable of a polar coordinate "chart" on codimension 1 submanifolds of $\mathcal{M}$ (the range of such a "chart" will be $\left.\mathbb{R}_{+} \times \mathbb{S}^{d-1}\right)$. For instance, $(r, \sigma):\{t=0\} \rightarrow \mathbb{R}_{+} \times \mathbb{S}^{d-1}$ will denote the usual polar coordinate chart on the hyperplane $\{t=0\}$ of $\mathbb{R}^{d+1}$.

For any function $h$ on a subset $\mathcal{U}$ of a spacetime $\mathcal{M}$ covered by a polar coordinate chart $\left(u_{1}, u_{2}, \sigma\right): \mathcal{U} \rightarrow \mathbb{R}_{+} \times \mathbb{R}_{+} \times \mathbb{S}^{d-1}$ and any $\alpha_{1}, \alpha_{2} \in \mathbb{R}_{+}, h\left(\alpha_{1}, \alpha_{2}, \cdot\right)$ defines a function on $\mathbb{S}^{d-1}$. In this way, the $\nabla^{\mathbb{S}^{d-1}}$ differential operator on $\mathbb{S}^{d-1}$ is extended to a tangential differential operator on the hypersurfaces $\left\{u_{1}, u_{2}=\right.$ const $\}$ of $\mathcal{U}$. Notice, of course, that this operator is tied to the specific choice of the polar coordinate chart $\left(u_{1}, u_{2}, \sigma\right)$.

We will now introduce some schematic notation for derivatives on $\mathbb{S}^{d-1}$ (and the associated tangential operators on the hypersurfaces $\left\{u_{1}, u_{2}=\right.$ const $\}$ in a $\left(u_{1}, u_{2}, \sigma\right)$ coordinate chart on a spacetime $\mathcal{M}$ ). For any function $h$ on $\mathbb{S}^{d-1}$ and any $k \in \mathbb{N}$, we will frequently denote the $k$-th order derivative $\nabla_{\mathbb{S}^{d-1}}^{k} h$ as $\partial_{\sigma}^{k} h$, and we will also use the following notation for the norm of this tensor:

$$
\left|\partial_{\sigma}^{k} h\right| \doteq\left|\nabla_{\mathbb{S}^{d-1}}^{k} h\right|_{\mathbb{S}^{d-1}}
$$

Moreover, for any symmetric $(k, 0)$-tensor $a$ on $\mathbb{S}^{d-1}$, we will use the following schematic notation for the contraction of $\left(\nabla^{\mathbb{S}^{d-1}}\right)^{k} h$ with $a$ :

$$
a \cdot \partial_{\sigma}^{k} h \doteq a^{\iota_{1} \ldots \iota_{k}}\left(\nabla_{\mathbb{S}^{d-1}}^{k}\right)_{\iota_{1} \ldots l_{k}} h
$$

(see the previous section for the notations on powers of covariant derivatives and the abstract index notation). We will use the same notation for the contraction of the product of derivatives of two or more functions: For any set of $n$ functions $h_{1}, \ldots, h_{n}$ on $\mathbb{S}^{d-1}$ and any set $\left(j_{1}, \ldots j_{n}\right)$ of non negative integers, for any $\left(\sum_{k=1}^{n} j_{k}, 0\right)$-tensor $a$ on $\mathbb{S}^{d-1}$ which is symmetric in any pair of indices lying in the same one of the intervals $I_{m}=\left(\sum_{k=1}^{m-1} j_{k}+1, \sum_{k=1}^{m} j_{k}\right)$ for each $m \in\{1, \ldots n\}$ (but not necessarily symmetric in pairs of indices lying in different $I_{m}$ 's), we will denote 


$$
a \cdot \partial_{\sigma}^{j_{1}} h_{1} \cdots \partial_{\sigma}^{j_{n}} h_{n} \doteq a^{\iota_{1} \ldots \iota \sum_{k=1}^{n} j_{k}} \cdot\left(\nabla_{\mathbb{S}^{d}-1}^{j_{1}}\right)_{\iota_{1} \ldots \iota_{j_{1}}} h_{1} \cdots\left(\nabla_{\mathbb{S}^{d-1}}^{j_{n}}\right)_{\iota_{\sum_{k=1}^{n-1} j_{k}+1}^{n} \ldots \sum_{k=1}^{n} j_{k}} h_{n} .
$$

The same notation (2.3) and (2.4) will also apply when $h, h_{1}, \ldots, h_{n}$ are tensor fields on $\mathbb{S}^{d-1}$.

Depending on the context, $d \sigma$ will be used to denote either the usual volume form on $\left(\mathbb{S}^{d-1}, g_{\mathbb{S}^{d-1}}\right)$ or a 1 -form on $\mathbb{S}^{d-1}$ satisfying for any $k \in \mathbb{N}$ the bound $\left|\left(\nabla^{\mathbb{S}^{d-1}}\right)^{k} d \sigma\right|_{g_{\mathbb{S} d-1}} \leq 10^{k}$. Similarly, $d \sigma d \sigma$ will denote a symmetric $(2,0)$-tensor on $\mathbb{S}^{d-1}$ satisfying for any $k \in \mathbb{N}$ the bound $\left|\left(\nabla^{\mathbb{S}^{d-1}}\right)^{k}(d \sigma d \sigma)\right|_{g_{\mathbb{S} d-1}} \leq 10^{k}$.

As an example, the above notation will allow us to perform the following integration by parts procedure on $\mathbb{S}^{d-1}$ for any function $f$ and any tensor $a$ with the aforementioned symmetries:

$$
\int_{\mathbb{S}^{d-1}} a \cdot \partial_{\sigma} f \cdot \partial_{\sigma} \partial_{\sigma} f d \sigma=-\frac{1}{2} \int_{\mathbb{S}^{d-1}}\left(\tilde{e}_{1} \partial_{\sigma} a+\tilde{e}_{2} a\right) \cdot \partial_{\sigma} f \cdot \partial_{\sigma} f d \sigma
$$

for some smooth contracting tensors $\tilde{e}_{1}, \tilde{e}_{2}$ which are bounded (in any $C^{k}$ norm) with bounds depending only on the tensor type of $a$.

We will frequently use the notation (2.3) and (2.4) in cases where we do not have an explicit form for the contracting tensor $a$, but we merely have bounds for the norm of $a$ and its derivatives. It is for this reason that we choose to use a notation which apparently loses information regarding the structure of the underlying expression.

Notice that in a polar coordinate chart $\left(u_{1}, u_{2}, \sigma\right): \mathcal{U} \rightarrow \mathbb{R}_{+} \times \mathbb{R}_{+} \times \mathbb{S}^{d-1}$, the following commutation relation holds for any function $h$ :

$$
\mathcal{L}_{\partial_{u_{i}}} \nabla^{\mathbb{S}^{d-1}} h=\nabla^{\mathbb{S}^{d-1}} \partial_{u_{i}} h
$$

where $\partial_{u_{i}}$ is the coordinate vector field associated to the coordinate function $u_{i}, i=$ 1,2 . Therefore, we will frequently denote

$$
\mathcal{L}_{\partial_{u_{i}}} \nabla^{\mathbb{S}^{d-1}} h \doteq \partial_{u_{i}} \partial_{\sigma} h
$$

and this will allow us to commute $\partial_{u_{i}}$ with $\partial_{\sigma}$, as if $\partial_{\sigma}$ was a regular coordinate vector field.

\subsection{Convention on the $O(\cdot)$ Notation}

For the conventions regarding the use the $O(\cdot)$ notation on asymptotically flat spacetimes in this paper, see the beginning Section 3. 


\subsection{Conventions on Integration}

When we integrate over open subsets of a Lorentzian manifold $(\mathcal{M}, g)$ using the natural volume form $\omega$ associated to $g$, we will often drop the volume form in the expression for the integral. Recall that $\omega$ is expressed as

$$
\omega=\sqrt{-\operatorname{det}(g)} d x^{0} \cdots d x^{d}
$$

in any local coordinate chart $\left(x^{0}, x^{1}, x^{2}, \ldots, x^{d}\right)$. The same rule will apply when integrating over any spacelike hypersurface $\mathcal{S}$ of $(\mathcal{M}, g)$ using the natural volume form of its induced Riemannian metric.

\subsection{Notations for Vector Field Multipliers and Currents}

Since we will use the language of currents and vector field multipliers in order to establish the desired estimates, let us briefly recall first the required notation: On any Lorentzian manifold $(\mathcal{M}, g)$, associated to the wave operator $\square_{g}=$ $\frac{1}{\sqrt{-\operatorname{det}(g)}} \partial_{\mu}\left(\sqrt{-\operatorname{det}(g)} \cdot g^{\mu \nu} \partial_{\nu}\right)$ is the energy momentum tensor $T$, which for any smooth function $\psi: \mathcal{M} \rightarrow \mathbb{C}$ takes the form

$$
T_{\mu \nu}(\psi)=\frac{1}{2}\left(\partial_{\mu} \psi \cdot \partial_{\nu} \bar{\psi}+\partial_{\mu} \bar{\psi} \cdot \partial_{\nu} \psi\right)-\frac{1}{2}\left(\partial^{\lambda} \psi \cdot \partial_{\lambda} \bar{\psi}\right) g_{\mu \nu}
$$

Given any continuous and piecewise $C^{1}$ vector field $X$ on $\mathcal{M}$, we can define almost everywhere the associated currents

$$
\begin{gathered}
J_{\mu}^{X}(\psi)=T_{\mu \nu}(\psi) X^{\mu}, \\
K^{X}(\psi)=T_{\mu \nu}(\psi) \nabla^{\mu} X^{\psi} .
\end{gathered}
$$

The following divergence identity then holds almost everywhere:

$$
\nabla^{\mu} J_{\mu}^{X}(\psi)=K^{X}(\psi)+\operatorname{Re}\left\{\left(\square_{g} \psi\right) \cdot X \bar{\psi}\right\}
$$

\section{Geometry of the Asymptotically Flat Regions $\left(\mathcal{N}_{a f}, g\right)$}

In Sections 4, 5 and 6, we will work in $d+1$ dimensional, smooth and time oriented Lorentzian manifolds $\left(\mathcal{N}_{a f}^{d+1}, g\right)$ diffeomorphic to $\mathbb{R} \times\left(\mathbb{R}^{d} \backslash B_{R}\right)$ for $d \geq 3$ (where $B_{R}$ is the closed Euclidian ball of radius $R$ ). The manifolds $\left(\mathcal{N}_{a f}, g\right)$ will serve as models of the asymptotically flat region of more general asymptotically flat spacetimes, and will appear as open subsets of the Lorentzian manifolds studied in Sections 7, 8 and 9. Let us notice that $\left(\mathcal{N}_{a f}, g\right)$ will not in general be globally hyperbolic.

We will fix a global coordinate chart $(t, r, \sigma): \mathcal{N}_{a f} \rightarrow \mathbb{R} \times\left(\mathbb{R}^{d} \backslash B_{R}\right)$, where $t \in \mathbb{R}$ is the projection to the first factor of $\mathbb{R} \times\left(\mathbb{R}^{d} \backslash B_{R}\right)$, and $(r, \sigma) \in\left(\mathbb{R}^{+}, \mathbb{S}^{d-1}\right)$ are the 
usual polar coordinates on $\mathbb{R}^{d} \backslash B_{R}$. Moreover, it will be useful for us to define the coordinate function $u=t-r$, and introduce the coordinate system $(u, r, \sigma)$.

In the $(u, r, \sigma)$ coordinate system, we will adopt the following notation for the derivatives of functions $h$ on $\mathcal{N}_{a f}$ : We will write

$$
h=O\left(r^{b}\right)
$$

for some $b \in \mathbb{R}$ if for any integer $k \geq 0$ we can bound:

$$
\sum_{k_{1}+k_{2}+k_{3}=k}\left|r^{k_{1}} \partial_{r}^{k_{1}} \partial_{\sigma}^{k_{2}} \partial_{u}^{k_{3}} h\right| \leq C_{k} \cdot r^{b}
$$

for some constant $C_{k}$ depending only on $k$ and the function $h$ itself. By replacing the coordinate derivatives in (3.2) with the connection derivatives in the associated directions, the same $O(\cdot)$ notation will apply when $h$ takes values in some vector bundle $\mathcal{E}$ over $\mathcal{N}_{a f}$ with a fixed Hermitian metric to measure the size of the norms in the left hand side of (3.2) and a fixed compatible connection. For instance, the case when $\mathcal{E}=\otimes^{n_{1}} T \mathbb{S}^{d-1} \otimes^{n_{2}} T^{*} \mathbb{S}^{d-1}$ (equipped with the natural metric and connection arising from the standard round $g_{\mathbb{S}^{d-1}}$ ) will appear in the text.

In the coordinate chart $(u, r, \sigma)$ on $\mathcal{N}_{a f}$, the Lorentzian metric $g$ will be assumed to take the following radiative form for some $0 \leq a \leq 1$ :

$$
\begin{aligned}
g= & -4\left(1-\frac{2 M(u, \sigma)}{r}+O\left(r^{-1-a}\right)\right) d u^{2}-\left(4+O\left(r^{-1-a}\right)\right) d u d r \\
& +r^{2} \cdot\left(g_{\mathbb{S}^{d-1}}+h_{\mathbb{S}^{d-1}}\right)\left(h_{3}^{a s}(u, \sigma)+O\left(r^{-a}\right)\right) d u d \sigma \\
& +O\left(r^{-a}\right) d r d \sigma+O\left(r^{-2-a}\right) d r^{2},
\end{aligned}
$$

where $M(u, \sigma)$ and $h_{3}^{a s}(u, \sigma)$ are real functions on $\mathbb{R} \times \mathbb{S}^{d-1}$ with all their derivatives uniformly bounded and $h_{\mathbb{S}^{d-1}}$ is a symmetric $(2,0)$-tensor on $\mathbb{S}^{d-1}$ satisfying the bound $h_{\mathbb{S}^{d-1}}=O\left(r^{-1}\right)$.

Notice that this class of metrics includes the Bondi radiating spacetimes (see i.e. $[6,33])$. Due to the form (3.3) of the metric, the vector field $\partial_{r}$ in the $(u, r, \sigma)$ coordinate system is almost null, but not necessarily null, and $u$ is not necessarily an optical function.

It will also be convenient to express the metric in an almost double null coordinate system $(u, v, \sigma)$, where $v=u+r$. We easily calculate from (3.3) that in the $(u, v, \sigma)$ coordinate system, the metric has the following form:

$$
\begin{aligned}
g= & -\left(4+O\left(r^{-1-a}\right)\right) d v d u+r^{2} \cdot\left(g_{\mathbb{S}^{d-1}}+h_{\mathbb{S}^{d-1}}\right)+\left(h^{a s}(u, \sigma)+O\left(r^{-a}\right)\right) d u d \sigma \\
& +O\left(r^{-a}\right) d v d \sigma+4\left(-\frac{2 M(u, \sigma)}{r}+O\left(r^{-1-a}\right)\right) d u^{2}+O\left(r^{-2-a}\right) d v^{2}
\end{aligned}
$$

where $h_{\mathbb{S}^{d-1}}=O\left(r^{-1}\right)$. 
Notice that differentiation with respect to $\partial_{v}$ in the $(u, v, \sigma)$ coordinate system is the same as differentiation with respect to $\partial_{r}$ in the $(u, r, \sigma)$ chart. Notice also that differentiation with respect to $\partial_{u}$ is not the same in the two coordinate systems.

Let us also define the function $t \doteq 2 u+r=u+v$, which has spacelike level sets, at least for $r \gg 1$. In the $(t, r, \sigma)$ coordinate system, we easily calculate from (3.3) that the metric $g$ has the following expression:

$$
\begin{aligned}
g= & -\left(1-\frac{2 M(u, \sigma)}{r}+O\left(r^{-1-a}\right)\right) \cdot d t^{2}-\left(\frac{4 M(u, \sigma)}{r}+O\left(r^{-1-a}\right)\right) d t d r \\
& +\left(1+\frac{2 M(u, \sigma)}{r}+O\left(r^{-1-a}\right)\right) d r^{2}+r^{2} \cdot\left(g_{\mathbb{S}^{d-1}}+O\left(r^{-1}\right)\right) \\
& +\left(-\frac{1}{2} h^{a s}(u, \sigma)+O\left(r^{-a}\right)\right)(d r-d t) d \sigma+O\left(r^{-a}\right) d t d \sigma .
\end{aligned}
$$

Notice that the vector field $T=\partial_{t}$ in the $(t, r, \sigma)$ coordinate chart will not necessarily be a Killing vector field for $\mathcal{N}_{a f}$, but it will certainly be timelike for $r \gg 1$. Notice also that in this coordinate system, differentiation with respect to either $\partial_{t}$ or $\partial_{r}$ does not improve the decay rate in $r$ of $O\left(r^{-c}\right)$ functions $\left(\partial_{r}\right.$ in this system is different than $\partial_{r}$ in the $(u, r, \sigma)$ chart $)$.

It is important to remark that if $R \gg 1$, in the region $\{r \geq R\}$ of $\mathcal{N}_{a f}$ we can estimate $d u d v d \sigma \sim r^{-(d-1)} \cdot d v o l_{g}$. In this region, we will also use the notation

$$
|\partial \varphi|^{2} \doteq\left|\partial_{v} \varphi\right|^{2}+\left|\partial_{u} \varphi\right|^{2}+\left|r^{-1} \partial_{\sigma} \varphi\right|^{2}
$$

for the "coordinate Euclidean norm" of the gradient of any differentiable function $\varphi: \mathcal{N}_{a f} \rightarrow \mathbb{C}$.

\subsection{Spacelike Hyperboloidal Hypersurfaces Terminating at $\mathcal{I}^{+}$}

It will be convenient to introduce a family of spacelike hypersurfaces "terminating at future null infinity" (in a sense that will be made precise shortly). These hypersurfaces will make easier the extraction of information regarding the radiating properties of solutions $\varphi$ to the wave equation $\square_{g} \varphi=0$ on $\left(\mathcal{N}_{a f}, g\right)$.

We first introduce the following definition:

Definition 3.1 We define the future null infinity $\mathcal{I}^{+}$of $\left(\mathcal{N}_{a f}, g\right)$ as the abstract limit of the hypersurfaces $\left\{v=v_{n}\right\}$ as $v_{n} \rightarrow+\infty$ in the $(u, v, \sigma)$ coordinate system. In particular, a function $\Psi$ on $\mathcal{I}^{+}$will always be defined as the $\operatorname{limit} \lim _{v \rightarrow+\infty} \tilde{\Psi}$ in the $(u, v, \sigma)$ coordinate system for some function $\tilde{\Psi}$ on $\mathcal{N}_{a f}$. We will also set $\mathcal{I}^{+}\left(\tau_{1}, \tau_{2}\right)=\mathcal{I}^{+} \cap\left\{\tau_{1} \leq u \leq t_{2}\right\}$, which is to be understood as the abstract limit of the hypersurfaces $\left\{v=v_{n}\right\} \cap\left\{\tau_{1} \leq u \leq \tau_{2}\right\}$ as $v_{n} \rightarrow+\infty$ in the $(u, v, \sigma)$ coordinate system.

We will now define the notion of a hypersurface terminating at $\mathcal{I}^{+}$: 
Definition 3.2 Let $\mathcal{S}$ be an achronal inextendible hypersurface of $\left(\mathcal{N}_{a f}, g\right)$. We will say that $\mathcal{S}$ terminates at future null infinity $\mathcal{I}^{+}$if the coordinate function $u$ restricted on $\mathcal{S} \cap\{r \gg 1\}$ is bounded.

Notice that due to the form (3.3) of the metric in the $(u, r, \sigma)$, and in particular due to the fact that in this coordinate system $g\left(\partial_{r}, \partial_{r}\right)=O\left(r^{-2-a}\right)$ and $g\left(\partial_{r}, \partial_{\sigma}\right)=O\left(r^{-a}\right)$, we can easily calculate that for any $R \gg 1$ and $\tau \in \mathbb{R}$ the boundary hypersurface $\partial J^{+}(\{r \leq R\} \cap\{t=\tau\})$ is an achronal hypersurface terminating at future null infinity.

We can now construct a function $\bar{t}: \mathcal{N}_{a f} \rightarrow \mathbb{R}$ with level sets that are spacelike hypersurfaces ${ }^{9}$ terminating at future null infinity. More precisely, we define for any fixed $0<\eta^{\prime}<1+a$

$$
\bar{t}_{\eta^{\prime}} \doteq u-\frac{1}{1+r^{\eta^{\prime}}} .
$$

The level sets of $\bar{t}_{\eta^{\prime}}$ are spacelike hypersurfaces for $r$ large enough depending on $\eta^{\prime}$. This follows from the computation:

$$
\begin{aligned}
g^{\mu \nu} \partial_{\mu} \bar{t}_{\eta^{\prime}} \cdot \partial_{\nu} \bar{t}_{\eta^{\prime}}= & g^{\mu \nu} \partial_{\mu} u \cdot \partial_{\nu} u+2 g^{\mu \nu} \partial_{\mu} u \cdot \partial_{\nu}\left(\frac{-1}{1+r^{\eta^{\prime}}}\right) \\
& +g^{\mu \nu} \partial_{\mu}\left(\frac{-1}{1+r^{\eta^{\prime}}}\right) \cdot \partial_{\nu}\left(\frac{-1}{1+r^{\eta^{\prime}}}\right) \\
= & -2 \eta^{\prime} r^{-1-\eta^{\prime}}+O_{\eta^{\prime}}\left(r^{-2-a}+r^{-2-\eta^{\prime}}\right)<0 .
\end{aligned}
$$

for $r$ large enough in terms of $\eta^{\prime}$.

Moreover, $\left|\bar{t}_{\eta^{\prime}}-u\right| \leq 1$, and hence the level sets of $\bar{t}_{\eta^{\prime}}$ are spacelike hypersurfaces terminating at future null infinity, according to the definition 3.2.

We will frequently simply write $\bar{t}$ in place of $\bar{t}_{\eta^{\prime}}$, since $\eta^{\prime}$ will be considered fixed throughout the next Sections.

For any $\tau \in \mathbb{R}$ and $\tau_{1} \leq \tau_{2} \in \mathbb{R}$, we will denote

$$
\mathcal{S}_{\tau} \doteq\{\bar{t}=\tau\} \cap\left\{r \geq R_{3}\right\}
$$

and

$$
\mathcal{R}\left(\tau_{1}, \tau_{2}\right) \doteq\left\{\tau_{1} \leq \bar{t} \leq \tau_{2}\right\} \cap\left\{r \geq R_{3}\right\},
$$

where $R_{3}=R_{3}\left(\eta^{\prime}\right)$ is fixed large enough so that in the region $\left\{r \geq R_{3}\right\}$ the level sets of $\bar{t}_{\eta^{\prime}}$ are spacelike.

Due to the definition of $\bar{t}_{\eta^{\prime}}$, the image of $\mathcal{S}_{\tau_{1}}$ under the flow of the vector field $T=\partial_{t}$ in the $(t, r, \sigma)$ coordinate system for time $\tau$ is precisely $\mathcal{S}_{\tau_{1}+\tau}$.

Note that the $\mathcal{S}_{\tau}$ can be regularly parametrized both by $(u, \sigma)$ and by $(v, \sigma)$, and the corresponding coordinate volume forms $d u d \sigma$ and $d v d \sigma$ satisfy the relation

$$
d u d \sigma \sim_{\eta^{\prime}} r^{-1-\eta^{\prime}} d v d \sigma .
$$

9 At least for $r \gg 1$. 


\subsection{Expression of the Wave Equation in the Coordinate Chart $(u, v, \sigma)$}

In the coordinate chart $(u, v, \sigma)$ on $\mathcal{N}_{a f}$, the metric $g$ takes the form (3.4), and we can easily compute that in these coordinates:

$$
\operatorname{det}(g)=-4 r^{2(d-1)} \cdot\left(1+O\left(r^{-1}\right)\right)
$$

and the inverse of the metric has the following form:

$$
\begin{aligned}
g^{-1}= & -\left(1+O\left(r^{-1-a}\right)\right) \partial_{u} \partial_{v}+r^{-2}\left(g_{\mathbb{S}^{d-1}}^{-1}-h_{\mathbb{S}^{d-1}}^{i n v}\right) \\
& +r^{-2}\left(\frac{1}{2} h^{a s}(u, \sigma)+O\left(r^{-a}\right)\right) \partial_{v} \partial_{\sigma} \\
& +O\left(r^{-2-a}\right) \partial_{u} \partial_{\sigma}+\left(-\frac{2 M(u, \sigma)}{r}+O\left(r^{-1-a}\right)\right) \partial_{v}^{2}+O\left(r^{-2-a}\right) \partial_{u}^{2},
\end{aligned}
$$

where $h_{\mathbb{S}^{d-1}}^{i n v}=O\left(r^{-1}\right)$.

Setting

$$
\Omega \doteq\left(-\frac{1}{4} \operatorname{det}(g)\right)^{\frac{1}{4}}=r^{\frac{d-1}{2}}\left(1+O\left(r^{-1}\right)\right)
$$

the wave operator then takes the following form:

$$
\begin{aligned}
\Omega \cdot \square \varphi= & -\left(1+O\left(r^{-1-a}\right)\right) \cdot \partial_{u} \partial_{v}(\Omega \varphi)+r^{-2} \Delta_{g_{\mathbb{S} d-1}+h_{\mathbb{S}^{d}-1}}(\Omega \varphi) \\
& -\frac{(d-1)(d-3)}{4} r^{-2} \cdot(\Omega \varphi)+\operatorname{Err}(\Omega \varphi),
\end{aligned}
$$

where the $\operatorname{Err}(\Phi)$ term is of the form (in our schematic notation of Section 2):

$$
\begin{aligned}
\operatorname{Err}(\Phi)= & O\left(r^{-2-a}\right) \cdot \partial_{u}^{2} \Phi+O\left(r^{-1}\right) \cdot \partial_{v}^{2} \Phi+O\left(r^{-2-a}\right) \partial_{u} \partial_{\sigma} \Phi+O\left(r^{-2}\right) \partial_{v} \partial_{\sigma} \Phi \\
& +O\left(r^{-3-a}\right) \partial_{\sigma} \partial_{\sigma} \Phi+O\left(r^{-2-a}\right) \partial_{u} \Phi+O\left(r^{-1-a}\right) \partial_{v} \Phi \\
& +O\left(r^{-2-a}\right) \cdot \partial_{\sigma} \Phi+O\left(r^{-3}\right) \Phi
\end{aligned}
$$

Notice the similarity of the expression (3.14) with the expression of the wave operator in the double null coordinates of Minkowski spacetime.

\section{Some $\partial_{r}$ - Morawetz Type and Energy Boundedness Estimates}

In this section, we will establish some estimates of Morawetz type, controlling the behaviour of solutions to $\square \varphi=F$ on our asymptotically flat model $\left(\mathcal{N}_{a f}, g\right)$.

Recall that in the $(u, v, \sigma)$ coordinate system, the wave equation takes the form (3.14): 


$$
\begin{aligned}
\Omega \cdot \square \varphi= & -\left(1+O\left(r^{-1-a}\right)\right) \cdot \partial_{u} \partial_{v}(\Omega \varphi)+r^{-2} \Delta_{g_{\mathbb{S} d-1}+h_{\mathbb{S} d-1}}(\Omega \varphi) \\
& -\frac{(d-1)(d-3)}{4} r^{-2} \cdot(\Omega \varphi)+\operatorname{Err}(\Omega \varphi),
\end{aligned}
$$

where

$$
\Omega=r^{\frac{d-1}{2}}\left(1+O\left(r^{-1}\right)\right)
$$

and the $\operatorname{Err}(\Phi)$ term is of the form:

$$
\begin{aligned}
\operatorname{Err}(\Phi)= & O\left(r^{-2-a}\right) \cdot \partial_{u}^{2} \Phi+O\left(r^{-1}\right) \cdot \partial_{v}^{2} \Phi+O\left(r^{-2-a}\right) \partial_{u} \partial_{\sigma} \Phi \\
& +O\left(r^{-2}\right) \partial_{v} \partial_{\sigma} \Phi+O\left(r^{-3-a}\right) \partial_{\sigma} \partial_{\sigma} \Phi+O\left(r^{-2-a}\right) \partial_{u} \Phi \\
& +O\left(r^{-1-a}\right) \partial_{v} \Phi+O\left(r^{-2-a}\right) \cdot \partial_{\sigma} \Phi+O\left(r^{-3}\right) \Phi .
\end{aligned}
$$

We will also adopt the following convention: We will say that a function $\varphi: N_{a f} \rightarrow$ $\mathbb{C}$ has compact support in space if there exists a continuous function $h: \mathbb{R} \rightarrow \mathbb{R}_{+}$ such that $\operatorname{supp}(\varphi) \subseteq\{r \leq h(t)\}$.

\subsection{A First $\partial_{r}$ - Morawetz Type Estimate}

We will establish the following Morawetz-type lemma in the region $\{r \gg 1\}$ :

Lemma 4.1 For any given $0<\eta<a$, there exists an $R=R(\eta)>0$ and constants $C(\eta), c(\eta)>0$ such that for any smooth function $\varphi: \mathcal{N}_{a f} \rightarrow \mathbb{C}$, any $\tau_{1} \leq \tau_{2}$, any $T^{*}>0$ and any smooth cut-off function $\chi: \mathcal{N}_{a f} \rightarrow[0,1]$ supported in $\{r \geq R\}$ we can bound:

$$
\begin{aligned}
& \int_{\mathcal{R}\left(\tau_{1}, \tau_{2}\right) \cap\left\{t \leq T^{*}\right\}} \chi \cdot\left(r^{-1-\eta}\left(\left|\partial_{u} \varphi\right|^{2}+\left|\partial_{v} \varphi\right|^{2}\right)+r^{-1}\left|r^{-1} \partial_{\sigma} \varphi\right|^{2}+r^{-3-\eta}|\varphi|^{2}\right) \\
& \leq C(\eta) \cdot \int_{\{\operatorname{supp}(\partial \chi)\} \cap \mathcal{R}\left(\tau_{1}, \tau_{2}\right) \cap\left\{t \leq T^{*}\right\}}|\partial \chi| \cdot\left(|\partial \varphi|^{2}+r^{-2}|\varphi|^{2}\right) \\
& \quad+C(\eta) \cdot \sum_{i=1}^{2} \int_{\mathcal{S}_{\tau_{i}} \cap\left\{t \leq T^{*}\right\}} \chi\left(\left|\partial_{v} \varphi\right|^{2}+\left|r^{-1} \partial_{\sigma} \varphi\right|^{2}\right. \\
& \left.+r^{-1-\eta^{\prime}}\left|\partial_{u} \varphi\right|^{2}+r^{-2}|\varphi|^{2}\right) r^{d-1} d v d \sigma \\
& +C(\eta) \cdot \int_{\left\{t=T^{*}\right\} \cap \mathcal{R}\left(\tau_{1}, \tau_{2}\right)} \chi\left(|\partial \varphi|^{2}+r^{-2}|\varphi|^{2}\right) r^{d-1} d v d \sigma \\
& +\int_{\mathcal{R}\left(\tau_{1}, \tau_{2}\right) \cap\left\{t \leq T^{*}\right\}} \chi \cdot \operatorname{Re}\left\{\left(O(1)\left(\partial_{v}-\partial_{u}\right) \bar{\varphi}+O\left(r^{-1}\right) \bar{\varphi}\right) \cdot \square_{g} \varphi\right\} \\
& +C(\eta) \int_{\mathcal{R}\left(\tau_{1}, \tau_{2}\right) \cap\left\{t \leq T^{*}\right\}} \chi \cdot r^{-1}\left(\left|\partial_{v} \varphi\right|^{2}+\left|r^{-1} \partial_{\sigma} \varphi\right|^{2}+r^{-2}|\varphi|^{2}\right)
\end{aligned}
$$

Remark In case the radiative components of the metric satisfy the bounds $\partial_{u} M \leq 0$ and $\left|\partial_{u} h_{a s}\right|+\left|r \partial_{u} h_{\mathbb{S}^{d-1}}\right| \ll-\left(\partial_{u} M\right)+O\left(r^{-a}\right)$ (which includes the non-radiating 
case $\partial_{u} M=0, h_{a s}=0$ and $\left.h_{\mathbb{S}^{d-1}}=O\left(r^{-1-a}\right)\right)$, the last term of the right hand side of (4.4) can be omitted. Furthermore, in case the $T$ vector field satisfies (9.6) for $m=1$, then the last term of the right hand side of (4.13) is replaced by

$$
\int_{\mathcal{R}\left(\tau_{1}, \tau_{2}\right) \cap\left\{t \leq T^{*}\right\}} \chi \cdot \bar{t}^{-\delta 0} r^{-1}\left(\left|\partial_{v} \varphi\right|^{2}+\left|r^{-1} \partial_{\sigma} \varphi\right|^{2}+r^{-2}|\varphi|^{2}\right) .
$$

Proof Without loss of generality, we can assume that $\varphi$ is real valued.

Let us consider the function $f:[0,+\infty) \rightarrow(0,+\infty)$ defined as:

$$
f(r) \doteq \frac{r^{\eta}}{1+r^{\eta}}
$$

Setting $\Phi=\Omega \varphi$ and multiplying equation (4.1) with

$$
\chi \cdot f(r) \cdot\left(\partial_{v}-\partial_{u}\right) \Phi,
$$

we obtain after integrating over $\mathcal{R}\left(\tau_{1}, \tau_{2}\right) \cap\left\{t \leq T^{*}\right\}$ (with $d u d v d \sigma$ used as a volume form):

$$
\begin{aligned}
& \int_{\mathcal{R}\left(\tau_{1}, \tau_{2}\right) \cap\left\{t \leq T^{*}\right\}} \chi f(r) \cdot\left(\partial_{v}-\partial_{u}\right) \Phi \cdot \Omega \square \varphi d u d v d \sigma \\
& =\int_{\mathcal{R}\left(\tau_{1}, \tau_{2}\right) \cap\left\{t \leq T^{*}\right\}} \chi f(r) \cdot\left(\partial_{v}-\partial_{u}\right) \Phi \\
& \quad \cdot\left\{-\left(1+O_{1}\left(r^{-1-a}\right)\right) \cdot \partial_{u} \partial_{v} \Phi+r^{-2} \Delta_{g_{\mathbb{S} d-1}+h_{\mathbb{S} d-1}} \Phi\right. \\
& \left.\quad-\frac{(d-1)(d-3)}{4} r^{-2} \cdot \Phi+\operatorname{Err}(\Phi)\right\} d u d v d \sigma .
\end{aligned}
$$

Using the expression

$$
\Delta_{g_{\mathbb{S}} d-1}+h_{\mathbb{S}^{d-1}}=\Delta_{g_{\mathbb{S}^{d-1}}}+O\left(r^{-1}\right) \partial_{\sigma} \partial_{\sigma}+O\left(r^{-1}\right) \partial_{\sigma},
$$

we obtain after integrating by parts in $\partial_{u}, \partial_{v}$ and $\partial_{\sigma}$ and absorbing the error terms in the $E r r$ summand (and recalling that $\partial_{v} r=-\partial_{u} r=1$ ):

$$
\begin{aligned}
& -\int_{\mathcal{R}\left(\tau_{1}, \tau_{2}\right) \cap\left\{t \leq T^{*}\right\}} \chi f(r) \cdot\left(\partial_{v}-\partial_{u}\right) \Phi \cdot \Omega \square \varphi d u d v d \sigma \\
& =\int_{\mathcal{R}\left(\tau_{1}, \tau_{2}\right) \cap\left\{t \leq T^{*}\right\}} \chi \cdot \frac{1}{2}\left\{\left(f^{\prime}+O\left(r^{-1-a}\right) f\right)\left|\partial_{v} \Phi\right|^{2}+\left(f^{\prime}+O\left(r^{-1-a}\right) f\right)\left|\partial_{u} \Phi\right|^{2}\right. \\
& \quad+2\left(2 r^{-1}\left(1+O\left(r^{-1}\right)\right) f-\left(1+O\left(r^{-a}\right)\right) f^{\prime}\right) \\
& \left.\quad \times\left(\left|r^{-1} \partial_{\sigma} \Phi\right|^{2}+\frac{(d-1)(d-3)}{4}\left|r^{-1} \Phi\right|^{2}\right)\right\} d u d v d \sigma \\
& \quad+\int_{\mathcal{R}\left(\tau_{1}, \tau_{2}\right) \cap\left\{t \leq T^{*}\right\}} \chi f \cdot\left(\partial_{v}-\partial_{u}\right) \Phi \cdot\left(\operatorname{Err}(\Phi)+O\left(r^{-3}\right) \partial_{\sigma} \partial_{\sigma} \Phi\right) d u d v d \sigma
\end{aligned}
$$




$$
\begin{aligned}
& +\int_{\mathcal{R}\left(\tau_{1}, \tau_{2}\right) \cap\left\{t \leq T^{*}\right\}} O(|\partial \chi|) \cdot\left(|\partial \Phi|^{2}+r^{-2}|\Phi|^{2}\right) d u d v d \sigma+\sum_{i=1}^{2} \int_{\mathcal{S}_{\tau_{1}} \cap\left\{t \leq T^{*}\right\}} \chi \\
& \cdot O(1)\left(\left|\partial_{v} \Phi\right|^{2}+\left|r^{-1} \partial_{\sigma} \Phi\right|^{2}+r^{-1-\eta^{\prime}}\left|\partial_{u} \Phi\right|^{2}+r^{-2}|\Phi|^{2}\right) d v d \sigma \\
& +\int_{\mathcal{R}\left(\tau_{1}, \tau_{2}\right) \cap\left\{t=T^{*}\right\} \cap\{r \geq R\}} O(1)\left(|\partial \Phi|^{2}+r^{-2}|\Phi|^{2}\right) d v d \sigma
\end{aligned}
$$

Since $\Phi=\Omega \varphi$, we calculate by applying the product rule and expanding the square:

$$
\begin{aligned}
& \int_{\mathcal{R}\left(\tau_{1}, \tau_{2}\right) \cap\left\{t \leq T^{*}\right\}} \chi \cdot \frac{1}{2}\left(f^{\prime}+O\left(r^{-1-a}\right) f\right)\left|\partial_{v} \Phi\right|^{2} d u d v d \sigma \\
& =\int_{\mathcal{R}\left(\tau_{1}, \tau_{2}\right) \cap\left\{t \leq T^{*}\right\}} \chi \cdot \frac{1}{2}\left(f^{\prime}+O\left(r^{-1-a}\right) f\right)\left(\left(\partial_{v} \varphi\right)^{2}+r^{-2}\left(\frac{(d-1)^{2}}{4}\right.\right. \\
& \left.\left.+O\left(r^{-1}\right)\right) \varphi^{2}+r^{-1}\left(\frac{d-1}{2}+O\left(r^{-1}\right)\right) \partial_{v}\left(\varphi^{2}\right)\right) \Omega^{2} d u d v d \sigma .
\end{aligned}
$$

By integrating by parts in the $\partial_{v}\left(\varphi^{2}\right)$ term, since for all $x>0$ :

$$
-\frac{d}{d x}\left(x^{d-2} \frac{d f}{d x}\right)+\frac{d-1}{2} x^{d-3} \frac{d f}{d x} \geq c_{\eta} x^{d-4+\eta}\left(1+x^{\eta}\right)^{-2}-C_{\eta}(d-3) x^{d-4} f,
$$

we obtain for any $\delta_{0} \leq 1$ due to the form of $f$ if $R$ is large enough in terms of $\eta$ :

$$
\begin{aligned}
& \int_{\mathcal{R}\left(\tau_{1}, \tau_{2}\right) \cap\left\{t \leq T^{*}\right\}} \chi \cdot \frac{1}{2}\left(f^{\prime}+O\left(r^{-1-a}\right) f\right)\left|\partial_{v} \Phi\right|^{2} d u d v d \sigma \\
& \geq \delta_{0}\left(c_{\eta} \int_{\mathcal{R}\left(\tau_{1}, \tau_{2}\right) \cap\left\{t \leq T^{*}\right\}} \chi \cdot r^{-1-\eta}\left(\left(\partial_{v} \varphi\right)^{2}+r^{-2} \varphi^{2}\right) \Omega^{2} d u d v d \sigma\right. \\
& \quad-C_{\eta}(d-3) \int_{\mathcal{R}\left(\tau_{1}, \tau_{2}\right) \cap\left\{t \leq T^{*}\right\}} \chi \cdot r^{-3}|\Phi|^{2} d u d v d \sigma \\
& \quad-C \int_{\mathcal{R}\left(\tau_{1}, \tau_{2}\right) \cap\left\{t \leq T^{*}\right\}}|\partial \chi| \cdot r^{-2}|\Phi|^{2} d u d v d \sigma \\
& \left.\quad-C \sum_{i=1}^{2} \int_{\mathcal{S}_{\tau_{i}} \cap\left\{t \leq T^{*}\right\}} \chi \cdot r^{-2}|\Phi|^{2} d v d \sigma-C \int_{\mathcal{R}\left(\tau_{1}, \tau_{2}\right) \cap\left\{t=T^{*}\right\}} \chi \cdot r^{-2}|\Phi|^{2} d v d \sigma\right) .
\end{aligned}
$$

Notice that the $(d-3) \int \chi \cdot r^{-3}|\Phi|^{2}$ error term in the right hand side (4.11) can be controlled by the corresponding term in the right hand side of (4.8), provided that $\delta_{0}$ is small enough in terms of $\eta$.

Using, now the expression (4.3) for $\operatorname{Err}(\Phi)$, we can readily bound after integrating by parts in the highest order terms (and in the $\Phi \partial_{u} \Phi=\frac{1}{2} \partial_{u}\left(\Phi^{2}\right)$ term) and using a Cauchy-Schwarz inequality: 


$$
\begin{aligned}
& \int_{\mathcal{R}\left(\tau_{1}, \tau_{2}\right) \cap\left\{t \leq T^{*}\right\}} \chi f(r) \cdot\left(\partial_{v}-\partial_{u}\right) \Phi \cdot\left(\operatorname{Err}(\Phi)+O\left(r^{-3}\right) \partial_{\sigma}^{2} \Phi\right) d u d v d \sigma \\
& \leq C_{\eta} \int_{\mathcal{R}\left(\tau_{1}, \tau_{2}\right) \cap\left\{t \leq T^{*}\right\}} \chi\left\{O\left(r^{-1-a}\right)\left|\partial_{u} \Phi\right|^{2}\right. \\
& \left.\quad+O\left(r^{-1}\right)\left(\left|\partial_{v} \Phi\right|^{2}+\left|r^{-1} \partial_{\sigma} \Phi\right|^{2}+r^{-2}|\Phi|^{2}\right)\right\} d u d v d \sigma \\
& \quad+C_{\eta} \int_{\mathcal{R}\left(\tau_{1}, \tau_{2}\right) \cap\left\{t \leq T^{*}\right\}}|\partial \chi|\left(|\partial \Phi|^{2}+r^{-2}|\Phi|^{2}\right) d u d v d \sigma+C_{\eta} \sum_{i=1}^{2} \int_{\mathcal{S}_{\tau_{1}} \cap\left\{t \leq T^{*}\right\}} \chi \\
& \quad O(1)\left(\left|\partial_{v} \Phi\right|^{2}+\left|r^{-1} \partial_{\sigma} \Phi\right|^{2}+r^{-1-\eta^{\prime}}\left|\partial_{u} \Phi\right|^{2}+r^{-2}|\Phi|^{2}\right) d v d \sigma \\
& \quad+C_{\eta} \int_{\mathcal{R}\left(\tau_{1}, \tau_{2}\right) \cap\left\{t=T^{*}\right\}} \chi \cdot\left(|\partial \Phi|^{2}+r^{-2}|\Phi|^{2}\right) d v d \sigma .
\end{aligned}
$$

Therefore, from (4.8), (4.11) and (4.12) for $\delta_{0}$ small enough in terms of $\eta$ we obtain the desired result (4.29) if $R$ is large enough in terms of $\eta$.

We can also establish the following variant of Lemma 4.1 in the region bounded by a pair of $\{t=$ const $\}$ hypersurfaces:

Lemma 4.2 For any given $0<\eta<a$, there exists an $R=R(\eta)>0$ and constants $C(\eta), c(\eta)>0$ such that for any smooth function $\varphi: \mathcal{N}_{a f} \rightarrow \mathbb{C}$ with compact support in space, any $t_{1} \leq t_{2}$ and any smooth cut-off function $\chi: \mathcal{N}_{a f} \rightarrow[0,1]$ supported in $\{r \geq R\}$ we can bound:

$$
\begin{aligned}
& \int_{\left\{t_{1} \leq t \leq t_{2}\right\}} \chi \cdot\left(r^{-1-\eta}\left(\left|\partial_{u} \varphi\right|^{2}+\left|\partial_{v} \varphi\right|^{2}\right)+r^{-1}\left|r^{-1} \partial_{\sigma} \varphi\right|^{2}+r^{-3-\eta}|\varphi|^{2}\right) \\
& \leq C_{\eta} \int_{\left\{t_{1} \leq t \leq t_{2}\right\}}|\partial \chi| \cdot\left(|\partial \varphi|^{2}+r^{-2}|\varphi|^{2}\right)+C_{\eta} \sum_{i=1}^{2} \int_{\left\{t=t_{i}\right\} \cap\{r \geq R\}} J_{\mu}^{T}(\varphi) n^{\mu} \\
& \quad+\int_{\left\{t_{1} \leq t \leq t_{2}\right\}} \chi \cdot \operatorname{Re}\left\{\left(O_{\eta}(1)\left(\partial_{v}-\partial_{u}\right) \bar{\varphi}+O_{\eta}\left(r^{-1}\right) \bar{\varphi}\right) \cdot \square_{g} \varphi\right\} \\
& \quad+C_{\eta} \int_{\left\{t_{1} \leq t \leq t_{2}\right\}} \chi \cdot r^{-1}\left(\left|\partial_{v} \varphi\right|^{2}+\left|r^{-1} \partial_{\sigma} \varphi\right|^{2}+r^{-2}|\varphi|^{2}\right)
\end{aligned}
$$

Remark In case the radiative components of the metric satisfy the bounds $\partial_{u} M \leq 0$ and $\left|\partial_{u} h_{a s}\right|+\left|r \partial_{u} h_{\mathbb{S}^{d}-1}\right| \ll-\left(\partial_{u} M\right)+O\left(r^{-a}\right)$ (which includes the non-radiative case $\partial_{u} M=0, h_{a s}=0$ and $\left.h_{\mathbb{S}^{d-1}}=O\left(r^{-1-a}\right)\right)$, the last term of the right hand side of (4.13) can be omitted.

The proof of this lemma is identical to the one for Lemma 4.1 (the only difference being the domain of $\mathcal{N}_{a f}$ over which integrations by parts take place, and an application of a Hardy type inequality for the boundary terms at $\left.t=t_{1}, t_{2}\right)$. Hence, the proof will be omitted. 


\subsection{An Improved $\partial_{r}$ - Morawetz Type Estimate}

By a more careful choice of the function $f$ used in the proof of Lemma 4.1, we can obtain improved control of the spacetime integral of $|\nabla \varphi|^{2},|\varphi|^{2}$ over any given compact subset of $\mathcal{R}\left(\tau_{1}, \tau_{2}\right) \cap\{r \geq R\}$, at the expense of having to introduce a larger constant in the dependence on the initial energy of $\varphi$, but without such a loss in the $r \sim R$ boundary terms. A related microlocal construction in the case of the subextremal Kerr family can be seen in [16].

Lemma 4.3 For any given $0<\eta<a$, and any $R>0$ sufficiently large in terms of $\eta$, any $R_{c} \geq R$, any $\tau_{1} \leq \tau_{2}$, any $T^{*}>0$, any function $\chi: \mathcal{N}_{a f} \rightarrow[0,1]$ supported in $\{r \geq R\}$ with $\partial \chi$ supported in $\left\{R \leq r \leq R_{c}\right\}$ and any smooth function $\varphi: \mathcal{N}_{a f} \rightarrow \mathbb{C}$ we can bound:

$$
\begin{aligned}
& \int_{\mathcal{R}\left(\tau_{1}, \tau_{2}\right) \cap\left\{t \leq T^{*}\right\} \cap\left\{r \leq R_{c}\right\}} \chi \cdot\left(\left(\left|\partial_{u} \varphi\right|^{2}+\left|\partial_{v} \varphi\right|^{2}\right)+\left|r^{-1} \partial_{\sigma} \varphi\right|^{2}+r^{-2}|\varphi|^{2}\right) \\
& +R_{c} \int_{\mathcal{R}\left(\tau_{1}, \tau_{2}\right) \cap\left\{t \leq T^{*}\right\} \cap\left\{r \geq R_{c}\right\}} \chi \cdot\left(r^{-1-\eta}\left(\left|\partial_{u} \varphi\right|^{2}+\left|\partial_{v} \varphi\right|^{2}\right)\right. \\
& \left.+r^{-1}\left|r^{-1} \partial_{\sigma} \varphi\right|^{2}+r^{-3-\eta}|\varphi|^{2}\right) \\
& \leq C(\eta) \cdot \int_{\mathcal{R}\left(\tau_{1}, \tau_{2}\right) \cap\left\{t \leq T^{*}\right\}}|\partial \chi| \cdot r \cdot\left(|\partial \varphi|^{2}+r^{-2}|\varphi|^{2}\right)+C\left(\eta, R_{c}\right) \\
& \cdot \sum_{i=1}^{2} \int_{\mathcal{S}_{\tau_{i}} \cap\left\{t \leq T^{*}\right\}} \chi\left(\left|\partial_{v} \varphi\right|^{2}+\left|r^{-1} \partial_{\sigma} \varphi\right|^{2}+r^{-1-\eta^{\prime}}\left|\partial_{u} \varphi\right|^{2}+r^{-2}|\varphi|^{2}\right) r^{d-1} d v d \sigma \\
& +C\left(\eta, R_{c}\right) \cdot \int_{\left\{t=T^{*}\right\} \cap \mathcal{R}\left(\tau_{1}, \tau_{2}\right)} \chi\left(|\partial \varphi|^{2}+r^{-2}|\varphi|^{2}\right) r^{d-1} d v d \sigma \\
& +\int_{\mathcal{R}\left(\tau_{1}, \tau_{2}\right) \cap\left\{t \leq T^{*}\right\}} \chi \cdot \operatorname{Re}\left\{\left(O_{R_{c}, \eta}(1)\left(\partial_{v}-\partial_{u}\right) \bar{\varphi}+O_{R_{c}, \eta}\left(r^{-1}\right) \bar{\varphi}\right) \cdot \square_{g} \varphi\right\} \\
& +C(\eta) R_{c} \cdot \int_{\mathcal{R}\left(\tau_{1}, \tau_{2}\right) \cap\left\{t \leq T^{*}\right\}} \chi \cdot r^{-1}\left(\left|\partial_{v} \varphi\right|^{2}+\left|r^{-1} \partial_{\sigma} \varphi\right|^{2}+r^{-2}|\varphi|^{2}\right) \text {. }
\end{aligned}
$$

Remark In case the radiative components of the metric satisfy the bounds $\partial_{u} M \leq 0$ and $\left|\partial_{u} h_{a s}\right|+\left|r \partial_{u} h_{\mathbb{S} d-1}\right| \ll-\left(\partial_{u} M\right)+O\left(r^{-a}\right)$ (which includes the non-radiative case $\partial_{u} M=0, h_{a s}=0$ and $\left.h_{\mathbb{S}^{d-1}}=O\left(r^{-1-a}\right)\right)$, the last term of the right hand side of (4.3) can be omitted. Furthermore, in case the $T$ vector field satisfies (9.6) for $m=1$, then the last term of the right hand side of (4.14) is replaced by

$$
\int_{\mathcal{R}\left(\tau_{1}, \tau_{2}\right) \cap\left\{t \leq T^{*}\right\}} \chi \cdot \bar{t}^{-\delta 0} r^{-1}\left(\left|\partial_{v} \varphi\right|^{2}+\left|r^{-1} \partial_{\sigma} \varphi\right|^{2}+r^{-2}|\varphi|^{2}\right) .
$$

Note that the constant in front of the boundary term in the $r \sim R$ region does not depend on $R_{c}$. This is where the importance of this lemma lies, and this is where Lemma (4.1) would fail to give a similar statement.

Proof Without loss of generality, we will assume that $\varphi$ is real valued. 
Let us consider the smooth function $f_{R_{c}}: \mathcal{N}_{a f} \rightarrow(0,+\infty)$ defined as $f_{R_{c}}=$ $R_{c} g \circ\left(\frac{r}{R_{c}}\right)$, where $g:(0,+\infty) \rightarrow(0,+\infty)$ is a smooth, increasing and concave function satisfying:

$$
g(x)= \begin{cases}x, & x \leq 1 \\ 2, & x \geq 2\end{cases}
$$

We will follow the proof of Lemma 4.1, but we will use as a multiplier for equation (4.1) the function

$$
f_{i m p}=f \cdot f_{R_{c}},
$$

where

$$
f=\frac{r^{\eta}}{1+r^{\eta}}
$$

is the seed function used in the proof of Lemma 4.1.

Notice that for $f_{i m p}$ we can calculate $\left(\right.$ since $\left.\Omega^{2}=r^{d-1}\left(1+O\left(r^{-1}\right)\right)\right)$ :

$$
\partial_{v} f_{i m p}=-\partial_{u} f_{i m p}=\left(1+O\left(r^{-\eta}\right)\right) \cdot g^{\prime}\left(\frac{r}{R_{c}}\right)+\eta r^{-1-\eta} R_{c}\left(1+O\left(r^{-1}\right)\right) \cdot g\left(\frac{r}{R_{c}}\right)
$$

and:

$$
\begin{aligned}
\partial_{v}\left(r^{-1} \Omega^{2} \partial_{u} f_{i m p}\right)= & -r^{d-2} R_{c}^{-1}\left(1+O\left(r^{-1}\right)\right) \cdot g^{\prime \prime}\left(\frac{r}{R_{c}}\right) \\
& -\left((d-2) r^{d-3}+2 \eta r^{d-3-\eta}\right) \cdot\left(1+O\left(r^{-1}\right)\right) \cdot g^{\prime}\left(\frac{r}{R_{c}}\right) \\
& +\eta(\eta-(d-3)) r^{d-4-\eta} R_{c}\left(1+O\left(r^{-1}\right)\right) \cdot g\left(\frac{r}{R_{c}}\right) .
\end{aligned}
$$

Therefore, since $g$ was assumed to be increasing and concave and satisfies (4.16), we can bound:

$$
\begin{aligned}
& \partial_{v}\left(r^{-1} \Omega^{2} \partial_{u} f_{i m p}\right)-r^{-2} \Omega^{2} \partial_{u} f_{i m p} \\
& \quad \geq \begin{cases}\left(-(d-3) r^{-2}+\eta r^{-2-\eta}+O\left(r^{-3}\right)\right) \Omega^{2}, & r \leq R_{c} \\
R_{c}\left(-(d-3) r^{-3}+\eta^{2} r^{-3-\eta}+O\left(r^{-4}\right)\right) \Omega^{2}, & r \geq R_{c} .\end{cases}
\end{aligned}
$$

Repeating the proof of Lemma 4.1, setting $\Phi=\Omega \varphi$ and multiplying equation (4.1) with $\chi \cdot f_{\text {imp }} \cdot\left(\partial_{v}-\partial_{u}\right) \Phi$, we obtain after integrating over $\mathcal{R}\left(\tau_{1}, \tau_{2}\right) \cap\left\{t \leq T^{*}\right\}$ (with $d u d v d \sigma$ used as a volume form) and integrating by parts in $\partial_{u}, \partial_{v}$ and $\partial_{\sigma}$ :

$$
\begin{aligned}
& -\int_{\mathcal{R}\left(\tau_{1}, \tau_{2}\right) \cap\left\{t \leq T^{*}\right\}} \chi f_{i m p} \cdot\left(\partial_{v}-\partial_{u}\right) \Phi \cdot \Omega \square \varphi d u d v d \sigma \\
& =\int_{\mathcal{R}\left(\tau_{1}, \tau_{2}\right) \cap\left\{t \leq T^{*}\right\}} \chi \cdot \frac{1}{2}\left\{\left(-\partial_{u} f_{i m p}+O\left(r^{-1-a}\right) f_{i m p}\right)\left|\partial_{v} \Phi\right|^{2}\right.
\end{aligned}
$$




$$
\begin{aligned}
& +\left(\partial_{v} f_{i m p}+O\left(r^{-1-a}\right) f_{i m p}\right)\left|\partial_{u} \Phi\right|^{2} \\
& \left.+\mathcal{A}_{i m p}\left(\left|r^{-1} \partial_{\sigma} \Phi\right|^{2}+\frac{(d-1)(d-3)}{4}\left|r^{-1} \Phi\right|^{2}\right)\right\} d u d v d \sigma \\
& +\int_{\mathcal{R}\left(\tau_{1}, \tau_{2}\right) \cap\left\{t \leq T^{*}\right\}} \chi f_{i m p} \cdot\left(\partial_{v}-\partial_{u}\right) \Phi \cdot\left(\operatorname{Err}(\Phi)+O\left(r^{-3}\right) \partial_{\sigma}^{2} \Phi\right) d u d v d \sigma \\
& +\int_{\mathcal{R}\left(\tau_{1}, \tau_{2}\right) \cap\left\{t \leq T^{*}\right\}} O\left(f_{i m p}|\partial \chi|\right) \cdot\left(|\partial \Phi|^{2}+r^{-2}|\Phi|^{2}\right) d u d v d \sigma \\
& +\sum_{i=1}^{2} \int_{\mathcal{S}_{\tau_{i}} \cap\left\{t \leq T^{*}\right\}} \chi O\left(f_{\text {imp }}\right)\left(\left|\partial_{v} \Phi\right|^{2}+\left|r^{-1} \partial_{\sigma} \Phi\right|^{2}+r^{-1-\eta^{\prime}}\left|\partial_{u} \Phi\right|^{2}+r^{-2}|\Phi|^{2}\right) d v d \sigma \\
& +\int_{\mathcal{R}\left(\tau_{1}, \tau_{2}\right) \cap\left\{t=T^{*}\right\}} O\left(f_{i m p}\right)\left(|\partial \Phi|^{2}+r^{-2}|\Phi|^{2}\right) d v d \sigma
\end{aligned}
$$

where

$$
\mathcal{A}_{i m p} \doteq 2\left(2 r^{-1}\left(1+O\left(r^{-1}\right)\right) f_{i m p}-\frac{1}{2}\left(1+O\left(r^{-a}\right)\right)\left(\partial_{v}-\partial_{u}\right) f_{i m p}\right) .
$$

Since $\Phi=\Omega \varphi$, we calculate by applying the product rule and expanding the square:

$$
\begin{aligned}
& \int_{\mathcal{R}\left(\tau_{1}, \tau_{2}\right) \cap\left\{t \leq T^{*}\right\}} \chi \cdot \frac{1}{2}\left(-\partial_{u} f_{i m p}+O\left(r^{-1-a}\right) f_{i m p}\right)\left|\partial_{v} \Phi\right|^{2} d u d v d \sigma \\
& =\int_{\mathcal{R}\left(\tau_{1}, \tau_{2}\right) \cap\left\{t \leq T^{*}\right\}} \chi \cdot\left(-\partial_{u} f_{i m p}+O\left(r^{-1-a}\right) f_{i m p}\right) \\
& \quad \times\left\{\left(\partial_{v} \varphi\right)^{2}+r^{-2}\left(1+O\left(r^{-1}\right)\right) \varphi^{2}+r^{-1}\left(1+O\left(r^{-1}\right)\right) \partial_{v}\left(\varphi^{2}\right)\right\} \Omega^{2} d u d v d \sigma .
\end{aligned}
$$

By integrating by parts in the $\partial_{v}\left(\varphi^{2}\right)$ term, we thus obtain (due to (4.20)) for any $\delta_{0} \leq 1$ :

$$
\begin{aligned}
& \int_{\mathcal{R}\left(\tau_{1}, \tau_{2}\right) \cap\left\{t \leq T^{*}\right\}} \chi \cdot \frac{1}{2}\left(-\partial_{u} f_{i m p}+O\left(r^{-1-a}\right) f_{i m p}\right)\left|\partial_{v} \Phi\right|^{2} d u d v d \sigma \\
& \geq \delta_{0}\left\{c_{\eta} \int_{\mathcal{R}\left(\tau_{1}, \tau_{2}\right) \cap\left\{t \leq T^{*}\right\} \cap\left\{r \leq R_{c}\right\}} \chi \cdot\left(\left(\partial_{v} \varphi\right)^{2}+r^{-2-\eta} \varphi^{2}\right) \Omega^{2} d u d v d \sigma\right. \\
& +c_{\eta} R_{c} \int_{\mathcal{R}\left(\tau_{1}, \tau_{2}\right) \cap\left\{t \leq T^{*}\right\} \cap\left\{r \geq R_{c}\right\}} \chi \cdot r^{-1-\eta}\left(\left(\partial_{v} \varphi\right)^{2}+r^{-2} \varphi^{2}\right) \Omega^{2} d u d v d \sigma \\
& -C_{\eta}(d-3) \int_{\mathcal{R}\left(\tau_{1}, \tau_{2}\right) \cap\left\{t \leq T^{*}\right\} \cap\left\{r \leq R_{c}\right\}} \chi \cdot r^{-2}|\Phi|^{2} d u d v d \sigma \\
& -C_{\eta}(d-3) R_{c} \int_{\mathcal{R}\left(\tau_{1}, \tau_{2}\right) \cap\left\{t \leq T^{*}\right\} \cap\left\{r \geq R_{c}\right\}} \chi \cdot r^{-3}|\Phi|^{2} d u d v d \sigma
\end{aligned}
$$




$$
\begin{aligned}
& -\int_{\mathcal{R}\left(\tau_{1}, \tau_{2}\right) \cap\left\{t \leq T^{*}\right\}} O\left(f_{\text {imp }}|\partial \chi|\right) \cdot r^{-2}|\Phi|^{2} d u d v d \sigma \\
& \left.-C\left(R_{c}\right) \sum_{i=1}^{2} \int_{\mathcal{S}_{\tau_{i}} \cap\left\{t \leq T^{*}\right\}} \chi r^{-2}|\Phi|^{2} d v d \sigma-C\left(R_{c}\right) \int_{\mathcal{R}\left(\tau_{1}, \tau_{2}\right) \cap\left\{t=T^{*}\right\}} \chi r^{-2}|\Phi|^{2} d v d \sigma\right\} .
\end{aligned}
$$

Moreover, using a trivial Cauchy-Schwarz inequality for the $\varphi \partial_{v} \varphi$ term we can bound from (4.23):

$$
\begin{aligned}
& \int_{\mathcal{R}\left(\tau_{1}, \tau_{2}\right) \cap\left\{t \leq T^{*}\right\} \cap\left\{r \leq R_{c}\right\}} \chi \cdot \frac{1}{2}\left(-\partial_{u} f_{i m p}+O\left(r^{-1-a}\right) f_{i m p}\right)\left|\partial_{v} \Phi\right|^{2} d u d v d \sigma \\
& \geq \int_{\mathcal{R}\left(\tau_{1}, \tau_{2}\right) \cap\left\{t \leq T^{*}\right\} \cap\left\{r \leq R_{c}\right\}} \chi \cdot\left(c_{\eta} r^{-2} \varphi^{2}-C_{\eta}\left(\partial_{v} \varphi\right)^{2}\right) \Omega^{2} d u d v d \sigma,
\end{aligned}
$$

and thus by adding to (4.24) a small multiple of (4.25) we obtain:

$$
\begin{aligned}
& \int_{\mathcal{R}\left(\tau_{1}, \tau_{2}\right) \cap\left\{t \leq T^{*}\right\}} \chi \cdot \frac{1}{2}\left(-\partial_{u} f_{i m p}+O\left(r^{-1-a}\right) f_{i m p}\right)\left|\partial_{v} \Phi\right|^{2} d u d v d \sigma \\
& \geq \delta_{0}\left\{c_{\eta} \int_{\mathcal{R}\left(\tau_{1}, \tau_{2}\right) \cap\left\{t \leq T^{*}\right\} \cap\left\{r \leq R_{c}\right\}} \chi \cdot\left(\left(\partial_{v} \varphi\right)^{2}+r^{-2} \varphi^{2}\right) \Omega^{2} d u d v d \sigma\right. \\
& \quad+c_{\eta} R_{C} \int_{\mathcal{R}\left(\tau_{1}, \tau_{2}\right) \cap\left\{t \leq T^{*}\right\} \cap\left\{r \geq R_{c}\right\}} \chi \cdot r^{-1-\eta}\left(\left(\partial_{v} \varphi\right)^{2}+r^{-2} \varphi^{2}\right) \Omega^{2} d u d v d \sigma \\
& -C_{\eta}(d-3) \int_{\mathcal{R}\left(\tau_{1}, \tau_{2}\right) \cap\left\{t \leq T^{*}\right\} \cap\left\{r \leq R_{c}\right\}} \chi \cdot r^{-2}|\Phi|^{2} d u d v d \sigma \\
& -C_{\eta}(d-3) R_{C} \int_{\mathcal{R}\left(\tau_{1}, \tau_{2}\right) \cap\left\{t \leq T^{*}\right\} \cap\left\{r \geq R_{c}\right\}} \chi \cdot r^{-3}|\Phi|^{2} d u d v d \sigma \\
& -\int_{\mathcal{R}\left(\tau_{1}, \tau_{2}\right) \cap\left\{t \leq T^{*}\right\}} O\left(f_{i m p}|\partial \chi|\right) \cdot r^{-2}|\Phi|^{2} d u d v d \sigma \\
& -C\left(R_{c}\right) \sum_{i=1}^{2} \int_{\mathcal{S}_{\tau_{i}} \cap\left\{t \leq T^{*}\right\}} \chi r^{-2}|\Phi|^{2} d v d \sigma \\
& \left.-C\left(R_{c}\right) \int_{\mathcal{R}\left(\tau_{1}, \tau_{2}\right) \cap\left\{t=T^{*}\right\}} \chi r^{-2}|\Phi|^{2} d v d \sigma\right\} .
\end{aligned}
$$

Using, now the expression (4.3) for $\operatorname{Err}(\Phi)$, we can readily bound after integrating by parts in the highest order terms (and in the $\Phi \partial_{u} \Phi=\frac{1}{2} \partial_{u}\left(\Phi^{2}\right)$ term) and using a Cauchy-Schwarz inequality:

$$
\begin{aligned}
& \int_{\mathcal{R}\left(\tau_{1}, \tau_{2}\right) \cap\left\{t \leq T^{*}\right\}} \chi f_{i m p} \cdot\left(\partial_{v}-\partial_{u}\right) \Phi \cdot\left(\operatorname{Err}(\Phi)+O\left(r^{-3}\right) \partial_{\sigma}^{2} \Phi\right) d u d v d \sigma \\
& \quad \leq C_{\eta} \int_{\mathcal{R}\left(\tau_{1}, \tau_{2}\right) \cap\left\{t \leq T^{*}\right\} \cap\left\{r \leq R_{c}\right\}} \chi\left\{O\left(r^{-a}\right)\left|\partial_{u} \Phi\right|^{2}\right.
\end{aligned}
$$




$$
\begin{aligned}
& \left.+\left(\left|\partial_{v} \Phi\right|^{2}+\left|r^{-1} \partial_{\sigma} \Phi\right|^{2}+r^{-2}|\Phi|^{2}\right)\right\} d u d v d \sigma \\
& +C_{\eta} R_{c} \int_{\mathcal{R}\left(\tau_{1}, \tau_{2}\right) \cap\left\{t \leq T^{*}\right\} \cap\left\{r \geq R_{c}\right\}} \chi \cdot\left\{O\left(r^{-1-a}\right)\left|\partial_{u} \Phi\right|^{2}\right. \\
& \left.+O\left(r^{-1}\right)\left(\left|\partial_{v} \Phi\right|^{2}+\left|r^{-1} \partial_{\sigma} \Phi\right|^{2}+r^{-2}|\Phi|^{2}\right)\right\} d u d v d \sigma \\
& +C_{\eta} \int_{\mathcal{R}\left(\tau_{1}, \tau_{2}\right) \cap\left\{t \leq T^{*}\right\}} O\left(f_{i m p}|\partial \chi|\right)\left(|\partial \Phi|^{2}+r^{-2}|\Phi|^{2}\right) d u d v d \sigma \\
& +C_{\eta}\left(R_{c}\right) \sum_{i=1}^{2} \int_{\mathcal{S}_{\tau_{i}} \cap\left\{t \leq T^{*}\right\}} \chi O\left(f_{i m p}\right)\left(\left|\partial_{v} \Phi\right|^{2}+\left|r^{-1} \partial_{\sigma} \Phi\right|^{2}\right. \\
& \left.+r^{-1-\eta^{\prime}}\left|\partial_{u} \Phi\right|^{2}+r^{-2}|\Phi|^{2}\right) d v d \sigma \\
& +C_{\eta}\left(R_{c}\right) \int_{\mathcal{R}\left(\tau_{1}, \tau_{2}\right) \cap\left\{t=T^{*}\right\}} O\left(f_{i m p}\right)\left(|\partial \Phi|^{2}+r^{-2}|\Phi|^{2}\right) d v d \sigma .
\end{aligned}
$$

Therefore, from (4.21), (4.24) and (4.27) for $\delta_{0}$ small enough in terms of $\eta$, we obtain the desired inequality if $R$ is large enough in terms of $\eta$ :

$$
\begin{aligned}
& \int_{\mathcal{R}\left(\tau_{1}, \tau_{2}\right) \cap\left\{t \leq T^{*}\right\} \cap\left\{r \leq R_{c}\right\}} \chi \cdot\left(\left(\left|\partial_{u} \varphi\right|^{2}+\left|\partial_{v} \varphi\right|^{2}\right)+\left|r^{-1} \partial_{\sigma} \varphi\right|^{2}+r^{-2}|\varphi|^{2}\right) \\
& +R_{c} \int_{\mathcal{R}\left(\tau_{1}, \tau_{2}\right) \cap\left\{t \leq T^{*}\right\} \cap\left\{r \geq R_{c}\right\}} \chi \cdot\left(r^{-1-\eta}\left(\left|\partial_{u} \varphi\right|^{2}+\left|\partial_{v} \varphi\right|^{2}\right)\right. \\
& \left.+r^{-1}\left|r^{-1} \partial_{\sigma} \varphi\right|^{2}+r^{-3-\eta}|\varphi|^{2}\right) \\
& \leq C(\eta) \cdot \int_{\mathcal{R}\left(\tau_{1}, \tau_{2}\right) \cap\left\{t \leq T^{*}\right\}}|\partial \chi| \cdot r \cdot\left(|\partial \varphi|^{2}+r^{-2}|\varphi|^{2}\right) \\
& +C\left(\eta, R_{c}\right) \cdot \sum_{i=1}^{2} \int_{\mathcal{S}_{\tau_{i}} \cap\left\{t \leq T^{*}\right\}} \chi\left(\left|\partial_{v} \varphi\right|^{2}+\left|r^{-1} \partial_{\sigma} \varphi\right|^{2}\right. \\
& \left.+r^{-1-\eta^{\prime}}\left|\partial_{u} \varphi\right|^{2}+r^{-2}|\varphi|^{2}\right) r^{d-1} d v d \sigma \\
& +C\left(\eta, R_{c}\right) \cdot \int_{\left\{t=T^{*}\right\} \cap \mathcal{R}\left(\tau_{1}, \tau_{2}\right)} \chi\left(|\partial \varphi|^{2}+r^{-2}|\varphi|^{2}\right) r^{d-1} d v d \sigma \\
& +\int_{\mathcal{R}\left(\tau_{1}, \tau_{2}\right) \cap\left\{t \leq T^{*}\right\}} \chi \cdot\left(O_{R_{c}, \eta}(1)\left(\partial_{v}-\partial_{u}\right) \varphi+O_{R_{c}, \eta}\left(r^{-1}\right) \varphi\right) \cdot \square_{g} \varphi \\
& +C(\eta) R_{C} \cdot \int_{\mathcal{R}\left(\tau_{1}, \tau_{2}\right) \cap\left\{t \leq T^{*}\right\}} \chi \cdot r^{-1}\left(\left|\partial_{v} \varphi\right|^{2}+\left|r^{-1} \partial_{\sigma} \varphi\right|^{2}+r^{-2}|\varphi|^{2}\right) \text {. }
\end{aligned}
$$

In the same way, we can establish a similar lemma in the region spanned by two hypersurfaces of the form $\{t=$ const $\}$.

Lemma 4.4 For any given $0<\eta<a$, and any $R>0$ sufficiently large in terms of $\eta$, any $R_{c} \geq R$, any $t_{1} \leq t_{2}$, any function $\chi: \mathcal{N}_{\text {af }} \rightarrow[0,1]$ supported in $\{r \geq R\}$ with 
$\partial \chi$ supported in $\left\{R \leq r \leq R_{c}\right\}$ and any smooth function $\varphi: \mathcal{N}_{a f} \rightarrow \mathbb{C}$ with compact support in space we can bound:s

$$
\begin{aligned}
& \int_{\left\{t_{1} \leq t \leq t_{2}\right\} \cap\left\{r \leq R_{c}\right\}} \chi \cdot\left(\left(\left|\partial_{u} \varphi\right|^{2}+\left|\partial_{v} \varphi\right|^{2}\right)+\left|r^{-1} \partial_{\sigma} \varphi\right|^{2}+r^{-2}|\varphi|^{2}\right) \\
& +R_{c} \int_{\left\{t_{1} \leq t \leq t_{2}\right\} \cap\left\{r \geq R_{c}\right\}} \chi \cdot\left(r^{-1-\eta}\left(\left|\partial_{u} \varphi\right|^{2}+\left|\partial_{v} \varphi\right|^{2}\right)+r^{-1}\left|r^{-1} \partial_{\sigma} \varphi\right|^{2}+r^{-3-\eta}|\varphi|^{2}\right) \\
& \leq C(\eta) \cdot \int_{\left\{t_{1} \leq t \leq t_{2}\right\}}|\partial \chi| \cdot r \cdot\left(|\partial \varphi|^{2}+r^{-2}|\varphi|^{2}\right) \\
& +C\left(\eta, R_{c}\right) \cdot \int_{\left\{t=t_{1}\right\} \cap\{r \geq R\}}|\partial \varphi|^{2}+C\left(\eta, R_{C}\right) \cdot \int_{\left\{t=t_{2}\right\} \cap\{r \geq R\}}|\partial \varphi|^{2} \\
& +\int_{\left\{t_{1} \leq t \leq t_{2}\right\}} \chi \cdot \operatorname{Re}\left\{\left(O_{R_{c}, \eta}(1)\left(\partial_{v}-\partial_{u}\right) \bar{\varphi}+O_{R_{c}, \eta}\left(r^{-1}\right) \bar{\varphi}\right) \cdot \square_{g} \varphi\right\} \\
& +C(\eta) R_{c} \cdot \int_{\left\{t_{1} \leq t \leq t_{2}\right\}} \chi \cdot r^{-1}\left(\left|\partial_{v} \varphi\right|^{2}+\left|r^{-1} \partial_{\sigma} \varphi\right|^{2}+r^{-2}|\varphi|^{2}\right) .
\end{aligned}
$$

Remark In case the radiative components of the metric satisfy the bounds $\partial_{u} M \leq 0$ and $\left|\partial_{u} h_{a s}\right|+\left|r \partial_{u} h_{\mathbb{S} d-1}\right| \ll-\left(\partial_{u} M\right)+O\left(r^{-a}\right)$ (which includes the non-radiative case $\partial_{u} M=0, h_{a s}=0$ and $\left.h_{\mathbb{S}^{d-1}}=O_{1}\left(r^{-1-a}\right)\right)$, the last term of the right hand side of (4.4) can be omitted.

\subsection{Estimates for the $J^{T}$-Energy}

In this Section, we will establish some useful estimates for the energy associated to the timelike vector field $T=\partial_{u}+\partial_{v}$ in the coordinate chart $(u, v, \sigma)$. Since we have not assumed $\left(\mathcal{N}_{a f}, g\right)$ to be stationary, the $J^{T}$-energy current will not be in general conserved.

Lemma 4.5 For any smooth function $\varphi: \mathcal{N}_{\text {af }} \rightarrow \mathbb{C}$, any $\chi: \mathcal{N}_{a f} \rightarrow[0,1]$ supported on $\{r \geq R\}$ for some $R>0$ large in terms of the geometry of $\left(\mathcal{N}_{a f}, g\right)$, any $\tau_{1} \leq \tau_{2}$ and any $T^{*}>0$ the following estimate is true:

$$
\begin{aligned}
\int_{\mathcal{S}_{\tau_{2}} \cap\left\{t \leq T^{*}\right\}} \chi \cdot J_{\mu}^{T}(\varphi) \bar{n}^{\mu}+\int_{\left\{t=T^{*}\right\} \cap \mathcal{R}\left(\tau_{1}, \tau_{2}\right)}\left(|\partial \varphi|^{2}+r^{-2}|\varphi|^{2}\right) \\
\leq C \cdot\left\{\int_{\mathcal{S}_{\tau_{i}} \cap\left\{t \leq T^{*}\right\}} \chi \cdot J_{\mu}^{T}(\varphi) \bar{n}^{\mu}\right. \\
\quad+\int_{\mathcal{R}\left(\tau_{1}, \tau_{2}\right) \cap\left\{t \leq T^{*}\right\}} \chi \cdot r^{-1}\left(\left|\partial_{v} \varphi\right|^{2}+\left|r^{-1} \partial_{\sigma} \varphi\right|^{2}+\left|r^{-1} \varphi\right|^{2}\right) \\
\quad+\int_{\mathcal{R}\left(\tau_{1}, \tau_{2}\right) \cap\left\{t \leq T^{*}\right\}} O(|\partial \chi|) \cdot\left(|\partial \varphi|^{2}+r^{-1}|\varphi|^{2}\right)+\int_{\mathcal{R}\left(\tau_{1}, \tau_{2}\right) \cap\left\{t \leq T^{*}\right\}} \chi O(1) \\
\left.\quad \times \operatorname{Re}\left\{\Omega^{-1}\left(\partial_{v}+\partial_{u}\right)(\Omega \bar{\varphi}) \cdot \square \varphi\right\}\right\} .
\end{aligned}
$$


for some constant $C>0$ depending only on the geometry of $\left(\mathcal{N}_{a f}, g\right)$. In the above, $\bar{n}$ and $n$ denote the future directed unit normals to the hypersurfaces $\mathcal{S}_{\tau}$ and $\{t=$ const $\}$ respectively.

Remark In case the radiative components of the metric satisfy the bounds $\partial_{u} M \leq 0$ and $\left|\partial_{u} h_{a s}\right|+\left|r \partial_{u} h_{\mathbb{S}^{d-1}}\right| \ll-\left(\partial_{u} M\right)+O\left(r^{-a}\right)$ (which includes the non-radiating case $\partial_{u} M=0, h_{a s}=0$ and $h_{\mathbb{S}^{d-1}}=O\left(r^{-1-a}\right)$ ), the second term of the right hand side of (4.30) can be omitted. Furthermore, in case the $T$ vector field satisfies (9.6) for $m=1$, then the second term of the right hand side of (4.30) is replaced by

$$
\int_{\mathcal{R}\left(\tau_{1}, \tau_{2}\right) \cap\left\{t \leq T^{*}\right\}} \chi \cdot \bar{t}^{-\delta_{0}} r^{-1}\left(\left|\partial_{v} \varphi\right|^{2}+\left|r^{-1} \partial_{\sigma} \varphi\right|^{2}+r^{-2}|\varphi|^{2}\right) .
$$

Proof Without loss of generality, we will assume that $\varphi$ is real valued.

Setting $\Phi=\Omega \varphi$ and multiplying equation (4.1) with $\chi \cdot\left(\partial_{v}+\partial_{u}\right) \Phi$, we obtain after integrating over $\mathcal{R}\left(\tau_{1}, \tau_{2}\right) \cap\left\{t \leq T^{*}\right\}$ (with $d u d v d \sigma$ used as a volume form):

$$
\begin{aligned}
& \int_{\mathcal{R}\left(\tau_{1}, \tau_{2}\right) \cap\left\{t \leq T^{*}\right\}} \chi\left(\partial_{v}+\partial_{u}\right) \Phi \cdot \Omega \square \varphi d u d v d \sigma \\
& =\int_{\mathcal{R}\left(\tau_{1}, \tau_{2}\right) \cap\left\{t \leq T^{*}\right\}} \chi\left(\partial_{v}+\partial_{u}\right) \Phi \cdot\left\{-\left(1+O_{1}\left(r^{-1-a}\right)\right) \cdot \partial_{u} \partial_{v} \Phi\right. \\
& +r^{-2} \chi_{g_{\mathbb{S} d-1}+h_{\mathbb{S} d-1}} \Phi-\int_{\mathcal{R}\left(\tau_{1}, \tau_{2}\right) \cap\left\{t \leq T^{*}\right\}} \chi\left(\partial_{v}+\partial_{u}\right) \Phi \\
& \left.\times\left\{-\frac{(d-1)(d-3)}{4} r^{-2} \cdot \Phi+\operatorname{Err}(\Phi)\right\}\right\} d u d v d \sigma .
\end{aligned}
$$

Using the expression

$$
\Delta_{g_{\mathbb{S}^{d}-1}+h_{\mathbb{S}^{d-1}}}=\Delta_{g_{\mathbb{S}^{d-1}}}+O_{1}\left(r^{-1}\right) \partial_{\sigma} \partial_{\sigma}+O\left(r^{-1}\right) \partial_{\sigma},
$$

we obtain after integrating by parts in $\partial_{u}, \partial_{v}$ and $\partial_{\sigma}$ and absorbing the error terms in the $E r r$ summand (and recalling that $\partial_{v} r=-\partial_{u} r=1$ ):

$$
\begin{aligned}
& -\int_{\mathcal{R}\left(\tau_{1}, \tau_{2}\right) \cap\left\{t \leq T^{*}\right\}} \chi\left(\partial_{v}+\partial_{u}\right) \Phi \cdot \Omega \square \varphi d u d v d \sigma \\
& =\int_{\mathcal{S}_{\tau_{2} \cap\left\{t \leq T^{*}\right\}}} \frac{1}{2} \chi\left(\left(1+O\left(r^{-1}\right)\right) \cdot\left(\partial_{v} \Phi\right)^{2}+\left|r^{-1} \partial_{\sigma} \Phi\right|^{2}+\frac{(d-1)(d-3)}{4}\left|r^{-1} \Phi\right|^{2}\right) d v d \sigma \\
& \quad+\int_{\mathcal{S}_{\tau_{2} \cap\left\{t \leq T^{*}\right\}}} \frac{1}{2} \chi\left(\left(1+O\left(r^{-1}\right)\right) \cdot\left(\partial_{u} \Phi\right)^{2}+\left|r^{-1} \partial_{\sigma} \Phi\right|^{2}+\frac{(d-1)(d-3)}{4}\left|r^{-1} \Phi\right|^{2}\right) d u d \sigma \\
& \quad+\int_{\left\{t=T^{*}\right\} \cap \mathcal{R}\left(\tau_{1}, \tau_{2}\right)} \frac{1}{2} \chi\left(\left(1+O\left(r^{-1}\right)\right) \cdot\left(\partial_{v} \Phi\right)^{2}+\left|r^{-1} \partial_{\sigma} \Phi\right|^{2}\right.
\end{aligned}
$$




$$
\begin{aligned}
& \left.+\frac{(d-1)(d-3)}{4}\left|r^{-1} \Phi\right|^{2}\right) d v d \sigma \\
& +\int_{\left\{t=T^{*}\right\} \cap \mathcal{R}\left(\tau_{1}, \tau_{2}\right)} \frac{1}{2} \chi\left(\left(1+O\left(r^{-1}\right)\right) \cdot\left(\partial_{u} \Phi\right)^{2}+\left|r^{-1} \partial_{\sigma} \Phi\right|^{2}\right. \\
& \left.+\frac{(d-1)(d-3)}{4}\left|r^{-1} \Phi\right|^{2}\right) d u d \sigma \\
& -\int_{\mathcal{S}_{\tau_{1}} \cap\left\{t \leq T^{*}\right\}} \frac{1}{2} \chi\left(\left(1+O\left(r^{-1}\right)\right) \cdot\left(\partial_{v} \Phi\right)^{2}+\left|r^{-1} \partial_{\sigma} \Phi\right|^{2}\right. \\
& \left.+\frac{(d-1)(d-3)}{4}\left|r^{-1} \Phi\right|^{2}\right) d v d \sigma \\
& -\int_{\mathcal{S}_{\tau_{1}} \cap\left\{t \leq T^{*}\right\}} \frac{1}{2} \chi\left(\left(1+O\left(r^{-1}\right)\right) \cdot\left(\partial_{u} \Phi\right)^{2}+\left|r^{-1} \partial_{\sigma} \Phi\right|^{2}+\frac{(d-1)(d-3)}{4}\left|r^{-1} \Phi\right|^{2}\right) d u d \sigma \\
& +\int_{\mathcal{R}\left(\tau_{1}, \tau_{2}\right) \cap\left\{t \leq T^{*}\right\}} \chi \cdot\left\{O\left(r^{-1-a}\right)\left|\partial_{v} \Phi\right|^{2}+O\left(r^{-1-a}\right)\left|\partial_{u} \Phi\right|^{2}\right. \\
& \left.+\int_{\mathcal{R}\left(\tau_{1}, \tau_{2}\right) \cap\left\{t \leq T^{*}\right\}} \chi \cdot+O\left(r^{-1}\right)\left(\left|r^{-1} \partial_{\sigma} \Phi\right|^{2}+\frac{(d-1)(d-3)}{2}\left|r^{-1} \Phi\right|^{2}\right)\right\} d u d v d \sigma \\
& +\int_{\mathcal{R}\left(\tau_{1}, \tau_{2}\right) \cap\left\{t \leq T^{*}\right\}} \chi\left(\partial_{v}+\partial_{u}\right) \Phi \cdot \operatorname{Err}(\Phi) d u d v d \sigma \\
& +\int_{\mathcal{R}\left(\tau_{1}, \tau_{2}\right) \cap\left\{t \leq T^{*}\right\}} O(|\partial \chi|) \cdot\left(|\partial \Phi|^{2}+r^{-2}|\Phi|^{2}\right) d u d v d \sigma .
\end{aligned}
$$

Since on $\mathcal{S}_{\tau_{i}}$ we have $d v d \sigma \sim r^{-1-\eta^{\prime}} d u d \sigma$, we obtain from (4.33):

$$
\begin{aligned}
& \int_{\mathcal{S}_{\tau_{2}} \cap\left\{t \leq T^{*}\right\}} \chi\left(\left(\partial_{v} \Phi\right)^{2}+r^{-1-\eta^{\prime}}\left(\partial_{u} \Phi\right)^{2}+\left|r^{-1} \partial_{\sigma} \Phi\right|^{2}+\frac{(d-1)(d-3)}{4}\left|r^{-1} \Phi\right|^{2}\right) d v d \sigma \\
& +\int_{\left\{t=T^{*}\right\} \cap \mathcal{R}\left(\tau_{1}, \tau_{2}\right)}\left(\left(\partial_{v} \Phi\right)^{2}+\left(\partial_{u} \Phi\right)^{2}+\left|r^{-1} \partial_{\sigma} \Phi\right|^{2}+\frac{(d-1)(d-3)}{4}\left|r^{-1} \Phi\right|^{2}\right) d v d \sigma \\
& \leq C \cdot\left\{\int_{\mathcal{S}_{\tau_{1}} \cap\left\{t \leq T^{*}\right\}} \chi\left(\left(\partial_{v} \Phi\right)^{2}+r^{-1-\eta^{\prime}}\left(\partial_{u} \Phi\right)^{2}+\left|r^{-1} \partial_{\sigma} \Phi\right|^{2}+\frac{(d-1)(d-3)}{4}\left|r^{-1} \Phi\right|^{2}\right) d v d \sigma\right. \\
& +\int_{\mathcal{R}\left(\tau_{1}, \tau_{2}\right) \cap\left\{t \leq T^{*}\right\}} \chi \cdot\left\{O\left(r^{-1-a}\right)\left|\partial_{v} \Phi\right|^{2}+O\left(r^{-1-a}\right)\left|\partial_{u} \Phi\right|^{2}\right. \\
& \left.+O\left(r^{-1}\right)\left(\left|r^{-1} \partial_{\sigma} \Phi\right|^{2}+\frac{(d-1)(d-3)}{4}\left|r^{-1} \Phi\right|^{2}\right)\right\} d u d v d \sigma \\
& +\int_{\mathcal{R}\left(\tau_{1}, \tau_{2}\right) \cap\left\{t \leq T^{*}\right\}} \chi\left(\partial_{v}+\partial_{u}\right) \Phi \cdot \operatorname{Err}(\Phi) d u d v d \sigma \\
& +\int_{\mathcal{R}\left(\tau_{1}, \tau_{2}\right) \cap\left\{t \leq T^{*}\right\}} O(|\partial \chi|) \cdot\left(|\partial \Phi|^{2}+r^{-2}|\Phi|^{2}\right) d u d v d \sigma \\
& \left.+\int_{\mathcal{R}\left(\tau_{1}, \tau_{2}\right) \cap\left\{t \leq T^{*}\right\}} \chi O(1) \cdot\left(\partial_{v}+\partial_{u}\right) \Phi \cdot \Omega \square \varphi d u d v d \sigma\right\} .
\end{aligned}
$$

Using a Hardy type inequality of the form established in Lemma C.1 (notice that it is a 1-dimesnional Hardy inequality, since the volume form is $d v d \sigma$ ), we can bound: 


$$
\begin{aligned}
& \int_{\mathcal{S}_{\tau_{i}} \cap\left\{t \leq T^{*}\right\}} \chi r^{-2}|\Phi|^{2} d v d \sigma+\int_{\mathcal{S}_{\tau_{i}} \cap\left\{t=T^{*}\right\}} r^{-1}|\Phi|^{2} d \sigma \\
& \leq C \cdot\left\{\int_{\mathcal{S}_{\tau_{i}} \cap\left\{t \leq T^{*}\right\}} \chi\left(\left(\partial_{v} \Phi\right)^{2}+r^{-1-\eta^{\prime}}\left(\partial_{u} \Phi\right)^{2}\right) d v d \sigma+\int_{\mathcal{S}_{\tau_{i}}}|\partial \chi| \cdot r^{-1}|\Phi|^{2} d v d \sigma\right\}
\end{aligned}
$$

and

$$
\begin{aligned}
\int_{\left\{t=T^{*}\right\} \cap \mathcal{R}\left(\tau_{1}, \tau_{2}\right)} r^{-2}|\Phi|^{2} d v d \sigma \leq & C \cdot\left\{\int_{\left\{t=T^{*}\right\} \cap \mathcal{R}\left(\tau_{1}, \tau_{2}\right)}\left(\left(\partial_{v} \Phi\right)^{2}+\left(\partial_{u} \Phi\right)^{2}\right) d v d \sigma\right. \\
& \left.+\sum_{i=1}^{2} \int_{\mathcal{S}_{\tau_{i}} \cap\left\{t=T^{*}\right\}} r^{-1}|\Phi|^{2} d \sigma\right\}
\end{aligned}
$$

Therefore, in view of (4.35) and (4.36), (4.34) can be improved into

$$
\begin{aligned}
\int_{\mathcal{S}_{\tau_{2}} \cap\left\{t \leq T^{*}\right\}} \chi\left(\left(\partial_{v} \Phi\right)^{2}+r^{-1-\eta^{\prime}}\left(\partial_{u} \Phi\right)^{2}+\left|r^{-1} \partial_{\sigma} \Phi\right|^{2}+\left|r^{-1} \Phi\right|^{2}\right) d v d \sigma \\
+\int_{\left\{t=T^{*}\right\} \cap \mathcal{R}\left(\tau_{1}, \tau_{2}\right)}\left(\left(\partial_{v} \Phi\right)^{2}+\left(\partial_{u} \Phi\right)^{2}+\left|r^{-1} \partial_{\sigma} \Phi\right|^{2}+\left|r^{-1} \Phi\right|^{2}\right) d v d \sigma \\
\leq C \cdot\left\{\int_{\mathcal{S}_{\tau_{1}} \cap\left\{t \leq T^{*}\right\}} \chi\left(\left(\partial_{v} \Phi\right)^{2}+r^{-1-\eta^{\prime}}\left(\partial_{u} \Phi\right)^{2}+\left|r^{-1} \partial_{\sigma} \Phi\right|^{2}+\left|r^{-1} \Phi\right|^{2}\right) d v d \sigma\right. \\
+\int_{\mathcal{R}\left(\tau_{1}, \tau_{2}\right) \cap\left\{t \leq T^{*}\right\}} \chi \cdot\left\{O\left(r^{-1-a}\right)\left|\partial_{v} \Phi\right|^{2}\right. \\
\left.+O\left(r^{-1-a}\right)\left|\partial_{u} \Phi\right|^{2}+O\left(r^{-1}\right)\left(\left|r^{-1} \partial_{\sigma} \Phi\right|^{2}+\frac{(d-1)(d-3)}{4}\left|r^{-1} \Phi\right|^{2}\right)\right\} d u d v d \sigma \\
+\int_{\mathcal{R}\left(\tau_{1}, \tau_{2}\right) \cap\left\{t \leq T^{*}\right\}} \chi\left(\partial_{v}+\partial_{u}\right) \Phi \cdot E r r(\Phi) d u d v d \sigma \\
+\int_{\mathcal{R}\left(\tau_{1}, \tau_{2}\right) \cap\left\{t \leq T^{*}\right\}} O(|\partial \chi|) \cdot\left(|\partial \Phi|^{2}+r^{-2}|\Phi|^{2}\right) d u d v d \sigma \\
+\sum_{i=1}^{2} \int_{\mathcal{S}_{\tau_{i}} \cap\left\{t \leq T^{*}\right\}}|\partial \chi| \cdot|\Phi|^{2} d v d \sigma \\
\left.+\int_{\mathcal{R}\left(\tau_{1}, \tau_{2}\right) \cap\left\{t \leq T^{*}\right\}} \chi O(1) \cdot\left(\partial_{v}+\partial_{u}\right) \Phi \cdot \Omega \square \varphi d u d v d \sigma\right\} .
\end{aligned}
$$

Using the expression (4.3) for $\operatorname{Err}(\Phi)$, we can readily bound after integrating by parts in the highest order terms (and in the $\Phi \partial_{u} \Phi=\frac{1}{2} \partial_{u}\left(\Phi^{2}\right)$ term) and using a Cauchy-Schwarz inequality:

$$
\begin{aligned}
& \int_{\mathcal{R}\left(\tau_{1}, \tau_{2}\right) \cap\left\{t \leq T^{*}\right\}} \chi\left(\partial_{v}+\partial_{u}\right) \Phi \cdot \operatorname{Err}(\Phi) d u d v d \sigma \\
& \quad \leq C_{\eta} \int_{\mathcal{R}\left(\tau_{1}, \tau_{2}\right) \cap\left\{t \leq T^{*}\right\}} \chi\left\{O\left(r^{-1-a}\right)\left|\partial_{u} \Phi\right|^{2}+O\left(r^{-1}\right)\right.
\end{aligned}
$$




$$
\begin{aligned}
& \left.\times\left(\left|\partial_{v} \Phi\right|^{2}+\delta_{1}\left|r^{-1} \partial_{\sigma} \Phi\right|^{2}+r^{-2}|\Phi|^{2}\right)\right\} d u d v d \sigma \\
& +C_{\eta}\left(\int_{\mathcal{R}\left(\tau_{1}, \tau_{2}\right) \cap\left\{t \leq T^{*}\right\}}|\partial \chi|\left(|\partial \Phi|^{2}+r^{-2}|\Phi|^{2}\right) d u d v d \sigma\right) \\
& +\int_{\mathcal{S}_{\tau_{2}} \cap\left\{t \leq T^{*}\right\}} \chi O\left(r^{-a}\right)\left(\left(\partial_{v} \Phi\right)^{2}+r^{-1-\eta^{\prime}}\left(\partial_{u} \Phi\right)^{2}+\left|r^{-1} \partial_{\sigma} \Phi\right|^{2}+\left|r^{-1} \Phi\right|^{2}\right) d v d \sigma \\
& +\int_{\left\{t=T^{*}\right\} \cap \mathcal{R}\left(\tau_{1}, \tau_{2}\right)} O\left(r^{-a}\right)\left(\left(\partial_{v} \Phi\right)^{2}+\left(\partial_{u} \Phi\right)^{2}+\left|r^{-1} \partial_{\sigma} \Phi\right|^{2}+\left|r^{-1} \Phi\right|^{2}\right) d v d \sigma \\
& +\int_{\mathcal{S}_{\tau_{1}} \cap\left\{t \leq T^{*}\right\}} \chi O\left(r^{-a}\right)\left(\left(\partial_{v} \Phi\right)^{2}+r^{-1-\eta^{\prime}}\left(\partial_{u} \Phi\right)^{2}+\left|r^{-1} \partial_{\sigma} \Phi\right|^{2}+\left|r^{-1} \Phi\right|^{2}\right) d v d \sigma .
\end{aligned}
$$

Therefore, from (4.37), (4.38), (4.35) and the trace inequality

$$
\begin{aligned}
& \sum_{i=1}^{2} \int_{\mathcal{S}_{\tau_{i}} \cap\left\{t \leq T^{*}\right\}}|\partial \chi| \cdot r^{-1}|\Phi|^{2} d v d \sigma \\
& \quad \leq \int_{\mathcal{R}\left(\tau_{1}, \tau_{2}\right) \cap\left\{t \leq T^{*}\right\}} O(|\partial \chi|) \cdot\left(r^{-1}|\partial \Phi|^{2}+r^{-1}|\Phi|^{2}\right) d u d v d \sigma,
\end{aligned}
$$

we obtain if $R$ is large enough in terms of the geometry of $\left(\mathcal{N}_{a f}, g\right)$ :

$$
\begin{aligned}
\int_{\mathcal{S}_{\tau_{2}} \cap\left\{t \leq T^{*}\right\}} \chi \cdot J_{\mu}^{T}(\varphi) \bar{n}^{\mu}+\int_{\left\{t=T^{*}\right\} \cap \mathcal{R}\left(\tau_{1}, \tau_{2}\right)}\left(J_{\mu}^{T}(\varphi) n^{\mu}+r^{-2}|\varphi|^{2}\right) \\
\leq C \cdot\left\{\int_{\mathcal{S}_{\tau_{1}} \cap\left\{t \leq T^{*}\right\}} \chi \cdot J_{\mu}^{T}(\varphi) \bar{n}^{\mu}+\int_{\mathcal{R}\left(\tau_{1}, \tau_{2}\right) \cap\left\{t \leq T^{*}\right\}} \chi\right. \\
\quad \cdot\left\{r^{-1-a} J_{\mu}^{T}(\varphi) n^{\mu}+r^{-1}\left(\left|\partial_{v} \varphi\right|^{2}+\left|r^{-1} \partial_{\sigma} \varphi\right|^{2}+\left|r^{-1} \varphi\right|^{2}\right)\right\} \\
+\int_{\mathcal{R}\left(\tau_{1}, \tau_{2}\right) \cap\left\{t \leq T^{*}\right\}} O(|\partial \chi|) \cdot\left(|\partial \varphi|^{2}+r^{-1}|\varphi|^{2}\right)+\int_{\mathcal{R}\left(\tau_{1}, \tau_{2}\right) \cap\left\{t \leq T^{*}\right\}} \chi O(1) \\
\left.\quad \cdot \operatorname{Re}\left\{\Omega^{-1}\left(\partial_{v}+\partial_{u}\right)(\Omega \bar{\varphi}) \cdot \square \varphi\right\}\right\} .
\end{aligned}
$$

Using (4.4) for some fixed $\eta<a$, we thus obtain (4.30) from (4.40) provided $R$ is large in terms of the geometry of $\left(\mathcal{N}_{a f}, g\right)$.

We can also establish the following generalisation of Lemma 4.5:

Lemma 4.6 For any smooth function $\varphi: \mathcal{N}_{a f} \rightarrow \mathbb{C}$, any $\chi: \mathcal{N}_{a f} \rightarrow[0,1]$ supported on $\{r \geq R\}$ for some $R>0$ large in terms of the geometry of $\left(\mathcal{N}_{a f}, g\right)$, and any two smooth, spacelike hypersurfaces $\mathcal{S}_{1}, \mathcal{S}_{2}$ of $\mathcal{N}_{\text {af }}$ intersecting the region $\{r \leq R\}$ such 
that $\mathcal{S}_{2}$ lies in the future domain of dependence of $\mathcal{S}_{1} \cup\{r \leq R\}$, the following estimate is true:

$$
\begin{aligned}
\int_{\mathcal{S}_{2}} & \chi \cdot J_{\mu}^{T}(\varphi) n_{\mathcal{S}_{2}}^{\mu} \\
& \leq C \cdot\left\{\int_{\mathcal{S}_{1}} \chi \cdot J_{\mu}^{T}(\varphi) n_{\mathcal{S}_{1}}^{\mu}+\int_{J^{+}\left(\mathcal{S}_{1}\right) \cap J^{-}\left(\mathcal{S}_{2}\right)} \chi \cdot r^{-1}\left(\left|\partial_{v} \varphi\right|^{2}+\left|r^{-1} \partial_{\sigma} \varphi\right|^{2}+\left|r^{-1} \varphi\right|^{2}\right)\right. \\
& +\int_{J^{+}\left(\mathcal{S}_{1}\right) \cap J^{-}\left(\mathcal{S}_{2}\right)} O(|\partial \chi|) \cdot\left(|\partial \varphi|^{2}+r^{-1}|\varphi|^{2}\right) \\
& \left.+\int_{J^{+}\left(\mathcal{S}_{1}\right) \cap J^{-}\left(\mathcal{S}_{2}\right)} \chi O(1) \cdot \operatorname{Re}\left\{\Omega^{-1}\left(\partial_{v}+\partial_{u}\right)(\Omega \bar{\varphi}) \cdot \square \varphi\right\}\right\}
\end{aligned}
$$

for some constant $C>0$ depending only on the geometry of $\left(\mathcal{N}_{a f}, g\right)$ and the precise form of $\mathcal{S}_{1}, \mathcal{S}_{2}$ but independent of translation of these hypersurfaces by the flow of $T=\partial_{u}+\partial_{v}$. In the above, $n_{\mathcal{S}_{i}}$ is the future directed unit normal of $\mathcal{S}_{i}$. Integration over $\mathcal{S}_{i}$ is performed using the volume form of the induced metric, while integration over $J^{+}\left(\mathcal{S}_{1}\right) \cap J^{-}\left(\mathcal{S}_{2}\right)$ is performed using the natural volume form of $g$.

Remark Again, in case the radiative components of the metric satisfy the bounds $\partial_{u} M \leq 0$ and $\left|\partial_{u} h_{a s}\right|+\left|r \partial_{u} h_{\mathbb{S}^{d-1}}\right| \ll-\left(\partial_{u} M\right)+O\left(r^{-a}\right)$ (which includes the nonradiating case $\partial_{u} M=0, h_{a s}=0$ and $\left.h_{\mathbb{S} d-1}=O\left(r^{-1-a}\right)\right)$, the second term of the right hand side of (4.41) can be omitted. Furthermore, in case the $T$ vector field satisfies (9.6) for $m=1$, then the second term of the right hand side of (4.41) is replaced by

$$
\int_{J^{+}\left(\mathcal{S}_{1}\right) \cap J^{-}\left(\mathcal{S}_{2}\right)} \chi \cdot \bar{t}^{-\delta 0} r^{-1}\left(\left|\partial_{v} \varphi\right|^{2}+\left|r^{-1} \partial_{\sigma} \varphi\right|^{2}+r^{-2}|\varphi|^{2}\right) .
$$

Proof The proof of Lemma 4.6 follows in exactly the same way as that of Lemma 4.5 , by integrating $\chi\left(\partial_{v}+\partial_{u}\right) \Phi \cdot \Omega \square \varphi$ over $J^{+}\left(\mathcal{S}_{1}\right) \cap J^{-}\left(\mathcal{S}_{2}\right)$ in place of $\mathcal{R}\left(\tau_{1}, \tau_{2}\right)$. Hence, the details will be omitted.

Finally, we will also establish the following improvement of Lemma 4.5 for higher order derivatives of $\varphi$, which will be used in Section 8:

Lemma 4.7 For any smooth function $\varphi: \mathcal{N}_{\text {af }} \rightarrow \mathbb{C}$, any $\chi: \mathcal{N}_{a f} \rightarrow[0,1]$ supported on $\{r \geq R\}$ for some $R>0$ large in terms of the geometry of $\left(\mathcal{N}_{a f}, g\right)$, any $\tau_{1} \leq \tau_{2}$ and any $T^{*}>0$ the following estimate is true:

$$
\begin{aligned}
& \sum_{j=0}^{k} \sum_{j_{1}+j_{2}+j_{3}=j}\left\{\int_{\mathcal{S}_{\tau_{2}} \cap\left\{t \leq T^{*}\right\}} \chi \cdot r^{2 j_{1}} J_{\mu}^{T}\left(\Omega^{-1} \partial_{v}^{j_{1}} \partial_{\sigma}^{j_{2}} \partial_{u}^{j_{3}}(\Omega \varphi)\right) \bar{n}^{\mu}\right. \\
& \left.\quad+\int_{\left\{t=T^{*}\right\} \cap \mathcal{R}\left(\tau_{1}, \tau_{2}\right)} r^{2 j_{1}}\left(J_{\mu}^{T}\left(\Omega^{-1} \partial_{v}^{j_{1}} \partial_{\sigma}^{j_{2}} \partial_{u}^{j_{3}}(\Omega \varphi)\right) n^{\mu}\right)\right\} \\
& \leq C_{k} \cdot \sum_{j=0}^{k} \sum_{j_{1}+j_{2}+j_{3}=j}\left\{\int_{\mathcal{S}_{\tau_{1}} \cap\left\{t \leq T^{*}\right\}} \chi \cdot r^{2 j_{1}} J_{\mu}^{T}\left(\Omega^{-1} \partial_{v}^{j_{1}} \partial_{\sigma}^{j_{2}} \partial_{u}^{j_{3}}(\Omega \varphi)\right) \bar{n}^{\mu}\right.
\end{aligned}
$$




$$
\begin{aligned}
& +\int_{\mathcal{R}\left(\tau_{1}, \tau_{2}\right) \cap\left\{t \leq T^{*}\right\}} \chi \cdot r^{2 j_{1}-1} \Omega^{-2} \\
& \times\left(\left|\partial_{v}^{j_{1}+1} \partial_{\sigma}^{j_{2}} \partial_{u}^{j_{3}}(\Omega \varphi)\right|^{2}+r^{-2}\left|\partial_{v}^{j_{1}} \partial_{\sigma}^{j_{2}+1} \partial_{u}^{j_{3}}(\Omega \varphi)\right|^{2}+r^{-2}\left|\partial_{v}^{j_{1}} \partial_{\sigma}^{j_{2}} \partial_{u}^{j_{3}}(\Omega \varphi)\right|^{2}\right) \\
& +\int_{\mathcal{R}\left(\tau_{1}, \tau_{2}\right) \cap\left\{t \leq T^{*}\right\}} O(|\partial \chi|) \cdot r^{2 j_{1}} \Omega^{-2}\left(\left|\partial \partial_{v}^{j_{1}} \partial_{\sigma}^{j_{2}} \partial_{u}^{j_{3}}(\Omega \varphi)\right|^{2}+r^{-1}\left|\partial_{v}^{j_{1}} \partial_{\sigma}^{j_{2}} \partial_{u}^{j_{3}}(\Omega \varphi)\right|^{2}\right) \\
& +\int_{\mathcal{R}\left(\tau_{1}, \tau_{2}\right) \cap\left\{t \leq T^{*}\right\}} \chi O(1) r^{2 j_{1}} \Omega^{-2} \\
& \left.\cdot \operatorname{Re}\left\{\left(\partial_{v}+\partial_{u}\right)\left(\partial_{v}^{j_{1}} \partial_{\sigma}^{j_{2}} \partial_{u}^{j_{3}}(\Omega \bar{\varphi})\right) \cdot \partial_{v}^{j_{1}} \partial_{\sigma}^{j_{2}} \partial_{u}^{j_{3}}(\Omega \square \varphi)\right\}\right\} .
\end{aligned}
$$

for some constant $C_{k}>0$ depending only on the geometry of $\left(\mathcal{N}_{a f}, g\right)$ and $k$.

Remark Again, in case the radiative components of the metric satisfy the bounds $\partial_{u} M \leq 0$ and $\left|\partial_{u} h_{a s}\right|+\left|r \partial_{u} h_{\mathbb{S} d-1}\right| \ll-\left(\partial_{u} M\right)+O\left(r^{-a}\right)$ (which includes the nonradiating case $\partial_{u} M=0, h_{a s}=0$ and $h_{\mathbb{S}^{d-1}}=O\left(r^{-1-a}\right)$ ), the second term of the right hand side of (4.43) can be omitted. Furthermore, in case the $T$ vector field satisfies (9.6) for $m=1$, then the integrand of the second term of the right hand side of (4.43) has an extra $\bar{t}^{-\delta_{0}}$ factor.

Proof The proof of (4.43) follows in exactly the same way as the proof of (4.30), by integrating (for real $\varphi$ ) for any $0 \leq j \leq k$ and any partition $j_{1}, j_{2}, j_{3}$ of $j$ :

$$
\chi \cdot\left(\partial_{v}+\partial_{u}\right)\left(r^{j_{1}} \partial_{v}^{j_{1}} \partial_{\sigma}^{j_{2}} \partial_{u}^{j_{3}}(\Omega \varphi)\right) \cdot \Omega \square\left(\Omega^{-1} r^{j_{1}} \partial_{v}^{j_{1}} \partial_{\sigma}^{j_{2}} \partial_{u}^{j_{3}}(\Omega \varphi)\right)
$$

over $\mathcal{R}\left(\tau_{1}, \tau_{2}\right) \cap\left\{t \leq T^{*}\right\}$ with $d u d v d \sigma$ as volume form, and then summing the resulting estimates over all possible $j_{1}, j_{2}, j_{3}$, using Lemma 6.3 to obtain an expression the commutator of $\square$ with $\partial_{v}, \partial_{\sigma}, \partial_{u}$.

\section{The Extension of the $r^{p}$-Weighted Energy Hierarchy}

In this section, we will generalise the $r^{p}$-weighted energy hierarchy introduced in [11], so as to apply to the asymptotically flat region of a broad class of stationary and asymptotically flat spacetimes, modeled on the spacetime $\left(\mathcal{N}_{a f}, g\right)$. This will be achieved by repeating the main steps of the method of [11] in the the case of Minkowski spacetime and controlling the error terms with the use of Lemmas 4.1 and 4.5 .

\subsection{Statement of the $r^{p}$-Weighted Energy Hierarchy}

We will establish the following decay estimate for solutions to $\square \varphi=F$ on $\left(\mathcal{N}_{a f}, g\right)$ :

Theorem 5.1 For any $0<p \leq 2$, any given $0<\eta<a$ and $0<\delta<1$, any $R>0$ large enough in terms of $p, \eta, \delta$ and any $\tau_{1} \leq \tau_{2}$, the following inequality is true 
for any smooth function $\varphi: \mathcal{N}_{a f} \rightarrow \mathbb{C}$ and any smooth cut-off $\chi_{R}: \mathcal{N}_{a f} \rightarrow[0,1]$ supported in $\{r \geq R\}$ :

$$
\begin{aligned}
& \mathcal{E}_{\text {bound }, R ; \delta}^{(p)}[\varphi]\left(\tau_{2}\right)+\int_{\tau_{1}}^{\tau_{2}} \mathcal{E}_{\text {bulk,R, }, ; \delta}^{(p-1)}[\varphi](\tau) d \tau+\limsup _{T^{*} \rightarrow+\infty} \mathcal{E}_{\mathcal{I}^{+}, T^{*} ; \delta}^{(p)}[\varphi]\left(\tau_{1}, \tau_{2}\right) \lesssim p, \eta, \delta \\
& \lesssim_{p, \eta, \delta} \mathcal{E}_{\text {bound }, R ; \delta}^{(p)}[\varphi]\left(\tau_{1}\right)+\int_{\mathcal{R}\left(t_{1}, t_{2}\right)}\left|\partial \chi_{R}\right| \cdot\left(r^{p}|\partial \varphi|^{2}+r^{p-2} \cdot|\varphi|^{2}\right) \\
& +\int_{\mathcal{R}\left(t_{1}, t_{2}\right)} \chi_{R} \cdot\left(r^{p+1}+r^{1+\eta}\right) \cdot\left|\square_{g} \varphi\right|^{2} \Omega^{2} d u d v d \sigma .
\end{aligned}
$$

In the above, the constants implicit in the $\lesssim p, \eta, \delta$ notation depend only on $p, \eta, \delta$ and on the geometry of $\left(\mathcal{N}_{a f}, g\right)$, and the $p$-weighted energies are defined as follows:

$$
\begin{aligned}
& \mathcal{E}_{\text {bound }, R ; \delta}^{(p)}[\varphi](\tau) \\
& =\int_{\mathcal{S}_{\tau}} \chi_{R}\left(r^{p}\left|\partial_{v}(\Omega \varphi)\right|^{2}+r^{-1-\eta^{\prime}}\left(r^{p}\left|r^{-1} \partial_{\sigma}(\Omega \varphi)\right|^{2}\right.\right. \\
& \left.\left.\quad+\left((d-3) r^{p-2}+\min \left\{r^{p-2}, r^{-\delta}\right\}\right)|\Omega \varphi|^{2}\right)\right) d v d \sigma+\int_{\mathcal{S}_{\tau}} \chi_{R} J_{\mu}^{T}(\varphi) \bar{n}^{\mu} \\
& \mathcal{E}_{\text {bulk,R, }{ }^{(p-1)}[\varphi](\tau)} \\
& =\int_{\mathcal{S}_{\tau}} \chi_{R}\left(p r^{p-1}\left|\partial_{v}(\Omega \varphi)\right|^{2}\right. \\
& \quad+\left\{\left((2-p) r^{p-1}+r^{p-1-\delta}\right)\left|r^{-1} \partial_{\sigma}(\Omega \varphi)\right|^{2}\right. \\
& \left.\left.\quad+\left((2-p)(d-3) r^{p-3}+\min \left\{r^{p-3}, r^{-1-\delta}\right\}\right)|\Omega \varphi|^{2}\right\}\right) d v d \sigma \\
& \quad+\int_{\mathcal{S}_{\tau}} \chi_{R} r^{-1-\eta}\left|\partial_{u}(\Omega \varphi)\right|^{2} d v d \sigma
\end{aligned}
$$

and

$$
\begin{aligned}
\mathcal{E}_{\mathcal{I}^{+}, T^{*}, \delta}^{(p)}[\varphi]\left(\tau_{1}, \tau_{2}\right)= & \int_{\mathcal{R}\left(\tau_{1}, \tau_{2}\right) \cap\left\{t=T^{*}\right\}}\left(r^{p}\left|r^{-1} \partial_{\sigma}(\Omega \varphi)\right|^{2}\right. \\
& \left.+\left((d-3) r^{p-2}+\min \left\{r^{p-2}, r^{-\delta}\right\}\right)|\Omega \varphi|^{2}\right) d v d \sigma \\
& +\int_{\mathcal{R}\left(\tau_{1}, \tau_{2}\right) \cap\left\{t=T^{*}\right\}} J_{\mu}^{T}(\varphi) n^{\mu}
\end{aligned}
$$

Remark In fact, it follows from the proof of Theorem 5.1 that (5.1) can be improved into: 


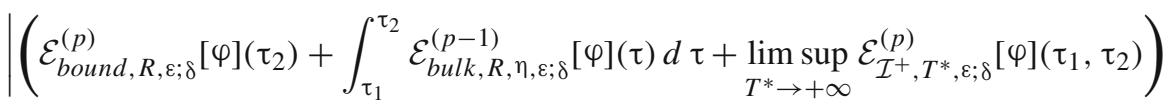

$$
\begin{aligned}
& -\mathcal{E}_{\text {bound }, R, \varepsilon ; \delta}^{(p)}[\varphi]\left(\tau_{1}\right) \\
& \leq C_{p, \eta, \delta} \cdot \varepsilon \cdot \mathcal{E}_{\text {bound }, R, \varepsilon ; \delta}^{(p)}[\varphi]\left(\tau_{1}\right)+C_{p, \eta, \delta, \varepsilon} \int_{\mathcal{R}\left(t_{1}, t_{2}\right)}\left|\partial \chi_{R}\right| \cdot\left(r^{p}|\partial \varphi|^{2}+r^{p-2} \cdot|\varphi|^{2}\right) \\
& +C_{p, \eta, \delta, \varepsilon} \int_{\mathcal{R}\left(t_{1}, t_{2}\right)} \chi_{R} \cdot\left(r^{p+1}+r^{1+\eta}\right) \cdot\left|\square_{g} \varphi\right|^{2} \Omega^{2} d u d v d \sigma
\end{aligned}
$$

for any $0<\delta<a$ and $0<\varepsilon<1$, where

$$
\begin{aligned}
\mathcal{E}_{\text {bound }, R, \varepsilon ; \delta}^{(p)}[\varphi](\tau)= & \int_{\mathcal{S}_{\tau}} \chi_{R}\left(r^{p}\left|\partial_{v}(\Omega \varphi)\right|^{2}+r^{-1-\eta^{\prime}}\left(r^{p}\left|r^{-1} \partial_{\sigma}(\Omega \varphi)\right|^{2}\right.\right. \\
& \left.\left.+\left((d-3) r^{p-2}+\varepsilon \cdot \min \left\{r^{p-2}, r^{-\delta}\right\}\right)|\Omega \varphi|^{2}\right)\right) d v d \sigma \\
& +\int_{\mathcal{S}_{\tau}} \chi_{R} J_{\mu}^{T}(\varphi) \bar{n}^{\mu} \\
\mathcal{E}_{\text {bulk,R, }, \varepsilon ; \delta}^{(p-1)}[\varphi](\tau)= & \int_{\mathcal{S}_{\tau}} \chi_{R}\left(p r^{p-1}\left|\partial_{v}(\Omega \varphi)\right|^{2}\right. \\
& +\left((2-p) r^{p-1}+r^{p-1-\delta}\right)\left|r^{-1} \partial_{\sigma}(\Omega \varphi)\right|^{2} \\
& \left.+\left((2-p)(d-3) r^{p-3}+\varepsilon \cdot \min \left\{r^{p-3}, r^{-1-\delta}\right\}\right)|\Omega \varphi|^{2}\right) d v d \sigma \\
& +\int_{\mathcal{S}_{\tau}} \chi_{R} r^{-1-\eta}\left|\partial_{u}(\Omega \varphi)\right|^{2} d v d \sigma
\end{aligned}
$$

and

$$
\begin{aligned}
\mathcal{E}_{\mathcal{I}^{+}, T^{*}, \varepsilon ; \delta}^{(p)}[\varphi]\left(\tau_{1}, \tau_{2}\right)= & \int_{\mathcal{R}\left(\tau_{1}, \tau_{2}\right) \cap\left\{t=T^{*}\right\}}\left(r^{p}\left|r^{-1} \partial_{\sigma}(\Omega \varphi)\right|^{2}\right. \\
& \left.+\left((d-3) r^{p-2}+\varepsilon \cdot \min \left\{r^{p-2}, r^{-\delta}\right\}\right)|\Omega \varphi|^{2}\right) d v d \sigma \\
& +\int_{\mathcal{R}\left(\tau_{1}, \tau_{2}\right) \cap\left\{t=T^{*}\right\}} J_{\mu}^{T}(\varphi) n^{\mu}
\end{aligned}
$$

Using Lemma 6.3 on the commutator of $\square$ with $\partial_{v}, \partial_{\sigma}$ and $\partial_{u}$, we can also obtain the following corollary of Theorem 5.1:

Corollary 5.2 Using the notations of Theorem 5.1, for any $0<p \leq 2$, any integer $m \geq 0$, any given $0<\eta<a$ and $0<\delta<1$, any $R>0$ large enough in terms of $p, \eta, \delta$ and any $\tau_{1} \leq \tau_{2}$, the following inequality is true for any smooth function $\varphi: \mathcal{N}_{a f} \rightarrow \mathbb{C}$ and any smooth cut-off $\chi_{R}: \mathcal{N}_{a f} \rightarrow[0,1]$ supported in $\{r \geq R\}:$

$$
\sum_{j=0}^{m-1} \sum_{j_{1}+j_{2}+j_{3}=j}\left\{\mathcal{E}_{\text {bound }, R ; \delta}^{(p)}\left[r^{-j_{2}} \partial_{v}^{j_{1}} \partial_{\sigma}^{j_{2}} \partial_{u}^{j_{3}} \varphi\right]\left(\tau_{2}\right)\right.
$$




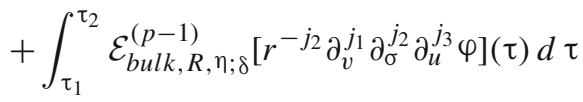

$$
\begin{aligned}
& \left.+\limsup _{T^{*} \rightarrow+\infty} \mathcal{E}_{\mathcal{I}^{+}, T^{*} ; \delta}^{(p)}\left[r^{-j_{2}} \partial_{v}^{j_{1}} \partial_{\sigma}^{j_{2}} \partial_{u}^{j_{3}} \varphi\right]\left(\tau_{1}, \tau_{2}\right)\right\}
\end{aligned}
$$

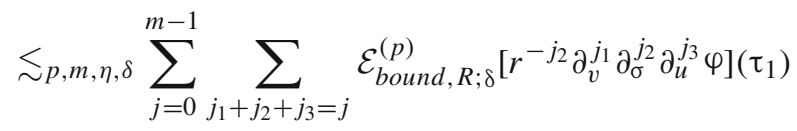

$$
\begin{aligned}
& +\sum_{j=0}^{m-1} \int_{\mathcal{R}\left(t_{1}, t_{2}\right)}\left|\partial \chi_{R}\right| \cdot\left(r^{p}\left|\partial^{j+1} \varphi\right|^{2}+r^{p-2} \cdot\left|\partial^{j} \varphi\right|^{2}\right) \\
& +\sum_{j=0}^{m-1} \int_{\mathcal{R}\left(t_{1}, t_{2}\right)} \chi_{R} \cdot\left(r^{p+1}+r^{1+\eta}\right) \cdot\left|\partial^{j}\left(\square_{g} \varphi\right)\right|^{2} \Omega^{2} d u d v d \sigma .
\end{aligned}
$$

The proof of Corollary 5.2 will be omitted. Notice that in comparison to the improved hierarchy statement (6.1), the estimate (5.9) holds for smaller values of $p$ and the terms of all orders in $\varphi$ appear with the same weight.

We can also establish the following variant of Theorem 5.1 in the region bounded by two hypersurfaces of the form $\{t=$ const $\}$ :

Theorem 5.3 For any $0<p \leq 2$, any given $0<\eta<a$ and $0<\delta<1$, any $R>0$ large enough in terms of $p, \eta, \delta$ and any $t_{1} \leq t_{2}$, the following inequality is true for any smooth function $\varphi: \mathcal{N}_{a f} \rightarrow \mathbb{C}$ with compact support in space and any smooth cut-off $\chi_{R}: \mathcal{N}_{\text {af }} \rightarrow[0,1]$ supported in $\{r \geq R\}$ :

$$
\begin{aligned}
\int_{\left\{t=t_{2}\right\}} \chi_{R} \cdot\left(r^{p}\left|\partial_{v}(\Omega \varphi)\right|^{2}+r^{p}\left|\frac{1}{r} \partial_{\sigma}(\Omega \varphi)\right|^{2}+\left((d-3) r^{p-2}\right.\right. \\
\left.\left.\quad+\min \left\{r^{p-2}, r^{-\delta}\right\}\right)|\Omega \varphi|^{2}\right) d v d \sigma+\int_{\left\{t=t_{2}\right\}} \chi_{R} J_{\mu}^{T}(\varphi) n^{\mu} \\
\quad+\int_{\left\{t_{1} \leq t \leq t_{2}\right\}} \chi_{R} \cdot\left(p r^{p-1}\left|\partial_{v}(\Omega \varphi)\right|^{2}+\left\{\left((2-p) r^{p-1}+r^{p-1-\delta}\right)\left|r^{-1} \partial_{\sigma}(\Omega \varphi)\right|^{2}\right.\right. \\
\left.\quad+\left((2-p)(d-3) r^{p-3}+\min \left\{r^{p-3}, r^{-1-\delta}\right\}\right)|\Omega \varphi|^{2}\right\} \\
\left.\quad+r^{-1-\eta}\left|\partial_{u}(\Omega \varphi)\right|^{2}\right) d u d v d \sigma \\
\lesssim \\
\quad+, \eta, \delta \int_{\left\{t=t_{1}\right\}} \chi_{R} \cdot\left(r^{p}\left|\partial_{v}(\Omega \varphi)\right|^{2}+r^{p}\left|\frac{1}{r} \partial_{\sigma}(\Omega \varphi)\right|^{2}\right. \\
\left.\left.\quad+(d-3) r^{p-2}+\min \left\{r^{p-2}, r^{-\delta}\right\}\right)|\Omega \varphi|^{2}\right) d v d \sigma \\
\quad+\int_{\left\{t=t_{1}\right\}} \chi_{R} J_{\mu}^{T}(\varphi) n^{\mu}+\int_{\left\{t_{1} \leq t \leq t_{2}\right\}}\left|\partial \chi_{R}\right| \cdot\left(r^{p}|\partial \varphi|^{2}+r^{p-2} \cdot|\varphi|^{2}\right) \\
\quad+\int_{\left\{t_{1} \leq t \leq t_{2}\right\}} \chi_{R} \cdot\left(r^{p+1}+r^{1+\eta}\right) \cdot\left|\square_{g} \varphi\right|^{2} \Omega^{2} d u d v d \sigma .
\end{aligned}
$$


In the above, the constants implicit in the $\lesssim_{p, \eta, \delta}$ notation depend only on $p, \eta, \delta$ and on the geometry of $\left(\mathcal{N}_{a f}, g\right)$.

The proof of Theorem 5.3 is identical to the proof of Theorem 5.1 (the only difference being the domain over which integrations are performed), and hence it will be omitted.

\subsection{Proof of Theorem 5.1}

For the proof of Theorem 5.1, we will need to introduce the following energy norms on the hyperboloids $\{\bar{t}=\tau\}$ :

$$
\begin{aligned}
& \mathcal{E}_{\text {bound }, R, T^{*}}^{(p)}[\varphi](\tau)=\int_{\mathcal{S}_{\tau} \cap\left\{t \leq T^{*}\right\}} \chi_{R}\left(r^{p}\left|\partial_{v}(\Omega \varphi)\right|^{2}+r^{-1-\eta^{\prime}}\left(r^{p}\left|r^{-1} \partial_{\sigma}(\Omega \varphi)\right|^{2}\right.\right. \\
& \left.\left.+\frac{(d-1)(d-3)}{4} r^{p-2}|\Omega \varphi|^{2}\right)\right) d v d \sigma, \\
& \mathcal{E}_{\text {bulk,R,T* }}^{(p-1)}[\varphi](\tau)=\int_{\mathcal{S}_{\tau} \cap\left\{t \leq T^{*}\right\}} \chi_{R}\left(p r^{p-1}\left|\partial_{v}(\Omega \varphi)\right|^{2}+(2-p)\left(r^{p-1}\left|r^{-1} \partial_{\sigma}(\Omega \varphi)\right|^{2}\right.\right. \\
& \left.\left.+\frac{(d-1)(d-3)}{4} r^{p-3}|\Omega \varphi|^{2}\right)\right) d v d \sigma,
\end{aligned}
$$

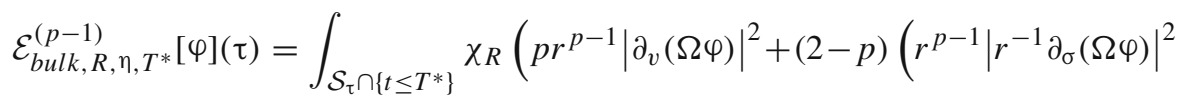

$$
\begin{aligned}
& \left.\left.+\frac{(d-1)(d-3)}{4} r^{p-3}|\Omega \varphi|^{2}\right)\right) d v d \sigma \\
& +\int_{\mathcal{S}_{\tau} \cap\left\{t \leq T^{*}\right\}} \chi_{R} r^{-1-\eta}\left|\partial_{u}(\Omega \varphi)\right|^{2} d v d \sigma
\end{aligned}
$$

and

$$
\begin{aligned}
\mathcal{E}_{\mathcal{I}^{+}, R, T^{*}}^{(p)}[\varphi]\left(\tau_{1}, \tau_{2}\right)= & \int_{\mathcal{R}\left(\tau_{1}, \tau_{2}\right) \cap\left\{t=T^{*}\right\}} \chi_{R}\left(r^{p}\left|r^{-1} \partial_{\sigma}(\Omega \varphi)\right|^{2}\right. \\
& \left.+\frac{(d-1)(d-3)}{4} r^{p-2}|\Omega \varphi|^{2}\right) d v d \sigma .
\end{aligned}
$$

The main step in establishing Theorem 5.1 is contained in the following Lemma:

Lemma 5.4 For any $0<p \leq 2$, any given $0<\eta<a$, any $0<\varepsilon<1$, any $R>0$ large enough in terms of $p, \eta$ and $\varepsilon$, any $\tau_{1} \leq \tau_{2}$ and any $T^{*} \geq 0$, the following inequality holds for any smooth function $\varphi: \mathcal{N}_{a f} \rightarrow \mathbb{C}$ and any smooth cut-off $\chi_{R}: \mathcal{N}_{a f} \rightarrow[0,1]$ supported in $\{r \geq R\}:$

$$
\begin{aligned}
& \mathcal{E}_{\text {bound }, R, T^{*}}^{(p)}[\varphi]\left(\tau_{2}\right)+\int_{\tau_{1}}^{\tau_{2}} \mathcal{E}_{\text {bulk,R, }, T^{*}}^{(p-1)}[\varphi](\tau) d \tau+\mathcal{E}_{\mathcal{I}^{+}, R, T^{*}}^{(p)}[\varphi]\left(\tau_{1}, \tau_{2}\right) \\
& \leq\left(1+O_{p, \eta}(\varepsilon)\right) \mathcal{E}_{\text {bound }, R, T^{*}}^{(p)}[\varphi]\left(\tau_{1}\right)
\end{aligned}
$$




$$
\begin{aligned}
& +C_{p, \eta, \varepsilon} \int_{\mathcal{R}\left(\tau_{1}, \tau_{2}\right) \cap\left\{t \leq T^{*}\right\}}\left|\partial \chi_{R}\right| \cdot\left(r^{p}|\partial(\Omega \varphi)|^{2}+r^{p-2}|\Omega \varphi|^{2}\right) d u d v d \sigma \\
& +C_{p, \eta, \varepsilon} \int_{\mathcal{S}_{\tau_{1}} \cap\left\{t \leq T^{*}\right\}} \chi_{R} J_{\mu}^{T}(\varphi) \bar{n}^{\mu}+C_{p, \eta, \varepsilon} \sum_{i=1}^{2} \int_{\mathcal{S}_{\tau_{i}} \cap\left\{t \leq T^{*}\right\}} \chi_{R} \cdot r^{p-2-a}|\Omega \varphi|^{2} d v d \sigma \\
& +C_{p, \eta, \varepsilon} \int_{\mathcal{R}\left(\tau_{1}, \tau_{2}\right) \cap\left\{t=T^{*}\right\}} \chi_{R} \cdot r^{p-2-a}|\Omega \varphi|^{2} d v d \sigma \\
& +C_{p, \eta, \varepsilon} \int_{\mathcal{R}\left(\tau_{1}, \tau_{2}\right) \cap\left\{t \leq T^{*}\right\}} \chi_{R} \cdot \max \left\{r^{p-3-a}, r^{-3}\right\}|\Omega \varphi|^{2} d u d v d \sigma \\
& +C_{p, \eta, \varepsilon} \int_{\mathcal{R}\left(\tau_{1}, \tau_{2}\right) \cap\left\{t \leq T^{*}\right\}} \chi_{R} \cdot\left(r^{p+1}+r^{1+\eta}\right)|\Omega \square \varphi|^{2} d u d v d \sigma
\end{aligned}
$$

where $\bar{n}$ is the future directed unit normal to the hyperboloids $\mathcal{S}_{\tau}$.

Proof Without loss of generality we will assume that $\varphi$ is real valued. We will set

$$
\Phi \doteq \Omega \cdot \varphi
$$

Following the approach of [11], we start by multiplying both sides of (3.14) with $\chi_{R} \cdot r^{p} \partial_{v} \Phi$. We therefore compute, after applying the product rule for derivatives:

$$
\begin{aligned}
\chi_{R} \cdot r^{p} \partial_{v} \Phi \cdot \Omega \square \varphi= & -\frac{1}{2} \partial_{u}\left\{\chi_{R} \cdot r^{p}\left(1+O_{1}\left(r^{-1-a}\right)\right)\left|\partial_{v} \Phi\right|^{2}\right\} \\
& +\frac{1}{2}\left(\partial_{u}\left\{\chi_{R} \cdot r^{p}\left(1+O_{1}\left(r^{-1-a}\right)\right)\right\}\left|\partial_{v} \Phi\right|^{2}\right. \\
& -\frac{1}{2} \partial_{v}\left\{\chi_{R} \cdot\left(\frac{(d-1)(d-3)}{4} r^{p-2}\right) \cdot \Phi^{2}\right\} \\
& +\frac{1}{2} \partial_{v}\left\{\chi_{R} \cdot\left(\frac{(d-1)(d-3)}{4} r^{p-2}\right)\right\} \Phi^{2} \\
& +\chi_{R} \cdot r^{p-2} \cdot \partial_{v} \Phi \cdot \Delta_{g_{\mathbb{S} d-1}+h_{\mathbb{S} d-1} \Phi} \\
& +\chi_{R} \cdot r^{p} \partial_{v} \Phi \cdot \operatorname{Err}(\Phi)
\end{aligned}
$$

Integration of (5.17) over $\left\{\tau_{1} \leq \bar{t} \leq \tau_{2}\right\} \cap\left\{t \leq T^{*}\right\}$ using the coordinate volume form $d u d v d \sigma$ (in place of the geometric volume form $\Omega^{2} d u d v d \sigma$ ) readily yields:

$$
\begin{aligned}
& \int_{\mathcal{S}_{\tau_{2} \cap\left\{t \leq T^{*}\right\}}} \frac{1}{2} \chi_{R} \cdot\left(r^{p}\left(1+O\left(r^{-1-a}\right)\right) \cdot\left|\partial_{v} \Phi\right|^{2}\right) d v d \sigma \\
& +\int_{\mathcal{R}\left(\tau_{1}, \tau_{2}\right) \cap\left\{t=T^{*}\right\}} \frac{1}{2} \chi_{R} \cdot\left(r^{p}\left(1+O\left(r^{-1-a}\right)\right) \cdot\left|\partial_{v} \Phi\right|^{2}\right) d v d \sigma \\
& +\int_{\mathcal{S}_{\tau_{2} \cap\left\{t \leq T^{*}\right\}}} \frac{1}{2} \chi_{R} \cdot\left(\frac{(d-1)(d-3)}{4} r^{p-2}+O\left(r^{p-3}\right)\right) \Phi^{2} d u d \sigma \\
& +\int_{\mathcal{R}\left(\tau_{1}, \tau_{2}\right) \cap\left\{t=T^{*}\right\}} \frac{1}{2} \chi_{R} \cdot\left(\frac{(d-1)(d-3)}{4} r^{p-2}+O\left(r^{p-3}\right)\right) \Phi^{2} d u d \sigma
\end{aligned}
$$




$$
\begin{aligned}
& +\int_{\mathcal{R}\left(t_{1}, t_{2}\right) \cap\left\{t \leq T^{*}\right\}} \chi_{R} \cdot\left\{\frac{1}{2} p r^{p-1}\left\{1+O\left(r^{-1-a}\right)\right\}\left|\partial_{v} \Phi\right|^{2}\right. \\
& \left.+\frac{1}{2} \chi_{R} \cdot\left((2-p) \frac{(d-1)(d-3)}{4} r^{p-3}+O\left(r^{p-4}\right)\right) \Phi^{2}\right\} d u d v d \sigma \\
& +\int_{\mathcal{R}\left(t_{1}, t_{2}\right) \cap\left\{t \leq T^{*}\right\}} \chi_{R} \cdot r^{p-2} \cdot \partial_{v} \Phi \cdot \Delta_{\mathbb{S}^{d-1}}+h_{\mathbb{S} d-1} \Phi d u d v d \sigma \\
& +\int_{\mathcal{R}\left(t_{1}, t_{2}\right) \cap\left\{t \leq T^{*}\right\}} \chi_{R} \cdot r^{p} \partial_{v} \Phi \cdot \operatorname{Err}(\Phi) d u d v d \sigma \\
& =\int_{\mathcal{S}_{\tau_{1}} \cap\left\{t \leq T^{*}\right\}} \frac{1}{2} \chi_{R} \cdot\left(r^{p}\left(1+O\left(r^{-1-a}\right)\right) \cdot\left|\partial_{v} \Phi\right|^{2}\right) d v d \sigma \\
& +\int_{\mathcal{S}_{\tau_{1} \cap\left\{t \leq T^{*}\right\}}} \frac{1}{2} \chi_{R} \cdot\left(\frac{(d-1)(d-3)}{4} r^{p-2}+O\left(r^{p-3}\right)\right) \Phi^{2} d u d \sigma \\
& +\int_{\mathcal{R}\left(t_{1}, t_{2}\right) \cap\left\{t \leq T^{*}\right\}} O\left(\left|\partial \chi_{R}\right|\right) \cdot\left(O\left(r^{p}\right) \cdot\left|\partial_{v} \Phi\right|^{2}+O\left(r^{p-2}\right) \cdot \Phi^{2}\right) d u d v d \sigma \\
& -\int_{\mathcal{R}\left(t_{1}, t_{2}\right) \cap\left\{t \leq T^{*}\right\}} \chi_{R} \cdot r^{p} \partial_{v} \Phi \cdot \Omega \square \varphi d u d v d \sigma .
\end{aligned}
$$

Moreover, we obtain after integrating by parts in the spherical directions (recalling that $\left.h_{\mathbb{S}^{d-1}}=O\left(r^{-1}\right)\right)$ :

$$
\begin{aligned}
& \int_{\mathcal{R}\left(t_{1}, t_{2}\right) \cap\left\{t \leq T^{*\}}\right.} \chi_{R} \cdot r^{p-2} \cdot \partial_{v} \Phi \cdot \Delta_{g_{\mathbb{S}^{d}-1}+h_{\mathbb{S} d-1}} \Phi d u d v d \sigma \\
& =\int_{\mathcal{R}\left(t_{1}, t_{2}\right) \cap\left\{t \leq T^{*}\right\}} \chi_{R} \cdot r^{p-2} \cdot \frac{1}{2} \partial_{v}\left\{\left|\partial_{\sigma} \Phi\right|^{2}\right\} d u d v d \sigma \\
& +\int_{\mathcal{R}\left(t_{1}, t_{2}\right) \cap\left\{t \leq T^{*}\right\}} \chi_{R} \cdot O\left(r^{p-3}\right) \cdot \partial_{v} \Phi \cdot \partial_{\sigma} \Phi d u d v d \sigma \\
& +\int_{\mathcal{R}\left(t_{1}, t_{2}\right) \cap\left\{t \leq T^{*}\right\}} O\left(\left|\partial_{\sigma} \chi_{R}\right|\right) \cdot r^{p-2} \cdot \partial_{v} \Phi \cdot \partial_{\sigma} \Phi d u d v d \sigma .
\end{aligned}
$$

Hence, integrating by parts in the first term of the right hand side of (5.19), substituting in (5.18) and absorbing the $\int \chi_{R} \cdot O\left(r^{p-3}\right) \cdot \partial_{v} \Phi \cdot \partial_{\sigma} \Phi$ summand of the right hand side of (5.19) into the $\operatorname{Err}$ term, we infer (provided $R$ is large enough in terms of $\varepsilon$ ):

$$
\begin{aligned}
& \mathcal{E}_{\text {bound }, R, T^{*}}^{(p)}[\varphi]\left(\tau_{2}\right)+\int_{\tau_{1}}^{\tau_{2}} \mathcal{E}_{\text {bulk,R,T*}}^{(p-1)}[\varphi](\tau) d \tau+\mathcal{E}_{\mathcal{I}^{+}, R, T^{*}}^{(p)}[\varphi]\left(\tau_{1}, \tau_{2}\right) \\
& \leq(1+\varepsilon) \mathcal{E}_{\text {bound }, R, T^{*}}^{(p)}[\varphi]\left(\tau_{1}\right)+\int_{\mathcal{R}\left(\tau_{1}, \tau_{2}\right) \cap\left\{t \leq T^{*}\right\}}\left|\partial \chi_{R}\right| \\
& \quad \times\left(O\left(r^{p}\right)|\partial(\Omega \varphi)|^{2}+O\left(r^{p-2}\right)|\Omega \varphi|^{2}\right) d u d v d \sigma \\
& +C \cdot \sum_{i=1}^{2} \int_{\mathcal{S}_{\tau_{i}} \cap\left\{t \leq T^{*}\right\}} \chi_{R} J_{\mu}^{T}(\varphi) \bar{n}^{\mu}+C \cdot \int_{\mathcal{R}\left(\tau_{1}, \tau_{2}\right) \cap\left\{t=T^{*}\right\}} \chi_{R} J_{\mu}^{T}(\varphi) \bar{n}^{\mu}
\end{aligned}
$$




$$
\begin{aligned}
& +\sum_{i=1}^{2} \int_{\mathcal{S}_{\tau_{i}} \cap\left\{t \leq T^{*}\right\}} \chi_{R} \cdot O\left(r^{p-2-a}\right)|\Omega \varphi|^{2} d v d \sigma \\
& +\int_{\mathcal{R}\left(\tau_{1}, \tau_{2}\right) \cap\left\{t=T^{*}\right\}} \chi_{R} \cdot O\left(r^{p-2-a}\right)|\Omega \varphi|^{2} d v d \sigma \\
& +\int_{\mathcal{R}\left(\tau_{1}, \tau_{2}\right) \cap\left\{t \leq T^{*}\right\}} \chi_{R} \cdot O\left(r^{p-3-a}\right)|\Omega \varphi|^{2} d u d v d \sigma \\
& +\int_{\mathcal{R}\left(t_{1}, t_{2}\right) \cap\left\{t \leq T^{*}\right\}} \chi_{R} \cdot O\left(r^{p}\right) \partial_{v} \Phi \cdot \operatorname{Err}(\Phi) d u d v d \sigma \\
& +\int_{\mathcal{R}\left(\tau_{1}, \tau_{2}\right) \cap\left\{t \leq T^{*}\right\}} \chi_{R} \cdot O\left(r^{p}\right) \partial_{v} \Phi \cdot \Omega \square \varphi d u d v d \sigma,
\end{aligned}
$$

where we denote for simplicity $|\partial \Phi|^{2}=\left|\partial_{v} \Phi\right|^{2}+\left|\partial_{u} \Phi\right|^{2}+\left|\frac{1}{r} \partial_{\sigma} \Phi\right|^{2}$. Notice that we have used the asymptotic relation $d u d \sigma \sim r^{-1-\eta^{\prime}} d v d \sigma$ on the hyperboloids $\mathcal{S}_{\tau}$.

The positive $\int_{\mathcal{R}\left(t_{1}, t_{2}\right) \cap\left\{t \leq T^{*}\right\}}(2-p) \chi_{R} r^{p-1}\left|\frac{1}{r} \partial_{\sigma} \Phi\right|^{2}$ term of the left hand side of (5.20) vanishes for $p=2$. Since it will prove useful to retain some control over angular derivatives in the left hand side of (5.20) even in the $p=2$ case, we will add to (5.20) the same inequality but for $p^{\prime}=p-a$ in place of $p$ in case $p \geq 1$, obtaining:

$$
\begin{aligned}
& \mathcal{E}_{\text {bound }, R, T^{*}}^{(p)}[\varphi]\left(\tau_{2}\right)+\int_{\tau_{1}}^{\tau_{2}}\left(\mathcal{E}_{\text {bulk,R,T* }}^{(p-1)}[\varphi](\tau)\right. \\
& \left.+\mathcal{E}_{\text {bulk,R,T*}}^{(p-1-a)}[\varphi](\tau)\right) d \tau+\mathcal{E}_{\mathcal{I}^{+}, R, T^{*}}^{(p)}[\varphi]\left(\tau_{1}, \tau_{2}\right) \\
& \leq(1+\varepsilon) \mathcal{E}_{\text {bound }, R, T^{*}}^{(p)}[\varphi]\left(\tau_{1}\right)+\int_{\mathcal{R}\left(\tau_{1}, \tau_{2}\right) \cap\left\{t \leq T^{*}\right\}}\left|\partial \chi_{R}\right| \cdot\left(O\left(r^{p}\right)|\partial(\Omega \varphi)|^{2}\right. \\
& \left.+O\left(r^{p-2}\right)|\Omega \varphi|^{2}\right) d u d v d \sigma+C \sum_{i=1}^{2} \int_{\mathcal{S}_{\tau_{i}} \cap\left\{t \leq T^{*}\right\}} \chi_{R} J_{\mu}^{T}(\varphi) \bar{n}^{\mu} \\
& +C \int_{\mathcal{R}\left(\tau_{1}, \tau_{2}\right) \cap\left\{t=T^{*}\right\}} \chi_{R} J_{\mu}^{T}(\varphi) \bar{n}^{\mu} \\
& +\sum_{i=1}^{2} \int_{\mathcal{S}_{\tau_{i}} \cap\left\{t \leq T^{*}\right\}} \chi_{R} \times O\left(r^{p-2-a}\right)|\Omega \varphi|^{2} d v d \sigma \\
& +\int_{\mathcal{R}\left(\tau_{1}, \tau_{2}\right) \cap\left\{t=T^{*}\right\}} \chi_{R} \cdot O\left(r^{p-2-a}\right)|\Omega \varphi|^{2} d v d \sigma \\
& +\int_{\mathcal{R}\left(\tau_{1}, \tau_{2}\right) \cap\left\{t \leq T^{*}\right\}} \chi_{R} \cdot O\left(r^{p-3-a}\right)|\Omega \varphi|^{2} d u d v d \sigma \\
& +\int_{\mathcal{R}\left(t_{1}, t_{2}\right) \cap\left\{t \leq T^{*}\right\}} \chi_{R} \cdot O\left(r^{p}\right) \partial_{v} \Phi \cdot \operatorname{Err}(\Phi) d u d v d \sigma \\
& +\int_{\mathcal{R}\left(\tau_{1}, \tau_{2}\right) \cap\left\{t \leq T^{*}\right\}} \chi_{R} \cdot O\left(r^{p}\right) \partial_{v} \Phi \cdot \Omega \square \varphi d u d v d \sigma .
\end{aligned}
$$


In order to reach (5.15) it only remains to add to (5.21) the estimate of Lemma (4.2) and suitably absorb the $\operatorname{Err}(\Phi)$ term in (5.21) into the left hand side. Moreover, the energy boundary terms on $\mathcal{S}_{\tau_{2}}$ and $\left\{t=T^{*}\right\}$ will be dealt with by using Lemma 4.5. In particular, assuming without loss of generality that $\varepsilon \ll_{p, \eta} 1$, if $R$ is large enough in terms of $\varepsilon, p, \eta$ the following estimate holds:

$$
\int_{\mathcal{R}\left(t_{1}, t_{2}\right) \cap\left\{t \leq T^{*}\right\}} \chi_{R} \cdot O\left(r^{p}\right) \partial_{v} \Phi \cdot \operatorname{Err}(\Phi) d u d v d \sigma \lesssim_{p, \eta} \mathcal{B}_{E r r, \eta, \varepsilon}^{(p)}[\varphi]\left(\tau_{1}, \tau_{2}\right)
$$

where

$$
\begin{aligned}
& \mathcal{B}_{E r r, \eta, \varepsilon}^{(p)}[\varphi]\left(\tau_{1}, \tau_{2}\right) \doteq \varepsilon\left\{\int_{\tau_{1}}^{\tau_{2}}\left(\mathcal{E}_{\text {bulk,R,T* }}^{(p-1)}[\varphi](\tau)+\mathcal{E}_{\text {bulk,R,T* }}^{(p-1-a)}[\varphi](\tau)\right) d \tau\right. \\
& \left.+\sum_{i=1}^{2} \mathcal{E}_{\text {bound }, R, T^{*}}^{(p)}[\varphi]\left(\tau_{i}\right)+\mathcal{E}_{\mathcal{I}^{+}, R, T^{*}}^{(p)}[\varphi]\left(\tau_{1}, \tau_{2}\right)\right\} \\
& +\int_{\mathcal{R}\left(\tau_{1}, \tau_{2}\right) \cap\left\{t \leq T^{*}\right\}}\left|\partial \chi_{R}\right| \cdot\left(r^{p}|\partial(\Omega \varphi)|^{2}+r^{p-2}|\Omega \varphi|^{2}\right) d u d v d \sigma \\
& +\sum_{i=1}^{2} \int_{\mathcal{S}_{\tau_{i}} \cap\left\{t \leq T^{*}\right\}} \chi_{R} J_{\mu}^{T}(\varphi) \bar{n}^{\mu}+\int_{\mathcal{R}\left(\tau_{1}, \tau_{2}\right) \cap\left\{t=T^{*}\right\}} \chi_{R} J_{\mu}^{T}(\varphi) \bar{n}^{\mu} \\
& +\sum_{i=1}^{2} \int_{\mathcal{S}_{\tau_{i}} \cap\left\{t \leq T^{*}\right\}} \chi_{R} \cdot r^{p-2-a}|\Omega \varphi|^{2} d v d \sigma \\
& +\int_{\mathcal{R}\left(\tau_{1}, \tau_{2}\right) \cap\left\{t=T^{*}\right\}} \chi_{R} \cdot r^{p-2-a}|\Omega \varphi|^{2} d v d \sigma \\
& +\int_{\mathcal{R}\left(\tau_{1}, \tau_{2}\right) \cap\left\{t \leq T^{*}\right\}} \chi_{R} \cdot \max \left\{r^{p-3-a}, r^{-3}\right\}|\Omega \varphi|^{2} d u d v d \sigma \\
& +\int_{\mathcal{R}\left(\tau_{1}, \tau_{2}\right) \cap\left\{t \leq T^{*}\right\}} \chi_{R} \cdot\left(r^{p+1}+r^{1+\eta}\right)|\Omega \square \varphi|^{2} d u d v d \sigma .
\end{aligned}
$$

Notice that in view of Lemma 4.5 and the fact that $0<p \leq 2$, the following bound also holds:

$$
\begin{aligned}
& \int_{\mathcal{S}_{\tau_{2}} \cap\left\{t \leq T^{*}\right\}} \chi_{R} J_{\mu}^{T}(\varphi) \bar{n}^{\mu}+\int_{\mathcal{R}\left(\tau_{1}, \tau_{2}\right) \cap\left\{t=T^{*}\right\}} \chi_{R} J_{\mu}^{T}(\varphi) \bar{n}^{\mu} \\
& \lesssim \eta \int_{\mathcal{S}_{\tau_{1} \cap\left\{t \leq T^{*}\right\}}} \chi_{R} J_{\mu}^{T}(\varphi) \bar{n}^{\mu} \\
& \quad+\varepsilon \int_{\tau_{1}}^{\tau_{2}}\left(\mathcal{E}_{\text {bulk,R,T* }}^{(p-1)}[\varphi](\tau)+\mathcal{E}_{\text {bulk,R,T* }}^{(p-1-a)}[\varphi](\tau)\right) d \tau \\
& \quad+\int_{\mathcal{R}\left(\tau_{1}, \tau_{2}\right) \cap\left\{t \leq T^{*}\right\}} \chi_{R} \cdot r^{-3}|\Omega \varphi|^{2} d u d v d \sigma
\end{aligned}
$$




$$
\begin{aligned}
& +\int_{\mathcal{R}\left(\tau_{1}, \tau_{2}\right) \cap\left\{t \leq T^{*}\right\}}\left|\partial \chi_{R}\right| \cdot\left(r^{p}|\partial(\Omega \varphi)|^{2}+r^{p-2}|\Omega \varphi|^{2}\right) d u d v d \sigma \\
& +\int_{\mathcal{R}\left(\tau_{1}, \tau_{2}\right) \cap\left\{t \leq T^{*}\right\}} \chi_{R} \cdot\left(r^{p+1}+r^{1+\eta}\right)|\Omega \square \varphi|^{2} d u d v d \sigma .
\end{aligned}
$$

Using (5.21), (5.26), (5.23), (5.22) and (5.24), inequality (5.15) follows readily, provided $\varepsilon$ has been chosen small enough in terms of $p$ and $\eta$ so that the first line of the right hand side of (5.23) can be absorbed into the left hand side of (5.21).

Notice that

$$
\begin{aligned}
& \int_{\mathcal{R}\left(t_{1}, t_{2}\right) \cap\left\{t \leq T^{*}\right\}} \chi_{R} \cdot O\left(r^{p}\right) \partial_{v} \Phi \cdot \operatorname{Err}(\Phi) d u d v d \sigma \\
& =\int_{\mathcal{R}\left(t_{1}, t_{2}\right) \cap\left\{t \leq T^{*}\right\}} \chi_{R} \partial_{v} \Phi \cdot\left\{O\left(r^{p-1}\right) \partial_{v}^{2} \Phi\right. \\
& \quad+O\left(r^{p-2}\right) \partial_{v} \partial_{\sigma} \Phi+O\left(r^{p-2-a}\right) \partial_{v}^{2} \Phi+O\left(r^{p-2-a}\right) \partial_{u} \partial_{\sigma} \Phi+O\left(r^{p-3-a}\right) \partial_{\sigma} \partial_{\sigma} \Phi \\
& \left.\quad+O\left(r^{p-2-a}\right) \partial_{\sigma} \Phi+O\left(r^{p-1-a}\right) \partial_{v} \Phi+O\left(r^{p-2-a}\right) \partial_{u} \Phi+O\left(r^{p-3}\right) \Phi\right\} d u d v d \sigma .
\end{aligned}
$$

In view of Lemma 4.1 and the fact that $0<p \leq 2$, we can bound for any $\varepsilon>0$ provided $R$ is large enough in terms of $\varepsilon, \eta, p$ :

$$
\begin{aligned}
& \int_{\mathcal{R}\left(\tau_{1}, \tau_{2}\right) \cap\left\{t \leq T^{*}\right\}} \chi_{R} \cdot r^{p-3-a}\left|\partial_{u} \Phi\right|^{2} d u d v d \sigma \\
& \quad \leq \int_{\mathcal{R}\left(\tau_{1}, \tau_{2}\right) \cap\left\{t \leq T^{*}\right\}} \chi_{R} \cdot r^{-1-\eta}\left|\partial_{u} \Phi\right|^{2} d u d v d \sigma \lesssim_{p, \eta} \\
& \quad \lesssim p, \eta \mathcal{B}_{E r r, \eta, \varepsilon}^{(p)}[\varphi]\left(\tau_{1}, \tau_{2}\right) .
\end{aligned}
$$

We can also trivialy bound (if $R$ is large enough in terms of $\varepsilon, \eta, p$ ):

$$
\begin{aligned}
& \int_{\mathcal{R}\left(\tau_{1}, \tau_{2}\right) \cap\left\{t \leq T^{*}\right\}} \chi_{R} \cdot r^{p-1-a}\left(\left|\partial_{v} \Phi\right|^{2}+\left|r^{-1} \partial_{\sigma} \Phi\right|^{2}+r^{-2}|\Phi|^{2}\right) d u d v d \sigma \\
& \quad \lesssim_{p, \eta} \mathcal{B}_{E r r, \eta, \varepsilon}^{(p)}[\varphi]\left(\tau_{1}, \tau_{2}\right) .
\end{aligned}
$$

Therefore, applying a Cauchy-Schwarz ineqality, all the terms of (5.25) which do not contain second order derivatives of $\Phi$ can be bounded by $\mathcal{B}_{E r r, \eta, \varepsilon}^{(p)}[\varphi]\left(\tau_{1}, \tau_{2}\right)$.

It thus remains to estimate the terms of $\int_{\mathcal{R}\left(t_{1}, t_{2}\right)} \chi_{R} \cdot r^{p} \cdot \partial_{v} \Phi \cdot \operatorname{Err}(\Phi) d u d v d \sigma$ which contain second order derivatives of $\Phi$, i.e. we have to bound (provided again that $R$ is large enough in terms of $p, \eta, \varepsilon)$ : 


$$
\begin{aligned}
& \int_{\mathcal{R}\left(\tau_{1}, \tau_{2}\right) \cap\left\{t \leq T^{*}\right\}} \chi_{R} \cdot\left\{O\left(r^{p-1}\right) \partial_{v} \Phi \cdot \partial_{v}^{2} \Phi+O\left(r^{p-2-a}\right) \partial_{v} \Phi \cdot \partial_{u}^{2} \Phi\right. \\
& \quad+O\left(r^{p-3-a}\right) \partial_{v} \Phi \cdot \partial_{\sigma} \partial_{\sigma} \Phi+O\left(r^{p-2-a}\right) \partial_{v} \Phi \cdot \partial_{u} \partial_{\sigma} \Phi \\
& \left.+O\left(r^{p-2}\right) \partial_{v} \Phi \cdot \partial_{v} \partial_{\sigma} \Phi\right\} d u d v d \sigma \lesssim_{p, \eta} \mathcal{B}_{E r r, \eta, \varepsilon}^{(p)}[\varphi]\left(\tau_{1}, \tau_{2}\right) .
\end{aligned}
$$

In order to estimate these terms, we will perform some integrations by parts over $\mathcal{R}\left(\tau_{1}, \tau_{2}\right) \cap\left\{t \leq T^{*}\right\}$ which will reduce the maximum number of derivatives of $\Phi$ appearing in these expressions to just one.

In particular, we proceed as follows:

1. For the part of $\int_{\mathcal{R}\left(t_{1}, t_{2}\right)} \chi_{R} \cdot r^{p} \cdot \partial_{v} \Phi \cdot \operatorname{Err}(\Phi)$ consisiting of terms of the form $\partial_{v} \Phi \cdot \partial_{i} \partial_{v} \Phi$, namely:

$$
\begin{aligned}
\operatorname{Err}_{v, i, v}^{(p)}[\varphi]\left(\tau_{1}, \tau_{2}\right) \doteq & \int_{\mathcal{R}\left(\tau_{1}, \tau_{2}\right) \cap\left\{t \leq T^{*}\right\}} \chi_{R} \\
& \times\left\{O\left(r^{p-1}\right) \partial_{v} \Phi \cdot \partial_{v}^{2} \Phi+O\left(r^{p-2}\right) \cdot \partial_{v} \Phi \cdot \partial_{v} \partial_{\sigma} \Phi\right\} d u d v d \sigma
\end{aligned}
$$

after putting each summand in the form $\frac{1}{2} \partial_{i}\left\{\left(\partial_{v} \Phi\right)^{2}\right\}$ and integrating by parts in $\partial_{i}$ we obtain

$$
\begin{aligned}
\operatorname{Err}_{v, i, v}^{(p)}[\varphi]\left(\tau_{1}, \tau_{2}\right) \lesssim & \int_{\mathcal{R}\left(\tau_{1}, \tau_{2}\right) \cap\left\{t \leq T^{*}\right\}} \chi_{R} \cdot r^{p-2} \cdot\left|\partial_{v} \Phi\right|^{2} d u d v d \sigma \\
& +\int_{\mathcal{R}\left(\tau_{1}, \tau_{2}\right) \cap\left\{t \leq T^{*}\right\}}\left|\partial \chi_{R}\right| \cdot r^{p-1} \cdot\left|\partial_{v} \Phi\right|^{2} d u d v d \sigma \\
& +\sum_{i=1}^{2} \int_{\mathcal{S}_{\tau_{i}} \cap\left\{t \leq T^{*}\right\}} \chi_{R} \cdot r^{p-2}\left|\partial_{v} \Phi\right|^{2} d v d \sigma \\
& +\int_{\mathcal{R}\left(\tau_{1}, \tau_{2}\right) \cap\left\{t=T^{*}\right\}} \chi_{R} \cdot r^{p-2}\left|\partial_{v} \Phi\right|^{2} d v d \sigma
\end{aligned}
$$

Thus, since $p \leq 2$, we immediately infer (provided again that $R$ is large enough in terms of $p, \eta, \varepsilon)$ that

$$
\operatorname{Err}_{v, i, v}^{(p)}[\varphi]\left(\tau_{1}, \tau_{2}\right) \lesssim_{p, \eta} \mathcal{B}_{E r r, \eta, \varepsilon}^{(p)}[\varphi]\left(\tau_{1}, \tau_{2}\right)
$$

2. For the part of $\int_{\mathcal{R}\left(t_{1}, t_{2}\right)} \chi_{R} \cdot r^{p} \cdot \partial_{v} \Phi \cdot \operatorname{Err}(\Phi)$ consisiting of terms of the form $\partial_{v} \Phi \partial_{i}^{2} \Phi$, namely

$$
\begin{aligned}
\operatorname{Err}_{v, i, i}^{(p)}[\varphi]\left(\tau_{1}, \tau_{2}\right) \doteq & \int_{\mathcal{R}\left(\tau_{1}, \tau_{2}\right) \cap\left\{t \leq T^{*}\right\}} \chi_{R} \cdot\left\{O\left(r^{p-2-a}\right) \cdot \partial_{v} \Phi \cdot \partial_{u}^{2} \Phi\right. \\
& \left.+O\left(r^{p-3-a}\right) \cdot \partial_{v} \Phi \cdot \partial_{\sigma} \partial_{\sigma} \Phi\right\} d u d v d \sigma
\end{aligned}
$$


we will perform two integrations by parts: By first integrating by parts schematically as $\partial_{v} \Phi \partial_{i}^{2} \Phi \rightarrow \partial_{i} \partial_{v} \Phi \partial_{i} \Phi=\frac{1}{2} \partial_{v}\left\{\partial_{i} \Phi\right\}^{2}$ and then we integrating in $\partial_{v}$ as before, we can readily bound recalling that $d u d \sigma \sim r^{-1-\eta^{\prime}} d v d \sigma$ on $\mathcal{S}_{\tau}$ ):

$$
\begin{aligned}
E r r_{v, i, i}^{(p)}[\varphi]\left(\tau_{1}, \tau_{2}\right) & \lesssim \int_{\mathcal{R}\left(\tau_{1}, \tau_{2}\right) \cap\left\{t \leq T^{*}\right\}} \chi_{R} \cdot\left\{r^{p-3-a}\left|\partial_{u} \Phi\right|^{2}+r^{p-2-a}\left|\partial_{u} \Phi\right| \cdot\left|\partial_{v} \Phi\right|^{2}\right. \\
& \left.+r^{p-4-a}\left|\partial_{\sigma} \Phi\right|^{2}+r^{p-3-a}\left|\partial_{v} \Phi\right| \cdot\left|\partial_{\sigma} \Phi\right|\right\} d u d v d \sigma \\
& +\int_{\mathcal{R}\left(\tau_{1}, \tau_{2}\right) \cap\left\{t \leq T^{*}\right\}}\left|\partial \chi_{R}\right| \cdot r^{p-1} \cdot|\partial \Phi|^{2} d u d v d \sigma \\
& +\sum_{i=1}^{2} \int_{\mathcal{S}_{\tau_{i}} \cap\left\{t \leq T^{*}\right\}} \chi_{R}\left\{r^{p-2-a}\left|\partial_{v} \Phi\right| \cdot\left|\partial_{u} \Phi\right|+r^{p-4-a-\eta^{\prime}}\left|\partial_{\sigma} \Phi\right|^{2}\right\} d v d \sigma \\
& +\int_{\mathcal{R}\left(\tau_{1}, \tau_{2}\right) \cap\left\{t=T^{*}\right\}} \chi_{R}\left\{r^{p-2-a}\left|\partial_{v} \Phi\right| \cdot\left|\partial_{u} \Phi\right|+r^{p-3-a}\left|\partial_{\sigma} \Phi\right|^{2}\right\} d v d \sigma .
\end{aligned}
$$

Using a Cauchy-Schwarz inequality as well as inequality (5.26), the first two lines of the right hand side of (5.33) can be bounded by $C_{p, \eta} \cdot \mathcal{B}_{E r r, \eta, \varepsilon}^{(p)}[\varphi]\left(\tau_{1}, \tau_{2}\right)$. Moreover, the last two lines of the right hand side of (5.33) can also be bounded by the boundry terms appearing in $\mathcal{B}_{E r r, \eta, \varepsilon}^{(p)}[\varphi]\left(\tau_{1}, \tau_{2}\right)$. Thus, provided again that $R$ is large enough in terms of $p, \eta, \varepsilon$, we have

$$
\operatorname{Err}_{v, i, i}^{(p)}[\varphi]\left(\tau_{1}, \tau_{2}\right) \lesssim_{p, \eta} \mathcal{B}_{E r r, \eta, \varepsilon}^{(p)}[\varphi]\left(\tau_{1}, \tau_{2}\right)
$$

3. Finally, it remains to bound the $\int O\left(r^{p-2-a}\right) \cdot \partial_{v} \Phi \cdot \partial_{u} \partial_{\sigma} \Phi$ summand in the expression (5.25). We will perform three integrations by parts schematically as

$$
\partial_{v} \Phi \cdot \partial_{\sigma} \partial_{u} \Phi \rightarrow-\partial_{u} \partial_{v} \Phi \cdot \partial_{\sigma} \Phi \rightarrow \partial_{u} \Phi \cdot \partial_{v} \partial_{\sigma} \Phi \rightarrow-\partial_{\sigma} \partial_{u} \Phi \cdot \partial_{v} \Phi
$$

and then move the resulting $\int O\left(r^{p-2-a}\right) \cdot \partial_{v} \Phi \cdot \partial_{u} \partial_{\sigma} \Phi$ bulk term (which is equal to -1 times the initial $O\left(r^{p-2-a}\right) \cdot \partial_{v} \Phi \cdot \partial_{u} \partial_{\sigma} \Phi$ term that we started with) from the right hand side to the left hand side. This will provide an estimate of $\int O\left(r^{p-2-a}\right) \cdot \partial_{v} \Phi \cdot \partial_{u} \partial_{\sigma} \Phi$ by bulk and boundary terms which contain only first order derivatives in $\Phi$. In particular, proceeding as described we infer ${ }^{10}$ that

$$
\begin{aligned}
& \int_{\mathcal{R}\left(\tau_{1}, \tau_{2}\right) \cap\left\{t \leq T^{*}\right\}} \chi_{R} O\left(r^{p-2-a}\right) \cdot \partial_{v} \Phi \cdot \partial_{u} \partial_{\sigma} \Phi d u d v d \sigma \\
& \quad \lesssim \int_{\mathcal{R}\left(\tau_{1}, \tau_{2}\right) \cap\left\{t \leq T^{*}\right\}} \chi_{R} r^{p-2-a}\left|\partial_{v} \Phi\right| \cdot\left|\partial_{\sigma} \Phi\right| d u d v d \sigma
\end{aligned}
$$

10 Using again the fact that $d u d \sigma \sim r^{-1-\eta^{\prime}} d v d \sigma$ on $\mathcal{S}_{\tau}$. 


$$
\begin{aligned}
& +\int_{\mathcal{R}\left(\tau_{1}, \tau_{2}\right) \cap\left\{t \leq T^{*}\right\}} \chi_{R} r^{p-3-a}\left|\partial_{u} \Phi\right| \cdot\left|\partial_{\sigma} \Phi\right| d u d v d \sigma \\
& +\int_{\mathcal{R}\left(\tau_{1}, \tau_{2}\right) \cap\left\{t \leq T^{*}\right\}} \chi_{R} r^{p-2-a}\left|\partial_{v} \Phi\right| \cdot\left|\partial_{u} \Phi\right| d u d v d \sigma \\
& +\int_{\mathcal{R}\left(\tau_{1}, \tau_{2}\right) \cap\left\{t \leq T^{*}\right\}}\left|\partial \chi_{R}\right| r^{p}|\partial \Phi|^{2} d u d v d \sigma \\
& +\sum_{i=1}^{2} \int_{\mathcal{S}_{\tau_{i}} \cap\left\{t \leq T^{*}\right\}} \chi_{R} r^{p-2-a}\left(\left|\partial_{v} \Phi\right|+r^{-1-\eta^{\prime}}\left|\partial_{u} \Phi\right|\right) \cdot\left|\partial_{\sigma} \Phi\right| d v d \sigma \\
& +\int_{\mathcal{R}\left(\tau_{1}, \tau_{2}\right) \cap\left\{t=T^{*}\right\}} \chi_{R} r^{p-2-a}\left(\left|\partial_{v} \Phi\right|+\left|\partial_{u} \Phi\right|\right) \cdot\left|\partial_{\sigma} \Phi\right| d v d \sigma .
\end{aligned}
$$

Thus, after using a Cauchy-Schwarz inequality (as well as the estimate (5.26)), we obtain provided that $R$ is large enough in terms of $p, \eta, \varepsilon$ :

$$
\begin{aligned}
& \int_{\mathcal{R}\left(\tau_{1}, \tau_{2}\right) \cap\left\{t \leq T^{*}\right\}} \chi_{R} O\left(r^{p-2-a}\right) \cdot \partial_{v} \Phi \cdot \partial_{u} \partial_{\sigma} \Phi d u d v d \sigma \\
& \quad \lesssim_{p, \eta} \mathcal{B}_{E r r, \eta, \varepsilon}^{(p)}[\varphi]\left(\tau_{1}, \tau_{2}\right) .
\end{aligned}
$$

Inequalities (5.31), (5.34) and (5.37) yield (5.22). Therefore, the proof of the Lemma is complete.

In order to control the zeroth order terms appearing in the right hand side of (5.15), we will make use of the following Hardy type inequality:

Lemma 5.5 For any $0<p<2$, any given $0<\eta<a$, any $R>0$ large enough in terms of $p, \eta$, any $\tau_{1} \leq \tau_{2}$ and any $T^{*} \geq 0$, the following inequality is true for any smooth function $\varphi: \mathcal{N}_{a f} \rightarrow \mathbb{C}$ with compact support in space and any smooth cut-off $\chi_{R}: \mathcal{N}_{a f} \rightarrow[0,1]$ supported in $\{r \geq R\}$ :

$$
\begin{aligned}
& \int_{\mathcal{S}_{\tau_{2}} \cap\left\{t \leq T^{*}\right\}} \chi_{R} \cdot r^{p-2}|\Omega \varphi|^{2} r^{-1-\eta^{\prime}} d v d \sigma \\
& +\int_{\mathcal{R}\left(\tau_{1}, \tau_{2}\right) \cap\left\{t=T^{*}\right\}} \chi_{R} \cdot r^{p-2} \cdot|\Omega \varphi|^{2} d v d \sigma \\
& +\int_{\mathcal{R}\left(\tau_{1}, \tau_{2}\right) \cap\left\{t \leq T^{*}\right\}} \chi_{R} \cdot r^{p-3}|\Omega \varphi|^{2} d u d v d \sigma \lesssim p, \eta \mathcal{E}_{\text {bound }, R, T^{*}}^{(p)}[\varphi]\left(\tau_{1}\right) \\
& +\int_{\mathcal{S}_{\tau_{1}}} \chi_{R} \cdot r^{p-2}|\Omega \varphi|^{2} r^{-1-\eta^{\prime}} d v d \sigma \\
& +\int_{\mathcal{R}\left(\tau_{1}, \tau_{2}\right) \cap\left\{t \leq T^{*}\right\}}\left|\partial \chi_{R}\right| \cdot\left(r^{p}|\partial(\Omega \varphi)|^{2}+r^{p-2}|\Omega \varphi|^{2}\right) d u d v d \sigma \\
& +\int_{\mathcal{S}_{\tau_{1}} \cap\left\{t \leq T^{*}\right\}} \chi_{R} J_{\mu}^{T}(\varphi) \bar{n}^{\mu} \\
& +\int_{\mathcal{R}\left(\tau_{1}, \tau_{2}\right) \cap\left\{t \leq T^{*}\right\}} \chi_{R} \cdot\left(r^{p+1}+r^{1+\eta}\right)|\Omega \square \varphi|^{2} d u d v d \sigma,
\end{aligned}
$$


where $\bar{n}$ is the future directed unit normal on the hyperboloids $\mathcal{S}_{\tau}$. In the above, the constants implicit in the $\lesssim p, \eta$ notation depend only on $p, \eta$ and on the geometry of $\left(\mathcal{N}_{a f}, g\right)$.

Proof As in the proof of Lemma (5.4), we can assume that $\varphi$ is real valued. We will also set $\Phi \doteq \Omega \varphi$.

In dimensions $d \geq 4$ (5.38) follows immediately from Lemma (5.4), since for $d \geq 4$ and for $R$ large enough, the left hand side of (5.15) controls the left hand side of (5.38) and the zeroth order terms in the right hand side of (5.38) can be absorbed into the left hand side. Therefore it only remains to treat the case $d=3$.

The proof of this lemma follows the standard steps of proving a Hardy-type inequality. Since $\partial_{v} r=1+o(1)$, we can write:

$$
\begin{aligned}
& \int_{\mathcal{R}\left(\tau_{1}, \tau_{2}\right) \cap\left\{t \leq T^{*}\right\}} \chi_{R} \cdot r^{p-3}|\Phi|^{2} d u d v d \sigma \\
& =\int_{\mathcal{R}\left(\tau_{1}, \tau_{2}\right) \cap\left\{t \leq T^{*}\right\}} \chi_{R} \cdot\left\{\frac{1}{p-2} \partial_{v}\left(r^{p-2}\right)+o\left(r^{p-3}\right)\right\}|\Phi|^{2} d u d v d \sigma
\end{aligned}
$$

and hence, if $R \gg 1$ :

$$
\begin{aligned}
& \int_{\mathcal{R}\left(\tau_{1}, \tau_{2}\right) \cap\left\{t \leq T^{*}\right\}} \chi_{R} \cdot r^{p-3}(1+o(1))|\Phi|^{2} d u d v d \sigma \\
& =\frac{1}{p-2} \int_{\mathcal{R}\left(\tau_{1}, \tau_{2}\right) \cap\left\{t \leq T^{*}\right\}} \chi_{R} \cdot \partial_{v}\left(r^{p-2}\right)|\Phi|^{2} d u d v d \sigma \\
& =\frac{1}{p-2}\left\{\int_{\mathcal{S}_{\tau_{2} \cap\left\{t \leq T^{*}\right\}}} \chi_{R} \cdot r^{p-2}|\Phi|^{2} d u d \sigma\right. \\
& +\int_{\mathcal{R}\left(\tau_{1}, \tau_{2}\right) \cap\left\{t=T^{*}\right\}} \chi_{R} \cdot r^{p-2}|\Phi|^{2} d u d \sigma \\
& -\int_{\mathcal{S}_{\tau_{1}} \cap\left\{t \leq T^{*}\right\}} \chi_{R} \cdot r^{p-2}|\Phi|^{2} d u d \sigma \\
& -\int_{\mathcal{R}\left(\tau_{1}, \tau_{2}\right) \cap\left\{t \leq T^{*}\right\}}\left(\partial_{v} \chi_{R}\right) \cdot r^{p-2}|\Phi|^{2} d u d v d \sigma \\
& \left.+\int_{\mathcal{R}\left(\tau_{1}, \tau_{2}\right) \cap\left\{t \leq T^{*}\right\}} \chi_{R} \cdot r^{p-2} 2 \cdot \partial_{v} \Phi \cdot \Phi d u d v d \sigma\right\},
\end{aligned}
$$

the last equality following after performing an integration by parts in $\partial_{v}$.

Notice that $\frac{1}{p-2}<0$ in the right hand side of (5.40). Therefore, after applying a Cauchy-Schwarz inequality we obtain:

$$
\begin{aligned}
& \int_{\mathcal{S}_{\tau_{2}} \cap\left\{t \leq T^{*}\right\}} \chi_{R} \cdot r^{p-2}|\Phi|^{2} d u d \sigma+\int_{\mathcal{R}\left(\tau_{1}, \tau_{2}\right) \cap\left\{t=T^{*}\right\}} \chi_{R} \cdot r^{p-2}|\Phi|^{2} d u d \sigma \\
& \quad+\int_{\mathcal{R}\left(\tau_{1}, \tau_{2}\right) \cap\left\{t \leq T^{*}\right\}} \chi_{R} \cdot r^{p-3}|\Phi|^{2} d u d v d \sigma
\end{aligned}
$$




$$
\begin{aligned}
\leq & C(p)\left\{\int_{\mathcal{S}_{\tau_{1} \cap\left\{t \leq T^{*}\right\}}} \chi_{R} \cdot r^{p-2}|\Phi|^{2} d u d \sigma\right. \\
& +\int_{\mathcal{R}\left(\tau_{1}, \tau_{2}\right) \cap\left\{t \leq T^{*}\right\}}\left|\partial \chi_{R}\right| \cdot r^{p-2}|\Phi|^{2} d u d v d \sigma \\
& +\left(\int_{\mathcal{R}\left(\tau_{1}, \tau_{2}\right) \cap\left\{t \leq T^{*}\right\}} \chi_{R} \cdot r^{p-1}\left|\partial_{v} \Phi\right|^{2} d u d v d \sigma\right)^{1 / 2} \\
& \left.\times\left(\int_{\mathcal{R}\left(\tau_{1}, \tau_{2}\right) \cap\left\{t \leq T^{*}\right\}} \chi_{R} \cdot r^{p-3}|\Phi|^{2} d u d v d \sigma\right)^{1 / 2}\right\} .
\end{aligned}
$$

The second factor of the last term of the right hand side of (5.41) can be absorbed into the left hand side, while the first factor of the same term can be bounded by the left hand side of (5.15), and thus:

$$
\begin{aligned}
& \int_{\mathcal{S}_{\tau_{2}} \cap\left\{t \leq T^{*}\right\}} \chi_{R} \cdot r^{p-2}|\Phi|^{2} d u d \sigma \\
& +\int_{\mathcal{R}\left(\tau_{1}, \tau_{2}\right) \cap\left\{t=T^{*}\right\}} \chi_{R} \cdot r^{p-2}|\Phi|^{2} d u d \sigma \\
& +\int_{\mathcal{R}\left(\tau_{1}, \tau_{2}\right) \cap\left\{t \leq T^{*}\right\}} \chi_{R} \cdot r^{p-3}|\Phi|^{2} d u d v d \sigma \\
& \lesssim p \int_{\mathcal{S}_{\tau_{1} \cap\left\{t \leq T^{*}\right\}}} \chi_{R} \cdot r^{p-2}|\Phi|^{2} d u d \sigma+\mathcal{E}_{\text {bound }, R, T^{*}}^{(p)}[\varphi]\left(\tau_{1}\right) \\
& +\int_{\mathcal{S}_{\tau_{1} \cap\left\{t \leq T^{*}\right\}}} \chi_{R} J_{\mu}^{T}(\varphi) \bar{n}^{\mu} \\
& +\int_{\mathcal{R}\left(\tau_{1}, \tau_{2}\right) \cap\left\{t \leq T^{*}\right\}}\left|\partial \chi_{R}\right| \cdot\left(r^{p}|\partial(\Omega \varphi)|^{2}+r^{p-2}|\Omega \varphi|^{2}\right) d u d v d \sigma \\
& +\int_{\mathcal{R}\left(\tau_{1}, \tau_{2}\right) \cap\left\{t \leq T^{*}\right\}} \chi_{R} \cdot\left(r^{p+1}+r^{1+\eta}\right)|\Omega \square \varphi|^{2} d u d v d \sigma \\
& +\int_{\mathcal{S}_{\tau_{2} \cap\left\{t \leq T^{*}\right\}}} \chi_{R} \cdot r^{p-2-a}|\Phi|^{2} d v d \sigma+\int_{\mathcal{R}\left(\tau_{1}, \tau_{2}\right) \cap\left\{t=T^{*}\right\}} \chi_{R} \cdot r^{p-2-a}|\Phi|^{2} d v d \sigma \\
& +\int_{\mathcal{R}\left(\tau_{1}, \tau_{2}\right) \cap\left\{t \leq T^{*}\right\}} \chi_{R} \cdot \max \left\{r^{p-3-a}, r^{-3}\right\}|\Phi|^{2} d u d v d \sigma .
\end{aligned}
$$

If $R$ is large enough in terms of $p$, the last two lines of the right hand side of (5.42) can be absorbed into the left hand side, yielding the desired inequality (5.38).

Proof of Theorem 5.1. The proof of Theorem 5.1 follows readily by adding inequalities (5.15) for the given value of $p$ and (5.38) for $\min \{p, 2-\delta\}$ in place of $p$, using also (4.30) and (4.4), and letting $T^{*} \rightarrow+\infty$. 


\section{The Improved $r^{p}$-Weighted Energy Hierarchy for Higher Order Derivatives}

In [34], Schlue established that on Schwarzschild exterior spacetimes, commutation of the wave equation (1.1) with the outgoing null vector field $\partial_{v}$ and the generators of the spherical isometries leads to an improved $r^{p}$-weighted hierarchy for $\partial_{v}(\Omega \varphi)$ and $r^{-1} \partial_{\sigma}(\Omega \varphi)$. This improvement of the $r$-weights in the hierarchy (1.7) for $\partial_{v}(\Omega \varphi)$, $r^{-1} \partial_{\sigma}(\Omega \varphi)$ was fundamental in obtaining improved decay rates in $u$ for $T \varphi$, and subsequently for $\varphi$ itself ( $T$ being the stationary Killing vector field of Schwarzschild spacetime). See [34] for more details.

In this Section, we will extend and improve the results of [34] to spacetime regions $\left(\mathcal{N}_{a f}, g\right)$ with $g$ of the form (3.3). In particular, we will establish that higher order derivatives of $\Omega \varphi$ in directions tangential to the hyperboloids $\{\bar{t}=$ const $\}$ satisfy an $r^{p}$-weighted hierarchy similar to the one established in the previous section for $\Omega \varphi$, but for $p$ taking values larger than 2 . These improved estimates will be crucial in the establishment of improved polynomial decay rates for $\varphi$ in Section 9.

Theorem 6.1 For any $k \in \mathbb{N}$, any $2 k-2<p \leq 2 k$, any given $0<\eta<a$ and $0<\delta<1$, any $R>0$ large enough in terms of $p, \eta, \delta, k$ and any $\tau_{1} \leq \tau_{2}$, the following inequality is true for any smooth function $\varphi: \mathcal{M} \rightarrow \mathbb{C}$ solving $\square_{g} \varphi=F$ and any smooth cut-off $\chi_{R}: \mathcal{M} \rightarrow[0,1]$ supported in $\{r \geq R\}$ :

$$
\begin{aligned}
& \mathcal{E}_{\text {bound }, R ; \delta}^{(p, k)}[\varphi]\left(\tau_{2}\right)
\end{aligned}
$$

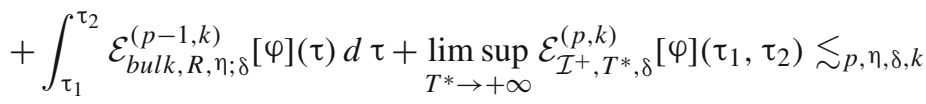

$$
\begin{aligned}
& \lesssim_{p, \eta, \delta, k} \mathcal{E}_{\text {bound }, R ; \delta}^{(p, k)}[\varphi]\left(\tau_{2}\right)+\sum_{j=0}^{k} \int_{\mathcal{R}\left(\tau_{1}, \tau_{2}\right)}\left|\partial \chi_{R}\right| \cdot r^{p-2(k-j)}\left|\partial^{j} \varphi\right|^{2} \\
& +\sum_{j=1}^{k} \sum_{k_{1}+k_{2}+k_{3}=j-1} \int_{\mathcal{R}\left(\tau_{1}, \tau_{2}\right)} \chi_{R} \cdot\left(r^{p+1-2 k_{3}-2(k-j)}+r^{1+\eta}\right) \\
& \times\left(\left|r^{-k_{2}} \partial_{v}^{k_{1}} \partial_{\sigma}^{k_{2}} \partial_{u}^{k_{3}}(\Omega F)\right|^{2}\right) d u d v d \sigma .
\end{aligned}
$$

In the above, the constants implicit in the $\lesssim_{p, \eta, k}$ notation depend only on $p, \eta, k$ and on the geometry of $(\mathcal{M}, g)$, and the higher order p-energy norms are defined as

$$
\begin{aligned}
& \mathcal{E}_{\text {bound }, R ; \delta}^{(p, k)}[\varphi](\tau) \\
& =\sum_{j=1}^{k} \sum_{k_{1}+k_{2}+k_{3}=j-1}\left\{\int _ { \mathcal { S } _ { \tau } } \chi _ { R } \left(r^{p-2(k-j)}\left|r^{-k_{2}-k_{3}} \partial_{v}^{k_{1}+1} \partial_{\sigma}^{k_{2}} \partial_{u}^{k_{3}}(\Omega \varphi)\right|^{2}\right.\right. \\
& \quad+r^{-1-\eta^{\prime}}\left(r^{p-2(k-j)}\left|r^{-k_{2}-k_{3}-1} \partial_{v}^{k_{1}} \partial_{\sigma}^{k_{2}+1} \partial_{u}^{k_{3}}(\Omega \varphi)\right|^{2}\right.
\end{aligned}
$$




$$
\begin{aligned}
& +\int_{\mathcal{S}_{\tau}}+\left((d-3) r^{p-2-2(k-j)}+\min \left\{r^{p-2-2(k-j)}, r^{-\delta-2(k-j)}\right\}\right) \\
& \left.\left.\left.\left|r^{-k_{2}-k_{3}} \partial_{v}^{k_{1}} \partial_{\sigma}^{k_{2}} \partial_{u}^{k_{3}}(\Omega \varphi)\right|^{2}\right)\right) d v d \sigma+\int_{\mathcal{S}_{\tau}} \chi_{R} J_{\mu}^{T}\left(r^{-k_{2}} \partial_{v}^{k_{1}} \partial_{\sigma}^{k_{2}} \partial_{u}^{k_{3}} \varphi\right) \bar{n}^{\mu}\right\} \\
& \mathcal{E}_{\text {bulk,R, } \eta ; \delta}^{(p-1, k)}[\varphi](\tau) \\
& =\sum_{j=1}^{k} \sum_{k_{1}+k_{2}+k_{3}=j-1}\left\{\int _ { \mathcal { S } _ { \tau } } \chi _ { R } \left(\left\{p r^{p-1-2(k-j)}\left|r^{-k_{2}-k_{3}} \partial_{v}^{k_{1}+1} \partial_{\sigma}^{k_{2}} \partial_{u}^{k_{3}}(\Omega \varphi)\right|^{2}\right.\right.\right. \\
& +\left((2 k-p) r^{p-1-2(k-j)}+r^{p-1-\delta-2(k-j)}\right)\left|r^{-k-1} \partial_{\sigma}^{k_{1}+k_{2}+1} \partial_{u}^{k_{3}}(\Omega \varphi)\right|^{2} \\
& +\left((2 k-p)(d-3) r^{p-3-2(k-j)}\right. \\
& \left.\left.\left.+\min \left\{r^{p-3-2(k-j)}, r^{-1-\delta-2(k-j)}\right\}\right)\left|r^{-k_{2}-k_{3}} \partial_{v}^{k_{1}} \partial_{\sigma}^{k_{2}} \partial_{u}^{k_{3}}(\Omega \varphi)\right|^{2}\right\}\right) d v d \sigma \\
& \left.+\int_{\mathcal{S}_{\tau}} \chi_{R} r^{-1-\eta}\left|\partial_{u}^{j+1}(\Omega \varphi)\right|^{2} d v d \sigma\right\}
\end{aligned}
$$

and

$$
\begin{aligned}
& \mathcal{E}_{\mathcal{I}^{+}, T^{*}, \delta}^{(p, k)}[\varphi]\left(\tau_{1}, \tau_{2}\right) \\
& =\sum_{j=1}^{k} \sum_{k_{1}+k_{2}+k_{3}=j-1}\left\{\int _ { \mathcal { R } ( \tau _ { 1 } , \tau _ { 2 } ) \cap \{ t = T ^ { * } \} } \left(r^{p-2(k-j)}\left|r^{-k_{2}-k_{3}-1} \partial_{v}^{k_{1}} \partial_{\sigma}^{k_{2}+1} \partial_{u}^{k_{3}}(\Omega \varphi)\right|^{2}\right.\right. \\
& \quad+\left((d-3) r^{p-2-2(k-j)}+\min \left\{r^{p-2-2(k-j)}, r^{-\delta-2(k-j)}\right\}\right) \\
& \left.\quad \times\left|r^{-k_{2}-k_{3}} \partial_{v}^{k_{1}} \partial_{\sigma}^{k_{2}} \partial_{u}^{k_{3}}(\Omega \varphi)\right|^{2}\right) d v d \sigma \\
& \left.\quad+\int_{\mathcal{R}\left(\tau_{1}, \tau_{2}\right) \cap\left\{t=T^{*}\right\}} J_{\mu}^{T}\left(r^{-k_{2}} \partial_{v}^{k_{1}} \partial_{\sigma}^{k_{2}} \partial_{u}^{k_{3}} \varphi\right) n^{\mu}\right\}
\end{aligned}
$$

Applying Theorem 6.1 for $\partial^{j} \varphi, j=0, \ldots m-1$ in place of $\varphi$ using Lemma 6.3, we will deduce the following estimate (the proof of which is straightforward and will be omitted)

Corollary 6.2 Keeping the same notations as in Theorem 6.1, for any any integer $m \geq 0$, any $k \in \mathbb{N}$, any $2 k-2<p \leq 2 k$, any given $0<\eta<a$ and $0<\delta<1$, any $R>0$ large enough in terms of $p, \eta, \delta, k, m$ and any $\tau_{1} \leq \tau_{2}$, the following inequality is true for any smooth function $\varphi: \mathcal{M} \rightarrow \mathbb{C}$ solving $\square_{g} \varphi=F$ and any smooth cut-off $\chi_{R}: \mathcal{M} \rightarrow[0,1]$ supported in $\{r \geq R\}:$ 


$$
\begin{aligned}
& \sum_{j=0}^{m-1} \sum_{j_{1}+j_{2}+j_{3}=j}\left\{\mathcal{E}_{\text {bound }, R ; \delta}^{(p, k)}\left[r^{-j_{2}} \partial_{v}^{j_{1}} \partial_{\sigma}^{j_{2}} \partial_{u}^{j_{3}} \varphi\right]\left(\tau_{2}\right)\right. \\
& +\int_{\tau_{1}}^{\tau_{2}} \mathcal{E}_{b u l k, R, \eta ; \delta}^{(p-1, k)}\left[r^{-j_{2}} \partial_{v}^{j_{1}} \partial_{\sigma}^{j_{2}} \partial_{u}^{j_{3}} \varphi\right](\tau) d \tau \\
& \left.+\limsup _{T^{*} \rightarrow+\infty} \mathcal{E}_{\mathcal{I}^{+}, T^{*}, \delta}^{(p, k)}\left[r^{-j_{2}} \partial_{v}^{j_{1}} \partial_{\sigma}^{j_{2}} \partial_{u}^{j_{3}} \varphi\right]\left(\tau_{1}, \tau_{2}\right)\right\} \\
& \lesssim_{p, \eta, \delta, k, m} \sum_{j=0}^{m-1} \sum_{j_{1}+j_{2}+j_{3}=j} \mathcal{E}_{\text {bound }, R ; \delta}^{(p, k)}\left[r^{-j_{2}} \partial_{v}^{j_{1}} \partial_{\sigma}^{j_{2}} \partial_{u}^{j_{3}} \varphi\right]\left(\tau_{2}\right) \\
& +\sum_{j=0}^{k} \sum_{i=0}^{m-1} \int_{\mathcal{R}\left(\tau_{1}, \tau_{2}\right)}\left|\partial \chi_{R}\right| \cdot r^{p-2(k-j)}\left|\partial^{j+i} \varphi\right|^{2} \\
& +\sum_{i_{1}+i_{2}+i_{3} \leq m-1} \sum_{j=1}^{k} \sum_{k_{1}+k_{2}+k_{3}=j-1} \int_{\mathcal{R}\left(\tau_{1}, \tau_{2}\right)} \chi_{R} \cdot\left(r^{p+1-2 k_{3}-2(k-j)}+r^{1+\eta}\right) \\
& \times\left(\left|r^{-k_{2}-i_{2}} \partial_{v}^{k_{1}+i_{1}} \partial_{\sigma}^{k_{2}+i_{2}} \partial_{u}^{k_{3}+i_{3}}(\Omega F)\right|^{2}\right) d u d v d \sigma .
\end{aligned}
$$

The proof of Theorem 6.1 will be presented in Section 6.2. Before that, we will obtain an expression for the equation satisfied by the derivatives of $\Omega \varphi$ when $\varphi$ solves $\square \varphi=F$. This will be accomplished in the following section.

\subsection{Commutator Expressions}

In this section, we will commute the wave operator $\square_{g}$ with the coordinate vector fields $\partial_{u}$ and $\partial_{v}$ in the $(u, v, \sigma)$ coordinate system, as well as the first order operator $r^{-1} \nabla^{\mathbb{S}^{d-1}}\left(\nabla^{\mathbb{S}^{d-1}}\right.$ denoting here the gradient of a function on $\left.\left(\mathbb{S}^{d-1}, g_{\mathbb{S}^{d-1}}\right)\right)$ in the asymptotically flat region $\{r \gg 1\}$.

We will establish the following lemma:

Lemma 6.3 For any smooth function $\varphi: \mathcal{M} \rightarrow \mathbb{C}$ the following expressions are true in the region $\{r \gg 1\}$ for any $l \in \mathbb{N}$ :

$$
\begin{aligned}
& \Omega \square\left(\Omega^{-1} \partial_{v}^{l}(\Omega \varphi)\right)=\partial_{v}^{l}(\Omega \square \varphi)+\sum_{j=1}^{l}(-1)^{j+1}\left\{\left(\begin{array}{l}
l \\
j
\end{array}\right)(1+j) ! \cdot r^{-2-j}\right. \\
& \left.\times\left(\Delta_{g_{\mathbb{S}} d-1}+h_{\mathbb{S}^{d-1}} \partial_{v}^{l-j}(\Omega \varphi)-\frac{(d-1)(d-3)}{4} \partial_{v}^{l-j}(\Omega \varphi)\right)\right\} \\
& +\sum_{j=0}^{l} r^{-(l-j)} \operatorname{Err}\left(\partial_{v}^{j}(\Omega \varphi)\right), \\
& \Omega \square\left(\Omega^{-1}\left(r^{-1} \nabla^{\mathbb{S}^{d-1}}\right)^{l}(\Omega \varphi)\right)=\left(r^{-1} \nabla^{\mathbb{S}^{d-1}}\right)^{l}(\Omega \square \varphi)+l \cdot r^{-l-1} \partial_{u}\left(\left(\nabla^{\mathbb{S}^{d-1}}\right)^{l}(\Omega \varphi)\right)
\end{aligned}
$$




$$
\begin{aligned}
& -l \cdot r^{-l-1} \partial_{v}\left(\left(\nabla^{\mathbb{S}^{d-1}}\right)^{l}(\Omega \varphi)\right)+l(l+1) r^{-l-2}\left(\nabla^{\mathbb{S}^{d-1}}\right)^{l}(\Omega \varphi) \\
& +\sum_{j=0}^{l} r^{-(l-j)} \operatorname{Err}\left(\left(r^{-1} \nabla^{\mathbb{S}^{d-1}}\right)^{j}(\Omega \varphi)\right)
\end{aligned}
$$

and

$$
\begin{aligned}
\Omega \square\left(\Omega^{-1} \partial_{u}^{l}(\Omega \varphi)\right)= & \left(1+O\left(r^{-1-a}\right)\right) \cdot \partial_{u}^{l}\left(\left(1+O\left(r^{-1-a}\right)\right) \cdot \Omega \square \varphi\right) \\
& +\sum_{j=0}^{l-1} O\left(r^{-3}\right) \partial_{\sigma} \partial_{\sigma}\left(\partial_{u}^{j}(\Omega \varphi)\right)+\sum_{j=0}^{l} \operatorname{Err}\left(\partial_{u}^{j}(\Omega \varphi)\right),
\end{aligned}
$$

where $\nabla^{\mathbb{S}^{d-1}}$ denotes the gradient on $\left(\mathbb{S}^{d-1}, g_{\mathbb{S}^{d-1}}\right)$ and the Err terms are of the form:

$$
\begin{aligned}
\operatorname{Err}(\Phi)= & O\left(r^{-2-a}\right) \cdot \partial_{u}^{2} \Phi+O\left(r^{-1}\right) \cdot \partial_{v}^{2} \Phi+O\left(r^{-2-a}\right) \partial_{u} \partial_{\sigma} \Phi+O\left(r^{-2}\right) \partial_{v} \partial_{\sigma} \Phi \\
& +O\left(r^{-3-a}\right) \partial_{\sigma} \partial_{\sigma} \Phi+O\left(r^{-2-a}\right) \partial_{u} \Phi \\
& +O\left(r^{-1-a}\right) \partial_{v} \Phi+O\left(r^{-2-a}\right) \cdot \partial_{\sigma} \Phi+O\left(r^{-3}\right) \Phi .
\end{aligned}
$$

Proof Let us set

$$
\Phi \doteq \Omega \varphi
$$

In $(u, v, \sigma)$ coordinates in the region $\{r \gg 1\}$, the wave operator takes the following form according to (3.14):

$$
\begin{aligned}
\Omega \cdot \square \varphi= & -\left(1+O\left(r^{-1-a}\right)\right) \cdot \partial_{u} \partial_{v} \Phi+r^{-2} \Delta_{g_{\mathbb{S} d-1}+h_{\mathbb{S} d-1}} \Phi \\
& -\frac{(d-1)(d-3)}{4} r^{-2} \cdot \Phi+\operatorname{Err}(\Phi) .
\end{aligned}
$$

Differentiating (6.11) $l$ times with respect to $\partial_{v}$, we readily obtain:

$$
\begin{aligned}
& \partial_{v}^{l}(\Omega \square \varphi)=-\left(1+O\left(r^{-1-a}\right)\right) \cdot \partial_{u} \partial_{v}\left(\partial_{v}^{l} \Phi\right) \\
& +\sum_{j=0}^{l}(-1)^{j}\left\{\left(\begin{array}{l}
l \\
j
\end{array}\right)(1+j) ! \cdot r^{-2-j}\right. \\
& \left.\times\left(\Delta_{g_{\mathbb{S} d-1}+h_{\mathbb{S} d-1}} \partial_{v}^{l-j} \Phi-\frac{(d-1)(d-3)}{4} \partial_{v}^{l-j} \Phi\right)\right\} \\
& +\sum_{j=0}^{l} r^{-(l-j)} \operatorname{Err}\left(\partial_{v}^{j} \Phi\right)
\end{aligned}
$$


which (due to the expression (6.11) for the wave operator) can be rewritten in the desired form (6.6) as:

$$
\begin{aligned}
\Omega \square\left(\Omega^{-1} \partial_{v} \Phi\right)= & \partial_{v}(\Omega \square \varphi)+\sum_{j=1}^{l}(-1)^{j+1}\left\{\left(\begin{array}{l}
l \\
j
\end{array}\right)(1+j) ! \cdot r^{-2-j}\right. \\
& \left.\times\left(\Delta_{g_{\mathbb{S}}-1+h_{\mathbb{S} d-1}} \partial_{v}^{l-j} \Phi-\frac{(d-1)(d-3)}{4} \partial_{v}^{l-j} \Phi\right)\right\} \\
& +\sum_{j=0}^{l} r^{-(l-j)} \operatorname{Err}\left(\partial_{v}^{j} \Phi\right) .
\end{aligned}
$$

Similarly, applying to (6.11) $l$ times the rescaled angular gradient $r^{-1} \nabla^{\mathbb{S}^{d-1}}$ to (6.11) (notice that $\nabla^{\mathbb{S}^{d-1}}$ commutes with $\Delta_{g_{\mathbb{S} d-1}}, \partial_{u}, \partial_{v}$ and $r$ ), we calculate:

$$
\begin{aligned}
\left(r^{-1} \nabla^{\mathbb{S}^{d-1}}\right)^{l}(\Omega \square \varphi)= & -\left(1+O\left(r^{-1-a}\right)\right) \cdot \partial_{u} \partial_{v}\left(r^{-l}\left(\nabla^{\mathbb{S}^{d-1}}\right)^{l} \Phi\right) \\
& +r^{-2} \Delta_{g_{\mathbb{S}^{d}-1}+h_{\mathbb{S}^{d-1}}\left(r^{-l}\left(\nabla^{\mathbb{S}^{d-1}}\right)^{l} \Phi\right)} \\
& -\frac{(d-1)(d-3)}{2} r^{-2} \cdot\left(r^{-l}\left(\nabla^{\mathbb{S}^{d-1}}\right)^{l} \Phi\right) \\
& -l \cdot r^{-l-1} \partial_{u}\left(\left(\nabla^{\mathbb{S}^{d-1}}\right)^{l} \Phi\right)+l \cdot r^{-l-1} \partial_{v}\left(\left(\nabla^{\mathbb{S}^{d-1}}\right)^{l} \Phi\right) \\
& -l(l+1) r^{-l-2}\left(\nabla^{\mathbb{S}^{d-1}}\right)^{l} \Phi \\
& +\sum_{j=0}^{l} r^{-(l-j)} \operatorname{Err}\left(\left(r^{-1} \nabla^{\left.\left.\left.\mathbb{S}^{d-1}\right)\right)^{j} \Phi\right),}\right.\right.
\end{aligned}
$$

(where the $O(\cdot)$ terms in the $r^{-1} \operatorname{Err}(\Phi)$ summand should be considered to denote vector fields on $\mathbb{S}^{d-1}$ rather than functions). Thus, rearranging the terms we deduce that:

$$
\begin{aligned}
\Omega \square\left(\Omega^{-1}\left(r^{-1} \nabla^{\mathbb{S}^{d-1}}\right)^{l} \Phi\right)= & \left(r^{-1} \nabla^{\mathbb{S}^{d-1}}\right)^{l}(\Omega \square \varphi)+l \cdot r^{-l-1} \partial_{u}\left(\left(\nabla^{\mathbb{S}^{d-1}}\right)^{l} \Phi\right) \\
& -l \cdot r^{-l-1} \partial_{v}\left(\left(\nabla^{\mathbb{S}^{d-1}}\right)^{l} \Phi\right) \\
& +l(l+1) r^{-l-2}\left(\nabla^{\mathbb{S}^{d-1}}\right)^{l} \Phi \\
& +\sum_{j=0}^{l} r^{-(l-j)} \operatorname{Err}\left(\left(r^{-1} \nabla^{\mathbb{S}^{d-1}}\right)^{j} \Phi\right)
\end{aligned}
$$

After multiplying (6.11) with $1+O\left(r^{-1-a}\right)$ so as to make the coefficient of $\partial_{u} \partial_{v} \Phi$ equal to 1 , differentiating $l$ times with respect to $\partial_{u}$ we eventually obtain (since $\partial_{u} r=$ $-1)$ that 


$$
\begin{aligned}
\Omega \square\left(\Omega^{-1} \partial_{u}^{l} \Phi\right)= & \left(1+O\left(r^{-1-a}\right)\right) \cdot \partial_{u}^{l}\left(\left(1+O\left(r^{-1-a}\right)\right) \cdot \Omega \square \varphi\right) \\
& +\sum_{j=0}^{l-1} O\left(r^{-3}\right) \partial_{\sigma} \partial_{\sigma}\left(\partial_{u}^{j} \Phi\right)+\sum_{j=0}^{l} \operatorname{Err}\left(\partial_{u}^{j} \Phi\right),
\end{aligned}
$$

\subsection{Proof of Theorem 6.1}

Without loss of generality, we will assume that $\varphi$ is real valued. We will set

$$
\Phi \doteq \Omega \varphi .
$$

In the case $k=1$ the statement of Theorem 6.1 reduces to the statement of Theorem 5.1. Thus, it suffices to assume that $k \geq 2$. In order to avoid unnecessarily complicated notations, we will only establish the case $k=2$, since the case $k>2$ can be treated in exactly the same way (through induction on $k$ ).

Fix an $\varepsilon>0$ small enough in terms of $p, \eta, \delta$. We will assume without loss of generality that $R$ in terms of $\varepsilon$. Repeating the proof of Lemma 5.4 for $\Omega^{-1} \partial_{v} \Phi$ in place of $\varphi$, but without absorbing terms of the form $\int O\left(r^{p-1-a}\right)\left|r^{-1} \partial_{\sigma} \partial_{v} \Phi\right|^{2}$ by the left hand side of the resulting inequality, and without using a Cauchy-Schwarz inequality to bound terms of the form $\partial^{2} \Phi \cdot \square\left(\Omega \partial_{v} \Phi\right)$, we readily obtain the following inequality for any $T^{*} \geq 0$ (using the energy norm notation of the proof of Theorem $5.1)$ :

$$
\begin{aligned}
& \mathcal{E}_{\text {bound }, R, T^{*}}^{(p)}\left[\Omega^{-1} \partial_{v} \Phi\right]\left(\tau_{2}\right)+\int_{\tau_{1}}^{\tau_{2}} \mathcal{E}_{\text {bulk,R, }, T^{*}}^{(p-1)}\left[\Omega^{-1} \partial_{v} \Phi\right](\tau) d \tau \\
& +\mathcal{E}_{\mathcal{I}^{+}, R, T^{*}}^{(p)}\left[\Omega^{-1} \partial_{v} \Phi\right]\left(\tau_{1}, \tau_{2}\right) \\
& \leq \\
& \left.+O_{p, \eta}(\varepsilon)\right) \cdot \mathcal{E}_{\text {bound }, R, T^{*}}^{(p)}\left[\Omega^{-1} \partial_{v} \Phi\right]\left(\tau_{1}\right) \\
& +C_{p, \eta, \varepsilon} \int_{\mathcal{R}\left(\tau_{1}, \tau_{2}\right) \cap\left\{t \leq T^{*}\right\}}\left|\partial \chi_{R}\right| \cdot\left(r^{p}\left|\partial \partial_{v} \Phi\right|^{2}+r^{p-2}\left|\partial_{v} \Phi\right|^{2}\right) d u d v d \sigma \\
& +C_{p, \eta, \varepsilon} \int_{\mathcal{R}\left(\tau_{1}, \tau_{2}\right) \cap\left\{t \leq T^{*}\right\}} \chi_{R} \cdot\left(r^{p-1-a}\left|r^{-1} \partial_{\sigma} \partial_{v} \Phi\right|^{2}+r^{p-3-a}\left|\partial_{u} \partial_{v} \Phi\right|^{2}\right. \\
& \left.+\max \left\{r^{p-3-a}, r^{-3}\right\}\left|\partial_{v} \Phi\right|^{2}\right) d u d v d \sigma \\
& +C_{p, \eta, \varepsilon} \int_{\mathcal{R}\left(\tau_{1}, \tau_{2}\right) \cap\left\{t \leq T^{*}\right\}} \chi_{R} \cdot\left(\frac{1}{2} f(r)\left(\partial_{v}-\partial_{u}\right)\left(\Omega^{-1} \partial_{v} \Phi\right)\right. \\
& \left.+\frac{(d-1) f(r)}{r}\left(\Omega^{-1} \partial_{v} \Phi\right)\right) \cdot \square\left(\Omega^{-1} \partial_{v} \Phi\right) \Omega^{2} d u d v d \sigma \\
& +C_{p, \eta, \varepsilon} \int_{\mathcal{R}\left(\tau_{1}, \tau_{2}\right) \cap\left\{t \leq T^{*}\right\}} \chi_{R} \cdot\left(\partial_{v}+\partial_{u}\right)\left(\partial_{v} \Phi\right) \cdot \Omega \square\left(\Omega^{-1} \partial_{v} \Phi\right) d u d v d \sigma
\end{aligned}
$$




$$
\begin{aligned}
& +\int_{\mathcal{R}\left(\tau_{1}, \tau_{2}\right) \cap\left\{t \leq T^{*}\right\}} \chi_{R} \cdot O_{p, \eta, \varepsilon}\left(r^{p-2-a}\right) \cdot \partial_{\sigma} \partial_{v} \Phi \cdot \Omega \square\left(\Omega^{-1} \partial_{v} \Phi\right) d u d v d \sigma \\
& -\int_{\mathcal{R}\left(\tau_{1}, \tau_{2}\right) \cap\left\{t \leq T^{*}\right\}} \chi_{R} \cdot r^{p} \partial_{v}^{2} \Phi \cdot \Omega \square\left(\Omega^{-1} \partial_{v} \Phi\right) d u d v d \sigma \\
& +C_{p, \eta, \varepsilon} \operatorname{Bound}_{p, T^{*}}^{\partial_{v}}[\varphi]\left(\tau_{1}, \tau_{2}\right)
\end{aligned}
$$

where $f(r)=\frac{r^{\eta}}{1+r^{\eta}}$ is the function used in the proof of Lemma 4.1 and

$$
\begin{aligned}
& \operatorname{Bound}_{p, T^{*}}^{\partial_{v}}[\varphi]\left(\tau_{1}, \tau_{2}\right)=\sum_{i=1}^{2} \int_{\mathcal{S}_{\tau_{i}} \cap\left\{t \leq T^{*}\right\}} \chi_{R} \cdot r^{p-2-a}\left(\left|\partial_{u} \partial_{v} \Phi\right|^{2}+\left|r^{-1} \partial_{\sigma} \partial_{v} \Phi\right|^{2}\right. \\
& \left.+\left|\partial_{v} \Phi\right|^{2}+r^{-2} \Phi^{2}\right) d v d \sigma \\
& +\int_{\mathcal{R}\left(\tau_{1}, \tau_{2}\right) \cap\left\{t=T^{*}\right\}} \chi_{R} \cdot r^{p-2-a}\left(\left|\partial_{u} \partial_{v} \Phi\right|^{2}+\left|r^{-1} \partial_{\sigma} \partial_{v} \Phi\right|^{2}\right. \\
& \left.+\left|\partial_{v} \Phi\right|^{2}+r^{-2} \Phi^{2}\right) d v d \sigma \\
& +\int_{\mathcal{S}_{\tau_{1}} \cap\left\{t \leq T^{*}\right\}} \chi_{R} J_{\mu}^{T}\left(\Omega^{-1} \partial_{v} \Phi\right) \bar{n}^{\mu} .
\end{aligned}
$$

Notice that since $2<p \leq 4$, the left hand side of (6.18) is not positive definite, since the $\mathcal{E}_{b u l k, R, T^{*}}^{(p-1)}$ term contains a summand of the form

$$
\int_{\mathcal{R}\left(\tau_{1}, \tau_{2}\right) \cap\left\{t \leq T^{*}\right\}}(2-p)\left(r^{p-1}\left|r^{-1} \partial_{\sigma} \partial_{v} \Phi\right|^{2}+\frac{(d-1)(d-3)}{4} r^{p-3}\left|\partial_{v} \Phi\right|^{2}\right) d u d v d \sigma
$$

which has a negative sign.

We will show that the last term of the right hand side of (6.18) can provide us with extra control over bulk terms of the form $\int r^{p-1}\left|r^{-1} \partial_{\sigma} \partial_{v} \Phi\right|^{2}$. These terms will then be moved to the left hand side, rendering it positive definite for $2<p \leq 4$.

According to Lemma $6.3, \Omega^{-1} \partial_{v} \Phi$ satisfies the following equation:

$$
\begin{aligned}
\Omega \square\left(\Omega^{-1} \partial_{v} \Phi\right)= & \partial_{v}(\Omega F)+2 r^{-3} \Delta_{g_{\mathbb{S} d-1}+h_{\mathbb{S} d-1}} \Phi-\frac{(d-1)(d-3)}{2} r^{-3} \Phi \\
& +\operatorname{Err}\left(\partial_{v} \Phi\right)+r^{-1} \operatorname{Err}(\Phi)
\end{aligned}
$$

Therefore (omitting the $d u d v d \sigma$ volume form for the next few lines):

$$
\begin{gathered}
\int_{\mathcal{R}\left(\tau_{1}, \tau_{2}\right) \cap\left\{t \leq T^{*}\right\}} \chi_{R} \cdot r^{p} \partial_{v}^{2} \Phi \cdot \Omega \square\left(\Omega^{-1} \partial_{v} \Phi\right) \\
=\int_{\mathcal{R}\left(\tau_{1}, \tau_{2}\right) \cap\left\{t \leq T^{*}\right\}} \chi_{R} \cdot r^{p} \partial_{v}^{2} \Phi \cdot \partial_{v}(\Omega F)
\end{gathered}
$$




$$
\begin{aligned}
& +2 \int_{\mathcal{R}\left(\tau_{1}, \tau_{2}\right) \cap\left\{t \leq T^{*}\right\}} \chi_{R} \cdot r^{p-3} \partial_{v}^{2} \Phi \cdot \Delta_{g_{\mathbb{S}} d-1}+h_{\mathbb{S}^{d-1}} \Phi \\
& -\frac{(d-1)(d-3)}{2} \int_{\mathcal{R}\left(\tau_{1}, \tau_{2}\right) \cap\left\{t \leq T^{*}\right\}} \chi_{R} \cdot r^{p-3} \partial_{v}^{2} \Phi \cdot \Phi \\
& +\int_{\mathcal{R}\left(\tau_{1}, \tau_{2}\right) \cap\left\{t \leq T^{*}\right\}} \chi_{R} \cdot r^{p} \partial_{v}^{2} \Phi \cdot\left(\operatorname{Err}\left(\partial_{v} \Phi\right)+r^{-1} \operatorname{Err}(\Phi)\right) .
\end{aligned}
$$

Integrating by parts in $\partial_{\sigma}, \partial_{v}$, we have: (note, again, that the volume form used here is $d u d v d \sigma)$

$$
\begin{aligned}
& \int_{\mathcal{R}\left(\tau_{1}, \tau_{2}\right) \cap\left\{t \leq T^{*}\right\}} \chi_{R} \cdot r^{p-3} \partial_{v}^{2} \Phi \cdot \Delta_{g_{\mathbb{S} d-1}+h_{\mathbb{S} d-1}} \Phi \\
& =\int_{\mathcal{R}\left(\tau_{1}, \tau_{2}\right) \cap\left\{t \leq T^{*}\right\}} \chi_{R} \cdot r^{p-1}\left|r^{-1} \partial_{\sigma} \partial_{v} \Phi\right|^{2} \\
& \quad-\frac{(p-3)(p-4)}{2} \int_{\mathcal{R}\left(\tau_{1}, \tau_{2}\right) \cap\left\{t \leq T^{*}\right\}} \chi_{R} \cdot r^{p-3}\left|r^{-1} \partial_{\sigma} \Phi\right|^{2} \\
& \quad+\int_{\mathcal{R}\left(\tau_{1}, \tau_{2}\right) \cap\left\{t \leq T^{*}\right\}}\left|\partial \chi_{R}\right| \cdot\left(O\left(r^{p}\right)\left|\partial^{2} \Phi\right|^{2}+O\left(r^{p-2}\right)|\partial \Phi|^{2}\right) \\
& \quad+\int_{\mathcal{R}\left(\tau_{1}, \tau_{2}\right) \cap\left\{t \leq T^{*}\right\}} \chi_{R} \cdot O\left(r^{p}\right) \partial_{v} \Phi \cdot \operatorname{Err}(\Phi)+O_{p, \eta}(1) \cdot \operatorname{Bound}_{p, T^{*}, \varepsilon}^{(n)}[\varphi]\left(\tau_{1}, \tau_{2}\right)
\end{aligned}
$$

and

$$
\begin{aligned}
& \int_{\mathcal{R}\left(\tau_{1}, \tau_{2}\right) \cap\left\{t \leq T^{*}\right\}} \chi_{R} \cdot r^{p-3} \partial_{v}^{2} \Phi \cdot \Phi \\
& =-\int_{\mathcal{R}\left(\tau_{1}, \tau_{2}\right) \cap\left\{t \leq T^{*}\right\}} \chi_{R} \cdot r^{p-3}\left|\partial_{v} \Phi\right|^{2} \\
& \quad-\frac{(p-3)(p-4)}{2} \int_{\mathcal{R}\left(\tau_{1}, \tau_{2}\right) \cap\left\{t \leq T^{*}\right\}} \chi_{R} \cdot r^{p-5}|\Phi|^{2} \\
& \quad+\int_{\mathcal{R}\left(\tau_{1}, \tau_{2}\right) \cap\left\{t \leq T^{*}\right\}}\left|\partial \chi_{R}\right| \cdot\left(O\left(r^{p-2}\right)|\partial \Phi|^{2}+O\left(r^{p-4}\right)|\Phi|^{2}\right) \\
& \quad+\int_{\mathcal{R}\left(\tau_{1}, \tau_{2}\right) \cap\left\{t \leq T^{*}\right\}} \chi_{R} \cdot O\left(r^{p}\right) \partial_{v} \Phi \cdot \operatorname{Err}(\Phi)+O_{p, \eta}(1) \cdot \operatorname{Bound}_{p, T^{*}, \varepsilon}^{(n)}[\varphi]\left(\tau_{1}, \tau_{2}\right),
\end{aligned}
$$

where

$$
\begin{aligned}
& \operatorname{Bound}_{p, T^{*}, \varepsilon}^{(n)}[\varphi]\left(\tau_{1}, \tau_{2}\right) \\
& \quad=\varepsilon \cdot \sum_{i=1}^{2}\left(\mathcal{E}_{\text {bound }, R, T^{*}}^{(p)}\left[\Omega^{-1} \partial_{v} \Phi\right]\left(\tau_{i}\right)+\mathcal{E}_{\text {bound }, R, T^{*}}^{(p)}\left[\Omega^{-1} r^{-1} \partial_{\sigma} \Phi\right]\left(\tau_{i}\right)\right.
\end{aligned}
$$




$$
\left.+\mathcal{E}_{\text {bound }, R, T^{*}}^{(p-2)}\left[\Omega^{-1} \partial_{u} \Phi\right]\left(\tau_{i}\right)\right)+{ }^{-1} \sum_{i=1}^{2} \mathcal{E}_{\text {bound }, R, T^{*}}^{(p-2)}[\varphi]\left(\tau_{i}\right)
$$

Therefore, using (6.21), (6.22), (6.23) and (6.24), as well as an integration by parts scheme similar to the one implemented in the proof of Lemma 5.4, we can estimate:

$$
\begin{aligned}
& C_{p, \eta, \varepsilon} \int_{\mathcal{R}\left(\tau_{1}, \tau_{2}\right) \cap\left\{t \leq T^{*}\right\}} \chi_{R} \cdot\left(\frac{1}{2} f(r)\left(\partial_{v}-\partial_{u}\right)\left(\Omega^{-1} \partial_{v} \Phi\right)+\frac{(d-1) f(r)}{r}\left(\Omega^{-1} \partial_{v} \Phi\right)\right) \\
& \times \square\left(\Omega^{-1} \partial_{v} \Phi\right) \Omega^{2} d u d v d \sigma \\
& +C_{p, \eta, \varepsilon} \int_{\mathcal{R}\left(\tau_{1}, \tau_{2}\right) \cap\left\{t \leq T^{*}\right\}} \chi_{R} \cdot\left(\partial_{v}+\partial_{u}\right)\left(\partial_{v} \Phi\right) \cdot \Omega \square\left(\Omega^{-1} \partial_{v} \Phi\right) d u d v d \sigma \\
& +\int_{\mathcal{R}\left(\tau_{1}, \tau_{2}\right) \cap\left\{t \leq T^{*}\right\}} \chi_{R} \cdot O\left(r^{p-2-a}\right) \cdot \partial_{\sigma} \partial_{v} \Phi \cdot \Omega \square\left(\Omega^{-1} \partial_{v} \Phi\right) d u d v d \sigma \\
& -\int_{\mathcal{R}\left(\tau_{1}, \tau_{2}\right) \cap\left\{t \leq T^{*}\right\}} \chi_{R} \cdot r^{p} \partial_{v}^{2} \Phi \cdot \Omega \square\left(\Omega^{-1} \partial_{v} \Phi\right) d u d v d \sigma \\
& \leq-2 \int_{\mathcal{R}\left(\tau_{1}, \tau_{2}\right) \cap\left\{t \leq T^{*}\right\}} \chi_{R} \cdot r^{p-1}\left|r^{-1} \partial_{\sigma} \partial_{v} \Phi\right|^{2} d u d v d \sigma \\
& -\frac{(d-1)(d-3)}{2} \int_{\mathcal{R}\left(\tau_{1}, \tau_{2}\right) \cap\left\{t \leq T^{*}\right\}} \chi_{R} \cdot r^{p-3}\left|\partial_{v} \Phi\right|^{2} \\
& +(p-3)(p-4) \int_{\mathcal{R}\left(\tau_{1}, \tau_{2}\right) \cap\left\{t \leq T^{*}\right\}} \chi_{R} \cdot\left(r^{p-3}\left|r^{-1} \partial_{\sigma} \Phi\right|^{2}\right. \\
& \left.+\frac{(d-1)(d-3)}{4} r^{p-5}|\Phi|^{2}\right) d u d v d \sigma \\
& +C_{p, \eta, \varepsilon} \int_{\mathcal{R}\left(\tau_{1}, \tau_{2}\right) \cap\left\{t \leq T^{*}\right\}}\left|\partial \chi_{R}\right| \cdot\left(r^{p}\left|\partial^{2} \Phi\right|^{2}+r^{p-2}|\partial \Phi|^{2}\right) d u d v d \sigma \\
& +C_{p, \eta, \varepsilon} \int_{\mathcal{R}\left(\tau_{1}, \tau_{2}\right) \cap\left\{t \leq T^{*}\right\}} \chi_{R} \cdot\left(r^{1+\eta}+r^{p+1}\right) \cdot\left|\partial_{v}(\Omega F)\right|^{2} d u d v d \sigma \\
& +C_{p, \eta, \varepsilon} \text { Bound }_{p, T^{*}}^{\partial_{v}}[\varphi]\left(\tau_{1}, \tau_{2}\right)+C_{p, \eta} \text { Bound }_{p, T^{*}, \varepsilon}^{(n)}[\varphi]\left(\tau_{1}, \tau_{2}\right) \\
& +\mathcal{B}_{p, T^{*}, \eta, \varepsilon}^{\left(\partial_{v}\right)}[\varphi]\left(\tau_{1}, \tau_{2}\right),
\end{aligned}
$$

where we have set:

$$
\begin{aligned}
& \mathcal{B}_{p, T^{*}, \eta, \varepsilon}^{\left(\partial_{v}\right)}[\varphi]\left(\tau_{1}, \tau_{2}\right) \\
& =C_{p, \eta, \varepsilon} \int_{\mathcal{R}\left(\tau_{1}, \tau_{2}\right) \cap\left\{t \leq T^{*}\right\}} \chi_{R} \cdot\left(r^{1+\eta}+r^{p-1-a}\right) \cdot r^{-2}\left|r^{-2} \partial_{\sigma} \partial_{\sigma} \Phi\right|^{2} d u d v d \sigma \\
& \quad+C_{p, \eta, \varepsilon} \int_{\mathcal{R}\left(\tau_{1}, \tau_{2}\right) \cap\left\{t \leq T^{*}\right\}} \chi_{R} \cdot r^{p-1-a}\left|r^{-1} \partial_{\sigma} \partial_{v} \Phi\right|^{2} d u d v d \sigma \\
& \quad+C_{p, \eta, \varepsilon} \int_{\mathcal{R}\left(\tau_{1}, \tau_{2}\right) \cap\left\{t \leq T^{*}\right\}} \chi_{R} \cdot r^{p-3-a}\left|\partial_{u} \partial_{v} \Phi\right|^{2} d u d v d \sigma
\end{aligned}
$$




$$
\begin{aligned}
& +C_{p, \eta, \varepsilon} \int_{\mathcal{R}\left(\tau_{1}, \tau_{2}\right) \cap\left\{t \leq T^{*}\right\}} \chi_{R} \cdot\left(r^{1+\eta}+r^{p-1-a}\right) \cdot r^{-2}|\operatorname{Err}(\Phi)|^{2} d u d v d \sigma \\
& +\int_{\mathcal{R}\left(\tau_{1}, \tau_{2}\right) \cap\left\{t \leq T^{*}\right\}} \chi_{R} \cdot O_{p, \eta, \varepsilon}\left(r^{p}\right) \partial_{v}^{2} \Phi \cdot \operatorname{Err}\left(\partial_{v} \Phi\right) d u d v d \sigma \\
& +\int_{\mathcal{R}\left(\tau_{1}, \tau_{2}\right) \cap\left\{t \leq T^{*}\right\}} \chi_{R} \cdot O_{p, \eta, \varepsilon}\left(r^{p-2-a}\right) \partial_{v} \partial_{\sigma} \Phi \cdot \operatorname{Err}(\Phi) d u d v d \sigma \\
& +\int_{\mathcal{R}\left(\tau_{1}, \tau_{2}\right) \cap\left\{t \leq T^{*}\right\}} \chi_{R} \cdot O_{p, \eta, \varepsilon}(f(r)) \partial_{u} \partial_{v} \Phi \cdot \operatorname{Err}\left(\partial_{v} \Phi\right) d u d v d \sigma \\
& +O_{p, \eta(\varepsilon)} \int_{\mathcal{R}\left(\tau_{1}, \tau_{2}\right) \cap\left\{t \leq T^{*}\right\}} \chi_{R} \cdot r^{p-1}\left(\left|\partial_{v}^{2} \Phi\right|^{2}+r^{-2}\left|\partial_{v} \Phi\right|^{2}\right) d u d v d \sigma .
\end{aligned}
$$

Returning to (6.18), (6.26) implies that

$$
\begin{aligned}
& \mathcal{E}_{\text {bound }, R, T^{*}}^{(p)}\left[\Omega^{-1} \partial_{v} \Phi\right]\left(\tau_{2}\right)+\int_{\tau_{1}}^{\tau_{2}} \mathcal{E}_{\text {bulk,R, }, T^{*}}^{(p-1)}\left[\Omega^{-1} \partial_{v} \Phi\right](\tau) d \tau \\
& +\mathcal{E}_{\mathcal{I}^{+}, R, T^{*}}^{(p)}\left[\Omega^{-1} \partial_{v} \Phi\right]\left(\tau_{1}, \tau_{2}\right) \\
& \leq\left(1+O_{p, \eta}(\varepsilon)\right) \mathcal{E}_{\text {bound }, R, T^{*}}^{(p)}\left[\Omega^{-1} \partial_{v} \Phi\right]\left(\tau_{1}\right)+C_{p, \eta, \varepsilon} \int_{\mathcal{R}\left(\tau_{1}, \tau_{2}\right) \cap\left\{t \leq T^{*}\right\}}\left|\partial \chi_{R}\right| \\
& \times\left(r^{p}\left|\partial \partial_{v} \Phi\right|^{2}+r^{p-2}\left|\partial_{v} \Phi\right|^{2}\right) d u d v d \sigma \\
& +C_{p, \eta, \varepsilon} \int_{\mathcal{R}\left(\tau_{1}, \tau_{2}\right) \cap\left\{t \leq T^{*}\right\}} \chi_{R} \cdot \max \left\{r^{p-3-a}, r^{-3}\right\}\left|\partial_{v} \Phi\right|^{2} d u d v d \sigma \\
& +C_{p, \eta, \varepsilon} \int_{\mathcal{R}\left(\tau_{1}, \tau_{2}\right) \cap\left\{t \leq T^{*}\right\}} \chi_{R} \cdot\left(r^{1+\eta}+r^{p+1}\right) \cdot\left|\partial_{v}(\Omega F)\right|^{2} d u d v d \sigma \\
& +(p-3)(p-4) \int_{\mathcal{R}\left(\tau_{1}, \tau_{2}\right) \cap\left\{t \leq T^{*}\right\}} \chi_{R} \\
& \times\left(r^{p-3}\left|r^{-1} \partial_{\sigma} \Phi\right|^{2}+\frac{(d-1)(d-3)}{4} r^{p-5}|\Phi|^{2}\right) d u d v d \sigma \\
& +C_{p, \eta, \varepsilon} \text { Bound }_{p, T^{*}}^{\partial_{v}}[\varphi]\left(\tau_{1}, \tau_{2}\right) \\
& +C_{p, \eta} \text { Bound }_{p, T^{*}, \varepsilon}^{(n)}[\varphi]\left(\tau_{1}, \tau_{2}\right)+\mathcal{B}_{p, T^{*}, \eta, \varepsilon}^{\left(\partial_{v}\right)}[\varphi]\left(\tau_{1}, \tau_{2}\right) \\
& -2 \int_{\mathcal{R}\left(\tau_{1}, \tau_{2}\right) \cap\left\{t \leq T^{*}\right\}} \chi_{R} \cdot r^{p-1}\left|r^{-1} \partial_{\sigma} \partial_{v} \Phi\right|^{2} d u d v d \sigma \\
& -\frac{(d-1)(d-3)}{2} \int_{\mathcal{R}\left(\tau_{1}, \tau_{2}\right) \cap\left\{t \leq T^{*}\right\}} \chi_{R} \cdot r^{p-1}\left|\partial_{v} \Phi\right|^{2} .
\end{aligned}
$$

Notice that the last two terms of the right hand side of (6.28) can be moved to the left hand side, thus providing us with extra control over bulk terms of the form $\int \chi_{R}$. $r^{p-1}\left|r^{-1} \partial_{\sigma} \partial_{v} \Phi\right|^{2}$ and (in dimensions $d>3$ ) $\int \chi_{R} \cdot r^{p-3}\left|\partial_{v} \Phi\right|^{2}$ : 


$$
\begin{aligned}
& \mathcal{E}_{\text {bound }, R, T^{*}}^{(p)}\left[\Omega^{-1} \partial_{v} \Phi\right]\left(\tau_{2}\right)+\int_{\tau_{1}}^{\tau_{2}} \mathcal{E}_{\text {bulk,R, }, T^{*}}^{\left(p-1, \partial_{v}\right)}\left[\Omega^{-1} \partial_{v} \Phi\right](\tau) d \tau \\
& +\mathcal{E}_{\mathcal{I}^{+}, R, T^{*}}^{(p)}\left[\Omega^{-1} \partial_{v} \Phi\right]\left(\tau_{1}, \tau_{2}\right) \\
& \leq\left(1+O_{p, \eta}(\varepsilon)\right) \mathcal{E}_{\text {bound }, R, T^{*}}^{(p)}\left[\Omega^{-1} \partial_{v} \Phi\right]\left(\tau_{1}\right) \\
& +C_{p, \eta, \varepsilon} \int_{\mathcal{R}\left(\tau_{1}, \tau_{2}\right) \cap\left\{t \leq T^{*}\right\}}\left|\partial \chi_{R}\right| \cdot\left(r^{p}\left|\partial \partial_{v} \Phi\right|^{2}+r^{p-2}\left|\partial_{v} \Phi\right|^{2}\right) d u d v d \sigma \\
& +C_{p, \eta, \varepsilon} \int_{\mathcal{R}\left(\tau_{1}, \tau_{2}\right) \cap\left\{t \leq T^{*}\right\}} \chi_{R} \cdot \max \left\{r^{p-3-a}, r^{-3}\right\}\left|\partial_{v} \Phi\right|^{2} d u d v d \sigma \\
& +C_{p, \eta, \varepsilon} \int_{\left\{\tau_{1} \leq \bar{t} \leq \tau_{2}\right\} \cap\left\{t \leq T^{*}\right\}} \chi_{R} \cdot\left(r^{1+\eta}+r^{p+1}\right) \cdot\left|\partial_{v}(\Omega F)\right|^{2} d u d v d \sigma \\
& +(p-3)(p-4) \int_{\mathcal{R}\left(\tau_{1}, \tau_{2}\right) \cap\left\{t \leq T^{*}\right\}} \chi_{R} \cdot\left(r^{p-3}\left|r^{-1} \partial_{\sigma} \Phi\right|^{2}\right. \\
& \left.+\frac{(d-1)(d-3)}{4} r^{p-5}|\Phi|^{2}\right) d u d v d \sigma \\
& +C_{p, \eta, \varepsilon} \text { Bound }_{p, T^{*}}^{\partial_{v}}[\varphi]\left(\tau_{1}, \tau_{2}\right)+C_{p, \eta} \text { Bound }_{p, T^{*}, \varepsilon}^{(n)}[\varphi]\left(\tau_{1}, \tau_{2}\right) \\
& +\mathcal{B}_{p, T^{*}, \eta, \varepsilon}^{\left(\partial_{v}\right)}[\varphi]\left(\tau_{1}, \tau_{2}\right)
\end{aligned}
$$

where we have set

$$
\begin{aligned}
& \mathcal{E}_{\text {bulk,R, }}^{\left(p-1, \partial_{v}\right)}, T^{*}[\Psi](\tau) \\
& \doteq \int_{\mathcal{S}_{\tau} \cap\left\{t \leq T^{*}\right\}} \chi_{R}\left(p r^{p-1}\left|\partial_{v}(\Omega \Psi)\right|^{2}\right. \\
& \left.\quad+(6-p)\left(r^{p-1}\left|r^{-1} \partial_{\sigma}(\Omega \Psi)\right|^{2}+\frac{(d-1)(d-3)}{4} r^{p-3}|\Omega \Psi|^{2}\right)\right) d v d \sigma \\
& \quad+\int_{\mathcal{S}_{\tau} \cap\left\{t \leq T^{*}\right\}} \chi_{R} r^{-1-\eta}\left|\partial_{u}(\Omega \Psi)\right|^{2} d v d \sigma .
\end{aligned}
$$

Notice the $(6-p)$ factor in the $\mathcal{E}_{b u l k, R, \eta, T^{*}}^{\left(p-1, \partial_{v}\right)}$ bulk energy norm which makes it positive definite for $2<p \leq 4$.

We can extract a similar inequality for $r^{-1} \partial_{\sigma} \Phi$ in place of $\partial_{v} \Phi$. Repeating as before the proof of Lemma 5.4 for $\Omega^{-1}\left(r^{-1} \nabla^{\mathbb{S}^{d-1}} \Phi\right)$ in place of $\varphi$, we obtain:

$$
\begin{aligned}
& \mathcal{E}_{\text {bound }, R, T^{*}}^{(p)}\left[\Omega^{-1} r^{-1} \partial_{\sigma} \Phi\right]\left(\tau_{2}\right)+\int_{\tau_{1}}^{\tau_{2}} \mathcal{E}_{\text {bulk,R, }, T^{*}}^{(p-1)}\left[\Omega^{-1} r^{-1} \partial_{\sigma} \Phi\right](\tau) d \tau \\
& \quad+\mathcal{E}_{\mathcal{I}^{+}, R, T^{*}}^{(p)}\left[\Omega^{-1} r^{-1} \partial_{\sigma} \Phi\right]\left(\tau_{1}, \tau_{2}\right) \\
& \leq\left(1+O_{p, \eta}(\varepsilon)\right) \mathcal{E}_{\text {bound }, R, T^{*}}^{(p)}\left[\Omega^{-1} r^{-1} \partial_{\sigma} \Phi\right]\left(\tau_{1}\right) \\
& \quad+C_{p, \eta, \varepsilon} \int_{\mathcal{R}\left(\tau_{1}, \tau_{2}\right) \cap\left\{t \leq T^{*}\right\}}\left|\partial \chi_{R}\right| \cdot\left(r^{p-2}\left|\partial \partial_{\sigma} \Phi\right|^{2}+r^{p-4}\left|\partial_{\sigma} \Phi\right|^{2}\right) d u d v d \sigma
\end{aligned}
$$




$$
\begin{aligned}
& +C_{p, \eta, \varepsilon} \int_{\mathcal{R}\left(\tau_{1}, \tau_{2}\right) \cap\left\{t \leq T^{*}\right\}} \chi_{R} \cdot\left(r^{p-1-a}\left|r^{-2} \partial_{\sigma} \partial_{\sigma} \Phi\right|^{2}+r^{p-3-a}\left|r^{-1} \partial_{u} \partial_{\sigma} \Phi\right|^{2}\right. \\
& \left.+\max \left\{r^{p-3-a}, r^{-3}\right\}\left|r^{-1} \partial_{\sigma} \Phi\right|^{2}\right) d u d v d \sigma \\
& +C_{p, \eta, \varepsilon} \int_{\mathcal{R}\left(\tau_{1}, \tau_{2}\right) \cap\left\{t \leq T^{*}\right\}} \chi_{R} \cdot \frac{1}{2} f \cdot\left(\partial_{v}-\partial_{u}\right)\left(\Omega^{-1} r^{-1} \nabla^{\mathbb{S}^{d-1}} \Phi\right) \\
& \times \square\left(\Omega^{-1} r^{-1} \nabla^{\mathbb{S}^{d-1}} \Phi\right) \Omega^{2} d u d v d \sigma \\
& +C_{p, \eta, \varepsilon} \int_{\mathcal{R}\left(\tau_{1}, \tau_{2}\right) \cap\left\{t \leq T^{*}\right\}} \chi_{R} \cdot \frac{(d-1) f}{r}\left(\Omega^{-1} r^{-1} \nabla^{\mathbb{S}^{d-1}} \Phi\right) \\
& \times \square\left(\Omega^{-1} r^{-1} \nabla^{\mathbb{S}^{d-1}} \Phi\right) \Omega^{2} d u d v d \sigma \\
& +C_{p, \eta, \varepsilon} \int_{\mathcal{R}\left(\tau_{1}, \tau_{2}\right) \cap\left\{t \leq T^{*}\right\}} \chi_{R} \cdot\left(\partial_{v}+\partial_{u}\right)\left(r^{-1} \nabla^{\mathbb{S}^{d-1}} \Phi\right) \\
& \times \Omega \square\left(\Omega^{-1} r^{-1} \nabla^{\mathbb{S}^{d-1}} \Phi\right) d u d v d \sigma \\
& +\int_{\mathcal{R}\left(\tau_{1}, \tau_{2}\right) \cap\left\{t \leq T^{*\}}\right.} \chi_{R} \cdot O\left(r^{p-3-a}\right) \cdot \partial_{\sigma}^{2} \Phi \cdot \Omega \square\left(\Omega^{-1} r^{-1} \partial_{\sigma} \Phi\right) d u d v d \sigma \\
& -\int_{\mathcal{R}\left(\tau_{1}, \tau_{2}\right) \cap\left\{t \leq T^{*}\right\}} \chi_{R} \cdot r^{p} \partial_{v}\left(r^{-1} \nabla^{\mathbb{S}^{d-1}} \Phi\right) \cdot \Omega \square\left(\Omega^{-1} r^{-1} \nabla^{\mathbb{S}^{d-1}} \Phi\right) d u d v d \sigma \\
& +C_{p, \eta, \varepsilon} \operatorname{Bound}_{p, T^{*}}^{r^{-1} \partial_{\sigma}}[\varphi]\left(\tau_{1}, \tau_{2}\right),
\end{aligned}
$$

where the multiplication between derivatives of the $\mathbb{S}^{d-1}$ gradient $\nabla^{\mathbb{S}^{d-1}} \Phi$ of $\Phi$ in the last lines of (6.31) is performed with respect to the usual metric $g_{\mathbb{S}^{d-1}}$ of $\mathbb{S}^{d-1}$, and

$$
\begin{aligned}
\text { Bound }_{p, T^{*}}^{r^{-1} \partial_{\sigma}[\varphi]\left(\tau_{1}, \tau_{2}\right)=} & \sum_{i=1}^{2} \int_{\mathcal{S}_{\tau_{i}} \cap\left\{t \leq T^{*}\right\}} \chi_{R} \cdot r^{p-2-a}\left(\left|r^{-1} \partial_{u} \partial_{\sigma} \Phi\right|^{2}+\left|r^{-2} \partial_{\sigma} \partial_{\sigma} \Phi\right|^{2}\right. \\
& \left.+\left|r^{-1} \partial_{\sigma} \Phi\right|^{2}+r^{-2} \Phi^{2}\right) d v d \sigma \\
& +\int_{\mathcal{R}\left(\tau_{1}, \tau_{2}\right) \cap\left\{t=T^{*}\right\}} \chi_{R} \cdot r^{p-2-a}\left(\left|r^{-1} \partial_{u} \partial_{\sigma} \Phi\right|^{2}\right. \\
& \left.+\left|r^{-2} \partial_{\sigma} \partial_{\sigma} \Phi\right|^{2}+\left|r^{-1} \partial_{\sigma} \Phi\right|^{2}+r^{-2} \Phi^{2}\right) d v d \sigma \\
& +\int_{\mathcal{S}_{\tau_{1}} \cap\left\{t \leq T^{*}\right\}} \chi_{R} J_{\mu}^{T}\left(\Omega^{-1} r^{-1} \partial_{\sigma} \Phi\right) \bar{n}^{\mu}
\end{aligned}
$$

Using Lemma 6.3, we have:

$$
\begin{aligned}
\Omega \square\left(\Omega^{-1}\left(r^{-1} \partial_{\sigma} \Phi\right)\right)= & r^{-1} \partial_{\sigma}(\Omega \square \varphi)+r^{-2} \partial_{u} \partial_{\sigma} \Phi-r^{-2} \partial_{v} \partial_{\sigma} \Phi \\
& +\operatorname{Err}\left(r^{-1} \partial_{\sigma} \Phi\right)+r^{-1} \operatorname{Err}(\Phi) .
\end{aligned}
$$


Remark The term $r^{-2} \partial_{u} \partial_{\sigma} \Phi^{11}$ in (6.33) will provide us with improved control over $\left|\partial_{\sigma} \partial_{\sigma} \Phi\right|^{2}$ bulk terms, in the same way that the term $r^{-3} \Delta_{g_{\mathbb{S} d-1}+h_{\mathbb{S} d-1}} \Phi$ in (6.21) eventually provided us with improved control over $\left|\partial_{\sigma} \partial_{v} \Phi\right|^{2}$ bulk terms through (6.22) and (6.23). We should also notice that the term $-r^{-2} \partial_{v} \partial_{\sigma} \Phi$ has a bad sign, and will result in the appearence of bulk terms of the form $\int \chi_{R} r^{p-1}\left|\partial_{\sigma} \partial_{v} \Phi\right|^{2}$ with a nonconvenient sign. All the terms with a bad sign, however, will be controlled by the corresponding terms in the left hand side of (6.29) plus a multiple of (5.1) for $p-2$ in place of $p$, provided $p \leq 4$.

Integrating by parts in $\partial_{v}$ and the spherical directions (omitting the $d u d v d \sigma$ volume form in the next few lines), and then once again in $\partial_{u}$ for the error term, we compute:

$$
\begin{aligned}
& \int_{\mathcal{R}\left(\tau_{1}, \tau_{2}\right) \cap\left\{t \leq T^{*}\right\}} \chi_{R} \cdot\left(1+C \cdot r^{-a}\right) r^{p-2} \partial_{v}\left(r^{-1} \nabla^{\mathbb{S}^{d-1}} \Phi\right) \cdot \partial_{u} \nabla^{\mathbb{S}^{d-1}} \Phi \\
& =\int_{\mathcal{R}\left(\tau_{1}, \tau_{2}\right) \cap\left\{t \leq T^{*}\right\}} \chi_{R} \cdot\left(1+O\left(r^{-a}\right)\right) r^{p-1}\left(r^{-2} \Delta_{g_{\mathbb{S}} d-1} \Phi\right) \cdot \partial_{u} \partial_{v} \Phi \\
& \quad+\int_{\mathcal{R}\left(\tau_{1}, \tau_{2}\right) \cap\left\{t \leq T^{*}\right\}} \chi_{R} \cdot\left(\frac{(p-2)(4-p)}{2} r^{p-3}+O\left(r^{p-3-a}\right)\right)\left|r^{-1} \partial_{\sigma} \Phi\right|^{2} \\
& \quad+\int_{\mathcal{R}\left(\tau_{1}, \tau_{2}\right) \cap\left\{t \leq T^{*}\right\}}\left|\partial \chi_{R}\right| \cdot\left(O\left(r^{p}\right)\left|\partial^{2} \Phi\right|^{2}+O\left(r^{p-2}\right)|\partial \Phi|^{2}\right) \\
& \quad+O_{p, \eta}(1) \cdot \text { Bound }_{p, T^{*}, \varepsilon}^{(n)}[\varphi]\left(\tau_{1}, \tau_{2}\right) .
\end{aligned}
$$

However, since $\square_{g} \varphi=F$, the following equality holds:

$$
\begin{aligned}
\left(1+O\left(r^{-1-a}\right)\right) \partial_{u} \partial_{v} \Phi= & -\Omega F+r^{-2} \Delta_{g_{\mathbb{S}^{d}-1}+h_{\mathbb{S}^{d}-1} \Phi} \\
& -\left(\frac{(d-1)(d-3)}{4} \cdot r^{-2}\right) \Phi+\operatorname{Err}(\Phi)
\end{aligned}
$$

Using (6.35) to substitute $\partial_{u} \partial_{v} \Phi$ in (6.34), as well as the following elliptic-type estimate on $\mathbb{S}^{d-1}$

$$
\int_{\mathbb{S}^{d-1}}\left|\Delta_{g_{\mathbb{S}^{d-1}}} \Phi\right|^{2} d \sigma \geq \int_{\mathbb{S}^{d-1}}\left|\partial_{\sigma} \partial_{\sigma} \Phi\right|^{2} d \sigma+(d-2) \int_{\mathbb{S}^{d-1}}\left|\partial_{\sigma} \Phi\right|^{2},
$$

we infer after integrating by parts in $\partial_{\sigma}$ in the term $\int \chi_{R} \cdot \frac{(d-1)(d-3)}{2} \cdot r^{p-5} \Delta_{g_{\mathbb{S}} d-1} \Phi \cdot \Phi$ :

$$
\begin{aligned}
& \int_{\mathcal{R}\left(\tau_{1}, \tau_{2}\right) \cap\left\{t \leq T^{*}\right\}} \chi_{R} \cdot r^{p-2} \partial_{v}\left(r^{-1} \nabla^{\mathbb{S}^{d-1}} \Phi\right) \cdot \partial_{u} \nabla^{\mathbb{S}^{d-1}} \Phi \\
& \geq \int_{\mathcal{R}\left(\tau_{1}, \tau_{2}\right) \cap\left\{t \leq T^{*}\right\}} \chi_{R} \cdot r^{p-1}\left|r^{-2} \partial_{\sigma} \partial_{\sigma} \Phi\right|^{2}
\end{aligned}
$$

$\overline{11 \text { Recall that this term is actually } r^{-2} \mathcal{L}_{\partial_{u}}\left(\nabla^{\mathbb{S}^{d-1}} \Phi\right) \text {, see Section } 2 .}$ 


$$
\begin{aligned}
& +\int_{\mathcal{R}\left(\tau_{1}, \tau_{2}\right) \cap\left\{t \leq T^{*}\right\}} \chi_{R} \cdot r^{p-1-a} \cdot O\left(\left|r^{-2} \partial_{\sigma} \partial_{\sigma} \Phi\right|^{2}+\left|r^{-1} \partial_{\sigma} \Phi\right|^{2}\right) \\
& +\int_{\mathcal{R}\left(\tau_{1}, \tau_{2}\right) \cap\left\{t \leq T^{*}\right\}} \chi_{R} \cdot\left(\frac{(d-1)(d-3)+2(p-2)(4-p)}{4}\right. \\
& \left.+(d-2)+O\left(r^{-a}\right)\right) \cdot r^{p-3}\left|r^{-1} \partial_{\sigma} \Phi\right|^{2} \\
& +\int_{\mathcal{R}\left(\tau_{1}, \tau_{2}\right) \cap\left\{t \leq T^{*}\right\}} O\left(\left|\partial \chi_{R}\right|\right) \cdot\left(O\left(r^{p}\right)\left|\partial^{2} \Phi\right|^{2}+O\left(r^{p-2}\right)|\partial \Phi|^{2}\right) \\
& +\int_{\mathcal{R}\left(\tau_{1}, \tau_{2}\right) \cap\left\{t \leq T^{*}\right\}} \chi_{R} \cdot O\left(r^{p-1}\right)\left(r^{-2} \partial_{\sigma} \partial_{\sigma} \Phi\right) \cdot \operatorname{Err}(\Phi) \\
& +\int_{\mathcal{R}\left(\tau_{1}, \tau_{2}\right) \cap\left\{t \leq T^{*}\right\}} \chi_{R} \cdot O\left(r^{p-1}\right) \cdot \partial_{v}\left(r^{-1} \partial_{\sigma} \Phi\right) \cdot \Omega F \\
& +C_{p, \eta, \varepsilon} \text { Bound }_{p, T^{*}}^{r^{-1} \partial_{\sigma}}[\varphi]\left(\tau_{1}, \tau_{2}\right)+C_{p, \eta} \text { Bound }_{p, T^{*}, \varepsilon}^{(n)}[\varphi]\left(\tau_{1}, \tau_{2}\right) .
\end{aligned}
$$

This should be considered as the analogue of (6.23). Proceeding therefore as before, from (6.31), (6.33) and (6.37) we can extract the following analogue of (6.29):

$$
\begin{aligned}
& \mathcal{E}_{\text {bound }, R, T^{*}}^{(p)}\left[\Omega^{-1} r^{-1} \partial_{\sigma} \Phi\right]\left(\tau_{2}\right)+\int_{\tau_{1}}^{\tau_{2}} \mathcal{E}_{\text {bulk,R, }, T^{*}}^{\left(p-1, \partial_{\sigma}\right)}\left[\Omega^{-1} r^{-1} \partial_{\sigma} \Phi\right](\tau) d \tau \\
& +\mathcal{E}_{\mathcal{I}^{+}, R, T^{*}}^{(p)}\left[\Omega^{-1} r^{-1} \partial_{\sigma} \Phi\right]\left(\tau_{1}, \tau_{2}\right) \\
& \leq\left(1+O_{p, \eta}(\varepsilon)\right) \mathcal{E}_{\text {bound }, R, T^{*}}^{(p)}\left[\Omega^{-1} r^{-1} \partial_{\sigma} \Phi\right]\left(\tau_{1}\right) \\
& +C_{p, \eta, \varepsilon} \int_{\mathcal{R}\left(\tau_{1}, \tau_{2}\right) \cap\left\{t \leq T^{*}\right\}}\left|\partial \chi_{R}\right| \cdot\left(r^{p-2}\left|\partial \partial_{\sigma} \Phi\right|^{2}+r^{p-4}\left|\partial_{\sigma} \Phi\right|^{2}\right) d u d v d \sigma \\
& +C_{p, \eta, \varepsilon} \int_{\mathcal{R}\left(\tau_{1}, \tau_{2}\right) \cap\left\{t \leq T^{*}\right\}} \chi_{R} \cdot \max \left\{r^{p-3-a}, r^{-3}\right\}\left|r^{-1} \partial_{\sigma} \Phi\right|^{2} d u d v d \sigma \\
& +C_{p, \eta, \varepsilon} \int_{\mathcal{R}\left(\tau_{1}, \tau_{2}\right) \cap\left\{t \leq T^{*}\right\}} \chi_{R} \cdot\left(r^{1+\eta}+r^{p+1}\right) \\
& \cdot\left(\left|r^{-1} \partial_{\sigma}(\Omega F)\right|^{2}+r^{-2}|\Omega F|^{2}\right) d u d v d \sigma \\
& +C_{p, \eta, \varepsilon} \cdot \int_{\mathcal{R}\left(\tau_{1}, \tau_{2}\right) \cap\left\{t \leq T^{*}\right\}} \chi_{R} \cdot r^{p-1-a}\left|r^{-1} \partial_{\sigma} \partial_{v} \Phi\right|^{2} d u d v d \sigma \\
& +\int_{\mathcal{R}\left(\tau_{1}, \tau_{2}\right) \cap\left\{t \leq T^{*}\right\}} \chi_{R} \cdot\left(1+O\left(r^{-a}\right)\right) r^{p-1}\left(r^{-1} \partial_{v} \nabla^{\mathbb{S}^{d-1}} \Phi\right) \\
& \cdot\left(\partial_{v}\left(r^{-1} \nabla^{\mathbb{S}^{d-1}} \Phi\right)\right) d u d v d \sigma \\
& +C_{p, \eta, \varepsilon} \text { Bound }_{p, T^{*}}^{r^{-1} \partial_{\sigma}}[\varphi]\left(\tau_{1}, \tau_{2}\right)+C_{p, \eta} \text { Bound }_{p, T^{*}, \varepsilon}^{(n)}[\varphi]\left(\tau_{1}, \tau_{2}\right) \\
& +\mathcal{B}_{p, T^{*}, \eta, \varepsilon}^{\left(r^{-1} \partial_{\sigma}\right)}[\varphi]\left(\tau_{1}, \tau_{2}\right),
\end{aligned}
$$


where we have set

$$
\begin{aligned}
& \mathcal{E}_{\text {bulk, } R, \eta, T^{*}}^{\left(p-1, \partial_{\sigma}\right)}[\Psi](\tau) \\
& \doteq \int_{\mathcal{S}_{\tau} \cap\left\{t \leq T^{*}\right\}} \chi_{R}\left(p r^{p-1}\left|\partial_{v}(\Omega \Psi)\right|^{2}+\max \left\{(4-p), r^{-\frac{a}{2}}\right\}\right. \\
& \left.\times\left(r^{p-1}\left|r^{-1} \partial_{\sigma}(\Omega \Psi)\right|^{2}+\frac{(d-1)(d-3)}{4} r^{p-3}|\Omega \Psi|^{2}\right)\right) d v d \sigma \\
& +\int_{\mathcal{S}_{\tau} \cap\left\{t \leq T^{*}\right\}} \chi_{R} r^{-1-\eta}\left|\partial_{u}(\Omega \Psi)\right|^{2} d v d \sigma
\end{aligned}
$$

and $\mathcal{B}_{p, T^{*}, \eta, \varepsilon}^{\left(r^{-1} \partial_{\sigma}\right)}[\varphi]\left(\tau_{1}, \tau_{2}\right)$ is of the form:

$$
\begin{aligned}
& \mathcal{B}_{p, T^{*}, \eta, \varepsilon}^{\left(r^{-1} \partial_{\sigma}\right)}[\varphi]\left(\tau_{1}, \tau_{2}\right) \\
& =C_{p, \eta, \varepsilon} \int_{\mathcal{R}\left(\tau_{1}, \tau_{2}\right) \cap\left\{t \leq T^{*}\right\}} \chi_{R} \cdot r^{-1+\eta}\left(\left|r^{-1} \partial_{\sigma} \partial_{u} \Phi\right|^{2}+\left|r^{-1} \partial_{\sigma} \partial_{v} \Phi\right|^{2}\right) d u d v d \sigma \\
& \quad+C_{p, \eta, \varepsilon} \int_{\mathcal{R}\left(\tau_{1}, \tau_{2}\right) \cap\left\{t \leq T^{*}\right\}} \chi_{R} \cdot r^{p-1-a}\left(\left|r^{-2} \partial_{\sigma} \partial_{\sigma} \Phi\right|^{2}+\left|r^{-1} \partial_{\sigma} \Phi\right|^{2}\right) d u d v d \sigma \\
& \quad+C_{p, \eta, \varepsilon} \int_{\mathcal{R}\left(\tau_{1}, \tau_{2}\right) \cap\left\{t \leq T^{*}\right\}} \chi_{R} r^{p-3-a}\left(\left|r^{-1} \partial_{u} \partial_{\sigma} \Phi\right|^{2}\right) d u d v d \sigma \\
& \quad+C_{p, \eta, \varepsilon} \int_{\mathcal{R}\left(\tau_{1}, \tau_{2}\right) \cap\left\{t \leq T^{*}\right\}} \chi_{R} \cdot\left(r^{1+\eta}+r^{p-1-a}\right) r^{-2}|\operatorname{Err}(\Phi)|^{2} d u d v d \sigma \\
& \quad+\int_{\mathcal{R}\left(\tau_{1}, \tau_{2}\right) \cap\left\{t \leq T^{*}\right\}} \chi_{R} \cdot O_{p, \eta, \varepsilon}\left(r^{p-1}\right) \partial_{v} \partial_{\sigma} \Phi\left(\operatorname{Err}\left(r^{-1} \partial_{\sigma} \Phi\right)+r^{-1} \operatorname{Err}(\Phi)\right) d u d v d \sigma \\
& +\int_{\mathcal{R}\left(\tau_{1}, \tau_{2}\right) \cap\left\{t \leq T^{*}\right\}} \chi_{R} \cdot O_{p, \eta, \varepsilon}\left(r^{p-3}\right) \partial_{\sigma} \partial_{\sigma} \Phi \cdot \operatorname{Err}(\Phi) d u d v d \sigma \\
& \quad+C_{p, \eta, \varepsilon} \int_{\mathcal{R}\left(\tau_{1}, \tau_{2}\right) \cap\left\{t \leq T^{*}\right\}} \chi_{R} \cdot f(r) \partial_{u}\left(r^{-1} \partial_{\sigma} \Phi\right) \operatorname{Err}\left(r^{-1} \partial_{\sigma} \Phi\right) d u d v d \sigma .
\end{aligned}
$$

We can now add (6.29) and (6.38) so as to obtain

$$
\begin{aligned}
& \mathcal{E}_{\text {bound }, R, T^{*}}^{(p)}\left[\Omega^{-1} \partial_{v} \Phi\right]\left(\tau_{2}\right)+\mathcal{E}_{\text {bound }, R, T^{*}}^{(p)}\left[\Omega^{-1} r^{-1} \partial_{\sigma} \Phi\right]\left(\tau_{2}\right)
\end{aligned}
$$

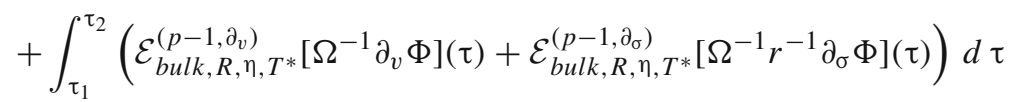

$$
\begin{aligned}
& +\mathcal{E}_{\mathcal{I}^{+}, R, T^{*}}^{(p)}\left[\Omega^{-1} \partial_{v} \Phi\right]\left(\tau_{1}, \tau_{2}\right)+\mathcal{E}_{\mathcal{I}^{+}, R, T^{*}}^{(p)}\left[\Omega^{-1} r^{-1} \partial_{\sigma} \Phi\right]\left(\tau_{1}, \tau_{2}\right) \\
& \leq\left(1+O_{p, \eta}(\varepsilon)\right)\left(\mathcal{E}_{\text {bound }, R, T^{*}}^{(p)}\left[\Omega^{-1} \partial_{v} \Phi\right]\left(\tau_{1}\right)+\mathcal{E}_{\text {bound }, R, T^{*}}^{(p)}\left[\Omega^{-1} r^{-1} \partial_{\sigma} \Phi\right]\left(\tau_{1}\right)\right) \\
& +C_{p, \eta, \varepsilon} \int_{\mathcal{R}\left(\tau_{1}, \tau_{2}\right) \cap\left\{t \leq T^{*}\right\}}\left|\partial \chi_{R}\right| \cdot\left(r^{p}\left|\partial^{2} \Phi\right|^{2}+r^{p-2}|\partial \Phi|^{2}\right) d u d v d \sigma \\
& +C_{p, \eta, \varepsilon} \int_{\mathcal{R}\left(\tau_{1}, \tau_{2}\right) \cap\left\{t \leq T^{*}\right\}} \chi_{R} \cdot \max \left\{r^{p-3-a}, r^{-3}\right\}\left(\left|\partial_{v} \Phi\right|^{2}+\left|r^{-1} \partial_{\sigma} \Phi\right|^{2}\right) d u d v d \sigma
\end{aligned}
$$




$$
\begin{aligned}
& +C_{p, \eta, \varepsilon} \int_{\mathcal{R}\left(\tau_{1}, \tau_{2}\right) \cap\left\{t \leq T^{*}\right\}} \chi_{R} \\
& \cdot\left(r^{1+\eta}+r^{p+1}\right) \cdot\left(\left|\partial_{v}(\Omega F)\right|^{2}+\left|r^{-1} \partial_{\sigma}(\Omega F)\right|^{2}+r^{-2}|\Omega F|^{2}\right) d u d v d \sigma \\
& +(p-3)(p-4) \int_{\mathcal{R}\left(\tau_{1}, \tau_{2}\right) \cap\left\{t \leq T^{*}\right\}} \chi_{R} \\
& \cdot\left(r^{p-3}\left|r^{-1} \partial_{\sigma} \Phi\right|^{2}+\frac{(d-1)(d-3)}{4} r^{p-5}|\Phi|^{2}\right) d u d v d \sigma \\
& +\int_{\mathcal{R}\left(\tau_{1}, \tau_{2}\right) \cap\left\{t \leq T^{*}\right\}} \chi_{R} \cdot\left(1+O\left(r^{-a}\right)\right) r^{p-1}\left(r^{-1} \partial_{v} \nabla^{\mathbb{S}^{d-1}} \Phi\right) \\
& \cdot\left(\partial_{v}\left(r^{-1} \nabla^{\mathbb{S}^{d-1}} \Phi\right)\right) d u d v d \sigma \\
& +C_{p, \eta, \varepsilon} \operatorname{Bound}_{p, T^{*}}^{\partial_{v}, \partial_{\sigma}}[\varphi]\left(\tau_{1}, \tau_{2}\right)+C_{p, \eta} \text { Bound }_{p, T^{*}, \varepsilon}^{(n)}[\varphi]\left(\tau_{1}, \tau_{2}\right) \\
& +\mathcal{B}_{p, T^{*}, \eta, \varepsilon}^{\left(\partial_{v}\right)}[\varphi]\left(\tau_{1}, \tau_{2}\right)+\mathcal{B}_{p, T^{*}, \eta, \varepsilon}^{\left(r^{-1} \partial_{\sigma}\right)}[\varphi]\left(\tau_{1}, \tau_{2}\right),
\end{aligned}
$$

where

$$
\begin{aligned}
& \text { Bound }_{p, T^{*}}^{\partial_{v}, \partial_{\sigma}}[\varphi]\left(\tau_{1}, \tau_{2}\right) \\
& =C_{p, \eta, \varepsilon} \sum_{i=1}^{2} \int_{\mathcal{S}_{\tau_{i}} \cap\left\{t \leq T^{*}\right\}} \chi_{R} \cdot r^{p-2-a} \\
& \times\left(\left|\partial_{u} \partial_{v} \Phi\right|^{2}+\left|r^{-1} \partial_{\sigma} \partial_{v} \Phi\right|^{2}+\left|\partial_{v} \Phi\right|^{2}+r^{-2}|\Phi|^{2}\right) d v d \sigma \\
& +C_{p, \eta, \varepsilon} \sum_{i=1}^{2} \int_{\mathcal{S}_{\tau_{i}} \cap\left\{t \leq T^{*}\right\}} \chi_{R} \cdot r^{p-2-a} \\
& \times\left(\left|r^{-1} \partial_{u} \partial_{\sigma} \Phi\right|^{2}+\left|r^{-2} \partial_{\sigma} \partial_{\sigma} \Phi\right|^{2}+\left|r^{-1} \partial_{\sigma} \Phi\right|^{2}\right) d v d \sigma \\
& +C_{p, \eta, \varepsilon} \int_{\mathcal{R}\left(\tau_{1}, \tau_{2}\right) \cap\left\{t=T^{*}\right\}} \chi_{R} \cdot r^{p-2-a} \\
& \times\left(\left|\partial_{u} \partial_{v} \Phi\right|^{2}+\left|r^{-1} \partial_{\sigma} \partial_{v} \Phi\right|^{2}+\left|\partial_{v} \Phi\right|^{2}+r^{-2}|\Phi|^{2}\right) d v d \sigma \\
& +C_{p, \eta, \varepsilon} \int_{\mathcal{R}\left(\tau_{1}, \tau_{2}\right) \cap\left\{t=T^{*}\right\}} \chi_{R} \cdot r^{p-2-a} \\
& \times\left(\left|r^{-1} \partial_{u} \partial_{\sigma} \Phi\right|^{2}+\left|r^{-2} \partial_{\sigma} \partial_{\sigma} \Phi\right|^{2}+\left|r^{-1} \partial_{\sigma} \Phi\right|^{2}\right) d v d \sigma \\
& +C_{p, \eta, \varepsilon} \int_{\mathcal{S}_{\tau_{1} \cap\left\{t \leq T^{*}\right\}}} \chi_{R} J_{\mu}^{T}\left(\Omega^{-1} \partial_{v} \Phi\right) \bar{n}^{\mu} \\
& +C_{p, \eta, \varepsilon} \int_{\mathcal{S}_{\tau_{1} \cap\left\{t \leq T^{*}\right\}}} \chi_{R} J_{\mu}^{T}\left(\Omega^{-1} r^{-1} \partial_{\sigma} \Phi\right) \bar{n}^{\mu} \text {. }
\end{aligned}
$$

Since $2<p \leq 4$, the second to the end line of the right hand side of (6.48) can be absorbed by the left hand side after using a Cauchy-Schwarz inequality, since 


$$
\begin{aligned}
& \int_{\mathcal{R}\left(\tau_{1}, \tau_{2}\right) \cap\left\{t \leq T^{*}\right\}} \chi_{R} \cdot\left(1+O\left(r^{-a}\right)\right) r^{p-1}\left(r^{-1} \partial_{v} \nabla^{\mathbb{S}^{d-1}} \Phi\right) \\
& \quad \cdot\left(\partial_{v}\left(r^{-1} \nabla^{\mathbb{S}^{d-1}} \Phi\right)\right) d u d v d \sigma \\
& \leq \frac{1}{2} \int_{\mathcal{R}\left(\tau_{1}, \tau_{2}\right) \cap\left\{t \leq T^{*}\right\}} \chi_{R} \cdot\left((6-p) r^{p-1}\left|r^{-1} \partial_{\sigma} \partial_{v} \Phi\right|^{2}\right. \\
& \left.\quad+p r^{p-1}\left|\partial_{v}\left(r^{-1} \partial_{\sigma} \Phi\right)\right|^{2}\right) d v d \sigma
\end{aligned}
$$

Moreover, the third to the end line of the right hand side of (6.48) can be bounded by the left hand side of (6.45) for $p-2$ in place of $p$ (notice the importance of the $(p-4)$ factor appearing in front of this term). Therefore, after adding to (6.48) a large multiple of (5.15) for $p-2$ in place of $p$ and a large multiple of (5.38) for $p-2-\delta$ in place of $p$, we obtain:

$$
\begin{aligned}
& \mathcal{E}_{\text {bound }, R, T^{*}}^{(p)}\left[\Omega^{-1} \partial_{v} \Phi\right]\left(\tau_{2}\right)+\mathcal{E}_{\text {bound }, R, T^{*}}^{(p)}\left[\Omega^{-1} r^{-1} \partial_{\sigma} \Phi\right]\left(\tau_{2}\right)+\mathcal{E}_{\text {bound }, R, T^{*}}^{(p-2)}[\varphi]\left(\tau_{2}\right)
\end{aligned}
$$

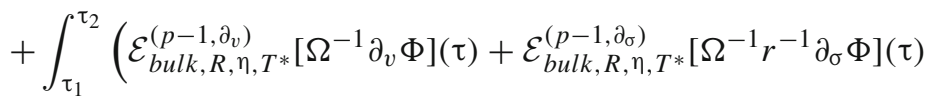

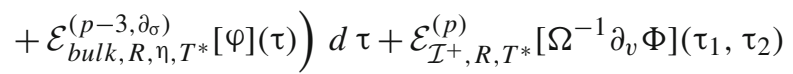

$$
\begin{aligned}
& +\mathcal{E}_{\mathcal{I}^{+}, R, T^{*}}^{(p)}\left[\Omega^{-1} r^{-1} \partial_{\sigma} \Phi\right]\left(\tau_{1}, \tau_{2}\right)+\mathcal{E}_{\mathcal{I}^{+}, R, T^{*}}^{(p-2)}[\varphi]\left(\tau_{1}, \tau_{2}\right) \\
& \leq\left(1+O_{p, \eta}(\varepsilon)\right)\left(\mathcal{E}_{\text {bound }, R, T^{*}}^{(p)}\left[\Omega^{-1} \partial_{v} \Phi\right]\left(\tau_{1}\right)+\mathcal{E}_{\text {bound }, R, T^{*}}^{(p)}\left[\Omega^{-1} r^{-1} \partial_{\sigma} \Phi\right]\left(\tau_{1}\right)\right. \\
& \left.+C \cdot \mathcal{E}_{\text {bound }, R, T^{*}}^{(p-2)}[\varphi]\left(\tau_{1}\right)\right) \\
& +C_{p, \eta, \varepsilon} \int_{\mathcal{R}\left(\tau_{1}, \tau_{2}\right) \cap\left\{t \leq T^{*}\right\}}\left|\partial \chi_{R}\right| \cdot\left(r^{p}\left|\partial^{2} \Phi\right|^{2}+r^{p-2}|\partial \Phi|^{2}+r^{p-4}|\Phi|^{2}\right) d u d v d \sigma \\
& +C_{p, \eta, \varepsilon} \int_{\mathcal{R}\left(\tau_{1}, \tau_{2}\right) \cap\left\{t \leq T^{*}\right\}} \chi_{R} \cdot\left(r^{1+\eta}+r^{p+1}\right) \\
& \times\left(\left|\partial_{v}(\Omega F)\right|^{2}+\left|r^{-1} \partial_{\sigma}(\Omega F)\right|^{2}+r^{-2}|\Omega F|^{2}\right) d u d v d \sigma \\
& +C_{p, \eta, \varepsilon} \text { Bound }_{p, T^{*}}^{\partial_{v}, \partial_{\sigma}}[\varphi]\left(\tau_{1}, \tau_{2}\right)+C_{p, \eta B_{\text {ound }}}^{(n) T^{*}, \varepsilon}[\varphi]\left(\tau_{1}, \tau_{2}\right) \\
& +\mathcal{B}_{p, T^{*}, \eta, \varepsilon}^{\left(\partial_{v}\right)}[\varphi]\left(\tau_{1}, \tau_{2}\right)+\mathcal{B}_{p, T^{*}, \eta, \varepsilon}^{\left(r^{-1} \partial_{\sigma}\right)}[\varphi]\left(\tau_{1}, \tau_{2}\right) .
\end{aligned}
$$

Finally, in order to absorb the error terms of the last line the right hand side of (6.44) into the left hand side, we will need to add to (6.44) a constant multiple of the estimate (5.1) for $\Omega^{-1} \partial_{u} \Phi$ in place of $\Phi$. By following again the proof of Lemma 5.4 for $\Omega^{-1} \partial_{u} \Phi$ in place of $\varphi$ for $p-2$ in place of $p$, we obtain:

$$
\begin{aligned}
& \mathcal{E}_{\text {bound }, R, T^{*}}^{(p-2)}\left[\Omega^{-1} \partial_{u} \Phi\right]\left(\tau_{2}\right)+\int_{\tau_{1}}^{\tau_{2}} \mathcal{E}_{\text {bulk,R, }, T^{*}}^{(p-3)}\left[\Omega^{-1} \partial_{u} \Phi\right](\tau) d \tau \\
& \quad+\mathcal{E}_{\mathcal{I}^{+}, R, T^{*}}^{(p-2)}\left[\Omega^{-1} \partial_{u} \Phi\right]\left(\tau_{1}, \tau_{2}\right)
\end{aligned}
$$




$$
\begin{aligned}
& \leq\left(1+O_{p, \eta}(\varepsilon)\right) \mathcal{E}_{\text {bound }, R, T^{*}}^{(p-2)}\left[\Omega^{-1} \partial_{u} \Phi\right]\left(\tau_{1}\right)+C_{p, \eta, \varepsilon} \int_{\mathcal{R}\left(\tau_{1}, \tau_{2}\right) \cap\left\{t \leq T^{*}\right\}}\left|\partial \chi_{R}\right| \\
& \times\left(r^{p-2}\left|\partial \partial_{u} \Phi\right|^{2}+r^{p-4}\left|\partial_{u} \Phi\right|^{2}\right) d u d v d \sigma \\
& +C_{p, \eta, \varepsilon} \int_{\mathcal{R}\left(\tau_{1}, \tau_{2}\right) \cap\left\{t \leq T^{*}\right\}} \chi_{R} \cdot\left(r^{p-3-a}\left|r^{-1} \partial_{\sigma} \partial_{u} \Phi\right|^{2}\right. \\
& \left.+\max \left\{r^{p-5-a}, r^{-3}\right\}\left|\partial_{u} \Phi\right|^{2}\right) d u d v d \sigma \\
& +C_{p, \eta, \varepsilon} \int_{\mathcal{R}\left(\tau_{1}, \tau_{2}\right) \cap\left\{t \leq T^{*}\right\}} \chi_{R} \cdot\left(\frac{1}{2} f(r)\left(\partial_{v}-\partial_{u}\right)\left(\Omega^{-1} \partial_{u} \Phi\right)\right. \\
& \left.+\frac{(d-1) f(r)}{r}\left(\Omega^{-1} \partial_{u} \Phi\right)\right) \cdot \square\left(\Omega^{-1} \partial_{u} \Phi\right) \Omega^{2} d u d v d \sigma \\
& +C_{p, \eta, \varepsilon} \int_{\mathcal{R}\left(\tau_{1}, \tau_{2}\right) \cap\left\{t \leq T^{*}\right\}} \chi_{R} \cdot\left(\partial_{v}+\partial_{u}\right)\left(\partial_{u} \Phi\right) \cdot \Omega \square\left(\Omega^{-1} \partial_{u} \Phi\right) d u d v d \sigma \\
& +\int_{\mathcal{R}\left(\tau_{1}, \tau_{2}\right) \cap\left\{t \leq T^{*}\right\}} \chi_{R} \cdot O\left(r^{p-4-a}\right) \cdot \partial_{\sigma} \partial_{u} \Phi \cdot \Omega \square\left(\Omega^{-1} \partial_{u} \Phi\right) d u d v d \sigma \\
& -\int_{\mathcal{R}\left(\tau_{1}, \tau_{2}\right) \cap\left\{t \leq T^{*}\right\}} \chi_{R} \cdot r^{p-2} \partial_{v} \partial_{u} \Phi \cdot \Omega \square\left(\Omega^{-1} \partial_{u} \Phi\right) d u d v d \sigma \\
& +C_{p, \eta, \varepsilon} \text { Bound }_{p-2, T^{*}}^{\partial_{u}}[\varphi]\left(\tau_{1}, \tau_{2}\right) \text {, }
\end{aligned}
$$

where

$$
\begin{aligned}
\text { Bound }_{p-2, T^{*}}^{\partial_{u}}[\varphi]\left(\tau_{1}, \tau_{2}\right)= & \sum_{i=1}^{2} \int_{\mathcal{S}_{\tau_{i} \cap\left\{t \leq T^{*}\right\}}} \chi_{R} \cdot r^{p-4-a}\left|\partial_{u} \Phi\right|^{2} d v d \sigma \\
& +\int_{\mathcal{R}\left(\tau_{1}, \tau_{2}\right) \cap\left\{t=T^{*}\right\}} \chi_{R} \cdot r^{p-4-a}\left|\partial_{u} \Phi\right|^{2} d v d \sigma \\
& +\int_{\mathcal{S}_{\tau_{1} \cap\left\{t \leq T^{*}\right\}}} \chi_{R} J_{\mu}^{T}\left(\Omega^{-1} \partial_{u} \Phi\right) \bar{n}^{\mu}
\end{aligned}
$$

Adding to (6.44) a large multiple of the estimate (6.45) (implementing also a Hardy type inequality for the $\partial_{v} \partial_{u} \Phi$ term), and using the fact that according to Lemma 6.3

$$
\begin{aligned}
\Omega \square\left(\Omega^{-1} \partial_{u} \Phi\right)= & \left(1+O\left(r^{-1-a}\right)\right) \cdot\left(\partial_{u}(\Omega F)+O\left(r^{-1-a}\right) \Omega F\right) \\
& +O\left(r^{-3}\right) \partial_{\sigma} \partial_{\sigma} \Phi+\sum_{j=0}^{1} \operatorname{Err}\left(\partial_{u}^{j} \Phi\right),
\end{aligned}
$$

we thus obtain:

$$
\begin{aligned}
& \mathcal{E}_{\text {bound }, R, T^{*}}^{(p)}\left[\Omega^{-1} \partial_{v} \Phi\right]\left(\tau_{2}\right)+\mathcal{E}_{\text {bound }, R, T^{*}}^{(p)}\left[\Omega^{-1} r^{-1} \partial_{\sigma} \Phi\right]\left(\tau_{2}\right) \\
& \quad+\mathcal{E}_{\text {bound }, R, T^{*}}^{(p-2)}\left[\Omega^{-1} \partial_{u} \Phi\right]\left(\tau_{2}\right)+\mathcal{E}_{\text {bound }, R, T^{*}}^{(p-2)}[\varphi]\left(\tau_{2}\right)
\end{aligned}
$$




$$
\begin{aligned}
& +\int_{\tau_{1}}^{\tau_{2}}\left(\mathcal{E}_{\text {bulk,R, }, T^{*}}^{\left(p-1, \partial_{v}\right)}\left[\Omega^{-1} \partial_{v} \Phi\right](\tau)+\mathcal{E}_{b u l k, R, \eta, T^{*}}^{\left(p-1, \partial_{\sigma}\right)}\left[\Omega^{-1} r^{-1} \partial_{\sigma} \Phi\right](\tau)\right.
\end{aligned}
$$

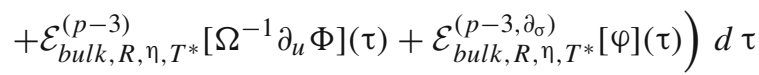

$$
\begin{aligned}
& +\mathcal{E}_{\mathcal{I}^{+}, R, T^{*}}^{(p)}\left[\Omega^{-1} \partial_{v} \Phi\right]\left(\tau_{1}, \tau_{2}\right)+\mathcal{E}_{\mathcal{I}^{+}, R, T^{*}}^{(p)}\left[\Omega^{-1} r^{-1} \partial_{\sigma} \Phi\right]\left(\tau_{1}, \tau_{2}\right) \\
& +\mathcal{E}_{\mathcal{I}^{+}, R, T^{*}}^{(p-2)}\left[\Omega^{-1} \partial_{u} \Phi\right]\left(\tau_{1}, \tau_{2}\right)+\mathcal{E}_{\mathcal{I}^{+}, R, T^{*}}^{(p-2)}[\varphi]\left(\tau_{1}, \tau_{2}\right) \\
& \leq\left(1+O_{p, \eta}(\varepsilon)\right)\left(\mathcal{E}_{\text {bound }, R, T^{*}}^{(p)}\left[\Omega^{-1} \partial_{v} \Phi\right]\left(\tau_{1}\right)+\mathcal{E}_{\text {bound }, R, T^{*}}^{(p)}\left[\Omega^{-1} r^{-1} \partial_{\sigma} \Phi\right]\left(\tau_{1}\right)\right) \\
& +C_{p, \eta} \cdot\left(\mathcal{E}_{\text {bound }, R, T^{*}}^{(p-2)}[\varphi]\left(\tau_{1}\right)+\mathcal{E}_{\text {bound }, R, T^{*}}^{(p-2)}\left[\Omega^{-1} \partial_{u} \Phi\right]\left(\tau_{1}\right)\right) \\
& +C_{p, \eta, \varepsilon} \int_{\mathcal{R}\left(\tau_{1}, \tau_{2}\right) \cap\left\{t \leq T^{*}\right\}}\left|\partial \chi_{R}\right| \cdot\left(r^{p}\left|\partial^{2} \Phi\right|^{2}+r^{p-2}|\partial \Phi|^{2}+r^{p-4}|\Phi|^{2}\right) d u d v d \sigma \\
& +C_{p, \eta, \varepsilon} \int_{\mathcal{R}\left(\tau_{1}, \tau_{2}\right) \cap\left\{t \leq T^{*}\right\}} \chi_{R} \cdot r^{p+1}\left(\left|\partial_{v}(\Omega F)\right|^{2}+\left|r^{-1} \partial_{\sigma}(\Omega F)\right|^{2}\right. \\
& \left.+r^{-2}\left|\partial_{u}(\Omega F)\right|^{2}+r^{-2}|\Omega F|^{2}\right) d u d v d \sigma \\
& +C_{p, \eta, \varepsilon} \int_{\mathcal{R}\left(\tau_{1}, \tau_{2}\right) \cap\left\{t \leq T^{*}\right\}} \chi_{R} \cdot r^{1+\eta}\left(\left|\partial_{v}(\Omega F)\right|^{2}+\left|r^{-1} \partial_{\sigma}(\Omega F)\right|^{2}\right. \\
& \left.+\left|\partial_{u}(\Omega F)\right|^{2}+|\Omega F|^{2}\right) d u d v d \sigma \\
& +C_{p, \eta, \varepsilon} \text { Bound }_{p-2, T^{*}}^{\partial_{u}}[\varphi]\left(\tau_{1}, \tau_{2}\right)+C_{p, \eta, \varepsilon} \text { Bound }_{p, T^{*}}^{\partial_{v}, \partial_{\sigma}}[\varphi]\left(\tau_{1}, \tau_{2}\right) \\
& +C_{p, \eta} \text { Bound }_{p, T^{*}, \varepsilon}^{(n)}[\varphi]\left(\tau_{1}, \tau_{2}\right) \\
& +\mathcal{B}_{p, T^{*}, \eta, \varepsilon}^{\left(\partial_{v}\right)}[\varphi]\left(\tau_{1}, \tau_{2}\right)+\mathcal{B}_{p, T^{*}, \eta, \varepsilon}^{\left(r^{-1} \partial_{\sigma}\right)}[\varphi]\left(\tau_{1}, \tau_{2}\right)+\mathcal{B}_{p-2, T^{*}, \eta, \varepsilon}^{\left(\partial_{u}\right)}[\varphi]\left(\tau_{1}, \tau_{2}\right) .
\end{aligned}
$$

where

$$
\begin{aligned}
& \mathcal{B}_{p-2, T^{*}, \eta, \varepsilon}^{\left(\partial_{u}\right)}[\varphi]\left(\tau_{1}, \tau_{2}\right) \\
& =C_{p, \eta, \varepsilon} \int_{\mathcal{R}\left(\tau_{1}, \tau_{2}\right) \cap\left\{t \leq T^{*}\right\}} \chi_{R} \cdot f(r)\left\{\left(\partial_{v}-\partial_{u}\right)\left(\Omega^{-1} \partial_{u} \Phi\right)+O\left(r^{-1}\right)\left(\Omega^{-1} \partial_{u} \Phi\right)\right\} \\
& \quad \times\left\{O\left(r^{-3}\right) \partial_{\sigma} \partial_{\sigma} \Phi+\operatorname{Err}\left(\partial_{u} \Phi\right)+\operatorname{Err}(\Phi)\right\} \Omega d u d v d \sigma \\
& +\int_{\mathcal{R}\left(\tau_{1}, \tau_{2}\right) \cap\left\{t \leq T^{*}\right\}} \chi_{R} \cdot O\left(r^{p-3-a}\right) \partial_{\sigma} \partial_{u} \Phi \cdot\left\{O\left(r^{-3}\right) \partial_{\sigma} \partial_{\sigma} \Phi\right. \\
& \left.+\operatorname{Err}\left(\partial_{u} \Phi\right)+\operatorname{Err}(\Phi)\right\} d u d v d \sigma \\
& -\int_{\mathcal{R}\left(\tau_{1}, \tau_{2}\right) \cap\left\{t \leq T^{*}\right\}} \chi_{R} \cdot O\left(r^{p-2}\right) \partial_{v} \partial_{u} \Phi \cdot\left\{O\left(r^{-3}\right) \partial_{\sigma} \partial_{\sigma} \Phi+\operatorname{Err}\left(\partial_{u} \Phi\right)\right. \\
& +\operatorname{Err}(\Phi)\} d u d v d \sigma .
\end{aligned}
$$

After adding to (6.48) a large (in terms of $p, \eta, \varepsilon$ ) multiple of (5.15) for $p-2$ in place of $p$ and of (5.38) for $\min \{p-2,2-\delta\}$ in place of $p$, the 


$$
\begin{aligned}
& C_{p, \eta} \text { Bound }_{p, T^{*}, \varepsilon}^{(n)}[\varphi]\left(\tau_{1}, \tau_{2}\right)+\mathcal{B}_{p, T^{*}, \eta, \varepsilon}^{\left(\partial_{v}\right)}[\varphi]\left(\tau_{1}, \tau_{2}\right)+\mathcal{B}_{p, T^{*}, \eta, \varepsilon}^{\left(r^{-1} \partial_{\sigma}\right)}[\varphi]\left(\tau_{1}, \tau_{2}\right) \\
& \quad+\mathcal{B}_{p-2, T^{*}, \eta, \varepsilon}^{\left(\partial_{u}\right)}[\varphi]\left(\tau_{1}, \tau_{2}\right)
\end{aligned}
$$

summand of the right hand side of (6.48) can be absorbed by the positive terms of the left hand side using an integration by parts scheme similar to the one used in the proof of Theorem 5.1 for the top order terms and a simple Cauchy-Schwarz inequality for the lower order terms. Moreover (in view also of Lemma 4.6, as well as the fact that $\left.\eta^{\prime}<1+a\right)$, the summand

$$
C_{p, \eta, \varepsilon} \text { Bound }_{p-2, T^{*}}^{\partial_{u}}[\varphi]\left(\tau_{1}, \tau_{2}\right)+C_{p, \eta, \varepsilon} \text { Bound }_{p, T^{*}}^{\partial_{v}, \partial_{\sigma}}[\varphi]\left(\tau_{1}, \tau_{2}\right)
$$

can be bounded by

$$
\int_{\mathcal{S}_{\tau_{1}}} \chi_{R} J_{\mu}^{T}\left(r^{-k_{2}} \partial_{v}^{k_{1}} \partial_{\sigma}^{k_{2}} \partial_{u}^{k_{3}} \varphi\right) \bar{n}^{\mu}
$$

plus $O_{p, \eta}(\varepsilon)$ times the existing terms in the left and right hand side of (6.48) and (5.38) (for $\min \{p-2,2-\delta\}$ in place of $p$ ). Therefore, provided $\varepsilon$ has been fixed small in terms of $p, \eta, \delta$ and $R$ is large enough in terms of $p, \eta, \delta$, the desired inequality (6.1) for $k=1$ readily follows from (6.48), (5.15) (for $p-2$ in place of $p$ ) and (5.38) (for $\min \{p-2,2-\delta\}$ in place of $p$ ) after letting $T^{*} \rightarrow+\infty$.

\section{Friedlander Radiation Field on $\mathcal{I}^{+}$}

In this section, we will establish the existence of the Friedlander radiation field on future null infinity $\mathcal{I}^{+}$for solutions $\varphi$ to $\square \varphi=F$ on general asymptotically flat spacetimes $\left(\mathcal{M}^{d+1}, g\right), d \geq 3$, with the asymptotics (3.3), provided the source term $F$ decays suitably fast in terms of $r$. This result is essentially a "soft" corollary of the results of the previous sections.

\subsection{Assumptions on the Spacetimes Under Consideration}

Let $\left(\mathcal{M}^{d+1}, g\right), d \geq 3$, be a time oriented smooth Lorentzian manifold, possibly with non empty piecewise smooth boundary $\partial \mathcal{M}$. We will assume the following condition on the asymptotics of $(\mathcal{M}, g)$ :

(G1) Asymptotic flatness There exists an open subset $\mathcal{N}_{a f, \mathcal{M}} \subset \mathcal{M}$ such that each connected component of $\mathcal{N}_{a f, \mathcal{M}}$ is diffeomorphic to $\mathbb{R} \times(1,+\infty) \times \mathbb{S}^{d-1}$. Fixing such a diffeomorphism (i.e. a coordinate chart) on each component, we will denote with $(u, r, \sigma)$ the associated coordinate functions. Furthermore, we assume that on each component of $\mathcal{N}_{a f, \mathcal{M}}$, the metric $g$ takes the form (3.3): 


$$
\begin{aligned}
g= & -4\left(1-\frac{2 M(u, \sigma)}{r}+O\left(r^{-1-a}\right)\right) d u^{2}-\left(4+O\left(r^{-1-a}\right)\right) d u d r \\
& +r^{2} \cdot\left(g_{\mathbb{S}^{d-1}}+O\left(r^{-1}\right)\right) \\
& +\left(h_{3}^{a s}(u, \sigma)+O\left(r^{-a}\right)\right) d u d \sigma+O\left(r^{-a}\right) d r d \sigma+O\left(r^{-2-a}\right) d r^{2}
\end{aligned}
$$

Thus, $\left(\mathcal{N}_{a f, \mathcal{M}}, g\right)$ should be thought of as a disjoint union of copies of the manifold $\left(\mathcal{N}_{a f}, g\right)$ of Section 3 .

Remark We do not assume that $(\mathcal{M}, g)$ is globally hyperbolic.

We will also set $t=u+r$ and $v=t-r$ on $\mathcal{N}_{a f, \mathcal{M}}$. Let also $T$ denote the vector field $\partial_{t}$ in the $(t, r, \sigma)$ coordinate system on each component of $\mathcal{N}_{a f, \mathcal{M}}$. We extend $r$ as a smooth function on the whole of $\mathcal{M}$ by the requirement that $0 \leq r \leq 2$ on $\mathcal{M} \backslash \mathcal{N}_{a f, \mathcal{M}}$. Finally, we also construct the function $\bar{t}_{\eta^{\prime}}:\{r \gg 1\} \subset \mathcal{N}_{a f, \mathcal{M}} \rightarrow \mathbb{R}$ for some fixed $0<\eta^{\prime}<1+a$ as in Section 3 .

\subsection{Existence of the Friedlander Radiation Field on Future Null Infinity}

We will prove the following result:

Theorem 7.1 Let $\left(\mathcal{M}^{d+1}, g\right), d \geq 3$ satisfy the assumption $(G 1)$, and let $R_{0}>0$ be large in terms of the geometry of $(\mathcal{M}, g)$ (this implies that $\left.\left\{r \geq R_{0}\right\} \subset \mathcal{N}_{a f, \mathcal{M}}\right)$. Let $\varphi: \mathcal{M} \rightarrow \mathbb{C}$ be a smooth function solving $\square_{g} \varphi=F$, such that for some $0<\delta<1$ and for any integer $0 \leq j \leq\left\lceil\frac{d}{2}\right\rceil$ and $\tau \in \mathbb{R}$ the following quantity is finite on each connected component of $\mathcal{N}_{a f, \mathcal{M}}$ :

$$
\begin{aligned}
\mathcal{E}_{i n}^{(1+\delta+2 j, j+1)}[\varphi](0) & +\sum_{i=1}^{j+1} \sum_{k_{1}+k_{2}+k_{3}=i-1} \int_{J^{+}(\{t=0\}) \cap J^{-}(\{\bar{t}=\tau\}) \cap\left\{r \geq R_{0}\right\}} r^{\delta+2 i} \\
& \times\left(\left|r^{-k_{2}-k_{3}} \partial_{v}^{k_{1}} \partial_{\sigma}^{k_{2}} \partial_{u}^{k_{3}}(\Omega F)\right|^{2}\right) d u d v d \sigma<+\infty
\end{aligned}
$$

where

$$
\begin{aligned}
& \mathcal{E}_{i n}^{(p, k)}[\varphi](0) \\
& =\sum_{j=1}^{k} \sum_{k_{1}+k_{2}+k_{3}=j-1}\left\{\int _ { \{ t = 0 \} \cap \{ r \geq R _ { 0 } \} } \left(r^{p-2(k-j)}\left|r^{-k_{2}-k_{3}} \partial_{v}^{k_{1}+1} \partial_{\sigma}^{k_{2}} \partial_{u}^{k_{3}}(\Omega \varphi)\right|^{2}\right.\right. \\
& \quad+r^{-1-\eta^{\prime}}\left(r^{p-2(k-j)}\left|r^{-k_{2}-k_{3}-1} \partial_{v}^{k_{1}} \partial_{\sigma}^{k_{2}+1} \partial_{u}^{k_{3}}(\Omega \varphi)\right|^{2}\right. \\
& \quad+\left((d-3) r^{p-2-2(k-j)}+\min \left\{r^{p-2-2(k-j)}, r^{-\delta-2(k-j)}\right\}\right) \\
& \left.\left.\quad \times\left|r^{-k_{2}-k_{3}} \partial_{v}^{k_{1}} \partial_{\sigma}^{k_{2}} \partial_{u}^{k_{3}}(\Omega \varphi)\right|^{2}\right)\right) d v d \sigma \\
& \left.\quad+\int_{\{t=0\} \cap\left\{r \geq R_{0}\right\}} J_{\mu}^{T}\left(r^{-k_{2}} \partial_{v}^{k_{1}} \partial_{\sigma}^{k_{2}} \partial_{u}^{k_{3}} \varphi\right) \bar{n}^{\mu}\right\}
\end{aligned}
$$


and the derivatives are considered with respect to the $(u, v, \sigma)$ coordinate system on each connected component of $\left\{r \geq R_{0}\right\}$. Then for each connected component of $\mathcal{N}_{a f, \mathcal{M}}$, the Friedlander radiation field

$$
\Phi_{\mathcal{I}^{+}}(u, \sigma) \doteq \lim _{v \rightarrow+\infty} \Omega \cdot \varphi(u, v, \sigma)
$$

exists on $\mathbb{R} \times \mathbb{S}^{d-1}$.

Moreover, if (7.2) holds for all integers $0 \leq j \leq l+\left\lceil\frac{d}{2}\right\rceil$ for some integer $l \geq 0$, then $\Phi_{\mathcal{I}^{+}} i$ is $C^{l-1}$ in $(u, \sigma)$ and for all integers $j_{1}, j_{2}, j_{3} \geq 0$ with $j_{1}+j_{2}+j_{3} \leq l$ and any $(u, \sigma) \in \mathbb{R} \times \mathbb{S}^{d-1}$ the limit

$$
\lim _{v \rightarrow+\infty} r^{j_{1}} \partial_{v}^{j_{1}} \partial_{\sigma}^{j_{3}} \partial_{u}^{j_{3}}(\Omega \varphi)(u, v, \sigma)
$$

is finite.

In particular, if (7.2) holds for all integers $j \geq 0$ (for instance when $\varphi$ solves $\square \varphi=0$ with smooth and compactly supported initial data on $\{t=0\})$, then $\Phi_{\mathcal{I}^{+}}$is smooth and (7.5) exists for all integers $j_{1}, j_{2}, j_{3} \geq 0$.

Remark In the case $j_{1} \geq 1$ we actually expect that the limit (7.5) is identically 0 when $\varphi$ solves $\square \varphi=0$ with compactly supported initial data. This expectation is justified by the fact follows from the fact that on spacetimes admitting a smooth conformal compactification of future null infinity, the following stronger statement (in comparison to (7.5)) holds for all integers $j_{1}, j_{2}, j_{3} \geq 0$ :

$$
\lim _{v \rightarrow+\infty} r^{2 j_{1}} \partial_{v}^{j_{1}} \partial_{\sigma}^{j_{3}} \partial_{u}^{j_{3}}(\Omega \varphi)(u, v, \sigma)<+\infty
$$

Notice, however, that in our case we are also including spacetimes which do not necessarily admit a smooth conformal compactification at $\mathcal{I}^{+}$.

Proof We will assume without loss of generality that $\varphi$ is real valued. We will also work on a fixed connected component of $\mathcal{N}_{a f, \mathcal{M}}$.

Let $\left\{v_{n}\right\}_{n \in \mathbb{N}}$ be an increasing sequence of positive real numbers tending to $+\infty$. Let us also fix a smooth function $\chi_{R}: \mathcal{M} \rightarrow[0,1]$ such that $\chi_{R} \equiv 0$ on $\{r \leq R\}$ and $\chi_{R} \equiv 1$ on $\{r \geq R+1\}$ for some large fixed constant $R \geq R_{0} \gg 1$.

By repeating the proof of Theorem 6.1 for $p=1+\delta$ in the spacetime region

$$
\mathcal{D}_{0, \tau} \doteq J^{+}(\{t=0\}) \cap J^{-}(\{\bar{t}=\tau\}) \cap\left\{r \geq R_{0}\right\}
$$

(instead of the region bounded by two hyperboloidal hypersurfaces) for any $\tau \in \mathbb{R}$, we can readily bound for any $k \in \mathbb{N}$ and $\tau \in \mathbb{R}$ :

$$
\begin{aligned}
& \mathcal{E}_{\text {bound }, R ; \delta}^{(1+\delta+2(k-1), k)}[\varphi](\tau) \\
& \lesssim_{k, \delta} \mathcal{E}_{\text {in }}^{(1+\delta+2(k-1), k)}[\varphi](0)+\sum_{j=0}^{k} \int_{\mathcal{D}_{0, \tau}}\left|\partial \chi_{R}\right| \cdot r^{\delta+2 j-1}\left|\partial^{j} \varphi\right|^{2}
\end{aligned}
$$




$$
+\sum_{j=1}^{k} \sum_{k_{1}+k_{2}+k_{3}=j-1} \int_{\mathcal{D}_{0, \tau}} \chi_{R} \cdot r^{\delta+2 j-2 k_{3}}\left(\left|r^{-k_{2}} \partial_{v}^{k_{1}} \partial_{\sigma}^{k_{2}} \partial_{v}^{k_{1}} \partial_{u}^{k_{3}}(\Omega F)\right|^{2}\right) d u d v d \sigma
$$

where

$$
\begin{aligned}
& \mathcal{E}_{\text {bound }, R ; \delta}^{(p, k)}[\varphi](\tau) \\
& =\sum_{j=1}^{k} \sum_{k_{1}+k_{2}+k_{3}=j-1}\left\{\int _ { \{ \overline { t } = \tau \} } \chi _ { R } \left(r^{p-2(k-j)}\left|r^{-k_{2}-k_{3}} \partial_{v}^{k_{1}+1} \partial_{\sigma}^{k_{2}} \partial_{u}^{k_{3}}(\Omega \varphi)\right|^{2}\right.\right. \\
& \quad+\left(r^{p-2(k-j)}\left|r^{-k_{2}-k_{3}-1} \partial_{v}^{k_{1}} \partial_{\sigma}^{k_{2}+1} \partial_{u}^{k_{3}}(\Omega \varphi)\right|^{2}\right. \\
& \quad+\left((d-3) r^{p-2-2(k-j)}+\min \left\{r^{p-2-2(k-j)}, r^{-\delta-2(k-j)}\right\}\right) \\
& \left.\left.\left.\quad \times\left|r^{-k_{2}-k_{3}} \partial_{v}^{k_{1}} \partial_{\sigma}^{k_{2}} \partial_{u}^{k_{3}}(\Omega \varphi)\right|^{2}\right)\right) d v d \sigma+\int_{\{\bar{t}=\tau\}} \chi_{R} J_{\mu}^{T}\left(r^{-k_{2}} \partial_{v}^{k_{1}} \partial_{\sigma}^{k_{2}} \partial_{u}^{k_{3}} \varphi\right) \bar{n}^{\mu}\right\}
\end{aligned}
$$

(the $\partial_{v}, \partial_{\sigma}, \partial_{u}$ coordinate vector fields are a priori only defined in the region $\{r \geq R\}$, but since the integrand is multiplied with the cut-off $\chi_{R}$ the expression in (7.9) is well defined).

Using the fundamental theorem of calculus and the expression (3.7) for $\bar{t}$, we can bound for any $C^{1}$ function $\Psi: \mathcal{M} \rightarrow \mathbb{R}$ and any $\tau \in \mathbb{R}, n_{0} \in \mathbb{N}$ :

$$
\begin{aligned}
& \sum_{n=1}^{n_{0}} \int_{\mathbb{S}^{d-1}}\left|\chi_{R} \Omega \Psi\right|_{\{\bar{t}=\tau\}}\left(v_{n+1}, \sigma\right)-\left.\chi_{R} \Omega \Psi\right|_{\{\bar{t}=\tau\}}\left(v_{n}, \sigma\right) \mid d \sigma \\
& \quad \lesssim \int_{\{\bar{t}=\tau\} \cap\left\{v \leq v_{n_{0}}\right\}}\left(\left|\partial_{v}\left(\chi_{R} \Omega \Psi\right)\right|+r^{-1}\left|\partial_{u}\left(\chi_{R} \Omega \Psi\right)\right|\right) d v d \sigma \lesssim \delta \\
& \quad \lesssim \delta \int_{\{\bar{t}=\tau\}}\left|\partial \chi_{R}\right| \cdot|\Omega \Psi| d \sigma \\
& \quad+\left(\int_{\{\bar{t}=\tau\} \cap\left\{v \leq v_{n_{0}}\right\}} r^{1+\delta} \chi_{R}\left(\left|\partial_{v}(\Omega \Psi)\right|^{2}+r^{-2}\left|\partial_{u}(\Omega \Psi)\right|^{2}\right) d v d \sigma\right)^{1 / 2} .
\end{aligned}
$$

In the above, $\left.\chi_{R} \Omega \Psi\right|_{\{\bar{t}=\tau\}}$ is considered as a function of $(v, \sigma)$, since $(v, \sigma)$ is a valid parametrisation of $\{\bar{t}=\tau\} \cap\{r \geq R\}$ where $\chi_{R} \Omega \Psi$ is supported. Moreover, the coordinate derivatives $\partial_{u}, \partial_{v}$ in the right hand side are defined in the $(u, v, \sigma)$ coordinate system in the region $\{r \geq R\}$. 
Therefore, from (7.8), (7.9) and (7.10) for $\Omega^{-1} r^{j_{1}} \partial_{v}^{j_{1}} \partial_{\sigma}^{j_{3}} \partial_{u}^{j_{3}}(\Omega \varphi)$ in place of $\Psi$ we readily deduce that for any $k \in \mathbb{N}$ and any $n_{0} \in \mathbb{N}$ :

$$
\begin{aligned}
& \left(\sum_{n=1}^{n_{0}} \sum_{j=0}^{k-1} \sum_{j_{1}+j_{2}+j_{3}=j} \int_{\mathbb{S}^{d}-1}\left|\chi_{R} r^{j_{1}} \partial_{v}^{j_{1}} \partial_{\sigma}^{j_{3}} \partial_{u}^{j_{3}}(\Omega \varphi)\right|_{\{\bar{t}=\tau\}}\left(v_{n+1}, \sigma\right)\right. \\
& \left.\quad-\left.\chi_{R} r^{j_{1}} \partial_{v}^{j_{1}} \partial_{\sigma}^{j_{3}} \partial_{u}^{j_{3}}(\Omega \varphi)\right|_{\{\bar{t}=\tau\}}\left(v_{n}, \sigma\right) \mid d \sigma\right)^{2} \lesssim_{k, \delta} \\
& \lesssim_{k, \delta} \mathcal{E}_{i n}^{(1+\delta+2(k-1), k)}[\varphi](0)+\sum_{j=0}^{k} \int_{\mathcal{D}_{0, \tau}}\left|\partial \chi_{R}\right| \cdot r^{\delta+2 j-1}\left|\partial^{j} \varphi\right|^{2} \\
& \quad+\left(\sum_{j=0}^{k-1} \int_{\{\bar{t}=\tau\}}\left|\partial \chi_{R}\right| r^{j}\left|\partial^{j} \varphi\right|\right)^{2} \\
& \quad+\sum_{j=1}^{k} \sum_{k_{1}+k_{2}+k_{3}=j-1} \int_{\mathcal{D}_{0, \tau}} \chi_{R} \cdot r^{\delta+2 j-2 k_{3}}\left(\left|r^{-k_{2}} \partial_{v}^{k_{1}} \partial_{\sigma}^{k_{2}} \partial_{v}^{k_{1}} \partial_{u}^{k_{3}}(\Omega F)\right|^{2}\right) d u d v d \sigma .
\end{aligned}
$$

Using the Sobolev inequality on $\mathbb{S}^{d-1}$

$$
\|\Psi\|_{L^{\infty}\left(\mathbb{S}^{d-1}\right)}^{2} \lesssim \sum_{j=0}^{\left\lceil\frac{d}{2}\right\rceil} \int_{\mathbb{S}^{d-1}}\left|\partial_{\sigma}^{j} \Psi\right|^{2} d \sigma
$$

from (7.11) (and the fact that $\|\Psi\|_{L^{1}\left(\mathbb{S}^{d-1}\right)} \lesssim d\|\Psi\|_{L^{2}\left(\mathbb{S}^{d-1}\right)}$ ) we infer that for any $k_{0}, n_{0} \in \mathbb{N}$ :

$$
\begin{aligned}
& \sup _{\sigma \in \mathbb{S}^{d-1}}\left\{\sum_{n=1}^{n_{0}} \sum_{j=0}^{k_{0}} \sum_{j_{1}+j_{2}+j_{3}=j}\left|\chi_{R} r^{j_{1}} \partial_{v}^{j_{1}} \partial_{\sigma}^{j_{3}} \partial_{u}^{j_{3}}(\Omega \varphi)\right|_{\{\bar{t}=\tau\}}\left(v_{n+1}, \sigma\right)\right. \\
& \left.\quad-\left.\left.\chi_{R} r^{j_{1}} \partial_{v}^{j_{1}} \partial_{\sigma}^{j_{3}} \partial_{u}^{j_{3}}(\Omega \varphi)\right|_{\{\bar{t}=\tau\}}\left(v_{n}, \sigma\right)\right|^{2}\right\} \lesssim_{k, \delta} \\
& \lesssim_{k, \delta} \mathcal{E}_{i n}^{\left(1+\delta+2\left(k_{0}+\left\lceil\frac{d}{2}\right\rceil\right), k_{0}+\left\lceil\frac{d+2}{2}\right\rceil\right)}[\varphi](0)+\sum_{j=0}^{k_{0}+\left\lceil\frac{d+2}{2}\right\rceil} \int_{\mathcal{D}_{0, \tau}}\left|\partial \chi_{R}\right| \cdot r^{\delta+2 j-1}\left|\partial^{j} \varphi\right|^{2} \\
& \quad+\left(\sum_{j=0}^{k_{0}+\left\lceil\frac{d}{2}\right\rceil} \int_{\{\bar{t}=\tau\}}\left|\partial \chi_{R}\right| r^{j}\left|\partial^{j} \varphi\right|\right)^{k_{0}+\left\lceil\frac{d+2}{2}\right\rceil} \sum_{j=1}^{j} \sum_{k_{1}+k_{2}+k_{3}=j-1} \int_{\mathcal{D}_{0, \tau}} \chi_{R} \cdot r^{\delta+2 j-2 k_{3}} \\
& \quad \times\left(\left|r^{-k_{2}} \partial_{v}^{k_{1}} \partial_{\sigma}^{k_{2}} \partial_{v}^{k_{1}} \partial_{u}^{k_{3}}(\Omega F)\right|^{2}\right) d u d v d \sigma .
\end{aligned}
$$


In view of (7.2) and the fact that $\mathcal{D}_{0, \tau} \cap \operatorname{supp}\left(\partial \chi_{R}\right)$ and $\{\bar{t}=\tau\} \cap \operatorname{supp}\left(\partial \chi_{R}\right)$ are compact subsets of $\mathcal{M}$, from (7.13) we deduce that for all $\tau_{0} \in \mathbb{R}$ and all $k_{0} \in \mathbb{N}$ such that (7.2) holds for all $k \leq k_{0}+\left\lceil\frac{d}{2}\right\rceil$, the following quantity is finite independently of $n_{0}$ :

$$
\begin{aligned}
& \sup _{\sigma \in \mathbb{S}^{d-1}, \tau \leq \tau_{0}}\left\{\sum_{n=1}^{n_{0}} \sum_{j=0}^{k_{0}} \sum_{j_{1}+j_{2}+j_{3}=j}\left|\chi_{R} r^{j_{1}} \partial_{v}^{j_{1}} \partial_{\sigma}^{j_{3}} \partial_{u}^{j_{3}}(\Omega \varphi)\right|_{\{\bar{t}=\tau\}}\left(v_{n+1}, \sigma\right)\right. \\
& \left.-\left.\left.\chi_{R} r^{j_{1}} \partial_{v}^{j_{1}} \partial_{\sigma}^{j_{3}} \partial_{u}^{j_{3}}(\Omega \varphi)\right|_{\{\bar{t}=\tau\}}\left(v_{n}, \sigma\right)\right|^{2}\right\} \leq C_{k_{0}, \delta}[\varphi]\left(\tau_{0}\right)<+\infty .
\end{aligned}
$$

Therefore, by letting $n_{0} \rightarrow+\infty$ (7.14) yields that for any $\tau \in \mathbb{R}, \sigma \in \mathbb{S}^{d-1}$, any $k_{0} \in \mathbb{N}$ such that (7.2) holds for all $k \leq k_{0}+\left\lceil\frac{d}{2}\right\rceil$ and any $j_{1}+j_{2}+j_{3}=k_{0}$, the sequence

$$
\left\{\left.r^{j_{1}} \partial_{v}^{j_{1}} \partial_{\sigma}^{j_{3}} \partial_{u}^{j_{3}}(\Omega \varphi)\right|_{\{\bar{t}=\tau\}}\left(v_{n}, \sigma\right)\right\}_{n \in \mathbb{N}}
$$

is a Cauchy sequence (and hence (7.5) follows).

Moreover, since $\bar{t}-u=O\left(r^{-\eta^{\prime}}\right)$, from (7.14) we infer the limit

$$
\Phi_{\mathcal{I}^{+}}(u, \sigma)=\lim _{v \rightarrow+\infty} \Omega \varphi(v, u, \sigma)
$$

exists and is a $C^{k_{0}-1}$ function of $(u, \sigma)\left(\right.$ if $\left.k_{0} \neq 0\right)$.

\subsection{Estimates for $\Phi_{\mathcal{I}^{+}}$Provided by Lemma 5.1}

The following corollary is a straightforward consequence of Theorems 6.1 and 7.1:

Corollary 7.2 Let $\mathcal{N}$ be any connected component of $\mathcal{N}_{a f, \mathcal{M}}$. Then for any $k \in \mathbb{N}$, any $2 k-2<p \leq 2 k$, any given $0<\eta<a$ and $0<\delta<1$, any $R>0$ large enough in terms of $p, \eta, \delta, k$, any $\tau_{1} \leq \tau_{2}$ and any smooth cut-off $\chi_{R}: \mathcal{M} \rightarrow[0,1]$ supported in $\{r \geq R\} \cap \mathcal{N}$, the following inequality holds for any smooth function $\varphi: \mathcal{M} \rightarrow \mathbb{C}$ solving $\square_{g} \varphi=F$ with suitably decaying initial data on $\{t=0\}$ :

$$
\begin{aligned}
& \sum_{k_{1}+k_{2}=k} \int_{\mathcal{I}^{+} \cap\left\{\tau_{1} \leq u \leq \tau_{2}\right\}} r^{p-2 k}\left|\partial_{\sigma}^{k_{1}} \partial_{u}^{k_{2}} \Phi_{\mathcal{I}^{+}}\right|^{2} d u d \sigma \lesssim p, \eta, \delta, k \\
& \lesssim_{p, \eta, \delta, k} \mathcal{E}_{\text {bound }, R ; \delta}^{(p, k)}[\varphi]\left(\tau_{2}\right)+\sum_{j=0}^{k} \int_{\mathcal{R}\left(\tau_{1}, \tau_{2}\right)}\left|\partial \chi_{R}\right| \cdot r^{p-2(k-j)}\left|\partial^{j} \varphi\right|^{2} \\
& +\sum_{j=1}^{k} \sum_{k_{1}+k_{2}+k_{3}=j-1} \int_{\mathcal{R}\left(\tau_{1}, \tau_{2}\right)} \chi_{R} \cdot\left(r^{p+1-2 k_{3}-2(k-j)}+r^{1+\eta}\right) \\
& \left(\left|r^{-k_{2}} \partial_{v}^{k_{1}} \partial_{\sigma}^{k_{2}} \partial_{v}^{k_{1}} \partial_{u}^{k_{3}}(\Omega F)\right|^{2}\right) d u d v d \sigma,
\end{aligned}
$$


where we have adopted the convention

$$
\left.r^{p-2 k}\right|_{\mathcal{I}^{+}}= \begin{cases}1, & p=2 k \\ 0, & p<2 k\end{cases}
$$

\section{Polynomial Decay $\bar{t}^{-1}$ for Solutions to $\square_{g} \varphi=0$}

In this Section, we will generalise the results of [11] by showing that on asymptotically flat spacetimes $\mathcal{M}$ with possibly non-empty timelike boundary $\partial_{\text {tim }} \mathcal{M}$, a $\bar{t}^{-1}$ polynomial decay rate hols for solutions $\varphi$ to $\square \varphi=0$ with suitable boundary conditions on $\partial_{\text {tim }} \mathcal{M}$, provided some specific geometric conditions on the interior region of $(\mathcal{M}, g)$ hold and assuming that an integrated local energy decay statement (possibly with loss of derivatives) holds for $\varphi$.

\subsection{Assumptions on the Class of Spacetimes $(\mathcal{M}, g)$ Under Consideration}

\subsubsection{Geometric Assumptions on $(\mathcal{M}, g)$ and Related Geometric Constructions}

Let $\left(\mathcal{M}^{d+1}, g\right), d \geq 3$, be a smooth Lorentzian manifold with possibly non empty piecewise smooth boundary $\partial \mathcal{M}$. We assume that $(\mathcal{M}, g)$ satisfies the Assumption (G1) on asymptotic flatness. We will now proceed to state a few more assumptions on the geometric structure of $(\mathcal{M}, g)$, and present some geometric constructions that will be used later.

Assumptions on the causal structure of $(\mathcal{M}, g)$ and $\left(\partial \mathcal{M},\left.g\right|_{\partial \mathcal{M}}\right)$ Since we will need to establish some global estimates for solutions to the wave equation (1.1) on $(\mathcal{M}, g)$, we will need to impose some conditions on the causal structure of $(\mathcal{M}, g)$ and its boundary.

(G2) Partition of the boundary. We assume that the boundary $\partial \mathcal{M}$ (if non-empty) can be split into two components (not necessarily connected)

$$
\partial \mathcal{M}=\partial_{\text {tim }} \mathcal{M} \cup \partial_{\text {hor }} \mathcal{M}
$$

where $\left(\partial_{\text {tim }} \mathcal{M},\left.g\right|_{\partial_{\text {tim }} \mathcal{M}}\right)$ is a smooth Lorentzian manifold (i.e. $\partial_{\text {tim }} \mathcal{M}$ is a smooth timelike hypersurface with respect to $g$ ) and ( $\left.\partial_{\text {hor }} \mathcal{M},\left.g\right|_{\partial_{h o r}} \mathcal{M}\right)$ is piecewise smooth and degenerate pseudo-Riemannian manifold (i.e. $\partial_{\text {hor }} \mathcal{M}$ is a null hypersurface with respect to $g$ ).

(G3) Global Hyperbolicity. Let $\tilde{\mathcal{M}}_{\text {tim }}$ denote the double of $\mathcal{M}$ along $\partial_{\text {tim }} \mathcal{M}$. We will denote as $i_{\tilde{r}}: \mathcal{M} \rightarrow \tilde{\mathcal{M}}_{\text {tim }}$ the natural inclusion of $\mathcal{M}$ into $\tilde{\mathcal{M}}_{\text {tim }}$, while $i_{\text {ref }}: \mathcal{M} \rightarrow \tilde{\mathcal{M}}_{\text {tim }}$ will denote the reflection map along $\partial_{\text {tim }} \mathcal{M}$. We assume that $\tilde{\mathcal{M}}_{\text {tim }}$ is globally hyperbolic. Let $\tilde{\Sigma}$ be a Cauchy hypersurface of $\tilde{\mathcal{M}}_{\text {tim }}$. We will denote with $\Sigma$ the restriction of $\tilde{\Sigma}$ on $\mathcal{M}$. We will also fix a time function $t$ associated with $\tilde{\Sigma}$ on $\tilde{\mathcal{M}}_{t i m}$, i.e. $g(\nabla t, \nabla t)<0$ on $\tilde{\mathcal{M}}_{t i m}$ and $\tilde{\Sigma} \equiv\{t=0\}$. Notice that with the help of $t$ we can identify $\tilde{\mathcal{M}}_{\text {tim }}$ with $\mathbb{R} \times \tilde{\Sigma}$ and $\mathcal{M}$ with $\mathbb{R} \times \Sigma$. 
(G4) Domain of outer communications. Let $\mathcal{N}_{\text {af, } \mathcal{M}}$ be the open subset of $\mathcal{M}$ defined in Assumption (G1), where $g$ has the asymptotically flat form (7.1), and let $\tilde{\mathcal{N}}_{a f, \mathcal{M}}=i_{\text {or }}\left(\mathcal{N}_{a f, \mathcal{M}}\right) \cup i_{\text {ref }}\left(\mathcal{N}_{a f, \mathcal{M}}\right)$. Having identified $\tilde{\mathcal{M}}_{\text {tim }}$ with $\mathbb{R} \times \tilde{\Sigma}$, we assume that $\tilde{\mathcal{N}}_{a f, \mathcal{M}}=\mathbb{R} \times(\tilde{\Sigma} \backslash K)$ for some compact $K \subset \tilde{\Sigma}$, and that $\tilde{\mathcal{N}}_{a f, \mathcal{M}}$ has a finite number of connected components. ${ }^{12}$ Moreover, we assume that the domain of dependence of $\mathbb{R} \times(\tilde{\Sigma} \backslash K)$ is the whole of $\tilde{\mathcal{M}}_{\text {tim }}$.

In view of Assumption $(\mathrm{G} 4), i_{\text {or }}\left(\partial_{\text {hor }} \mathcal{M}\right) \cup i_{\text {ref }}\left(\partial_{\text {hor }} \mathcal{M}\right)$ (if non-empty) constitutes the event horizon of $\tilde{\mathcal{M}}_{\text {tim }}$. From now on we will use the notation $\mathcal{H}$ for $\partial_{\text {hor }} \mathcal{M}$ and we will call $\mathcal{H}$ the event horizon of $\mathcal{M}$. Using the fact that $i_{\text {or }}(\mathcal{H}) \cup i_{\text {ref }}(\mathcal{H})$ constitutes the event horizon of a globally hyperbolic spacetime, we can define the future event horizon $\mathcal{H}^{+}$and the past event horizon $\mathcal{H}^{-}$of $\mathcal{M}$ by the requirement that

- $\mathcal{H}^{+}, \mathcal{H}^{-}$are piecewise smooth achronal hypersurfaces (possibly with boundary and not necessarily connected) such that $\mathcal{H}^{+} \cup \mathcal{H}^{-}=\mathcal{H}$

- $\mathcal{H}^{-} \subset J^{-}\left(\mathcal{H}^{+}\right)$

We will also assume that $\mathcal{H}^{+}$is smooth (if non-empty).

Assumptions on the existence of a well behaved foliation by hyperboloidal hypersurfaces Fixing $\eta^{\prime}=1$, we will assume that the function $\bar{t}_{\eta^{\prime}}$ originally defined on the subset $\{r \gg 1\}$ of $\mathcal{N}_{a f, \mathcal{M}}$ can be extended as a smooth function on $\mathcal{M}$ satisfying the following conditions:

(G5) $\bar{t}$ is given by the relation (3.7) in the region $\{r \geq R\}$ of each connected component of $\mathcal{N}_{a f, \mathcal{M}}$ for some $R \gg 1$.

(G6) For any $0 \leq \tau_{1} \leq \tau_{2},\left\{\bar{t}=\tau_{2}\right\}$ is contained in the future domain of dependence of $\left\{\bar{t}=\tau_{1}\right\}$. $^{13}$

(G7) $g(\nabla \bar{t}, \nabla \bar{t})<0$ everywhere on $\mathcal{M} \cap\{\bar{t} \geq 0\}$, where $\nabla \bar{t}$ denotes the gradient of $\bar{t}$ with respect to $g$. Moreover, $-C \leq g(\nabla \bar{t}, \nabla \bar{t}) \leq-c<0$ in the region $\{\bar{t} \geq 0\} \backslash \mathcal{N}_{a f, \mathcal{M}}$, for some $C, c>0$.

It will be convenient to have a globally defined future directed timelike vector field $N$ adjusted to the choice of our foliation $\{\bar{t}=$ const $\}$. Therefore, we will fix $N$ to be a timelike future directed vector field on $\mathcal{M}$ such that $N \equiv(-g(\nabla \bar{t}, \nabla \bar{t}))^{-1} \cdot \nabla \bar{t}$ on $\{\bar{t} \geq 0\} \backslash \mathcal{N}_{a f, \mathcal{M}}, N \equiv T$ in the region $\{\bar{t} \geq 0\} \cap\{r \geq 2 R\}$ of $\mathcal{N}_{a f, \mathcal{M}}$, and the relations $-C \leq g(N, N) \leq-C^{-1}<0$ and $d \bar{t}(N)=1$ hold everywhere on $\mathcal{M}$ for some $C>0$. The existence of such a vector field follows from time orientability of $\mathcal{M}$ and the convexity of the set

$$
\mathfrak{F}_{p}=\left\{X \in T_{p} \mathcal{M} \mid g(X, X)<0 \text { and } d \bar{t}(X)=1\right\}
$$

for each $p \in \mathcal{M}$.

\footnotetext{
12 If $\partial \mathcal{M} \neq \emptyset$, then it is necessary that $K \cap \partial \mathcal{M} \neq \emptyset$.

13 The future domain of dependence $\mathcal{D}^{+}(\mathcal{B})$ of a set $\mathcal{B} \subset \mathcal{M}$ is defined as the set of all points $p \in \mathcal{M}$ such that all past inextendible causal curves $\gamma$ emanating from $p$ intersect $\mathcal{B}$, where now $\gamma$ is not considered past inextendible if it has a past endpoint $q$ on $\partial_{\text {tim }} \mathcal{M}$, since from $q$ one can further extend $\gamma$ by a causal path inside $J^{-}(q) \backslash \partial_{\text {tim }} \mathcal{M}$.
} 
We also extend the function $r$ (defined originally in the region $\mathcal{N}_{a f, \mathcal{M}}$ ) as a Morse function (but not necessarily as a coordinate function) on the whole of $\mathcal{M}$, under the assumption that $r \geq 0$ everywhere on $\mathcal{M}, r \equiv 0$ on $\partial \mathcal{M}$ and $N(r)=0$. In this way, the asymptotically flat region $\mathcal{N}_{a f, \mathcal{M}}$ of $\mathcal{M}$ will correspond to the region $\{r \gg 1\}$.

Assumption (G7) implies that, if $d g_{\bar{t}}$ denotes the volume form of the induced Riemannian metric $g_{\bar{t}}$ on the $\{\bar{t}=$ const $\}$ hypersurfaces, then there exists a $C>0$ such that for any measurable function $f: \mathcal{M} \rightarrow[0,+\infty)$ and any $0 \leq \tau_{1} \leq \tau_{2}$ we have the equivalence

$$
\int_{\left\{\tau_{1} \leq \bar{t} \leq \tau_{2}\right\} \cap\{r \leq R\}} f d \operatorname{vol}_{g} \sim_{R} \int_{\tau 1}^{\tau_{2}}\left(\int_{\{\bar{t}=\sigma\} \cap\{r \leq R\}} f d g_{\bar{t}}\right) d \sigma .
$$

Notice that Assumptions (G6) and (G7) also imply that for $\bar{t} \geq 0$ the level sets of the extended $\bar{t}$ intersect transversely $\mathcal{H}^{+}$(if $\left.\mathcal{H}^{+} \neq \emptyset\right)$. ${ }^{14}$ It will be useful to denote

$$
\mathcal{H}_{\tau} \doteq \mathcal{H}^{+} \cap\{\bar{t}=\tau\}
$$

We will also denote

$$
\partial_{\text {tim }} \mathcal{M}^{\tau} \doteq \partial_{\text {tim }} \mathcal{M} \cap\{\bar{t}=\tau\}
$$

and

$$
\partial \mathcal{M}_{\tau} \doteq \mathcal{H}_{\tau} \cup \partial_{\text {tim }} \mathcal{M}^{\tau}
$$

Without loss of generality, we also assume that the function $r$ has been extended in such a way in the region $\{r \lesssim 1\}$ so that $d r \neq 0$ on $\mathcal{H} \cap\{\bar{t} \geq 0\}$. We will also use the shorthand notation

$$
r_{+} \doteq\left(1+r^{2}\right)^{1 / 2}
$$

Finally, we will also need to assume that the deformation tensor of $N$ and its derivatives are bounded on $\{\bar{t} \geq 0\}$ when measured with the reference Riemannian metric (8.11) (that will be constructed in a moment):

(G8) For any $l \in \mathbb{N}$, there exists a $C_{l}>0$ such that

$$
\sup _{\{\bar{t} \geq 0\}} \sum_{j=0}^{l}\left|\nabla_{g}^{l-j} \mathcal{L}_{N}^{j} g\right|_{h} \leq C_{l}
$$

Remark Assumption (G8) holds in the case when the spacetime $\mathcal{M}$ is near stationary or time periodic. Moreover, this is an assumption regarding the structure of the foliation in the region $\{r \lesssim 1\}$.

\footnotetext{
14 As an example, on Schwarzschild exterior the function $\bar{t}$ could not have been chosen to coincide with the coordinate function $t$ in a neighborhood horizon, but it can coincide with $t^{*}$ (see i.e. [13]).
} 
It will be convenient to fix a vector field $Y$ in a neighborhood of $\mathcal{H}^{+} \cup \partial_{\text {tim }} \mathcal{M}$ so that for any $\tau \geq 0, Y$ is tangent to $\{\bar{t}=\tau\}$, orthogonal to $\mathcal{H}_{\tau}$ and $\partial_{\text {tim }} \mathcal{M}^{\tau}$ and satisfies $g(Y, Y) \mid \mathcal{H}_{\tau}=1$ and $\left.g(Y, Y)\right|_{\partial_{\text {tim }} \mathcal{M}^{\tau}}=1$.

Boundary conditions on $\partial_{\text {tim }} \mathcal{M}$ for $\square \varphi=F$ Assumptions (G6) and (G7) guarantee that for any $\tau \geq 0$, we can solve the inhomogeneous wave equation

$$
\square_{g} \varphi=F
$$

on $J^{+}(\{\bar{t}=\tau\})$ with Cauchy initial data on $\{\bar{t}=\tau\}$, provided suitable boundary conditions (e.g. Dirichlet conditions) have been imposed on $\partial_{t i m} \mathcal{M}$. In particular, we will introduce the following definition:

Definition We define the class of admissible boundary conditions on $\partial_{\text {tim }} \mathcal{M}$ to be the set $\mathcal{C}_{a d m}$ of all families of linear functions

$$
\mathcal{F}_{\tau}: C^{\infty}\left(\partial_{\text {tim }} \mathcal{M}^{\tau}\right) \times C^{\infty}\left(\partial_{\text {tim }} \mathcal{M}^{\tau}\right) \rightarrow C^{\infty}\left(\partial_{\text {tim }} \mathcal{M}^{\tau}\right)
$$

depending smoothly on $\tau \geq 0$ such that for any $\tau_{0} \geq 0$, any $F \in C^{\infty}\left(\left\{\bar{t} \geq \tau_{0}\right\}\right)$ and any $\varphi_{0}, \varphi_{1} \in C^{\infty}\left(\left\{\bar{t}=\tau_{0}\right\}\right)$, the inititial-boundary value problem

$$
\begin{cases}\square \varphi=F & \text { on }\left\{\bar{t} \geq \tau_{0}\right\} \\ \left(\left.\varphi\right|_{\bar{t}=\tau_{0}},\left.N \varphi\right|_{\bar{t}=\tau_{0}}\right)=\left(\varphi_{0}, \varphi_{1}\right) & \\ \mathcal{F}_{\tau}\left(\left.\varphi\right|_{\partial_{\text {tim }} \mathcal{M}},\left.Y \varphi\right|_{\partial_{\text {tim }} \mathcal{M}}\right)=0 & \text { for } \tau \geq \tau_{0}\end{cases}
$$

is well posed.

Notice that the usual Dirichlet and Neumann boundary condition belong to the class $\mathcal{C}_{a d m}$, corresponding to $\mathcal{F}_{\tau}=I d \oplus 0$ and $\mathcal{F}_{\tau}=0 \oplus I d$ respectively.

Construction of the reference Riemannian metric $h$ on $\mathcal{M}$ In Section A of the Appendix we establish the existence of a natural Riemannian metric $h_{\tau, N}$ defined on the hypersurfaces $\{\bar{t}=\tau\}$ (wich we will sometimes denote with $h_{N}$ for simplicity), associated to $g$ and $N$ (and distinct from the induced metric $g_{\bar{t}}$, which degenerates as on approaches $\mathcal{I}^{+}$).

Remark Notice that $h_{N}$ is non singular up to $\mathcal{H}_{\tau}$, since $N$ is timelike everywhere up to $\mathcal{H}^{+}$. Thus, in the language of Section B of the Appendix, $h_{N}$ corresponds to the $\tilde{h}$ metric of that section.

We will extend $h_{N}$ to a Riemannian metric $h$ on $\mathcal{M}$ by setting

$$
h \doteq(d \bar{t})^{2}+h_{N}
$$

This Riemannian metric will be used to measure the norms of tensors on $\mathcal{M}$. Moreover, we will denote with $h_{\mathcal{H}_{\tau}}$ the Riemannian metric induced by $h$ on $\mathcal{H}_{\tau}$.

Due to the expression (3.4) for $g$ in the region $\{r \gg 1\}$ and (A.2) for $\square_{g}$, we can bound for any smooth $\varphi: \mathcal{M} \rightarrow \mathbb{C}$ and any $l \in \mathbb{N}$ : 


$$
\begin{aligned}
& \left|\nabla_{h_{\tau, N}}^{l}\left(\Delta_{h_{\tau, N}, N} \varphi\right)\right|_{h_{\tau, N}}^{2} \\
& \leq C(\tau)\left\{\sum_{j=1}^{2} r_{+}^{-2}\left|\nabla_{h_{\tau, N}}^{l}\left(N^{j} \varphi\right)\right|_{h_{\tau, N}}^{2}+\sum_{i=0}^{l}\left(\left|\nabla_{h_{\tau, N}}^{i+1}(N \varphi)\right|_{h_{\tau, N}}^{2}+\left|\nabla_{h_{\tau, N}}^{i+1} \varphi\right|_{h_{\tau, N}}^{2}\right)\right. \\
& \left.\quad+\left|\nabla_{h_{\tau, N}}^{l}(\square \varphi)\right|_{h_{\tau, N}}^{2}\right\}
\end{aligned}
$$

where the operator $\Delta_{h_{\tau, N}, N}$ on $\{\bar{t}=\tau\}$ is defined as:

$$
\Delta_{h_{\tau, N}, N}=\frac{1}{\sqrt{-g(N, N)}} d i v_{h_{\tau, N}}(\sqrt{-g(N, N)} d) .
$$

Notice that in the right hand side of (8.12) there is no term of order $l+2$ in the spatial derivatives (i.e. $\nabla_{h_{\tau, N}}$ ).

Assumptions on the uniformity of elliptic, Poincare and Sobolev type estimates on the leaves of the foliation $\{\bar{t}=\tau\}$ We will also need to ensure that we can establish elliptic, Poincare and Sobolev type estimates on the leaves of the foliation $\{\bar{t}=\tau\}$ with constants that do not depend on $\tau$. We will assume the following uniformity condition on $Y$ :

(G9) For any $l \in \mathbb{N}$ the following uniform bound holds:

$$
\sup _{\{\bar{t} \geq 0\}}\left|\nabla_{g}^{l} Y\right|_{h} \leq C_{l}
$$

According to Proposition B.2 of the Appendix and the estimate (8.12), without imposing any extra assumptions the following statement holds:

Lemma For any integer $l \geq 2$ and any $\beta \in[0,1)$ we can bound for any $\tau \geq 0$ and any $\varphi \in C^{\infty}(\mathcal{M})$ satisfying for any $j_{1}+j_{2} \leq l$ the finite radiation field condition $\lim \sup _{r \rightarrow+\infty}\left|r^{\frac{d-1}{2}+j_{1}} \nabla_{h_{\tau, N}}^{j_{1}}\left(N^{j_{2}} \varphi\right)\right|_{h_{\tau, N}}<+\infty$ :

$$
\begin{aligned}
& \int_{\{\bar{t}=\tau\}} r_{+}^{-\beta}\left|\nabla_{g}^{l} \varphi\right|_{h}^{2} d h_{N} \leq C_{\beta, n_{0}}(\tau)\left\{\int _ { \{ \overline { t } = \tau \} } r _ { + } ^ { - \beta } \left\{\sum_{j=1}^{l} \sum_{i=0}^{l-j} r_{+}^{-2}\left|\nabla_{h_{\tau, N}}^{i}\left(N^{j} \varphi\right)\right|_{h_{\tau, N}}^{2}\right.\right. \\
& +\sum_{0 \leq j_{1}+j_{2} \leq l-2}\left(\left|\nabla_{h_{\tau, N}}^{j_{1}+1}\left(N^{j_{2}+1} \varphi\right)\right|_{h_{\tau, N}}^{2}\right. \\
& \left.\left.\quad+\left|\nabla_{h_{\tau, N}}^{j_{1}+1}\left(N^{j_{2}} \varphi\right)\right|_{h_{\tau, N}}^{2}+\left|\nabla_{h_{\tau, N}}^{j_{1}}\left(N^{j_{2}} \square \varphi\right)\right|_{h_{\tau, N}}^{2}\right)\right\} d h_{N} \\
& \left.\quad+\sum_{j=0}^{l-1}\left|\int_{\partial \mathcal{M}_{\tau}} h_{\partial \mathcal{M}_{\tau}}\left(\nabla_{h_{\partial \mathcal{M}}}^{j}(Y u), \nabla_{h_{\partial \mathcal{M}}}^{j} u\right) d h_{\partial \mathcal{M}_{\tau}}\right|\right\}
\end{aligned}
$$

Our final assumtions on the geometry of $(\mathcal{M}, g)$ in the region $\{\bar{t} \geq 0\}$ will be the following: 
(G10) The constants appearing in the right hand side of (8.15) do not depend on $\tau \geq 0$.

(G11) The following elliptic estimate holds for any $l \in \mathbb{N}$ and any smooth function $u$ on the submanifolds $\partial \mathcal{M}_{\tau}$ with a constant $C_{l}$ not depending on $\tau \geq 0$ :

$$
\sum_{j=1}^{l} \int_{\partial \mathcal{M}_{\tau}}\left|\nabla_{h_{\partial \mathcal{M} \tau}}^{j} u\right|_{h_{\partial \mathcal{M} \tau}}^{2} d h_{\partial \mathcal{M}_{\tau}} \leq C_{l} \cdot \int_{\partial \mathcal{M}_{\tau}}\left|\nabla_{h_{\partial \mathcal{M} \tau}}^{l-2}\left(\Delta_{h_{\partial \mathcal{M}} \tau} u\right)\right|_{h_{\partial \mathcal{M} \tau}}^{2} d h_{\partial \mathcal{M}_{\tau}}
$$

(G12) For $C^{1}$ functions $u$ on $\mathcal{M}$, the Poincare inequality

$$
\begin{aligned}
& \int_{\{\bar{t}=\tau\} \cap\{r \leq R\}}|u|^{2} d h_{N} \leq C(R) \cdot\left(\int_{\{\bar{t}=\tau\} \cap\{r \leq R\}}\left|\nabla_{h_{N}} u\right|_{h_{N}}^{2} d h_{N}\right. \\
& \left.+\int_{\{\bar{t}=\tau\} \cap\{R \leq r \leq 2 R\}}|u|^{2} d h_{N}\right)
\end{aligned}
$$

and the trace inequality

$$
\begin{aligned}
\int_{\partial \mathcal{M}_{\tau}}\left|\left(\nabla_{h_{\partial \mathcal{M}}}\right)^{1 / 2} u\right|^{2} d h_{\partial \mathcal{M}_{\tau}} \leq & C(\varepsilon) \cdot\left(\int_{\partial \mathcal{M} \cap\{\tau-\varepsilon \leq \bar{t} \leq \tau\}}\left|\nabla_{h_{\partial \mathcal{M}}} u\right|_{h_{\partial \mathcal{M}}}^{2} d h_{\partial \mathcal{M}}\right. \\
& \left.+\int_{\partial \mathcal{M} \cap\{\tau-\varepsilon \leq \bar{t} \leq \tau\}}|u|^{2} d h_{\partial \mathcal{M}}\right)
\end{aligned}
$$

(where $h_{\partial \mathcal{M}}$ is the Riemannian metric on $\partial \mathcal{M}$ induced by $h$ ) hold for constants $C(R)$ and $C(\varepsilon)$ that do not depend on $\tau \geq 0$. In the above

(G13) The following Sobolev inequality holds for smooth and compactly supported functions $u$ on the hypersurfaces $\{\bar{t}=\tau\}_{\tau \geq 0}$

$$
\sup _{\{\bar{t}=\tau\}}|u|^{2} \leq C \cdot \sum_{j=0}^{\left\lceil\frac{d+1}{2}\right\rceil} \int_{\{\bar{t}=\tau\}}\left|\nabla_{h_{N}}^{j} u\right|_{h_{N}}^{2} d h_{N}
$$

(see [19]) with the constant $C$ in the right hand side independent of $\tau$.

Remark Assumptions (G10)-(G13) are automatically satisfied in the case the spacetime $\mathcal{M}$ is near stationary or time periodic. Moreover, these assumptions are only tied to the structure of the foliation in the region $\{r \lesssim 1\}$.

\subsubsection{Integrated Local Energy Decay Statement on $(\mathcal{M}, g)$}

We assume that the following integrated local energy decay statement holds on the spacetime $(\mathcal{M}, g)$ under consideration:

(ILED1) Integrated local energy decay with loss of derivatives: We assume that there exists a (non-empty) class $\mathcal{C}_{I L E D}$ of boundary conditions on $\partial_{\text {tim }} \mathcal{M}$, which 
is contained in the class of admissible boundary conditions $\mathcal{C}_{a d m}$, so that the following statement holds: There exists an integer $k \geq 0$ such that for any $R, R_{f}>0$, any integer $m \geq 0$, any $0<\eta<a$, any smooth $\varphi: \mathcal{M} \rightarrow \mathbb{C}$ solving $\square_{g} \varphi=F$ satisfying boundary conditions on $\partial_{\text {tim }} \mathcal{M}$ belonging to the class $\mathcal{C}_{I L E D}$ and any $0 \leq \tau_{1} \leq \tau_{2}$ we can bound:

$$
\begin{aligned}
& \sum_{j=1}^{m} \int_{\left\{\tau_{1} \leq \bar{t} \leq \tau_{2}\right\} \cap\{r \leq R\}}\left(\left|\nabla_{g}^{j} \varphi\right|_{h}^{2}+|\varphi|^{2}\right)+\sum_{j=1}^{m} \int_{\left\{\tau_{1} \leq \bar{t} \leq \tau_{2}\right\} \cap \partial_{t i m} \mathcal{M}}\left(\left|\nabla_{g}^{j} \varphi\right|_{h}^{2}+|\varphi|^{2}\right) \\
& \leq C_{m, \eta}\left(R, R_{f}\right) \sum_{j=0}^{m+k-1}\left(\int_{\left\{\bar{t}=\tau_{1}\right\}} J_{\mu}^{N}\left(N^{j} \varphi\right) \bar{n}^{\mu}+\int_{\left\{\tau_{1} \leq \bar{t} \leq \tau_{2}\right\}} r_{+}^{1+\eta}\left|\nabla_{g}^{j} F\right|_{h}^{2} d h\right) \\
& \leq+C_{m, \eta} \sum_{j=0}^{m-1} \sum_{j_{1}+j_{2}=j} \int_{\left\{\tau_{1} \leq \bar{t} \leq \tau_{1}\right\} \cap\left\{r \geq R_{f}\right\}} r_{+}^{-1}\left(\left|\nabla_{h_{\tau, N}}^{j_{1}+1}\left(N^{j_{2}} \varphi\right)\right|^{2}\right. \\
& \left.\quad+r_{+}^{-2}\left|\nabla_{h_{\tau, N}}^{j_{1}}\left(N^{j_{2}} \varphi\right)\right|^{2}+r_{+}^{-2}\left|N^{j+1} \varphi\right|^{2}\right) d g
\end{aligned}
$$

Using Lemma 4.1 and 4.5, as well as a trace theorem for $\int_{\partial_{\text {tim }} \mathcal{M}}|\varphi|^{2},(8.19)$ can be improved into the statement that for any $0<\eta<a$ and $R \gg 1$ :

$$
\begin{aligned}
& \int_{\left\{\tau_{1} \leq \bar{t} \leq \tau_{2}\right\}} r_{+}^{-1-\eta}\left(\sum_{j=1}^{m}\left|\nabla_{g}^{j} \varphi\right|_{h}^{2}+r^{-2}|\varphi|^{2}\right)+\sum_{j=1}^{m} \int_{\left\{\tau_{1} \leq \bar{t} \leq \tau_{2}\right\} \cap \partial_{t i m} \mathcal{M}}\left(\left|\nabla_{g}^{j} \varphi\right|_{h}^{2}+|\varphi|^{2}\right) \\
& \leq C_{m, \eta}(R) \sum_{j=0}^{m+k-1}\left(\int_{\left\{\bar{t}=\tau_{1}\right\}} J_{\mu}^{N}\left(N^{j} \varphi\right) \bar{n}^{\mu}+\int_{\left\{\tau_{1} \leq \bar{t} \leq \tau_{2}\right\}} r_{+}^{1+\eta}\left|\nabla_{g}^{j} F\right|_{h}^{2} d h\right) \\
& \quad+C_{m, \eta} \cdot \sum_{j=0}^{m-1} \sum_{j_{1}+j_{2}=j} \int_{\left\{\tau_{1} \leq \bar{t} \leq \tau_{1}\right\} \cap\{r \geq R\}} r_{+}^{-1}\left(\left|\nabla_{h_{\tau, N}}^{j_{1}+1}\left(N^{j_{2}} \varphi\right)\right|^{2}\right. \\
& \left.\quad+r_{+}^{-2}\left|\nabla_{h_{\tau, N}}^{j_{1}}\left(N^{j_{2}} \varphi\right)\right|^{2}+r_{+}^{-2}\left|N^{j+1} \varphi\right|^{2}\right) d g .
\end{aligned}
$$

We should also notice that the results of this section can be readily established in case one replaces Assumption (ILED1) by the following pair of integrated local energy decay statements:

Alternative integrated local energy decay statement: With the notations as in Assumption (ILED1), we assume that there exists an integer $k \geq 0$ and an $R_{c}>0$ such that for any $R, R_{f}>0$, any integer $m \geq 0$, any $0<\eta<a$, any smooth $\varphi$ : $\mathcal{M} \rightarrow \mathbb{C}$ solving $\square_{g} \varphi=F$ satisfying boundary conditions on $\partial_{\text {tim }} \mathcal{M}$ belonging to the class $\mathcal{C}_{I L E D}$ and any $0 \leq \tau_{1} \leq \tau_{2}$ we can bound:

$$
\sum_{j=1}^{m} \int_{\left\{\tau_{1} \leq \bar{t} \leq \tau_{2}\right\} \cap\{r \leq R\}}\left(\left|\nabla_{g}^{j} \varphi\right|_{h}^{2}+|\varphi|^{2}\right)+\sum_{j=1}^{m} \int_{\left\{\tau_{1} \leq \bar{t} \leq \tau_{2}\right\} \cap \partial_{t i m} \mathcal{M}}\left(\left|\nabla_{g}^{j} \varphi\right|_{h}^{2}+|\varphi|^{2}\right)
$$




$$
\begin{aligned}
\leq & C_{m, \eta}\left(R, R_{f}\right) \sum_{j=0}^{m+k-1}\left(\int_{\left\{\bar{t}=\tau_{1}\right\}} J_{\mu}^{N}\left(N^{j} \varphi\right) \bar{n}^{\mu}+\int_{\left\{\tau_{1} \leq \bar{t} \leq \tau_{2}\right\}} r_{+}^{1+\eta}\left|\nabla_{g}^{j} F\right|_{h}^{2} d h\right) \\
& +C_{m, \eta} \sum_{j=0}^{m+k-1} \sum_{j_{1}+j_{2}=j} \int_{\left\{\tau_{1} \leq \bar{t} \leq \tau_{1}\right\} \cap\left\{r \geq R_{f}\right\}} r_{+}^{-1} \\
& \times\left(\left|\nabla_{h_{\tau, N}}^{j_{1}+1}\left(N^{j_{2}} \varphi\right)\right|^{2}+r_{+}^{-2}\left|\nabla_{h_{\tau, N}}^{j_{1}}\left(N^{j_{2}} \varphi\right)\right|^{2}+r_{+}^{-2}\left|N^{j+1} \varphi\right|^{2}\right) d g
\end{aligned}
$$

and

$$
\begin{aligned}
& \sum_{j=1}^{m} \int_{\left\{\tau_{1} \leq \bar{t} \leq \tau_{2}\right\} \cap\left\{R_{c} \leq r \leq R\right\}}\left(\left|\nabla_{g}^{j} \varphi\right|_{h}^{2}+|\varphi|^{2}\right)+\sum_{j=1}^{m} \int_{\left\{\tau_{1} \leq \bar{t} \leq \tau_{2}\right\} \cap \partial_{t i m} \mathcal{M}}\left(\left|\nabla_{g}^{j} \varphi\right|_{h}^{2}+|\varphi|^{2}\right) \\
& \leq C_{m, \eta}\left(R, R_{f}\right) \sum_{j=0}^{m-1}\left(\int_{\left\{\bar{t}=\tau_{1}\right\}} J_{\mu}^{N}\left(N^{j} \varphi\right) \bar{n}^{\mu}+\int_{\left\{\tau_{1} \leq \bar{t} \leq \tau_{2}\right\}} r_{+}^{1+\eta}\left|\nabla_{g}^{j} F\right|_{h}^{2} d h\right) \\
& \quad+C_{m, \eta} \sum_{j=0}^{m-1} \sum_{j_{1}+j_{2}=j} \int_{\left\{\tau_{1} \leq \bar{t} \leq \tau_{1}\right\} \cap\left\{r \geq R_{f}\right\}} r_{+}^{-1}\left(\left|\nabla_{h_{\tau, N}}^{j_{1}+1}\left(N^{j_{2}} \varphi\right)\right|^{2}\right. \\
& \left.\quad+r_{+}^{-2}\left|\nabla_{h_{\tau, N}}^{j_{1}}\left(N^{j_{2}} \varphi\right)\right|^{2}+r_{+}^{-2}\left|N^{j+1} \varphi\right|^{2}\right) d g .
\end{aligned}
$$

However, we will not pursue this issue again in the paper.

\subsection{A First Polynomial Decay Result}

On any spacetime $(\mathcal{M}, g)$ satisfying the geometric assumptions (G1)-(G13) and Assumption (ILED1) on integrated local energy decay with loss of derivatives, we will establish the following polynomial decay estimates:

Theorem 8.1 Let $\left(\mathcal{M}^{d+1}, g\right), d \geq 3$, satisfy Assumptions (G1)-(G13) and (ILED1). For any smooth solution $\varphi$ to $\square_{g} \varphi=F$ on $J^{+}(\{\bar{t}=0\})$ with suitably decaying initial data on $\{\bar{t}=0\}$ (and satisfying boundary conditions on $\partial_{\text {tim }} \mathcal{M}$ belonging to the class $\left.\mathcal{C}_{I L E D}\right)$, the following decay estimates hold for any $\tau \geq 0$, any integer $m \geq 0$ and any $\varepsilon>0,0<\eta<a$, provided (7.2) holds for all $0 \leq j \leq m+1+d+3 k$ :

$$
\begin{aligned}
& \sum_{j=0}^{m-1} \sum_{j_{1}+j_{2}=j} \int_{\{\bar{t}=\tau\}}\left(\left|\nabla_{h_{\tau, N}}^{j_{1}+1}\left(N^{j_{2}} \varphi\right)\right|^{2}+r_{+}^{-2}\left|N^{j+1} \varphi\right|^{2}+r_{+}^{-2}|\varphi|^{2}\right) d h_{N} \\
& \quad \sum_{m, \varepsilon, \eta} \tau^{-2+\varepsilon} \mathcal{E}_{\text {bound }}^{(2, m+3 k)}[\varphi](0)+\mathcal{F}_{\eta, \varepsilon}^{(2, m, k)}[F](\tau), \\
& \sup _{\{\bar{t}=\tau\}}\left|r_{+}^{\frac{d-2}{2}} \nabla_{g}^{m} \varphi\right|_{h}^{2} \lesssim_{m, \varepsilon, \eta} \tau^{-2+\varepsilon} \mathcal{E}_{\text {bound }}^{\left(2, m+\left\lceil\frac{d+2}{2}\right\rceil+3 k\right)}[\varphi](0) \mathcal{F}_{\eta, \varepsilon}^{\left(2, m+\left\lceil\frac{d+2}{2}\right\rceil, k\right)}[F](\tau)
\end{aligned}
$$


and

$$
\begin{aligned}
& \sup _{\{\bar{t}=\tau\}}\left|r_{+}^{\frac{d-1}{2}} \nabla_{g}^{m} \varphi\right|_{h}^{2} \lesssim m, \eta \\
& \quad \tau^{-1} \mathcal{E}_{\text {bound }}^{\left(2, m+\left\lceil\frac{d+2}{2}\right\rceil+2 k\right)}[\varphi](0)+\mathcal{F}_{\eta}^{\left(1, m+\left\lceil\frac{d+2}{2}\right\rceil, k\right)}[F](\tau) .
\end{aligned}
$$

In the above, $k$ is the integer measuring the derivative loss in the integrated local energy decay statement (8.19) and for some fixed $R, C>1$ :

$$
\begin{aligned}
\mathcal{E}_{\text {bound }}^{(p, m)}[\varphi](\tau)= & \sum_{j=0}^{m} \int_{\{\bar{t}=\tau\} \cap\{r \leq R+1\}}\left|\nabla_{h_{\tau, N}}^{j_{1}}\left(N^{j_{2}} \varphi\right)\right|_{h_{\tau, N}}^{2} d h_{N} \\
& +\sum_{\substack{\text { components } \\
\text { of } \mathcal{N}_{\text {af }, \mathcal{M}}}} \sum_{j=0}^{m-1} \sum_{j_{1}+j_{2}+j_{3}=j} \mathcal{E}_{\text {bound }, R ; \delta}^{(p, 1)}\left[r^{-j_{2}} \partial_{v}^{j_{1}} \partial_{\sigma}^{j_{2}} \partial_{u}^{j_{3}} \varphi\right](\tau)
\end{aligned}
$$

(where the derivatives in the second term are with respect to the $(u, v, \sigma)$ coordinate charts over each connected component of $\mathcal{N}_{\text {af, }} \mathcal{M}$ ),

$$
\begin{aligned}
\mathcal{F}_{\eta, \varepsilon}^{(2, m, k)}[F](\tau)= & \tau^{-2+\varepsilon} \sum_{j=0}^{m+3 k-1} \int_{\{0 \leq \bar{t} \leq \tau\}} r^{3}\left|\nabla_{g}^{j} F\right|_{h}^{2} d g \\
& +\tau^{-1+\varepsilon} \sum_{j=0}^{m+2 k-1} \int_{\left\{C^{-1} \tau \leq \bar{t} \leq \tau\right\}} r^{2}\left|\nabla_{g}^{j} F\right|_{h}^{2} d g \\
& +\sum_{j=0}^{m+2 k-1} \int_{\left\{C^{-1} \tau \leq \bar{t} \leq \tau\right\}}\left(r^{1+\varepsilon}+r^{1+\eta}\right)\left|\nabla_{g}^{j} F\right|_{h}^{2} d g
\end{aligned}
$$

and

$$
\begin{aligned}
\mathcal{F}_{\eta}^{(1, m, k)}[F](\tau)= & \tau^{-1} \sum_{j=0}^{m+2 k-1} \int_{\{0 \leq \bar{t} \leq \tau\}} r^{3}\left|\nabla_{g}^{j} F\right|_{h}^{2} d g \\
& +\sum_{j=0}^{m+2 k-1} \int_{\left\{C^{-1} \tau \leq \bar{t} \leq \tau\right\}} r^{2}\left|\nabla_{g}^{j} F\right|_{h}^{2} d g .
\end{aligned}
$$

Remark In case there exists some small $\delta_{0}>0$ such that the deformation tensor of the vector field $T$ in the region $\{r \gg 1\}$ satisfies the following bound for any integer $m \geq 1$ :

$$
\begin{aligned}
\mathcal{L}_{T}^{m} g= & O\left(\bar{t}^{-\delta 0}\right)\left\{O\left(r^{-1-a}\right) d v d u+O(r) d \sigma d \sigma+O(1) d u d \sigma\right. \\
& \left.+O\left(r^{-a}\right) d v d \sigma+O\left(r^{-1}\right) d u^{2}+O\left(r^{-2-a}\right) d v^{2}\right\}
\end{aligned}
$$


and the last term in the right hand side of the integrated local energy decay estimate (8.19) is replaced by

$$
\begin{aligned}
& C_{m, \eta} \sum_{j=0}^{m-1} \sum_{j_{1}+j_{2}=j} \int_{\left\{\tau_{1} \leq \bar{t} \leq \tau_{1}\right\} \cap\left\{r \geq R_{f}\right\}} \bar{t}^{-\delta 0} r_{+}^{-1}\left(\left|\nabla_{h_{\tau, N}}^{j_{1}+1}\left(N^{j_{2}} \varphi\right)\right|^{2}\right. \\
& \left.+r_{+}^{-2}\left|\nabla_{h_{\tau, N}}^{j_{1}}\left(N^{j_{2}} \varphi\right)\right|^{2}+r_{+}^{-2}\left|N^{j+1} \varphi\right|^{2}\right) d g
\end{aligned}
$$

(this is consistent with the remark below Lemma 4.1), then the $\varepsilon$ loss in the exponent of $\tau$ in (8.23) and (8.24) can be removed. This follows readily the fact that, in this case, the second summand of the right hand side of (4.30) comes with a $O\left(\bar{t}^{-\delta 0}\right)$ factor, which enables us to deduce (8.23) from (8.55) and (8.56) for some $\varepsilon \ll \delta_{0}$.

The proof of Theorem 8.1 will be presented in Section 8.4.

We will also establish the following generalisation of Theorem 8.1 with improved weights in $r$ associated to higher derivatives of $\varphi$ :

Theorem 8.2 Let $\left(\mathcal{M}^{d+1}, g\right), d \geq 3$, satisfy Assumptions (G1)-(G13) and (ILED1). For any smooth solution $\varphi$ to $\square_{g} \varphi=F$ on $J^{+}(\{\bar{t}=0\}) \subset(\mathcal{M}, g)$ with suitably decaying initial data on $\{\bar{t}=0\}$ (and satisfying boundary conditions on $\partial_{\text {tim }} \mathcal{M}$ belonging to the class $\left.\mathcal{C}_{I L E D}\right)$, the following decay estimates hold for any $\tau \geq 0$, any integers $q, m \geq 1$ and any $\varepsilon>0,0<\eta<$ a provided (7.2) holds for all $0 \leq j \leq q+m+d+3 k$ :

$$
\begin{aligned}
& \sum_{0 \leq i_{1}+i_{2} \leq m-1} \sum_{j=0}^{q-1} \sum_{j_{1}+j_{2}=j} \int_{\{\bar{t}=\tau\}}\left(r^{2 j_{1}}\left|\nabla_{h_{\tau, N}}^{i_{1}+j_{1}+1}\left(N^{i_{2}+j_{2}} \varphi\right)\right|^{2}\right. \\
& \left.+r_{+}^{-2}\left|N^{j+i_{1}+i_{2}+1} \varphi\right|^{2}+r_{+}^{-2}|\varphi|^{2}\right) d h_{N} \lesssim_{m, q, \varepsilon, \eta} \\
& \lesssim_{m, q, \varepsilon, \eta} \tau^{-2+\varepsilon} \mathcal{E}_{\text {bound }}^{(2 q, q, m-1+3 k)}[\varphi](0)+\mathcal{F}_{\eta, \varepsilon}^{(2, q, m-1+k)}[F](\tau), \\
& \sum_{0 \leq i_{1}+i_{2} \leq m-1} \sum_{j_{1}+j_{2}=q} \sup _{\{\bar{t}=\tau\}}\left|r_{+}^{\frac{d-2}{2}+j_{1}} \nabla_{h_{\tau, N}}^{j_{1}+i_{1}}\left(N^{j_{2}+i_{2}} \varphi\right)\right|_{h}^{2} \\
& \left.\lesssim_{m, q, \varepsilon, \eta} \tau^{-2+\varepsilon} \mathcal{E}_{\text {bound }}^{\left(2 q, q, m-1+\left\lceil\frac{d+2}{2}\right\rceil+3 k\right)}[\varphi](0)+\mathcal{F}_{\eta, \varepsilon}^{\left(2, q, m-1+\left\lceil\frac{d+2}{2}\right\rceil, k\right)}[F](\tau) 8.32\right)
\end{aligned}
$$

and

$$
\begin{gathered}
\sum_{0 \leq i_{1}+i_{2} \leq m-1} \sum_{j_{1}+j_{2}=q} \sup _{\{\bar{t}=\tau\}}\left|r_{+}^{\frac{d-1}{2}+j_{1}} \nabla_{h_{\tau, N}}^{j_{1}+i_{1}}\left(N^{j_{2}+i_{2}} \varphi\right)\right|_{h}^{2} \lesssim_{m, q, \eta} \\
\tau^{-1} \mathcal{E}_{\text {bound }}^{\left(2 q, q, m-1+\left\lceil\frac{d+2}{2}\right\rceil+2 k\right)}[\varphi](0)+\mathcal{F}_{\eta}^{\left(1, q, m-1+\left\lceil\frac{d+2}{2}\right\rceil, k\right)}[F](\tau) .
\end{gathered}
$$

In the above, $k$ is the integer measuring the derivative loss in the integrated local energy decay statement (8.19) and for some fixed $R, C>1$ : 


$$
\begin{gathered}
\mathcal{E}_{\text {bound }}^{(p, q, m)}[\varphi](\tau)=\sum_{j=0}^{q+m-1} \int_{\{\bar{t}=\tau\} \cap\{r \leq R+1\}}\left|\nabla_{h_{\tau, N}}^{j_{1}}\left(N^{j_{2}} \varphi\right)\right|_{h_{\tau, N}}^{2} d h_{N} \\
+\sum_{\substack{\text { components } \\
\text { of } \mathcal{N}_{\text {af, } \mathcal{M}}}} \sum_{j=0}^{m-1} \sum_{j_{1}+j_{2}+j_{3}=j} \mathcal{E}_{\text {bound }, R ; \delta}^{(p, q)}\left[r^{-j_{2}} \partial_{v}^{j_{1}} \partial_{\sigma}^{j_{2}} \partial_{u}^{j_{3}} \varphi\right](\tau)
\end{gathered}
$$

(where the derivatives in the second term are with respect to the $(u, v, \sigma)$ coordinate charts over each connected component of $\mathcal{N}_{\text {af, }} \mathcal{M}$ ),

$$
\begin{aligned}
& \mathcal{F}_{\eta, \varepsilon}^{(2, q, m, k)}[F](\tau) \\
& =\tau^{-2+\varepsilon} \sum_{j=0}^{m+3 k-1} \sum_{j_{1}+j_{2}=j} \sum_{i_{1}+i_{2} \leq q-1} \int_{\{0 \leq \bar{t} \leq \tau\}} r^{3+2 i_{1}}\left|\nabla_{h_{\tau, N}}^{j_{1}+i_{1}}\left(N^{j_{2}+i_{2}} F\right)\right|_{h_{\tau, N}}^{2} d g \\
& \quad+\tau^{-1+\varepsilon} \sum_{j=0}^{m+2 k-1} \sum_{j_{1}+j_{2}=j} \sum_{i_{1}+i_{2} \leq q-1} \int_{\left\{C^{-1} \tau \leq \bar{t} \leq \tau\right\}} r^{2+2 i_{1}}\left|\nabla_{h_{\tau}, N}^{j_{1}+i_{1}}\left(N^{j_{2}+i_{2}} F\right)\right|_{h_{\tau, N}}^{2} d g \\
& \quad+\sum_{j=0}^{m+2 k-1} \sum_{j_{1}+j_{2}=j} \sum_{i_{1}+i_{2} \leq q-1} \int_{\left\{C^{-1} \tau \leq \bar{t} \leq \tau\right\}}\left(r^{1+\varepsilon+2 i_{1}}+r^{1+\eta}\right)\left|\nabla_{h_{\tau}, N}^{j_{1}+i_{1}}\left(N^{j_{2}+i_{2}} F\right)\right|_{h_{\tau, N}}^{2} d g
\end{aligned}
$$

and

$$
\begin{aligned}
& \mathcal{F}_{\eta}^{(1, q, m, k)}[F](\tau) \\
& =\tau^{-1} \sum_{j=0}^{m+2 k-1} \sum_{j_{1}+j_{2}=j} \sum_{i_{1}+i_{2} \leq q-1} \int_{\{0 \leq \bar{t} \leq \tau\}} r^{3+2 i_{1}}\left|\nabla_{h_{\tau, N}}^{j_{1}+i_{1}}\left(N^{j_{2}+i_{2}} F\right)\right|_{h_{\tau, N}}^{2} d g \\
& \quad+\sum_{j=0}^{m+2 k-1} \sum_{j_{1}+j_{2}=j} \sum_{i_{1}+i_{2} \leq q-1} \int_{\left\{C^{-1} \tau \leq \bar{t} \leq \tau\right\}} r^{2+2 i_{1}}\left|\nabla_{h_{\tau, N}}^{j_{1}+i_{1}}\left(N^{j_{2}+i_{2}} F\right)\right|_{h_{\tau, N}}^{2} d g .
\end{aligned}
$$

Remark Notice that each derivative of $\varphi$ tangential to $\{\bar{t}=\tau\}$ carries an extra $r$-weight. Again, as before, in case there exists some small $\delta_{0}>0$ such that the deformation tensor of the vector field $T$ in the region $\{r \gg 1\}$ satisfies the bound (8.29) for any $m \in \mathbb{N}$ and the last term in the right hand side of the integrated local energy decay estimate (8.19) is replaced by

$$
\begin{aligned}
& C_{m, \eta} \sum_{j=0}^{m-1} \sum_{j_{1}+j_{2}=j} \int_{\left\{\tau_{1} \leq \bar{t} \leq \tau_{1}\right\} \cap\left\{r \geq R_{f}\right\}} \bar{t}^{-\delta 0} r_{+}^{-1}\left(\left|\nabla_{h_{\tau, N}}^{j_{1}+1}\left(N^{j_{2}} \varphi\right)\right|^{2}\right. \\
& \left.+r_{+}^{-2}\left|\nabla_{h_{\tau, N}}^{j_{1}}\left(N^{j_{2}} \varphi\right)\right|^{2}+r_{+}^{-2}\left|N^{j+1} \varphi\right|^{2}\right) d g,
\end{aligned}
$$

then the $\varepsilon$ loss in the exponent of $\tau$ in (8.31) and (8.32) can be removed. 
The proof of this theorem follows exactly as that of Theorem 8.1, the only difference being that Corollary 6.2 is used in place of 5.2, and Lemma 4.7 in place of 4.5. Thus, the details of the proof will be omitted.

\subsection{Energy Boundedness with Loss of Derivatives}

The integrated local energy decay assumption (8.20) on $(\mathcal{M}, g)$ allows us to establish the following energy boundedness statement with loss of derivatives on $(\mathcal{M}, g)$ :

Lemma 8.3 For any $R>0$, any integer $m \geq 1$ and any $0<\eta<$ a we can bound for any $0 \leq \tau_{1} \leq \tau_{2}$ and any smooth function $\varphi$ satisfying $\square \varphi=F$ on $(\mathcal{M}, g)$ with finite energy norm on $\left\{\bar{t}=\tau_{1}\right\}$ (and satisfying boundary conditions on $\partial_{\text {tim }} \mathcal{M}$ belonging to the class $\mathcal{C}_{I L E D}$ ), we can bound provided (7.2) holds for all $0 \leq j \leq m+d+k$ :

$$
\begin{aligned}
& \sum_{j=0}^{m-1} \sum_{j_{1}+j_{2}=j} \int_{\mathcal{H}^{+}\left(\tau_{1}, \tau_{2}\right)}\left(\left|\nabla_{h_{\mathcal{H}}}^{j_{1}+1}\left(N^{j_{2}} \varphi\right)\right|_{h_{\mathcal{H}}}^{2}+|\varphi|^{2}\right) d h_{\mathcal{H}} \\
& +\sum_{j=0}^{m} \sum_{j_{1}+j_{2}=j} \int_{\partial_{t i m} \mathcal{M} \cap\left\{\tau_{1} \leq \bar{t} \leq \tau_{2}\right\}}\left|\nabla_{g}^{j} \varphi\right|_{h}^{2} d h_{\partial \mathcal{M}_{\text {tim }}} \\
& +\sum_{j=0}^{m-1} \sum_{j_{1}+j_{2}=j} \int_{\left\{\bar{t}=\tau_{2}\right\}}\left(\left|\nabla_{h_{N}}^{j_{1}+1}\left(N^{j_{2}} \varphi\right)\right|_{h_{N}}^{2}+r_{+}^{-2}\left|N^{j+1} \varphi\right|^{2}\right) d h_{N} \\
& \leq C_{m, R} \sum_{j=0}^{m+k-1} \sum_{j_{1}+j_{2}=j} \int_{\left\{\bar{t}=\tau_{1}\right\}}\left(\left|\nabla_{h_{N}}^{j_{1}+1}\left(N^{j_{2}} \varphi\right)\right|_{h_{N}}^{2}+r_{+}^{-2}\left|N^{j+1} \varphi\right|^{2}\right) d h_{N} \\
& +\sum_{j=0}^{m-1} \sum_{j_{1}+j_{2}=j} \int_{\left\{\tau_{1} \leq \bar{t} \leq \tau_{2}\right\} \cap\{r \geq R\}} r_{+}^{-1} \\
& \times\left(\left|\nabla_{h_{N}}^{j_{1}+1}\left(N^{j_{2}} \varphi\right)\right|_{h_{N}}^{2}+r_{+}^{-2}\left|N^{j+1} \varphi\right|^{2}+r_{+}^{-2}|\varphi|^{2}\right) d h \\
& +C_{m, R} \sum_{j=0}^{m+k-1} \int_{\left\{\tau_{1} \leq \bar{t} \leq \tau_{2}\right\}} r_{+}^{1+\eta}\left|\nabla_{g}^{j} F\right|_{h}^{2} d h .
\end{aligned}
$$

where $\mathcal{H}^{+}\left(\tau_{1}, \tau_{2}\right)=\mathcal{H}^{+} \cap\left\{\tau_{1} \leq \bar{t} \leq \tau_{2}\right\}$ and $k$ is the integer measuring the loss of derivatives in (8.19).

Proof Without loss of generality, we can assume that $R$ is large in terms of the geometry of $(\mathcal{M}, g)$. Let us fix a second smooth vector field $N_{1}$ on $(\mathcal{M}, g)$, such that:

- $N_{1} \equiv N$ on $\{r \geq 1\}$

- $\left|N_{1}\right|_{h} \leq 2$ on $(\mathcal{M}, g)$

- $g\left(N_{1}, N_{1}\right) \leq-c<0$ everywhere on $(\mathcal{M}, g)$

- For any $l \in \mathbb{N}:\left|\mathcal{L}_{N_{1}}^{l} g\right|_{h} \leq C_{l}{ }^{15}$

\footnotetext{
15 This is possible in view of Assumptions (G8) and (G9).
} 
- $N_{1}$ and $N$ are linearly independent on $\mathcal{H}^{+}$and their span intersects the tangent space of the surfaces $\mathcal{H}^{+} \cap\{\bar{t}=$ const $\}$ transversally

- $\left|\left(g\left(N_{1}, N\right)\right)^{2}-g\left(N_{1}, N_{1}\right) g(N, N)\right| \geq c>0$ on $\mathcal{H}^{+}$.

Notice that in the case $\mathcal{H}^{+}=\emptyset$ we can simply choose $N_{1}=N$.

Fix a smooth cut-off function $\chi:[0,+\infty) \rightarrow[0,1]$ such that $\chi(x)=1$ for $x \leq 1$ and $\chi(x)=0$ for $x \geq 2$, and define:

$$
\chi_{R}(r) \doteq \chi\left(\frac{r}{R}\right)
$$

Define also the energy current

$$
J_{\mu}^{m}[\varphi]=\sum_{j=0}^{m-1} \sum_{j_{1}+j_{2}=j}\left(J_{\mu}^{N}\left(N_{1}^{j_{1}} N^{j_{2}} \varphi\right)\right) .
$$

By integrating $\nabla_{g}^{\mu}\left(\chi_{R} J_{\mu}^{m}[\varphi]\right)$ over the region $\left\{\tau_{1} \leq \bar{t} \leq \tau_{2}\right\}$, we obtain (due to the boundedness of the derivatives of the deformation tensors of $N_{1}, N$ ):

$$
\begin{aligned}
& \int_{\mathcal{H}^{+}\left(\tau_{1}, \tau_{2}\right)} J_{\mu}^{m}[\varphi] n_{\mathcal{H}^{\mu}}^{\mu}+\int_{\left\{\bar{t}=\tau_{2}\right\} \cap\{r \leq R\}} J_{\mu}^{m}[\varphi] \bar{n}^{\mu} \\
& \leq \int_{\left\{\bar{t}=\tau_{1}\right\} \cap\{r \leq 2 R\}} J_{\mu}^{m}[\varphi] \bar{n}^{\mu}+C_{m} \int_{J^{+}\left(\left\{\bar{t}=\tau_{1}\right\}\right) \cap\{r \leq 2 R\}} \sum_{j=0}^{m}\left|\nabla_{g}^{j} \varphi\right|_{h}^{2} \\
& \quad+\left|\int_{\partial_{\text {tim }} \mathcal{M} \cap\left\{\tau_{1} \leq \bar{t} \leq \tau_{2}\right\}} J_{\mu}^{m}[\varphi] n_{\partial_{\text {tim }} \mathcal{M}}^{\mu}\right| .
\end{aligned}
$$

Adding to (8.41) the integrated local energy decay statement (8.20), and using Lemma 4.5, we obtain:

$$
\begin{aligned}
& \int_{\mathcal{H}^{+}\left(\tau_{1}, \tau_{2}\right)} J_{\mu}^{m}[\varphi] n_{\mathcal{H}}^{\mu}+\int_{\left\{\bar{t}=\tau_{2}\right\}} J_{\mu}^{m}[\varphi] \bar{n}^{\mu}+\int_{\left\{\tau_{1} \leq \bar{t} \leq \tau_{2}\right\} \cap\{r \leq 2 R\}} \sum_{j=0}^{m}\left|\nabla_{g}^{j} \varphi\right|_{h}^{2} d h \\
& \quad+\int_{\partial_{t i m} \mathcal{M} \cap\left\{\tau_{1} \leq \bar{t} \leq \tau_{2}\right\}} \sum_{j=0}^{m}\left|\nabla_{g}^{j} \varphi\right|_{h}^{2} d h_{\partial_{t i m} \mathcal{M}} \\
& \leq C_{m, R} \int_{\left\{\bar{t}=\tau_{1}\right\}} J_{\mu}^{m+k}[\varphi] \bar{n}^{\mu}+\sum_{j=0}^{m-1} \sum_{j_{1}+j_{2}=j} \int_{\left\{\tau_{1} \leq \bar{t} \leq \tau_{2}\right\} \cap\{r \geq 2 R\}} r_{+}^{-1}\left(\left|\nabla_{h_{N}}^{j_{1}+1}\left(N^{j_{2}} \varphi\right)\right|_{h_{N}}^{2}\right. \\
& \left.\quad+r_{+}^{-2}\left|N^{j+1} \varphi\right|^{2}+r_{+}^{-2}|\varphi|^{2}\right) d h+C_{m, R} \sum_{j=0}^{m+k-1} \int_{\left\{\tau_{1} \leq \bar{t} \leq \tau_{2}\right\}} r_{+}^{1+\eta}\left|\nabla_{g}^{j} F\right|_{h}^{2} d h .
\end{aligned}
$$

In view of the assumptions on $N_{1}, N$ (it is here that we make use of the fact that their span is transversal to $\mathcal{H}^{+} \cap\{\bar{t}=$ const $\}$ ), as well Assumption (G11) on the uniformity of the elliptic estimates on sections of $\mathcal{H}^{+}$(note that Theorem 7.1 applies 
to yield $\lim \sup _{r \rightarrow+\infty}\left|r^{\frac{d-1}{2}+j_{1}} \nabla_{h_{\tau, N}}^{j_{1}}\left(N^{j_{2}} \varphi\right)\right|_{h_{\tau, N}}<+\infty$ for all $\left.j_{1}+j_{2} \leq m+k\right)$, we can bound:

$$
\begin{aligned}
& \int_{\mathcal{H}^{+}\left(\tau_{1}, \tau_{2}\right)} J_{\mu}^{m}[\varphi] n_{\mathcal{H}}^{\mu}+\sum_{j=0}^{m-2} \int_{\mathcal{H}^{+}\left(\tau_{1}, \tau_{2}\right)}\left|\nabla_{g}^{j} F\right|_{h} d h_{\mathcal{H}} \\
& \geq c_{m} \cdot \sum_{j=0}^{m-1} \sum_{j_{1}+j_{2}=j} \int_{\mathcal{H}^{+}\left(\tau_{1}, \tau_{2}\right)}\left|\nabla_{h_{\mathcal{H}}}^{j_{1}+1}\left(N^{j_{2}} \varphi\right)\right|_{h_{\mathcal{H}}}^{2} d h_{\mathcal{H}}
\end{aligned}
$$

Thus, from (8.42) and (8.43), as well as a trace theorem on the horizon, we obtain:

$$
\begin{aligned}
& \sum_{j=0}^{m-1} \sum_{j_{1}+j_{2}=j} \int_{\mathcal{H}^{+}\left(\tau_{1}, \tau_{2}\right)}\left(\left|\nabla_{h_{\mathcal{H}}}^{j_{1}+1}\left(N^{j_{2}} \varphi\right)\right|_{h_{\mathcal{H}}}^{2}+|\varphi|^{2}\right) d h_{\mathcal{H}}+\int_{\left\{\bar{t}=\tau_{2}\right\}} J_{\mu}^{m}[\varphi] \bar{n}^{\mu} \\
& \quad+\int_{\left\{\tau_{1} \leq \bar{t} \leq \tau_{2}\right\} \cap\{r \leq 2 R\}} \sum_{j=0}^{m}\left|\nabla_{g}^{j} \varphi\right|_{h}^{2} d h+\int_{\partial_{t i m} \mathcal{M} \cap\left\{\tau_{1} \leq \bar{t} \leq \tau_{2}\right\}} \sum_{j=0}^{m}\left|\nabla_{g}^{j} \varphi\right|_{h}^{2} d h_{\partial_{\text {tim }} \mathcal{M}} \\
& \leq C_{m, R} \int_{\left\{\bar{t}=\tau_{1}\right\}} J_{\mu}^{m+k}[\varphi] \bar{n}^{\mu}+\sum_{j=0}^{m-1} \sum_{j_{1}+j_{2}=j} \int_{\left\{\tau_{1} \leq \bar{t} \leq \tau_{2}\right\} \cap\{r \geq R\}} r_{+}^{-1} \\
& \quad \times\left(\left|\nabla_{h_{N}}^{j_{1}+1}\left(N^{j_{2}} \varphi\right)\right|_{h_{N}}^{2}+r_{+}^{-2}\left|N^{j+1} \varphi\right|^{2}+r_{+}^{-2}|\varphi|^{2}\right) d h \\
& \quad+C_{m, R} \sum_{j=0}^{m-2} \int_{\mathcal{H}^{+}\left(\tau_{1}, \tau_{2}\right)}\left|\nabla_{g}^{j} F\right|_{h} d h_{\mathcal{H}} .
\end{aligned}
$$

Moreover, using Assumptions (G10), (G11) and (G12) on the uniformity of elliptic estimates and trace inequalities, we can bound:

$$
\begin{aligned}
& \sum_{j=0}^{m-1} \sum_{j_{1}+j_{2}=j} \int_{\left\{\bar{t}=\tau_{2}\right\}}\left(\left|\nabla_{h_{N}}^{j_{1}+1}\left(N^{j_{2}} \varphi\right)\right|_{h_{N}}^{2}+r_{+}^{-2}\left|N^{j+1} \varphi\right|^{2}\right) d h_{N} \leq C_{m}\left(\int_{\left\{\bar{t}=\tau_{2}\right\}} J_{\mu}^{m}[\varphi] \bar{n}^{\mu}\right. \\
& \quad+\sum_{j=0}^{m-1} \sum_{j_{1}+j_{2}=j} \int_{\mathcal{H}^{+}\left(\tau_{1}, \tau_{2}\right)}\left(\left|\nabla_{h_{\mathcal{H}}}^{j_{1}+1}\left(N^{j_{2}} \varphi\right)\right|_{h_{\mathcal{H}}}^{2}+|\varphi|^{2}\right) d h_{\mathcal{H}} \\
& \left.\quad+\int_{\partial_{\text {tim }} \mathcal{M} \cap\left\{\tau_{1} \leq \bar{t} \leq \tau_{2}\right\}} \sum_{j=0}^{m}\left|\nabla_{g}^{j} \varphi\right|_{h}^{2} d h_{\partial_{t i m} \mathcal{M}}+\sum_{j=0}^{m-2} \int_{\left\{\bar{t}=\tau_{2}\right\}}\left|\nabla_{g}^{j} F\right|_{h}^{2} d h_{N}\right)
\end{aligned}
$$

Thus, using (8.44) and (8.45) and a trace theorem for the terms

$$
\sum_{j=0}^{m-2}\left(\int_{\left\{\bar{t}=\tau_{2}\right\}}\left|\nabla_{g}^{j} F\right|_{h}^{2} d h_{N}+\int_{\mathcal{H}^{+}\left(\tau_{1}, \tau_{2}\right)}\left|\nabla_{g}^{j} F\right|_{h} d h_{\mathcal{H}}\right),
$$


we deduce the required energy boundedness estimate:

$$
\begin{aligned}
& \sum_{j=0}^{m-1} \sum_{j_{1}+j_{2}=j} \int_{\mathcal{H}^{+}\left(\tau_{1}, \tau_{2}\right)}\left(\left|\nabla_{h_{\mathcal{H}}}^{j_{1}+1}\left(N^{j_{2}} \varphi\right)\right|_{h_{\mathcal{H}}}^{2}\right. \\
& \left.+|\varphi|^{2}\right) d h_{\mathcal{H}}+\sum_{j=0}^{m-1} \sum_{j_{1}+j_{2}=j} \int_{\left\{\bar{t}=\tau_{2}\right\}}\left(\left|\nabla_{h_{N}}^{j_{1}+1}\left(N^{j_{2}} \varphi\right)\right|_{h_{N}}^{2}+r_{+}^{-2}\left|N^{j+1} \varphi\right|^{2}\right) d h_{N} \\
& +\int_{\partial_{t i m} \mathcal{M} \cap\left\{\tau_{1} \leq \bar{t} \leq \tau_{2}\right\}} \sum_{j=0}^{m}\left|\nabla_{g}^{j} \varphi\right|_{h}^{2} d h_{\partial_{t i m} \mathcal{M}} \\
& \leq C_{m, R} \sum_{j=0}^{m+k-1} \sum_{j_{1}+j_{2}=j} \int_{\left\{\bar{t}=\tau_{1}\right\}}\left(\left|\nabla_{h_{N}}^{j_{1}+1}\left(N^{j_{2}} \varphi\right)\right|_{h_{N}}^{2}+r_{+}^{-2}\left|N^{j+1} \varphi\right|^{2}\right) d h_{N} \\
& \quad+\sum_{j=0}^{m-1} \sum_{j_{1}+j_{2}=j} \int_{\left\{\tau_{1} \leq \bar{t} \leq \tau_{2}\right\} \cap\{r \geq R\}} r^{-1} \\
& \quad \times\left(\left|\nabla_{h_{N}}^{j_{1}+1}\left(N^{j_{2}} \varphi\right)\right|_{h_{N}}^{2}+r_{+}^{-2}\left|N^{j+1} \varphi\right|^{2}+r_{+}^{-2}|\varphi|^{2}\right) d h \\
& \quad+C_{m, R} \sum_{j=0}^{m+k-1} \int_{\left\{\tau_{1} \leq \bar{t} \leq \tau_{2}\right\}} r_{+}^{1+\eta}\left|\nabla_{g}^{j} F\right|_{h}^{2} d h .
\end{aligned}
$$

\subsection{Proof of Theorem 8.1}

We will assume without loss of generality that $\varphi$ is real valued. We will also set $\Phi=\Omega \varphi$.

Fix an $R>0$ large enough in terms of the geometry of $(\mathcal{M}, g)$. Fix also a smooth cut-off $\chi_{R}: \mathcal{M} \rightarrow[0,1]$ which is only a function of $r$, such that $\chi_{R} \equiv 0$ on $\{r \leq R\}$ and $\chi_{R} \equiv 1$ on $\{r \geq R+1\}$. Fix also a small number $0<\delta \ll 1$.

We will use the following notations for the $r^{p}$-weighted energy norms for any $\tau \geq 0$ :

$$
\begin{aligned}
& \mathcal{E}_{\text {bound }}^{(p, m)}[\varphi](\tau) \\
& =\sum_{j=0}^{m} \int_{\{\bar{t}=\tau\} \cap\{r \leq R+1\}}\left|\nabla_{h_{\tau, N}}^{j_{1}}\left(N^{j_{2}} \varphi\right)\right|_{h_{\tau, N}}^{2} d h_{N} \\
& +\sum_{\substack{\text { components } \\
\text { of } \mathcal{N}_{\text {af }, \mathcal{M}}}} \sum_{j=0}^{m-1} \sum_{j_{1}+j_{2}+j_{3}=j} \mathcal{E}_{\text {bound }, R ; \delta}^{(p, 0)}\left[r^{-j_{2}} \partial_{v}^{j_{1}} \partial_{\sigma}^{j_{2}} \partial_{u}^{j_{3}} \varphi\right](\tau),
\end{aligned}
$$




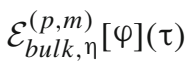

$$
\begin{aligned}
& =\sum_{j=0}^{m} \int_{\{\bar{t}=\tau\} \cap\{r \leq R+1\}}\left|\nabla_{h_{\tau, N}}^{j_{1}} N^{j_{2}} \varphi\right|_{h_{\tau, N}}^{2} d h_{N} \\
& +\sum_{j=1}^{m} \sum_{j_{1}+j_{2}=j-1} \int_{\{\bar{t}=\tau\} \cap \mathcal{H}}\left|\nabla_{h_{\mathcal{H}}}^{j_{1}+1}\left(N^{j_{2}} \varphi\right)\right|_{h_{\mathcal{H}}}^{2} d h_{\mathcal{H}_{\tau}} \\
& +\sum_{j=0}^{m} \int_{\{\bar{t}=\tau\} \cap \partial_{t i m} \mathcal{M}}\left|\nabla_{g}^{j} \varphi\right|_{h}^{2} d h_{\partial_{t i m} \mathcal{M}, \tau} \\
& +\sum_{\substack{\text { components } \\
\text { of } \mathcal{N}_{a f, \mathcal{M}}}} \sum_{j=0}^{m-1} \sum_{j_{1}+j_{2}+j_{3}=j} \mathcal{E}_{\text {bulk,R, } \eta ; \delta}^{(p, 0)}\left[r^{-j_{2}} \partial_{v}^{j_{1}} \partial_{\sigma}^{j_{2}} \partial_{u}^{j_{3}} \varphi\right](\tau)
\end{aligned}
$$

and

$$
\begin{aligned}
& \mathcal{E}_{\text {en }}^{(m)}[\varphi](\tau)=\sum_{j=1}^{m} \sum_{j_{1}+j_{2}=j-1} \int_{\{\bar{t}=\tau\}}\left(\left|\nabla_{h_{\tau, N}}^{j_{1}+1}\left(N^{j_{3}} \varphi\right)\right|_{h_{\tau, N}}^{2}\right. \\
& \left.+r_{+}^{-2}\left(\left|N^{j} \varphi\right|^{2}+|\varphi|^{2}\right)\right) d h_{N} .
\end{aligned}
$$

We will also use the norm

$$
\mathcal{F}_{\eta}^{(p, m)}[\varphi](\tau)=\sum_{j=0}^{m-1} \int_{\{\bar{t}=\tau\}}\left(r^{p+1}+r^{1+\eta}\right)\left|\nabla_{g}^{j}(\square \varphi)\right|_{h}^{2} d h_{N}
$$

Using Corollary 5.2 and Lemma 8.3, we can bound for any integer $m \geq 0$, any $0 \leq \tau_{1} \leq \tau_{2}$ and any $0<p \leq 2$ :

$$
\begin{aligned}
& \mathcal{E}_{\text {bound }}^{(p, m)}[\varphi]\left(\tau_{2}\right)+\int_{\tau_{1}}^{\tau_{2}} \mathcal{E}_{\text {bulk, }, \eta}^{(p-1, m)}[\varphi](\tau) d \tau \\
& \lesssim_{p, m, \eta} \mathcal{E}_{\text {bound }}^{(p, m+k)}[\varphi]\left(\tau_{1}\right)+\int_{\tau_{1}}^{\tau_{2}} \mathcal{F}_{\eta}^{(p, m+k)}[\varphi](\tau) d \tau .
\end{aligned}
$$

Starting from (8.51) for $\tau_{1}=0$ and letting $\tau_{2} \rightarrow+\infty$, we obtain for any $m \in \mathbb{N}$ and any $T \geq 0$ :

$$
\int_{0}^{T} \mathcal{E}_{\text {bulk, },[\varphi]}^{(1, m)}[\varphi](\tau) d \tau \lesssim m, \eta \mathcal{E}_{\text {bound }}^{(2, m+k)}[\varphi](0)+\int_{0}^{T} \mathcal{F}_{\eta}^{(2, m+k)}[\varphi](\tau) d \tau .
$$

An application of the pigeonhole principle on (8.52) readily yields that there exists a sequence of positive numbers $\left\{\tau_{n}\right\}_{n \in \mathbb{N}}$ with $\tau_{0} \geq 1$ and $2 \tau_{n} \leq \tau_{n+1} \leq 4 \tau_{n}$ such that

$$
\mathcal{E}_{\text {bulk, },[}^{(1, m)}[\varphi]\left(\tau_{n}\right) \lesssim_{m, \eta} \tau_{n}^{-1}\left(\mathcal{E}_{\text {bound }}^{(2, m+k)}[\varphi](0)+\int_{0}^{\tau_{n}} \mathcal{F}_{\eta}^{(2, m+k)}[\varphi](\tau) d \tau\right) .
$$


Applying (8.51) in the intevals $\left\{\tau_{n} \leq \bar{t} \leq \tau_{n+1}\right\}$ for $p=1$ and using (8.53) for $m+k$ in place of $m$ to bound the first term of the right hand side, ${ }^{16}$ we obtain for any $\bar{\tau} \in\left[\tau_{n}, \tau_{n+1}\right]:$

$$
\begin{aligned}
& \int_{\tau_{n}}^{\bar{\tau}} \mathcal{E}_{\text {bulk, },}^{(0, m)}[\varphi](\tau) d \tau \lesssim m, \eta \tau_{n}^{-1}\left(\mathcal{E}_{\text {bound }}^{(2, m+2 k)}[\varphi](0)\right. \\
& \left.+\int_{0}^{\tau_{n}} \mathcal{F}_{\eta}^{(2, m+2 k)}[\varphi](\tau) d \tau\right)+\int_{\tau_{n}}^{\bar{\tau}} \mathcal{F}_{\eta}^{(1, m+k)}[\varphi](\tau) d \tau .
\end{aligned}
$$

Using the mean value theorem on the intervals $\left\{\tau_{n} \leq \bar{t} \leq \tau_{n+1}\right\}$, we thus obtain on a possibly different sequence $\bar{\tau}_{n} \in\left[\tau_{n}+\frac{\tau_{n+1}-\tau_{n}}{4}, \tau_{n+1}-\frac{\tau_{n+1}-\tau_{n}}{4}\right]$ :

$$
\begin{aligned}
& \mathcal{E}_{\text {bulk, },[}^{(0, m)}[\varphi]\left(\bar{\tau}_{n}\right) \lesssim m, \eta \bar{\tau}_{n}^{-2}\left(\mathcal{E}_{\text {bound }}^{(2, m+2 k)}[\varphi](0)+\int_{0}^{\bar{\tau}_{n}} \mathcal{F}_{\eta}^{(2, m+2 k)}[\varphi](\tau) d \tau\right) \\
& +\bar{\tau}_{n}^{-1} \int_{\frac{1}{4} \bar{\tau}_{n}}^{\bar{\tau}_{n}} \mathcal{F}_{\eta}^{(1, m+k)}[\varphi](\tau) d \tau
\end{aligned}
$$

Notice that by interpolating between (8.55) for $p=2$ on the intervals $\left\{\bar{\tau}_{n} \leq \bar{t} \leq\right.$ $\left.\bar{\tau}_{n+1}\right\}$, we can also bound:

$$
\begin{gathered}
\mathcal{E}_{\text {bound }, \eta}^{(\varepsilon, m)}[\varphi]\left(\bar{\tau}_{n}\right) \lesssim{ }_{m, \varepsilon} \bar{\tau}_{n}^{-2+\varepsilon}\left(\mathcal{E}_{\text {bound }}^{(2, m+2 k)}[\varphi](0)+\int_{0}^{\bar{\tau}_{n}} \mathcal{F}_{\eta}^{(2, m+2 k)}[\varphi](\tau) d \tau\right) \\
+\bar{\tau}_{n}^{-1+\varepsilon} \int_{\frac{1}{4} \bar{\tau}_{n}}^{\bar{\tau}_{n}} \mathcal{F}_{\eta}^{(1, m+k)}[\varphi](\tau) d \tau
\end{gathered}
$$

Applying (8.51) for $p=\varepsilon$ on the intervals $\left\{\bar{\tau}_{n} \leq \bar{t} \leq \bar{\tau}_{n+1}\right\}$ and using (8.56), we obtain for any $\bar{\tau} \in\left[\bar{\tau}_{n}, \bar{\tau}_{n+1}\right]$ :

$$
\begin{aligned}
\int_{\bar{\tau}_{n}}^{\bar{\tau}} \mathcal{E}_{\text {bulk, }}^{(-1+\varepsilon, m)}[\varphi](\tau) d \tau \lesssim & \bar{\tau}_{m, \varepsilon, \eta} \bar{\tau}_{n}^{-2+\varepsilon}\left(\mathcal{E}_{\text {bound }}^{(2, m+2 k)}[\varphi](0)+\int_{0}^{\bar{\tau}_{n}} \mathcal{F}_{\eta}^{(2, m+2 k)}[\varphi](\tau) d \tau\right) \\
& +\bar{\tau}_{n}^{-1+\varepsilon} \int_{\frac{1}{4} \bar{\tau}_{n}}^{\bar{\tau}_{n}} \mathcal{F}_{\eta}^{(1, m+k)}[\varphi](\tau) d \tau \\
& +\int_{\bar{\tau}_{n}}^{\bar{\tau}} \mathcal{F}_{\eta}^{(\varepsilon, m+k)}[\varphi](\tau) d \tau
\end{aligned}
$$

Using the fact that

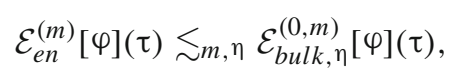

$\overline{16 \text { Notice the trivial inequality } \mathcal{E}_{\text {bound }}^{(p, m)}[\varphi](\tau) \lesssim p, m, \eta \mathcal{E}_{b u l k, \eta}^{(p, m)}[\varphi](\tau)}$. 
by applying Lemma 8.3 on the intervals $\left\{\bar{\tau}_{n} \leq \bar{t} \leq \bar{\tau}_{n+1}\right\}$ and using (8.55) and (8.57) (for $m+k$ in place of $k$ ), we obtain (8.23) for some $C>1$ and any $\tau \geq 1$ in view of the fact that $\frac{5}{4} \bar{\tau}_{n} \leq \bar{\tau}_{n+1} \leq 8 \bar{\tau}_{n}$ :

$$
\begin{aligned}
\mathcal{E}_{\text {en }}^{(m)}[\varphi](\tau) \lesssim & m, \varepsilon, \eta \tau^{-2+\varepsilon}\left(\mathcal{E}_{\text {bound }}^{(2, m+3 k)}[\varphi](0)+\int_{0}^{\tau} \mathcal{F}_{\eta}^{(2, m+3 k)}[\varphi](\tau) d \tau\right) \\
& +\tau^{-1+\varepsilon} \int_{C^{-1} \tau}^{\tau} \mathcal{F}_{\eta}^{(1, m+2 k)}[\varphi](\tau) d \tau+\int_{C^{-1} \tau}^{\tau} \mathcal{F}_{\eta}^{(\varepsilon, m+2 k)}[\varphi](\tau) d \tau
\end{aligned}
$$

In view of Assumption (G13), we obtain from (8.59) for any integer $m \geq 0$ and any $\tau \geq 0$ using the Sobolev embedding theorem:

$$
\begin{aligned}
& \sup _{\{\bar{t}=\tau\}}\left|\nabla^{m} \varphi\right|_{h}^{2} \\
& \lesssim m, \varepsilon, \eta \tau^{-2+\varepsilon}\left(\mathcal{E}_{\text {bound }}^{\left(2, m+\left\lceil\frac{d+1}{2}\right\rceil+3 k\right)}[\varphi](0)+\int_{0}^{\tau} \mathcal{F}_{\eta}^{\left(2, m+\left\lceil\frac{d+1}{2}\right\rceil+3 k\right)}[\varphi](\tau) d \tau\right) \\
& \quad+\tau^{-1+\varepsilon} \int_{C^{-1} \tau}^{\tau} \mathcal{F}_{\eta}^{\left(1, m+\left\lceil\frac{d+1}{2}\right\rceil+2 k\right)}[\varphi](\tau) d \tau+\int_{C^{-1} \tau}^{\tau} \mathcal{F}_{\eta}^{\left(\varepsilon, m+\left\lceil\frac{d+1}{2}\right\rceil+2 k\right)}[\varphi](\tau) d \tau .
\end{aligned}
$$

Using the fundamental theorem of calculus and applying the product rule for derivatives, we can bound for any function $\Psi$ on $\mathcal{M}$ and any $1 \ll R_{1}<R_{2}$ :

$$
\begin{aligned}
\int_{\{\bar{t}=\tau\} \cap\left\{r=R_{2}\right\}}\left|r^{\frac{d-2}{2}} \Psi\right|^{2} d \sigma \lesssim & \int_{\{\bar{t}=\tau\} \cap\left\{r=R_{1}\right\}}\left|r^{\frac{d-2}{2}} \Psi\right|^{2} d \sigma \\
& +\int_{\{\bar{t}=\tau\} \cap\left\{R_{1} \leq r \leq R_{2}\right\}}\left|r^{\frac{d-3}{2}} \Psi\right|\left(\left|\partial_{v}\left(r^{\frac{d-1}{2}} \Psi\right)\right|\right. \\
& \left.+r^{-2}\left|\partial_{u}\left(r^{\frac{d-1}{2}} \Psi\right)\right|+\left|r^{\frac{d-3}{2}} \Psi\right|\right) d v d \sigma
\end{aligned}
$$

Hence, using a Sobolev inequality on $\mathbb{S}^{d-1}$, a trace inequality for the first term of the right hand side of (8.61) and a Cauchy-Schwarz inequality for the second term, we infer from (8.61) for any integer $m \geq 0$ :

$$
\sup _{\{\bar{t}=\tau\}}\left|r_{+}^{\frac{d-2}{2}} \nabla_{g}^{m} \varphi\right|_{h}^{2} \lesssim m \mathcal{E}_{e n}^{\left(m+\left\lceil\frac{d+2}{2}\right\rceil\right)}[\varphi](\tau) .
$$

Thus, (8.59) yields:

$$
\begin{aligned}
& \sup _{\{\bar{t}=\tau\}}\left|r_{+}^{\frac{d-2}{2}} \nabla_{g}^{m} \varphi\right|_{h}^{2} \lesssim_{m, \varepsilon, \eta} \tau^{-2+\varepsilon}\left(\mathcal{E}_{\text {bound }}^{\left(2, m+\left\lceil\frac{d+2}{2}\right\rceil+3 k\right)}[\varphi](0)\right. \\
& \left.\quad+\int_{0}^{\tau} \mathcal{F}_{\eta}^{\left(2, m+\left\lceil\frac{d+2}{2}\right\rceil+3 k\right)}[\varphi](\tau) d \tau\right)
\end{aligned}
$$




$$
\begin{aligned}
& +\tau^{-1+\varepsilon} \int_{C^{-1} \tau}^{\tau} \mathcal{F}_{\eta}^{\left(1, m+\left\lceil\frac{d+2}{2}\right\rceil+2 k\right)}[\varphi](\tau) d \tau \\
& +\int_{C^{-1} \tau}^{\tau} \mathcal{F}_{\eta}^{\left(\varepsilon, m+\left\lceil\frac{d+2}{2}\right\rceil+2 k\right)}[\varphi](\tau) d \tau .
\end{aligned}
$$

Using (8.53) and (8.51) for $p=1$ on the intervals $\left\{\tau_{n} \leq \bar{t} \leq \tau_{n+1}\right\}$, we can bound for any integer $m \geq 0$ and any $\tau \geq 0$ (for some fixed $C>1$ ):

$$
\begin{aligned}
\mathcal{E}_{\text {bound }}^{(1, m)}[\varphi](\tau) \lesssim & m, \eta \tau^{-1}\left(\mathcal{E}_{\text {bound }}^{(2, m+2 k)}[\varphi](0)+\int_{0}^{\tau} \mathcal{F}_{\eta}^{(2, m+2 k)}[\varphi](\tau) d \tau\right) \\
& +\int_{C^{-1} \tau}^{\tau} \mathcal{F}_{\eta}^{(1, m+2 k)}[\varphi](\tau) d \tau .
\end{aligned}
$$

Again, using the fundamental theorem of calculus we can bound for any any function $\Psi$ on $\mathcal{M}$ and any $1 \ll R_{1}<R_{2}$ :

$$
\begin{aligned}
& \int_{\{\bar{t}=\tau\} \cap\left\{r=R_{2}\right\}}\left|r^{\frac{d-1}{2}} \Psi\right|^{2} d \sigma \lesssim \int_{\{\bar{t}=\tau\} \cap\left\{r=R_{1}\right\}}\left|r^{\frac{d-1}{2}} \Psi\right|^{2} d \sigma \\
& \quad+\int_{\{\bar{t}=\tau\} \cap\left\{R_{1} \leq r \leq R_{2}\right\}}\left|r^{\frac{d-1}{2}} \Psi\right|\left(\left|\partial_{v}\left(r^{\frac{d-1}{2}} \Psi\right)\right|+r^{-2}\left|\partial_{u}\left(r^{\frac{d-1}{2}} \Psi\right)\right|\right) d v d \sigma
\end{aligned}
$$

and thus from (8.64), (8.65) and a Sobolev inequality on $\mathbb{S}^{d-1}$, we obtain the desired decay rate for $r^{\frac{d-1}{2}} \varphi$ :

$$
\begin{aligned}
& \sup _{\{\bar{t}=\tau\}}\left|r_{+}^{\frac{d-1}{2}} \nabla_{g}^{m} \varphi\right|_{h}^{2} \\
& \lesssim_{m, \eta} \tau^{-1}\left(\mathcal{E}_{\text {bound }}^{\left(2, m+\left\lceil\frac{d+2}{2}\right\rceil+2 k\right)}[\varphi](0)+\int_{0}^{\tau} \mathcal{F}_{\eta}^{\left(2, m+\left\lceil\frac{d+2}{2}\right\rceil+2 k\right)}[\varphi](\tau) d \tau\right) \\
& \quad+\int_{C^{-1} \tau}^{\tau} \mathcal{F}_{\eta}^{\left(1, m+\left\lceil\frac{d+2}{2}\right\rceil+2 k\right)}[\varphi](\tau) d \tau .
\end{aligned}
$$

\section{Improved Polynomial Decay $\bar{t}^{-\frac{d}{2}}$ for Solutions to $\square_{g} \varphi=0$ in Dimensions $d \geq 3$}

In this section, we will establish $\bar{t}^{-\frac{d}{2}}$ polynmial decay estimates for solutions $\varphi$ to $\square \varphi=0$ on spacetimes $\left(\mathcal{M}^{d+1}, g\right), d \geq 3$, satisfying Assumptions (G1)-(G13) and (ILED1), which in addition possess two "almost Killing" vector fields $T, K$ (not necessarily distinct) with timelike span on $\mathcal{M} \backslash \mathcal{H}^{+}$and for which $\mathcal{H}^{+}$becomes a nondegenerate "almost Killing" horizon. These estimates extend the $\bar{t}^{-\frac{3}{2}+\varepsilon}$ decay rate established in the region $\{r \lesssim 1\}$ of Schwarzschild spacetime by Schlue in [34]. 


\subsection{Further Assumptions and Geometric Constructions on $(\mathcal{M}, g)$}

Let $\left(\mathcal{M}^{d+1}, g\right), d \geq 3$, be a smooth Lorentzian manifold with possibly non-empty piecewise smooth boundary $\partial \mathcal{M}$. We assume that $(\mathcal{M}, g)$ satisfies the geometric assumptions (G1)-(G13) stated in Sections 7 and 8, as well as Assumption (ILED1) on integrated local energy decay with loss of derivatives. We will use the same notation as in Section 8 for the subsets $\mathcal{N}_{a f, \mathcal{M}}, \mathcal{H}, \mathcal{H}^{+}, \partial_{\text {tim }} \mathcal{M}, \mathcal{H}_{\tau}, \partial_{\text {tim }} \mathcal{M}^{\tau}$ of $\mathcal{M}$, the functions $r, r_{+}, \bar{t}$ on $\mathcal{M}$ and the vectorfields $N, T, Y$.

Recall that in the coordinate chart $(u, v, \sigma)$ on each connected component of the region $\{r \gg 1\}$, the metric $g$ takes the form:

$$
\begin{aligned}
g= & -\left(4+O\left(r^{-1-a}\right)\right) d v d u+r^{2} \cdot\left(g_{\mathbb{S}^{d-1}}+h_{\mathbb{S}^{d-1}}\right)+\left(h^{a s}(u, \sigma)+O\left(r^{-a}\right)\right) d u d \sigma \\
& +O\left(r^{-a}\right) d v d \sigma+4\left(-\frac{2 M(u, \sigma)}{r}+O\left(r^{-1-a}\right)\right) d u^{2}+O\left(r^{-2-a}\right) d v^{2},
\end{aligned}
$$

with $h_{\mathbb{S} d-1}=O\left(r^{-1}\right)$.

Assumptions on the vector fields $T, K$ We will also assume that $(\mathcal{M}, g)$ possesses two smooth vector fields, $T$ and $K$, not necessarily distinct, such that:

(EG1) The following equality holds: $d \bar{t}(K)=d \bar{t}(T)=1$

(EG2) In the region $\{r \gg 1\}, T$ is as in Section 7 and $K=\Phi+T$, where $\Phi$ is a generator of a rotation of $\mathbb{S}^{d-1}$ (possibly being identically 0 ).

(EG3) The span of $T$ and $K$ everywhere on $\mathcal{M} \backslash \mathcal{H}$ contains a timelike direction.

(EG4) The span of $\{T, K\}$ is tangential to $\mathcal{H}$. Moreover, $\mathcal{H}^{+}$is non degenerate with respect to $K$, in the sense that $g(K, K)=0$ and $d(g(K, K)) \neq 0$ on $\mathcal{H}^{+} \cap$ $\{\bar{t} \geq 0\}$ and the following red-shift type estimate holds for some $r_{1}>0$, any $0 \leq \tau_{1} \leq \tau_{2}$, any $l \in \mathbb{N}$ and any $\varphi \in C^{\infty}(\mathcal{M})$ (see also [9]):

$$
\begin{aligned}
& \sum_{j=1}^{l} \int_{\left\{\tau_{1} \leq \bar{t} \leq \tau_{2}\right\} \cap\left\{r \leq r_{1}\right\}}\left|\nabla^{j} \varphi\right|_{h}^{2} d g \leq C_{l}\left\{\sum_{j=1}^{l} \int_{\left\{\bar{t}=\tau_{1}\right\} \cap\left\{r \leq 2 r_{1}\right\}}\left|\nabla^{j} \varphi\right|_{h}^{2} d h_{N}\right. \\
& \quad+\sum_{j=1}^{l} \int_{\left\{\tau_{1} \leq \bar{t} \leq \tau_{2}\right\} \cap\left\{r_{1} \leq r \leq 2 r_{1}\right\}}\left|\nabla^{j} \varphi\right|_{h}^{2} d g \\
& \left.\quad+\sum_{j=0}^{l-1} \int_{\left\{\tau_{1} \leq \bar{t} \leq \tau_{2}\right\} \cap\left\{r \leq 2 r_{1}\right\}}\left|\nabla^{j}(\square \varphi)\right|_{h}^{2} d g\right\} .
\end{aligned}
$$

Convention Since we have not assumed that $T, K$ commute in the near region $\{r \lesssim 1\}$, iterated Lie derivatives in the directions of $T, K$ will not necessarily commute. Hence, it will be useful to introduce the following pointwise norms for smooth tensors $\mathfrak{m}$ on $\mathcal{M}$ for any $l \in \mathbb{N}$ and any two vector fields $X^{(0)}, X^{(1)}$ : 


$$
|\mathfrak{m}|_{X^{(0)}, X^{(1)}}^{(l)} \doteq\left(\sum_{\left(e_{1}, \ldots e_{d}\right) \in\{0,1\}^{l}}\left|\mathcal{L}_{\left.X^{\left(e_{1}\right.}\right)} \ldots \mathcal{L}_{X^{\left(e_{d}\right)}} \mathfrak{m}\right|_{h}^{2}\right)^{1 / 2}
$$

and for any $l, n \in \mathbb{N}$ :

$$
|\mathfrak{m}|_{X^{(0)}, X^{(1)}}^{(l ; n)} \doteq\left(\sum_{j=0}^{n} \sum_{\left(e_{1}, \ldots e_{d}\right) \in\{0,1\}^{l}}\left|\nabla_{g}^{j}\left(\mathcal{L}_{\left.X^{\left(e_{1}\right.}\right)} \ldots \mathcal{L}_{\left.X^{\left(e_{d}\right.}\right)} \mathfrak{m}\right)\right|_{h}^{2}\right)^{1 / 2}
$$

(EG5) We assume that we can bound for some small $\delta_{0}>0$, any pair of integers $j_{1}, j_{2} \geq 0$ and any $\tau \geq 0$ :

$$
\sup _{\{\bar{t}=\tau\}}|g|_{T, K}^{\left(j_{1} ; j_{2}\right)} \lesssim j_{1}, j_{2}(1+\tau)^{-\max \left\{0, j_{1}+\delta_{0}-1\right\}} .
$$

Moreover, in the $(u, v, \sigma)$ coordinate chart on each connected component of the region $\{r \gg 1\}$ the following precise bounds on the derivatives of $g$ are assumed to hold for $j_{1} \geq 1$ and any $\left(e_{1}, \ldots, e_{j_{1}}\right) \in\{0,1\}^{j_{1}}$ :

$$
\begin{aligned}
& \mathcal{L}_{\left.X^{\left(e_{1}\right.}\right)} \ldots \mathcal{L}_{X^{\left(e_{1}\right)}} g \\
& =O\left(\bar{t}^{-\left(j_{1}+\delta_{0}-1\right)}\right)\left\{O\left(r^{-1-a}\right) d v d u+O(r) d \sigma d \sigma+O(1) d u d \sigma\right. \\
& \left.\quad+O\left(r^{-a}\right) d v d \sigma+O\left(r^{-1}\right) d u^{2}+O\left(r^{-2-a}\right) d v^{2}\right\}
\end{aligned}
$$

where $X^{(0)}=T$ and $X^{(1)}=K$.

Remark For any $m_{0} \in \mathbb{N}$ and $\delta_{0} \in(0,1)$, inequalities (9.5) and (9.6) can be relaxed to hold only for $j_{1}, j_{2}$ less than some large constant $M=M\left(m_{0}, \delta_{0}\right) \in \mathbb{N}$ depending on $m_{0}$, and then Theorem 9.1 will still hold provided $m$ is restricted to take values up to $m_{0}$. This fact will also apply to all the assumptions regarding estimates on the derivatives of the metric $g$ appearing in the text, and will not be highlighted again. In all the assumptions that are stated in this section, the number of derivatives of the metric $M=M\left(m_{0}, \delta_{0}\right)$ that need to appear in the related estimates can be bounded from above by

$$
m_{0}+12\left\lceil\delta_{0}^{-1} \cdot d\right\rceil d \cdot k
$$

where $k$ is the number expressing the loss of derivatives in Assumption (ILED1).

Let us define the vector field

$$
\Phi \doteq K-T
$$

on $\mathcal{M}$. Recall that $\Phi$ is a rotation vector field in the region $\{r \gg 1\}$ in view of Assumption (EG2). Notice that (9.5) and (9.6) hold for $\Phi$ in place of $K$. Moreover, due to Assumption (EG1) we have $d \bar{t}(\Phi)=0$, and thus $\Phi$ is tangent to the level sets of $\bar{t}$. 
Definition of the $K_{R_{c}}$ vector field and the $|\cdot|_{T, K, R_{c}}^{(l)}$ norm Let us introduce the (large) parameter $R_{c}>0$. This parameter will be fixed after Lemma 9.4 has been established. Fixing a smooth cut-off function $\chi:[0,+\infty) \rightarrow[0,1]$ such that $\chi \equiv 1$ on $[0,1]$ and $\chi \equiv 0$ on $[2,+\infty)$, and defining $\chi_{R_{c}}: \mathcal{M} \rightarrow[0,1]$ by the relation

$$
\chi_{R_{c}} \doteq \chi\left(\frac{r}{R_{c}}\right)
$$

we introduce the following vector field:

$$
K_{R_{c}} \doteq \chi_{R_{c}} K+\left(1-\chi_{R_{c}}\right) T
$$

Notice that for any $\tau \geq 0$, the set $\{\bar{t}=\tau\} \cap\left\{g\left(K_{R_{c}}, K_{R_{c}}\right)>0\right\}$ is precompact and its closure does not intersect the horizon.

Provided $R_{c} \gg 1$, due to (9.5) we can bound for any pair of integers $j_{1}, j_{2} \geq 0$ (using the (9.4) norm)

$$
\sup _{\{\bar{t}=\tau\} \cap\left\{r \notin\left[R_{c}, 2 R_{c}\right]\right\}}|g|_{T, K_{R_{C}}}^{\left(j_{1} ; j_{2}\right)} \lesssim_{j_{1}, j_{2}}(1+\tau)^{-\max \left\{0, j_{1}+\delta_{0}-1\right\}},
$$

while when restricted in the region $\{r \gg 1\}$, the following refined bounds hold (in the $(u, v, \sigma)$ coordinate chart on each connected component of $\{r \gg 1\})$ due to (9.6) for $j_{1} \geq 1$ and any $\left(e_{1}, \ldots, e_{j_{1}}\right) \in\{0,1\}^{j_{1}}\left(\text { with } X^{(0)}=T \text { and } X^{(1)}=K_{R_{c}}\right)^{17}$ :

- For $r \notin\left[R_{c}, 2 R_{c}\right]$ :

$$
\begin{aligned}
\mathcal{L}_{\left.X^{\left(e_{1}\right.}\right) \ldots \mathcal{L}_{X}{ }^{\left(e_{j_{1}}\right)} g=} & O\left(\tau^{-\left(j_{1}+\delta_{0}-1\right)}\right) \\
& \times\left\{O\left(r^{-1-a}\right) d v d u+O(r) d \sigma d \sigma+O(1) d u d \sigma\right. \\
& \left.+O\left(r^{-a}\right) d v d \sigma+O\left(r^{-1}\right) d u^{2}+O\left(r^{-2-a}\right) d v^{2}\right\} .
\end{aligned}
$$

- For $\left\{R_{c} \leq r \leq 2 R_{c}\right\}$ :

$$
\begin{aligned}
\mathcal{L}_{\left.X^{\left(e_{1}\right.}\right)} \ldots \mathcal{L}_{X^{\left(e_{j_{1}}\right)}} g= & \left(O\left(r^{-1}\right)+O\left(\tau^{-\left(j_{1}+\delta_{0}-1\right)}\right)\right) \\
& \times\left\{O(r) d v d u+O(r) d \sigma d \sigma+O\left(r^{2}\right) d u d \sigma\right. \\
& \left.+O\left(r^{2}\right) d v d \sigma+O(r) d u^{2}+O(r) d v^{2}\right\} .
\end{aligned}
$$

Moreover, it will be convenient to introduce the following truncated version of the pointwise norm (9.3) for any smooth tensor $k$ on $\mathcal{M}$ :

$$
|k|_{T, K, R_{c}}^{(l)} \doteq\left|\chi_{R_{c}} k\right|_{T, K}^{(l)}+\left|\mathcal{L}_{T}^{l} k\right|_{h}
$$

\footnotetext{
17 For the calculation of the Lie derivatives in the direction of $K_{R_{C}}$ it is convenient to use the formula $\mathcal{L}_{f \cdot X}(\omega)=f \mathcal{L}_{X}(\omega)+d f \cdot i_{X}(\omega)$ for any smooth function $f$, vector field $X$ and 1-form $\omega$.
} 
The Riemannian metric $h_{\tau, K_{R_{C}}, \Phi}$ on the hyperboloids $\{\bar{t}=\tau\}$ According to Section A of the Appendix, for any $\tau \geq 0$ we can define the Riemannian metric $h_{\tau, K_{R_{c}}, \Phi}$ on the hypersurfaces $\{\bar{t}=\tau\}$ associated to $K_{R_{c}}$, $\Phi$. Due to the fact that $g(K, K)=0$ and $d g(K, K) \neq 0$ on $\mathcal{H}^{+}$, for any $\tau \geq 0$ there exists some $r_{0}(\tau)>0$ such that in a neighborhood of $\mathcal{H}_{\tau}$ of the form $\left\{r \leq r_{0}(\tau)\right\} \cap\{\bar{t}=\tau\}$ the metric $h_{\tau, K_{R_{C}}}$, has the form:

$$
h_{\tau, K_{R_{c}}, \Phi}=\left(r^{-1}+O(1)\right) d r^{2}+h_{\mathcal{H}_{\tau}, r}
$$

where $h_{\mathcal{H}_{\tau}, r}$ is a symmetric $(0,2)$-tensor on $\left\{r \leq r_{0}(\tau)\right\} \cap\{\bar{t}=\tau\}$, smooth up to $\mathcal{H}_{\tau}$, satisfying $h_{\mathcal{H}_{\tau}, r}(Y, \cdot) \equiv 0$ (where the vector field $Y$ is transversal to $\mathcal{H}_{\tau}$ and was defined in Section 8.1). Hence, in the language of Section B of the Appendix, $h_{\tau, K_{R_{c}}, \Phi}$ corresponds to the singular metric $h$. For notational simplicity, from now on we will adopt the shorthand notation $h_{R_{c}}=h_{\tau, K_{R_{c}}, \Phi}$.

While $h_{R_{c}}$ will be useful to define certain elliptic operators, we will mostly measure the norms of tensors on the $\{\bar{t}=\tau\}$ hypersurfaces with the previously defined non singular metric $h_{\tau, N}$ associated to $N$.

In view of the aforementioned assumptions on the almost-Killing vector fields $K, T, \Phi$, as well as the expression (9.1) for the asymptotics of $g$ and the expression (A.15) for $\square_{g}$, we can bound for any smooth $\varphi: \mathcal{M} \rightarrow \mathbb{C}$ and any integer $l \geq 0$ and $R_{c} \gg 1$ :

$$
\begin{aligned}
& \left|\nabla_{h_{\tau, N}}^{l}\left(\Delta_{h_{R_{c}}, \bmod } \varphi\right)\right|_{h_{\tau, N}}^{2} \\
& \quad \leq C\left(\tau, R_{c}\right)\left|\nabla_{h_{\tau, N}}^{l}(\square \varphi)\right|_{h_{\tau, N}}^{2}+C\left(\tau, R_{C}\right) \cdot \mathcal{T}_{T, K, R_{c}}^{(l+2)}[\varphi] \\
& \quad+C(\tau) \chi_{r \sim R_{c}} \sum_{j_{1}=0}^{l+1} \sum_{j_{2}=1}^{l+2-j_{1}}\left(\left|\nabla_{h_{\tau, N}}^{j_{2}} \varphi\right|_{T, K, R_{c}}^{\left(j_{1}\right)}\right)^{2},
\end{aligned}
$$

where

$$
\begin{aligned}
& \mathcal{T}_{T, K, R_{c}}^{(m)}[\varphi] \\
& =r_{+}^{-2}\left(|\varphi|_{T, K, R_{c}}^{(m)}\right)^{2}+\sum_{j_{1}+j_{2}=m-2}\left(\left|\nabla_{h_{\tau, N}}^{j_{1}+1} \varphi\right|_{T, K, R_{c}}^{\left(j_{2}+1\right)}\right)^{2} \\
& \quad+\sum_{0 \leq j_{1}+j_{2} \leq m-2}\left(r_{+}^{-2\left(m-j_{1}-j_{2}-1\right)}+r_{+}^{-2} \tau^{-2\left(\delta_{0}+m-j_{1}-j_{2}-2\right)}\right)\left(\left|\nabla_{h_{\tau, N}}^{j_{1}} \varphi\right|_{T, K, R_{c}}^{\left(j_{2}+1\right)}\right)^{2} \\
& \quad+\sum_{0 \leq j \leq m-2}\left(r_{+}^{-2-2(m-2-j)} \tau^{-2 \delta_{0}}+r_{+}^{-2} \tau^{-2\left(\delta_{0}+m-2-j\right)}\right)\left|\nabla_{h_{\tau, N}}^{j+1} \varphi\right|_{h_{\tau, N}}^{2},
\end{aligned}
$$

$\chi_{r \sim R_{c}}$ is identically 1 on $\left\{R_{c} \leq r \leq 2 R_{c}\right\}$ and 0 elsewhere, and the elliptic operator $\Delta_{h_{R_{c}}, \bmod }$ on $\{\bar{t}=\tau\} \backslash \mathcal{H}_{\tau}$ is defined as:

$$
\Delta_{h_{R_{c}}, \bmod } \Psi=\mathfrak{w}_{\tau, K_{R_{c}}, \Phi}^{-1} \cdot d i v_{h_{R_{c}}}\left(\mathfrak{w}_{\tau, K_{R_{c}}, \Phi} \cdot d \Psi\right)
$$


with $\mathfrak{w}_{\tau, K_{R_{C}}, \Phi}>0$ on $\{\bar{t}=\tau\} \backslash \mathcal{H}_{\tau}$ satisfying $\mathfrak{w}_{\tau, K_{R_{c}}, \Phi} \sim_{\tau} r^{\frac{1}{2}}$ near $\mathcal{H}^{+}$and $\mathfrak{w}_{\tau, K_{R_{C}}, \Phi} \sim_{R_{C}} 1$ in the region $r \gg 1$ (for its precise form, see Section A of the Appendix). ${ }^{18}$

It will also be convenient to introduce the higher order pointwise norm

$$
\mathcal{T}_{T, K, R_{c}}^{(m, n)}[\varphi] \doteq \sum_{j_{1}+j_{2} \leq n} \mathcal{T}_{T, K, R_{c}}^{(m)}\left[\nabla_{h_{\tau, N}}^{j_{1}}\left(T^{j_{2}} \varphi\right)\right]
$$

for any pair of integers $m, n \geq 0$ and $\varphi \in C^{\infty}(\mathcal{M})$.

Similarly, in the region $\left\{r \geq R_{0}\right\}$ for some $R_{0} \gg 1$ we can bound:

$$
\left|\nabla_{h_{\tau, N}}^{l}\left(\Delta_{h_{\tau, T}, T} \varphi\right)\right|_{h_{\tau, N}}^{2} \leq C(\tau)\left|\nabla_{h_{\tau, N}}^{l}(\square \varphi)\right|_{h_{\tau, N}}^{2}+C(\tau) \cdot \mathcal{T}_{T}^{(l+2)}[\varphi],
$$

where

$$
\begin{aligned}
& \mathcal{T}_{T}^{(m)}[\varphi] \\
& =r_{+}^{-2}\left|T^{m} \varphi\right|^{2}+\sum_{j_{1}+j_{2}=m-2}\left|\nabla_{h_{\tau, N}}^{j_{1}+1}\left(T^{j_{2}+1} \varphi\right)\right|_{h_{\tau, N}}^{2} \\
& \quad+\sum_{0 \leq j_{1}+j_{2} \leq m-2}\left(r_{+}^{-2\left(m-j_{1}-j_{2}-1\right)}+r_{+}^{-2} \tau^{-2\left(\delta_{0}+m-j_{1}-j_{2}-2\right)}\right)\left|\nabla_{h_{\tau, N}}^{j_{1}}\left(T^{j_{2}+1} \varphi\right)\right|_{h_{\tau, N}}^{2} \\
& \quad+\sum_{0 \leq j \leq m-2}\left(r_{+}^{-2-2(m-2-j)} \tau^{-2 \delta_{0}}+r_{+}^{-2} \tau^{-2\left(\delta_{0}+m-2-j\right)}\right)\left|\nabla_{h_{\tau, N}}^{j+1} \varphi\right|_{h_{\tau, N}}^{2}
\end{aligned}
$$

and the operator $\Delta_{h_{\tau, T}, T}$ on $\{\bar{t}=\tau\} \cap\left\{r \geq R_{0}\right\}$ is defined as:

$$
\Delta_{h_{\tau, T}, T} \Psi=(\sqrt{-g(T, T)})^{-1} \cdot \operatorname{div}_{h_{\tau, T}}(\sqrt{-g(T, T)} \cdot d \Psi)
$$

The following higher order pointwise norm will also be useful:

$$
\mathcal{T}_{T}^{(m, n)}[\varphi] \doteq \sum_{j_{1}+j_{2} \leq n} \mathcal{T}_{T}^{(m)}\left[\nabla_{h_{\tau, N}}^{j_{1}}\left(T^{j_{2}} \varphi\right)\right]
$$

for any pair of integers $m, n \geq 0$ and $\varphi \in C^{\infty}(\mathcal{M})$.

Assumptions on the uniformity of the degenerate elliptic estimates on the hyperboloids $\{\bar{t}=\tau\}$ Let us introduce the functions $r_{\text {hor }}, r_{\text {tim }}: \mathcal{M} \rightarrow[0,1]$ by the relations

$$
r_{h o r}(x)=\frac{\operatorname{dist}_{h}(x, \mathcal{H})}{1+\operatorname{dist}_{h}(x, \mathcal{H})}
$$

\footnotetext{
18 It is obivious that we do not need all the terms of $\mathcal{T}_{T, K, R_{C}}^{(m)}[\varphi]$ to bound the left hand side of (9.16), but since this expression will appear frequently in what follows, we chose to introduce it here.
} 
and

$$
r_{t i m}(x)=\frac{\operatorname{dist}_{h}\left(x, \partial_{t i m} \mathcal{M}\right)}{1+\operatorname{dist}_{h}\left(x, \partial_{t i m} \mathcal{M}\right)} .
$$

Notice that this definition does not guarantee that $r_{h o r}$ and $r_{t i m}$ are smooth functions away from $\partial \mathcal{M}$. For this reason, we will mollify $r_{h o r}$ and $r_{t i m}$ way from $\partial \mathcal{M}$ so that they are smooth functions on $\mathcal{M}$, and we will replace the original $r_{h o r}$ and $r_{\text {tim }}$ by the corresponding mollified functions.

Using Propositions B.1 and B.3 of the Appendix, together with (9.15), (9.16) and (9.20), we can deduce the following elliptic estimate:

Lemma For any $l \in \mathbb{N}$ with $l \leq\left\lfloor\frac{d+1}{2}\right\rfloor$, any $n_{0} \in \mathbb{N}$, any $\beta \in\left(-\bar{\delta}_{n_{0}}, 1\right)$ (for some $\bar{\delta}_{n_{0}}>0$ depending on $n_{0}$ and $\tau$ ) and any $R_{0} \gg 1$ large in terms of the geometry of $(\mathcal{M}, g)$, we can bound for any $\varphi \in C^{\infty}(\mathcal{M})$ satisfying for all $j_{1}+j_{2} \leq l+n_{0}$ the finite radiation field condition $\lim \sup _{r \rightarrow+\infty}\left|r^{\frac{d-1}{2}+j_{1}} \nabla_{h_{\tau, N}}^{j_{1}}\left(N^{j_{2}} \varphi\right)\right|_{h_{\tau, N}}<+\infty$ :

$$
\begin{aligned}
& \sum_{n=0}^{n_{0}} \sum_{j=0}^{l-1} \int_{\{\bar{t}=\tau\}} r_{+}^{-\beta-2 j}\left|\nabla_{h_{\tau, N}}^{n+l-j} \varphi\right|_{\left(1-\log \left(r_{t i m}\right)\right) \cdot h_{R_{c}}}^{2} d h_{N} \\
& \leq C_{\beta, n_{0}}\left(\tau, R_{c}\right) \int_{\{\bar{t}=\tau\}} r_{+}^{-\beta}\left\{\sum_{n=0}^{n_{0}}\left|\nabla_{h_{\tau, N}}^{n+l-2}(\square \varphi)\right|_{\left(1-\log \left(r_{\text {tim }}\right)\right) \cdot h_{\tau, N}}^{2}+\mathcal{T}_{T, K, R_{c}}^{\left(l, n_{0}\right)}[\varphi]\right\} d h_{N} \\
& \quad+C_{\beta, n_{0}}(\tau) \sum_{n=0}^{n_{0}} \sum_{j_{1}=0}^{l-1} \sum_{j_{2}=1}^{l-j_{1}} \int_{\{\bar{t}=\tau\} \cap\left\{R_{c} \leq r \leq 2 R_{c}\right\}} r_{+}^{-\beta}\left(\left|\nabla_{h_{\tau, N}}^{j_{2}+n} \varphi\right|_{T, K, R_{c}}^{\left(j_{1}\right)}\right)^{2} d h_{N} \\
& \quad+C_{\beta, n_{0}}(\tau) \sum_{j=0}^{1} \max \left\{-\operatorname{Re}\left\{\int_{\partial_{\text {tim }} \mathcal{M}^{\tau}} h_{\partial_{\text {tim }} \mathcal{M}^{\tau}}\left(\nabla_{h_{\partial_{\text {tim }} \mathcal{M}^{\tau}}^{j}}(Y \varphi), \nabla_{h_{\partial_{\text {tim }} \mathcal{M}^{\tau}}^{j}} \bar{\varphi}\right) d h_{\left.\left.\partial_{\text {tim }} \mathcal{M}^{\tau}\right\}, 0\right\}}\right.\right.
\end{aligned}
$$

and for any $0<\varepsilon \ll 1-\beta$ (provided that $R_{c} \gg R_{0}$ ):

$$
\begin{aligned}
& \sum_{n=0}^{n_{0}} \sum_{j=1}^{l} \int_{\{\bar{t}=\tau\} \cap\left\{R_{c} \leq r \leq 2 R_{c}\right\}} r_{+}^{2(j-1)-\beta+\frac{\varepsilon}{2}}\left|\nabla_{g}^{j+n} \varphi\right|_{h}^{2} d h_{N} \\
& \leq C_{\beta, \epsilon}, n_{0}\left(\tau, R_{c}\right) \int_{\{\bar{t}=\tau\} \cap\left\{r \geq 2 R_{0}\right\}} r_{+}^{-\beta}\left\{\sum_{n=0}^{n_{0}}\left|\nabla_{h_{\tau, N}}^{n+l-2}(\square \varphi)\right|_{h_{\tau, N}}^{2}+\mathcal{T}_{T}^{\left(l, n_{0}\right)}[\varphi]\right\} d h_{N} \\
& \quad+C_{\beta, \varepsilon, n_{0}}(\tau) R_{c}^{-\frac{\varepsilon}{2}} \sum_{j=1}^{n_{0}+l} \int_{\{\bar{t}=\tau\} \cap\left\{2 R_{0} \leq r \leq 4 R_{0}\right\}}\left|\nabla_{g}^{j} \varphi\right|_{h}^{2} d h_{N} .
\end{aligned}
$$

Our extra assumptions will be the following uniformity conditions on the elliptic estimates (9.26) and the Sobolev-type estimates of Lemmas 9.10 and 9.11:

(EG6) The constants $\bar{\delta}_{n_{0}}, C_{\beta, n_{0}}(\tau), C_{\beta, \varepsilon, n_{0}}(\tau)$ and $C_{\beta, \varepsilon, n_{0}}\left(\tau, R_{c}\right)$ in Lemma 9.1 can be chosen not to depend on $\tau$. 
(EG7) The constants in the estimates of Lemmas 9.10 and 9.11 can be chosen not to depend on $\tau$.

We will also need the following assumption regarding the volume of the domains $\{\bar{t}=\tau\} \cap\{r \leq 1\}$ as $\tau \rightarrow+\infty$ :

(EG8) There exists some $C>0$ such that for any $\tau \geq 0$ the $h_{\tau, N}$-volume of the $\{\bar{t}=\tau\} \cap\{r \leq 1\}$ region is uniformly bounded in $\tau$ :

$$
\operatorname{Vol}_{h_{\tau, N}}(\{\bar{t}=\tau\} \cap\{r \leq 1\}) \leq C .
$$

The higher order integrated local energy decay estimate assumption In Section 8.1.2 we defined the class $\mathcal{C}_{I L E D}$ of admissible boundary conditions on $\partial_{\text {tim }} \mathcal{M}$ for which the integrated local energy decay statement (8.19) holds, and we assumed that $\mathcal{C}_{I L E D} \neq \emptyset$. However, since the class $C_{I L E D}$ was not necessarily closed under differentiation with respect to the vector fields $T, K$ introduced in this section, we do not know a priori that (8.19) holds for $T$ and $K$ derivatives of functions belonging to $C_{I L E D}$. the In this section, therefore, we will assume that a slightly stronger form of the integrated local energy decay statement (8.19) (including also higher order $T$ and $K$ derivatives of $\varphi$ ) holds on $(\mathcal{M}, g)$, which further restricts the class of allowed boundary conditions imposed on $\partial_{\text {tim }} \mathcal{M}$ :

(ILED2) We assume that there exists a non empty class $\mathcal{C}_{I L E D}^{(T, K)}$ of boundary conditions on $\partial_{\text {tim }} \mathcal{M}$, which is contained in the class of admissible boundary conditions $\mathcal{C}_{a d m}$, so that the following integrated local energy decay statement holds on $(\mathcal{M}, g)$ : There exists an integer $k \geq 0$ such that for any $R, R_{f}>0$, any integers $m \geq 0$ and $j_{1}, j_{2} \geq 0$, any $0<\eta<a$, any smooth $\varphi: \mathcal{M} \rightarrow \mathbb{C}$ solving $\square_{g} \varphi=F$ satisfying boundary conditions on $\partial_{\text {tim }} \mathcal{M}$ belonging to the class $\mathcal{C}_{I L E D}^{(T, K)}$ and any $0 \leq \tau_{1} \leq \tau_{2}$, we can bound

$$
\begin{aligned}
& \sum_{j=0}^{m} \int_{\left\{\tau_{1} \leq \bar{t} \leq \tau_{2}\right\} \cap\{r \leq R\}}\left|\nabla_{g}^{j}\left(T^{j_{1}} K_{R_{c}}^{j_{2}} \varphi\right)\right|_{h}^{2}+\sum_{j=1}^{m} \int_{\left\{\tau_{1} \leq \bar{t} \leq \tau_{2}\right\} \cap \partial_{t i m} \mathcal{M}}\left|\nabla_{g}^{j}\left(T^{j_{1}} K_{R_{c}}^{j_{2}} \varphi\right)\right|_{h}^{2} \\
& \leq C_{m, \eta, i_{1}, i_{2}}\left(R, R_{f}\right) \cdot \sum_{j=0}^{m+k-1}\left(\int_{\left\{\bar{t}=\tau_{1}\right\}} J_{\mu}^{N}\left(N^{j}\left(T^{j_{1}} K_{R_{c}}^{j_{2}} \varphi\right)\right) \bar{n}^{\mu}\right. \\
& \left.\quad+\int_{\left\{\tau_{1} \leq \bar{t} \leq \tau_{2}\right\}} r_{+}^{1+\eta}\left|\nabla_{g}^{j}\left(\square_{g}\left(T^{j_{1}} K_{R_{c}}^{j_{2}} \varphi\right)\right)\right|_{h}^{2} d h\right) \\
& \quad+C_{m, \eta_{i_{1}, i_{2}}} \sum_{i=0}^{m-1} \sum_{i_{1}+i_{2}=i} \int_{\left\{\tau_{1} \leq \bar{t} \leq \tau_{1}\right\} \cap\left\{r \geq R_{f}\right\}} \bar{t}^{-\delta 0} r_{+}^{-1}\left(\left|\nabla_{h_{\tau, N}}^{i_{1}+1}\left(T^{i_{2}+j_{1}} K_{R_{c}}^{j_{2}} \varphi\right)\right|^{2}\right. \\
& \left.\quad+r_{+}^{-2}\left|\nabla_{h_{\tau, N}}^{i_{1}}\left(T^{i_{2}+j_{1}} K_{R_{c}}^{j_{2}} \varphi\right)\right|^{2}+r_{+}^{-2}\left|T^{i+j_{1}+1} K_{R_{c}}^{j_{2}} \varphi\right|^{2}\right) d h .
\end{aligned}
$$


Remark Notice that (9.29) follows trivially from (8.19) (provided that the last term in the right hand side of (8.19) is replaced by

$$
\begin{aligned}
& C_{m, \eta} \sum_{j=0}^{m-1} \sum_{j_{1}+j_{2}=j} \int_{\left\{\tau_{1} \leq \bar{t} \leq \tau_{1}\right\} \cap\left\{r \geq R_{f}\right\}} \bar{t}^{-\delta 0} r_{+}^{-1}\left(\left|\nabla_{h_{\tau}, N}^{j_{1}+1}\left(N^{j_{2}} \varphi\right)\right|^{2}\right. \\
& \left.+r_{+}^{-2}\left|\nabla_{h_{\tau, N}}^{j_{1}}\left(N^{j_{2}} \varphi\right)\right|^{2}+r_{+}^{-2}\left|N^{j+1} \varphi\right|^{2}\right) d g,
\end{aligned}
$$

which is consistent with the remark below Lemma 4.1 in view of the assumptions on the vector field $T$ ) in the case when $\partial_{\text {tim }} \mathcal{M}=\emptyset$, or in the case when $T$ and $K$ are tangential to $\partial_{\text {tim }} \mathcal{M}$ and $\mathcal{C}_{I L E D}$ is restricted to include only the Dirichlet boundary conditions. In these cases, $\mathcal{C}_{I L E D}^{(T, K)}=\mathcal{C}_{I L E D}$. Furthermore, the results of this section also hold if one replaces Assumption (ILED2) by a pair of alternative integrated local energy decay assumptions similar to (8.21) and (8.22), but we will not pursue this issue any further.

\subsection{Shorthand Notation for Energy Norms}

In order to state and prove Theorem 9.1 conveniently, it will be useful to introduce some shorthand notation for a variety of energy norms on the hyperboloids $\{\bar{t}=\tau\}$. More precisely, we will make use of the following notations for $p \in \mathbb{R}, \varepsilon \in(0,1)$ and $q, l, m, k \in \mathbb{N}$ :

1. The following energy norms will appear when using the $r^{p}$-weighted estimates of Sections 5 and 6:

$$
\begin{aligned}
& \mathcal{E}_{\text {bulk,R }}^{(p, q, m)}[\varphi](\tau) \\
& \quad \doteq \sum_{j=0}^{m} \sum_{0 \leq j_{1}+j_{2} \leq q+j} \int_{\{\bar{t}=\tau\} \cap\{r \geq R\}} r^{p-2\left(q+j-j_{1}\right)}\left|\nabla_{h_{\tau, N}}^{j_{1}} T^{j_{2}}(\Omega \varphi)\right|_{h_{\tau, N}}^{2} \Omega^{-2} d h_{N}
\end{aligned}
$$

and

$$
\begin{aligned}
& \mathcal{E}_{\text {bound, } R}^{(p, q, m)}[\varphi](\tau) \\
& \quad \doteq \sum_{j=0}^{m} \sum_{0 \leq j_{1}+j_{2} \leq q+j-1} \int_{\{\bar{t}=\tau\} \cap\{r \geq R\}} r^{p-2\left(q+j-j_{1}-1\right)}\left(\left|\nabla_{h_{\tau, N}}^{j_{1}} T^{j_{2}} \partial_{v}(\Omega \varphi)\right|_{h_{\tau, N}}^{2}\right. \\
& \quad+r^{-2}\left|\nabla_{h_{\tau, N}}^{j_{1}+1} T^{j_{2}}(\Omega \varphi)\right|_{h_{\tau, N}}^{2}+r^{-4}\left|\nabla_{h_{\tau, N}}^{j_{1}} T^{j_{2}+1}(\Omega \varphi)\right|_{h_{\tau, N}}^{2} \\
& \left.\quad+r^{-4}\left|\nabla_{h_{\tau, N}}^{j_{1}} T^{j_{2}}(\Omega \varphi)\right|_{h_{\tau, N}}^{2}\right) \Omega^{-2} d h_{N}
\end{aligned}
$$

2. The following weighted non degenerate energy norms on $\{\bar{t}=\tau\}$ will also appear frequently (fixing some $R_{1}$ large in terms of the geometry of $(\mathcal{M}, g)$ ): 


$$
\begin{aligned}
\mathcal{E}_{e n}^{(p, q, m)}[\varphi](\tau) \doteq & \sum_{j=0}^{m} \sum_{1 \leq j_{2}+j_{3} \leq q+j-1} \int_{\{\bar{t}=\tau\}} r_{+}^{p-2\left(q+j-1-j_{2}-j_{3}\right)} \\
& \times\left(\left|\nabla_{h_{\tau, N}}^{j_{2}+1}\left(N^{j_{3}} \varphi\right)\right|_{h_{\tau, N}}^{2}+r_{+}^{-2}\left|N^{q+j} \varphi\right|^{2}\right) d h_{N}, \\
\mathcal{E}_{\text {bulk }}^{(p, q, m)}[\varphi](\tau) \doteq & \mathcal{E}_{\text {bulk, } R_{1}}^{(p, q, m)}[\varphi](\tau) \\
& +\sum_{j=0}^{m} \sum_{0 \leq j_{1}+j_{2} \leq q+j} \int_{\{\bar{t}=\tau\} \cap\left\{r \leq R_{1}\right\}}\left|\nabla_{h_{\tau, N}}^{j_{1}} N^{j_{2}} \varphi\right|_{h_{\tau, N}}^{2} d h_{N}
\end{aligned}
$$

and

$$
\begin{aligned}
\mathcal{E}_{\text {bound }}^{(p, q, m)}[\varphi](\tau) \doteq & \mathcal{E}_{\text {bound }, R_{1}}^{(p, q), \varphi](\tau)} \\
& +\sum_{j=0}^{m} \sum_{0 \leq j_{1}+j_{2} \leq q+j} \int_{\{\bar{t}=\tau\} \cap\left\{r \leq R_{1}\right\}}\left|\nabla_{h_{\tau, N}}^{j_{1}} N^{j_{2}} \varphi\right|_{h_{\tau, N}}^{2} d h_{N} .
\end{aligned}
$$

3. The following energy norm (associated to the vector fields $K_{R_{c}}, \Phi$ ) which degenerates at $\mathcal{H}$ and $\partial_{\text {tim }} \mathcal{M}$ will also appear:

$$
\begin{aligned}
\mathcal{E}_{\text {en, deg }}^{(p, q, m)}[\varphi](\tau) \doteq & \sum_{j=0}^{m} \sum_{1 \leq j_{2}+j_{3} \leq l+j-1} \int_{\{\bar{t}=\tau\}} r_{+}^{p-2\left(l+j-1-j_{2}-j_{3}\right)} \\
& \times\left(\left|\nabla_{h_{\tau, N}}^{j_{2}+1}\left(K_{R_{c}}^{j_{3}} \varphi\right)\right|_{\left(1-\log \left(r_{\text {tim }}\right)\right) \cdot h_{R_{C}}}^{2}+r_{+}^{-2}\left|K_{R_{c}}^{l+j} \varphi\right|^{2}\right) d h_{N} .
\end{aligned}
$$

4. Finally, we will make use of the following spacetime norms for the source terms, for some $C=C_{l, q, m, k}>0$ that will be fixed in the statement of Theorem 9.1:

$$
\begin{aligned}
\mathcal{F}_{\eta, \varepsilon}^{(2 l, q, m, k)}[F](\tau) & \tau^{-2 l+\varepsilon} \sum_{j=0}^{m+(2 l+1) k-1} \sum_{j_{1}+j_{2}=j} \sum_{i_{1}+i_{2} \leq q-1} \int_{\{0 \leq \bar{t} \leq \tau\}} r^{3+2 i_{1}}\left|\nabla_{h_{\tau, N}}^{j_{1}+i_{1}}\left(N^{j_{2}+i_{2}} F\right)\right|_{h_{\tau, N}}^{2} d g \\
& +\sum_{s=1}^{l-1} \tau^{-2 s+\varepsilon} \sum_{j=0}^{m+(2 s+1) k-1} \sum_{j_{1}+j_{2}=j} \sum_{i_{1}=0}^{q-(l-s)-1} \sum_{i_{2}=0}^{l-s} \int_{\left\{C^{-1} \tau \leq \bar{t} \leq \tau\right\}} r^{3+2 i_{1}} \\
& \times\left|\nabla_{h_{\tau, N}}^{j_{1}+i_{1}}\left(N^{j_{2}+i_{2}} F\right)\right|_{h_{\tau, N}}^{2} d g \\
& +\sum_{s=1}^{l} \tau^{-2 s+1+\varepsilon} \sum_{j=0}^{m+2 s k-1} \sum_{j_{1}+j_{2}=j} \sum_{i_{1}=0} \sum_{i_{2}=0} \int_{\left\{C^{-1} \tau \leq \bar{t} \leq \tau\right\}} r^{2+2 i_{1}} \\
& \times\left|\nabla_{h_{\tau, N}}^{j_{1}+i_{1}}\left(N^{j_{2}+i_{2}} F\right)\right|_{h_{\tau, N}}^{2} d g
\end{aligned}
$$




$$
\begin{aligned}
& +\sum_{j=0}^{m+2 k-1} \sum_{j_{1}+j_{2}=j} \sum_{i_{1}=0}^{q-l} \sum_{i_{2}=0}^{l-1} \int_{\left\{C^{-1} \tau \leq \bar{t} \leq \tau\right\}}\left(r^{1+\varepsilon+2 i_{1}}+r^{1+\eta}\right) \\
& \times\left|\nabla_{h_{\tau, N}}^{j_{1}+i_{1}}\left(N^{j_{2}+i_{2}} F\right)\right|_{h_{\tau, N}}^{2} d g
\end{aligned}
$$

and

$$
\begin{aligned}
\mathcal{F}_{d e g, \eta}^{(2 l, q, m, k)}[F](\tau) & \tau^{-2 l} \sum_{j=0}^{m+(2 l+1) k-1} \sum_{j_{1}+j_{2}=j} \sum_{i_{1}+i_{2} \leq q-1} \int_{\{0 \leq \bar{t} \leq \tau\}} r^{3+2 i_{1}}\left|\nabla_{h_{\tau, N}}^{j_{1}+i_{1}}\left(K_{R_{c}}^{j_{2}+i_{2}} F\right)\right|_{h_{\tau, N}}^{2} d g \\
& +\sum_{s=1}^{l-1} \tau^{-2 s} \sum_{j=0}^{m+(2 s+1) k-1} \sum_{j_{1}+j_{2}=j} \sum_{i_{1}=0}^{q-(l-s)-1} \sum_{i_{2}=0}^{l-s} \int_{\left\{C^{-1} \tau \leq \bar{t} \leq \tau\right\}} r^{3+2 i_{1}} \\
& \times\left|\nabla_{h_{\tau, N}}^{j_{1}+i_{1}}\left(K_{R_{c}}^{j_{2}+i_{2}} F\right)\right|_{h_{\tau, N}}^{2} d g \\
& +\sum_{s=1}^{l} \tau^{-2 s+1} \sum_{j=0}^{m+2 s k-1} \sum_{j_{1}+j_{2}=j} \sum_{i_{1}=0} \sum_{i_{2}=0}^{l-(l-s)-1} \int_{\left\{C^{-1} \tau \leq \bar{t} \leq \tau\right\}} r^{2+2 i_{1}} \\
& \times\left|\nabla_{h_{\tau, N}}^{j_{1}+i_{1}}\left(K_{R_{c}}^{j_{2}+i_{2}} F\right)\right|_{h_{\tau, N}}^{2} d g \\
& +\sum_{j=2 k-1}^{m+2} \sum_{j_{1}+j_{2}=j} \sum_{i_{1}=0}^{l-l} \sum_{i_{2}=0}^{l-1} \int_{\left\{C^{-1}\right.} \sum_{\tau \leq \bar{t} \leq \tau\}}\left(r^{1+2 i_{1}}+r^{1+\eta}\right) \\
& \times\left|\nabla_{h_{\tau, N}}^{j_{1}+i_{1}}\left(K_{R_{c}}^{j_{2}+i_{2}} F\right)\right|_{h_{\tau, N}}^{2} d g .
\end{aligned}
$$

\subsection{Statement of the Results on Improved Polynomial Decay}

In this class of spacetimes $(\mathcal{M}, g)$ we will establish the following result:

Theorem 9.1 Assume that $\left(\mathcal{M}^{d+1}, g\right), d \geq 3$, satisfies Assumptions (G1)-(G13), (EG1)-(EG8) and (ILED2). Then for any smooth function $\varphi$ on $\mathcal{M}$ solving $\square_{g} \varphi=F$ on $\mathrm{J}^{+}(\{\bar{t}=0\})$ with suitably decaying initial data on $\{\bar{t}=0\}$ and satisfying boundary conditions on $\partial_{\text {tim }} \mathcal{M}$ belonging to the class $\mathcal{C}_{I L E D}^{(T, K)}$ and such that

$$
\begin{aligned}
& \operatorname{Re}\left\{\int_{\partial_{\text {tim }} \mathcal{M}^{\tau}} Y \varphi \cdot \bar{\varphi} d h_{\left.\partial_{\text {tim }} \mathcal{M}^{\tau}\right\} \geq 0 \text { and }}\right. \\
& \quad \operatorname{Re}\left\{\int _ { \partial _ { \text { tim } } \mathcal { M } ^ { \tau } } h _ { \partial _ { t i m } \mathcal { M } ^ { \tau } } \left(\nabla_{h_{\partial_{\text {tim }} \mathcal{M}^{\tau}}}(Y \varphi), \nabla_{\left.\left.h_{\partial_{t i m} \mathcal{M}^{\tau}} \bar{\varphi}\right) d h_{\partial_{t i m} \mathcal{M}^{\tau}}\right\} \geq 0,}\right.\right.
\end{aligned}
$$

the following bounds hold for any integer $1 \leq q \leq\left\lfloor\frac{d+1}{2}\right\rfloor$, any $0<\varepsilon \ll \delta_{0}$, any integer $m \geq 1$ and any $\tau \geq 0$, provided (7.2) holds for all $0 \leq j \leq q+\left\lceil\frac{d}{2}\right\rceil+m+$ $\left\lceil\delta_{0}^{-1} \cdot 2(q-1)\right\rceil(3 q+1) \cdot k$ : 


$$
\begin{aligned}
& \mathcal{E}_{\text {en }}^{(0, q, m)}[\varphi](\tau)+\mathcal{E}_{\text {bound }}^{(\varepsilon, q, m)}[\varphi](\tau)+\int_{\tau}^{+\infty} \mathcal{E}_{\text {en }}^{(-1+\varepsilon, q, m)}[\varphi](s) d s \\
& \lesssim_{m, \varepsilon} \tau^{-2 q+C_{m} \varepsilon} \mathcal{E}_{\text {bound }}^{\left(2 q, q, m+\left\lceil\delta_{0}^{-1} \cdot 2(q-1)\right\rceil(3 q+1) \cdot k\right)}[\varphi](0) \\
& \quad+\mathcal{F}_{\varepsilon, \varepsilon}^{\left(2 q, q, m+\left\lceil\delta_{0}^{-1} \cdot 2(q-1)\right\rceil(3 q+1) \cdot k, k\right)}[F](\tau)
\end{aligned}
$$

and

$$
\begin{aligned}
\mathcal{E}_{\text {en,deg }}^{(0, q, m)}[\varphi](\tau) \lesssim & { }_{m, \varepsilon} \tau^{-2 q} \mathcal{E}_{\text {bound }}^{\left(2 q, q, m+\left\lceil\delta_{0}^{-1} \cdot 2(q-1)\right\rceil(3 q+1) \cdot k\right)}[\varphi](0) \\
& +\mathcal{F}_{\text {deg, }, \varepsilon}^{\left(2 q, q, m+\left\lceil\delta_{0}^{-1} \cdot 2(q-1)\right\rceil(3 q+1) \cdot k, k\right)}[F](\tau) .
\end{aligned}
$$

See Section 9.2 for the notations on the energy norms used.

Remark Notice that the condition (9.39) is satisfied when $\varphi$ is subject to Dirichlet or Neumann boundary conditions. Theorem 9.1 still holds if one replaces the condition (9.39) with any boundary condition for which Lemma 9.5 can still be established.

Let us also remark in the case the integrated local energy decay statement in Assumption (ILED2) does not lose derivatives (i.e. $k=0$ ), we can replace Assumption (EG5) on the $\bar{t}^{-\delta_{0}}$ decay of the deformation tensors of $T$ and $K$ with the following $O\left(\varepsilon_{0}\right)$-smallness assumption:

Uniform smallness of the deformation tensor: There exists some (small) $\varepsilon_{0}>0$ so that:

$$
\sup _{\{\bar{t}=\tau\}}\left(\left|\mathcal{L}_{T} g\right|+\left|\mathcal{L}_{K} g\right|\right)=O\left(\varepsilon_{0}\right)
$$

and in the $(u, r, \sigma)$ coordinate chart on each connected component of the region $\mathcal{N}_{a f, \mathcal{M}}$ :

$$
\begin{aligned}
\mathcal{L}_{T} g= & O\left(\varepsilon_{0}\right)\left\{O\left(r^{-1-a}\right) d r d u+O(r) d \sigma d \sigma+O(1) d u d \sigma\right. \\
& \left.+O\left(r^{-a}\right) d r d \sigma+O\left(r^{-1}\right) d u^{2}+O\left(r^{-2-a}\right) d r^{2}\right\}
\end{aligned}
$$

and

$$
\begin{aligned}
\mathcal{L}_{K} g= & O\left(\varepsilon_{0}\right)\left\{O\left(r^{-1-a}\right) d r d u+O(r) d \sigma d \sigma+O(1) d u d \sigma\right. \\
& \left.+O\left(r^{-a}\right) d r d \sigma+O\left(r^{-1}\right) d u^{2}+O\left(r^{-2-a}\right) d r^{2}\right\}
\end{aligned}
$$

Moreover, any further Lie differentiation of $g$ in the direction of $T$ or $K$ should improve the above decay rates by a factor of $\bar{t}^{-1}$.

In this case, we also relax the integrated local energy decay assumption (ILED2), by replacing the $\bar{t}^{-\delta_{0}}$ factor in the last term of the right hand side of (9.29) with $\varepsilon_{0}$. Under these weaker assumptions, we can still obtain (9.40) and (9.41), but with an 
$O\left(\varepsilon_{0}\right)$ loss in the exponents of both inequalities. The proof in this case is similar, and actually easier.

The proof of Theorem 9.1 will be presented in Section 9.7.

As a Corollary of Theorem 9.1, we will establish improved pointwise decay rates for $\varphi$ and its derivatives:

Corollary 9.2 Assume that $\left(\mathcal{M}^{d+1}, g\right), d \geq 3$, satisfies Assumptions (G1)-(G13), (EG1)-(EG8) and (ILED2). Then for any smooth function $\varphi$ on $\mathcal{M}$ solving $\square_{g} \varphi=F$ on $\mathrm{J}^{+}(\{\bar{t}=0\})$ with suitably decaying initial data on $\{\bar{t}=0\}$ and satisfying boundary conditions on $\partial_{\text {tim }} \mathcal{M}$ belonging to the class $\mathcal{C}_{I L E D}^{(T, K)}$ and such that

$$
\begin{aligned}
& \operatorname{Re}\left\{\int_{\partial_{t i m} \mathcal{M}^{\tau}} Y \varphi \cdot \bar{\varphi} d h_{\partial_{t i m} \mathcal{M}^{\tau}}\right\} \geq 0 \text { and } \\
& \operatorname{Re}\left\{\int_{\partial_{t i m} \mathcal{M}^{\tau}} h_{\partial_{t i m} \mathcal{M}^{\tau}}\left(\nabla_{h_{\partial_{t i m}} \mathcal{M}^{\tau}}(Y \varphi), \nabla_{h_{\partial_{t i m} \mathcal{M}^{\tau}}} \bar{\varphi}\right) d h_{\left.\partial_{t i m} \mathcal{M}^{\tau}\right\} \geq 0,}\right.
\end{aligned}
$$

the following pointwise decay estimates hold for any $0<\varepsilon \ll \delta_{0}$, any integer $m \geq 0$ and any $\tau \geq 0$, provided (7.2) holds for all $0 \leq j \leq d+1+m+\left\lceil\delta_{0}^{-1} \cdot 2(q-1)\right\rceil(3 \bar{q}+$ $1) \cdot k$ :

1. In case the dimesion $d$ is odd, we can bound:

$$
\sup _{\{\bar{t}=\tau\}}|\varphi|^{2} \lesssim \varepsilon \tau^{-d} \cdot \mathcal{E}_{0, d}[\varphi](0)+\mathcal{F}_{d e g, \varepsilon, 0, d}[F](\tau),
$$

and if $m \geq 1$ :

$$
\sup _{\{\bar{t}=\tau\}}\left|\nabla_{g}^{m} \varphi\right|_{h}^{2} \lesssim m, \varepsilon \tau^{-d-1} \cdot \mathcal{E}_{m+2, d}[\varphi](0)+\mathcal{F}_{d e g, \varepsilon, m+2, d}[F](\tau)
$$

2. In case the dimension d is even, we can bound:

$$
\sup _{\{\bar{t}=\tau\}}\left|\nabla_{g}^{m} \varphi\right|_{h}^{2} \lesssim m, \varepsilon \tau^{-d+C_{m} \varepsilon} \cdot \mathcal{E}_{m, d}[\varphi](0)+\mathcal{F}_{\varepsilon, m, d}[F](\tau) .
$$

In the above,

$$
\begin{aligned}
\mathcal{E}_{m, d}[\varphi](0) & \doteq \mathcal{E}_{\text {bound }}^{\left(2\left\lceil\frac{d+1}{2}\right\rceil,\left\lceil\frac{d+1}{2}\right\rceil, m+\left\lceil\delta_{0}^{-1} \cdot 2\left(\left\lceil\frac{d+1}{2}\right\rceil-1\right)\right\rceil\left(3\left\lceil\frac{d+1}{2}\right\rceil+1\right) \cdot k\right)}[\varphi](0), \\
\mathcal{F}_{\text {deg }, \eta, m, d}[F](\tau) & \doteq \mathcal{F}_{\text {deg }, \eta}^{\left(2\left\lceil\frac{d+1}{2}\right\rceil,\left\lceil\frac{d+1}{2}\right\rceil, m+\left\lceil\delta_{0}^{-1} \cdot 2\left(\left\lceil\frac{d+1}{2}\right\rceil-1\right)\right\rceil(3 q+1) \cdot k, k\right)}[F](\tau)
\end{aligned}
$$

and

$$
\mathcal{F}_{\varepsilon, m, d}\left[F(\tau) \doteq \mathcal{F}_{\varepsilon, \varepsilon}^{\left(2\left\lceil\frac{d+1}{2}\right\rceil,\left\lceil\frac{d+1}{2}\right\rceil, m+\left\lceil\delta_{0}^{-1} \cdot 2\left(\left\lceil\frac{d+1}{2}\right\rceil-1\right)\right\rceil(3 q+1) \cdot k, k\right)}[F](\tau) .\right.
$$

See Section 9.2 for the notations on the energy norms used. 
Remark Again, Corollary 9.2 still holds if one replaces the condition (9.45) with any boundary condition for which Lemma 9.5 can still be established. Moreover, in case the integrated local energy decay statement in Assumption (ILED2) does not lose derivatives (i.e. $k=0$ ), Assumption (EG5) can be replaced by (9.42)-(9.44) and the integrated local energy decay assumption (ILED2) can be relaxed by replacing the $\bar{t}^{-\delta 0}$ factor in the last term of the right hand side of (9.29) with $\varepsilon_{0}$. In this case, inequalities (9.46), (9.47) and (9.48) still hold with an $O\left(\varepsilon_{0}\right)$ loss in the exponent of $\tau$.

The proof of Corollary 9.2 will be presented in Section 9.9.

\subsection{Sketch of the Proof of Theorem 9.1 and Corollary 9.2}

In this Section, we will first sketch the proof of Theorem 9.1 and Corollary 9.2 under some simplifying assumptions on the structure of the spacetime $(\mathcal{M}, g)$, and then we will highlight the main difficulties arising in the general case.

Let us assume first that $d=3, \partial \mathcal{M}=\emptyset, F=0, k=0$ (i.e. there is no derivative loss in the integrated local energy decay statement (8.19)), $m=1$ and the vector field $T$ is globally timelike and Killing. In this case, there is no condition on $\partial_{\text {tim }} \mathcal{M}$ that $\varphi$ is assumed to satisfy, and we can assume without loss of generality that the vector field $K$ has been fixed so that $T \equiv K$. Let us also note that in this case, the $\varepsilon$-loss in (9.40) can be dropped, and the estimates of Theorem 9.1 and Corollary 9.2 read as follows:

$$
\begin{gathered}
\sum_{i_{1}+i_{2}=1} \int_{\{\bar{t}=\tau\}}\left(\left|\nabla_{h_{\tau, T}}^{i_{1}+1}\left(T^{i_{2}} \varphi\right)\right|_{h_{T}}^{2}+r^{-2}\left|T^{2} \varphi\right|^{2}\right) d h_{T} \lesssim \tau^{-4} \mathcal{E}_{i n}[\varphi](0), \\
\sup _{\{\bar{t}=\tau\}}|\varphi| \lesssim \tau^{-\frac{3}{2}}
\end{gathered}
$$

and:

$$
\sup _{\{\bar{t}=\tau\}}\left|\nabla_{g} \varphi\right|_{h} \lesssim \tau^{-2}
$$

The main idea for the proof of (9.52) is the following (assuming without loss of generality that $\varphi$ is real valued): From Theorem 8.2 we deduce that:

$$
\sum_{i_{1}+i_{2}=1} \int_{\{\bar{t}=\tau\}}\left(r^{2}\left|\nabla_{h_{\tau, T}}^{2} \varphi\right|_{h_{T}}^{2}+\left|\nabla_{h_{\tau, T}}^{i_{1}}\left(T^{1+i_{2}} \varphi\right)\right|_{h_{T}}^{2}\right) d h_{T} \lesssim \tau^{-2} \mathcal{E}_{i n}[\varphi](0) .
$$

Let us fix a vector field $L$ on $\mathcal{M}$ so that $[T, L]=0$ and $L=\partial_{v}$ in the $(\bar{t}, v, \sigma)$ coordinate system on each connected component of the region $(r \gg 1)$. Using the expression for the equation $\square \varphi=0$, from (9.55) we deduce that

$$
\sum_{i_{1}+i_{2}=1} \int_{\{\bar{t}=\tau\}}\left(r^{2}|L(T \varphi)|^{2}+\left|\nabla_{h_{\tau, T}}^{i_{1}}\left(T^{1+i_{2}} \varphi\right)\right|_{h_{T}}^{2}\right) d h_{T} \lesssim \tau^{-2} \mathcal{E}_{i n}[\varphi](0) .
$$


Fixing a dyadic sequence $\left\{\tau_{n}\right\}_{n \in \mathbb{N}}$, by repeating the proof Theorem 8.1 on the intervals $\left\{\tau_{n} \leq \bar{t} \leq \tau_{n+1}\right\}$ with $T \varphi$ in place of $\varphi$ and using the estimate (9.56) (notice that for the sketch of the proof we have assumed that $T$ is Killing, and thus $\left[T, \square_{g}\right]=0$ ), we readily obtain:

$$
\int_{\{\bar{t}=\tau\}}\left(\left|\nabla_{h_{\tau}, T}(T \varphi)\right|_{h_{T}}^{2}+r^{-2}\left|T^{2} \varphi\right|^{2}\right) d h_{T} \lesssim \tau^{-4} \mathcal{E}_{i n}[\varphi](0) .
$$

Using again the expression for the wave equation $\square \varphi=0$, from (9.57) we deduce that

$$
\int_{\{\bar{t}=\tau\}}\left(\Delta_{e l l} \varphi\right)^{2} d h_{T} \lesssim \tau^{-4} \mathcal{E}_{i n}[\varphi](0)
$$

for a suitable elliptic operator $\Delta_{\text {ell }}$ on the hyperboloids $\{\bar{t}=\tau\}$. The elliptic estimates of Section B of the Appendix then yield:

$$
\int_{\{\bar{t}=\tau\}}\left|\nabla_{h_{\tau, T}}^{2} \varphi\right|_{h_{T}} d h_{T} \lesssim \tau^{-4} \mathcal{E}_{i n}[\varphi](0),
$$

which combined with (9.57) yields (9.52).

The estimate (9.54) for $\nabla_{g} \varphi$ follows readily from a Sobolev inequality applied on (9.52) for $\varphi$ and $T \varphi$ (combined with elliptic estimates). The zeroth order estimate (9.53), on the other hand, follows from (9.52), the decay estimate from Theorem 8.1:

$$
\int_{\{\bar{t}=\tau\}}\left(\left|\nabla_{h_{\tau}, T} \varphi\right|_{h_{T}}^{2}+r^{-2}|T \varphi|^{2}\right) d h_{T} \lesssim \tau^{-2} \mathcal{E}_{i n}[\varphi](0)
$$

and the following Gagliardo-Nirenberg type estimate on $\{\bar{t}=\tau\}$ (see Section 9.8):

$$
\begin{aligned}
\sup _{\{\bar{t}=\tau\}}|\varphi|^{2} \lesssim & \left(\int_{\{\bar{t}=\tau\}}\left(\left|\nabla_{h_{\tau}, T} \varphi\right|_{h_{T}}^{2}+r^{-2}|T \varphi|^{2}\right) d h_{T}\right)^{1 / 2} \\
& \times\left(\sum_{i_{1}+i_{2}=1} \int_{\{\bar{t}=\tau\}}\left(\left|\nabla_{h_{\tau, T}}^{i_{1}+1}\left(T^{i_{2}} \varphi\right)\right|_{h_{T}}^{2}+r^{-2}\left|T^{2} \varphi\right|^{2}\right) d h_{T}\right)^{1 / 2} \\
& +\sum_{i_{1}+i_{2}=1} \int_{\{\bar{t}=\tau\}}\left(\left|\nabla_{h_{\tau, T}}^{i_{1}+1}\left(T^{i_{2}} \varphi\right)\right|_{h_{T}}^{2}+r^{-2}\left|T^{2} \varphi\right|^{2}\right) d h_{T}
\end{aligned}
$$

One important difficulty arising in the proof of Theorem 9.1 in the more general class of spacetimes $(\mathcal{M}, g)$ under consideration comes from the fact that $T$ is not in general a Killing vector field, and in fact its deformation tensor decays only like $\bar{t}^{-\delta_{0}}$ for some small $\delta_{0}>0$. This results in a number of error terms appearing each time $\square$ is commuted with $T$, which can only be controlled in the final step of the estimates, using also some refined elliptic estimates leading to the $\varepsilon$-loss in (9.40) (however, we avoid this loss in (9.41)). Furthermore, the slow $O\left(\bar{t}^{-\delta 0}\right)$ decay of the deformation 
tensor of $T$ combined with the loss of derivatives in the integrated local energy decay statement (8.19) require an iteration of the above procedure $\sim \delta_{0}^{-1} \cdot k$ times, leading to the corresponding derivative loss in the estimates of Theorem 9.1. Notice, however, that in case where the integrated local energy decay statement (8.19) does not lose derivatives, the same steps can be applied (without the need of the extra iterations of the procedure) even when the deformation tensor of $T$ does not decay at all, but is, instead, merely bounded by some small constant $\varepsilon_{0}>0$.

We should also notice that in the general case where $T$ is not everywhere nonspacelike (and thus we necessarily have $K \neq T$ ), one extra difficulty arises from the fact that, in order to avoid losing unnecessary $r$-weights in the estimates of Theorem 9.1, we only commute $\square$ with $\chi K$ instead of $K$, for a cut-off function $\chi$ supported in the far away region $\{r \gg 1\}$ (notice that $g(K, K) \sim r^{2}$ in case $K \neq T$ in the far away region). However, commutation with $\chi K$ leads to additional error terms which do not decay in time. A key element in dealing with these terms are the elliptic estimates of Lemma B.3.

\subsection{Commutation with $T, K_{R_{c}}$ and Control of the Error Terms}

The following Lemma will provide us with some useful estimates for the commutator of $\square_{g}$ with the almost Killing vector field $T$ and the vector field $K_{R_{c}}$ which fails to be almost Killing in the region $r \sim R_{c}$ of $\mathcal{M}$.

Lemma 9.3 Provided that $1 \ll R_{0} \ll R_{c}$, let us fix $\chi_{R_{0}}, \chi_{\sim} R_{c}: \mathcal{M} \rightarrow[0,1]$ so that

$$
\chi_{R_{0}}=\chi \circ\left(\frac{r}{R_{0}}\right)
$$

for some smooth $\chi:[0,+\infty) \rightarrow[0,1]$ satisfying $\chi \equiv 1$ on $[0,1]$ and $\chi \equiv 0$ on $[2,+\infty)$ and

$$
\chi \sim R_{c}= \begin{cases}0, & \text { on }\left\{r \notin\left[R_{c}, 2 R_{c}\right]\right\} \\ 1, & \text { on }\left\{r \in\left[R_{c}, 2 R_{c}\right]\right\} .\end{cases}
$$

Then the following commutation relations hold for any $\varphi \in C^{\infty}(\mathcal{M})$ and any integer $l \geq 0$ :

$$
\begin{aligned}
\square_{g}\left(T^{l} \varphi\right)= & T^{l}\left(\square_{g} \varphi\right)+\chi_{R_{0}} \cdot \sum_{j=1}^{l} O\left(\tau^{-(l-j)-\delta 0}\right)\left(\left|\nabla^{j+1} \varphi\right|_{h}+\left|\nabla^{j} \varphi\right|_{h}\right) \\
& +\left(1-\chi_{R_{0}}\right) \mathscr{E}_{(T, \ldots T)}^{(l)}[\varphi]
\end{aligned}
$$

and for any $\left(e_{1}, \ldots e_{l}\right) \in\{0,1\}^{l}$ setting $X^{(0)}=T$ and $X^{(1)}=K_{R_{c}}$ :

$$
\square_{g}\left(X^{\left(e_{1}\right)} \cdots X^{\left(e_{l}\right)} \varphi\right)=X^{\left(e_{1}\right)} \cdots X^{\left(e_{l}\right)}\left(\square_{g} \varphi\right)+\chi_{R_{0}}
$$




$$
\begin{aligned}
& \times \sum_{j=1}^{l} O\left(\tau^{-(l-j)-\delta 0}\right)\left(\left|\nabla^{j+1} \varphi\right|_{h}+\left|\nabla^{j} \varphi\right|_{h}\right) \\
& +\left(1-\chi_{R_{0}}\right)\left(1-\chi_{r \sim R_{c}}\right) \mathscr{E}_{\left(X^{\left(e_{1}\right)}, \ldots X^{\left(e_{l}\right)}\right)}^{(l)}[\varphi] \\
& +\chi_{r \sim R_{c}} \sum_{j=0}^{l} O\left(r^{j-1}\right)\left|\nabla^{j+1} \varphi\right|_{h},
\end{aligned}
$$

where (setting for simplicity $\varphi_{i_{1} \ldots i_{j}}=X^{\left(e_{i_{1}}\right)} \cdots X^{\left(e_{i_{j}}\right)} \varphi$ )

$$
\begin{aligned}
& \stackrel{\mathscr{E}_{\left(X^{\left(e_{1}\right)}, \ldots X^{\left(e_{l}\right)}\right)}^{(l)}[\varphi]}{=} \sum_{\substack{\text { components } \\
\text { of } \mathcal{N}_{a f, \mathcal{M}}}} \sum_{j=0}^{l-1} \sum_{\left\{i_{1}, \ldots i_{j}\right\} \subset\{1, \ldots l\}} O\left(\tau^{-(l-j-1)-\delta 0}\right) \\
& \quad \times\left\{O\left(r^{-1}\right) \partial_{v}^{2}\left(\varphi_{i_{1} \ldots i_{j}}\right)+O\left(r^{-2}\right) \partial_{v} \partial_{\sigma}\left(\varphi_{i_{1} \ldots i_{j}}\right)\right. \\
& \quad+O\left(r^{-3}\right) \partial_{\sigma} \partial_{\sigma}\left(\varphi_{i_{1} \ldots i_{j}}\right)+O\left(r^{-1-a}\right) \partial_{u} \partial_{v}\left(\varphi_{i_{1} \ldots i_{j}}\right) \\
& \quad+O\left(r^{-2-a}\right) \partial_{u} \partial_{\sigma}\left(\varphi_{i_{1} \ldots i_{j}}\right)+O\left(r^{-2-a}\right) \partial_{u}^{2}\left(\varphi_{i_{1} \ldots i_{j}}\right) \\
& \left.\quad+O\left(r^{-1-a}\right) \partial_{v}\left(\varphi_{i_{1} \ldots i_{j}}\right)+O\left(r^{-2-a}\right) \partial_{\sigma}\left(\varphi_{i_{1} \ldots i_{j}}\right)+O\left(r^{-2-a}\right) \partial_{u}\left(\varphi_{i_{1} \ldots i_{j}}\right)\right\} .
\end{aligned}
$$

Proof The relations (9.63) and (9.64) follow readily by differnetiating the expression for the wave equation

$$
\square \varphi=g^{\mu \nu} \partial_{\mu} \partial_{\nu} \varphi+\frac{1}{\sqrt{-\operatorname{det}(g)}} \partial_{\mu}\left(\sqrt{-\operatorname{det}(g)} \cdot g^{\mu \nu}\right) \partial_{\nu} \varphi
$$

with respect to $T$ and $K_{R_{C}}$, using Assumption (EG5) on the almost Killing vector fields $T, K$ and the relation $K_{R_{c}}=\chi_{R_{c}} K+\left(1-\chi_{R_{c}}\right) T$.

The error term obtained from the commutation of $\square$ with $K_{R_{C}}$ on the region $r \sim R_{C}$ (where $K_{R_{c}}$ fails to be almost Killing) will be controlled with the use of suitable elliptic estimates. In particular, we will establish the following Lemma:

Lemma 9.4 For any $l \in \mathbb{N}$ with $l \leq\left\lfloor\frac{d+1}{2}\right\rfloor$, any $n_{0} \in \mathbb{N}$, any $\beta \in\left(-\bar{\delta}_{n_{0}}, 1\right)$ (for some $\bar{\delta}_{n_{0}}>0$ depending on $\left.n_{0}\right)$ and any $0<\varepsilon \ll 1-\beta$, if $R_{c}$ is large in terms of $\beta$, $\varepsilon$ and the geometry of $(\mathcal{M}, g)$ we can bound for any $\varphi \in C^{\infty}(\mathcal{M})$ satisfying for all $j_{1}+j_{2} \leq$ $l+n_{0}$ the finite radiation field condition $\lim \sup _{r \rightarrow+\infty}\left|r^{\frac{d-1}{2}+j_{1}} \nabla_{h_{\tau, N}}^{j_{1}}\left(N^{j_{1}} \varphi\right)\right|_{h_{\tau, N}}<$ $+\infty$ :

$$
\begin{aligned}
& \sum_{n=0}^{n_{0}} \sum_{j=0}^{l-3} \int_{\{\bar{t}=\tau\}} r_{+}^{-\beta-2 j}\left|\nabla_{h_{\tau}, N}^{n+l-j} \varphi\right|_{\left(1-\log \left(r_{t i m}\right)\right) \cdot h_{R_{c}}}^{2} d h_{N} \\
& \quad+\sum_{n=0}^{n_{0}} \sum_{j=1}^{l} \int_{\{\bar{t}=\tau\} \cap\left\{R_{c} \leq r \leq 2 R_{c}\right\}} r_{+}^{2(j-1)-\beta+2 \varepsilon}\left|\nabla_{g}^{j+n} \varphi\right|_{h}^{2} d h_{N}
\end{aligned}
$$




$$
\begin{aligned}
\leq & C_{\beta, n_{0}}\left(R_{c}\right) \int_{\{\bar{t}=\tau\}} r_{+}^{-\beta}\left\{\sum_{n=0}^{n_{0}}\left|\nabla_{h_{\tau, N}}^{n+l-2}(\square \varphi)\right|_{\left(1-\log \left(r_{t i m}\right)\right) \cdot h_{\tau, N}}^{2}+\mathcal{T}_{T, K, R_{c}}^{\left(l, n_{0}\right)}[\varphi]\right\} d h_{N} \\
& +C_{\beta, n_{0}}\left(R_{c}\right) \sum_{j=0}^{1} \max \left\{-\operatorname{Re}\left\{\int_{\partial_{\text {tim }} \mathcal{M}^{\tau}} h_{\partial_{\text {tim }} \mathcal{M}^{\tau}}\left(\nabla_{h_{\partial_{\text {tim }} \mathcal{M}^{\tau}}^{j}}(Y \varphi), \nabla_{h_{\partial_{\text {tim }}} \mathcal{M}^{\tau}}^{j} \bar{\varphi}\right) d h_{\left.\left.\partial_{\text {tim }} \mathcal{M}^{\tau}\right\}, 0\right\},}\right.\right.
\end{aligned}
$$

where $\mathcal{T}_{T, K, R_{C}}^{(l, k)}[\varphi]$ is given by (9.17).

Remark From now on, we will assume that $R_{c}$ has been fixed large in terms of $\beta$ and the geometry of $(\mathcal{M}, g)$. Hence, we will drop the dependence of constants on $R_{c}$ (replacing it with dependence on the parameters on which $\beta$ will depend on).

Proof Inequality (9.67) follows readily after adding (9.26) and (9.27) (for $4 \varepsilon$ in place of $\varepsilon$ ), and absorbing the last term of the right hand side of (9.27) into the left hand side of (9.26), which can be done provided $R_{c}$ has been fixed large in terms of $\beta, R_{0}$ and $0<\varepsilon \ll 1-\beta$. Recall that in view of Assumption (EG6), the constants in the right hand sides of (9.26) and (9.27) do not depend on $\tau$.

\subsection{Integrated Local Energy Decay After Commuting with $T, K_{R_{c}}$}

Let us fix a vector field $L$ on $\mathcal{M}$ such that $[L, T]=0$ everywhere, $L \equiv 0$ in the region $\left\{r \leq 2 R_{c}\right\}$ and $L \equiv \partial_{v}$ in the coordinate chart $(\bar{t}, v, \sigma)$ on each connected component of the region $\left\{r \geq 2 R_{c}+1\right\}$.

Lemma 9.5 For any $l \in \mathbb{N}$ with $l \leq\left\lfloor\frac{d+1}{2}\right\rfloor$, and any $0<\varepsilon<1$, if $R_{c}$ is large in terms of $\varepsilon$ and the geometry of $(\mathcal{M}, g)$ we can bound for any integer $m \geq 1$, any $0 \leq \tau_{1} \leq \tau_{2}$ and any $\varphi \in C^{\infty}(\mathcal{M})$ solving $\square \varphi=F$ satisfying boundary conditions on $\partial_{\text {tim }} \overline{\mathcal{M}}$ belonging to the class $\mathcal{C}_{I L E D}^{(T, K)}$ and such that

$$
\begin{aligned}
& \operatorname{Re}\left\{\int_{\partial_{\text {tim }} \mathcal{M}^{\tau}} Y \varphi \cdot \bar{\varphi} d h_{\left.\partial_{\text {tim }} \mathcal{M}^{\tau}\right\}}\right\} 0 \text { and }
\end{aligned}
$$

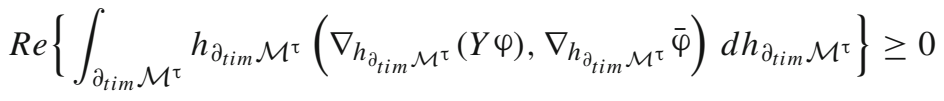

(provided (7.2) holds for all $0 \leq j \leq m+l+k+\left\lceil\frac{d}{2}\right\rceil$ ):

$$
\begin{aligned}
& \sum_{j=0}^{m-1} \int_{\left\{\tau_{1} \leq \bar{t} \leq \tau_{2}\right\}}\left(\sum_{j_{2}+j_{3}=l+j-1} r_{+}^{-1+\varepsilon}\left|\nabla_{h_{\tau, N}}^{j_{2}+1}\left(N^{j_{3}} \varphi\right)\right|_{h_{\tau, N}}^{2}\right. \\
& \left.\quad+\sum_{1 \leq j_{2}+j_{3} \leq l+j-1} r_{+}^{-1+\varepsilon-2\left(l+j-j_{2}-j_{3}\right)}\left|\nabla_{h_{\tau, N}}^{j_{2}}\left(N^{j_{3}} \varphi\right)\right|_{h_{\tau, N}}^{2}+r_{+}^{-1-\varepsilon}\left|N^{l+j} \varphi\right|^{2}\right) d g \\
& \quad+\sum_{j=0}^{m-1} \sum_{1 \leq j_{2}+j_{3} \leq l+j-1} \int_{\left\{\bar{t}=\tau_{2}\right\}} r_{+}^{-2\left(l+j-1-j_{2}-j_{3}\right)}\left(r_{+}^{\varepsilon}\left|\mathcal{L}_{L} \nabla_{h_{\tau, N}}^{j_{2}}\left(N^{j_{3}} \varphi\right)\right|^{2}\right.
\end{aligned}
$$




$$
\begin{aligned}
& \left.+\left|\nabla_{h_{\tau, N}}^{j_{2}+1}\left(N^{j_{3}} \varphi\right)\right|_{h_{\tau, N}}^{2}+r_{+}^{-2}\left|N^{l+j} \varphi\right|^{2}\right) d h_{N} \\
& +\sum_{j=0}^{m-1} \sum_{1 \leq j_{2}+j_{3} \leq l+j-1} \int_{\mathcal{H}^{+}\left(\tau_{1}, \tau_{2}\right)}\left|\nabla_{h_{\mathcal{H}}}^{j_{2}+1}\left(K_{R_{c}}^{j_{3}} \varphi\right)\right|_{h_{\mathcal{H}}}^{2} d h_{\mathcal{H}} \\
& \leq C_{\varepsilon, m}\left\{\sum_{j=0}^{k+m-1} \sum_{1 \leq j_{2}+j_{3} \leq l+j-1} \int_{\left\{\bar{t}=\tau_{1}\right\}} r_{+}^{\varepsilon-2\left(l+j-1-j_{2}-j_{3}\right)}\right. \\
& \times\left(\left|\nabla_{h_{\tau, N}}^{j_{2}+1}\left(N^{j_{3}} \varphi\right)\right|_{h_{\tau, N}}^{2}+r_{+}^{-2}\left|N^{l+j} \varphi\right|^{2}\right) d h_{N} \\
& \left.+\tau_{1}^{-\delta 0} \int_{\left\{\tau_{1} \leq \bar{t} \leq \tau_{2}\right\}}^{r_{+}^{-1+\varepsilon}} \mathcal{T}_{T, K, R_{c}, s l}^{(l, m+k-1)}[\varphi] d g+\sum_{j=l-1}^{m+k+l-2} \int_{\left\{\tau_{1} \leq \bar{t} \leq \tau_{2}\right\}} r_{+}^{1+\varepsilon}\left|\nabla_{g}^{j} F\right|_{h}^{2} d g\right\}
\end{aligned}
$$

(where $\mathcal{L}_{L}$ denotes the Lie derivative in the direction of the outgoing vector field $L$ ) and

$$
\begin{aligned}
\sum_{j=0}^{m-1} & \sum_{1 \leq j_{2}+j_{3} \leq l+j-1} \int_{\left\{\bar{t}=\tau_{2}\right\}} r_{+}^{-2\left(l+j-1-j_{2}-j_{3}\right)} \\
& \times\left(\left|\nabla_{h_{\tau, N}}^{j_{2}+1}\left(K_{R_{c}}^{j_{3}} \varphi\right)\right|_{\left(1-\log \left(r_{t i m}\right)\right) \cdot h_{R_{c}}}^{2}+r_{+}^{-2}\left|K_{R_{c}}^{l+j} \varphi\right|^{2}\right) d h_{N} \\
\leq & C_{\varepsilon, m}\left\{\sum_{j=0}^{k+m-1} \sum_{1 \leq j_{2}+j_{3} \leq l+j-1} \int_{\left\{\bar{t}=\tau_{1}\right\}} r_{+}^{-2\left(l+j-1-j_{2}-j_{3}\right)}\right. \\
& \times\left(\left|\nabla_{h_{\tau, N}}^{j_{2}+1}\left(N^{j_{3}} \varphi\right)\right|_{h_{\tau, N}}^{2}+r_{+}^{-2}\left|N^{l+j} \varphi\right|^{2}\right) d h_{N} \\
& +\tau_{1}^{-\delta 0} \sum_{j=0}^{m-1} \sum_{1 \leq j_{2}+j_{3} \leq l+j-1} \int_{\left\{\bar{t}=\tau_{1}\right\}} r_{+}^{\varepsilon-2\left(l+j-1-j_{2}-j_{3}\right)} \\
& \times\left(\left|\nabla_{h_{\tau, N}}^{j_{2}+1}\left(N^{j_{3}} \varphi\right)\right|_{h_{\tau, N}}^{2}+r_{+}^{-2}\left|N^{l+j} \varphi\right|^{2}\right) d h_{N} \\
& \left.+\tau_{1}^{-\delta 0} \int_{\left\{\tau_{1} \leq \bar{t} \leq \tau_{2}\right\}}^{r_{+}^{-1+\varepsilon}} \mathcal{T}_{T, K, R_{c}, s l}^{(l, m+k-1)}[\varphi] d g+\sum_{j=l-1}^{m+k+l-2} \int_{\left\{\tau_{1} \leq \bar{t} \leq \tau_{2}\right\}} r_{+}^{1+\varepsilon}\left|\nabla_{g}^{j} F\right|_{h}^{2} d g\right\}
\end{aligned}
$$

In the above,

$$
\begin{aligned}
& \mathcal{T}_{T, K, R_{c}, s l}^{(l, m+k-1)}[\varphi] \\
& \doteq \mathcal{T}_{T, K, R_{c}}^{(l, m+k-1)}[\varphi]+\sum_{0 \leq i_{1}+i_{2} \leq m} \sum_{0 \leq j \leq l-2} \int_{\left\{\tau_{1} \leq \bar{t} \leq \tau_{2}\right\}}\left(r_{+}^{-2-2(l-2-j)}\right.
\end{aligned}
$$




$$
\begin{aligned}
& \left.+r_{+}^{-2} \tau^{-2(l-2-j)}\right)\left|\nabla_{h_{\tau, N}}^{j+i_{1}+1}\left(K_{R_{c}}^{i_{2}} \varphi\right)\right|_{h_{\tau, N}}^{2} d g \\
& +\sum_{0 \leq i_{1}+i_{2} \leq m} \sum_{0 \leq j_{1}+j_{2} \leq l-2} \int_{\left\{\tau_{1} \leq \bar{t} \leq \tau_{2}\right\}} r_{+}^{-2} \tau^{-2\left(l-j_{1}-j_{2}-2\right)}\left(\left|\nabla_{h_{\tau, N}}^{j_{1}+i_{1}} \varphi\right|_{T, K, R_{c}}^{\left(j_{2}+i_{2}+1\right)}\right)^{2} d g .
\end{aligned}
$$

Remark Notice that in comparison to (9.69), the left hand side of (9.70) controls only a degenerate energy norm of $\varphi$ on $\left\{\bar{t}=\tau_{2}\right\}$ and no bulk terms. However, in the right hand side of (9.70) the dependence on the $r^{\varepsilon}$-weighted initial energy comes with a factor decaying polynomially in time.

Proof Without loss of generality, we will assume that $\tau_{1}$ is large in terms of $\varepsilon, m$. Moreover, we will assume that $\varphi$ is real valued.

Recall that according to Assumption (G8), in each connected component of the region $\{r \gg 1\}$ we have:

$$
\begin{aligned}
\mathcal{L}_{T} g= & O\left(\tau^{-\delta 0}\right)\left\{O\left(r^{-1-a}\right) d v d u+O(r) d \sigma d \sigma+O(1) d u d \sigma\right. \\
& \left.+O\left(r^{-a}\right) d v d \sigma+O\left(r^{-1}\right) d u^{2}+O\left(r^{-2-a}\right) d v^{2}\right\} .
\end{aligned}
$$

Thus, in view of the integrated local energy decay assumption (ILED2) (which is satisfied in view of our assumption that $\varphi$ has boundary conditions on $\partial_{\text {tim }} \mathcal{M}$ belonging to the class $\mathcal{C}_{I L E D}^{(T, K)}$ ) and Lemma 4.1 in the region $\{r \gg 1\}$ (see the remark below that Lemma for the case when $T$ has deformation tensor with slow polynomial decay in $\bar{t})$, we can bound for any $\left(e_{1}, \ldots e_{l-1}\right) \in\{0,1\}^{l-1}$ and any $R_{f}>0$ to be fixed later $\left(\right.$ setting $X^{(0)}=T$ and $\left.X^{(1)}=K_{R_{c}}\right)$ :

$$
\begin{aligned}
& \sum_{j=1}^{m} \int_{\left\{\tau_{1} \leq \bar{t} \leq \tau_{2}\right\}} r_{+}^{-1-\varepsilon}\left(\left|\nabla_{g}^{j}\left(\mathcal{L}_{X^{\left(e_{1}\right)} \ldots X^{\left(e_{l-1}\right)}} \varphi\right)\right|_{h}^{2}\right. \\
& \quad+r_{+}^{-2} \mid \mathcal{L}_{\left.\left.X^{\left(e_{1}\right)} \ldots X^{\left(e_{l-1}\right)} \varphi\right|^{2}\right) d g} \\
& \quad+\sum_{j=0}^{m} \sum_{j_{1}+j_{2}=j} \int_{\partial_{t i m} \mathcal{M} \cap\left\{\tau_{1} \leq \bar{t} \leq \tau_{2}\right\}}\left|\nabla_{g}^{j}\left(\mathcal{L}_{X^{\left(e_{1}\right)} \ldots X^{\left(e_{l-1}\right)}} \varphi\right)\right|_{h}^{2} d h_{\partial_{t i m} \mathcal{M}} \\
& \leq C_{\varepsilon, m, R_{f}} \sum_{j=0}^{m+k-1} \sum_{j_{1}+j_{2}=j} \int_{\left\{\bar{t}=\tau_{1}\right\}}\left(\mid \nabla_{h_{N}}^{j_{1}+1}\left(N^{j_{2}} \mathcal{L}_{\left.\left.X^{\left(e_{1}\right)} \ldots X^{\left(e_{l-1}\right)} \varphi\right)\left.\right|_{h_{N}} ^{2}\right)}\right.\right. \\
& \quad+r_{+}^{-2}\left|N^{j+1} \mathcal{L}_{X^{\left(e_{1}\right)} \ldots X^{\left(e_{l-1}\right)}} \varphi\right|^{2} d h_{N}{ }^{m-1} \sum_{j=0} \sum_{j_{1}+j_{2}=j} \int_{\left\{\tau_{1} \leq \bar{t} \leq \tau_{2}\right\} \cap\{r \gtrsim 1\}}{ }^{-1}\left(\left|\nabla_{h_{\tau, N}}^{1+j_{1}}\left(T^{j_{2}}\left(\mathcal{L}_{X^{\left(e_{1}\right)} \ldots X^{\left(e_{l-1}\right)}} \varphi\right)\right)\right|_{h_{\tau, N}}^{2}\right.
\end{aligned}
$$




$$
\begin{aligned}
& \left.+r_{+}^{-2}\left(\left|T^{m}\left(\mathcal{L}_{X^{\left(e_{1}\right)} \ldots X^{\left(e_{l-1}\right)}} \varphi\right)\right|^{2}+\left|\mathcal{L}_{X^{\left(e_{1}\right)} \ldots X^{\left(e_{l-1}\right)}} \varphi\right|^{2}\right)\right) d g \\
& +C_{\varepsilon, m, R_{f}} \sum_{j=0}^{k+m-1} \int_{\left\{\tau_{1} \leq \bar{t} \leq \tau_{2}\right\}} r_{+}^{1+\varepsilon}\left|\nabla_{g}^{j}\left(\square \mathcal{L}_{X^{\left(e_{1}\right)} \ldots X^{\left(e_{l-1}\right)}} \varphi\right)\right|_{h}^{2} d g .
\end{aligned}
$$

Using Lemma 8.3 for $\mathcal{L}_{X^{\left(e_{1}\right)} \ldots X^{\left(e_{l-1}\right)}} \varphi$ in place of $\varphi$ (and adapting the proof a bit so as to use (9.73) instead of simply (8.19) and repeating the proof of Lemma 4.1), we obtain the following energy boundedness statement for any $\gamma_{0}>0$ to be fixed small later:

$$
\begin{aligned}
& \sum_{j=0}^{m-1} \sum_{j_{1}+j_{2}=j} \int_{\left\{\bar{t}=\tau_{2}\right\}}\left(\left|\nabla_{h_{N}}^{j_{1}+1}\left(N^{j_{2}} \mathcal{L}_{X^{\left(e_{1}\right)} \ldots X^{\left(e_{l-1}\right)}} \varphi\right)\right|_{h_{N}}^{2}+r_{+}^{-2}\left|N^{j+1} \mathcal{L}_{X^{\left(e_{1}\right)} \ldots X^{\left(e_{l-1}\right)}} \varphi\right|^{2}\right) d h_{N} \\
& \leq C_{m, \varepsilon}\left\{\sum_{j=0}^{m+k-1} \sum_{j_{1}+j_{2}=j} \int_{\left\{\bar{t}=\tau_{1}\right\}}\left(\left|\nabla_{h_{N}}^{j_{1}+1}\left(N^{j_{2}} \mathcal{L}_{X^{\left(e_{1}\right)} \ldots X^{\left(e_{l-1}\right)}} \varphi\right)\right|_{h_{N}}^{2}+r_{+}^{-2}\left|N^{j+1} \mathcal{L}_{X^{\left(e_{1}\right)} \ldots X^{\left(e_{l-1}\right)}} \varphi\right|^{2}\right) d h_{N}\right. \\
& +\tau_{1}^{-\delta 0} \sum_{j=0}^{m-1} \sum_{j_{1}+j_{2}=j} \int_{\left\{\tau_{1} \leq \bar{t} \leq \tau_{2}\right\}} r_{+}^{-1+\varepsilon} \\
& \times\left(\left|\nabla_{h_{N}}^{j_{1}+1}\left(N^{j_{2}} \mathcal{L}_{X^{\left(e_{1}\right)} \ldots X^{\left(e_{l-1}\right)}} \varphi\right)\right|_{h_{N}}^{2}+r_{+}^{-2}\left(\left|N^{j+1}\left(\mathcal{L}_{X^{\left(e_{1}\right)} \ldots X^{\left(e_{l-1}\right)}} \varphi\right)\right|^{2}\right.\right. \\
& \left.\left.\left.+\left|\mathcal{L}_{X^{\left(e_{1}\right)} \ldots X^{\left(e_{l-1}\right)}} \varphi\right|^{2}\right)\right) d g+\sum_{j=0}^{m+k-1} \int_{\left\{\tau_{1} \leq \bar{t} \leq \tau_{2}\right\}} r_{+}^{1+\varepsilon}\left|\nabla_{g}^{j}\left(\square \mathcal{L}_{X^{\left(e_{1}\right)} \ldots X^{\left(e_{l}-1\right)}} \varphi\right)\right|_{h}^{2} d g\right\} .
\end{aligned}
$$

Fixing some $\delta, \eta>0$ small in terms of $\varepsilon$, we obtain from (5.1) for $p=\varepsilon$ and for $T^{j} \varphi$ in place of $\varphi$ for $j=l-1, \ldots m+l-2$ :

$$
\begin{aligned}
& \sum_{j=l-1}^{m+l-2} \int_{\left\{\tau_{1} \leq \bar{t} \leq \tau_{2}\right\} \cap\left\{r \geq 2 R_{c}\right\}}\left(r_{+}^{-1+\varepsilon}\left|\nabla_{h_{\tau, N}}\left(T^{j} \varphi\right)\right|_{h_{\tau, N}}^{2}+r_{+}^{-1-\varepsilon}\left|T^{j+1} \varphi\right|^{2}+r_{+}^{-3+\varepsilon}\left|T^{j} \varphi\right|^{2}\right) d g \\
& +\sum_{j=l-1}^{m+l-2} \int_{\left\{\bar{t}=\tau_{2}\right\} \cap\left\{r \geq 2 R_{c}\right\}} r_{+}^{\varepsilon}\left(\left|L\left(T^{j} \varphi\right)\right|^{2}+r_{+}^{-2}\left|\nabla_{h_{\tau, N}}\left(T^{j} \varphi\right)\right|_{h_{\tau, N}}^{2}+r_{+}^{-2}\left|T^{j} \varphi\right|^{2}\right) d h_{N} \\
& \leq C_{\varepsilon, m}\left\{\sum_{j=l-1}^{m+l-2} \int_{\left\{\bar{t}=\tau_{1}\right\} \cap\left\{r \geq R_{c}\right\}}\left(r_{+}^{\varepsilon}\left|\nabla_{h_{\tau, N}}\left(T^{j} \varphi\right)\right|_{h_{\tau, N}}^{2}+r_{+}^{-2}\left|T^{j+1} \varphi\right|^{2}+r_{+}^{-2+\varepsilon}\left|T^{j} \varphi\right|^{2}\right) d h_{N}\right. \\
& +\sum_{j=l-1}^{m+l-2} \int_{\left\{\tau_{1} \leq \bar{t} \leq \tau_{2}\right\} \cap\left\{R_{c} \leq r \leq 2 R_{c}\right\}} r_{+}^{-1+\varepsilon}\left(\left|\nabla_{h_{\tau, N}}\left(T^{j} \varphi\right)\right|_{h_{\tau, N}}^{2}+\left|T^{j+1} \varphi\right|^{2}+r_{+}^{-2}\left|T^{j} \varphi\right|^{2}\right) d g \\
& \left.+\sum_{j=l-1}^{m+l-2} \int_{\left\{\tau_{1} \leq \bar{t} \leq \tau_{2}\right\}} r_{+}^{1+\varepsilon}\left|\square\left(T^{j} \varphi\right)\right|^{2} d g\right\} .
\end{aligned}
$$


Using the elliptic estimate (9.67) repeatedly together with the expression (9.64) for the commutation of $\square$ with $\mathcal{L}_{X^{\left(e_{i}\right)}}$ at each step (notice that Theorem 7.1 applies to yield $\lim \sup _{r \rightarrow+\infty}\left|r^{\frac{d-1}{2}+j_{1}} \nabla_{h_{\tau, N}}^{j_{1}}\left(T^{j_{2}} \varphi\right)\right|_{h_{\tau, N}}<+\infty$ for all $\left.j_{1}+j_{2} \leq l+m+k\right)$, in view of the condition (9.68) for $\varphi$ at $\partial_{\text {tim }} \mathcal{M}$, we can bound after summing over all possible combinations of $\left(e_{1}, \ldots e_{l-1}\right) \in\{0,1\}^{l-1}$ :

$$
\begin{aligned}
& \sum_{0 \leq j_{1}+j_{2} \leq m-1} \sum_{0 \leq i_{1}+i_{2} \leq l-1} \int_{\left\{\tau_{1} \leq \bar{t} \leq \tau_{2}\right\}}\left(r_{+}^{-1+\varepsilon-2\left(l-1-i_{1}-i_{2}\right)}\left|\nabla_{h_{\tau, N}}^{i_{1}+j_{1}+1}\left(K_{R_{c}}^{i_{2}+j_{2}} \varphi\right)\right|_{\left(1-\log \left(r_{t i m}\right)\right) \cdot h_{R_{c}}}^{2}\right. \\
& \left.+r_{+}^{-1-\varepsilon}\left|K_{R}^{l+j_{1}+j_{2}} \varphi\right|^{2}\right) d g \\
& \leq \sum_{\left(e_{1}, \ldots e_{l-1}\right) \in\{0,1\}^{l-1}}\left\{\sum_{j=1}^{m} \int_{\left\{\tau_{1} \leq \bar{t} \leq \tau_{2}\right\}} r_{+}^{-1-\varepsilon}\left(\left|\nabla_{g}^{j}\left(\mathcal{L}_{X^{\left(e_{1}\right)} \ldots X^{\left(e_{l-1}\right)}} \varphi\right)\right|_{h}^{2}+r_{+}^{-2}\left|\mathcal{L}_{X^{\left(e_{1}\right)} \ldots X^{\left(e_{l}-1\right)}} \varphi\right|^{2}\right) d g\right\} \\
& +C_{\varepsilon, m} \sum_{j=l-1}^{m+l-2} \int_{\left\{\tau_{1} \leq \bar{t} \leq \tau_{2}\right\} \cap\left\{r \geq 2 R_{c}\right\}} r_{+}^{-1+\varepsilon}\left(\left|\nabla_{h_{\tau, N}}\left(T^{j} \varphi\right)\right|_{h_{\tau, N}}^{2}+r_{+}^{-2}\left|T^{j} \varphi\right|^{2}\right) d g \\
& +C_{\varepsilon, m} \sum_{0 \leq i_{1}+i_{2} \leq m-1} \sum_{0 \leq j \leq l-2} \int_{\left\{\tau_{1} \leq \bar{t} \leq \tau_{2}\right\}} r_{+}^{-1+\varepsilon}\left(r_{+}^{-2-2(l-2-j)} \tau^{-2 \delta 0}\right. \\
& \left.+r_{+}^{-2} \tau^{-2\left(\delta_{0}+l-2-j\right)}\right)\left|\nabla_{h_{\tau, N}}^{j+i_{1}+1}\left(K_{R_{c}}^{i_{2}} \varphi\right)\right|_{h_{\tau, N}}^{2} d g \\
& +C_{\varepsilon, m} \sum_{0 \leq i_{1}+i_{2} \leq m-1} \sum_{0 \leq j_{1}+j_{2} \leq l-2} \int_{\left\{\tau_{1} \leq \bar{t} \leq \tau_{2}\right\}} r_{+}^{-3+\varepsilon} \tau^{-2\left(\delta_{0}+l-j_{1}-j_{2}-2\right)}\left(\left|\nabla_{h_{\tau, N}}^{j_{1}+i_{1}} \varphi\right|_{T, K, R_{c}}^{\left(j_{2}+i_{2}+1\right)}\right)^{2} d g \\
& +C_{\varepsilon, m} \sum_{j=l-2}^{m+l-3} \int_{\left\{\tau_{1} \leq \bar{\tau} \leq \tau_{2}\right\}} r_{+}^{-1+\varepsilon}\left|\nabla_{g}^{j} F\right|_{h}^{2} d g,
\end{aligned}
$$

and

$$
\begin{aligned}
& \quad \sum_{0 \leq j_{1}+j_{2} \leq m-1} \sum_{0 \leq i_{1}+i_{2} \leq l-1} \int_{\left\{\bar{t}=\tau_{2}\right\}} r_{+}^{-2\left(l-1-i_{1}-i_{2}\right)}\left(\left|\nabla_{h_{\tau}, N}^{i_{1}+j_{1}+1}\left(K_{R_{c}}^{i_{2}+j_{2}} \varphi\right)\right|_{\left(1-\log \left(r_{t i m}\right)\right) \cdot h_{R_{c}}}^{2}\right. \\
& \left.+r_{+}^{-2}\left|K_{R_{c}}^{l+j} \varphi\right|^{2}\right) d h_{N} \\
& \leq C_{m} \sum_{\left(e_{1}, \ldots e_{l-1}\right) \in\{0,1\}^{l-1}}\left\{\sum _ { j = 0 } \sum _ { j _ { 1 } + j _ { 2 } = j } \int _ { \{ \overline { t } = \tau _ { 2 } \} } \left(\left|\nabla_{h_{N}}^{j_{1}+1}\left(N^{j_{2}} \mathcal{L}_{X^{\left(e_{1}\right)} \ldots X^{\left(e_{l}-1\right)}} \varphi\right)\right|_{h_{N}}^{2}\right.\right. \\
& \left.\left.+r_{+}^{-2}\left|N^{j} \mathcal{L}_{X^{\left(e_{1}\right)} \ldots X^{\left(e_{l-1}\right)}} \varphi\right|^{2}\right) d h_{N}\right\} \\
& +C_{m} \sum_{0 \leq i_{1}+i_{2} \leq m-1} \sum_{0 \leq j \leq l-2} \int_{\left\{\bar{t}=\tau_{2}\right\}}\left(r_{+}^{-2-2(l-2-j)} \tau^{-2 \delta_{0}}+r_{+}^{-2} \tau^{-2\left(\delta_{0}+l-2-j\right)}\right) \\
& \quad \times\left.\nabla_{h_{\tau, N}}^{j+i_{1}+1}\left(K_{R_{c}}^{i_{2}} \varphi\right)\right|_{h_{\tau, N}} ^{2} d h_{N} \\
& +C_{m} \sum_{0 \leq i_{1}+i_{2} \leq m-1} \sum_{0 \leq j_{1}+j_{2} \leq l-2} \int_{\left\{\bar{t}=\tau_{2}\right\}} r_{+}^{-2} \tau^{-2\left(\delta 0+l-j_{1}-j_{2}-2\right)}\left(\left|\nabla_{h_{\tau, N}}^{j_{1}+i_{1}} \varphi\right|_{T, K, R_{c}}^{\left(j_{2}+i_{2}+1\right)}\right)^{2} d h_{N} \\
& +C_{m} \sum_{j=l-2}^{m+l-3} \int_{\left\{\bar{t}=\tau_{2}\right\}}\left|\nabla_{g}^{j} F\right|_{h}^{2} d h_{N} .
\end{aligned}
$$


Moreover, using the expression (3.14) for the wave equation in the region $\{r \gg 1\}$ and Lemma 6.3 to estimate the commutator of $\square$ with $L$ (and recalling that $L$ is supported in the region $\left\{r \geq 2 R_{c}\right\}$ ), we can also bound in view of Lemma 9.4:

$$
\begin{aligned}
& \sum_{j=0}^{m-1} \sum_{1 \leq j_{2}+j_{3} \leq l+j-1} \int_{\left\{\bar{t}=\tau_{2}\right\}} r_{+}^{\varepsilon-2\left(l+j-1-j_{2}-j_{3}\right)}\left|\mathcal{L}_{L} \nabla_{h_{\tau, N}}^{j_{2}}\left(K_{R_{c}}^{j_{3}} \varphi\right)\right|_{\left(1-\log \left(r_{t i m}\right)\right) \cdot h_{R_{c}}}^{2} d h_{N} \\
& \leq C_{\varepsilon, m}\left\{\sum _ { j = l - 1 } ^ { m + l - 2 } \int _ { \{ \overline { t } = \tau _ { 2 } \} \cap \{ r \geq 2 R _ { c } \} } r _ { + } ^ { \varepsilon } \left(\left|L\left(T^{j} \varphi\right)\right|^{2}+r_{+}^{-2}\left|\nabla_{h_{\tau, N}}\left(T^{j} \varphi\right)\right|_{h_{\tau, N}}^{2}+r_{+}^{-2-\varepsilon}\left|T^{j+1} \varphi\right|^{2} d h_{N}\right.\right. \\
& \quad+\sum_{\left(e_{1}, \ldots e_{l-1}\right) \in\{0,1\}^{l-1}} \sum_{j=0}^{m-1} \sum_{j_{1}+j_{2}=j} \int_{\left\{\bar{t}=\tau_{2}\right\}}\left(\left|\nabla_{h_{N}}^{j_{1}+1}\left(N^{j_{2}} \mathcal{L}_{X^{\left(e_{1}\right)} \ldots X^{\left(e_{l-1}\right)}} \varphi\right)\right|_{h_{N}}^{2}\right. \\
& +r_{+}^{-2} \mid N^{j} \mathcal{L}_{\left.\left.X^{\left(e_{1}\right)} \ldots X^{\left(e_{l-1}\right)} \varphi\right|^{2}\right) d h_{N}} \\
& \quad+\sum_{0 \leq i_{1}+i_{2} \leq m-1} \sum_{0 \leq j \leq l-2} \int_{\left\{\bar{t}=\tau_{2}\right\}}\left(r_{+}^{\varepsilon-2-2(l-2-j)} \tau^{-2 \delta_{0}}+r_{+}^{\varepsilon-2} \tau^{-2\left(\delta_{0}+l-2-j\right)}\right) \\
& \quad \times\left|\nabla_{h_{\tau, N}}^{j+i_{1}+1}\left(K_{R_{c}}^{i_{2}} \varphi\right)\right|_{h_{\tau, N}}^{2} d h_{N} \\
& \quad+\sum_{0 \leq i_{1}+i_{2} \leq m-1} \sum_{m_{0 \leq j}+j_{2} \leq l-2} \int_{\left\{\bar{t}=\tau_{2}\right\}} r_{+}^{\varepsilon-2} \tau^{-2\left(\delta_{0}+l-j_{1}-j_{2}-2\right)}\left(\left|\nabla_{h_{\tau, N}}^{j_{1}+i_{1}} \varphi\right|_{T, K, R_{c}}^{\left(j_{2}+i_{2}+1\right)}\right)^{2} d h_{N} \\
& \left.\quad+\sum_{j=l-2}^{m+l-3} \int_{\left\{\bar{t}=\tau_{2}\right\}} r_{+}^{\varepsilon}\left|\nabla_{g}^{j} F\right|_{h}^{2} d h_{N}\right\} .
\end{aligned}
$$

Therefore, fixing $R_{f}$ large enough in terms of $\varepsilon, m$, after adding (for all possible combinations of $\left.\left(e_{1}, \ldots e_{l-1}\right) \in\{0,1\}^{l-1}\right)$ the estimates (9.73), (9.74) and a small multiple (in terms of $\varepsilon, m$ ) of (9.75), and using the expression (9.64) for the commutation of $\square$ with $\mathcal{L}_{X^{\left(e_{i}\right)}}$, we obtain in view of (9.76), (9.77) and (9.78) as well as a trace-type inequality for the terms $\int_{\left\{\bar{t}=\tau_{2}\right\}}\left|\nabla^{j} F\right|^{2}, \int_{\left\{\bar{t}=\tau_{2}\right\}}\left|\nabla_{h_{\tau, N}}^{j+i_{1}+1}\left(K_{R_{c}}^{i_{2}} \varphi\right)\right|_{h_{\tau, N}}^{2}$ and $\int_{\left\{\bar{t}=\tau_{2}\right\}}\left(\left|\nabla_{h_{\tau, N}}^{j_{1}+i_{1}} \varphi\right|_{T, K, R_{c}}^{\left(j_{2}+i_{2}+1\right)}\right)^{2}$ (and recalling that $\tau_{1}$ was assumed to be large in terms of $\varepsilon, m)$ :

$$
\begin{aligned}
& \sum_{j=0}^{m-1} \int_{\left\{\tau_{1} \leq \bar{t} \leq \tau_{2}\right\}}\left(\sum_{j_{2}+j_{3}=l+j-1} r_{+}^{-1+\varepsilon}\left|\nabla_{h_{\tau, N}}^{j_{2}+1}\left(K_{R_{c}}^{j_{3}} \varphi\right)\right|_{\left(1-\log \left(r_{\text {tim }}\right)\right) \cdot h_{R_{c}}}^{2}\right. \\
& \left.+\sum_{1 \leq j_{2}+j_{3} \leq l+j-1} r_{+}^{-1+\varepsilon-2\left(l+j-j_{2}-j_{3}\right)}\left|\nabla_{h_{\tau, N}}^{j_{2}}\left(K_{R_{c}}^{j_{3}} \varphi\right)\right|_{\left(1-\log \left(r_{\text {tim }}\right)\right) \cdot h_{R_{c}}}^{2}+r_{+}^{-1-\varepsilon}\left|K_{R_{c}}^{l+j} \varphi\right|^{2}\right) d g \\
& \quad+\sum_{j=0}^{m-1} \sum_{1 \leq j_{2}+j_{3} \leq l+j-1} \int_{\left\{\bar{t}=\tau_{2}\right\}} r_{+}^{-2\left(l+j-1-j_{2}-j_{3}\right)}\left(r^{\varepsilon}\left|\mathcal{L}_{L} \nabla_{h_{\tau, N}}^{j_{2}}\left(K_{R_{c}}^{j_{3}} \varphi\right)\right|_{\left(1-\log \left(r_{t i m}\right)\right) \cdot h_{R_{c}}}^{2}\right.
\end{aligned}
$$




$$
\begin{aligned}
& \left.+\left|\nabla_{h_{R_{c}}}^{j_{2}+1}\left(K_{R_{c}}^{j_{3}} \varphi\right)\right|_{\left(1-\log \left(r_{t i m}\right)\right) \cdot h_{R_{c}}}^{2}+r_{+}^{-2}\left|K_{R_{c}}^{l+j} \varphi\right|^{2}\right) d h_{N} \\
\leq & C_{\varepsilon, m}\left\{\sum_{j=0}^{k+m-1} \sum_{1 \leq j_{2}+j_{3} \leq l+j-1} \int_{\left\{\bar{t}=\tau_{1}\right\}} r_{+}^{\varepsilon-2\left(l+j-1-j_{2}-j_{3}\right)}\left(\left|\nabla_{h_{\tau}, N}^{j_{2}+1}\left(N^{j_{3}} \varphi\right)\right|_{h_{\tau, N}}^{2}+r_{+}^{-2}\left|N^{l+j} \varphi\right|^{2}\right) d h_{N}\right. \\
& \left.+\tau_{1}^{-\delta_{0}} \int_{\left\{\tau_{1} \leq \bar{t} \leq \tau_{2}\right\}} r_{+}^{-1+\varepsilon} \mathcal{T}_{T, K, R_{c}, s l}^{(l, m+k-1)}[\varphi] d g+\sum_{j=l-1}^{m+k+l-2} \int_{\left\{\tau_{1} \leq \bar{t} \leq \tau_{2}\right\}} r_{+}^{1+\varepsilon}\left|\nabla_{g}^{j} F\right|_{h}^{2} d g\right\}
\end{aligned}
$$

Let $r_{1}>0$ be the small constant appearing in (9.2), and fix a smooth cut-off function $\chi_{r_{1}}: \mathcal{M} \rightarrow[0,1]$ such that $\chi_{r_{1}}$ is a function of $r$ satisfying $\chi_{r_{1}} \equiv 1$ on $\left\{r \leq r_{1}\right\}$ and $\chi_{r_{1}} \equiv 0$ on $\left\{r \geq 2 r_{1}\right\}$. Integrating $\nabla^{\mu}\left(\chi_{r_{1}} J_{\mu}^{m+l}[\varphi]\right.$ ) over $\mathcal{M}$ (where $J_{\mu}^{m+l}[\varphi]$ is given by 8.40 ), and using the red shift estimate (9.2), we obtain:

$$
\begin{aligned}
& \int_{\mathcal{H}^{+}\left(\tau_{1}, \tau_{2}\right)} J_{\mu}^{m+l}[\varphi] n_{\mathcal{H}}^{\mu}+\int_{\left\{\bar{t}=\tau_{2}\right\} \cap\left\{r \leq r_{1}\right\}} J_{\mu}^{m+l}[\varphi] \bar{n}^{\mu}+\sum_{j=1}^{m+l-1} \int_{\left\{\tau_{1} \leq \bar{t} \leq \tau_{2}\right\} \cap\left\{r \leq r_{1}\right\}}\left|\nabla_{g}^{j} \varphi\right|_{h}^{2} d g \\
& \leq C_{m}\left\{\sum_{j=1}^{m+l-1} \int_{\left\{\bar{t}=\tau_{1}\right\} \cap\left\{r \leq 2 r_{1}\right\}}\left|\nabla_{g}^{j} \varphi\right|_{h}^{2} d h_{N}+\sum_{j=1}^{m+l-1} \int_{\left\{\tau_{1} \leq \bar{t} \leq \tau_{2}\right\} \cap\left\{r_{1} \leq r \leq 2 r_{1}\right\}}\left|\nabla_{g}^{j} \varphi\right|_{h}^{2} d g\right. \\
& \left.+\sum_{j=0}^{m+l-2} \int_{\left\{\tau_{1} \leq \bar{t} \leq \tau_{2}\right\} \cap\left\{r \leq 2 r_{1}\right\}}\left|\nabla_{g}^{j} F\right|_{h}^{2} d g\right\} .
\end{aligned}
$$

Adding to (9.79) a small multiple of the non degenerate estimate 9.80, and using Assumptions (G10) and (G11) (together with a Hardy type inequality for the terms $\sum_{j=0}^{l-2} \int_{\left\{r \leq 2 r_{1}\right\}}\left|\nabla_{g}^{j} F\right|_{h}^{2} d g$ ), we obtain (9.69):

$$
\begin{aligned}
& \sum_{j=0}^{m-1} \int_{\left\{\tau_{1} \leq \bar{t} \leq \tau_{2}\right\}}\left(\sum_{j_{2}+j_{3}=l+j-1} r_{+}^{-1+\varepsilon}\left|\nabla_{h_{\tau, N}}^{j_{2}+1}\left(N^{j_{3}} \varphi\right)\right|_{h_{\tau, N}}^{2}\right. \\
& \left.+\sum_{1 \leq j_{2}+j_{3} \leq l+j-1} r_{+}^{-1+\varepsilon-2\left(l+j-j_{2}-j_{3}\right)}\left|\nabla_{h_{\tau, N}}^{j_{2}}\left(N^{j_{3}} \varphi\right)\right|_{h_{\tau, N}}^{2}+r_{+}^{-1-\varepsilon}\left|N^{l+j} \varphi\right|^{2}\right) d g \\
& +\sum_{j=0}^{m-1} \sum_{1 \leq j_{2}+j_{3} \leq l+j-1} \int_{\left\{\bar{t}=\tau_{2}\right\}} r_{+}^{-2\left(l+j-1-j_{2}-j_{3}\right)}\left(r_{+}^{\varepsilon}\left|\mathcal{L}_{L} \nabla_{h_{\tau, N}}^{j_{2}}\left(N^{j_{3}} \varphi\right)\right|^{2}\right. \\
& \left.+\left|\nabla_{h_{\tau, N}}^{j_{2}+1}\left(N^{j_{3}} \varphi\right)\right|_{h_{\tau, N}}^{2}+r_{+}^{-2}\left|N^{l+j} \varphi\right|^{2}\right) d h_{N} \\
& +\sum_{j=0}^{m-1} \sum_{1 \leq j_{2}+j_{3} \leq l+j-1} \int_{\mathcal{H}^{+}\left(\tau_{1}, \tau_{2}\right)}\left|\nabla_{h_{\mathcal{H}}}^{j_{2}+1}\left(K_{R_{c}}^{j_{3}} \varphi\right)\right|_{h_{\mathcal{H}}}^{2} d h_{\mathcal{H}}
\end{aligned}
$$




$$
\begin{aligned}
\leq & C_{\varepsilon, m}\left\{\sum_{j=0}^{k+m-1} \sum_{1 \leq j_{2}+j_{3} \leq l+j-1} \int_{\left\{\bar{t}=\tau_{1}\right\}} r_{+}^{\varepsilon-2\left(l+j-1-j_{2}-j_{3}\right)}\right. \\
& \times\left(\left|\nabla_{h_{\tau, N}}^{j_{2}+1}\left(N^{j_{3}} \varphi\right)\right|_{h_{\tau, N}}^{2}+r_{+}^{-2}\left|N^{l+j} \varphi\right|^{2}\right) d h_{N} \\
& \left.+\tau_{1}^{-\delta 0} \int_{\left\{\tau_{1} \leq \bar{t} \leq \tau_{2}\right\}} r_{+}^{-1+\varepsilon} \mathcal{T}_{T, K, R_{c}, s l}^{(l, m+k-1)}[\varphi] d g+\sum_{j=l-1}^{m+k+l-2} \int_{\left\{\tau_{1} \leq \bar{t} \leq \tau_{2}\right\}} r_{+}^{1+\varepsilon}\left|\nabla_{g}^{j} F\right|_{h}^{2} d g\right\} .
\end{aligned}
$$

Moreover, by adding (for all possible combinations of $\left(e_{1}, \ldots e_{l-1}\right) \in\{0,1\}^{l-1}$ ) the estimates $(9.73),(9.74)$ and $\tau_{1}^{-\delta_{0}}$ times a small multiple of $(9.75)$, we also obtain the degenerate energy boundedness statement (9.70):

$$
\begin{aligned}
& \sum_{j=0}^{m-1} \sum_{1 \leq j_{2}+j_{3} \leq l+j-1} \int_{\left\{\bar{t}=\tau_{2}\right\}} r_{+}^{-2\left(l+j-1-j_{2}-j_{3}\right)}\left(\left|\nabla_{h_{\tau, N}}^{j_{2}+1}\left(K_{R_{c}}^{j_{3}} \varphi\right)\right|_{\left(1-\log \left(r_{t i m}\right)\right) \cdot h_{R_{c}}}^{2}+r_{+}^{-2}\left|K_{R_{c}}^{l+j} \varphi\right|^{2}\right) d h_{N} \\
& \leq C_{\varepsilon, m}\left\{\sum_{j=0}^{k+m-1} \sum_{1 \leq j_{2}+j_{3} \leq l+j-1} \int_{\left\{\bar{t}=\tau_{1}\right\}} r_{+}^{-2\left(l+j-1-j_{2}-j_{3}\right)}\left(\left|\nabla_{h_{\tau}, N}^{j_{2}+1}\left(N^{j_{3}} \varphi\right)\right|_{h_{\tau, N}}^{2}+r_{+}^{-2}\left|N^{l+j} \varphi\right|^{2}\right) d h_{N}\right. \\
& +\tau_{1}^{-\delta 0} \sum_{j=0}^{m-1} \sum_{1 \leq j_{2}+j_{3} \leq l+j-1} \int_{\left\{\bar{t}=\tau_{1}\right\}} r_{+}^{\varepsilon-2\left(l+j-1-j_{2}-j_{3}\right)}\left(\left|\nabla_{h_{\tau, N}}^{j_{2}+1}\left(N^{j_{3}} \varphi\right)\right|_{h_{\tau, N}}^{2}+r_{+}^{-2}\left|N^{l+j} \varphi\right|^{2}\right) d h_{N} \\
& \left.+\tau_{1}^{-\delta_{0}} \int_{\left\{\tau_{1} \leq \bar{i} \leq \tau_{2}\right\}}^{r_{+}^{-1+\varepsilon}} \mathcal{T}_{T, K, R_{c}, s l}^{(l, m+k-1)}[\varphi] d g+\sum_{j=l-1}^{m+k+l-2} \int_{\left\{\tau_{1} \leq \bar{t} \leq \tau_{2}\right\}} r_{+}^{1+\varepsilon}\left|\nabla_{g}^{j} F\right|_{h}^{2} d g\right\} .
\end{aligned}
$$

\subsection{Proof of Theorem 9.1 on Improved Polynomial Decay}

Without loss of generality, we will assume that $\varphi$ is real valued. Moreover, in order to avoid confusion with unnecessarily complicated notations, we will assume that $F \equiv 0$, since the proof of (9.40) and (9.41) in the case $F \neq 0$ follows by repeating exactly the same steps.

The proof of Theorem 9.1 will proceed by induction on $q$, from $q=1$ up to $q=\left\lfloor\frac{d+1}{2}\right\rfloor$.

More precisely, we will assume the following inductive hypothesis for some integer $1 \leq q_{0} \leq\left\lfloor\frac{d+1}{2}\right\rfloor$ :

Inductive hypothesis: For any integer $1 \leq q \leq q_{0}-1$, any $0<\varepsilon \ll \delta_{0}$, any integer $m \geq 1$, any $0 \leq p<2 q-1$ and any $0 \leq \tau_{1} \leq \tau_{2}$ the following bounds hold:

$$
\begin{aligned}
& \mathcal{E}_{\text {en }}^{(p, q, m)}[\varphi]\left(\tau_{2}\right)+\int_{\tau_{2}}^{\tau_{3}} \mathcal{E}_{\text {en }}^{(p-1, q, m)}[\varphi](\tau) d \tau \\
& \quad \lesssim m, \varepsilon\left(\tau_{2}-\tau_{1}\right)^{-2 q+p+C_{m} \varepsilon} \mathcal{E}_{\text {bound }}^{\left(2 q, q, m+\left\lceil\delta_{0}^{-1} \cdot 2(q-1)\right\rceil(3 q+1) \cdot k\right)}[\varphi]\left(\tau_{1}\right),
\end{aligned}
$$




$$
\begin{gathered}
\left\{\mathcal{E}_{\text {en }}^{(0, q, m)}[\varphi]\left(\tau_{2}\right)+\mathcal{E}_{\text {bound }}^{(\varepsilon, q, m)}[\varphi]\left(\tau_{2}\right)+\int_{\tau_{2}}^{+\infty} \mathcal{E}_{\text {en }}^{(-1+\varepsilon, q, m)}[\varphi](s) d s\right\} \\
\lesssim_{m, \varepsilon}\left(\tau_{2}-\tau_{1}\right)^{-2 q+C_{m} \varepsilon} \mathcal{E}_{\text {bound }}^{\left(2 q, q, m+\left\lceil\delta_{0}^{-1} \cdot 2(q-1)\right\rceil(3 q+1) \cdot k\right)}[\varphi]\left(\tau_{1}\right)
\end{gathered}
$$

and

$$
\mathcal{E}_{\text {en,deg }}^{(0, q, m)}[\varphi]\left(\tau_{2}\right) \lesssim_{m}\left(\tau_{2}-\tau_{1}\right)^{-2 q} \mathcal{E}_{\text {bound }}^{\left(2 q, q, m+\left\lceil\delta_{0}^{-1} \cdot 2(q-1)\right\rceil(3 q+1) \cdot k\right)}[\varphi]\left(\tau_{1}\right) .
$$

Granted this inductve hypothesis, the inductive step of our induction scheme will be the following:

Inductive step: For any $0<\varepsilon \ll \delta_{0}$, any integer $m \geq 1$, any $0 \leq p<2 q_{0}-1$ and any $0 \leq \tau_{1} \leq \tau_{2}$ the following bounds hold:

$$
\begin{aligned}
& \mathcal{E}_{\text {en }}^{\left(p, q_{0}, m\right)}[\varphi]\left(\tau_{2}\right)+\int_{\tau_{2}}^{\tau_{3}} \mathcal{E}_{\text {en }}^{\left(p-1, q_{0}, m\right)}[\varphi](\tau) d \tau \\
& \lesssim_{m, \varepsilon}\left(\tau_{2}-\tau_{1}\right)^{-2 q_{0}+p+C_{m} \varepsilon} \mathcal{E}_{\text {bound }}^{\left(2 q_{0}, q_{0}, m+\left\lceil\delta_{0}^{-1} \cdot 2\left(q_{0}-1\right)\right\rceil\left(3 q_{0}+1\right) \cdot k\right)}[\varphi]\left(\tau_{1}\right), \\
& \left\{\mathcal{E}_{\text {en }}^{\left(0, q_{0}, m\right)}[\varphi]\left(\tau_{2}\right)+\mathcal{E}_{\text {bound }}^{\left(\varepsilon, q_{0}, m\right)}[\varphi]\left(\tau_{2}\right)+\int_{\tau_{2}}^{+\infty} \mathcal{E}_{\text {en }}^{\left(-1+\varepsilon, q_{0}, m\right)}[\varphi](s) d s\right\} \\
& \lesssim_{m, \varepsilon}\left(\tau_{2}-\tau_{1}\right)^{-2 q_{0}+C_{m} \varepsilon} \mathcal{E}_{\text {bound }}^{\left(2 q_{0}, q_{0}, m+\left\lceil\delta_{0}^{-1} \cdot 2\left(q_{0}-1\right)\right\rceil\left(3 q_{0}+1\right) \cdot k\right)}[\varphi]\left(\tau_{1}\right)
\end{aligned}
$$

and

$$
\mathcal{E}_{\text {en,deg }}^{\left(0, q_{0}, m\right)}[\varphi]\left(\tau_{2}\right) \lesssim_{m}\left(\tau_{2}-\tau_{1}\right)^{-2 q_{0}} \mathcal{E}_{\text {bound }}^{\left(2 q_{0}, q_{0}, m+\left\lceil\delta_{0}^{-1} \cdot 2\left(q_{0}-1\right)\right\rceil\left(3 q_{0}+1\right) \cdot k\right)}[\varphi]\left(\tau_{1}\right) .
$$

Note that for $q=1$ Theorem 9.1 degenerates to Theorem 8.2, and thus the basis of the induction has already been established.

\subsubsection{Proof of the Inductive Step}

In order to establish inequalities (9.86), (9.87) and (9.88) of the inductive step, we will first need to prove a series of lemmas. Without loss of generality, we will assume that $\tau_{1}=0$ in (9.86), (9.87) and (9.88).

Lemma 9.6 There exists a $C>1$ such that for any integer $1 \leq l \leq q_{0}$, any integer $m \geq 1$, and any $\varphi \in C^{\infty}(\mathcal{M})$ solving $\square \varphi=0$, there exists a sequence $\left\{\tau_{n}\right\}_{n \in \mathbb{N}}$ of positive numbers satisfying $\left(1+C^{-1}\right) \tau_{n} \leq \tau_{n+1} \leq(1+C) \tau_{n}$ such that 


$$
\begin{aligned}
& \sum_{\left(e_{1}, \ldots e_{l-1}\right) \in\{0,1\}^{l-1}} \mathcal{E}_{\text {bulk }}^{\left(2\left(q_{0}-l\right), q_{0}-l+1, m\right)}\left[\mathcal{L}_{\left.X^{\left(e_{1}\right)} \ldots X^{\left(e_{l}-1\right.}\right)} \varphi\right]\left(\tau_{n}\right) \\
& \lesssim_{m} \tau_{n}^{-2 l} \mathcal{E}_{\text {bound }}^{\left(2 q_{0}, q_{0}, m+3 l \cdot k\right)}[\varphi](0)+\tau_{n}^{-\delta 0} \sum_{i=1}^{2 l} \sum_{j=0}^{q_{0}-1} \tau_{n}^{-2 j-(2 l-i)} \mathcal{E}_{\text {en }}^{\left(2 q_{0}-i, q_{0}-j, m+3 l \cdot k\right)}[\varphi]\left(\tau_{n}\right) \\
& +\tau_{n}^{-\delta} \sum_{i=1}^{2 l} \sum_{j=0}^{q_{0}-1} \tau_{n}^{-2 j-(2 l-i)} \int_{\tau_{n-2}}^{\tau_{n+1}} \mathcal{E}_{\text {en }}^{\left(2 q_{0}-i-1, q_{0}-j, m+3 l \cdot k\right)}[\varphi](s) d s
\end{aligned}
$$

where $X^{(0)}=T$ and $X^{(1)}=K_{R_{c}}$.

Proof The proof of (9.89) will follow by induction on $l$.

Inequality (9.89) for $l=1$ follows directly from the proof of Lemma 8.2 (using also Hardy-type inequalities of the form established in Lemma C.3) adapted to the case when $\left|\mathcal{L}_{T}^{j} g\right|_{h} \lesssim_{j} \tau^{-\delta_{0}}$ for all integers $j \geq 0$ (see also the remark below Lemma 8.2). It thus remains to prove (9.89) for any $1<l_{\text {ind }} \leq q_{0}$, assuming the cases $l \leq l_{\text {ind }}-1$ have been established. Without loss of generality, we will assume that $l_{\text {ind }}=q_{0}$, since this is the hardest case. The case $l_{\text {ind }}<q_{0}$ will follow in the same way, and hence the relevant details will be omitted.

Since we have assumed that (9.89) holds for $l=q_{0}-1$ and any integer $m \geq 1$, we can bound on a sequence $\left\{\tau_{n}\right\}_{n \in \mathbb{N}}$ satisfying $\left(1+C^{-1}\right) \tau_{n} \leq \tau_{n+1} \leq(1+C) \tau_{n}$ :

$$
\begin{aligned}
& \sum_{\left(e_{1}, \ldots e_{q_{0}-2}\right) \in\{0,1\}^{q_{0}-2}} \mathcal{E}_{\text {bulk }}^{(2,2, m)}\left[\mathcal{L}_{X^{\left(e_{1}\right)} \ldots X^{\left(e_{q_{0}}-2\right)}} \varphi\right]\left(\tau_{n}\right) \\
& \lesssim_{m} \tau_{n}^{-2\left(q_{0}-1\right)} \mathcal{E}_{\text {bound }}^{\left(2 q_{0}, q_{0}, m+3\left(q_{0}-1\right) \cdot k\right)}[\varphi](0) \\
& +\tau_{n}^{-\delta 0} \sum_{i=1}^{2\left(q_{0}-1\right)} \sum_{j=0}^{q_{0}-1} \tau_{n}^{-2 j-\left(2\left(q_{0}-1\right)-i\right)} \mathcal{E}_{\text {en }}^{\left(2 q_{0}-i, q_{0}-j, m+3\left(q_{0}-1\right) \cdot k\right)}[\varphi]\left(\tau_{n}\right) \\
& +\tau_{n}^{-\delta 0} \sum_{i=1}^{2\left(q_{0}-1\right)} \sum_{j=0}^{q_{0}-1} \tau_{n}^{-2 j-\left(2\left(q_{0}-1\right)-i\right)} \int_{\tau_{n-2}}^{\tau_{n+1}} \mathcal{E}_{\text {en }}^{\left(2 q_{0}-i-1, q_{0}-j, m+3\left(q_{0}-1\right) \cdot k\right)}[\varphi](s) d s .
\end{aligned}
$$

Using the expression (A.2) for the wave operator in the region $\{r \gg 1\}$ (and hence morally interchanging $\nabla_{h_{\tau, N}}^{2}+\square \rightarrow \partial_{v} T$ ), we can bound (using also some Hardy type inequalities of the form established in Lemma C.3):

$$
\begin{gathered}
\sum_{\left(e_{1}, \ldots e_{q_{0}-1}\right) \in\{0,1\}^{q_{0}-1}} \mathcal{E}_{\text {bound }}^{(2,1, m)}\left[\mathcal{L}_{X^{\left(e_{1}\right)} \ldots X^{\left(e_{q_{0}-1}\right)}} \varphi\right]\left(\tau_{n}\right) \\
\lesssim_{\left(e_{1}, \ldots e_{\left.q_{0}-2\right) \in\{0,1\}^{q_{0}-2}}\right.} \sum_{\mathcal{E}_{\text {bulk }}^{(2,2, m)}}\left[\mathcal{L}_{X^{\left(e_{1}\right)} \ldots X^{\left(e_{q_{0}-2}\right)}} \varphi\right]\left(\tau_{n}\right)
\end{gathered}
$$




$$
\begin{aligned}
& +\sum_{\left(e_{1}, \ldots e_{\left.q_{0}-2\right) \in\{0,1\}^{q_{0}-2}}\right.} \sum_{j=0}^{m-1} \sum_{0 \leq j_{1}+j_{2} \leq j} \int_{\left\{\bar{t}=\tau_{n}\right\} \cap\left\{r \geq R_{c}\right\}} r^{2-2\left(q+j-2-j_{1}\right)} \\
& \times \mid \nabla_{h_{\tau, N}}^{j_{1}}\left(T ^ { j _ { 2 } } \square \left(\left.\mathcal{L}_{\left.\left.X^{\left(e_{1}\right)} \ldots X^{\left(e_{q_{0}-2}\right)} \varphi\right)\right)}\right|_{h_{\tau, N}} ^{2} d h_{N} .\right.\right.
\end{aligned}
$$

Thus, from (9.90) and (9.91), we obtain in view of the expression (9.64) for the commutator of $\square$ with $\mathcal{L}_{X^{\left(e_{i}\right)}}$ :

$$
\begin{gathered}
\sum_{\left(e_{1}, \ldots e_{q_{0}-1}\right) \in\{0,1\}^{q_{0}-1}} \mathcal{E}_{\text {bound }}^{(2,1, m)}\left[\mathcal{L}_{\left.X^{\left(e_{1}\right)} \ldots X^{(e} q_{0}-1\right)} \varphi\right]\left(\tau_{n}\right) \\
\lesssim_{m} \tau_{n}^{-2\left(q_{0}-1\right)} \mathcal{E}_{\text {bound }}^{\left(2 q_{0}, q_{0}, m+3\left(q_{0}-1\right) \cdot k\right)}[\varphi](0) \\
+\tau_{n}^{-\delta 0} \sum_{i=1}^{2\left(q_{0}-1\right)} \sum_{j=0}^{q_{0}-1} \tau_{n}^{-2 j-\left(2\left(q_{0}-1\right)-i\right)} \mathcal{E}_{\text {en }}^{\left(2 q_{0}-i, q_{0}-j, m+3\left(q_{0}-1\right) \cdot k\right)}[\varphi]\left(\tau_{n}\right) \\
+\tau_{n}^{-\delta 0} \sum_{i=1}^{2\left(q_{0}-1\right)} \sum_{j=0}^{q_{0}-1} \tau_{n}^{-2 j-\left(2\left(q_{0}-1\right)-i\right)} \int_{\tau_{n-2}}^{\tau_{n+1}} \mathcal{E}_{\text {en }}^{\left(2 q_{0}-i-1, q_{0}-j, m+3\left(q_{0}-1\right) \cdot k\right)}[\varphi](s) d s .
\end{gathered}
$$

Repeating the first steps of the proof of Theorem 8.1 for $\mathcal{L}_{X^{\left(e_{1}\right)}{ }_{\ldots X} X^{\left(e_{e_{0}-1}\right)}} \varphi$ in place of $\varphi$, using Lemma 9.5 (and in particular the estimate (9.69)) in place of the simple integrated local energy decay assumption 8.19, in view of (9.64) we can bound on a sequence $\left\{\tau_{n}\right\}_{n \in \mathbb{N}}$ with $\left(1+C^{-1}\right) \tau_{n} \leq \tau_{n+1} \leq(1+C) \tau_{n}$ (possibly different than the one appearing before):

$$
\begin{aligned}
& \sum_{\left(e_{1}, \ldots e_{q_{0}-1}\right) \in\{0,1\}^{q_{0}-1}} \mathcal{E}_{\text {bulk }}^{(0,1, m)}\left[\mathcal{L}_{X^{\left(e_{1}\right)} \ldots X^{\left(e_{q_{0}-2}\right)}} \varphi\right]\left(\tau_{n}\right) \\
& \lesssim_{m} \tau_{n}^{-2} \sum_{\left(e_{1}, \ldots e_{q_{0}-1}\right) \in\{0,1\}^{q_{0}-1}} \mathcal{E}_{\text {bound }}^{(2,1, m+2 k)}\left[\mathcal{L}_{X^{\left(e_{1}\right)} \ldots X^{\left(e_{q_{0}}-1\right)}} \varphi\right]\left(\tau_{n}\right) \\
& +\tau_{n}^{-\delta} \sum_{i=1}^{2 q_{0}} \sum_{j=0}^{q_{0}-1} \tau_{n}^{-2 j-\left(2 q_{0}-i\right)} \mathcal{E}_{\text {en }}^{\left(2 q_{0}-i, q_{0}-j, m+\left(3 q_{0}-1\right) \cdot k\right)}[\varphi]\left(\tau_{n}\right) \\
& +\tau_{n}^{-\delta 0} \sum_{i=1}^{2 q_{0}} \sum_{j=0}^{q_{0}-1} \tau_{n}^{-2 j-\left(2 q_{0}-i\right)} \int_{\tau_{n-2}}^{\tau_{n+1}} \mathcal{E}_{\text {en }}^{\left(2 q_{0}-i-1, q_{0}-j, m+\left(3 q_{0}-1\right) \cdot k\right)}[\varphi](s) d s .
\end{aligned}
$$

Using the bound (9.92) with $m+2 k$ in place of $m$, we finally obtain the desired inequality: 


$$
\begin{gathered}
\sum_{\left(e_{1}, \ldots e_{q_{0}-1} \in\{0,1\}^{q_{0}-1}\right.} \mathcal{E}_{\text {bulk }}^{(0,1, m)}\left[\mathcal{L}_{X^{\left(e_{1}\right)} \ldots X^{\left(e q_{0}-1\right)}} \varphi\right]\left(\tau_{n}\right) \\
\lesssim_{m} \tau_{n}^{-2 q_{0}} \mathcal{E}_{\text {bound }}^{\left(2 q_{0}, q_{0}, m+\left(3 q_{0}-1\right) \cdot k\right)}[\varphi](0) \\
+\tau_{n}^{-\delta} \sum_{i=1}^{2 q_{0}} \sum_{j=0}^{q_{0}-1} \tau_{n}^{-2 j-\left(2 q_{0}-i\right)} \mathcal{E}_{\text {en }}^{\left(2 q_{0}-i, q_{0}-j, m+\left(3 q_{0}-1\right) \cdot k\right)}[\varphi]\left(\tau_{n}\right) \\
+\tau_{n}^{-\delta 0} \sum_{i=1}^{2 q_{0}} \sum_{j=0}^{q_{0}-1} \tau_{n}^{-2 j-\left(2 q_{0}-i\right)} \int_{\tau_{n-2}}^{\tau_{n+1}} \mathcal{E}_{\text {en }}^{\left(2 q_{0}-i-1, q_{0}-j, m+\left(3 q_{0}-1\right) \cdot k\right)}[\varphi](s) d s .
\end{gathered}
$$

Thus, the proof of the Lemma is complete.

Lemma 9.7 For any integer $m \geq 1$, any $0<\varepsilon \ll \delta_{0}$ small in terms of $m$ and any smooth function $\varphi$ on $\mathcal{M}$ solving $\square \varphi=0$ we can bound:

$$
\begin{aligned}
& \left\{\mathcal{E}_{\text {en }}^{\left(0, q_{0}, m\right)}[\varphi](\tau)+\mathcal{E}_{\text {bound }}^{\left(\varepsilon, q_{0}, m\right)}[\varphi](\tau)+\int_{\tau}^{+\infty} \mathcal{E}_{\text {en }}^{\left(-1+\varepsilon, q_{0}, m\right)}[\varphi](s) d s\right\} \\
& \lesssim_{m, \varepsilon} \tau^{-2 q_{0}+C_{m} \cdot \varepsilon} \mathcal{E}_{\text {bound }}^{\left(2 q_{0}, q_{0}, m+\left(3 q_{0}+1\right) \cdot k\right)}[\varphi](0) \\
& \quad+\tau^{-\delta 0} \sum_{i=1}^{2 l} \sum_{j=0}^{q_{0}-1} \tau^{-2 j-\left(2 q_{0}-i\right)} \mathcal{E}_{\text {en }}^{\left(2 q_{0}-i+\varepsilon, q_{0}-j, m+\left(3 q_{0}+1\right) \cdot k\right)}[\varphi](\tau) \\
& +\tau^{-\delta 0} \sum_{i=1}^{2 l} \sum_{j=0}^{q_{0}-1} \tau^{-2 j-\left(2 q_{0}-i\right)} \int_{C_{\varepsilon, m}^{-1} \tau}^{+\infty} \mathcal{E}_{\text {en }}^{\left(2 q_{0}-i-1+\varepsilon, q_{0}-j, m+\left(3 q_{0}+1\right) \cdot k\right)}[\varphi](s) d s
\end{aligned}
$$

and

$$
\begin{aligned}
& \mathcal{E}_{\text {en }, \text { deg }}^{\left(0, q_{0}, m\right)}[\varphi](\tau) \lesssim m \tau^{-2 q_{0}} \mathcal{E}_{\text {bound }}^{\left(2 q_{0}, q_{0}, m+\left(3 q_{0}+1\right) \cdot k\right)}[\varphi](0) \\
& +\tau^{-\delta 0} \sum_{i=1}^{2 l} \sum_{j=0}^{q_{0}-1} \tau^{-2 j-\left(2 q_{0}-i\right)} \mathcal{E}_{\text {en }}^{\left(2 q_{0}-i+\varepsilon, q_{0}-j, m+\left(3 q_{0}+1\right) \cdot k\right)}[\varphi](\tau) \\
& +\tau^{-\delta 0} \sum_{i=1}^{2 l} \sum_{j=0}^{q_{0}-1} \tau^{-2 j-\left(2 q_{0}-i\right)} \int_{C_{\varepsilon, m}^{-1} \tau}^{+\infty} \mathcal{E}_{\text {en }}^{\left(2 q_{0}-i-1+\varepsilon, q_{0}-j, m+\left(3 q_{0}+1\right) \cdot k\right)}[\varphi](s) d s .
\end{aligned}
$$

Proof From Lemma (9.6), we can bound on a sequence $\left\{\tau_{n}\right\}_{n \in \mathbb{N}}$ satisfying (1+ $\left.C^{-1}\right) \tau_{n} \leq \tau_{n+1} \leq(1+C) \tau_{n}$ for some large $C>0$ :

$$
\begin{aligned}
& \sum_{\left(e_{1}, \ldots e_{l-1}\right) \in\{0,1\}^{q_{0}-1}} \mathcal{E}_{\text {bulk }}^{(0,1, m)}\left[\mathcal{L}_{X^{\left(e_{1}\right)} \ldots X^{\left(e_{q_{0}}-1\right)}} \varphi\right]\left(\tau_{n}\right) \\
& \lesssim_{m} \tau_{n}^{-2 q_{0}} \mathcal{E}_{\text {bound }}^{\left(2 q_{0}, q_{0}, m+3 q_{0} \cdot k\right)}[\varphi](0)
\end{aligned}
$$




$$
\begin{aligned}
& +\tau_{n}^{-\delta} \sum_{i=1}^{2 q_{0}} \sum_{j=0}^{q_{0}-1} \tau_{n}^{-2 j-\left(2 q_{0}-i\right)} \mathcal{E}_{\text {en }}^{\left(2 q_{0}-i, q_{0}-j, m+3 q_{0} \cdot k\right)}[\varphi]\left(\tau_{n}\right) \\
& +\tau_{n}^{-\delta 0} \sum_{i=1}^{2 q_{0}} \sum_{j=0}^{q_{0}-1} \tau_{n}^{-2 j-\left(2 q_{0}-i\right)} \int_{\tau_{n-2}}^{\tau_{n+1}} \mathcal{E}_{e n}^{\left(2 q_{0}-i-1, q_{0}-j, m+3 q_{0} \cdot k\right)}[\varphi](s) d s .
\end{aligned}
$$

Using (9.67) successively for $\mathcal{L}_{X^{\left(e_{2}\right)} \ldots X^{\left(e_{q_{0}-1}\right)}} \varphi, \mathcal{L}_{X^{\left(e_{3}\right)} \ldots X^{\left(e_{q_{0}-1}\right)}} \varphi, \ldots, \varphi$ (in place of $\varphi$ ), making also use of (9.64) for the commutator of $\square$ with $\mathcal{L}_{X^{\left(e_{i}\right)}}$, we can bound (assuming without loss of generality that $\tau_{1}$ is large enough in terms of $m$ ):

$$
\mathcal{E}_{\text {en }}^{\left(0, q_{0}, m\right)}[\varphi]\left(\tau_{n}\right) \lesssim_{\left(e_{1}, \ldots e_{l-1}\right) \in\{0,1\}^{l-1}} \mathcal{E}_{b u l k}^{(0,1, m)}\left[\mathcal{L}_{X^{\left(e_{1}\right)} \ldots X^{\left(e_{q_{0}-1}\right)}} \varphi\right]\left(\tau_{n}\right) .
$$

Therefore, from (9.97) and (9.98) we obtain:

$$
\begin{aligned}
& \mathcal{E}_{\text {en }}^{\left(0, q_{0}, m\right)}[\varphi]\left(\tau_{n}\right) \lesssim m \tau_{n}^{-2 q_{0}} \mathcal{E}_{\text {bound }}^{\left(2 q_{0}, q_{0}, m+3 q_{0} \cdot k\right)}[\varphi](0) \\
& +\tau_{n}^{-\delta} \sum_{i=1}^{2 q_{0}} \sum_{j=0}^{q_{0}-1} \tau_{n}^{-2 j-\left(2 q_{0}-i\right)} \mathcal{E}_{\text {en }}^{\left(2 q_{0}-i, q_{0}-j, m+3 q_{0} \cdot k\right)}[\varphi]\left(\tau_{n}\right) \\
& +\tau_{n}^{-\delta} \sum_{i=1}^{2 q_{0}} \sum_{j=0}^{q_{0}-1} \tau_{n}^{-2 j-\left(2 q_{0}-i\right)} \int_{\tau_{n-2}}^{\tau_{n+1}} \mathcal{E}_{\text {en }}^{\left(2 q_{0}-i-1, q_{0}-j, m+3 q_{0} \cdot k\right)}[\varphi](s) d s
\end{aligned}
$$

From (6.1) and (8.19), we also obtain after repeating the first steps of the proof of Theorem 8.1 on a sequence $\left\{\bar{\tau}_{n}\right\}_{n \in \mathbb{N}}$ with $\left(1+C^{-1}\right) \bar{\tau}_{n} \leq \bar{\tau}_{n+1} \leq(1+C) \bar{\tau}_{n}$ :

$$
\mathcal{E}_{\text {bulk }}^{\left(2 q_{0}-1-\varepsilon, q_{0}, m\right)}[\varphi]\left(\bar{\tau}_{n}\right) \lesssim_{\varepsilon, m} \bar{\tau}_{n}^{-1} \mathcal{E}_{\text {bound }}^{\left(2 q_{0}, q_{0}, m+k\right)}[\varphi](0) .
$$

Notice that, a priori, $\left\{\bar{\tau}_{n}\right\}_{n \in \mathbb{N}}$ might be different than $\left\{\tau_{n}\right\}_{n \in \mathbb{N}}$. However, we can run the pigeonhole principle argument leading to the choice of these sequences more carefully and arrange so that $\tau_{n}=\bar{\tau}_{n}$ provided $C>0$ had been fixed large enough in terms of $\varepsilon, m$ and the geometry of $(\mathcal{M}, g)$ (henceforth we will thus assume without loss of generality that $\bar{\tau}_{n}=\tau_{n}$ ); this follows from the following general fact: If $f_{1}, f_{2}:(0,+\infty) \rightarrow(0,+\infty)$ are measurable functions satisfying

$$
\int_{0}^{+\infty} f_{i}(x) d x \leq C_{i}
$$

for $i=1,2$, then there exists a sequence $\left\{x_{n}\right\}_{n \in \mathbb{N}}$ with $2 x_{n} \leq x_{n+1} \leq 4 x_{n}$ such that for all $n \in \mathbb{N}$ and for $i=1,2$ :

$$
f_{i}\left(x_{n}\right) \leq \log ^{-1}\left(\frac{4}{3}\right) \cdot \frac{C_{i}}{x_{n}} .
$$


Proof of (9.102): This is established by contradiction: If there exists an interval $[a, 2 a] \subset(0,+\infty)$ such that the measurable sets

$$
I_{i}^{(a)}=\left\{x \in[a, 2 a] \mid f_{i}(x) \leq \log ^{-1}\left(\frac{4}{3}\right) \cdot \frac{C_{i}}{x}\right\}
$$

for $i=1,2$ are disjoint, then there exists an $i_{0} \in\{1,2\}$ such that the complement of $I_{i_{0}}^{(a)}$ on $[a, 2 a]$ is at least of half measure, i.e.

$$
m\left\{\left(I_{i_{0}}^{(a)}\right)^{c} \cap[a, 2 a]\right\} \geq \frac{a}{2} .
$$

But then one obtains

$$
\begin{aligned}
C_{i_{0}} & \geq \int_{0}^{+\infty} f_{i}(x) d x \geq \int_{\left(I_{i_{0}}^{(a)}\right)^{c} \cap[a, 2 a]} f_{i_{0}}(x) d x \\
& >\int_{\left(I_{i_{0}}^{(a)}\right)^{c} \cap[a, 2 a]} \log ^{-1}\left(\frac{4}{3}\right) \cdot \frac{C_{i_{0}}}{x} d x \geq \log ^{-1}\left(\frac{4}{3}\right) \cdot C_{i_{0}} \int_{\left[\frac{3 a}{2}, 2 a\right]} \frac{1}{x} d x=C_{i_{0}},
\end{aligned}
$$

(the second to last inequality following because the integral of $\frac{1}{x}$ over subsets of $[a, 2 a]$ of measure at least $\frac{a}{2}$ is minimized over $\left.\left[\frac{3 a}{2}, 2 a\right]\right)$, which is a contradiction. Thus, there exists an infinite sequence $\left\{x_{n}\right\}_{n \in \mathbb{N}}$ with $2 x_{n} \leq x_{n+1} \leq 4 x_{n}$ on which (9.102) is satisfied.

By interpolating between (9.99) and (9.100) (note again that we have assumed $\tau_{n}=\bar{\tau}_{n}$ ), we obtain:

$$
\begin{aligned}
& \mathcal{E}_{\text {en }}^{\left(\varepsilon, q_{0}, m\right)}[\varphi]\left(\tau_{n}\right) \lesssim m \\
& +\tau_{n}^{-\delta 0} \sum_{i=1}^{2 q_{0}} \sum_{j=0}^{q_{0}-1} \tau_{n}^{-2 j-\left(2 q_{0}+C_{m}-i\right)} \mathcal{E}_{\text {bound }}^{\left(2 q_{0}, q_{0}, m+3 q_{0} \cdot k\right)}[\varphi](0) \\
& \mathcal{E}_{\text {en }}^{\left(2 q_{0}-i, q_{0}-j, m+3 q_{0} \cdot k\right)}[\varphi]\left(\tau_{n}\right) \\
& +\tau_{n}^{-\delta 0} \sum_{i=1}^{2 q_{0}} \sum_{j=0}^{q_{0}-1} \tau_{n}^{-2 j-\left(2 q_{0}-i\right)} \int_{\tau_{n-2}}^{\tau_{n+1}} \mathcal{E}_{\text {en }}^{\left(2 q_{0}-i-1, q_{0}-j, m+3 q_{0} \cdot k\right)}[\varphi](s) d s(.9 .106)
\end{aligned}
$$

By applying Lemma 9.5 in the regions $\left\{\tau_{n} \leq \bar{t} \leq \tau_{n+1}\right\}$ and using (9.99), (9.106) and the fact that $\tau_{n} \sim_{\varepsilon, m} \tau_{n+1}$, we thus obtain provided $\varepsilon$ has been chosen small in terms of $m$ and $\delta_{0}$ :

$$
\begin{aligned}
& \left\{\mathcal{E}_{\text {en }}^{\left(0, q_{0}, m\right)}[\varphi](\tau)+\mathcal{E}_{\text {bound }}^{\left(\varepsilon, q_{0}, m\right)}[\varphi](\tau)+\int_{\tau}^{+\infty} \mathcal{E}_{\text {en }}^{\left(-1+\varepsilon, q_{0}, m\right)}[\varphi](s) d s\right\} \\
& \lesssim_{m, \varepsilon} \tau^{-2 q_{0}+C_{m} \varepsilon} \mathcal{E}_{\text {bound }}^{\left(2 q_{0}, q_{0}, m+\left(3 q_{0}+1\right) \cdot k\right)}[\varphi](0) \\
& \quad+\tau^{-\delta 0} \sum_{i=1}^{2 l} \sum_{j=0}^{q_{0}-1} \tau^{-2 j-\left(2 q_{0}-i\right)} \mathcal{E}_{\text {en }}^{\left(2 q_{0}-i+\varepsilon, q_{0}-j, m+\left(3 q_{0}+1\right) \cdot k\right)}[\varphi](\tau)
\end{aligned}
$$




$$
+\tau^{-\delta 0} \sum_{i=1}^{2 l} \sum_{j=0}^{q_{0}-1} \tau^{-2 j-\left(2 q_{0}-i\right)} \int_{C_{\varepsilon, m}^{-1} \tau}^{+\infty} \mathcal{E}_{e n}^{\left(2 q_{0}-i-1+\varepsilon, q_{0}-j, m+\left(3 q_{0}+1\right) \cdot k\right)}[\varphi](s) d s
$$

and

$$
\begin{aligned}
& \mathcal{E}_{\text {en }, \text { deg }}^{\left(0, q_{0}, m\right)}[\varphi](\tau) \\
& \lesssim_{m} \tau^{-2 q_{0}} \mathcal{E}_{\text {bound }}^{\left(2 q_{0}, q_{0}, m+\left(3 q_{0}+1\right) \cdot k\right)}[\varphi](0) \\
& \quad+\tau^{-\delta 0} \sum_{i=1}^{2 l} \sum_{j=0}^{q_{0}-1} \tau^{-2 j-\left(2 q_{0}-i\right)} \mathcal{E}_{\text {en }}^{\left(2 q_{0}-i+\varepsilon, q_{0}-j, m+\left(3 q_{0}+1\right) \cdot k\right)}[\varphi](\tau) \\
& \quad+\tau^{-\delta 0} \sum_{i=1}^{2 l} \sum_{j=0}^{q_{0}-1} \tau^{-2 j-\left(2 q_{0}-i\right)} \int_{C_{\varepsilon, m}^{-1} \tau}^{+\infty} \mathcal{E}_{\text {en }}^{\left(2 q_{0}-i-1+\varepsilon, q_{0}-j, m+\left(3 q_{0}+1\right) \cdot k\right)}[\varphi](s) d s
\end{aligned}
$$

We are now ready to establish inequalities (9.84) and (9.85) of the inductive step:

Lemma 9.8 For any integer $m \geq 1$, any $0<\varepsilon \ll \delta_{0}$ small in terms of $m$ and any smooth function $\varphi$ on $\mathcal{M}$ solving $\square \varphi=0$ we can bound:

$$
\begin{aligned}
& \left\{\mathcal{E}_{\text {en }}^{\left(0, q_{0}, m\right)}[\varphi](\tau)+\mathcal{E}_{\text {bound }}^{\left(\varepsilon, q_{0}, m\right)}[\varphi](\tau)+\int_{\tau}^{+\infty} \mathcal{E}_{\text {en }}^{\left(-1+\varepsilon, q_{0}, m\right)}[\varphi](s) d s\right\} \\
& \lesssim m, \varepsilon \tau^{-2 q_{0}+C_{m} \cdot \varepsilon} \mathcal{E}_{\text {bound }}^{\left(2 q_{0}, q_{0}, m+\left\lceil\delta_{0}^{-1} \cdot 2\left(q_{0}-1\right)\right\rceil\left(3 q_{0}+1\right) \cdot k\right)}[\varphi](0)
\end{aligned}
$$

and

$$
\mathcal{E}_{\text {en,deg }}^{\left(0, q_{0}, m\right)}[\varphi](\tau) \lesssim m \tau^{-2 q_{0}} \mathcal{E}_{\text {bound }}^{\left(2 q_{0}, q_{0}, m+\left\lceil\delta_{0}^{-1} \cdot 2\left(q_{0}-1\right)\right\rceil\left(3 q_{0}+1\right) \cdot k\right)}[\varphi](0) .
$$

Proof From Lemma 9.6 we can bound:

$$
\begin{aligned}
& \left\{\mathcal{E}_{\text {en }}^{\left(0, q_{0}, m\right)}[\varphi](\tau)+\mathcal{E}_{\text {bound }}^{\left(\varepsilon, q_{0}, m\right)}[\varphi](\tau)+\int_{\tau}^{+\infty} \mathcal{E}_{\text {en }}^{\left(-1+\varepsilon, q_{0}, m\right)}[\varphi](s) d s\right\} \\
& \lesssim_{m, \varepsilon} \tau^{-2 q_{0}+C_{m} \cdot \varepsilon} \mathcal{E}_{\text {bound }}^{\left(2 q_{0}, q_{0}, m+\left(3 q_{0}+1\right) \cdot k\right)}[\varphi](0) \\
& \quad+\tau^{-\delta 0} \sum_{i=1}^{2 l} \sum_{j=0}^{q_{0}-1} \tau^{-2 j-(2 l-i)} \mathcal{E}_{\text {en }}^{\left(2 q_{0}-i+\varepsilon, q_{0}-j, m+\left(3 q_{0}+1\right) \cdot k\right)}[\varphi](\tau) \\
& \quad+\tau^{-\delta 0} \sum_{i=1}^{2 l} \sum_{j=0}^{q_{0}-1} \tau^{-2 j-\left(2 q_{0}-i\right)} \int_{C_{\varepsilon, m}^{-1} \tau}^{+\infty} \mathcal{E}_{\text {en }}^{\left(2 q_{0}-i-1+\varepsilon, q_{0}-j, m+\left(3 q_{0}+1\right) \cdot k\right)}[\varphi](s) d s
\end{aligned}
$$


and

$$
\begin{aligned}
& \mathcal{E}_{\text {en,deg }}^{\left(0, q_{0}, m\right)}[\varphi](\tau) \\
& \lesssim_{m} \tau^{-2 q_{0}} \mathcal{E}_{\text {bound }}^{\left(2 q_{0}, q_{0}, m+\left(3 q_{0}+1\right) \cdot k\right)}[\varphi](0) \\
& +\tau^{-\delta 0} \sum_{i=1}^{2 l} \sum_{j=0}^{q_{0}-1} \tau^{-2 j-\left(2 q_{0}-i\right)} \mathcal{E}_{\text {en }}^{\left(2 q_{0}-i+\varepsilon, q_{0}-j, m+\left(3 q_{0}+1\right) \cdot k\right)}[\varphi](\tau) \\
& +\tau^{-\delta 0} \sum_{i=1}^{2 l} \sum_{j=0}^{q_{0}-1} \tau^{-2 j-\left(2 q_{0}-i\right)} \int_{C_{\varepsilon, m}^{-1} \tau}^{+\infty} \mathcal{E}_{\text {en }}^{\left(2 q_{0}-i-1+\varepsilon, q_{0}-j, m+\left(3 q_{0}+1\right) \cdot k\right)}[\varphi](s) d s .
\end{aligned}
$$

Using the inductive hypothesis (i.e. (9.83), (9.84) and (9.85)) as well as Theorem 8.1 and the integrated local energy decay statement (8.19), we obtain from (9.111) and (9.112):

$$
\begin{aligned}
& \left\{\mathcal{E}_{\text {en }}^{\left(0, q_{0}, m\right)}[\varphi](\tau)+\mathcal{E}_{\text {bound }}^{\left(\varepsilon, q_{0}, m\right)}[\varphi](\tau)+\int_{\tau}^{+\infty} \mathcal{E}_{\text {en }}^{\left(-1+\varepsilon, q_{0}, m\right)}[\varphi](s) d s\right\} \\
& \lesssim m, \varepsilon \tau^{-\min \left\{2+\delta_{0}, 2 q_{0}-C_{m} \cdot \varepsilon\right\}} \mathcal{E}_{\text {bound }}^{\left(2 q_{0}, q_{0}, m+\left(3 q_{0}+1\right) \cdot k\right)}[\varphi](0)
\end{aligned}
$$

and

$$
\mathcal{E}_{\text {en,deg }}^{\left(0, q_{0}, m\right)}[\varphi](\tau) \lesssim m \tau^{-\min \left\{2+\delta 0,2 q_{0}\right\}} \mathcal{E}_{\text {bound }}^{\left(2 q_{0}, q_{0}, m+\left(3 q_{0}+1\right) \cdot k\right)}[\varphi](0) .
$$

Going back to (9.111) and (9.112) and using (9.113) (with $\left.m+\left(3 q_{0}+1\right) \cdot k\right)$ in place of $m$ ) for the error terms in the right hand side, combined with the inductive hypothesis' inequalities (9.83), (9.84) for the lower order terms, we obtain the following improvement of (9.113) and (9.114):

$$
\begin{aligned}
& \left\{\mathcal{E}_{\text {en }}^{\left(0, q_{0}, m\right)}[\varphi](\tau)+\mathcal{E}_{\text {bound }}^{\left(\varepsilon, q_{0}, m\right)}[\varphi](\tau)+\int_{\tau}^{+\infty} \mathcal{E}_{\text {en }}^{\left(-1+\varepsilon, q_{0}, m\right)}[\varphi](s) d s\right\} \\
& \lesssim m, \varepsilon \tau^{-\min \left\{2+2 \delta_{0}, 2 q_{0}-C_{m} \cdot \varepsilon\right\}} \mathcal{E}_{\text {bound }}^{\left(2 q_{0}, q_{0}, m+2\left(3 q_{0}+1\right) \cdot k\right)}[\varphi](0)
\end{aligned}
$$

and

$$
\mathcal{E}_{\text {en }, \text { deg }}^{\left(0, q_{0}, m\right)}[\varphi](\tau) \lesssim m \tau^{-\min \left\{2+2 \delta_{0}, 2 q_{0}\right\}} \mathcal{E}_{\text {bound }}^{\left(2 q_{0}, q_{0}, m+2\left(3 q_{0}+1\right) \cdot k\right)}[\varphi](0) .
$$

Repeating the same procedure $\left\lceil\delta_{0}^{-1} \cdot 2\left(q_{0}-1\right)\right\rceil$ times, we finally obtain the desired decay statement:

$$
\begin{aligned}
& \left\{\mathcal{E}_{\text {en }}^{\left(0, q_{0}, m\right)}[\varphi](\tau)+\mathcal{E}_{\text {bound }}^{\left(\varepsilon, q_{0}, m\right)}[\varphi](\tau)+\int_{\tau}^{+\infty} \mathcal{E}_{\text {en }}^{\left(-1+\varepsilon, q_{0}, m\right)}[\varphi](s) d s\right\} \\
& \lesssim_{m, \varepsilon} \tau^{-2 q_{0}+C_{m} \cdot \varepsilon} \mathcal{E}_{\text {bound }}^{\left(2 q_{0}, q_{0}, m+\left\lceil\delta_{0}^{-1} \cdot 2\left(q_{0}-1\right)\right\rceil\left(3 q_{0}+1\right) \cdot k\right)}[\varphi](0)
\end{aligned}
$$


and

$$
\mathcal{E}_{\text {en }, \text { deg }}^{\left(0, q_{0}, m\right)}[\varphi](\tau) \lesssim m \tau^{-2 q_{0}} \mathcal{E}_{\text {bound }}^{\left(2 q_{0}, q_{0}, m+\left\lceil\delta_{0}^{-1} \cdot 2\left(q_{0}-1\right)\right\rceil\left(3 q_{0}+1\right) \cdot k\right)}[\varphi](0)
$$

Finally, we will establish inequality (9.83) of the inductive step:

Lemma 9.9 For any $0 \leq p \leq 2 q_{0}-2$, any integer $m \geq 1$, any $0<\varepsilon \ll \delta_{0}$ small in terms of $m$ and any smooth function $\varphi$ on $\mathcal{M}$ solving $\square \varphi=0$ we can bound:

$$
\begin{aligned}
& \mathcal{E}_{\text {en }}^{\left(p, q_{0}, m\right)}[\varphi](\tau)+\int_{\tau}^{+\infty} \mathcal{E}_{\text {en }}^{\left(p-1, q_{0}, m\right)}[\varphi](s) d s \\
& \quad{ }_{m, \varepsilon} \tau^{-2 q_{0}+p+C_{m} \varepsilon} \mathcal{E}_{\text {bound }}^{\left(2 q_{0}, q_{0}, m+\left\lceil\delta_{0}^{-1} \cdot 2\left(q_{0}-1\right)\right\rceil\left(3 q_{0}+1\right) \cdot k\right)}[\varphi](0) .
\end{aligned}
$$

Proof Inequality (9.119) follows readily after interpolating between (9.109) and (8.31).

\subsection{Gagliardo-Nirenberg Type Inequalities on the Hyperboloids $\{\bar{t}=$ const $\}$}

In the proof of Corollary 9.2, we need to obtain refined pointwise control for functions $\Psi$ on the hyperboloids $\{\bar{t}=$ const $\}$ by estimating the $L^{2}$ norms of higher order derivatives of them. To this end, we will make use of the following Gagliardo-Nirenberg type estimates:

Lemma 9.10 For any $r_{0} \geq 0$, any $\tau>0$ and any smooth function $\Psi: \mathcal{S}_{\tau, r_{0}} \rightarrow \mathbb{C}$ (where $\mathcal{S}_{\tau, r_{0}} \doteq\{\bar{t}=\tau\} \cap\left\{r \geq r_{0}\right\}$ ) satisfying $r^{\frac{d-1}{2}}\left|\nabla_{h_{\tau, N}}^{l} \Psi\right|=O(1)$ for $0 \leq l \leq$ $\left\lceil\frac{d+1}{2}\right\rceil-1$ as $r \rightarrow+\infty$ :

1. If $d$ is odd, we can bound ${ }^{19}$ :

$$
\begin{aligned}
\sup _{\mathcal{S}_{\tau, r_{0}}}|\Psi|^{2} \leq & C(\tau)\left\{\left(\int_{\mathcal{S}_{\tau, r_{0}}}\left|\nabla_{h_{\tau, N}}^{\frac{d-1}{2}} \Psi\right|_{h_{\tau, N}}^{2} d h_{N}\right)^{\frac{1}{2}}\left(\int_{\mathcal{S}_{\tau, r_{0}}}\left|\nabla_{h_{\tau, N}}^{\frac{d+1}{2}} \Psi\right|_{h_{\tau, N}}^{2} d h_{N}\right)^{\frac{1}{2}}\right. \\
& \left.+\int_{\mathcal{S}_{\tau, r_{0}}}\left|\nabla_{h_{\tau, N}}^{\frac{d+1}{2}} \Psi\right|_{h_{\tau, N}}^{2} d h_{N}\right\} .
\end{aligned}
$$

19 Recall that $d$ is the dimension of the hypersurface $\{\bar{t}=\tau\}$. 
and

$$
\begin{aligned}
\sup _{\mathcal{S}_{\tau, r_{0}}}|\Psi|^{2} \leq C(\tau) & \left\{\left(\int_{\mathcal{S}_{\tau, r_{0}}}\left|\nabla_{h_{\tau, N}} \Psi\right|_{h_{\tau, N}}^{2} d h_{N}\right)^{\frac{1}{d-1}}\left(\int_{\mathcal{S}_{\tau, r_{0}}}\left|\nabla_{h_{\tau, N}}^{\frac{d+1}{2}} \Psi\right|_{h_{\tau, N}}^{2} d h_{N}\right)^{\frac{d-2}{d-1}}\right. \\
+ & \left.\int_{\mathcal{S}_{\tau, r_{0}}}\left|\nabla_{h_{\tau, N}}^{\frac{d+1}{2}} \Psi\right|_{h_{\tau, N}}^{2} d h_{N}\right\}
\end{aligned}
$$

2. If $d$ is even, we can bound for any $\varepsilon>0$ :

$$
\begin{aligned}
& \sup _{\mathcal{S}_{\tau, r_{0}}}|\Psi|^{2} \\
& \leq C_{\varepsilon}(\tau)\left\{\left(\int_{\mathcal{S}_{\tau, r_{0}}}\left|\nabla_{h_{\tau, N}} \Psi\right|_{h_{\tau, N}}^{2} d h_{N}\right)^{\frac{\varepsilon}{d-2}}\right. \\
& \quad \times\left(\int_{\mathcal{S}_{\tau, r_{0}}}\left(\left|\nabla_{h_{\tau, N}}^{\frac{d}{2}+1} \Psi\right|_{h_{\tau, N}}^{2}+\left|\nabla_{h_{\tau, N}}^{\frac{d}{2}} \Psi\right|_{h_{\tau, N}}^{2}\right) d h_{N}\right)^{1-\frac{\varepsilon \cdot(2 d-2)}{2(d-2)}} \\
& \left.\quad+\int_{\mathcal{S}_{\tau, r_{0}}}\left(\left|\nabla_{h_{\tau, N}}^{\frac{d}{2}+1} \Psi\right|_{h_{\tau, N}}^{2}+\left|\nabla_{h_{\tau, N}}^{\frac{d}{2}} \Psi\right|_{h_{\tau, N}}^{2}\right) d h_{N}+\int_{\mathcal{S}_{\tau, r_{0}}} r_{+}^{\varepsilon}\left|L^{\frac{d}{2}} \Psi\right|^{2} d h_{N}\right\} .
\end{aligned}
$$

In the above, $L$ is a vector field which is identically 0 in the region $\{r \lesssim 1\}$ and equals the coordinate vector field $\partial_{v}$ of the coordinate system $(v, \sigma)$ on $\{\bar{t}=\tau\} \cap\{r \gg 1\}$.

Proof Let us fix an $R_{0}>0$ large in terms of the geometry of $(\mathcal{M}, g)$.

On $\mathbb{R}^{d}$, the following Gagliardo-Nirenberg type inequalities hold for $f \in C_{0}^{\infty}\left(\mathbb{R}^{d}\right)$ (see [30] and [36]):

1. If $d$ is odd:

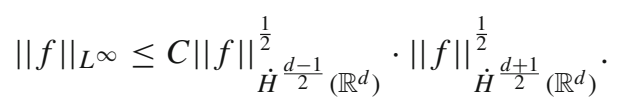

and

$$
\|f\|_{L^{\infty}} \leq C\|f\|_{\dot{H}^{1}\left(\mathbb{R}^{d}\right)}^{\frac{1}{d-1}} \cdot\|f\|_{\dot{H}^{\frac{d+1}{2}}\left(\mathbb{R}^{d}\right)}^{\frac{d-2}{d-1}}
$$

2. If $d$ is even:

$$
\|f\|_{L^{\infty}} \leq C_{\varepsilon}\|f\|_{\dot{H}^{\frac{d}{2}-\varepsilon}\left(\mathbb{R}^{d}\right)}^{\frac{1}{2}} \cdot\|f\|_{\dot{H}^{\frac{d}{2}+\varepsilon}\left(\mathbb{R}^{d}\right)}^{\frac{1}{\frac{1}{2}}} \cdot
$$

In the above, we have used the homogeneous norms $\dot{H}^{a}\left(\mathbb{R}^{d}\right)$ defined with the use of the Fourier transform as 


$$
\|f\|_{\dot{H}^{a}\left(\mathbb{R}^{d}\right)}^{2} \doteq \int_{\mathbb{R}^{d}}|\xi|^{2 a}|\hat{f}|^{2} d \xi
$$

where $\hat{f}$ is the Fourier transform of $f$.

Using a simple interpolation argument, we can also bound

$$
\|f\|_{\dot{H}^{\frac{d}{2}-\varepsilon}\left(\mathbb{R}^{d}\right)} \leq C_{\varepsilon}\|f\|_{\dot{H}^{1}\left(\mathbb{R}^{d}\right)}^{\frac{2 \varepsilon}{d-2}} \cdot\|f\|_{\dot{H}^{\frac{d}{2}}\left(\mathbb{R}^{d}\right)}^{\frac{d-2-2 \varepsilon}{d}}
$$

and

$$
\|f\|_{\dot{H}^{\frac{d}{2}+\varepsilon}\left(\mathbb{R}^{d}\right)} \leq C_{\varepsilon}\|f\|_{\dot{H}^{\frac{d}{2}}\left(\mathbb{R}^{d}\right)}^{1-\varepsilon} \cdot\|f\|_{\dot{H}^{\frac{d}{2}+1}\left(\mathbb{R}^{d}\right)}^{\varepsilon},
$$

and thus we obtain from (9.125) in the case $d$ is even:

$$
\|f\|_{L^{\infty}} \leq C_{\varepsilon}\|f\|_{\dot{H}^{1}\left(\mathbb{R}_{+}^{d}\right)}^{\frac{\varepsilon}{d-2}} \cdot\|f\|_{\dot{H}^{\frac{d}{2}}\left(\mathbb{R}_{+}^{d}\right)}^{1-\frac{\varepsilon \cdot d}{2(d-2)}} \cdot\|f\|_{\dot{H}^{\frac{d}{2}+1}\left(\mathbb{R}_{+}^{d}\right)}^{\frac{\varepsilon}{2}} \cdot
$$

Let $\chi_{R_{0}}:\{\bar{t}=\tau\} \rightarrow[0,1]$ be a smooth cut-off function such that $\chi_{R_{0}}$ is a function of $r$ satisfying $\chi_{R_{0}} \equiv 0$ on $\left\{r \leq R_{0}\right\}$ and $\chi_{R_{0}} \equiv 1$ on $\left\{r \geq 2 R_{0}\right\}$. Since $\chi_{R_{0}} \Psi$ is supported on $\left\{r \geq R_{0}\right\}$, by pulling back through the diffeomorphism $(r, \sigma)$ the operator $\nabla_{\mathbb{R}^{d}}$ on $\{\bar{t}=\tau\}$ and using the bound

$$
\left|\nabla_{\mathbb{R}^{d}}^{l} \varphi\right|_{h_{\tau, N}}^{2} \leq C_{l} \sum_{j=1}^{l} r^{-2(l-j)}\left|\nabla_{h_{\tau, N}} \varphi\right|_{h_{\tau, N}}^{2}
$$

we obtain from (9.123), (9.124) and (9.129):

1. In case $d$ is odd:

$$
\begin{aligned}
\sup _{\mathcal{S}_{\tau, r_{0}}}\left|\chi_{R_{0}} \Psi\right|^{2} \leq & C \cdot\left(\sum_{j=0}^{\frac{d-3}{2}} \int_{\mathcal{S}_{\tau, r_{0}}}\left|\nabla_{h_{\tau, N}}^{\frac{d-1}{2}-j}\left(\chi_{R_{0}} \Psi\right)\right|_{h_{\tau, N}}^{2} d h_{N}\right)^{\frac{1}{2}} \\
& \times\left(\sum_{j=0}^{\frac{d-1}{2}} \int_{\mathcal{S}_{\tau, r_{0}}} r^{-2 j}\left|\nabla_{h_{\tau, N}}^{\frac{d+1}{2}-j}\left(\chi_{R_{0}} \Psi\right)\right|_{h_{\tau, N}}^{2} d h_{N}\right)^{\frac{1}{2}} .
\end{aligned}
$$

and

$$
\begin{aligned}
\sup _{\mathcal{S}_{\tau, r_{0}}}\left|\chi_{R_{0}} \Psi\right|^{2} \leq & C \cdot\left(\int_{\mathcal{S}_{\tau, r_{0}}}\left|\nabla_{h_{\tau, N}}\left(\chi_{R_{0}} \Psi\right)\right|_{h_{\tau, N}}^{2} d h_{N}\right)^{\frac{1}{d-1}} \\
& \times\left(\sum_{j=0}^{\frac{d-1}{2}} \int_{\mathcal{S}_{\tau, r_{0}}} r^{-2 j}\left|\nabla_{h_{\tau, N}}^{\frac{d+1}{2}-j}\left(\chi_{R_{0}} \Psi\right)\right|_{h_{\tau, N}}^{2} d h_{N}\right)^{\frac{d-2}{d-1}}
\end{aligned}
$$


2. In case $d$ is even:

$$
\begin{aligned}
\sup _{\mathcal{S}_{\tau, r_{0}}}\left|\chi_{R_{0}} \Psi\right|^{2} \leq & C_{\varepsilon} \cdot\left(\int_{\mathcal{S}_{\tau, r_{0}}}\left|\nabla_{h_{\tau, N}}\left(\chi_{R_{0}} \Psi\right)\right|_{h_{\tau, N}}^{2} d h_{N}\right)^{\frac{\varepsilon}{d-2}} \\
& \times\left(\sum_{j=0}^{\frac{d-2}{2}} \int_{\mathcal{S}_{\tau, r_{0}}} r^{-2 j}\left|\nabla_{h_{\tau, N}}^{\frac{d}{2}-j}\left(\chi_{R_{0}} \Psi\right)\right|_{h_{\tau, N}}^{2} d h_{N}\right)^{1-\frac{\varepsilon \cdot d}{2(d-2)}} \\
& \times\left(\sum_{j=0}^{\frac{d}{2}} \int_{\mathcal{S}_{\tau, r_{0}}} r^{-2 j}\left|\nabla_{h_{\tau, N}}^{\frac{d}{2}+1-j}\left(\chi_{R_{0}} \Psi\right)\right|_{h_{\tau, N}}^{2} d h_{N}\right)^{\frac{\varepsilon}{2}}
\end{aligned}
$$

By applying the Leibnitz rule and using Hardy type inequalities of the form established in Lemma C.3, we obtain from (9.131), (9.132) and (9.133):

1. In case $d$ is odd:

$$
\begin{aligned}
\sup _{\mathcal{S}_{\tau, r_{0}}}\left|\chi_{R_{0}} \Psi\right|^{2} \leq & C \cdot\left\{\left(\int_{\mathcal{S}_{\tau, r_{0}}}\left|\nabla_{h_{\tau, N}}^{\frac{d-1}{2}} \Psi\right|_{h_{\tau, N}}^{2} d h_{N}\right)^{\frac{1}{2}}\left(\int_{\mathcal{S}_{\tau, r_{0}}}\left|\nabla_{h_{\tau, N}}^{\frac{d+1}{2}} \Psi\right|_{h_{\tau, N}}^{2} d h_{N}\right)^{\frac{1}{2}}\right. \\
& \left.+\int_{\mathcal{S}_{\tau, r_{0}} \cap\left\{R_{0} \leq r \leq 2 R_{0}\right\}}|\Psi|^{2} d h_{N}\right\} .
\end{aligned}
$$

and

$$
\begin{aligned}
\sup _{\mathcal{S}_{\tau, r_{0}}}\left|\chi_{R_{0}} \Psi\right|^{2} \leq & C \cdot\left\{\left(\int_{\mathcal{S}_{\tau, r_{0}}}\left|\nabla_{h_{\tau, N}} \Psi\right|_{h_{\tau, N}}^{2} d h_{N}\right)^{\frac{1}{d-1}}\left(\int_{\mathcal{S}_{\tau, r_{0}}}\left|\nabla_{h_{\tau, N}}^{\frac{d+1}{2}} \Psi\right|_{h_{\tau, N}}^{2} d h_{N}\right)^{\frac{d-2}{d-1}}\right. \\
& \left.+\int_{\mathcal{S}_{\tau, r_{0}} \cap\left\{R_{0} \leq r \leq 2 R_{0}\right\}}|\Psi|^{2} d h_{N}\right\}
\end{aligned}
$$

2. In case $d$ is even:

$$
\begin{aligned}
& \sup _{\mathcal{S}_{\tau, r_{0}}}\left|\chi_{R_{0}} \Psi\right|^{2} \leq C_{\varepsilon} \cdot\left\{\left(\int_{\mathcal{S}_{\tau, r_{0}}}\left|\nabla_{h_{\tau, N}} \Psi\right|_{h_{\tau, N}}^{2} d h_{N}\right)^{\frac{\varepsilon}{d-2}}\right. \\
& \left(\int_{\mathcal{S}_{\tau, r_{0}}}\left(\left|\nabla_{h_{\tau, N}}^{\frac{d}{2}+1} \Psi\right|_{h_{\tau, N}}^{2}+\left|\nabla_{h_{\tau, N}}^{\frac{d}{2}} \Psi\right|_{h_{\tau, N}}^{2}\right) d h_{N}\right)^{1-\frac{\varepsilon \cdot(2 d-2)}{2(d-2)}} \\
& \left.+\int_{\mathcal{S}_{\tau, r_{0}} \cap\left\{R_{0} \leq r \leq 2 R_{0}\right\}}|\Psi|^{2} d h_{N}\right\} .
\end{aligned}
$$


Since $\left(1-\chi_{R_{0}}\right)$ is supported in the compact region $\{\bar{t}=\tau\} \cap\left\{r \leq 2 R_{0}\right\}$ and $R_{0}$ is fixed in terms of the geometry of $\{\bar{t}=\tau\}$, using the Sobolev inequality (see e.g. [19])

$$
\sup _{\mathcal{S}_{\tau, r_{0}}}\left|\left(1-\chi_{R_{0}}\right) \Psi\right|^{2} \leq C(\tau) \sum_{j=0}^{\left\lceil\frac{d+1}{2}\right\rceil} \int_{\mathcal{S}_{\tau, r_{0}} \cap\left\{r \leq 2 R_{0}\right\}}\left|\nabla_{h_{\tau, N}}^{j} \Psi\right|_{h_{\tau, N}}^{2} d h_{N}
$$

and the Poincare inequality

$$
\begin{aligned}
\int_{\mathcal{S}_{\tau, r_{0}} \cap\left\{r \leq 2 R_{0}\right\}}|\Psi|^{2} d h_{N} \leq & C(\tau) \int_{\mathcal{S}_{\tau, r_{0}} \cap\left\{r \leq 2 R_{0}\right\}}\left|\nabla_{h_{\tau, N}} \Psi\right|_{h_{\tau, N}}^{2} d h_{N} \\
& +\int_{\mathcal{S}_{\tau, r_{0}} \cap\left\{R_{0} \leq r \leq 2 R_{0}\right\}}|\Psi|^{2} d h_{N},
\end{aligned}
$$

we obtain from (9.134), (9.135) and (9.136):

1. In case $d$ is odd:

$$
\begin{aligned}
\sup _{\mathcal{S}_{\tau, r_{0}}}|\Psi|^{2} \leq & C(\tau)\left\{\left(\int_{\mathcal{S}_{\tau, r_{0}}}\left|\nabla_{h_{\tau, N}}^{\frac{d-1}{2}} \Psi\right|_{h_{\tau, N}}^{2} d h_{N}\right)^{\frac{1}{2}}\left(\int_{\mathcal{S}_{\tau, r_{0}}}\left|\nabla_{h_{\tau, N}}^{\frac{d+1}{2}} \Psi\right|_{h_{\tau, N}}^{2} d h_{N}\right)^{\frac{1}{2}}\right. \\
& \left.+\int_{\mathcal{S}_{\tau, r_{0}}}\left|\nabla_{h_{\tau, N}}^{\frac{d+1}{2}} \Psi\right|_{h_{\tau, N}}^{2} d h_{N}+\int_{\mathcal{S}_{\tau, r_{0}} \cap\left\{R_{0} \leq r \leq 2 R_{0}\right\}}|\Psi|^{2} d h_{N}\right\} .
\end{aligned}
$$

and

$$
\begin{aligned}
\sup _{\mathcal{S}_{\tau, r_{0}}|\Psi|^{2} \leq} & C(\tau)\left\{\left(\int_{\mathcal{S}_{\tau, r_{0}}}\left|\nabla_{h_{\tau, N}} \Psi\right|_{h_{\tau, N}}^{2} d h_{N}\right)^{\frac{1}{d-1}}\left(\int_{\mathcal{S}_{\tau, r_{0}}}\left|\nabla_{h_{\tau, N}}^{\frac{d+1}{2}} \Psi\right|_{h_{\tau, N}}^{2} d h_{N}\right)^{\frac{d-2}{d-1}}\right. \\
& \left.+\int_{\mathcal{S}_{\tau, r_{0}}}\left|\nabla_{h_{\tau, N}}^{\frac{d+1}{2}} \Psi\right|_{h_{\tau, N}}^{2} d h_{N}+\int_{\mathcal{S}_{\tau, r_{0}} \cap\left\{R_{0} \leq r \leq 2 R_{0}\right\}}|\Psi|^{2} d h_{N}\right\}
\end{aligned}
$$

2. In case $d$ is even:

$$
\begin{aligned}
\sup _{\mathcal{S}_{\tau, r_{0}}}|\Psi|^{2} \leq & C_{\varepsilon}(\tau)\left\{\left(\int_{\mathcal{S}_{\tau, r_{0}}}\left|\nabla_{h_{\tau, N}} \Psi\right|_{h_{\tau, N}}^{2} d h_{N}\right)^{\frac{\varepsilon}{d-2}}\right. \\
& \times\left(\int_{\mathcal{S}_{\tau, r_{0}}}\left(\left|\nabla_{h_{\tau, N}}^{\frac{d}{2}+1} \Psi\right|_{h_{\tau, N}}^{2}+\left|\nabla_{h_{\tau, N}}^{\frac{d}{2}} \Psi\right|_{h_{\tau, N}}^{2}\right) d h_{N}\right)^{1-\frac{\varepsilon(2 d-2)}{2(d-2)}} \\
& \left.+\int_{\mathcal{S}_{\tau, r_{0}}}\left(\left|\nabla_{h_{\tau, N}}^{\frac{d}{2}+1} \Psi\right|_{h_{\tau, N}}^{2}+\left|\nabla_{h_{\tau, N}}^{\frac{d}{2}} \Psi\right|_{h_{\tau, N}}^{2}\right) d h_{N}+\int_{\mathcal{S}_{\tau, r_{0}} \cap\left\{R_{0} \leq r \leq 2 R_{0}\right\}}|\Psi|^{2} d h_{N}\right\} .
\end{aligned}
$$


Finally, in case $d$ is odd, using Lemma C.4 (as well as Lemma C.3) we can bound

$$
\begin{aligned}
\int_{\mathcal{S}_{\tau, r_{0}} \cap\left\{R_{0} \leq r \leq 2 R_{0}\right\}}|\Psi|^{2} d h_{N} \leq & C \cdot\left(\int_{\mathcal{S}_{\tau, r_{0}}}\left|\nabla_{h_{\tau, N}} \Psi\right|_{h_{\tau, N}}^{2} d h_{N}\right)^{\frac{1}{2}} \\
& \times\left(\int_{\mathcal{S}_{\tau, r_{0}}}\left|\nabla_{h_{\tau, N}}^{\frac{d+1}{2}} \Psi\right|_{h_{\tau, N}}^{2} d h_{N}\right)^{\frac{1}{2}},
\end{aligned}
$$

and

$$
\begin{aligned}
\int_{\mathcal{S}_{\tau, r_{0}} \cap\left\{R_{0} \leq r \leq 2 R_{0}\right\}}|\Psi|^{2} d h_{N} \leq & C \cdot\left(\int_{\mathcal{S}_{\tau, r_{0}}}\left|\nabla_{h_{\tau, N}} \Psi\right|_{h_{\tau, N}}^{2} d h_{N}\right)^{\frac{1}{d-1}} \\
& \times\left(\int_{\mathcal{S}_{\tau, r_{0}}}\left|\nabla_{h_{\tau, N}}^{\frac{d+1}{2}} \Psi\right|_{h_{\tau, N}}^{2} d h_{N}\right)^{\frac{d-2}{d-1}}
\end{aligned}
$$

and thus (9.140) and (9.139) become

$$
\begin{aligned}
\sup _{\mathcal{S}_{\tau, r_{0}}}|\Psi|^{2} \leq & C(\tau)\left\{\left(\int_{\mathcal{S}_{\tau, r_{0}}}\left|\nabla_{h_{\tau, N}}^{\frac{d-1}{2}} \Psi\right|_{h_{\tau, N}}^{2} d h_{N}\right)^{\frac{1}{2}}\left(\int_{\mathcal{S}_{\tau, r_{0}}}\left|\nabla_{h_{\tau, N}}^{\frac{d+1}{2}} \Psi\right|_{h_{\tau, N}}^{2} d h_{N}\right)^{\frac{1}{2}}\right. \\
& \left.+\int_{\mathcal{S}_{\tau, r_{0}}}\left|\nabla_{h_{\tau, N}}^{\frac{d+1}{2}} \Psi\right|_{h_{\tau, N}}^{2} d h_{N}\right\}
\end{aligned}
$$

and

$$
\begin{aligned}
\sup _{\mathcal{S}_{\tau, r_{0}}}|\Psi|^{2} \leq & C(\tau)\left\{\left(\int_{\mathcal{S}_{\tau, r_{0}}}\left|\nabla_{h_{\tau, N}} \Psi\right|_{h_{\tau, N}}^{2} d h_{N}\right)^{\frac{1}{d-1}}\left(\int_{\mathcal{S}_{\tau, r_{0}}}\left|\nabla_{h_{\tau, N}}^{\frac{d+1}{2}} \Psi\right|_{h_{\tau, N}}^{2} d h_{N}\right)^{\frac{d-2}{d-1}}\right. \\
& \left.+\int_{\mathcal{S}_{\tau, r_{0}}}\left|\nabla_{h_{\tau, N}}^{\frac{d+1}{2}} \Psi\right|_{h_{\tau, N}}^{2} d h_{N}\right\}
\end{aligned}
$$

In case $d$ is even, on the other hand, from Lemma C.3 we can readily bound

$$
\int_{\mathcal{S}_{\tau, r_{0}} \cap\left\{R_{0} \leq r \leq 2 R_{0}\right\}}|\Psi|^{2} d h_{N} \leq C_{\varepsilon} \int_{\mathcal{S}_{\tau, r_{0}}} r_{+}^{\varepsilon}\left|L^{\frac{d}{2}} \Psi\right|^{2} d h_{N},
$$


and hence (9.141) becomes:

$$
\begin{aligned}
\sup _{\mathcal{S}_{\tau, r_{0}}|\Psi|^{2} \leq} & C_{\varepsilon}(\tau)\left\{\left(\int_{\mathcal{S}_{\tau, r_{0}}}\left|\nabla_{h_{\tau, N}} \Psi\right|_{h_{\tau, N}}^{2} d h_{N}\right)^{\frac{\varepsilon}{d-2}}\right. \\
& \times\left(\int_{\mathcal{S}_{\tau, r_{0}}}\left(\left|\nabla_{h_{\tau, N}}^{\frac{d}{2}+1} \Psi\right|_{h_{\tau, N}}^{2}+\left|\nabla_{h_{\tau, N}}^{\frac{d}{2}} \Psi\right|_{h_{\tau, N}}^{2}\right) d h_{N}\right)^{1-\frac{\varepsilon \cdot(2 d-2)}{2(d-2)}} \\
& \left.+\int_{\mathcal{S}_{\tau, r_{0}}}\left(\left|\nabla_{h_{\tau, N}}^{\frac{d}{2}+1} \Psi\right|_{h_{\tau, N}}^{2}+\left|\nabla_{h_{\tau, N}}^{\frac{d}{2}} \Psi\right|_{h_{\tau, N}}^{2}\right) d h_{N}+\int_{\mathcal{S}_{\tau, r_{0}}} r_{+}^{\varepsilon}\left|L^{\frac{d}{2}} \Psi\right|^{2} d h_{N}\right\} .
\end{aligned}
$$

Lemma 9.11 For any $\tau>0$ and any smooth function $\Psi:\{\bar{t}=\tau\} \cap\{r \leq 1\} \rightarrow \mathbb{C}$ we can bound

$$
\begin{aligned}
& \sup _{\{\bar{t}=\tau\} \cap\left\{r \leq \frac{1}{2}\right\}}\left((-\log (r)+1)^{-2\left\lceil\frac{d-1}{2}\right\rceil} \cdot|\Psi|^{2}\right) \\
& \leq C(\tau)\left\{\int_{\{\bar{t}=\tau\} \cap\{r \leq 1\}}\left|\nabla_{h_{\tau, N}}^{\left\lceil\frac{d+1}{2}\right\rceil} \Psi\right|_{\left(1-\log \left(r_{\text {tim }}\right)\right) \cdot h_{R_{C}}}^{2} d h_{N}\right. \\
& \left.+\sum_{j=0}^{\left\lceil\frac{d-1}{2}\right\rceil} \int_{\{\bar{t}=\tau\} \cap\left\{\frac{1}{2} \leq r \leq 1\right\}}\left|\nabla_{h_{\tau, N}}^{j} \Psi\right|_{h_{\tau, N}}^{2} d h_{N}\right\} .
\end{aligned}
$$

Remark Notice that the energy norm in the right hand side of (9.148) degenerates polynomially at $\mathcal{H}^{+}$and logarithmically at $\partial_{\text {tim }} \mathcal{M}$.

Proof Using the fundamental theorem of calculus and a Cauchy-Schwarz inequality, we can bound for any smooth $\varphi:[0,1] \rightarrow \mathbb{C}$ and any $x_{0} \in(0,1]$ :

$$
\begin{aligned}
& \left|\varphi\left(x_{0}\right)\right| \leq \int_{x_{0}}^{1}\left|\frac{d \varphi}{d x}\right| d x+|\varphi(1)| \leq \\
& \leq\left(\int_{x_{0}}^{1} x^{-1} d x\right)^{\frac{1}{2}}\left(\int_{x_{0}}^{1} x\left|\frac{d \varphi}{d x}\right|^{2} d x\right)^{\frac{1}{2}}+|\varphi(1)| \\
& \quad \leq\left(-\log \left(x_{0}\right)\right) \cdot\left(\int_{0}^{1} x\left|\frac{d \varphi}{d x}\right|^{2} d x\right)^{\frac{1}{2}}+|\varphi(1)| .
\end{aligned}
$$

Let us fix a small $r_{0}=r_{0}(\tau)$, so that $\{\bar{t}=\tau\} \cap\left\{r \leq 2 r_{0}\right\}$ is diffeomorphic to $\left[0,2 r_{0}\right] \times \mathcal{H}_{\tau}$ and $h_{R_{c}}$ has the form (9.15) there. It then readily follows in view of (9.149) and a Sobolev inequality on the surfaces $\{r=$ const $\}$ that 


$$
\begin{aligned}
& \sup _{\{\bar{t}=\tau\} \cap\left\{r \leq r_{0}\right\}}\left((-\log (r))^{-2\left\lceil\frac{d-1}{2}\right\rceil} \cdot|\Psi|^{2}\right) \\
\leq & C(\tau)\left\{\sum_{j=0}^{\left\lceil\frac{d-1}{2}\right\rceil} \int_{\{\bar{t}=\tau\} \cap\left\{r \leq 2 r_{0}\right\}} r_{h o r}\left(-\log \left(r_{\text {tim }}\right)\right)^{-2\left\lceil\frac{d-1}{2}\right\rceil}\left|\nabla_{\mathcal{H}_{\tau}}^{j}\left(\partial_{r} \Psi\right)\right|^{2} d h_{N}\right. \\
& \left.+\sum_{j=0}^{\left\lceil\frac{d-1}{2}\right\rceil} \int_{\{\bar{t}=\tau\} \cap\left\{r_{0} \leq r \leq 2 r_{0}\right\}}\left|\nabla_{h_{\tau, N}}^{j} \Psi\right|_{h_{\tau, N}}^{2} d h_{N}\right\},
\end{aligned}
$$

where $\nabla_{\mathcal{H}_{\tau}}$ is the covariant derivative on the surfaces $\{r=$ const $\}$ associated to the metric $h_{\mathcal{H}_{\tau}}$ in (9.15). Inequality (9.148) now readily follows from (9.150) in view of the Sobolev-inequality in the region $\left\{r_{0} \leq r \leq \frac{1}{2}\right\}$ :

$$
\sup _{\{\bar{t}=\tau\} \cap\left\{r_{0} \leq r \leq \frac{1}{2}\right\}}|\Psi|^{2} \leq C(\tau) \sum_{j=0}^{\left\lceil\frac{d+1}{2}\right\rceil} \int_{\{\bar{t}=\tau\} \cap\left\{r_{0} \leq r \leq \frac{1}{2}\right\}}\left|\nabla_{h_{\tau, N}}^{j} \Psi\right|_{h_{\tau, N}}^{2} d h_{N}
$$

the Hardy-type inequality:

$$
\begin{aligned}
& \sum_{j=0}^{\left\lceil\frac{d-1}{2}\right\rceil} \int_{\{\bar{t}=\tau\} \cap\left\{r \leq 2 r_{0}\right\}} r_{h o r}\left(-\log \left(r_{\text {tim }}\right)\right)^{-2\left\lceil\frac{d-1}{2}\right\rceil}\left|\nabla_{\mathcal{H}_{\tau}}^{j}\left(\partial_{r} \Psi\right)\right|^{2} d h_{N} \\
& \leq C(\tau)\left\{\int_{\{\bar{t}=\tau\} \cap\{r \leq 1\}}\left|\nabla_{\left.h_{\tau, N} \frac{d+1}{2}\right\rceil} \Psi\right|_{\left(1-\log \left(r_{\text {tim }}\right)\right) \cdot h_{R_{C}}}^{2} d h_{N}\right. \\
& \left.+\sum_{j=0}^{\left\lceil\frac{d-1}{2}\right\rceil} \int_{\{\bar{t}=\tau\} \cap\left\{\frac{1}{2} \leq r \leq 1\right\}}\left|\nabla_{h_{\tau, N}}^{j} \Psi\right|_{h_{\tau, N}}^{2} d h_{N}\right\},
\end{aligned}
$$

and the Poincare-type inequality

$$
\begin{aligned}
& \sum_{j=0}^{\left\lceil\frac{d+1}{2}\right\rceil} \int_{\{\bar{t}=\tau\} \cap\left\{r_{0} \leq r \leq \frac{1}{2}\right\}}\left|\nabla_{h_{\tau, N}}^{j} \Psi\right|_{h_{\tau, N}}^{2} d h_{N} \\
& \leq C(\tau)\left\{\int_{\{\bar{t}=\tau\} \cap\{r \leq 1\}}\left|\nabla_{h_{\tau, N}}^{\left\lceil\frac{d+1}{2}\right\rceil} \Psi\right|_{h_{R_{C}}}^{2} d h_{N}\right. \\
& \left.\quad+\sum_{j=0}^{\left\lceil\frac{d-1}{2}\right\rceil} \int_{\{\bar{t}=\tau\} \cap\left\{\frac{1}{2} \leq r \leq 1\right\}}\left|\nabla_{h_{\tau, N}}^{j} \Psi\right|_{h_{\tau, N}}^{2} d h_{N}\right\} .
\end{aligned}
$$




\subsection{Proof of Corollary 9.2}

Inequality (9.48) for any dimension $d$ follows readily from the Gagliardo-Nirenberg type inequalities (9.121) and (9.122) for $r_{0}=0$, Assumption (EG7) and the decay estimate (9.40) of Theorem 9.1 for $q=1,\left\lfloor\frac{d+1}{2}\right\rfloor$ and $m+1$ in place of $m$.

In case the dimension $d$ is odd, after adding the Gagliardo-Nirenberg type inequalities (9.121) (for some small $r_{0}>0$ ) and (9.148), and using a Poincare type inequality and the "critical" Hardy-type estimate (C.8) (together with Lemma C.3) to bound

$$
\begin{aligned}
& \sum_{j=0}^{\left\lceil\frac{d-1}{2}\right\rceil} \int_{\{\bar{t}=\tau\} \cap\left\{\frac{1}{2} \leq r \leq 1\right\}}\left|\nabla_{h_{\tau, N}}^{j} \varphi\right|_{h_{\tau, N}}^{2} d h_{N} \\
& \leq C \cdot\left(\mathcal{E}_{\text {en, deg }}^{\left(0, \frac{d+1}{2}, 0\right)}[\varphi](\tau)+\left(\mathcal{E}_{\text {en,deg }}^{(0,1,0)}[\varphi](\tau)\right)^{\frac{1}{d-1}}\left(\mathcal{E}_{\text {en,deg }}^{\left(0, \frac{d+1}{2}, 0\right)}[\varphi](\tau)\right)^{\frac{d-2}{d-1}}\right),
\end{aligned}
$$

we obtain in view of Assumption (EG7):

$$
\begin{aligned}
& \sup _{\{\bar{t}=\tau\} \cap\left\{r \leq \frac{1}{2}\right\}}\left((-\log (r)+1)^{-2\left\lceil\frac{d-1}{2}\right\rceil} \cdot|\varphi|^{2}\right) \\
& \leq C \cdot\left\{\left(\mathcal{E}_{\text {en, deg }}^{(0,1,0)}[\varphi](\tau)\right)^{\frac{1}{d-1}}\left(\mathcal{E}_{\text {en, deg }}^{\left(0, \frac{d+1}{2}, 0\right)}[\varphi](\tau)\right)^{\frac{d-2}{d-1}}+\mathcal{E}_{\text {en, deg }}^{\left(0, \frac{d+1}{2}, 0\right)}[\varphi](\tau)\right\} .
\end{aligned}
$$

Therefore, from (9.155) and (9.41) we deduce that

$$
\begin{aligned}
& \sup _{\{\bar{t}=\tau\}}\left((-\log (r)+1)^{-2\left\lceil\frac{d-1}{2}\right\rceil} \cdot|\varphi|^{2}\right) \\
& \lesssim_{\varepsilon} \tau^{-d} \cdot \mathcal{E}_{\text {bound }}^{\left(d+1, \frac{d+1}{2},\left\lceil\delta_{0}^{-1} \cdot 2(d-1)\right\rceil\left(3 \frac{d+1}{2}+1\right) \cdot k\right)}[\varphi](0) \\
& \quad+\mathcal{F}_{\text {deg, }, \varepsilon}^{\left(d+1, \frac{d+1}{2}, m+\left\lceil\delta_{0}^{-1} \cdot(d-1)\right\rceil\left(3 \frac{d+1}{2}+1\right) \cdot k, k\right)}[F](\tau) .
\end{aligned}
$$

Moreover, using a standard Sobolev estimate we can also bound:

$$
\sup _{\{\bar{t}=\tau\} \cap\{r \leq 1\}}|\varphi|^{2} \leq C\left\{\mathcal{E}_{\text {en }}^{\left(0, \frac{d+1}{2}, 0\right)}[\varphi](\tau)+\int_{\{\bar{t}=\tau\} \cap\{r \leq 1\}}|\varphi|^{2} d h_{N}\right\},
$$

which, in view of a Poincare inequality and the Hardy inequality (C.8) (together with Lemma C.3), yields:

$\sup _{\{\bar{t}=\tau\} \cap\{r \leq 1\}}|\varphi|^{2} \leq C \cdot\left\{\mathcal{E}_{\text {en }}^{\left(0, \frac{d+1}{2}, 0\right)}[\varphi](\tau)+\left(\mathcal{E}_{\text {en, deg }}^{\left(0, \frac{d-1}{2}, 0\right)}[\varphi](\tau)\right)^{\frac{1}{2}}\left(\mathcal{E}_{\text {en, deg }}^{\left(0, \frac{d+1}{2}, 0\right)}[\varphi](\tau)\right)^{\frac{1}{2}}\right\}$ 
Therefore, from Theorem 9.1 and (9.158) we obtain

$$
\sup _{\{\bar{t}=\tau\} \cap\{r \leq 1\}}|\varphi|^{2} \lesssim \varepsilon \tau^{-\frac{d}{2}} \mathcal{E}_{\text {bound }}^{\left(d+1, \frac{d+1}{2},\left\lceil\delta_{0}^{-1} \cdot 2(d-1)\right\rceil\left(3 \frac{d+1}{2}+1\right) \cdot k\right)}[\varphi](0)+\mathcal{F}_{\text {deg, },, 0, d}[F](\tau),
$$

which combined with (9.156) yields the required estimate (9.46) for the pointwise decay of $\varphi$.

In case $m \geq 1$, we can bound in view of the Gagliardo-Nirenberg inequality (9.120) for some fixed small $r_{0}>0$ :

$$
\begin{aligned}
& \sum_{i=1}^{m} \sup _{\{\bar{t}=\tau\} \cap\left\{r \geq r_{0}\right\}}\left|\nabla_{g}^{i} \varphi\right|_{h}^{2} \leq C_{m, r_{0}}\left\{\left(\mathcal{E}_{\text {en,deg }}^{\left(0, \frac{d+1}{2}, m\right)}[\varphi](\tau)\right)^{\frac{1}{2}}\right. \\
& \left.\quad \times\left(\mathcal{E}_{\text {en, deg }}^{\left(0, \frac{d+1}{2}, m+1\right)}[\varphi](\tau)\right)^{\frac{1}{2}}+\mathcal{E}_{\text {en, deg }}^{\left(0, \frac{d+1}{2}, m+1\right)}[\varphi](\tau)\right\} .
\end{aligned}
$$

Therefore, from Theorem 9.1 and (9.160) we obtain:

$$
\begin{aligned}
& \sum_{i=1}^{m} \sup _{\{\bar{t}=\tau\} \cap\left\{r \geq r_{0}\right\}}\left|\nabla_{g}^{i} \varphi\right|_{h}^{2} \lesssim_{m, r_{0}, \varepsilon} \tau^{-d-1} \mathcal{E}_{\text {bound }}^{\left(d+1, \frac{d+1}{2}, m+1+\left\lceil\delta_{0}^{-1} \cdot 2(d-1)\right\rceil\left(3 \frac{d+1}{2}+1\right) \cdot k\right)}[\varphi](0) \\
& +\mathcal{F}_{d e g, \varepsilon, m+2, d}[F](\tau) .
\end{aligned}
$$

Moreover, using Lemma 9.11 for $\partial^{i} \varphi$ in place of $\Psi$, we can bound (in view also of Theorem 9.1):

$$
\begin{aligned}
& \sum_{i=1}^{m+1} \sup _{\{\bar{t}=\tau\} \cap\left\{r \leq \frac{1}{2}\right\}}\left((1-\log (r))^{-2\left\lceil\frac{d-1}{2}\right\rceil}\left|\nabla_{g}^{i} \varphi\right|_{h}^{2}\right) \lesssim_{m} \mathcal{E}_{\text {en, deg }}^{\left(0, \frac{d+1}{2}, m+2\right)}[\varphi](\tau) \\
& \lesssim m, \varepsilon \tau^{-d-1} \mathcal{E}_{\text {bound }}^{\left(d+1, \frac{d+1}{2}, m+2+\left\lceil\delta_{0}^{-1} \cdot 2(d-1)\right\rceil\left(3 \frac{d+1}{2}+1\right) \cdot k\right)}[\varphi](0)+\mathcal{F}_{\text {deg, }, m+1, d}[F](\tau)
\end{aligned}
$$

Since the function $(-\log (r))^{2\left\lceil\frac{d-1}{2}\right\rceil}$ is integrable near $r=0$, using the fundamental theorem of calculus and a Cauchy-Schwarz inequality we can bound for any smooth function $\Psi$ on $\{\bar{t}=\tau\} \cap\left\{r \leq \frac{1}{2}\right\}:^{20}$

\footnotetext{
${ }^{20}$ Here we have also used Assumption (EG8) on the boundedness of the volume of the region $\{\bar{t}=\tau\} \cap\{r \lesssim$ $1\}$.
} 


$$
\begin{aligned}
& \sup _{\{\bar{t}=\tau\} \cap\left\{r \leq \frac{1}{2}\right\}}|\Psi|^{2} \leq C\left\{\sup _{\{\bar{t}=\tau\} \cap\left\{r \leq \frac{1}{2}\right\}}\left((1-\log (r))^{-2\left\lceil\frac{d-1}{2}\right\rceil}\left|\nabla_{h_{\tau, N}} \Psi\right|_{h_{\tau, N}}^{2}\right)\right. \\
& \left.+\sup _{\{\bar{t}=\tau\} \cap\left\{\frac{1}{4} \leq r \leq \frac{1}{2}\right\}}|\Psi|^{2}\right\} .
\end{aligned}
$$

Thus, from (9.161), (9.162) and (9.163) (for $\partial^{i} \varphi$ in place of $\Psi$ ) we deduce the desired bound (9.47):

$$
\begin{aligned}
& \sum_{i=1}^{m} \sup _{\{\bar{t}=\tau\}}\left|\nabla^{m} \varphi\right|_{h}^{2} \lesssim m, \varepsilon \\
& \quad+\mathcal{F}_{\text {deg, }, \text { }, m+2, d}[F](\tau)
\end{aligned}
$$

Acknowledgments I would like to express my gratitude to my advisor Mihalis Dafermos for suggesting this problem to me, offering his advice and support and providing comments, ideas and assistance while this paper was being written. I would also like to thank Igor Rodnianski for his invaluable ideas and suggestions, as well as for our discussions on many aspects of the problem. Finally, I would like to thank Stefanos Aretakis, Yakov Shlapentokh-Rothman and Yannis Angelopoulos for many insightful conversations and important comments on preliminary versions of this paper.

Appendix A: Construction of the Natural Riemannian Metrics $h_{\tau, N}$ and $h_{\tau, K, \Phi}$

Let $\left(\mathcal{M}^{d+1}, g\right), d \geq 1$, be a Lorentzian manifold and $\bar{t}: \mathcal{M} \rightarrow \mathbb{R}$ a smooth function with acausal level sets. ${ }^{21}$ For any timelike vector field $N$ on $\mathcal{M}$ such that $d \bar{t}(N)=1$, there exists a special Riemannian metric $h_{\tau, N}$ defined on the $S_{\tau}=\{\bar{t}=\tau\}$ hypersurfaces naturally associated to the vector field $N$. This metric does not necessarily coincide with the induced metric on $S_{\tau}$, but its usefulness lies in the fact that the Laplace operator associated to $h_{\tau, N}$ appears naturally in a "useful" decomposition of the wave operator $\square_{g}$. A similar construction of a Riemannian metric $h_{\tau, K, \Phi}$ (not natural in this case) can be constructed in case one has two vector fields $K$, $\Phi$ with merely timelike span, such that $K$ satisfies $d \bar{t}(K)=1$ and becomes non timelike only on a set with compact intersection with each $S_{\tau}$, and $\Phi$ is tangent to the level sets of $\bar{t}$. We will now proceed with the details of the construction of these metrics.

\section{A.1 Construction of $h_{\tau, N}$}

Let $N$ be a timelike vector field on $\mathcal{M}$ with $d \bar{t}(N)=1$. Let $\mathcal{V}_{\mathcal{M}}$ denote vector bundle on $S_{\tau}$ defined as the pullback of $T \mathcal{M}$ through the inclusion $i: S_{\tau} \rightarrow \mathcal{M}$, the latter giving rise to the natural inclusion $T S_{\tau} \hookrightarrow \mathcal{V}_{\mathcal{M}}$. The vector bundle $\mathcal{V}_{\mathcal{M}}$ inherits from $T \mathcal{M}$ the Lorentzian metric $i^{*} g$ and the timelike section $i^{*} N$, which will be denoted as $g$ and $N$ respectively for notational simplicity. Similarly, the one form $d \bar{t}$ on $\mathcal{M}$

21 This manifold $\mathcal{M}$ will correspond to the manifold $\mathcal{M} \backslash \partial \mathcal{M}$ in the language of Sections 8-9. 
can also be viewed as a section of the dual bundle $\mathcal{V}_{\mathcal{M}}^{*}$. Let us denote with $g^{-1}$ the Lorentzian metric on $\mathcal{V}_{\mathcal{M}}^{*}$ associated to $g$ on $\mathcal{V}_{\mathcal{M}}$.

Let $\mathcal{K}_{N} \hookrightarrow \mathcal{V}_{\mathcal{M}}^{*}$ be the vector subbundle of $\mathcal{V}_{\mathcal{M}}^{*}$ defined as the set of $v \in \Gamma\left(\mathcal{V}_{\mathcal{M}}^{*}\right)$ such that $v(N)=0$. Then the Lorentzian metric $g^{-1}$ on $\mathcal{V}_{\mathcal{M}}^{*}$ induces a metric $h_{\text {inv }, \tau, N}$ on $\mathcal{K}_{N}$. It is easy to verify that $h_{i n t, \tau, N}$ is positive definite, owing to the fact that $g(N, N)<0$.

We can naturally identify $T^{*} S_{\tau}$ and $\mathcal{K}_{N}$ in the following way: Since $\mathcal{V}_{\mathcal{M}}$ can be split as $T S_{\tau} \oplus(N)$ (where $(N)$ is the line bundle spanned by $N$ ), any $\omega \in \Gamma\left(T^{*} S_{\tau}\right)$ can be extended to a section $\omega_{N} \in \Gamma\left(\mathcal{V}_{\mathcal{M}}^{*}\right)$ by demanding that $\left.\omega_{N}\right|_{T S_{\tau}}=\omega$ and $\omega_{N}(N)=0$. But then, since $\omega_{N}(N)=0, \omega_{N}$ is a section of $\mathcal{K}$. It is easy to verify that the mapping $\omega \rightarrow \omega_{N}$ is a vector bundle isomorphism. Thereofore, $T^{*} S_{\tau}$ inherits the Lorentzian metric $h_{\text {inv }, \tau, N}$ of $\mathcal{K}_{N}$.

Finally, we define the (positive definite) metric $h_{\tau, N}$ on $T S_{\tau}$ as the dual metric of $h_{\text {inv }, \tau, N}$ on $T^{*} S_{\tau}$. Thus, $\left(S_{\tau}, h_{\tau, N}\right)$ becomes a Riemannian manifold. Notice that the following relation holds:

$$
d v_{g}=\sqrt{-g(N, N)} \cdot d \bar{t} \wedge \operatorname{dvol}_{h_{\tau, N}}
$$

where $d_{\text {vol }}$ is the natural volume form on $\mathcal{M}$ associated with $g$, while $d v o l_{h_{\tau, N}}$ is the natural volume form on $\left(S_{\tau}, h_{\tau, N}\right)$ extended to a $d$-form on $\mathcal{M}$ by the requirement that $i_{N} d v o l_{h_{\tau, N}}=0$.

The connection of the metric $h_{\tau, N}$ with the wave operator $\square_{g}$ on $\mathcal{M}$ is the following: In any local coordinate system $\left(x^{1}, \ldots, x^{d}\right)$ on $S_{\tau}$, extended to a local coordinate system $\left(\bar{t}, x^{1}, \ldots, x^{d}\right)$ on $\mathcal{M}$ by the requirement that $N\left(x^{i}\right)=0$, the wave operator $\square_{g}$ on $\mathcal{M}$ around $S_{\tau}$ satisfies:

$$
\begin{aligned}
\square_{g}= & (\sqrt{-g})^{-1} N\left(\sqrt{-g} g^{\bar{t} \bar{t}} N\right)+(\sqrt{-g})^{-1} \partial_{x^{i}}\left(\sqrt{-g} g^{x^{i} \bar{t}} N\right) \\
& +(\sqrt{-g})^{-1} N\left(\sqrt{-g} g^{x^{i} \bar{t}} \partial_{x^{i}}\right)+\Delta_{h_{\tau}, N},
\end{aligned}
$$

where the operator $\Delta_{h_{\tau, N}, N}$ on $S_{\tau}$ is defined as:

$$
\Delta_{h_{\tau, N}, N}=\frac{1}{\sqrt{-g(N, N)}} d i v_{h_{\tau, N}}(\sqrt{-g(N, N)} d)
$$

and $d i v_{h_{\tau, N}}$ acting on the one form $\omega$ on $S_{\tau}$ is defined as the divergence (with respect to $h_{\tau, N}$ ) of the dual vector field $\omega^{\sharp} .{ }^{22}$ Equivalently, $\square_{g} \varphi$ takes the following covariant form for any $\Psi \in C^{\infty}(\mathcal{M})$ (assuming that $\mathcal{M}$ is orientable):

$$
\square_{g} \Psi=\operatorname{div}_{g}((N \Psi) \cdot d \bar{t})+\star \mathcal{L}_{N}\left(\left(N_{\text {tan }} \Psi\right) \cdot \operatorname{dvol}_{g}\right)+\Delta_{h_{\tau, N}, N} \Psi
$$

where $\star$ is the Hodge star operator on $(\mathcal{M}, g)$ and $N_{\text {tan }}$ is the projection of $\nabla \bar{t}$ on $S_{\tau}$ along $N$.

\footnotetext{
${ }^{22}$ Equivalently, it is the dual of the gradient operator on functions with respect to the inner product $\int_{S_{\tau}}\langle\cdot, \cdot\rangle_{h_{\tau, N}} d \operatorname{vol}_{h_{\tau, N}} \cdot$
} 


\section{A.2 Construction of $\boldsymbol{h}_{\tau, K, \Phi}$}

Let $K, \Phi$ be two vector fields on $\mathcal{M}$ with timelike span such that $d \bar{t}(K)=1$ and $\Phi$ is tangent to the leaves of the foliation $\left\{S_{\tau}\right\}_{\tau \in \mathbb{R}}$. Assume also that for any $\tau \in \mathbb{R}$, the set $\mathfrak{A}_{\tau}=\left\{p \in S_{\tau}: g(K, K) \geq 0\right\}$ is compact. In this case, we will also define a Riemannian metric $h_{\tau, K, \Phi}$ on $S_{\tau}$ which will prove helpful in decomposing the wave operator $\square_{g}$, but contrary to the metric $h_{\tau, N}$ constructed in the previous Section, the construction of $h_{\tau, K, \Phi}$ will not be natural.

Proceeding as in the previous Section, we can define the natural metric $h_{\tau, K}$ on $S_{\tau}$, but $h_{\tau, K}$ will now not be Riemannian on $\mathfrak{A}_{\tau}$. Moreover, $h_{\tau, K}$ will be singular at the points where $g(K, K)=0$, although its inverse (i.e. the associated metric on the dual bndle $T^{*} S_{\tau}$ ) will be smooth everywhere on $S_{\tau}$.

Since the span of $K, \Phi$ is everywhere timelike on $\mathcal{M}$ and $\mathfrak{A}_{\tau}$ is compact, for any $\tau \in \mathbb{R}$ there exists some $C_{\tau}>0$ such that everywhere on $S_{\tau}$ :

$$
\frac{g(K, K)}{g(\Phi, \Phi) g(K, K)-(g(\Phi, K))^{2}}>-C_{\tau} .
$$

Notice that the bound (A.5) holds trivially outside $\mathfrak{A}_{\tau}$. Thus, if $\Phi_{\perp}$ denotes the projecton of $\Phi$ on the orthogonal complement of $K$, we have

$$
\frac{1}{g\left(\Phi_{\perp}, \Phi_{\perp}\right)}=\frac{g(K, K)}{g(\Phi, \Phi) g(K, K)-(g(\Phi, K))^{2}}>-C_{\tau},
$$

and

$$
g\left(\Phi_{\perp}, \Phi_{\perp}\right) \leq 0
$$

on $\cup_{\tau} \mathfrak{A}_{\tau}$. Recall also that for any vector fields $X, Y$ with timelike (or null) span the following inverted Cauchy inequality holds pointwise:

$$
(g(X, Y))^{2} \geq|g(X, X)| \cdot|g(Y, Y)| .
$$

Thus, for any vector field $X$ on $\mathcal{M}$ with $g(X, K)=0$ and $g(X, X) \leq 0$ (notice that such a vector field must be identically 0 outside $\cup_{\tau} \mathfrak{A}_{\tau}$ ) we can bound due to (A.6), (A.7) and (A.8):

$$
g(X, X) \geq \frac{(g(X, \Phi))^{2}}{-\left|g\left(\Phi_{\perp}, \Phi_{\perp}\right)\right|}=\frac{(g(X, \Phi))^{2}}{g\left(\Phi_{\perp}, \Phi_{\perp}\right)}>-C_{\tau}(g(X, \Phi))^{2} .
$$

Recall that for any $\omega \in \Gamma\left(T^{*} S_{\tau}\right)$, extended to an element of $\Gamma\left(\mathcal{V}_{M}^{*}\right)$ by the condition $\omega(K)=0$, we have everywhere on $S_{\tau}$ :

$$
h_{\tau, K}^{-1}(\omega, \omega)=g^{-1}(\omega, \omega) .
$$

The bound (A.9) then readily implies that for any $\omega \in \Gamma\left(T^{*} S_{\tau}\right)$ we have

$$
h_{\tau, K}^{-1}(\omega, \omega)+C_{\tau}(\omega(\Phi))^{2}>0 .
$$


Therefore, the symmetric (2,0)-tensor

$$
h_{i n v, \tau, K, \Phi} \doteq h_{\tau, K}^{-1}+C_{\tau} \cdot \Phi \otimes \Phi
$$

is a positive definite metric on the dual bundle $T^{*} S_{\tau}$, and its inverse $h_{\tau, K, \Phi}$ is a Riemannian metric on $S_{\tau}$.

Notice that in this case, $S_{\tau}$ carries two volume forms, $d v o l_{h_{\tau, K} \Phi}$ and $i_{K} d v o l_{g}$, and their Radon-Nikodym derivative, i.e.

$$
\mathfrak{m}_{\tau, K, \Phi} \doteq \frac{\delta\left(i_{K} d v o l_{g}\right)}{\delta\left(d v o l_{h_{\tau, K, \Phi}}\right)}
$$

is a smooth function on $S_{\tau}$ satisfying

$$
\mathfrak{m}_{\tau, K, \Phi} \sim \sqrt{-\left(g(K, K)-\frac{(g(K, \Phi))^{2}}{g(\Phi, \Phi)}\right)}
$$

(compare with (A.1)).

Using the Riemannian metric $h_{\tau, K, \Phi}$, we can decompose the wave operator as:

$$
\begin{aligned}
\square_{g} \Psi= & \operatorname{div}_{g}((K \Psi) \cdot d \bar{t})+\star \mathcal{L}_{K}\left(\left(K_{\text {tan }} \Psi\right) \cdot \text { dvol }_{g}\right) \\
& -C_{\tau}\left(\star \mathcal{L}_{\Phi}\left((\Phi \Psi) \cdot \operatorname{dvol}_{g}\right)\right)+\Delta_{h_{\tau, K}, \Phi, \text { mod }} \Psi
\end{aligned}
$$

where $\star$ is the Hodge star operator on $(\mathcal{M}, g), K_{\text {tan }}$ is the projection of $\nabla \bar{t}$ on $S_{\tau}$ along $K$ and the elliptic operator $\Delta_{h_{\tau, K, \Phi}, \bmod }$ on $S_{\tau}$ is defined as

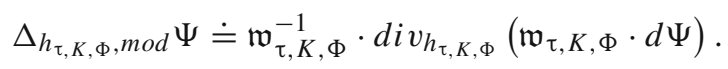

\section{Appendix B: Elliptic Estimates on Asymptotically Euclidean Riemannian Manifolds with Boundary}

In this section, we will establish some general elliptic estimates for asymptotically Euclidean Riemannian manifolds. This class of manifolds will include, in particular, the slices $\{\bar{t}=$ const $\}$ of the hyperboloidal foliation of the spacetimes $(\mathcal{M}, g)$ appearing in Sections 8 and 9, equipped with a Riemannian metric of the form that was introduced in Section A of the Appendix. However, the results of the current section might also be of independent interest.

Let $\mathcal{S}^{d}$ be a smooth manifold with boundary of dimension $d \geq 3$, with smooth compact boundary $\partial \mathcal{S}^{d-1}$ (allowed to be empty). We will assume that $\partial \mathcal{S}$ splits into two (not necessarily connected) components:

$$
\partial \mathcal{S}=\partial_{\text {tim }} \mathcal{S} \cup \partial_{\text {hor }} \mathcal{S} .
$$


The reason for assuming such a splitting for $\partial \mathcal{S}$ is that the hypersurfaces $\{\bar{t}=\tau\}$ of the spacetimes $(\mathcal{M}, g)$ in Sections 8 and 9 (i.e. the hypersurfaces on which the elliptic estimates of the current Section will be applied) have boundary $\{\bar{t}=\tau\} \cap \partial \mathcal{M}$, which is split as the disjoint union of $\{\bar{t}=\tau\} \cap \partial_{\text {tim }} \mathcal{M}$ and $\{\bar{t}=\tau\} \cap \mathcal{H}$.

Let $h$ be a Riemannian metric on $\mathcal{S} \backslash \partial \mathcal{S}$. We would like $h$ to model the Riemannian metric $h_{\tau, K_{R_{C}} \text {, }}$ on the hypersurfaces $\{\bar{t}=\tau\}$ of Section 9 (constructed as in the previous Section of the Appendix). To this end, we assume that $(\mathcal{S} \backslash \partial \mathcal{S}, h)$ is asymptotically flat in the sense that there exists a compact subset $\mathcal{K} \subset \mathcal{S}$ containing $\partial \mathcal{S}$ such that $\mathcal{S} \backslash \mathcal{K}$ has a finite number of connected components, each mapped diffeomorphically onto $\left(R_{0},+\infty\right) \times \mathbb{S}^{d-1}$ through a coordinate chart $(r, \sigma)$, and in this chart $h$ has the expression

$$
h=d r^{2}+r^{2} g_{\mathbb{S}^{d-1}}+h_{a s},
$$

with

$$
\left|\nabla^{m} h_{a s}\right|_{h}=O\left(r^{-m-1}\right)
$$

for all integers $m \geq 0$. In what follows, $\nabla$ will denote the covariant connection with respect to $h$.

We extend $r$ smoothly on the whole of $\mathcal{S}$, so that it is strictly positive on $\mathcal{S} \backslash \partial \mathcal{S}$ and satisfies $r=0$ and $d r \neq 0$ on $\partial \mathcal{S}$, if $\partial \mathcal{S} \neq \emptyset$. If $\partial \mathcal{S}=\emptyset$, we simply require that $r \geq 1$ everywhere on $\mathcal{S}$. Notice that the assumtion $d r \neq 0$ on $\partial \mathcal{S}$ together with the compactness of $\partial \mathcal{S}$ imply that for $\{r \ll 1\}$ the level sets of the function $r$ are smooth hypersurfaces of $\mathcal{S}$, and $r$ can be used as a coordinate function.

As for the behaviour of $h$ near the boundary $\partial \mathcal{S}$, we impose the following assumptions (in accordance with the behaviour of metric $h_{\tau, K_{R_{C}}, \Phi}$ on the hypersurfaces $\{\bar{t}=\tau\}$ of Section 9): Let us denote by $h_{\text {tan }}$ the induced metric on the $\{r=$ const $\}$ hypersurfaces for $\{0<r \ll 1\}$, and extend it to a symmetric $(0,2)$-form on $\mathcal{S} \backslash \partial \mathcal{S}$ by the requirement that $h_{\text {tan }}(\nabla r, \cdot) \equiv 0$. Then we assume that the metric $h$ in the region $\{r \ll 1\}$ takes the following form:

1. Near the $\partial_{\text {hor }} \mathcal{S}$ component of the boundary:

$$
h=\left(r^{-1}+O(1)\right) d r^{2}+h_{t a n}
$$

and $h_{\text {tan }}$ extends smoothly on $\partial_{h o r} \mathcal{S}$, with $\left.h_{\text {tan }}\right|_{\partial_{h o r} \mathcal{S}}$ being positive definite.

2. Near the $\partial_{\text {tim }} \mathcal{S}$ component of the boundary:

$$
h=(1+O(r)) d r^{2}+h_{t a n},
$$

and $h_{\text {tan }}$ extends smoothly on $\partial_{t i m} \mathcal{S}$, with $\left.h_{t a n}\right|_{\partial_{t i m} \mathcal{S}}$ being positive definite.

We will also assume that we are given a continuous function $\omega: \mathcal{S} \rightarrow[0,+\infty)$ which is smooth on $\mathcal{S} \backslash \partial_{\text {hor }} \mathcal{S}$, such that 
- $\omega=0$ only on $\partial_{\text {hor }} \mathcal{S}$

- $\omega=c r^{\frac{1}{2}}(1+O(r))$ for some $c>0$ near $\partial_{h o r} \mathcal{S}$

- $\omega>0$ near $\partial_{\text {tim }} \mathcal{S}$ and

- $\omega=1+O\left(r^{-1}\right)$ in the region $\{r \gg 1\}$.

For this class of Riemannian manifolds we will establish a series of elliptic estimates in the following sections. But before that, we have to carry out the construction of some geometric objects that will appear in our estimates.

\section{B.1 Geometric Constructions on $(\mathcal{S}, h)$}

We will define the perturbed Laplacian

$\Delta_{h, \omega}$ associated to $h, \omega$ by the relation:

$$
\Delta_{h, \omega}=\omega^{-1} \operatorname{div}_{h}(\omega \cdot d)
$$

This operator models the operator (A.16) associated to the metric $h_{\tau, K_{R_{c}}}, \Phi$ on the hypersurfaces $\{\bar{t}=\tau\}$ of the spacetimes $(\mathcal{M}, g)$ of Section 9 .

On each connected component of the region $\{r \gg 1\}$ in the $(r, \sigma)$ coordinate chart, (B.6) takes the form:

$$
\Delta_{h, \omega}=\Delta_{h}+O\left(r^{-2}\right) \partial_{r}+O\left(r^{-3}\right) \partial_{\sigma}
$$

On the other hand, near the boundary $\partial \mathcal{S}$ we have the following relations:

1. According to (B.4), if $Y=|\nabla r|_{h}^{-2} \cdot \nabla r,{ }^{23}$ near $\partial_{h o r} \mathcal{S}$ we have:

$$
\Delta_{h, \omega}=a^{-1} Y(r a(1+O(r)) \cdot Y)+\Delta_{h_{t a n}}+O(r) \cdot X,
$$

where $a$ is a positive function smooth up to $\partial_{h o r} \mathcal{S}, \Delta_{h_{\text {tan }}}$ is the Laplacian of the induced metric on the $\{r=$ const $\}$ hypersuerfaces and $X$ is a vector field in a neighborhood of $\partial \mathcal{S}$ smooth up to $\partial \mathcal{S}$. Hence, $\Delta_{h, \omega}$ is degenerate elliptic near $\partial_{\text {hor }} \mathcal{S}$.

2. According to (B.5), near $\partial_{\text {tim }} \mathcal{S}$ we have

$$
\Delta_{h, \omega}=Y((1+O(r)) \cdot Y)+\Delta_{h_{\text {tan }}}+X
$$

Hence, $\Delta_{h, \omega}$ is uniformly elliptic near $\partial_{t i m} \mathcal{S}$.

It will be convenient to have a canonical coordinate "chart" near the boundary of $\partial \mathcal{S}$. If $r_{1}$ is small enough, we can define the diffeomorphism

$$
\mathcal{J}:\left\{r \leq r_{1}\right\} \subset \mathcal{S} \rightarrow\left[0, r_{1}\right] \times \partial \mathcal{S}
$$

so that for any point $p \in\left\{r \leq r_{1}\right\}$ :

\footnotetext{
${ }^{23}$ Notice that $Y$ can be extended smoothly on $\partial \mathcal{S}$.
} 
- $\pi_{1}(\mathcal{J}(p))=r(p)$

- $\pi_{2}(\mathcal{J}(p))$ is the unique point on $\partial \mathcal{S}$ connected with $p$ through an integral line of the vector field $Y=|\nabla r|_{h}^{-2} \cdot \nabla r$.

In the above, $\pi_{1}:\left[0, r_{1}\right] \times \partial \mathcal{S} \rightarrow\left[0, r_{1}\right]$ and $\pi_{2}:\left[0, r_{1}\right] \times \partial \mathcal{S} \rightarrow \partial \mathcal{S}$ are the projections onto the first and second factor of $\left[0, r_{1}\right] \times \partial \mathcal{S}$ respectively.

Notice that through this map, the vector field $Y$ is mapped to the vector field $\partial_{r}$, i.e. the coordinate vector field on $\left[0, r_{1}\right] \times \partial \mathcal{S}$ which is tangent to the lines $\left\{\pi_{2}=\right.$ const $\}$ and is mapped to $\frac{d}{d r}$ by $\pi_{1}$. Moreover, $h_{\text {tan }}$ is a smooth non-degenerate Riemannian metric on the hypersurfaces $\left\{\pi_{1}=\rho\right\}$, varying smoothly with $\rho$. From now on, we will assume that $\left\{r \leq r_{1}\right\}$ and $\left[0, r_{1}\right] \times \partial \mathcal{S}$ have been identified through $\mathcal{J}$.

Construction of the auxilliary metrics $\tilde{h}, \tilde{h}_{t i m}$ Since $h$ is singular on $\partial_{h o r} \mathcal{S}$, it will be useful to have a second Riemannian metric $\tilde{h}$ on $\mathcal{S}$ that is smooth up to $\partial_{h o r} \mathcal{S},{ }^{24}$ so that we can measure the norms of tensors with the use of $\tilde{h}$. The metric $\tilde{h}$ will also be used to define covariant derivative operators and geometric volume forms which are regular up to $\partial_{h o r} \mathcal{S}$ (where the associated constructions with $h$ will either be singular or degenerate). This metric will model the metric $h_{\tau, N}$ on the hypersurfaces $\{\bar{t}=\tau\}$ of the spacetimes $(\mathcal{M}, g)$ of Sections 8 and 9. To this end, we define a Riemannian metric $\tilde{h}$ on $\mathcal{S}$ such that $\tilde{h} \equiv h$ on $\{r \geq 1\}$, and

$$
\tilde{h}=d r^{2}+h_{t a n}
$$

in the region $r \ll 1$.

It will be convenient to define the smooth functions

$$
r_{+}=\left(1+r^{2}\right)^{1 / 2}
$$

and

$$
r_{-}=\left(1+\frac{1}{r}\right)^{-1}
$$

Notice that $r_{+} \sim r$ for $r \gg 1$ and $r_{+} \sim 1$ for $r \lesssim 1$, while $r_{-} \sim r$ near $\partial \mathcal{S}$ and $r_{-} \sim 1$ away from $\partial \mathcal{S}$.

We also define the smooth funcions $r_{\text {hor }}, r_{\text {tim }}: \mathcal{S} \rightarrow[0,1]$ by the following requirements

- $r_{h o r}=r$ in the region $\left\{\operatorname{dist}_{\tilde{h}}\left(\cdot, \partial_{h o r} \mathcal{S}\right) \ll 1\right\}$ (where $\operatorname{dist}_{\tilde{h}}\left(\cdot, \partial_{h o r} \mathcal{S}\right)$ is smooth), $r_{\text {hor }}>0$ on $\mathcal{S} \backslash \partial_{\text {hor }} \mathcal{S}$ and $r_{\text {hor }} \equiv 1$ on $\left\{\operatorname{dist}_{\tilde{h}}\left(\cdot, \partial_{\text {hor }} \mathcal{S}\right) \geq 1\right\}$.

- $r_{\text {tim }}=r$ in the region $\left\{\operatorname{dist}_{\tilde{h}}\left(\cdot, \partial_{\text {tim }} \mathcal{S}\right) \ll 1\right\}, r_{\text {tim }}>0$ on $\mathcal{S} \backslash \partial_{\text {tim }} \mathcal{S}$ and $r_{\text {tim }} \equiv 1$ on $\left\{\operatorname{dist}_{\tilde{h}}\left(\cdot, \partial_{\text {tim }} \mathcal{S}\right) \geq 1\right\}$.

Finally, define the Riemannian metric $\tilde{h}_{t i m}$ on $\mathcal{S} \backslash \partial_{t i m} \mathcal{S}$ so that $\tilde{h}_{t i m} \equiv \tilde{h}$ on $\left\{r_{t i m} \geq \frac{1}{2}\right\}$ and

$$
\tilde{h}_{t i m}=r^{-2} d r^{2}+\left(-\log \left(r_{t i m}\right)\right) \cdot h_{t a n}
$$

$24 h$ is already smooth up to $\partial_{\text {tim }} \mathcal{S}$. 
in the region $\left\{r_{\text {tim }} \ll 1\right\}$. This metric will only be used to handle difficulties appearing near $\partial_{\text {tim }} \mathcal{S}$ in the derivation of the elliptic estimates in this Section, and is not associated with any geometric construction performed in Sections 8 and 9.

Remark We will raise and lower indices only with the use of the singular metric $h$. The non-singular metric $\tilde{h}$ will only be used to measure norms of tensors on $\mathcal{S}$. Covariant derivatives with respect to $h$ will be simply denoted by $\nabla$, while the ones associated to $\tilde{h}$ and $\tilde{h}_{\text {tim }}$ will be denoted by $\nabla^{(\tilde{h})}$ and $\nabla^{\left(\tilde{h}_{\text {tim }}\right)}$ respectively.

\section{B.2 Elliptic Estimates on $(\mathcal{S}, h)$}

In this Section, we will establish elliptic estimates on the Riemannian manifolds $(\mathcal{S}, h)$ associated to the elliptic operators (B.6) and (B.72). To this end, we will need some shorthand notation for weighted norms of derivatives of functions $u$ on $\mathcal{S}$, with weights which are either regular or degenerate on parts of the boundary $\partial \mathcal{S}$. These weights are tied naturally to the use of differential operators associated to the different Riemannian metrics $h, \tilde{h}$ and $\tilde{h}_{t i m}$ that we have already defined on $\mathcal{S} \backslash \partial \mathcal{S}$. With this motivation, after fixing some smooth cut-off functions $\chi_{\geq r_{1}}, \chi_{\leq r_{1}}: \mathcal{S} \rightarrow[0,1]$ so that $\chi_{\geq r_{1}} \equiv 1$ on $\left\{r \geq r_{1}\right\}$ and $\chi \geq r_{1} \equiv 0$ on $\left\{r \leq r_{1} / 2\right\}$ and $\chi \leq r_{1}=1-\chi \geq r_{1}$, we introduce the following definition:

Definition We will introduce the following pointwise norm on $\mathcal{S} \backslash \partial \mathcal{S}$ for any pair of Riemannian metrics $h_{1}, h_{2}$ on $\mathcal{S} \backslash \partial \mathcal{S}$ and any integer $m \geq 2$ :

$$
\begin{aligned}
& |u|_{h_{1}, h_{2} ; m}^{2} \\
& \doteq \chi_{\geq r_{1}}\left|\left(\nabla^{\left(h_{1}\right)}\right)^{m} u\right|_{h_{2}}^{2} \\
& \quad+\chi_{\leq r_{1}} \cdot r_{h o r}^{2}\left|\left(\nabla^{\left(h_{1}\right)}\right)^{m-2}\left(Y^{2} u\right)\right|_{h_{2}}^{2}+\chi_{\leq r_{1}} \cdot r_{h o r}\left|\left(\nabla^{\left(h_{1}\right)}\right)^{m-2}\left(i_{*} \nabla^{\left(h_{\text {tan }}\right)}(Y u)\right)\right|_{h_{2}}^{2} \\
& \quad+\chi_{\leq r_{1}} \cdot\left|\left(\nabla^{\left(h_{1}\right)}\right)^{m-2}\left(\left(i_{*} \nabla^{\left(h_{\text {tan }}\right)}\right)^{2} u\right)\right|_{h_{2}}^{2} .
\end{aligned}
$$

In the above the $i_{*}$ notation is used as follows: For any $(0, k)$-tensor $\mathfrak{m}$ on the surfaces $\{r=$ const $\}$ on $\left\{r \leq r_{1}\right\}$ varying smoothly with $r$ we denote with $i_{*} \mathfrak{m}$ the unique tensor on $\left\{r \leq r_{1}\right\}$ such that for any local frame $\left\{E_{0}, E_{1}, \ldots E_{d-1}\right\}$ on $\left\{r \leq r_{1}\right\}$ with $E_{0}=Y$ and $E_{1} r=\ldots=E_{d-1} r=0$ and any $i_{1}, \ldots i_{k} \in\{0, \ldots d-1\}$, the following relation holds:

$$
i_{*} \mathfrak{m}\left(E_{i_{1}}, \ldots E_{i_{k}}\right)= \begin{cases}0, & \text { if some of the } i_{k} \text { 's is } 0 \\ \mathfrak{m}\left(E_{i_{1}}, \ldots E_{i_{k}}\right) & \text { if none of the } i_{k} \text { 's is } 0\end{cases}
$$

Remark In most instances where the notation (B.15) will appear, $h_{1}$ will be the everywhere regular metric $\tilde{h}$, while $h_{2}$ will be a metric which is singular on $\partial_{\text {tim }} \mathcal{S}$.

We will establish the following lemma: 
Proposition B.1 (Degenerate elliptic estimates). For any $l \in \mathbb{N}$ with $2 \leq l \leq$ $\left\lfloor\frac{d+1}{2}\right\rfloor$, any $k_{0} \in \mathbb{N}$ and any $\beta \in\left(-\bar{\delta}_{k_{0}}, 1\right)$ for some $\bar{\delta}_{k_{0}}>0$ depending on $k_{0}$, there exists a (small) $r_{0}>0$ so that we can bound for any $u \in C^{\infty}(\mathcal{S})$ with $\lim \sup _{r \rightarrow+\infty}\left|r^{\frac{d-1}{2}+j} \nabla^{j} u\right|_{h}<+\infty$ for any $j \leq l+k_{0}$ :

$$
\begin{aligned}
& \sum_{k=0}^{k_{0}}\left\{\int_{\mathcal{S}} r_{+}^{-\beta}|u|_{\tilde{h}, \tilde{h}_{t i m} ; k+l}^{2} d v o l_{\tilde{h}}+\sum_{j=1}^{l-1} \int_{\left\{r \geq r_{0}\right\}} r_{+}^{-2 j-\beta} \cdot\left|\left(\nabla^{(\tilde{h})}\right)^{k+l-j} u\right|_{\tilde{h}}^{2} d v o l_{\tilde{h}}\right. \\
& \quad+\sum_{j=1}^{l-1} \int_{\left\{r \leq r_{0}\right\}}\left(\left|\left(\nabla^{(\tilde{h})}\right)^{k+l-j-1}(Y u)\right|_{\tilde{h}_{\text {tim }}}^{2}+r_{-}^{-1} \log ^{-2}\left(\frac{r_{-}}{2}\right)\right. \\
& \left.\left.\quad \cdot\left|\left(\nabla^{(\tilde{h})}\right)^{k+l-j-1}\left(i_{*}\left(\nabla^{\left(h_{\text {tan }}\right)} u\right)\right)\right|_{\tilde{h}_{\text {tim }}}^{2}\right) d v o l_{\tilde{h}}\right\} \\
& \leq C_{\beta, k_{0}} \sum_{k=0}^{k_{0}} \int_{\mathcal{S}} r_{+}^{-\beta}\left|\left(\nabla^{(\tilde{h})}\right)^{k+l-2}\left(\Delta_{h, \omega} u\right)\right|_{\tilde{h}_{\text {tim }}}^{2} d v o l_{\tilde{h}} \\
& \quad+C_{\beta, k_{0}} \sum_{j=0}^{1} \max \left\{-\operatorname{Re}\left\{\int_{\partial_{\text {tim }} \mathcal{S}} h_{\text {tan }}\left(\left(\nabla^{\left(h_{\text {tan }}\right)}\right)^{j}(Y u),\left(\nabla^{\left(h_{\text {tan }}\right)}\right)^{j} \bar{u}\right) d h_{\text {tan }}\right\}, 0\right\}
\end{aligned}
$$

and

$$
\begin{aligned}
& \sum_{k=0}^{k_{0}}\left\{\int_{\mathcal{S}} r_{+}^{-\beta}|u|_{\tilde{h},\left(1-\log \left(r_{\text {tim }}\right)\right) \cdot \tilde{h} ; k+l}^{2} d v o l_{\tilde{h}}+\sum_{j=1}^{l-1} \int_{\left\{r \geq r_{0}\right\}} r_{+}^{-2 j-\beta} \cdot\left|\left(\nabla^{(\tilde{h})}\right)^{k+l-j} u\right|_{\tilde{h}}^{2} d v o l_{\tilde{h}}\right. \\
& \quad+\sum_{j=1}^{l-1} \int_{\left\{r \leq r_{0}\right\}}\left(\left|\left(\nabla^{(\tilde{h})}\right)^{k+l-j-1}(Y u)\right|_{\left(1-\log \left(r_{\text {tim }}\right) \tilde{h}^{2}\right.}^{2}+r_{-}^{-1} \log ^{-2}\left(\frac{r_{-}}{2}\right)\right. \\
& \left.\left.\quad \cdot\left|\left(\nabla^{(\tilde{h})}\right)^{k+l-j-1}\left(i_{*}\left(\nabla^{\left(h_{\text {tan }}\right)} u\right)\right)\right|_{\left(1-\log \left(r_{\text {tim }}\right)\right) \tilde{h}}^{2}\right) d v o l_{\tilde{h}}\right\} \\
& \leq C_{\beta, k_{0}} \sum_{k=0}^{k_{0}} \int_{\mathcal{S}} r_{+}^{-\beta}\left|\left(\nabla^{(\tilde{h})}\right)^{k+l-2}\left(\Delta_{h, \omega} u\right)\right|_{\left(1-\log \left(r_{\text {tim }}\right)\right) \cdot \tilde{h}}^{2} d v o l_{\tilde{h}} \\
& \quad+C_{\beta, k_{0}} \sum_{j=0}^{1} \max \left\{-\operatorname{Re}\left\{\int_{\partial_{\text {tim }} \mathcal{S}} h_{\text {tan }}\left(\left(\nabla^{\left(h_{\text {tan }}\right)}\right)^{j}(Y u),\left(\nabla^{\left(h_{\text {tan }}\right)}\right)^{j} \bar{u}\right) d h_{\text {tan }}\right\}, 0\right\},
\end{aligned}
$$

where the constant $C_{\beta, k_{0}}$ of the right hand side depends only on $\beta, k_{0}$ and on the geometry of $(\mathcal{S}, h), \tilde{h}$ and $\omega$.

Remark Notice that the boundary terms on the right hand side of (B.17) and (B.18) contain only terms on the $\partial_{t i m} \mathcal{S}$ part of the boundary. These terms vanish when $u$ satisfies the Dirichlet or Neumann conditions on $\partial_{\text {tim }} \mathcal{S}$. 
Proof Withot loss of generality, we can assume that $u$ is real valued. We will treat $r_{0}$ and $\bar{\delta}_{k_{0}}$ as small parameters (which will be fixed later in the proof).

The region $\left\{r \leq 2 r_{0}\right\}$ will be identified with $\left[0,2 r_{0}\right] \times \partial \mathcal{S}$ through the diffeomorphism $\mathcal{J}$. We will assume that $r_{0}$ is small enough so that $\left[0,2 r_{0}\right] \times \partial_{\text {hor }} \mathcal{S}$ and $\left[0,2 r_{0}\right] \times \partial_{\text {tim }} \mathcal{S}$ are disjoint. Let us define two smooth cut-off functions $\chi_{\leq r_{0}}^{h o r}, \chi_{\leq r_{0}}^{t i m}$ : $\mathcal{S} \rightarrow[0,1]$ such that

$$
\begin{aligned}
& \text { 1. } \operatorname{supp}\left(\chi_{\leq r_{0}}^{\text {hor }}\right) \subseteq\left[0,2 r_{0}\right] \times \partial_{\text {hor }} \mathcal{S} \text { and } \chi_{\leq r_{0}}^{\text {hor }} \equiv 1 \text { on }\left[0, r_{0}\right] \times \partial_{\text {hor }} \mathcal{S} \text { and } \\
& \text { 2. } \operatorname{supp}\left(\chi_{\leq r_{0}}^{t i m}\right) \subseteq\left[0,2 r_{0}\right] \times \partial_{\text {tim }} \mathcal{S} \text { and } \chi_{\leq r_{0}}^{t i m} \equiv 1 \text { on }\left[0, r_{0}\right] \times \partial_{\text {tim }} \mathcal{S} .
\end{aligned}
$$

We will also set $\chi \geq r_{0}=1-\chi_{\leq r_{0}}^{\text {hor }}-\chi_{\leq r_{0}}^{\text {tim }}$. Let us also define $\chi_{\geq R_{0}}=1-\chi\left(\frac{r}{R_{0}}\right)$ for some $R_{0}$ large and fixed in terms of $\beta$ and the geometry of $(\mathcal{S}, \bar{h})$.

Without loss of generality, we can assume that $k_{0}=0$ (since the proof in the case $k_{0} \geq 1$ follows in exactly the same way). In order to present the necessary ideas in a simpler form, we will first establish the case when $l=2$.

Remark Notice that for $l=2$, it is most difficult to treat the case $d=3$, since in that case one is not able to obtain control over weighted $L^{2}$ norms of $u$ using only the finiteness of $\int_{\mathcal{S}} r_{+}^{-\beta}\left|\nabla^{2} u\right|^{2}$ dvol $_{\tilde{h}}$ together with Hardy and Poincare inequalities. The same difficulties occur generally in dimension $d$ for $l=\left\lfloor\frac{d+1}{2}\right\rfloor$.

We will start by establishing the following elliptic estimate on each connected component of the far away region $\{r \gg 1\}$ :

$$
\begin{aligned}
& \int_{\mathcal{S}} \chi \geq R_{0} r^{-\beta}\left(r^{-(d-1)} \partial_{r}\left(r^{d-1} \partial_{r} u\right)+r^{-2} \Delta_{\mathbb{S}^{d-1}} u\right)^{2} d v^{2} l_{h} \\
& \geq c_{\beta} \int_{\mathcal{S}} \chi \geq R_{0} r^{-\beta}\left(\left|\nabla^{2} u\right|_{h}^{2}+r^{-2}|\nabla u|_{h}^{2}\right) \text { dvol }_{h} \\
& \quad+\int_{\mathcal{S}} O\left(\left|\nabla \chi \geq R_{0}\right|+\left|\nabla^{2} \chi \geq R_{0}\right|\right)|\nabla u|_{h}^{2} \text { dvol }_{h} .
\end{aligned}
$$

Without loss of generality, it suffices to establish (B.19) in the case $0<1-\beta \ll 1$, since the case $\beta \in\left(-\bar{\delta}_{k_{0}}, 0\right]$ (provided $\bar{\delta}_{k_{0}}$ is small enough) follows by a straightforward integration by parts scheme (and thus (B.19) for intermediate values of $\beta$ will follow by an easy interpolation argument).

On each connected component of the region $\left\{r \geq R_{0}\right\}$ in the coordinate chart $(r, \sigma)$ we calculate

$$
\begin{aligned}
\Delta_{h, \omega} u= & r^{-(d-1)} \partial_{r}\left(r^{d-1} \partial_{r} u\right)+r^{-2} \Delta_{\mathbb{S}^{d-1}} u \\
& +O_{\mu \nu}\left(r^{-1}\right) \nabla^{\mu} \nabla^{v} u+O_{\mu}\left(r^{-2}\right) \nabla^{\mu} u .
\end{aligned}
$$

Assuming (B.19) has been established, from (B.19) and (B.20) the following estimate is readily deduced (provided that $R_{0}$ has been fixed large in terms of $\beta$ and the geometry of $(\mathcal{S}, h))$ : 


$$
\begin{aligned}
& \int_{\mathcal{S}} \chi_{\geq R_{0}} r^{-\beta}\left(\Delta_{h, \omega} u\right)^{2} \text { dvol }_{h} \geq c_{\beta} \\
& \int_{\mathcal{S}} \chi_{\geq R_{0}} r^{-\beta}\left(\left|\nabla^{2} u\right|_{h}^{2}+r^{-2}|\nabla u|_{h}^{2}\right) d \text { vol }_{h} \\
&+\int_{\mathcal{S}} O\left(\left|\nabla \chi_{\geq R_{0}}\right|+\left|\nabla^{2} \chi_{\geq R_{0}}\right|\right)|\nabla u|_{h}^{2} \text { dvol }_{h} .
\end{aligned}
$$

We will now establish (B.19). By expanding the square we can trivially infer for any $a_{s} \in[0,1]$ (which will be fixed later):

$$
\begin{aligned}
& \int_{\mathcal{S}} \chi \geq R_{0} r^{-\beta}\left(r^{-(d-1)} \partial_{r}\left(r^{d-1} \partial_{r} u\right)+r^{-2} \Delta_{\mathbb{S}^{d-1}} u\right)^{2} r^{d-1} d r d \sigma \\
& =a_{S} \int_{\mathcal{S}} \chi_{\geq R_{0}} r^{-\beta}\left\{\left(r^{-(d-1)} \partial_{r}\left(r^{d-1} \partial_{r} u\right)\right)^{2}+2 r^{-(d-1)} \partial_{r}\left(r^{d-1} \partial_{r} u\right)\right. \\
& \left.\cdot r^{-2} \Delta_{\mathbb{S} d-1} u+r^{-4}\left(\Delta_{\mathbb{S} d-1} u\right)^{2}\right\} r^{d-1} d r d \sigma \\
& +\left(1-a_{S}\right) \int_{\mathcal{S}} \chi_{\geq R_{0}} r^{-\beta}\left\{\left(r^{-(d-1)} \partial_{r}\left(r^{d-1} \partial_{r} u\right)\right)^{2}+2 r^{-(d-1)} \partial_{r}\left(r^{d-1} \partial_{r} u\right)\right. \\
& \left.\quad \cdot r^{-2} \Delta_{\mathbb{S} d-1} u+r^{-4}\left(\Delta_{\mathbb{S} d-1} u\right)^{2}\right\} r^{d-1} d r d \sigma .
\end{aligned}
$$

Using a Cauchy-Schwarz inequality

$$
\begin{aligned}
& 2 a_{S} r^{-(d-1)} \partial_{r}\left(r^{d-1} \partial_{r} u\right) \cdot r^{-2} \Delta_{\mathbb{S}^{d-1}} u \geq-\frac{a_{s}^{2}}{b_{s}}\left(r^{-(d-1)} \partial_{r}\left(r^{d-1} \partial_{r} u\right)\right)^{2} \\
& \quad-b_{s} r^{-4}\left(\Delta_{\mathbb{S} d-1} u\right)^{2}
\end{aligned}
$$

(for a parameter $b_{s}>0$ to be fixed later) in the first summand of the right hand side of (B.22), and then applying the product rule and an integration by parts in the $\partial_{r}$ and the angular directions for both summands, we obtain from (B.22):

$$
\begin{aligned}
\int_{\mathcal{S}} & \chi \geq R_{0} r^{-\beta}\left(r^{-(d-1)} \partial_{r}\left(r^{d-1} \partial_{r} u\right)+r^{-2} \Delta_{\mathbb{S}^{d-1}} u\right)^{2} r^{d-1} d r d \sigma \\
\geq & \int_{\mathcal{S}} \chi \geq R_{0} r^{-\beta}\left\{\left(1-\frac{a_{s}^{2}}{b_{S}}\right)\left(\left(\partial_{r}^{2} u\right)^{2}+(d-1)(1+\beta) r^{-2}\left(\partial_{r} u\right)^{2}\right)\right. \\
& +2\left(1-a_{s}\right) r^{-2}\left|\nabla_{\mathbb{S}^{d-1}} \partial_{r} u\right|_{g_{\mathbb{S}^{d-1}}}^{2} \\
& \left.+\left(1-b_{s}\right) r^{-4}\left(\Delta_{\mathbb{S}^{d-1}} u\right)^{2}\right\} r^{d-1} d r d \sigma \\
& -\int_{\mathcal{S}} \chi \geq R_{0} 2\left(1-a_{S}\right)(2+\beta) r^{-3-\beta} \cdot \partial_{r} u \cdot \Delta_{\mathbb{S}^{d-1}} u r^{d-1} d r d \sigma \\
& +\int_{\mathcal{S}} O\left(\left|\nabla \chi \geq R_{0}\right|\right)\left(\left(\partial_{r} u\right)^{2}+\partial_{r} u \cdot \Delta_{\mathbb{S}^{d-1}} u\right) r^{d-1} d r d \sigma .
\end{aligned}
$$


Using the Hardy type inequality

$$
\begin{aligned}
& \int_{\mathcal{S}} \chi \geq R_{0} r^{d-3-\beta}\left(\partial_{r} u\right)^{2} d r d \sigma \leq \frac{4}{(d-2-\beta)^{2}} \int_{\mathcal{S}} \chi \geq R_{0} r^{d-1-\beta}\left(\partial_{r}^{2} u\right)^{2} d r d \sigma \\
& +C_{\beta} \int_{\mathcal{S}} O\left(\left|\nabla \chi_{\geq} R_{0}\right|\right)\left(\partial_{r} u\right)^{2} r^{d-1} d r d \sigma,
\end{aligned}
$$

we obtain from (B.24) for any $0<\delta_{H}<1$ (also to be fixed later):

$$
\begin{aligned}
\int_{\mathcal{S}} & \chi \geq R_{0} r^{-\beta}\left(r^{-(d-1)} \partial_{r}\left(r^{d-1} \partial_{r} u\right)+r^{-2} \Delta_{\mathbb{S}^{d-1}} u\right)^{2} r^{d-1} d r d \sigma \\
\geq & \int_{\mathcal{S}} \chi \geq R_{0} r^{-\beta}\left\{\left(1-\frac{a_{s}^{2}}{b_{s}}\right)\left(\delta_{H}\left(\partial_{r}^{2} u\right)^{2}+\left\{\left(d-1-\frac{d-2-\beta}{2}\right)^{2}+O\left(\delta_{H}\right)\right\} r^{-2}\left(\partial_{r} u\right)^{2}\right)\right. \\
& \left.+2\left(1-a_{S}\right) r^{-2}\left|\nabla_{\mathbb{S}^{d-1}} \partial_{r} u\right|_{g_{\mathbb{S}^{d-1}}}^{2}+\left(1-b_{s}\right) r^{-4}\left(\Delta_{\mathbb{S}^{d-1}} u\right)^{2}\right\} r^{d-1} d r d \sigma \\
& -\int_{\mathcal{S}} \chi \geq R_{0} 2\left(1-a_{S}\right)(2+\beta) r^{-3-\beta} \cdot \partial_{r} u \cdot \Delta_{\mathbb{S}^{d-1}} u r^{d-1} d r d \sigma \\
& +\int_{\mathcal{S}} O\left(\left|\nabla \chi_{\geq}\right| R_{0} \mid\right)\left(\left(\partial_{r} u\right)^{2}+\partial_{r} u \cdot \Delta_{\mathbb{S}^{d-1}} u\right) r^{d-1} d r d \sigma .
\end{aligned}
$$

Setting

$$
u_{0} \doteq \frac{1}{\operatorname{Vol}\left(\mathbb{S}^{d-1}\right)} \int_{\mathbb{S}^{d-1}} u d \sigma
$$

and

$$
u_{\geq 1}=u-u_{0},
$$

and noting that $u_{0}$ and $u_{\geq 1}$ are orthogonal with respect to the $L^{2}\left(d g_{\mathbb{S} d-1}\right)$ inner product, we obtain from (B.26):

$$
\begin{aligned}
& \int_{\mathcal{S}} \chi \geq R_{0} r^{-\beta}\left(r^{-(d-1)} \partial_{r}\left(r^{d-1} \partial_{r} u\right)+r^{-2} \Delta_{\mathbb{S}^{d}-1} u\right)^{2} r^{d-1} d r d \sigma \geq\left(1-\frac{a_{s}^{2}}{b_{S}}\right) \delta_{H} \\
& \cdot \int_{\mathcal{S}} \chi \geq R_{0} r^{-\beta}\left(\partial_{r}^{2} u\right)^{2} r^{d-1} d r d \sigma \\
& +\int_{\mathcal{S}} \chi \geq R_{0} r^{-\beta}\left\{\left(1-\frac{a_{s}^{2}}{b_{s}}\right)\left(\left\{\left(d-1-\frac{d-2-\beta}{2}\right)^{2}+O\left(\delta_{H}\right)\right\} r^{-2}\left(\partial_{r} u_{\geq 1}\right)^{2}\right)\right. \\
& \left.+2\left(1-a_{S}\right) r^{-2}\left|\nabla_{\mathbb{S}^{d}-1} \partial_{r} u_{\geq 1}\right|_{g_{\mathbb{S}} d-1}^{2}+\left(1-b_{S}\right) r^{-4}\left(\Delta_{\mathbb{S}^{d-1}} u_{\geq 1}\right)^{2}\right\} r^{d-1} d r d \sigma \\
& -\int_{\mathcal{S}} \chi \geq R_{0} 2\left(1-a_{S}\right)(2+\beta) r^{-3-\beta} \cdot \partial_{r} u_{\geq 1} \cdot \Delta_{\mathbb{S}^{d-1}} u_{\geq 1} r^{d-1} d r d \sigma \\
& +\int_{\mathcal{S}} O\left(\left|\nabla \chi_{\geq R_{0}}\right|\right)\left(\left(\partial_{r} u\right)^{2}+\partial_{r} u \cdot \Delta_{\mathbb{S}^{d-1}} u\right) r^{d-1} d r d \sigma .
\end{aligned}
$$

Since the first non zero eigenvalue of $\Delta_{\mathbb{S}^{d-1}}$ equals $d-1$, we can bound (in view of (B.27) and (B.28)):

$$
\int_{\mathbb{S}^{d-1}}\left|\nabla_{\mathbb{S}^{d-1}} \partial_{r} u_{\geq 1}\right|_{g_{\mathbb{S}^{d-1}}}^{2} d s \geq(d-1) \int_{\mathbb{S}^{d-1}}\left(\partial_{r} u_{\geq 1}\right)^{2} d s
$$


and thus, since $d \geq 3$, from (B.29) we obtain after setting $\varepsilon_{\beta} \doteq 1-\beta>0$ :

$$
\begin{aligned}
\int_{\mathcal{S}} & \chi \geq R_{0} r^{-\beta}\left(r^{-(d-1)} \partial_{r}\left(r^{d-1} \partial_{r} u\right)+r^{-2} \Delta_{\mathbb{S}^{d-1}} u\right)^{2} r^{d-1} d r d \sigma \\
\geq & c\left(a_{s}, b_{s}, \delta_{H}\right) \int_{\mathcal{S}} \chi \geq R_{0} r^{-\beta}\left(\partial_{r}^{2} u\right)^{2} r^{d-1} d r d \sigma \\
& +\int_{\mathcal{S}} \chi_{\geq R_{0}} r^{-\beta}\left\{\left(1-\frac{a_{s}^{2}}{b_{s}}\right)\left(2-\frac{\varepsilon_{\beta}}{2}\right)^{2}+4\left(1-a_{S}\right)+O\left(\delta_{H}\right)\right\} r^{-2}\left(\partial_{r} u_{\geq 1}\right)^{2} \\
& +\left(1-b_{S}\right) r^{-4}\left(\Delta_{\mathbb{S}^{d-1}} u_{\geq 1}\right)^{2} r^{d-1} d r d \sigma \\
& -\int_{\mathcal{S}} \chi \geq R_{0} 2\left(1-a_{S}\right)\left(3-\varepsilon_{\beta}\right) r^{-3-\beta} \cdot \partial_{r} u_{\geq 1} \cdot \Delta_{\mathbb{S}^{d-1}} u_{\geq 1} r^{d-1} d r d \sigma \\
& +c\left(a_{S}, b_{S}\right) \int_{\mathcal{S}} \chi \geq R_{0} r^{-2-\beta}\left(\partial_{r} u_{0}\right)^{2} r^{d-1} d r d \sigma \\
& +\int_{\mathcal{S}} O\left(\left|\nabla \chi_{\geq}\right| R_{0} \mid\right)\left(\left(\partial_{r} u\right)^{2}+\partial_{r} u \cdot \Delta_{\mathbb{S}^{d-1}} u\right) r^{d-1} d r d \sigma .
\end{aligned}
$$

Using a Cauchy-Schwarz inequality for the third line of the right hand side of (B.31):

$$
\begin{aligned}
& \left|\int_{\mathcal{S}} \chi_{\geq R_{0}} 2\left(1-a_{S}\right)\left(3-\varepsilon_{\beta}\right) r^{-3-\beta} \cdot \partial_{r} u_{\geq 1} \cdot \Delta_{\mathbb{S}^{d-1}} u_{\geq 1} r^{d-1} d r d \sigma\right| \\
& \leq \int_{\mathcal{S}} \chi \geq R_{0}\left(1-a_{S}\right)^{2} \frac{\left(3-\varepsilon_{\beta}\right)^{2}}{\left(1-b_{S}-\delta_{H}\right)} r^{-2-\beta}\left(\partial_{r} u_{\geq 1}\right)^{2} r^{d-1} d r d \sigma \\
& \quad+\int_{\mathcal{S}} \chi_{\geq R_{0}}\left(1-b_{S}-\delta_{H}\right) \cdot r^{-4-\beta}\left(\Delta_{\mathbb{S}^{d-1}} u_{\geq 1}\right)^{2} r^{d-1} d r d \sigma
\end{aligned}
$$

we infer from (B.31):

$$
\begin{aligned}
\int_{\mathcal{S}} \chi \geq R_{0} r^{-\beta}\left(r^{-(d-1)} \partial_{r}\left(r^{d-1} \partial_{r} u\right)+r^{-2} \Delta_{\mathbb{S}^{d-1}} u\right)^{2} r^{d-1} d r d \sigma \\
\geq c\left(a_{S}, b_{s}, \delta H\right) \int_{\mathcal{S}} \chi \geq R_{0} r^{-\beta}\left(\partial_{r}^{2} u\right)^{2} r^{d-1} d r d \sigma \\
\quad+\int_{\mathcal{S}} \chi \geq R_{0} r^{-\beta}\left\{A_{c o} \cdot r^{-2}\left(\partial_{r} u_{\geq 1}\right)^{2}+\delta_{H} r^{-4}\left(\Delta_{\mathbb{S}^{d-1}} u_{\geq 1}\right)^{2}\right\} r^{d-1} d r d \sigma \\
\left.\quad+c\left(a_{S}, b_{s}\right) \int_{\mathcal{S}} \chi \geq R_{0} r^{-2-\beta}\left(\partial_{r} u_{0}\right)^{2}\right) r^{d-1} d r d \sigma \\
\quad+\int_{\mathcal{S}} O\left(\left|\nabla \chi_{\geq} R_{0}\right|\right)\left(\left(\partial_{r} u\right)^{2}+\partial_{r} u \cdot \Delta_{\mathbb{S}^{d-1}} u\right) r^{d-1} d r d \sigma
\end{aligned}
$$

where

$$
A_{c o} \doteq\left(1-\frac{a_{s}^{2}}{b_{s}}\right)\left(2-\frac{\varepsilon_{\beta}}{2}\right)^{2}+4\left(1-a_{s}\right)+O\left(\delta_{H}\right)-\left(1-a_{s}\right)^{2} \frac{\left(3-\varepsilon_{\beta}\right)^{2}}{\left(1-b_{s}-\delta_{H}\right)} .
$$


It thus remains to show that the parameters $a_{S}, b_{s}, \delta_{H}$ can be suitably chosen in terms of $\varepsilon_{\beta}$ (provided that $\varepsilon_{\beta} \ll 1$, which we have assumed without loss of generality for the proof of (B.19)) so that $A_{\text {coeff }}>0$, and then (B.19) will follow. Setting $a_{s}=1-\delta_{1}$ and $b_{s}=1-\frac{3}{2} \delta_{1}$, we can directly calculate from (B.34):

$$
\begin{aligned}
A_{\text {coeff }}= & \left(\frac{1}{2} \delta_{1}+O\left(\delta_{1}^{2}\right)\right)\left(4-2 \varepsilon_{\beta}+O\left(\varepsilon_{\beta}^{2}\right)\right)+4 \delta_{1}+O\left(\delta_{H}\right) \\
& -\frac{2}{3} \delta_{1}\left(9-6 \varepsilon_{\beta}+O\left(\varepsilon_{\beta}^{2}\right)\right)\left(1+O\left(\delta_{1}^{-1} \delta_{H}\right)\right) \\
= & +3 \varepsilon_{\beta} \delta_{1}\left(1+O\left(\varepsilon_{\beta}\right)+O\left(\varepsilon_{\beta}{ }^{-1} \delta_{1}\right)+O\left(\varepsilon_{\beta}{ }^{-1} \delta_{1}^{-1} \delta_{H}\right)\right),
\end{aligned}
$$

and thus it follows that $A_{\text {coeff }}>0$ provided that $\delta_{H} \ll \delta_{1} \ll \varepsilon_{\beta} \ll 1\left(\delta_{H}, \delta_{1}\right.$ can be fixed in terms of $\varepsilon_{\beta}$ ). Therefore, from (B.33) (using also an integration by parts for the last term of the right hand side of (B.33), as well as the fact that $r^{d-1} d r d \sigma \sim d v o l_{h}$ in that region) we finally obtain the desired estimate (B.19) on each connected component of the region $\{r \gg 1\}$ :

$$
\begin{aligned}
\int_{\mathcal{S}} & \chi \geq R_{0} r^{-\beta}\left(r^{-(d-1)} \partial_{r}\left(r^{d-1} \partial_{r} u\right)+r^{-2} \Delta_{\mathbb{S}^{d-1}} u\right)^{2} r^{d-1} d r d \sigma \\
\geq & c_{\beta} \int_{\mathcal{S}} \chi \geq R_{0} r^{-\beta}\left(\left|\nabla^{2} u\right|_{h}^{2}+r^{-2}|\nabla u|_{h}^{2}\right) d v o l_{h} \\
& +\int_{\mathcal{S}} O\left(\left|\nabla \chi_{\geq} R_{0}\right|+\left|\nabla^{2} \chi \geq R_{0}\right|\right)|\nabla u|_{h}^{2} \text { dvol }_{h} .
\end{aligned}
$$

We will now proceed to establish estimates in the region $\left\{r \lesssim R_{0}\right\}$. Proceeding through integrations by parts using the formula

$$
\begin{aligned}
& \nabla_{\mu} \nabla_{\nu} X_{\alpha_{1} \ldots \alpha_{k}}-\nabla_{\nu} \nabla_{\mu} X_{\alpha_{1} \ldots \alpha_{k}} \\
& =h^{\beta_{1} \gamma_{1}} \mathcal{R}_{\mu \nu \beta_{1} \alpha_{1}} X_{\gamma_{1} \alpha_{2} \ldots \alpha_{k}}+\ldots+h^{\beta_{k} \gamma_{k}} \mathcal{R}_{\mu \nu \beta_{k} \alpha_{k}} X_{\alpha_{1} \alpha_{2} \ldots \gamma_{k}}
\end{aligned}
$$

we readily obtain that:

$$
\begin{aligned}
& \int_{\mathcal{S}} \chi \geq r_{0}\left(1-\chi \geq R_{0}\right) \cdot\left(\Delta_{h, \sigma} u\right)^{2} \text { dvol }_{h} \\
& \quad=\int_{\mathcal{S}} \chi \geq r_{0}\left(1-\chi \geq R_{0}\right) \cdot\left(\nabla^{\mu} \nabla_{\mu} u+O_{\mu}\left(r^{-2}\right) \nabla^{\mu} u\right) \cdot\left(\nabla^{v} \nabla_{\nu} u+O_{v}\left(r^{-2}\right) \nabla^{v} u\right) d v o l_{h} \\
& \quad \geq \frac{1}{2} \int_{\mathcal{S}} \chi \geq r_{0}\left(1-\chi \geq R_{0}\right) \cdot\left|\nabla^{2} u\right|_{h}^{2} d v o l_{h}-C \int_{\left\{r_{0} \leq r \leq 2 R_{0}\right\}}|\nabla u|_{\tilde{h}}^{2} d v o l_{h} .
\end{aligned}
$$

Notice that here we have used the volume form associated with $h$ (in place of $\tilde{h}$ ).

Therefore, from (B.38) and (B.21), and recalling that in the region $\left\{r \geq r_{0}\right\}$ we have $|\cdot|_{h} \sim_{r_{0}}|\cdot|_{\tilde{h}}$ and $d$ vol $_{h} \sim_{r_{0}}$ dvol $_{\tilde{h}}$, we deduce that there exists some large $R_{1}>0$ depending only on on $\beta$ and on the geometry of $(\mathcal{S}, h)$ in the region $\{r \geq 1\}$ such that: 


$$
\begin{aligned}
& \int_{\mathcal{S}} \chi \geq r_{0} r_{+}^{-\beta}\left(\Delta_{h, \omega} u\right)^{2} \text { dvol }_{h} \\
& \geq c_{\beta} \int_{\mathcal{S}} \chi_{\geq r_{0}} r_{+}^{-\beta}\left|\nabla^{2} u\right|_{\tilde{h}}^{2} d v o l_{\tilde{h}}+c_{\beta} \cdot \int_{\left\{r \geq R_{1}\right\}} r^{-2-\beta}|\nabla u|_{h}^{2} d v o l_{\tilde{h}} \\
& \quad-C_{r_{0}, \beta} \int_{\left\{r_{0} \leq r \leq R_{1}\right\}}|\nabla u|_{h}^{2} \operatorname{dvol}_{h} .
\end{aligned}
$$

In the region $\left\{r \leq 2 r_{0}\right\}$ we will perform the same integration by parts procedure, but here we will use the explicit forms (B.8) and (B.9) for the Laplacian of $h$ near $\partial_{h o r} \mathcal{S}$ and $\partial_{\text {tim }} \mathcal{S}$ respectively, as well as the form (B.11) for the non-singular metric $\tilde{h}$.

On $\left[0,2 r_{0}\right] \times \partial_{h o r} \mathcal{S}$, the perturbed Laplacian $\Delta_{h, \omega}$ takes the form (B.8):

$$
\Delta_{h, \omega}=a^{-1} \partial_{r}\left(r \tilde{a} \cdot \partial_{r}\right)+\Delta_{h_{\text {tan }}}+O(r) \cdot X,
$$

where $\tilde{a}=a \cdot(1+O(r))$.

Let us define the weight function $w:\left[0,2 r_{0}\right] \times \partial_{h o r} \mathcal{S} \rightarrow(0,+\infty)$ by the relation:

$$
w(r, \vartheta)=a(r, \vartheta) \cdot\left(1+\int_{0}^{r} \tilde{a}^{-1}(\rho, \vartheta) d \rho\right) .
$$

Since $\chi_{\leq r_{0}}^{\text {hor }}$ is supported in $\left[0,2 r_{0}\right] \times \partial_{h o r} \mathcal{S}$, we calculate:

$$
\begin{aligned}
\int_{\mathcal{S}} & \chi_{\leq r_{0}}^{h o r} w\left(\Delta_{h, \omega} u\right)^{2} d v o l_{\tilde{h}} \\
= & \int_{0}^{2 r_{0}} \int_{\partial_{h o r} \mathcal{S}} \chi_{\leq r_{0}}^{h o r} w\left(a^{-1} \partial_{r}\left(r \tilde{a} \partial_{r} u\right)+\Delta_{h_{\text {tan }}} u+O(r) \cdot X u\right)^{2} d h_{\text {tan }} d r \\
= & \int_{0}^{2 r_{0}} \int_{\partial_{h o r} \mathcal{S}} \chi_{\leq r_{0}}^{h o r} w\left(a^{-2}\left(\partial_{r}\left(r \tilde{a} \partial_{r} u\right)\right)^{2}\right. \\
& \left.+2 a^{-1}\left(\partial_{r}\left(r \tilde{a} \partial_{r} u\right)\right)\left(\Delta_{h_{\text {tan }}} u\right)+\left(\Delta_{h_{\text {tan }}} u\right)^{2}\right) d h_{\text {tan }} d r \\
& \left.+\int_{0}^{2 r_{0}} \int_{\partial_{h o r} \mathcal{S}} \chi_{\leq r_{0}}^{h o r} O(r)\left|a^{-1} \partial_{r}\left(r \tilde{a} \partial_{r} u\right)+\Delta_{h_{\text {tan }}} u\right| \cdot|X u|+O\left(r^{2}\right)|X u|^{2}\right) d h_{\text {tan }} d r .
\end{aligned}
$$

Integrating by parts three times in the mixed $\partial_{r}\left(r \partial_{r} u\right) \cdot \Delta_{h_{\text {tan }}} u$ term, and using the fact that

$$
\partial_{r}\left(w a^{-1}\right)=\tilde{a}^{-1}
$$

we estimate (notice that the resulting boundary terms at $r=0$ vanish because $r \partial_{r} u$ and $r \nabla^{\left(h_{\text {tan }}\right)} u$ vanish there):

$$
\begin{aligned}
& \int_{0}^{2 r_{0}} \int_{\partial_{h o r} \mathcal{S}} \chi_{\leq r_{0}}^{h o r} w a^{-1}\left(\partial_{r}\left(r \tilde{a} \partial_{r} u\right)\right)\left(\Delta_{h_{\text {tan }}} u\right) d h_{\text {tan }} d r \\
& \geq \int_{0}^{2 r_{0}} \int_{\partial_{h o r} \mathcal{S}} \chi_{\leq r_{0}}^{h o r} \tilde{a} \cdot r(1+O(r)) \cdot\left|\nabla^{\left(h_{\text {tan }}\right)} \partial_{r} u\right|_{h_{s}}^{2} d h_{\text {tan }} d r
\end{aligned}
$$




$$
\begin{aligned}
& -C \int_{0}^{r_{0}} \int_{\partial_{h o r} \mathcal{S}}\left(r^{2}\left|\partial_{r} u\right|^{2}+\left|\nabla^{\left(h_{\text {tan }}\right)} u\right|_{h_{\text {tan }}}^{2}\right) d h_{\text {tan }} d r \\
& -C_{r_{0}} \int_{r_{0}}^{2 r_{0}} \int_{\partial_{h o r} \mathcal{S}}|\nabla u|_{\tilde{h}}^{2} d h_{\text {tan }} d r .
\end{aligned}
$$

Thus, from (B.42) and (B.43) we obtain:

$$
\begin{aligned}
& \int_{\mathcal{S}} \chi_{\leq r_{0}}^{\text {hor }}\left(\Delta_{h, \omega} u\right)^{2} d v o \tilde{h}_{\tilde{h}} \\
& \geq c \int_{0}^{2 r_{0}} \int_{\partial_{h o r} \mathcal{S}} \chi_{\leq r_{0}}^{h o r}\left(\left(\partial_{r}\left(r \tilde{a} \partial_{r} u\right)\right)^{2}+r\left|\nabla^{\left(h_{\text {tan }}\right)} \partial_{r} u\right|_{h_{\text {tan }}}^{2}+\left(\Delta_{h_{\text {tan }}} u\right)^{2}\right) d h_{\text {tan }} d r \\
& \quad-C \int_{0}^{r_{0}} \int_{\partial_{h o r} \mathcal{S}}\left(r^{2}\left|\partial_{r} u\right|^{2}+\left|\nabla^{\left(h_{\text {tan }}\right)} u\right|_{h_{\text {tan }}}^{2}\right) d h_{\text {tan }} d r-C_{r_{0}} \int_{r_{0}}^{2 r_{0}} \int_{\partial_{h o r} \mathcal{S}}|\nabla u|_{\tilde{h}}^{2} d h_{\text {tan }} d r \\
& \quad+\int_{0}^{2 r_{0}} \int_{\partial_{h o r} \mathcal{S}} \chi_{\leq r_{0}}^{\text {hor }}\left(O(r)\left|a^{-1} \partial_{r}\left(r \tilde{a} \partial_{r} u\right)+\Delta_{h_{\text {tan }}} u\right| \cdot|X u|+O\left(r^{2}\right)|X u|^{2}\right) d h_{\text {tan }} d r .
\end{aligned}
$$

By expanding

$$
\left(\partial_{r}\left(r \tilde{a} \partial_{r} u\right)\right)^{2}=r^{2} \tilde{a}^{2}\left(\partial_{r}^{2} u\right)^{2}+2 r \tilde{a}^{2}(1+O(r)) \partial_{r}^{2} u \cdot \partial_{r} u+\tilde{a}^{2}(1+O(r))\left(\partial_{r} u\right)^{2}
$$

and integrating by parts in the resulting $\partial_{r}^{2} u \partial_{r} u$ term, we obtain from (B.44):

$$
\begin{aligned}
& \int_{\mathcal{S}} \chi_{\leq r_{0}}^{h o r}\left(\Delta_{h, \omega} u\right)^{2} d v o l_{\tilde{h}} \\
& \geq c \int_{0}^{2 r_{0}} \int_{\partial_{h o r} \mathcal{S}} \chi_{\leq r_{0}}^{h o r}\left(r^{2}\left(\partial_{r}^{2} u\right)^{2}-C r \cdot\left(\partial_{r} u\right)^{2}+r\left|\nabla^{\left(h_{\text {tan }}\right)} \partial_{r} u\right|_{h_{\text {tan }}}^{2}+\left(\Delta_{h_{\text {tan }}} u\right)^{2}\right) d h_{\text {tan }} d r \\
& \quad-C \int_{0}^{r_{0}} \int_{\partial_{h o r} \mathcal{S}}\left(r^{2}\left|\partial_{r} u\right|^{2}+\left|\nabla^{\left(h_{\text {tan }}\right)} u\right|_{h_{\text {tan }}}^{2}\right) d h_{\text {tan }} d r-C_{r_{0}} \int_{r_{0}}^{2 r_{0}} \int_{\partial_{h o r} \mathcal{S}}|\nabla u|_{\tilde{h}}^{2} d h_{\text {tan }} d r \\
& \quad+\int_{0}^{2 r_{0}} \int_{\partial_{h o r} \mathcal{S}} \chi_{\leq r_{0}}^{h o r}\left(O(r)\left|a^{-1} \partial_{r}\left(r \tilde{a} \partial_{r} u\right)+\Delta_{h_{\text {tan }}} u\right| \cdot|X u|+O\left(r^{2}\right)|X u|^{2}\right) d h_{\text {tan }} d r .
\end{aligned}
$$

Using, now a Hardy type inequality (of the form established in Lemma C.1) for the $\partial_{r}^{2} u$ and $\nabla^{\left(h_{\text {tan }}\right)} \partial_{r} u$ terms in the right hand side of (B.44), as well as elliptic estimates for the $\Delta_{h_{\text {tan }}} u$ term (using here the compactness of the level sets of $r$ ) we obtain from (B.46):

$$
\begin{aligned}
& \int_{\mathcal{S}} \chi_{\leq r_{0}}^{h o r}\left(\Delta_{h, \omega} u\right)^{2} d v o l_{\tilde{h}} \\
& \geq c \int_{0}^{2 r_{0}} \int_{\partial_{h o r} \mathcal{S}} \chi_{\leq r_{0}}^{h o r}\left(r^{2}\left(\partial_{r}^{2} u\right)^{2}+r\left|\nabla^{\left(h_{\text {tan }}\right)} \partial_{r} u\right|_{h_{\text {tan }}}^{2}+\left|\left(\nabla^{\left(h_{\text {tan }}\right)}\right)^{2} u\right|_{h_{\text {tan }}}^{2}\right) d h_{\text {tan }} d r \\
& \quad+c \int_{0}^{2 r_{0}} \int_{\partial_{\text {hor }} \mathcal{S}} \chi_{\leq r_{0}}^{h o r}\left(\left(\partial_{r} u\right)^{2}+r^{-1} \log ^{-2}(r) \cdot\left|\left(\nabla^{\left(h_{\text {tan }}\right)}\right) u\right|_{h_{\text {tan }}}^{2}\right) d h_{\text {tan }} d r
\end{aligned}
$$




$$
\begin{aligned}
& -C \int_{0}^{r_{0}} \int_{\partial_{h o r} \mathcal{S}}\left(r^{2}\left|\partial_{r} u\right|^{2}+\left|\nabla^{\left(h_{\text {tan }}\right)} u\right|_{h_{\text {tan }}}^{2}\right) d h_{\text {tan }} d r-C_{r_{0}} \int_{r_{0}}^{2 r_{0}} \int_{\partial_{h o r} \mathcal{S}}|\nabla u|_{\tilde{h}}^{2} d h_{\text {tan }} d r \\
& +\int_{0}^{2 r_{0}} \int_{\partial_{h o r} \mathcal{S}} \chi_{\leq r_{0}}^{h o r}\left(O(r)\left|a^{-1} \partial_{r}\left(r \tilde{a} \partial_{r} u\right)+\Delta_{h_{\text {tan }}} u\right| \cdot|X u|+O\left(r^{2}\right)|X u|^{2}\right) d h_{\text {tan }} d r .
\end{aligned}
$$

Applying a Cauchy-Schwarz inequality on the last term of the right hand side of (B.47), and absorbing all lower order (with respect to decaying powers in $r$ ) terms into their top order counterparts, we obtain provided thar $r_{0}$ is small enough:

$$
\begin{aligned}
& \int_{\mathcal{S}} \chi_{\leq r_{0}}^{h o r}\left(\Delta_{h, \omega} u\right)^{2} d v^{\prime} l_{\tilde{h}} \\
& \geq c \int_{0}^{2 r_{0}} \int_{\partial_{h o r} \mathcal{S}} \chi_{\leq r_{0}}^{h o r}\left(r^{2}\left(\partial_{r}^{2} u\right)^{2}+r\left|\nabla^{\left(h_{\text {tan }}\right)} \partial_{r} u\right|_{h_{\text {tan }}}^{2}+\left|\left(\nabla^{\left(h_{\text {tan }}\right)}\right)^{2} u\right|_{h_{\text {tan }}}^{2}\right) d h_{\text {tan }} d r \\
& \quad+c \int_{0}^{2 r_{0}} \int_{\partial_{h o r} \mathcal{S}} \chi_{\leq r_{0}}^{h o r}\left(\left(\partial_{r} u\right)^{2}+r^{-1} \log ^{-2}(r) \cdot\left|\left(\nabla^{\left(h_{\text {tan }}\right)}\right) u\right|_{h_{\text {tan }}}^{2}\right) d h_{\text {tan }} d r \\
& \quad-C_{r_{0}} \int_{r_{0}}^{2 r_{0}} \int_{\partial_{h o r} \mathcal{S}}|\nabla u|_{\tilde{h}}^{2} d h_{\text {tan }} d r .
\end{aligned}
$$

In the region $\left[0,2 r_{0}\right] \times \partial_{\text {tim }} \mathcal{S}$, on the other hand, we have

$$
\Delta_{h, \omega}=\partial_{r}\left((1+O(r)) \partial_{r}\right)+\Delta_{h_{t a n}}+X .
$$

Hence, we calculate after expanding the square (and applying the product rule for derivatives):

$$
\begin{aligned}
\int_{\mathcal{S}} & \chi_{\leq r_{0}}^{\text {tim }}\left(\Delta_{h, \omega} u\right)^{2} d v \operatorname{vol}_{\tilde{h}} \\
& =\int_{0}^{2 r_{0}} \int_{\partial_{\text {tim }} \mathcal{S}} \chi_{\leq r_{0}}^{\text {tim }}\left(\partial_{r}\left((1+O(r)) \partial_{r} u\right)+\Delta_{h_{\text {tan }}} u+X u\right)^{2} d h_{\text {tan }} d r \\
\geq & \int_{0}^{2 r_{0}} \int_{\partial_{\text {tim }} \mathcal{S}} \chi_{\leq r_{0}}^{\text {tim }}\left((1+O(r))\left(\partial_{r}^{2} u\right)^{2}+2(1+O(r)) \partial_{r}^{2} u \cdot \Delta_{h_{\text {tan }}} u+\left(\Delta_{h_{\text {tan }}} u\right)^{2}\right) d h_{\text {tan }} d r \\
& -C \int_{0}^{2 r_{0}} \int_{\partial_{\text {tim }} \mathcal{S}} \chi_{\leq r_{0}}^{\text {tim }}\left(\left(\left|\partial_{r}^{2} u\right|+\left|\Delta_{h_{\text {tan }}} u\right|\right) \cdot\left(\left|\partial_{r} u\right|+\left|\nabla^{\left(h_{\text {tan }}\right)} u\right|_{h_{\text {tan }}}\right)\right. \\
& \left.+\left(\left|\partial_{r} u\right|^{2}+\left|\nabla^{\left(h_{\text {tan }}\right)} u\right|_{h_{\text {tan }}}^{2}\right)\right) d h_{\text {tan }} d r .
\end{aligned}
$$

After integrating by parts in the $\partial_{r}^{2} u \cdot \Delta_{h_{S}} u$ term and using elliptic estimates for $\Delta_{h_{S}}$ on the surfaces $\{r=$ const $\}$, we obtain from (B.50):

$$
\begin{aligned}
& \int_{\mathcal{S}} \chi_{\leq r_{0}}^{\text {tim }}\left(\Delta_{h, \omega} u\right)^{2} \operatorname{dvol}_{\tilde{h}} \\
& \geq c \int_{0}^{2 r_{0}} \int_{\partial_{\text {tim }} \mathcal{S}} \chi_{\leq r_{0}}^{\text {tim }}\left(\left(\partial_{r}^{2} u\right)^{2}+\left|\nabla^{\left(h_{\text {tan }}\right)} \partial_{r} u\right|_{h_{\text {tan }}}^{2}+\left|\left(\nabla^{\left(h_{\text {tan }}\right)}\right)^{2} u\right|_{h_{\text {tan }}}^{2}\right) d h_{\text {tan }} d r
\end{aligned}
$$




$$
\begin{aligned}
& +2 \int_{\partial_{\text {tim }} \mathcal{S}} h_{\text {tan }}\left(\nabla^{\left(h_{\text {tan }}\right)} \partial_{r} u, \nabla^{\left(h_{\text {tan }}\right)} u\right) d h_{\text {tan }} \\
& -C \int_{0}^{2 r_{0}} \int_{\partial_{\text {tim }} \mathcal{S}} \chi_{\leq r_{0}}^{\text {tim }}\left(\left(\left|\partial_{r}^{2} u\right|+\left|\Delta_{h_{\text {tan }}} u\right|\right)\right. \\
& \left.\times\left(\left|\partial_{r} u\right|+\left|\nabla^{\left(h_{\text {tan }}\right)} u\right|_{h_{\text {tan }}}\right)+\left(\left|\partial_{r} u\right|^{2}+\left|\nabla^{\left(h_{\text {tan }}\right)} u\right|_{h_{\text {tan }}}^{2}\right)\right) d h_{\text {tan }} d r \\
& -C \int_{0}^{2 r_{0}} \int_{\partial_{\text {tim }} \mathcal{S}} \chi_{\leq r_{0}}^{\text {tim }\left|\nabla^{2} u\right|_{\tilde{h}} \cdot|\nabla u|_{\tilde{h}} d h_{\text {tan }} d r} \\
& -C \int_{0}^{2 r_{0}} \int_{\partial_{\text {tim }} \mathcal{S}}\left(\left|\nabla^{2} \chi_{\leq r_{0}}^{\text {tim }}\right|+\left|\nabla \chi_{\leq r_{0}}^{\text {tim }}\right|+1\right)|\nabla u|_{\tilde{h}}^{2} d h_{\text {tan }} d r .
\end{aligned}
$$

Applying a Cauchy-Schwarz inequality for the second and fourth terms of the right hand side and using the Hardy type inequality

$$
\begin{aligned}
& \int_{0}^{2 r_{0}} \int_{\partial_{\text {tim }} \mathcal{S}} \chi_{\leq r_{0}}^{\text {tim }}\left(\left(\partial_{r}^{2} u\right)^{2}+\left|\nabla^{\left(h_{\text {tan }}\right)} \partial_{r} u\right|_{h_{\text {tan }}}^{2}\right) d h_{\text {tan }} d r \\
& \geq c \int_{0}^{r_{0}} \int_{\partial_{\text {tim }} \mathcal{S}} r_{-}^{-1} \log ^{-2}\left(r_{-}\right)\left(\left(\partial_{r} u\right)^{2}+\left|\nabla^{\left(h_{\text {tan }}\right)} u\right|_{h_{\text {tan }}}^{2}\right) d h_{\text {tan }} d r \\
& \quad-C_{r_{0}} \int_{r_{0}}^{2 r_{0}} \int_{\partial_{\text {tim }} \mathcal{S}}\left(\left(\partial_{r} u\right)^{2}+\left|\nabla^{\left(h_{\text {tan }}\right)} u\right|_{h_{\text {tan }}}^{2}\right) d h_{\text {tan }} d r
\end{aligned}
$$

we deduce from (B.51) provided $r_{0}$ is small enough:

$$
\begin{aligned}
& \int_{\mathcal{S}} \chi_{\leq r_{0}}^{\text {tim }}\left(\Delta_{h, \omega} u\right)^{2} d \operatorname{vol}_{\tilde{h}} \\
& \geq c \int_{0}^{2 r_{0}} \int_{\partial_{\text {tim }} \mathcal{S}} \chi_{\leq r_{0}}^{\text {tim }}\left(\left(\partial_{r}^{2} u\right)^{2}+\left|\nabla^{\left(h_{\text {tan }}\right)} \partial_{r} u\right|_{h_{\text {tan }}}^{2}+\left|\left(\nabla^{\left(h_{\text {tan }}\right)}\right)^{2} u\right|_{h_{\text {tan }}}^{2}\right) d h_{\text {tan }} d r \\
& \quad+c \int_{0}^{r_{0}} \int_{\partial_{\text {tim }} \mathcal{S}} r_{-}^{-1} \log ^{-2}\left(r_{-}\right)\left(\left(\partial_{r} u\right)^{2}+\left|\nabla^{\left(h_{\text {tan }}\right)} u\right|_{h_{\text {tan }}}^{2}\right) d h_{\text {tan }} d r \\
& \quad-C_{r_{0}} \int_{r_{0}}^{2 r_{0}} \int_{\partial_{\text {tim }} \mathcal{S}}\left(\left(\partial_{r} u\right)^{2}+\left|\nabla^{\left(h_{\text {tan }}\right)} u\right|_{h_{\text {tan }}}^{2}\right) d h_{\text {tan }} d r \\
& \quad-2 \int_{\partial_{\text {tim }} \mathcal{S}}^{h_{\text {tan }}\left(\nabla^{\left(h_{\text {tan }}\right)} \partial_{r} u, \nabla^{\left(h_{\text {tan }}\right)} u\right) d h_{\text {tan }} .}
\end{aligned}
$$

By adding (B.39), (B.48) and (B.53) we infer that:

$$
\begin{aligned}
& \int_{\mathcal{S}} r_{+}^{-\beta}\left(\Delta_{h, \omega} u\right)^{2} \text { dvol }_{\tilde{h}} \\
& \geq c \int_{\mathcal{S}} r_{+}^{-\beta}\left|\left(\nabla^{(\tilde{h})}\right)^{2} u\right|_{h}^{2} \operatorname{dvol}_{\tilde{h}} \\
& \quad+c \int_{\mathcal{S}} r_{+}^{-2-\beta}\left(\left|\left(\chi_{\leq r_{0}}^{\text {hor }}+\chi_{\leq r_{0}}^{\text {tim }}\right) Y u\right|^{2}+r_{-}^{-1} \log ^{-2}\left(\frac{r_{-}}{2}\right)\left|\nabla^{(\tilde{h})} u\right|_{h}^{2}\right) \text { dvol }_{\tilde{h}}
\end{aligned}
$$




$$
-C_{r_{0}} \int_{\left\{r_{0} \leq r \leq R_{1}\right\}}|\nabla u|_{\tilde{h}}^{2} \operatorname{dvol}_{\tilde{h}}+2 \int_{\partial_{\text {tim }} \mathcal{S}} h_{t a n}\left(\nabla^{\left(h_{t a n}\right)} Y u, \nabla^{\left(h_{t a n}\right)} u\right) d h_{t a n} .
$$

From now on, we will assume that $r_{0}$ has been fixed, and we will drop the dependence of constants on it.

Let us denote with $\mathcal{H}^{2}(\mathcal{S}, h, \tilde{h})$ the semi-norm space consisting of the functions $\Psi$ of $H_{l o c}^{2}(\mathcal{S})$ with $\|\Psi\|_{\mathcal{H}^{2}(\mathcal{S}, h, \tilde{h})}<+\infty$, where

$$
\begin{aligned}
& \|\Psi\|_{\mathcal{H}^{2}(\mathcal{S}, h, \tilde{h})}^{2} \doteq \int_{\mathcal{S}} r_{+}^{-\beta}\left|\left(\nabla^{(\tilde{h})}\right)^{2} \Psi\right|_{h}^{2} \text { dvol }_{\tilde{h}} \\
& \quad+\int_{\mathcal{S}} r_{+}^{-2-\beta}\left(\left|\left(\chi_{\leq r_{0}}^{h o r}+\chi_{\leq r_{0}}^{\text {tim }}\right) Y \Psi\right|^{2}+r_{-}^{-1} \log ^{-2}\left(\frac{r_{-}}{2}\right)|\nabla \Psi|_{h}^{2}\right) d v o l_{\tilde{h}}
\end{aligned}
$$

(this is the semi-norm appearing in the right hand side of (B.54)). Notice that $\|$. \|\|$_{\mathcal{H}^{2}(\mathcal{S}, h, \tilde{h})}$ becomes an actual norm if we mod out the constant functions (the resulting normed space being a Hilbert space). It will be also convenient to introduce the seminorm space $\mathcal{H}^{1}(\mathcal{S}, h, \tilde{h})$ defined by the norm:

$$
\|\Psi\|_{\mathcal{H}^{1}(\mathcal{S}, h, \tilde{h})}^{2} \doteq \int_{\mathcal{S}} r_{+}^{-\beta}|\nabla \Psi|_{h}^{2} \operatorname{dvol}_{\tilde{h}}
$$

Using the Rellich-Kondrachov theorem for smooth manifolds with boundary (see e.g. [19]), we infer that for any $\varepsilon_{0}>0$ (which will be fixed small with respect to all the constants, and their inverses, appearing in (B.54), as well as the restriction of the weights in the integrals of (B.54) over $\left\{r_{0} \leq r \leq R_{1}\right\}$ ), the set $\mathcal{D}_{\varepsilon_{0}}$ of functions $\Psi \in \mathcal{H}^{2}(\mathcal{S}, h, \tilde{h})$ satisfying

$$
\|\Psi\|_{\mathcal{H}^{1}(\mathcal{S}, h, \tilde{h})}^{2}=1
$$

and

$$
\int_{\left\{r_{0} \leq r \leq R_{1}\right\}}|\nabla \Psi|_{\tilde{h}}^{2} \operatorname{dvol}_{\tilde{h}} \geq \varepsilon_{0}|| \Psi \|_{\mathcal{H}^{2}(\mathcal{S}, h, \tilde{h})}^{2}
$$

is a precompact subset of the semi-norm space $\mathcal{H}^{1}(\mathcal{S}, h, \tilde{h})$.

From Lemma B.4 we deduce that any non-constant function $\Psi \in \mathcal{H}^{2}(\mathcal{S}, h, \tilde{h})$ satisfies for any $\xi \geq \beta$ :

$$
\left\|\Delta_{h, \omega} \Psi\right\|_{L_{\xi}^{2}(\mathcal{S}, \tilde{h})}+\max \left\{-\int_{\partial_{\text {tim }} \mathcal{S}} Y \Psi \cdot \Psi d h_{t a n}, 0\right\}>0
$$

where

$$
\|f\|_{L_{\xi}^{2}(\mathcal{S}, \tilde{h})}^{2} \doteq \int_{\mathcal{S}} r_{+}^{-\xi} f^{2} d v o l_{\tilde{h}}
$$

Therefore, since $\mathcal{D}_{\varepsilon_{0}}$ is precompact and no constant function lies in its closure in the seminorm space $\mathcal{H}^{1}(\mathcal{S}, h, \tilde{h})$ (due to (B.56)), we infer that we can bound for any $\Psi \in \mathcal{D}:$ 


$$
\int_{\left\{r_{0} \leq r \leq R_{1}\right\}}|\nabla \Psi|_{\tilde{h}}^{2} \operatorname{dvol}_{\tilde{h}} \leq C_{\varepsilon_{0}}\left(\left\|\Delta_{h, \omega} \Psi\right\|_{L_{\beta}^{2}(\mathcal{S}, \tilde{h})}^{2}+\max \left\{-\int_{\partial_{t i m} \mathcal{S}} Y \Psi \cdot \Psi d h_{t a n}, 0\right\}\right) .
$$

Thus, fixing $\varepsilon_{0}$ small enough in terms of $r_{0}, \beta$ and the geometry of $(\mathcal{S}, h)$, returning to our original function $u$ we distinguish between two cases:

1 In case $u$ is constant or $\|u\|_{\mathcal{H}^{1}(\mathcal{S}, h, \tilde{h})}^{-1} \cdot u \in \mathcal{D}_{\varepsilon_{0}}$, from (B.54) and (B.60) we can bound:

$$
\begin{aligned}
& \int_{\mathcal{S}} r_{+}^{-\beta}\left|\left(\nabla^{(\tilde{h})}\right)^{2} u\right|_{h}^{2} d v o l_{\tilde{h}}+\int_{\mathcal{S}} r_{+}^{-2-\beta}\left(\left|\left(\chi_{\leq r_{0}}^{h o r}+\chi_{\leq r_{0}}^{t i m}\right) Y u\right|^{2}+r_{-}^{-1} \log ^{-2}\left(\frac{r_{-}}{2}\right)|\nabla u|_{h}^{2}\right) d v o l_{\tilde{h}} \\
& \leq C_{\beta} \int_{\mathcal{S}} r_{+}^{-\beta}\left(\Delta_{h, \omega} u\right)^{2} d v o l_{\tilde{h}}+C_{\beta} \max \left\{-\int_{\partial_{t i m} \mathcal{S}} h_{t a n}\left(\nabla^{\left(h_{t a n}\right)} Y u, \nabla^{\left(h_{t a n}\right)} u\right) d h_{t a n}, 0\right\} \\
& \quad+C_{\beta} \max \left\{-\int_{\partial_{t i m} \mathcal{S}} Y \Psi \cdot \Psi d h_{t a n}, 0\right\} .
\end{aligned}
$$

2. In case $u$ is not constant and $\|u\|_{\mathcal{H}^{1}(\mathcal{S}, h, \tilde{h})}^{-1} \cdot u \notin \mathcal{D}_{\varepsilon_{0}}$, from the definition of $\mathcal{D}_{\varepsilon_{0}}$ (i.e. (B.57)) we can bound:

$$
\int_{\left\{r_{0} \leq r \leq R_{1}\right\}}|\nabla u|_{\tilde{h}}^{2} \operatorname{dvol}_{\tilde{h}} \leq \varepsilon_{0}\|u\|_{\mathcal{H}^{2}(\mathcal{S}, h, \tilde{h})}^{2} .
$$

Thus, if $\varepsilon_{0}$ has been fixed small enough in terms of $r_{0}, \beta$ and the geometry of $(\mathcal{S}, h)$, from (B.54) and (B.62) we deduce that:

$$
\begin{aligned}
& \int_{\mathcal{S}} r_{+}^{-\beta}\left|\left(\nabla^{(\tilde{h})}\right)^{2} u\right|_{h}^{2} d v o l_{\tilde{h}}+\int_{\mathcal{S}} r_{+}^{-2-\beta}\left(\left|\chi_{\leq r_{0}} Y u\right|^{2}+r_{-}^{-1} \log ^{-2}\left(\frac{r_{-}}{2}\right)|\nabla u|_{h}^{2}\right) d v o l_{\tilde{h}} \\
& \leq C_{\beta} \int_{\mathcal{S}} r_{+}^{-\beta}\left(\Delta_{h, \omega} u\right)^{2} d v_{\tilde{h}}+C_{\beta} \max \left\{-\int_{\partial_{\text {tim }} \mathcal{S}} h_{\text {tan }}\left(\nabla^{\left(h_{\text {tan }}\right)} Y u, \nabla^{\left(h_{\text {tan }}\right)} u\right) d h_{\text {tan }}, 0\right\} \\
& \quad+C_{\beta} \max \left\{-\int_{\partial_{\text {tim }} \mathcal{S}} Y \Psi \cdot \Psi d h_{\text {tan }}, 0\right\} .
\end{aligned}
$$

Therefore, the elliptic estimates (B.17) and (B.18) in the case $l=2$ (and $k_{0}=0$ ) have been established.

The case when $2<l \leq\left\lfloor\frac{d+1}{2}\right\rfloor$ follows in an analogous way: In order to derive the analogue of (B.54), one needs to commute $l-2$ times with $\nabla^{\left(\tilde{h}_{\text {tim }}\right)}$ (the curvature terms appearing in this way are treated exactly as we did for the simple curvature terms in the $l=2$ case using the flat asymptotics of $(\mathcal{S}, h)$ ). By applying a Hardy type inequality near $\partial \mathcal{S}$ (using the form of the metric $\tilde{h}_{\text {tim }}$ there) in order to obtain an estimate of the form

$$
\begin{aligned}
& \sum_{j=0}^{l-3} \int_{\left\{r \leq r_{0}\right\}}\left|\left(\nabla^{(\tilde{h})}\right)^{j}\left(\Delta_{h, \omega} u\right)\right|_{\tilde{h}_{t i m}}^{2} d v l_{\tilde{h}} \lesssim \int_{\left\{r \leq 2 r_{0}\right\}}\left|\left(\nabla^{(\tilde{h})}\right)^{l-2}\left(\Delta_{h, \omega} u\right)\right|_{\tilde{h}_{t i m}}^{2} d v o l_{\tilde{h}} \\
& +\sum_{j=0}^{l-3} \int_{\left\{r_{0} \leq r \leq 2 r_{0}\right\}}\left|\left(\nabla^{(\tilde{h})}\right)^{j}\left(\Delta_{h, \omega} u\right)\right|_{\tilde{h}_{t i m}}^{2} \operatorname{dvol}_{\tilde{h}},
\end{aligned}
$$


and then integrating by parts as before, one readily obtains the following estimate:

$$
\begin{aligned}
& \int_{\mathcal{S}} r_{+}^{-\beta}\left|\left(\nabla^{(\tilde{h})}\right)^{l-2}\left(\Delta_{h, \omega} u\right)\right|_{\tilde{h}_{\text {tim }}}^{2} d v o l_{\tilde{h}} \geq c \int_{\mathcal{S}} r_{+}^{-\beta}|u|_{\tilde{h}, \tilde{h}_{\text {tim }} ; l}^{2} d v o l_{\tilde{h}} \\
& +c \sum_{j=1}^{l-1} \int_{\left\{r \geq r_{0}\right\}} r_{+}^{-2 j-\beta} \cdot\left|\left(\nabla^{(\tilde{h})}\right)^{l-j} u\right|_{\tilde{h}}^{2} d v o l_{\tilde{h}} \\
& +c \sum_{j=1}^{l-1} \int_{\left\{r \leq r_{0}\right\}}\left(\left|\left(\nabla^{(\tilde{h})}\right)^{l-j-1}(Y u)\right|_{\tilde{h}_{\text {tim }}}^{2}+r_{-}^{-1} \log ^{-2}\left(\frac{r_{-}}{2}\right)\right. \\
& \left.\cdot\left|\left(\nabla^{(\tilde{h})}\right)^{l-j-1}\left(i_{*}\left(\nabla^{\left(h_{\text {tan }}\right)} u\right)\right)\right|_{\tilde{h}_{\text {tim }}}^{2}\right) d v o l_{\tilde{h}} \\
& -C_{r_{0}} \sum_{j=1}^{l-1} \int_{\left\{r_{0} \leq r \leq R_{1}\right\}}^{\left|\nabla^{l-j-1} u\right|_{\tilde{h}}^{2} d v o l_{\tilde{h}}} \\
& +c \cdot \min \left\{\int_{\partial_{\text {tim }} \mathcal{S}} h_{\text {tan }}\left(\left(\nabla^{\left(h_{\text {tan }}\right)}\right)(Y u),\left(\nabla^{\left(h_{\text {tan }}\right)}\right) u\right) d h_{\text {tan }}\right\}, 0 .
\end{aligned}
$$

Using the relation

$$
\Delta_{h, \omega} u=Y((1+O(r)) \cdot Y) u+\Delta_{h_{\text {tan }}} u+X u
$$

near $\partial_{\text {tim }} \mathcal{S}$, together with a Hardy-type inequality, we can immediately estimate:

$$
\begin{aligned}
\int_{\left\{r \leq r_{0}\right\}}|u|_{\tilde{h},\left(1-\log \left(r_{t i m}\right)\right) \tilde{h} ; l}^{2} d v_{\tilde{h}} \lesssim & \int_{\left\{r \leq 2 r_{0}\right\}}|u|_{\tilde{h}, \tilde{h}_{\text {tim }} ; l}^{2} d v o l_{\tilde{h}} \\
& +\int_{\left\{r \leq 2 r_{0}\right\}}\left|\left(\nabla^{(\tilde{h})}\right)^{l-2}\left(\Delta_{h, \omega} u\right)\right|_{\left(1-\log \left(r_{t i m}\right)\right) \tilde{h}}^{2} d v o l_{\tilde{h}} \\
& +\sum_{j=1}^{l-1} \int_{\left\{r_{0} \leq r \leq 2 r_{0}\right\}}\left|\nabla^{l-j-1} u\right|_{\tilde{h}}^{2} d v o l_{\tilde{h}}
\end{aligned}
$$

and thus from (B.65) we also obtain:

$$
\begin{aligned}
& \int_{\mathcal{S}} r_{+}^{-\beta}\left|\left(\nabla^{(\tilde{h})}\right)^{l-2}\left(\Delta_{h, \omega} u\right)\right|_{\left(1-\log \left(r_{\text {tim }}\right)\right) \tilde{h}}^{2} \operatorname{dvol}_{\tilde{h}} \geq c \int_{\mathcal{S}} r_{+}^{-\beta}|u|_{\tilde{h},\left(1-\log \left(r_{\text {tim }}\right)\right) \tilde{h} ; l}^{2}{ }^{2} \operatorname{sol}_{\tilde{h}} \\
& +c \sum_{j=1}^{l-1} \int_{\left\{r \geq r_{0}\right\}} r_{+}^{-2 j-\beta} \cdot\left|\left(\nabla^{(\tilde{h})}\right)^{l-j} u\right|_{\tilde{h}}^{2} \operatorname{dvol}_{\tilde{h}} \\
& +c \sum_{j=1}^{l-1} \int_{\left\{r \leq r_{0}\right\}}\left(\left|\left(\nabla^{(\tilde{h})}\right)^{l-j-1}(Y u)\right|_{\left(1-\log \left(r_{\text {tim }}\right)\right) \tilde{h}}^{2}+r_{-}^{-1} \log ^{-2}\left(\frac{r_{-}}{2}\right)\right. \\
& \left.\left.\quad \times\left|\left(\nabla^{(\tilde{h})}\right)^{l-j-1}\left(i_{*}\left(\nabla^{\left(h_{\text {tan }}\right)} u\right)\right)\right|_{\left(1-\log \left(r_{\text {tim }}\right)\right) \tilde{h}}^{2}\right) d v o l_{\tilde{h}}\right\}
\end{aligned}
$$




$$
\begin{aligned}
& -C_{r_{0}} \sum_{j=1}^{l-1} \int_{\left\{r_{0} \leq r \leq R_{1}\right\}}\left|\nabla^{l-j-1} u\right|_{\tilde{h}}^{2} d v_{\tilde{h}} \\
& \left.+c \cdot \min \left\{\int_{\partial_{\text {tim }} \mathcal{S}} h_{\text {tan }}\left(\left(\nabla^{\left(h_{\text {tan }}\right)}\right)(Y u),\left(\nabla^{\left(h_{\text {tan }}\right)}\right) u\right) d h_{\text {tan }}\right\}, 0\right\} .
\end{aligned}
$$

Using the same Fredholm-type technique as before, we can absorb the $\int_{\left\{r_{0} \leq r \leq R_{1}\right\}}$ $\left|\nabla^{l-j-1} u\right|_{\tilde{h}}^{2}$ terms in the right hand side of (B.65) and (B.69) after adding to the left hand side of each of these estimates a large multiple of

$$
\left.\int_{\mathcal{S}} r_{+}^{-\beta}\left|\left(\nabla^{(\tilde{h})}\right)^{l-2}\left(\Delta_{h, \omega} u\right)\right|_{\tilde{h}_{\text {tim }}}^{2} \operatorname{dvol}_{\tilde{h}}+\max \left\{-\int_{\partial_{\text {tim }} \mathcal{S}} Y u \cdot u d h_{\text {tan }}\right\}, 0\right\}
$$

and

$$
\left.\int_{\mathcal{S}} r_{+}^{-\beta}\left|\left(\nabla^{(\tilde{h})}\right)^{l-2}\left(\Delta_{h, \omega} u\right)\right|_{\left(1-\log \left(r_{t i m}\right)\right)}^{2} d v o l_{\tilde{h}}+\max \left\{-\int_{\partial_{t i m} \mathcal{S}} Y u \cdot u d h_{t a n}\right\}, 0\right\}
$$

respectively, thus obtaining inequalities (B.17) and (B.18). We will omit the details.

The case $k_{0} \geq 1$ follows in exactly the same way.

Let us assume that we are given a smooth function $\omega_{n d}: \mathcal{S} \rightarrow(0,+\infty)$ with $\omega_{n d}=1+O\left(r^{-1}\right)$ in $\{r \gg 1\}$ (notice that we necessarily have $\omega_{n d} \neq 0$ on $\partial \mathcal{S}$ ), and let us define the non-degenerate elliptic operator

$$
\Delta_{\tilde{h}, \omega_{n d}} \doteq \omega_{n d}^{-1} d i v_{\tilde{h}}\left(\omega_{n d} \cdot d\right)
$$

This operator will model the operator (A.3) associated to the metric $h_{\tau, N}$ on the hypersurfaces $\{\bar{t}=\tau\}$ of the spacetimes $(\mathcal{M}, g)$ of Section 8 . The following nondegenerate variant of Proposition B.1 holds for (B.72):

Proposition B.2 (Non-degenerate elliptic estimates). For any $l \in \mathbb{N}$ with $2 \leq l \leq$ $\left\lfloor\frac{d+1}{2}\right\rfloor$, any $k_{0} \in \mathbb{N}$ and any $\beta \in\left(-\bar{\delta}_{k_{0}}, 1\right)$ for some $\bar{\delta}_{k_{0}}>0$ depending on $k_{0}$, we can bound for any $u \in C^{\infty}(\mathcal{S})$ satisfying $\lim \sup _{r \rightarrow+\infty}\left|r^{\frac{d-1}{2}+j} \nabla^{j} u\right|_{h}<+\infty$ for any $j \leq l+k_{0}$ :

$$
\begin{aligned}
& \sum_{k=0}^{k_{0}}\left\{\int_{\mathcal{S}} r_{+}^{-\beta}\left|\left(\nabla^{(\tilde{h})}\right)^{k+l} u\right|_{\tilde{h}}^{2} d \operatorname{vol}_{\tilde{h}}+\sum_{j=1}^{l-1} \int_{\mathcal{S}} r_{+}^{-2 j-\beta} r_{-}^{-1} \log ^{-2}\left(\frac{r_{-}}{2}\right)\right. \\
& \left.\quad \cdot\left|\left(\nabla^{(\tilde{h})}\right)^{k+l-j} u\right|_{\tilde{h}}^{2} \operatorname{dvol}_{\tilde{h}}\right\} \leq C_{\beta, k_{0}} \sum_{k=0}^{k_{0}} \int_{\mathcal{S}} r_{+}^{-\beta}\left|\left(\nabla^{(\tilde{h})}\right)^{k+l-2}\left(\Delta_{\tilde{h}, \omega_{n d}} u\right)\right|_{\tilde{h}}^{2} d \operatorname{vol}_{\tilde{h}} \\
& \quad+C_{\beta, k_{0}} \sum_{j=0}^{k_{0}+l-1} \max \left\{-\operatorname{Re}\left\{\int_{\partial \mathcal{S}} h_{\text {tan }}\left(\left(\nabla^{\left(h_{\text {tan }}\right)}\right)^{j}(Y u),\left(\nabla^{\left(h_{\text {tan }}\right)}\right)^{j} \bar{u}\right) d h_{\text {tan }}\right\}, 0\right\},
\end{aligned}
$$


where $\nabla^{(\tilde{h})}$ denotes the covariant derivative with respect to the metric $\tilde{h}$ and the constant $C_{\beta}$ of the right hand side depends only on $\beta$ and on the geometry of $(\mathcal{S}, h)$, $\tilde{h}$ and $\omega_{n d}$.

Remark Notice that in contrast to (B.18), the right hand side of (B.73) contains terms on the whole of $\partial \mathcal{S}$.

The proof of Proposition B.2 follows in the same lines as that for Lemma B.1 (using everywhere the metric $\tilde{h}$ in place of $h$ and $\left.\tilde{h}_{t i m}\right)$, and hence it will be omitted.

It will also be useful to establish the following estimate in the region $\{r \gg 1\}$ (this estimate will be used in Section 9 to control the error terms arising when commuting $\square_{g}$ with the vector field $K_{R_{c}}$ which is not settling to a Killing field in the region $\left.\left\{r \sim R_{c}\right\}\right)$ :

Proposition B.3 (Improved control of derivatives in the far away region). There exists some $R_{0} \geq 1$ large in terms of the geometry of $(\mathcal{S}, h)$, such that for any $R>R_{0}$ the following bound holds for any $l \in \mathbb{N}$ with $l \leq\left\lfloor\frac{d+1}{2}\right\rfloor$, any $k_{0} \in \mathbb{N}$, any $\beta \in\left(-\bar{\delta}_{k_{0}}, 1\right)$ for some $\bar{\delta}_{k_{0}}>0$ depending on $k_{0}$, any $0<\varepsilon<1$ and any $u \in C^{\infty}(\mathcal{N})$ with $\sum_{k=0}^{k_{0}} \sum_{j=1}^{l} \int_{\left\{r \geq R_{0}\right\}} r^{-2(l-j)}\left|\nabla^{j+k} u\right|_{h}^{2}$ dvol $_{h}<\infty$ :

$$
\begin{aligned}
& \sum_{k=0}^{k_{0}}\left\{\sum_{j=1}^{l} \int_{\{R \leq r \leq 2 R\}} r^{2(j-1)-\beta+\varepsilon}\left|\nabla^{j+k} u\right|_{h}^{2} d v o l_{h}\right. \\
& \left.\quad+\sum_{j=1}^{l} \int_{\left\{r \geq R_{0}\right\}} r^{-2(l-j)-\beta}\left|\nabla^{j+k} u\right|_{h}^{2} d v o l_{h}\right\} \\
& \leq C_{R, \beta, \varepsilon, k_{0}} \sum_{k=0}^{k_{0}} \int_{\left\{r \geq R_{0}\right\}} r^{-\beta}\left|\nabla^{k+l-2}\left(\Delta_{h, \omega} u\right)\right|_{h}^{2} d v o l_{h} \\
& \quad+C_{\beta, \varepsilon, k_{0}} \sum_{j=1}^{l+k_{0}} \int_{\left\{R_{0} \leq r \leq 2 R_{0}\right\}}\left|\nabla^{j} u\right|_{h}^{2} \text { dvol }_{h} .
\end{aligned}
$$

Remark Notice that the constant in front of the last term of the right hand side of (B.74) does not depend on $R$.

Proof Without loss of generality, we can assume that $k_{0}=0$, since the proof in the case $k_{0} \geq 1$ follows in exactly the same way.

It suffices to establish the following estimate on $\mathbb{R}^{d}$ for $R_{0}, R, l, \beta, \varepsilon$ as above and $u \in C^{\infty}\left(\mathbb{R}^{d}\right)$ with $\lim \sup _{r \rightarrow+\infty}\left|r^{\frac{d-1}{2}+j} \nabla^{j} u\right|_{h}<+\infty$ for $j \leq l$ :

$$
\begin{gathered}
\sum_{j=1}^{l} \int_{\{R \leq r \leq 2 R\}} r^{2(j-1)-\beta+\varepsilon}\left|\nabla^{j} u\right|_{e}^{2} \text { dvol }_{e} \\
+\sum_{j=1}^{l} \int_{\left\{r \geq R_{0}\right\}} r^{-2(l-j)-\beta}\left|\nabla^{j} u\right|_{e}^{2} \text { dvol }_{e}
\end{gathered}
$$




$$
\begin{aligned}
\leq & C_{R, R_{0}, \beta, \varepsilon} \int_{\left\{r \geq R_{0}\right\}} r^{-\beta}\left|\nabla^{l-2}\left(\Delta_{\mathbb{R}^{d}} u\right)\right|_{e}^{2} \text { dvol }_{e} \\
& +C_{R_{0}, \beta, \varepsilon} \sum_{j=1}^{l} \int_{\left\{R_{0} \leq r \leq 2 R_{0}\right\}}\left|\nabla^{j} u\right|_{e}^{2} d v o l_{e} .
\end{aligned}
$$

Assuming that (B.75) holds, by substituting

$$
\Delta_{h, \omega} u=\Delta_{\mathbb{R}^{d}} u+O\left(r^{-1}\right) \nabla^{2} u+O\left(r^{-2}\right) \nabla u
$$

one obtains (B.74) by absorbing the resulting error terms into the left hand side, provided that $R_{0}$ has been fixed sufficiently large in terms of the geometry of $(\mathcal{S}, h)$ (in view also of the flat asymptotics of $(\mathcal{S}, h)$ ).

As we did in the proof of Proposition B.1, we will prove (B.75) in detail in the case $l=2$, and omit the details for the case $l>2$ (which follows in a similar, albeit notationally more complicated, way).

Fix a function $w:[0,+\infty) \rightarrow[0,+\infty)$ such that

- $w(x)=x^{2+\varepsilon}$ for $x \leq 1,,^{25}$

- $w(x)=1$ for $x \geq 2$ and

- $\frac{d w}{d x} \geq 0$ on $[0,+\infty)$.

We then define the function $w_{R}: \mathbb{R}^{d} \rightarrow(0,+\infty)$ by the relation

$$
w_{R}=R^{2+\varepsilon} w\left(\frac{r}{R}\right) \text {. }
$$

Fixing also a smooth cut-off $\chi_{R_{0}}: \mathbb{R}^{d} \rightarrow[0,1]$ such that $\chi_{R_{0}} \equiv 0$ on $\left\{r \leq R_{0}\right\}$ and $\chi_{R_{0}} \equiv 1$ on $\left\{r \geq R_{0}\right\}$, we obtain after integrating by parts:

$$
\begin{aligned}
& \int_{\mathbb{R}^{d}} \chi_{R_{0}} w_{R} \cdot r^{-\beta}\left(\Delta_{\mathbb{R}^{d}} u\right)^{2} \text { dvol }_{e}=\int_{\mathbb{R}^{d}} \chi_{R_{0}} w_{R} \cdot r^{-\beta}\left|\nabla^{2} u\right|_{e}^{2} \text { dvol }_{e} \\
& +\int_{\mathbb{R}^{d}} \chi_{R_{0}} w_{R} \cdot\left(\nabla_{\mu} \nabla_{\nu} r_{+}^{-\beta}-\frac{1}{2}\left(\Delta_{h} r_{+}^{-\beta}\right) h_{\mu v}\right) \cdot \nabla^{\mu} u \cdot \nabla^{v} u \text { dvol }_{e} \\
& +\int_{\mathbb{R}^{d}} O_{R_{0}}\left(\left|\nabla \chi_{R_{0}}\right|_{e}+\left|\nabla^{2} \chi_{R_{0}}\right|_{e}\right)|\nabla u|_{e}^{2} d v o l_{e} \\
& +\int_{\mathbb{R}^{d}} \chi_{R_{0}} O\left(\left|\nabla w_{R}\right|_{e} \cdot\left|\nabla r^{-\beta}\right|_{e}+\left|\nabla^{2} w_{R}\right|_{e} r^{-\beta}\right)|\nabla u|_{e}^{2} d v o l_{e} .
\end{aligned}
$$

Notice that the boudary terms at infinity obtained through this integration by parts procedure vanish. This follows from the fact that, because $\sum_{j=1}^{l} \int_{\left\{r \geq R_{0}\right\}} r^{-2(l-j)-\beta} \mid$ $\left.\nabla^{j} u\right|_{e} ^{2} d v^{\prime} l_{e}<\infty$, exactly as in the proof of Lemma B.4, we can find a sequence of positive numbers $\left\{R_{n}\right\}_{n \in \mathbb{N}}$ tending to $+\infty$ so that

$\overline{25}$ Let us note that for more general $l$, one should choose $w=x^{2(l-1)}+\varepsilon$ for $x \leq 1$. 


$$
\lim _{n \rightarrow \infty}\left(R_{n} \sum_{j=1}^{l} \int_{\left\{r=R_{n}\right\}} r^{-2(l-j)-\beta}\left|\nabla^{j} u\right|_{e}^{2} d \operatorname{vol}_{\left\{r=R_{n}\right\}}\right)=0 .
$$

Thus, in view of the relation

$$
\begin{aligned}
& \nabla_{\mu} \nabla_{\nu} r_{+}^{-\beta}-\frac{1}{2}\left(\Delta_{h} r_{+}^{-\beta}\right) h_{\mu \nu} \\
& \quad=\frac{1}{2} \beta\left((d-\beta-4) h_{\mu \nu}+2(\beta+2) d r_{\mu} \cdot d r_{\nu}\right) r^{-2-\beta}+o\left(r^{-2-\beta}\right),
\end{aligned}
$$

using a Hardy type inequality we readily obtain from (B.78) after adding to both sides of (B.78) the quantity $\int_{\left\{R_{0} \leq r \leq 2 R_{0}\right\}}\left(\left|\nabla^{2} u\right|_{e}^{2}+|\nabla u|_{e}^{2}\right.$ ) (notice that we have assumed $\bar{\delta}_{k_{0}}>0$ to be small enough):

$$
\begin{aligned}
& \int_{\{R \leq r \leq 2 R\}} r^{2+\varepsilon}\left(r^{-\beta}\left|\nabla^{2} u\right|_{e}^{2}+r^{-2-\beta}|\nabla u|_{e}^{2}\right) \text { dvol }_{e} \\
& \quad+\int_{\left\{r \geq R_{0}\right\}}\left(r^{-\beta}\left|\nabla^{2} u\right|_{e}^{2}+r^{-2-\beta}|\nabla u|_{e}^{2}\right) d v o l_{e} \\
& \leq C_{R, R_{0}, \beta, \varepsilon} \int_{\left\{r \geq R_{0}\right\}} r^{-\beta}\left(\Delta_{\mathbb{R}^{d}} u\right)^{2} \text { dvol }_{e} \\
& \quad+C_{R_{0}, \beta, \varepsilon} \int_{\left\{R_{0} \leq r \leq 2 R\right\}} r^{\varepsilon-\beta}|\nabla u|_{e}^{2} \text { dvol }_{e} \\
& \quad+C_{R_{0}, \beta, \varepsilon} \int_{\left\{R_{0} \leq r \leq 2 R_{0}\right\}}\left(\left|\nabla^{2} u\right|_{e}^{2}+|\nabla u|_{e}^{2}\right) \text { dvol }_{e} .
\end{aligned}
$$

Let us consider the seminorm space $\mathcal{H}_{R_{0}, \beta}^{2}$ defined as the completion of the space $C^{\infty}\left(\left\{r \geq R_{0}\right\}\right)$ with the seminorm

$$
\|\Psi\|_{\mathcal{H}_{R_{0}, \beta}^{2}}^{2} \doteq \int_{\left\{r \geq R_{0}\right\}}\left(r^{-\beta}\left|\nabla^{2} \Psi\right|_{e}^{2}+r^{-2-\beta}|\nabla \Psi|_{e}^{2}\right) d \text { vol }_{e} .
$$

Notice that $\mathcal{H}_{R_{0}, \beta}^{2}$ modulo the constant functions becomes a Hilbert space.

The subspace of harmonic functions

$$
\mathcal{V}_{h r m} \doteq\left\{\Psi \in \mathcal{H}_{R_{0}, \beta}^{2} \mid \Delta_{\mathbb{R}^{d}} \Psi=0\right\}
$$

is a closed subspace of $\mathcal{H}_{R_{0}, \beta}^{2}$. If we introduce the following semi-definite inner product on $\mathcal{H}_{R_{0}, \beta}^{2}$ :

$$
\left\langle\Psi_{1}, \Psi_{2}\right\rangle_{R_{0}} \doteq \int_{\left\{R_{0} \leq r \leq 2 R_{0}\right\}}\left(\nabla^{\mu} \nabla^{\nu} \Psi_{1} \cdot \nabla_{\mu} \nabla_{\nu} \Psi_{2}+\nabla^{\mu} P s i_{1} \cdot \nabla_{\mu} \Psi_{2}\right) \text { dvol }_{e}
$$


then $\langle\cdot, \cdot\rangle_{R_{0}}$ is continuous with respect to $\|\cdot\|_{\mathcal{H}_{R_{0}, \beta}^{2}}$, and for any $\Psi \in \mathcal{V}_{h r m}$ which is

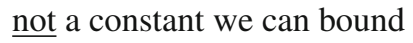

$$
\langle\Psi, \Psi\rangle_{R_{0}}>0
$$

This follows from the fact that if $\langle\Psi, \Psi\rangle_{R_{0}}=0$, i.e. if $\Psi$ is constant on $\left\{R_{0}<r<\right.$ $2 R_{0}$, and $\Psi$ is harmonic (i.e. belongs to $\mathcal{V}_{h r m}$ ), then $\Psi$ must be identically constant. Therefore, the orthogonal complement of $\mathcal{V}_{h r m}$ with respect to $\langle\cdot, \cdot\rangle_{R_{0}}$, that is the subspace

$$
\mathcal{V}_{\text {orth }} \doteq\left\{\Psi \in \mathcal{H}_{R_{0}, \beta}^{2} \mid \forall \varphi \in \mathcal{V}_{h r m}:\langle\Psi, \varphi\rangle_{R_{0}}=0\right\},
$$

is a closed subspace of $\mathcal{H}_{R_{0}, \beta}^{2}$, satisfying $\mathcal{V}_{\text {hrm }} \cap \mathcal{V}_{\text {orth }}=<1>$. Moreover, we can decompose any $\Psi \in \mathcal{H}_{R_{0}, \beta}^{2}$ as

$$
\Psi=\Psi_{h r m}+\Psi_{\text {orth }},
$$

uniquely modulo addition of some constant function, where $\Psi_{h r m} \in \mathcal{V}_{h r m}$ and $\Psi_{\text {orth }} \in$ $\mathcal{V}_{\text {orth }}$.

Remark We should emphasize that we will not need to establish that the resulting projection of $\mathcal{H}_{R_{0}, \beta}^{2} /<1>$ onto $\mathcal{V}_{\text {orth }} /<\overline{1>\text { along } \mathcal{V}_{\text {hrm }} /<1>\text { is continuous }}$ with respect to the topology of $\mathcal{H}_{R_{0}, \beta}^{2} /<1>$.

Let us introduce the semi-norm

$$
\|\Psi\|_{\mathcal{H}_{R_{0}, \beta}^{1}}^{2} \doteq \int_{\left\{r \geq R_{0}\right\}} r^{-\beta}|\nabla \Psi|_{e}^{2} \text { dvol }_{e} .
$$

Moreover, fixing a sufficiently small $\varepsilon_{0}>0$, using the Rellich-Kondrachov theorem (see e.g. [19]), we can establish that the subset $\mathcal{D}$ of functions $\Psi$ in $\mathcal{V}_{\text {orth }}$ satisfying

$$
\|\Psi\|_{\mathcal{H}_{R_{0}, \beta}^{1}}=1
$$

and

$$
\int_{\left\{R_{0} \leq r \leq 2 R\right\}}|\nabla \Psi|_{e}^{2} \text { dvol }_{e} \geq \varepsilon_{0} R^{-2-\varepsilon}\|\Psi\|_{\mathcal{H}_{R_{0}, \beta}^{2}}^{2}
$$

is a pre-compact subset of the semi-norm space $\mathcal{H}_{R_{0}, \beta}^{1}$. Therefore, since no constant function lies in the closure of $\mathcal{D}$ with the semi norm $\|\cdot\|_{\mathcal{H}_{R_{0}, \beta}^{1}}$ (due to (B.89)) and for any non constant $\Psi \in \mathcal{V}_{\text {orth }}$ we have $\left\|\Delta_{\mathbb{R}^{d}} \Psi\right\|_{L_{R_{0}, \beta}^{2}}>0$, where

$$
\|\varphi\|_{L_{R_{0}, \beta}^{2}} \doteq \int_{\left\{r \geq R_{0}\right\}} r^{-\beta} \varphi^{2} \text { dvol }_{e},
$$

there exists some large $C_{R}>0$ so that we can bound for any $\Psi \in \mathcal{D}$ :

$$
\int_{\left\{R_{0} \leq r \leq 2 R\right\}} r^{\varepsilon-\beta}|\nabla \Psi|_{e}^{2} \operatorname{dvol}_{e} \leq C_{R, R_{0}, \beta, \varepsilon}\left\|\Delta_{\mathbb{R}^{d}} \Psi\right\|_{L_{R_{0}, \beta}^{2}}^{2} .
$$


Therefore, for any $\Psi \in \mathcal{H}_{R_{0}, \beta}^{2}$, using (B.81) in case $\left.\Psi_{\text {orth }} \notin\{\lambda\} \mathcal{D} \mid \lambda \geq 0\right\}$ and (B.92) in case $\Psi_{\text {orth }} \in\{\lambda \mathcal{D} \mid \lambda \geq 0\}$ (and recalling the definition of $\mathcal{D}$ ), we obtain (provided $\varepsilon_{0}$ was chosen sufficiently small):

$$
\begin{aligned}
& \int_{\{R \leq r \leq 2 R\}} r^{2+\varepsilon}\left(r^{-\beta}\left|\nabla^{2} \Psi_{\text {orth }}\right|_{e}^{2}+r^{-2-\beta}\left|\nabla \Psi_{\text {orth }}\right|_{e}^{2}\right) d v o l_{e} \\
& \quad+\int_{\left\{r \geq R_{0}\right\}}\left(r^{-\beta}\left|\nabla^{2} \Psi_{\text {orth }}\right|_{e}^{2}+r^{-2-\beta}\left|\nabla \Psi_{\text {orth }}\right|_{e}^{2}\right) d v o l_{e} \\
& \leq C_{R, R_{0}, \beta, \varepsilon} \int_{\left\{r \geq R_{0}\right\}} r^{-\beta}\left(\Delta_{\mathbb{R}^{d}} \Psi_{\text {orth }}\right)^{2} d \text { vol }_{e}+C_{R_{0}, \beta, \varepsilon}<\Psi_{\text {orth }}, \Psi_{\text {orth }}>
\end{aligned}
$$

We will now establish the necessary estimates for functions belonging to $\mathcal{V}_{\mathrm{hrm}}$. For any function $\Psi$ solving $\Delta_{\mathbb{R}^{d}} \Psi=0$ on $\left\{r \geq R_{0}\right\}$ and having finite $\|\cdot\|_{\mathcal{H}_{R_{0}, \beta}^{2}}$ norm, we readily deduce after decomposing it into spherical harmonics and using (B.145) and (B.146) (as well as the fact that $2+\varepsilon-\beta<d$ ) that

$$
\begin{aligned}
& \int_{\{R \leq r \leq 2 R\}} r^{2-\beta+\varepsilon}\left(\left|\nabla^{2} \Psi\right|_{e}^{2}+r^{-2}|\nabla \Psi|_{e}^{2}\right) \text { dvol }_{e} \\
& \quad \leq C_{R_{0}, \beta, \varepsilon} \int_{\left\{R_{0} \leq r \leq 2 R_{0}\right\}}\left(\left|\nabla^{2} \Psi\right|_{e}^{2}+|\nabla \Psi|_{e}^{2}\right) \text { dvol }_{e}
\end{aligned}
$$

while the estimate

$$
\begin{aligned}
& \int_{\left\{r \geq R_{0}\right\}}\left(r^{-\beta}\left|\nabla^{2} \Psi\right|_{e}^{2}+r^{-2-\beta}|\nabla \Psi|_{e}^{2}\right) \text { dvol }_{e} \\
& \quad \leq C_{R_{0}, \beta} \int_{\left\{R_{0} \leq r \leq 2 R_{0}\right\}}\left(\left|\nabla^{2} \Psi\right|_{e}^{2}+|\nabla \Psi|_{e}^{2}\right) \text { dvol }_{e}
\end{aligned}
$$

follows readily after integrating by parts in the expression

$$
\int \chi_{R_{0}} r^{-\beta}\left(\Delta_{\mathbb{R}^{d}} \Psi\right)^{2} d \text { vol }_{e}=0
$$

Thus, for any $\Psi \in \mathcal{H}_{R_{0}, \beta}^{2}$ we can bound

$$
\begin{aligned}
& \int_{\{R \leq r \leq 2 R\}} r^{2-\beta+\varepsilon}\left(\left|\nabla^{2} \Psi_{h r m}\right|_{e}^{2}+r^{-2}\left|\nabla \Psi_{h r m}\right|_{e}^{2}\right) d v o l_{e} \\
& \quad+\int_{\left\{r \geq R_{0}\right\}}\left(r^{-\beta}\left|\nabla^{2} \Psi_{h r m}\right|_{e}^{2}+r^{-2-\beta}\left|\nabla \Psi_{h r m}\right|_{e}^{2}\right) d v o l_{e} \leq C_{R_{0}, \beta, \varepsilon}\left\langle\Psi_{h r m}, \Psi_{h r m}\right\rangle_{R_{0}} .
\end{aligned}
$$

Therefore, adding (B.93) and (B.97) for $u$ in place of $\Psi$ and using a triangle inequality and the fact that

$$
\langle u, u\rangle_{R_{0}}=\left\langle u_{\text {orth }}, u_{\text {orth }}\right\rangle_{R_{0}}+\left\langle u_{h r m}, u_{h r m}\right\rangle_{R_{0}},
$$


we readily deduce the desired bound (B.75) for $l=2$ :

$$
\begin{aligned}
& \int_{\{R \leq r \leq 2 R\}} r^{2+\varepsilon}\left(r^{-\beta}\left|\nabla^{2} u\right|_{e}^{2}+r^{-2-\beta}|\nabla u|_{e}^{2}\right) \text { dvol }_{e} \\
& \quad+\int_{\left\{r \geq R_{0}\right\}}\left(r^{-\beta}\left|\nabla^{2} u\right|_{e}^{2}+r^{-2-\beta}|\nabla u|_{e}^{2}\right) d \text { vol }_{e} \\
& \leq C_{R, R_{0}, \beta, \varepsilon} \int_{\left\{r \geq R_{0}\right\}} r^{-\beta}\left(\Delta_{\mathbb{R}^{d}} u\right)^{2} \text { dvol }_{e} \\
& \quad+C_{R_{0}, \beta, \varepsilon} \int_{\left\{R_{0} \leq r \leq 2 R_{0}\right\}}\left(\left|\nabla^{2} u\right|_{e}^{2}+|\nabla u|_{e}^{2}\right) d \text { vol }_{e}
\end{aligned}
$$

Inequality (B.75) for $2<l \leq\left\lfloor\frac{d+1}{2}\right\rfloor$ follows in a similar way, and hence the details will be omitted.

\section{B.3 Lemma on Harmonic Functions on $(\mathcal{S}, \boldsymbol{h})$}

Lemma B.4 Let $\bar{\delta}>0$ be small in terms of the geometry of $(\mathcal{S}, h)$. If a function $u: \mathcal{S} \rightarrow \mathbb{R}$ with

$$
\begin{aligned}
& \int_{\mathcal{S}} r_{+}^{-\beta}\left|\nabla^{2} u\right|_{h}^{2} d v o l_{\tilde{h}}+\int_{\mathcal{S}}\left(-\log \left(r_{-}\right)+1\right)^{-2} r_{-}^{-1} r_{+}^{-2-\beta}|\nabla u|_{h}^{2} d v o l_{\tilde{h}} \\
& \quad \times \int_{\left\{r \leq r_{0}\right\}}|Y u|^{2} \operatorname{dvol}_{\tilde{h}}<+\infty
\end{aligned}
$$

for some $\beta \in(-\bar{\delta}, 1)$ and $r_{0}>0$ solves

$$
\Delta_{h, \omega} u=0
$$

satisfying the following boundary condition on $\partial_{\text {tim }} \mathcal{S}$ :

$$
\int_{\partial_{\text {tim }} \mathcal{S}} u \cdot Y u d h_{t a n} \geq 0,
$$

then $u$ is necessarily a constant function.

Proof By standard elliptic regularity results (see i.e. [18]), $u \in C^{\infty}(\mathcal{S} \backslash \partial \mathcal{S})$. Let us fix some $\bar{\delta}<\varepsilon \ll 1$ small enough in terms of $1-\beta$ and the geometry of $(\mathcal{S}, h)$ (this is possible since $\bar{\delta}$ was considered small in terms of $(\mathcal{S}, h))$. In this way, $0<\beta+\varepsilon<1$.

Suppose first that

$$
\int_{\mathcal{S}}\left(r_{+}^{-\beta-\varepsilon}|\nabla u|_{h}^{2}+r_{+}^{-2-\beta-\varepsilon} u^{2}\right) d v o l_{h}<+\infty .
$$

In this case, we will show that $u \equiv 0$. 
We can define for $\rho \geq R_{0}$ (large in terms of the geometry of $(\mathcal{S}, h)$ ) the function:

$$
f(\rho) \doteq \int_{\{r=\rho\}} r^{-\beta-\varepsilon}\left(|\nabla u|_{h}^{2}+r^{-2} u^{2}\right) \operatorname{dvol}_{h,\{r=\rho\}}
$$

Due to the flat asymptotics of $(\mathcal{S}, h)$, from (B.102) we deduce that:

$$
\int_{R_{0}}^{+\infty} f(\rho) d \rho<+\infty
$$

Therefore, since the function $\frac{1}{\rho}$ is not integrable in $\{\rho \gg 1\}$, using the pigeonhole principle we deduce that there exists a sequence $\left\{R_{n}\right\}_{n \in \mathbb{N}}$ tending to $+\infty$ such that

$$
R_{n} \cdot f\left(R_{n}\right) \rightarrow 0
$$

Without loss of generality, we can assume that $r_{0}$ small in terms of the geometry of $(\mathcal{S}, h)$. In the $\left[0, r_{0}\right] \times \partial_{h o r} \mathcal{S}$ coordinate chart near $\partial_{h o r} \mathcal{S}, h$ takes the form

$$
h=\left(r^{-1}+O(1)\right) d r^{2}+h_{t a n},
$$

and thus our main assumption

$$
\int_{\mathcal{S}}\left(-\log \left(r_{-}\right)+1\right)^{-2} r_{-}^{-1} r_{+}^{-2-\beta}|\nabla u|_{h}^{2} d v o l_{\tilde{h}}+\int_{\left\{r \leq r_{0}\right\}}|Y u|^{2} d v o l_{\tilde{h}}<+\infty
$$

implies (through a Hardy inequality for the zeroth order term) that

$$
\int_{0}^{r_{0}} \int_{\partial_{h o r} \mathcal{S}} r^{-1}(-\log (r))^{-2}\left(r\left(\partial_{r} u\right)^{2}+\left|\nabla^{\left(h_{\text {tan }}\right)} u\right|_{h_{\text {tan }}}^{2}+|u|^{2}\right) d h_{\text {tan }} d r<+\infty .
$$

Similarly, in the $\left[0, r_{0}\right] \times \partial_{\text {tim }} \mathcal{S}$ region, $h$ takes the form

$$
h=(1+O(r)) d r^{2}+h_{t a n},
$$

and thus (B.107) implies that

$$
\int_{0}^{r_{0}} \int_{\partial_{\text {tim }} \mathcal{S}} r^{-1}(-\log (r))^{-2}\left(\left(\partial_{r} u\right)^{2}+\left|\nabla^{\left(h_{\text {tan }}\right)} u\right|_{h_{\text {tan }}}^{2}+|u|^{2}\right) d h_{\text {tan }} d r<+\infty .
$$

Setting for $\rho \ll 1$ :

$$
g(\rho) \doteq \int_{\{r=\rho\}}(-\log (r))^{-1}\left(r_{h o r}\left(\partial_{r} u\right)^{2}+\left|\nabla^{\left(h_{\text {tan }}\right)} u\right|_{h_{\text {tan }}}^{2}+|u|^{2}\right) d h_{\text {tan }}
$$

since $f(r)=r^{-1} \log ^{-1}(r)$ is not integrable around 0, from (B.108), (B.110) and a trivial pigeonhole principle argument we infer the existence of a sequence $\left\{r_{n}\right\}_{n \in \mathbb{N}}$ 
tending to 0 so that:

$$
g\left(r_{n}\right) \rightarrow 0
$$

Let us fix a $C^{1}$ and piecewise $C^{2}$ function $w_{R_{1}}: \mathcal{S} \rightarrow(0,1]$ which satisfies the following properties for some $c>0$ depending only on the geometry of $\tilde{h}$ :

- $w_{R_{1}}$ is a function of $r$,

- $w_{R_{1}}=1$ for $r<R_{1}$,

- $\partial_{r} w_{R_{1}} \leq 0, \partial_{r}^{2} w_{R_{1}}<-c \cdot R_{1}^{-2}$ for $R_{1}<r<2 R_{1}$ and

- $w_{R_{1}}=\left(\frac{r}{R_{1}}\right)^{-\beta-\varepsilon}$ for $r>2 R_{1}$.

Since $(\mathcal{S}, h)$ has a finite number of asymptotically flat regions, we compute that in the $(r, \sigma)$ coordinate system on each connected component of the region $\{r \gg 1\}$ :

$$
\Delta_{h, \omega} w_{R_{1}}=\left(1+O\left(r^{-1}\right)\right) \cdot \partial_{r}^{2} w_{R_{1}}+\left(d-1+O\left(r^{-1}\right)\right) r^{-1} \partial_{r} w_{R_{1}}
$$

On the other hand, in the region $\left\{r<R_{1}\right\}$ we have $\Delta_{h, \omega} w_{R_{1}} \equiv 0$ since $w_{R_{1}}$ is a constant there.

Therefore, if $R_{1}$ is fixed large and $r_{1}$ is fixed small in terms the geometry of $(\mathcal{S}, h)$ and $\omega$, from (B.113) and the properties of $w_{R_{1}}$ (and the fact that $0<\beta+\varepsilon<1$ ) we infer that

$$
\Delta_{h, \omega} w_{R_{1}} \leq 0
$$

almost everywhere on $\mathcal{S}$ (recall that $w_{R_{1}}$ is $C^{1}$ but only piecewise $C^{2}$ ).

Let us fix the vector fields $v_{1}=-|\nabla r|_{h}^{-1} \nabla r$ in the region $\{r \ll 1\}$ and $v_{2}=$ $|\nabla r|_{h}^{-1} \nabla r$ in the region $\{r \gg 1\}$. Since $\Delta_{h, \omega} u=0$ by assumption, after integrating by parts (and using the fact that $w_{R_{1}}$ is $C^{1}$ and piecewise $C^{2}$ ) we obtain for any integer $n$ sufficiently large:

$$
\begin{aligned}
0= & -\int_{\left\{r_{n} \leq r \leq R_{n}\right\}} w_{R_{1}} \omega \cdot \Delta_{h, \omega} u \cdot u \text { dvol }_{h} \\
= & \int_{\left\{r_{n} \leq r \leq R_{n}\right\}}\left(\omega \cdot w_{R_{1}}|\nabla u|_{h}^{2}-\frac{1}{2} \omega \cdot \Delta_{h, \omega} w_{R_{1}} \cdot u^{2}\right) d v o l_{h} \\
& -\int_{\left\{r=r_{n}\right\}}\left(\omega \cdot w_{R_{1}} v_{1} u \cdot u-\frac{1}{2} \omega\left(v_{1} w_{R_{1}}\right) \cdot u^{2}\right) \operatorname{dvol}_{h,\left\{r=r_{n}\right\}} \\
& -\int_{\left\{r=R_{n}\right\}}\left(\omega \cdot w_{R_{1}} v_{2} u \cdot u-\frac{1}{2} \omega\left(v_{2} w_{R_{1}}\right) \cdot u^{2}\right) \operatorname{dvol}_{h,\left\{r=R_{n}\right\}} .
\end{aligned}
$$

In view if the form of $h$ in the $\left[0, r_{0}\right] \times \partial \mathcal{S}$ coordinate chart (see (B.4) and (B.5)), we compute that

$$
\begin{aligned}
d \operatorname{vol}_{h,\left\{r=r_{n}\right\}} & =\left.d h_{\text {tan }}\right|_{r=r_{n}}, \\
v_{1} & =-r_{h o r}^{\frac{1}{2}}(1+O(r)) \partial_{r},
\end{aligned}
$$


and

$$
|\nabla u|_{h}^{2}=r_{h o r}\left(\partial_{r} u\right)^{2}+\left|\nabla^{\left(h_{\text {tan }}\right)} u\right|_{h_{\text {tan }}}^{2} .
$$

Since $\int_{0}^{r_{0}} \int_{\partial_{\text {tim }} \mathcal{S}}\left|\left(\nabla^{(\tilde{h})}\right)^{2} u\right|_{h}^{2} d h_{\text {tan }} d r<+\infty$ and $h$ is regular up to $\partial_{\text {tim }} \mathcal{S}$, by applying a trace theorem we infer that $Y u$ has a well defined limit on $L^{2}\left(\partial_{t i m} \mathcal{S}, h_{S}\right)$. Thus, since $w_{R_{1}}=1$ and $\omega \sim r_{\text {hor }}^{\frac{1}{2}}$ near $\partial \mathcal{S}$, we obtain in view of (B.111) and (B.112):

$$
\begin{aligned}
& \limsup _{n \rightarrow+\infty} \int_{\left\{r=r_{n}\right\}} \omega\left(w_{R_{1}} v_{1} u \cdot u-\frac{1}{2}\left(v_{1} w_{R_{1}}\right) \cdot u^{2}\right) d \operatorname{vol}_{h,\left\{r=r_{n}\right\}} \\
& \leq-\int_{\partial_{\text {tim }} \mathcal{S}} Y u \cdot u d h_{\text {tan }}+0 \leq 0,
\end{aligned}
$$

because of (B.101).

Similarly, by a Cauchy-Schwarz inequality we have because of (B.105) as $n \rightarrow$ $+\infty$ :

$$
\begin{aligned}
& \left|\int_{\left\{r=R_{n}\right\}} \omega\left(w_{r_{1}, R_{1}} v_{2} u \cdot u-\frac{1}{2}\left(v_{2} w_{r_{1}, R_{1}}\right) \cdot u^{2}\right) d \operatorname{vol}_{h,\left\{r=R_{n}\right\}}\right| \\
& \quad \leq C_{R_{1}, \varepsilon, \beta} \cdot \int_{\{r=\rho\}} r^{-\varepsilon-\beta}\left(|\nabla u|_{h} \cdot u+r^{-1} u^{2}\right) d v o l_{h,\{r=\rho\}} \\
& \quad \leq C_{R_{1}, \varepsilon, \beta} R_{n} \cdot f\left(R_{n}\right) \rightarrow 0
\end{aligned}
$$

Thus, by letti ng $n \rightarrow+\infty$ in (B.115), from (B.119), (B.120) and (B.114) we deduce that:

$$
\int_{\mathcal{S}} \omega\left(w_{R_{1}}|\nabla u|_{h}^{2}+w_{2} \cdot u^{2}\right) d v o l_{h} \leq 0
$$

for some suitably decaying $w_{2} \geq 0$ which is not identically 0 . Therefore, $u \equiv 0$.

In order to establish Lemma B.4, therefore, it suffices to establish that for any $u \in C^{\infty}(\mathcal{S})$ satisfying $\Delta_{h, \omega} u=0$ and (B.99), there exists some constant $c_{u}$ so that $u-c_{u}$ satisfies (B.102). In view of (B.99), it suffices to show that

$$
\int_{\left\{r \geq 2 R_{u}\right\}} r^{-\varepsilon-\beta}\left(|\nabla u|_{h}^{2}+r^{-2}\left(u-c_{u}\right)^{2}\right) d v o l_{h}<+\infty
$$

for some $R_{u}$ large depending on $u$ itself.

We will work in the $(r, \sigma)$ coordinate system on a single connected component $\mathcal{N}_{1}$ of the region $\{r \gg 1\}$ (since the proof for each component is identical, this is not actually a restriction). In this coordinate system on $\mathcal{N}_{1}$, we will define the coordinate flat metric

$$
e=d r^{2}+r^{2} g_{\mathbb{S}^{d-1}}
$$

and the associated flat Laplacian:

$$
\Delta_{\mathbb{R}^{d}} \doteq r^{-(d-1)} \partial_{r}\left(r^{d-1} \partial_{r}\right)+r^{-2} \Delta_{\mathbb{S}^{d-1}} .
$$


Since $\Delta_{h, \omega} u=0$ and $\Delta_{h, \omega}$ has the asymptotics (B.7), while $h$ is asymptotically of the form (B.2), $u$ satisfies the equation

$$
\Delta_{\mathbb{R}^{d}} u=F_{u},
$$

where

$$
F_{u}=O\left(r^{-1}\right) \nabla^{2} u+O\left(r^{-2}\right) \nabla u .
$$

We will first show that there exists a smooth solution $u_{1}$ to the boundary value problem

$$
\begin{cases}\Delta_{\mathbb{R}^{d}} u_{1}=F_{u}, & \left\{r \geq R_{u}\right\} \cap \mathcal{N}_{1} \\ u_{1}=0 & \text { on }\left\{r=R_{u}\right\} \cap \mathcal{N}_{1}\end{cases}
$$

with:

$$
\int_{\left\{r \geq R_{u}\right\} \cap \mathcal{N}_{1}} r^{-\beta}\left(\left|\nabla u_{1}\right|_{e}^{2}+r^{-2} u_{1}^{2}\right) d v o l_{e}<+\infty .
$$

To this end, considering the sequence of cut-off functions $\chi_{R_{n}}=\chi\left(\frac{r}{R_{n}}\right)$ for some dyadic sequence $\left\{R_{n}\right\}_{n \in \mathbb{N}}$ and some fixed smooth cut-off function $\chi: \mathbb{R} \rightarrow[0,1]$ satisfying $\chi(x)=1$ for $x \leq 1$ and $\chi(x)=0$ for $x \geq 2$, we first solve the boundary value problems:

$$
\begin{cases}\Delta_{\mathbb{R}^{d}} u_{1, n}=\chi_{R_{n}} \cdot F_{u}, & \left\{r>R_{u}\right\} \cap \mathcal{N}_{1} \\ u_{1, n}=0 & \text { on }\left\{r=R_{u}\right\} \cap \mathcal{N}_{1}\end{cases}
$$

The existence of solutions $u_{1, n}$ to (B.129) readily follows using the variational approach: Let us define the Hilbert space $\mathcal{H}_{R_{u}}^{1}$ as the completion of the vector space $C_{0, R_{u}}^{\infty}$

$$
C_{0, R_{u}}^{\infty}=\left\{\Psi \in C_{0}^{\infty}\left(\left\{r \geq R_{u}\right\} \cap \mathcal{N}_{1}\right)|\Psi|_{r=R_{u}}=0\right\}
$$

with the norm

$$
\|\Psi\|_{\mathcal{H}_{R_{u}}^{1}} \doteq\left(\int_{\left\{r>R_{u}\right\} \cap \mathcal{N}_{1}}\left(|\nabla \Psi|_{e}^{2}+r^{-2} \Psi^{2}\right) d v l_{e}\right)^{1 / 2} .
$$

Then the function $\mathcal{F}_{n}: C_{0, R_{u}}^{\infty} \rightarrow \mathbb{R}$ given by the relation

$$
\mathcal{F}_{n}(\Psi) \doteq \int_{\left\{r>R_{u}\right\} \cap \mathcal{N}_{1}}\left(\frac{1}{2}|\nabla \Psi|_{e}^{2}-\chi_{R_{n}} F_{u} \cdot \Psi\right) \text { dvol }_{e}
$$

extends to a continuous function on $\mathcal{H}_{R_{u}}^{1}$, since $\chi_{R_{n}} F_{u}$ is compactly supported. Thus, we can bound through a Cauchy-Schwarz inequality for any $v \in \mathcal{H}_{R_{u}}^{1}$

$$
\int_{\left\{r>R_{u}\right\} \cap \mathcal{N}_{1}}\left|\chi_{R_{n}} F_{u} \cdot v\right| d \operatorname{vol}_{e} \lesssim R_{n} \|\left. v\right|_{\mathcal{H}_{R_{u}}^{1}}
$$


Moreover, $\mathcal{F}_{n}$ is convex and satisfies $\mathcal{F}_{n}(\Psi) \rightarrow+\infty$ as $\|\Psi\|_{\mathcal{H}_{R_{u}}^{1}} \rightarrow+\infty$. By minimizing $\mathcal{F}_{n}$ over $\mathcal{H}_{R_{u}}^{1}$ using the usual techniques (and using elliptic regularity results), we arrive at a strong solution $u_{1, n}$ of (B.129) belonging to $\mathcal{H}_{R_{u}}^{1}$.

Since $\left\|u_{1, n}\right\|_{\mathcal{H}_{R_{u}}^{1}}<+\infty$, using the approach leading to (B.105) we deduce that there exists a dyadic sequence $\left\{\rho_{m}^{(n)}\right\}_{m \in \mathbb{N}}$ such that

$$
\rho_{m}^{(n)} \cdot \int_{\left\{r=\rho_{m}^{(n)}\right\} \cap \mathcal{N}_{1}}\left(|\nabla u|_{e}^{2}+r^{-2} u^{2}\right) \operatorname{dvol}_{e,\{r=\rho\}} \leq 1 .
$$

Hence, by multiplying (B.129) with $r^{-\beta} u_{1, n}$ and integrating by parts over $\left\{R_{u}<r<\right.$ $\left.\rho_{m}^{(n)}\right\} \cap \mathcal{N}_{1}$, we obtain after taking the limit $m \rightarrow+\infty$ :

$$
\begin{aligned}
& \int_{\left\{r>R_{u}\right\} \cap \mathcal{N}_{1}}\left(r^{-\beta}\left|\nabla u_{1, n}\right|_{e}^{2}-\frac{1}{2}\left(\Delta_{\mathbb{R}^{d}} r^{-\beta}\right) u_{1, n}^{2}\right) \text { dvol }_{e} \\
& \quad \leq \int_{\left\{r>R_{u}\right\} \cap \mathcal{N}_{1}} \chi_{R_{n}} r^{-\beta} F_{u} \cdot u_{1, n} d \text { vol }_{e} .
\end{aligned}
$$

Notice that

$$
\Delta_{\mathbb{R}^{d}} r^{-\beta}=-\beta(d-2-\beta) \cdot r^{-\beta-2} \leq \begin{cases}0, & \beta \in[0,1) \\ (d-1) \cdot \bar{\delta} r^{-\beta-2} & \beta \in(-\bar{\delta}, 0]\end{cases}
$$

since $d \geq 3$. Using also a Hardy-type inequality of the form established in Lemma (C.2), we deduce from (B.135) and (B.136) (and the assumption that $\bar{\delta}$ is small in terms of the geometry of $(\mathcal{S}, h))$ :

$$
\begin{aligned}
& \int_{\left\{r>R_{u}\right\} \cap \mathcal{N}_{1}}\left(r^{-\beta}\left|\nabla u_{1, n}\right|_{e}^{2}+r^{-2-\beta} u_{1, n}^{2}\right) d \text { vol }_{e} \\
& \leq C_{\beta} \int_{\left\{r>R_{u}\right\} \cap \mathcal{N}_{1}} \chi_{R_{n}} r^{-\beta} F_{u} \cdot u_{1, n} \text { dvol }_{e} .
\end{aligned}
$$

Using a Cauchy-Schwarz inequality, from (B.137) we infer that

$$
\begin{aligned}
& \int_{\left\{r>R_{u}\right\} \cap \mathcal{N}_{1}}\left(r^{-\beta}\left|\nabla u_{1, n}\right|_{e}^{2}+r^{-2-\beta} u_{1, n}^{2}\right) \text { dvol }_{e} \\
& \quad \leq C_{\beta} \int_{\left\{r>R_{u}\right\} \cap \mathcal{N}_{1}} \chi_{R_{n}} r^{2-\beta}\left|F_{u}\right|^{2} \text { dvol }_{e} \leq C_{\beta, u}<+\infty
\end{aligned}
$$

with the constants not depending on $n$. Similarly, we can also bound:

$$
\begin{aligned}
& \int_{\left\{r>R_{u}\right\} \cap \mathcal{N}_{1}}\left(r^{-\beta}\left|\nabla\left(u_{1, n+1}-u_{1, n}\right)\right|_{e}^{2}+r^{-2-\beta}\left(u_{1, n+1}-u_{1, n}\right)^{2}\right) d v l_{e} \\
& \leq C_{\beta} \int_{\left\{r>R_{u}\right\} \cap \mathcal{N}_{1}}\left(\chi_{R_{n+1}}-\chi_{R_{n}}\right) r^{2-\beta}\left|F_{u}\right|^{2} \text { dvol }_{e} .
\end{aligned}
$$


From (B.126) and (B.99) we can bound

$$
\int_{\left\{r>R_{u}\right\} \cap \mathcal{N}_{1}} r^{2-\beta}\left|F_{u}\right|^{2} \text { dvol }_{e} \leq C_{u}<\infty .
$$

Thus, from (B.138) and (B.139) we infer that the sequence of functions $u_{1, n}$ converges to a function $u_{1}$ which is a weak (and hence strong, due to elliptic regularity) solution of (B.127) satisfying (B.128).

Moreoever, after integrating by parts in the expression

$$
\begin{gathered}
\int_{\left\{r \geq R_{u}\right\} \cap \mathcal{N}_{1}} r^{-\beta}\left(1-\chi_{2 R_{u}}\right)\left(\Delta_{\mathbb{R}^{d}} u_{1}\right)^{2} \text { dvol }_{e} \\
=\int_{\left\{r \geq R_{u}\right\} \cap \mathcal{N}_{1}} r^{-\beta}\left(1-\chi_{2 R_{u}}\right) F_{u}^{2} \text { dvol }_{e}
\end{gathered}
$$

and using (B.128), (B.99) and the fact that $\left(\left\{r \geq R_{u}\right\} \cap \mathcal{N}_{1}, e\right)$ is flat, we obtain that:

$$
\int_{\left\{r \geq 2 R_{u}\right\} \cap \mathcal{N}_{1}} r^{-\beta}\left|\nabla^{2} u_{1}\right|_{e}^{2} \text { dvol }_{e}<+\infty .
$$

We will now return to our function $u \in C^{\infty}(\mathcal{S})$ solving $\Delta_{h, \omega} u=0$ and satisfying (B.99). Setting on $\left\{r \geq 2 R_{u}\right\} \cap \mathcal{N}_{1}$

$$
u_{2}=u-u_{1},
$$

the new function $u_{2}$ will satisfy on $\left\{r>2 R_{u}\right\} \cap \mathcal{N}_{1}$ :

$$
\Delta_{\mathbb{R}^{d}} u_{2}=0
$$

(being smooth by elliptic regularity) and

$$
\int_{\left\{r \geq 2 R_{u}\right\} \cap \mathcal{N}_{1}} r^{-\beta}\left(\left|\nabla^{2} u_{2}\right|_{e}^{2}+r^{-2}\left|\nabla u_{2}\right|_{e}^{2}\right) d v o l_{e}<+\infty
$$

because of (B.99), (B.141) and (B.128).

For any $\rho \geq 2 R_{u},\left.u_{2}\right|_{\{r=\rho\} \cap \mathcal{N}_{1}}$ is a smooth function on $\mathbb{S}^{d-1}$ (by elliptic regularity) and hence we can decompose $\left.u_{2}\right|_{\{r=\rho\} \cap \mathcal{N}_{1}}$ in spherical harmonics. This turns (B.143) into the following system of ODE's:

$$
r^{-(d-1)} \frac{d}{d r}\left(r^{d-1} \frac{d}{d r}\left(u_{2}\right)_{m}\right)-\Lambda_{m} r^{-2}\left(u_{2}\right)_{m}=0,
$$

where the integer $m$ corresponds to an enumeration of the spherical haromics, $\Psi_{m}$ denotes the projection of a function $\Psi \in L^{2}\left(\mathbb{S}^{d-1}\right)$ on $m$-th spherical harmonic, and 
$-\Lambda_{m}$ is the eigenvalue of $\Delta_{\mathbb{S}^{d-1}}$ corresponding to the $m$-th spherical harmonic (by convention $\Lambda_{m}$ is increasing in $m$ and $\Lambda_{0}=0$ ). Therefore, we can explicitly solve:

$$
\left(u_{2}\right)_{m}=c_{m} r^{a_{m}}+d_{m} r^{-(d-2)-a_{m}}
$$

where:

- $c_{m}, d_{m} \in \mathbb{R}$

- $a_{m} \in \mathbb{N}$ is an increasing sequence with $a_{0}=0$ and $a_{1}=1$.

By (B.144) we deduce that

$$
\begin{aligned}
& \sum_{m \in \mathbb{N}} \int_{r=2 R_{u}}^{+\infty} r^{-\beta}\left\{\left(\frac{d^{2}\left(u_{2}\right)_{m}}{d r^{2}}\right)^{2}\right. \\
& \left.+\left(\Lambda_{m}+1\right) r^{-2}\left(\frac{d\left(u_{2}\right)_{m}}{d r}\right)^{2}+\left(\Lambda_{m}^{2}+1\right) r^{-4}\left(u_{2}\right)_{m}^{2}\right\} r^{d-1} d r<+\infty
\end{aligned}
$$

which, in view of the fact that $\beta \in(-\bar{\delta}, 1)$, forces $c_{m}=0$ for $m \neq 0$ in (B.146). Therefore, from (B.146), (B.147) and the fact that $c_{m}=0$ for $m \neq 0$, we infer that for all $m \in \mathbb{N}$ :

$$
\sum_{m \in \mathbb{N}} \int_{r=2 R_{u}}^{+\infty} r^{-\beta-\varepsilon}\left\{\left(\frac{d\left(u_{2}\right)_{m}}{d r}\right)^{2}+\Lambda_{m} r^{-2}\left(u_{2}\right)_{m}^{2}\right\} r^{d-1} d r<+\infty
$$

(recall that $\Lambda_{0}=0$ ). Therefore

$$
\int_{\left\{r \geq 2 R_{u}\right\} \cap \mathcal{N}_{1}} r^{-\beta-\varepsilon}\left|\nabla u_{2}\right|_{e}^{2} \operatorname{dvol}_{e}<+\infty .
$$

Furthermore, using standard ode theory (and elliptic regularity), we can establish in this case that the series $\sum_{m}\left(u_{2}\right)_{m} e_{m}$ converges to $u_{2}$ pointwise (together with all its derivatives). Moreover, because of (B.146) and the fact that $c_{m}=0$ for $m \neq 0$, we deduce that:

$$
\lim _{r \rightarrow+\infty} u_{2}=c_{0}
$$

Thus, (B.149) implies that (through a Hardy-type inequality) that:

$$
\int_{\left\{r \geq 2 R_{u}\right\} \cap \mathcal{N}_{1}} r^{-\beta-\varepsilon}\left(\left|\nabla u_{2}\right|_{e}^{2}+r^{-2}\left(u_{2}-c_{0}\right)^{2}\right) d v o l_{e}<+\infty .
$$

All in all, from (B.128) and (B.151) and the fact that $u=u_{1}+u_{2}$, we finally infer (B.122) for $c_{u}=c_{0}$ :

$$
\int_{\left\{r \geq 2 R_{u}\right\} \cap \mathcal{N}_{1}} r^{-\beta-\varepsilon}\left(|\nabla u|_{e}^{2}+r^{-2}\left(u-c_{0}\right)^{2}\right) d v o l_{e}<+\infty .
$$

Hence, the proof of the Lemma is complete. 


\section{Appendix C: Hardy Type Inequalities}

In this Section, we will state the main Hardy type inequalities that are used throughout this paper. We will start with the following lemma:

Lemma C.1 For any strictly increasing function $g:(0,+\infty) \rightarrow \mathbb{R}$, the following inequality holds for any $u \in C^{1}((0,+\infty))$ and $0<a<b$ :

$$
\begin{aligned}
& \int_{a}^{b} \frac{d g}{d x} \cdot|u|^{2} d x+g(a)|u(a)|^{2} \\
& \quad \leq C_{g} \cdot\left(\int_{a}^{b} g^{2}\left(\frac{d g}{d x}\right)^{-1} \cdot\left|\frac{d u}{d x}\right|^{2} d x+g(b)|u(b)|^{2}\right)
\end{aligned}
$$

while for $g$ strictly decreasing we have:

$$
\begin{aligned}
& \int_{a}^{b}\left(-\frac{d g}{d x}\right)|u|^{2} d x+g(b)|u(b)|^{2} \\
& \quad \leq C_{g} \cdot\left(\int_{a}^{b}\left(-g^{2}\left(\frac{d g}{d x}\right)^{-1}\right) \cdot\left|\frac{d u}{d x}\right|^{2} d x+g(a)|u(a)|^{2}\right) .
\end{aligned}
$$

Proof The proof follows readily after performing an integration by parts in the terms $\int_{a}^{b} \frac{d g}{d x}|u|^{2}$ and $\int_{a}^{b}\left(-\frac{d g}{d x}\right)|u|^{2}$ respectively, and then using a Cauchy-Schwarz inequality.

Lemma C.2 For any $k \in \mathbb{N}$ and $a \in \mathbb{R}$, there exists a constant $C_{k, a}>0$ such that for any function $\Psi \in C^{\infty}\left(\mathbb{R}^{d}\right)$ and any $0<R_{1}<R_{2}$ we can bound

$$
\begin{aligned}
& \sum_{j=1}^{\min \left\{\left\lfloor\frac{d-1+a}{2}\right\rfloor, k\right\}}\left\{\int_{\left\{R_{1} \leq r \leq R_{2}\right\}} r^{a-2 j}\left|\partial_{r}^{k-j} \Psi\right|^{2} d v o l_{e}+\int_{\left\{r=R_{1}\right\}} r^{a+1-2 j}\left|\partial_{r}^{k-j} \Psi\right|^{2} d \operatorname{vol}_{\left\{r=R_{1}\right\}}\right\} \\
& \leq C_{k, a} \cdot\left\{\int_{\left\{R_{1} \leq r \leq R_{2}\right\}} r^{a}\left|\partial_{r}^{k} \Psi\right|^{2} \text { dvol }_{e}+\sum_{j=1}^{\left\lfloor\frac{d-1+a}{2}\right\rfloor} \int_{\left\{r=R_{2}\right\}} r^{a+1-2 j}\left|\partial_{r}^{k-j} \Psi\right|^{2} \operatorname{dvol}_{\left\{r=R_{2}\right\}}\right\},
\end{aligned}
$$

where $\partial_{r}$ denotes the gradient of the polar distance $r$ on $\mathbb{R}^{d}$, dvol $l_{e}$ is the natural volume form of the flat metric e on $\mathbb{R}^{d}$ and $d v o l_{\left\{r=R_{i}\right\}}$ is the natural volume form of the induced metric on the sphere $\left\{r=R_{i}\right\}$.

Proof From (C.1) for $g(x)=x^{b+1}$ for any $b>-1$, it follows that for any function $h \in C^{\infty}(\mathbb{R})$ :

$$
\int_{R_{1}}^{R_{2}} x^{b}|h|^{2} d x+x^{b+1}\left|h\left(R_{1}\right)\right|^{2} \leq C_{b} \cdot\left(\int_{R_{1}}^{R_{2}} x^{b+2}\left|\frac{d h}{d x}\right|^{2} d x+x^{b+1}\left|h\left(R_{2}\right)\right|^{2}\right)
$$


(with the constant $C_{b}$ depending only on $b$ ). Therefore, recalling that in polar coordinates $(r, \sigma)$ on $\mathbb{R}^{d}$ we can write for any $b>0$ and $h \in C^{\infty}\left(\mathbb{R}^{d}\right)$ :

$$
\int_{\left\{R_{1} \leq r \leq R_{2}\right\}} r^{b}|h|^{2}=\int_{R_{1}}^{R_{2}} r^{b+d-1}\left(\int_{\mathbb{S}^{d-1}} h(r, \sigma) d \sigma\right) d r
$$

inequality (C.3) follows readily after repeated applications of (C.4).

Let $(\mathcal{M}, g)$ be as in Section 8. Recall that we denote with $\nabla_{h_{\tau, N}}$ the covariant derivative associated to the Riemannian metric $h_{\tau, N}$ on the hyperboloid $\{\bar{t}=\tau\}$ (see Section 8). Let us also denote with $L$ the $\partial_{v}$ coordinate vector field in the $(v, \sigma)$ coordinate chart on each connected component of $\{\bar{t}=\tau\} \cap\{r \gg 1\}$ (notice that this vector field does not coincide with the $\partial_{v}$ vector field on $\mathcal{M}$ in the $(u, v, \sigma)$ coordinate chart). Using Lemma C.2, we can establish the following Hardy type inequality for functions $\Psi$ on the hyperboloids $\{\bar{t}=$ const $\}$ with tame behaviour near $\mathcal{I}^{+}$:

Lemma C.3 There exists an $R_{0}>0$ (large) such that for any $\tau>0$, any $k \in \mathbb{N}$, any integer $0 \leq m \leq k$, any $a \in \mathbb{R}$, any smooth tensor field $\Psi$ on $\{\bar{t}=\tau\}$ satisfying $\lim _{r \rightarrow+\infty} r^{\frac{d+a-2}{2}}\left|\mathcal{L}_{L}^{l} \Psi\right|_{h_{\tau, N}}=0$ for $0 \leq l \leq k$, and any $R_{1}>R_{0}$ we can bound

$$
\begin{aligned}
& \quad \sum_{j=1}^{\min \left\{\left\lfloor\frac{d-1+a}{2}\right\rfloor, k\right\}}\left\{\int_{\left\{r \geq R_{1}\right\} \cap\{\bar{t}=\tau\}} r^{a-2 j}\left|\mathcal{L}_{L}^{k-j} \Psi\right|^{2} \Omega^{2} d v d \sigma\right. \\
& \left.\quad+\int_{\left\{r=R_{1}\right\} \cap\{\bar{t}=\tau\}} r^{a+1-2 j}\left|\mathcal{L}_{L}^{k-j} \Psi\right|^{2} \Omega^{2} d \sigma\right\} \\
& \leq C_{k, a} \cdot \int_{\left\{r \geq R_{1}\right\} \cap\{\bar{t}=\tau\}} r^{a}\left|\mathcal{L}_{L}^{k} \Psi\right|^{2} \Omega^{2} d v d \sigma,
\end{aligned}
$$

where $\mathcal{L}_{L}$ denotes the Lie derivative in the direction of $L$.

Proof Fix an $R_{2}>R_{1}$. An application of Lemma C.2 on each connected component of $\left\{R_{1} \leq r \leq R_{2}\right\} \cap\{\bar{t}=\tau\}$ (using the coordinate chart $(v, \sigma)$ in the region $\left\{r \geq R_{1}\right\} \cap\{\bar{t}=$ $\tau$ \}, so that $\mathcal{L}_{L}$ becomes differentiation with respect to $\partial_{v}$ ) readily yields that:

$$
\begin{aligned}
& \sum_{j=1}^{\min \left\{\left\lfloor\frac{d-1+a}{2}\right\rfloor, k\right\}}\left\{\int_{\left\{R_{1} \leq r \leq R_{2}\right\} \cap\{\bar{t}=\tau\}} r^{a-2 j}\left|\mathcal{L}_{L}^{k-j} \Psi\right|^{2} \Omega^{2} d v d \sigma\right. \\
& \left.\quad+\int_{\left\{r=R_{1}\right\} \cap\{\bar{t}=\tau\}} r^{a+1-2 j}\left|\mathcal{L}_{L}^{k-j} \Psi\right|^{2} \Omega^{2} d \sigma\right\} \\
& \leq C_{k, a} \cdot\left(\int_{\left\{R_{1} \leq r \leq R_{2}\right\} \cap\{\bar{t}=\tau\}} r^{a}\left|\mathcal{L}_{L}^{k} \Psi\right|^{2} \Omega^{2} d v d \sigma\right. \\
& \quad+\sum_{j=1}^{\left\lfloor\int_{\left\{r=R_{2}\right\} \cap\{\bar{t}=\tau\}} r^{a+1-2 j}\left|\mathcal{L}_{L}^{k-j} \Psi\right|^{2} \Omega^{2} d \sigma\right) .}
\end{aligned}
$$


Since $\lim _{r \rightarrow+\infty} r^{\frac{d+a-2}{2}}\left|\mathcal{L}_{L}^{l} \Psi\right|_{h_{\tau, N}}=0$ for $0 \leq l \leq k$, we infer:

$$
\lim _{R_{2} \rightarrow+\infty} \sum_{j=1}^{\left\lfloor\frac{d-1+a}{2}\right\rfloor} \int_{\left\{r=R_{2}\right\} \cap\{\bar{t}=\tau\}} r^{a+1-2 j}\left|\mathcal{L}_{L}^{k-j} \Psi\right|^{2} \Omega^{2} d \sigma=0 .
$$

Thus, (C.5) follows from (C.6) after taking the limit $R_{2} \rightarrow+\infty$.

We will also need the following "critical" Hardy type inequality:

Lemma C.4 For any $R>0$ large in terms of the geometry of $\{\bar{t}=\tau\}$ and any smooth function $\Psi$ on $\{\bar{t}=\tau\}$ satisfying $\lim _{r \rightarrow+\infty}|\Psi| \rightarrow 0$ we can bound:

$$
\begin{aligned}
\int_{\{R \leq r \leq 2 R\} \cap\{\bar{t}=\tau\}} r^{-d}|\Psi|^{2} d h_{N} \leq & C \cdot\left(\int_{\{r \geq R\} \cap\{\bar{t}=\tau\}} r^{-d}|\Psi|^{2} d h_{N}\right)^{\frac{1}{2}} \\
& \times\left(\int_{\{r \geq R\} \cap\{\bar{t}=\tau\}} r^{-d+2}|L \Psi|^{2} d h_{N}\right)^{\frac{1}{2}} .
\end{aligned}
$$

Remark The exponent of the second term of the right hand side of (C.8) can not be increased, since then the inequality would not be satisfied by the function $\Psi_{R_{0}}(r)=$ $\frac{\log \left(R_{0}\right)}{\log (r)+\log \left(R_{0}\right)}$ for some large enough $R_{0}>0$.

Proof It suffices to establish the following inequality on $\mathbb{R}^{d}$ for any $R>0$ and any real $\Psi \in C^{\infty}\left(\mathbb{R}^{d}\right)$ sarisfying $\lim _{r \rightarrow+\infty}|\Psi| \rightarrow 0$ :

$$
\begin{aligned}
\int_{\{R \leq r \leq 2 R\}} r^{-d} \Psi^{2} \text { dvol }_{e} \leq & C \cdot\left(\int_{\{r \geq R\}} r^{-d} \Psi^{2} \text { dvol }_{e}\right)^{\frac{1}{2}} \\
& \times\left(\int_{\{r \geq R\}} r^{-d+2}\left(\partial_{r} \Psi\right)^{2} \text { dvol }_{e}\right)^{\frac{1}{2}} .
\end{aligned}
$$

In turn, (C.9) will follow (using polar coordinates) by the following "critical" Hardytype inequality on $\mathbb{R}$ for any function $\Psi \in C^{\infty}(\mathbb{R})$ with $\lim _{r \rightarrow+\infty}|\Psi| \rightarrow 0$ :

$$
\int_{R}^{2 R} r^{-1} \Psi^{2} d r \leq C \cdot\left(\int_{R}^{+\infty} r^{-1} \Psi^{2} d r\right)^{\frac{1}{2}}\left(\int_{R}^{+\infty} r \cdot\left(\partial_{r} \Psi\right)^{2} d r\right)^{\frac{1}{2}}
$$

In order to establish (C.10), let us fix a continuous and piecewise $C^{1}$ function $\chi_{1}:[0,+\infty) \rightarrow[0,1]$ by the relation:

$$
\chi_{1}(x)= \begin{cases}0, & x \leq 1 \\ x-1, & 1 \leq x \leq 2 \\ 1, & x \geq 2\end{cases}
$$


and define the function $\chi_{1, R}:[0,+\infty) \rightarrow[0,1]$ by the relation:

$$
\chi_{1, R}(r) \doteq \chi_{1}\left(\frac{r}{R}\right)
$$

Using the fact that $\chi_{1}$ is contnuous and piecewise $C^{1}$, we obtain after integrating by parts (in view of the fact that $\lim _{r \rightarrow+\infty} \Psi=0$ ):

$$
\begin{aligned}
\int_{0}^{+\infty} \partial_{r} \chi_{1, R} \cdot \Psi^{2} d r= & -2 \int_{0}^{+\infty} \chi_{1, R} \cdot \Psi \partial_{r} \Psi d r \\
& \leq\left(\int_{0}^{+\infty} \chi_{1, R} r^{-1} \Psi^{2} d r\right)^{\frac{1}{2}}\left(\int_{0}^{+\infty} \chi_{1, R} r \cdot\left(\partial_{r} \Psi\right)^{2} d r\right)^{\frac{1}{2}} .
\end{aligned}
$$

Thus, (C.10) follows from (1) in view of the fact that $\chi_{1, R}$ is supported on $[R,+\infty)$ and $\partial_{r} \chi_{1, R}$ is identically 1 on $[R, 2 R]$ and 0 elsewhere.

\section{References}

1. Andersson, L., Blue, P.: Hidden symmetries and decay for the wave equation on the Kerr spacetime. Ann. Math. 182(3), 787-853 (2015)

2. Angelopoulos, Y.: Global spherically symmetric solutions of non-linear wave equations with null condition on extremal Reissner-Nordström spacetimes. International Mathematics Research Notices (to appear)

3. Aretakis, S.: Stability and instability of extreme Reissner-Nordström black hole spacetimes for linear scalar perturbations II. Annales Henri Poincaré 12(8), 1491-1538 (2011)

4. Blue, P., Soffer, A.: Semilinear wave equations on the Schwarzschild manifold I: local decay estimates. Preprint. gr-qc/0310091 (2003)

5. Blue, P., Sterbenz, J.: Uniform decay of local energy and the semi-linear wave equation on Schwarzschild space. Commun. Math. Phys. 268(2), 481-504 (2006)

6. Bondi, H., Van der Burg, M., Metzner, A.: Gravitational waves in general relativity. VII. Waves from axi-symmetric isolated systems. Proc. R. Soc. Lond. Ser. A. Math. Phys. Sci. 269(1336), 21-52 (1962)

7. Christodoulou, D., and Klainerman, S.: The Global Nonlinear Stability of the Minkowski Space, vol. 1 of Princeton Mathematical Series. Princeton University Press, Princeton (1993)

8. Dafermos, M., Rodnianski, I.: A note on energy currents and decay for the wave equation on a Schwarzschild background. Preprint. arXiv:0710.0171 (2007)

9. Dafermos, M., Rodnianski, I.: The red-shift effect and radiation decay on black hole spacetimes. Commun. Pure Appl. Math. 62(7), 859-919 (2009)

10. Dafermos, M., Rodnianski, I.: Decay for solutions of the wave equation on Kerr exterior spacetimes I-II: The cases $|a| \ll M$ or axisymmetry. Preprint. arXiv:1010.5132 (2010)

11. Dafermos, M., Rodnianski, I.: A new physical-space approach to decay for the wave equation with applications to black hole spacetimes. In: XVIth International Congress on Mathematical, Physics, pp. 421-432 (2010)

12. Dafermos, M., Rodnianski, I.: A proof of the uniform boundedness of solutions to the wave equation on slowly rotating Kerr backgrounds. Invent. Math. 185(3), 467-559 (2011)

13. Dafermos, M., Rodnianski, I.: Lectures on black holes and linear waves. In: Evolution Equations, Clay Mathematics Proceedings, vol. 17. American Mathematical Society, Providence, pp. 97-205 (2013)

14. Dafermos, M., Holzegel, G., Rodnianski, I.: A scattering theory construction of dynamical vacuum black holes. Preprint. arXiv:1306.5364 (2013)

15. Dafermos, M., Holzegel, G. Rodnianski, I.: The linear stability of the Schwarzschild solution to gravitational perturbations. ArXiv Preprint arXiv:1601.06467 (2016)

16. Dafermos, M., Rodnianski, I., Shlapentokh-Rothman Y.: Decay for solutions of the wave equation on Kerr exterior spacetimes III: the full subextremal case $|a| \ll$ M. Preprint. arXiv:1402.7034 (2014) 
17. Friedlander, F.G.: Notes on the wave equation on asymptotically Euclidean manifolds. J. Funct. Anal. 184(1), 1-18 (2001)

18. Gilbarg, D., Trudinger, N.S.: Elliptic Partial Differential Equations of Second Order, vol. 224. Springer, Berlin (2001)

19. Hebey, E.: Nonlinear Analysis on Manifolds: Sobolev Spaces and Inequalities, vol. 5. American Mathematical Society, Providence (1999)

20. Gustav, H.: Ultimately Schwarzschildean spacetimes and the black hole stability problem. Preprint. arXiv:1010.3216 (2010)

21. Klainerman, S.: Uniform decay estimates and the Lorentz invariance of the classical wave equation. Commun. Pure Appl. Math. 38(3), 321-332 (1985)

22. Klainerman, S.: Remarks on the global Sobolev inequalities in the Minkowski space $R^{n+1}$. Commun. Pure Appl. Math. 40(1), 111-117 (1987)

23. Laul, P., Metcalfe, J., Tikare, S., Tohaneanu, M.: Localized energy estimates for wave equations on (1+4)-dimensional Myers-Perry space-times. SIAM J. Math. Anal. 47(3), 1933-1957 (2015)

24. Luk, J.: Improved decay for solutions to the linear wave equation on a Schwarzschild black hole. Annales Henri Poincaré 11(5), 805-880 (2010)

25. Luk, J.: A vector field method approach to improved decay for solutions to the wave equation on a slowly rotating Kerr black hole. Anal. PDE 5(3), 553-625 (2012)

26. Luk, J.: The null condition and global existence for nonlinear wave equations on slowly rotating Kerr spacetimes. J. Eur. Math. Soc. 15(5), 1629-1700 (2013)

27. Morawetz, C.S.: The decay of solutions of the exterior initial-boundary value problem for the wave equation. Commun. Pure Appl. Math. 14(3), 561-568 (1961)

28. Morawetz, C.S.: The limiting amplitude principle. Commun. Pure Appl. Math. 15(3), 349-361 (1962)

29. Moschidis, G.: Logarithmic local energy decay for scalar waves on a general class of asymptotically flat spacetimes. Preprint

30. Nirenberg, L.: On Elliptic Partial Differential Equations. Springer, New York (2011)

31. Oliver, J.: A vector field method for non-trapping, radiating space-times. Preprint. arXiv:1410.5154 (2014)

32. Ralston, J.V.: Trapped rays in spherically symmetric media and poles of the scattering matrix. Commun. Pure Appl. Math. 24(4), 571-582 (1971)

33. Sachs, R.K.: Gravitational waves in general relativity. VIII. Waves in asymptotically flat space-time. Proc. R. Soc. Lond. Ser. A. Math. Phys. Sci. 270(1340), 103-126 (1962)

34. Schlue, V.: Decay of linear waves on higher-dimensional Schwarzschild black holes. Anal. PDE 6(3), 515-600 (2013)

35. Sogge, C.D.: Lectures on Non-linear Wave Equations. International Press, Boston (2008)

36. Stein, Elias M, Weiss, Guido L.: Introduction to Fourier Analysis on Euclidean Spaces, vol. 1. Princeton University Press, Princeton (1971)

37. Tataru, D.: Local decay of waves on asymptotically flat stationary space-times. Am. J. Math. 135(2), 361-401 (2013)

38. Tataru, D., Tohaneanu, M.: A local energy estimate on Kerr black hole backgrounds. Int. Math. Res. Not. 2011(2), 248-292 (2011)

39. Yang, S.: Global solutions of nonlinear wave equations in time dependent inhomogeneous media. Arch. Ration. Mech. Anal. 209(2), 683-728 (2013)

40. Yang S.: On the quasilinear wave equations in time dependent inhomogeneous media. Preprint. arXiv:1312.7264 (2013)

41. Yang, S.: Global stability of solutions to nonlinear wave equations. Sel. Math. 21, 833-881 (2014) 